\title{
Dynamics and Mechanism of DNA-Bending Proteins in Binding Site Recognition
}

\author{
By \\ Velmurugu Yogambigai \\ B.S., University of Jaffna, Sri Lanka, 1999 \\ M.Phil, University of Peradeniya, Sri Lanka, 2005 \\ M.S., University of Illinois at Chicago, Chicago, 2007
}

THESIS

Submitted as partial fulfilment of the requirements

for the degree of Doctor of Philosophy in Physics

in the Graduate College of the

University of Illinois at Chicago, 2016

Chicago, Illinois

Defense committee:

Anjum Ansari, Chair and Advisor

Jung-Hyun Min, Department of Chemistry, UIC

Mark Schlossman

Phoebe Rice, Biochemistry and molecular Biophysics, University of Chicago

Fatemeh Khalili 
This thesis is affectionately dedicated to the memory of my farther Kumarasamy Velmurugu and my loving mother Karunadevi Velmurugu 


\section{ACKNOWLEDGMENT}

First and foremost, I offer my deepest gratitude to my advisor, Professor Anjum Ansari for her excellent guidance and care throughout in every aspect of this research. She is an exceptional advisor in all possible ways. She was there for me all the many years, never short of alternative ideas or guidance to pick me up whenever I faltered. Her patience and consistent support helped me overcome many adverse situations and complete this dissertation. There are many things I have learned from working with her about personal integrity, high expectations of excellence, and much, much more - that extends far beyond the laboratory. No matter where I go or what I do, I will be carry on the lessons which she taught me and be better because of it.

I would like to thank the rest of my thesis committee: Prof. Mark Schlossman, Prof. Phoebe Rice, Prof. Jung-Hyun Min, Prof. Fatemeh Khalili, for all of their encouragement and insightful comments. A special thanks to Dr. Serguei Kouznetsov for teaching me the experimental technique involved in this study. His dedication and perfectionism helped me to learn the ability of not only just appreciate the success of an experimental end results but also to be equally passionate about the experimental design and procedures. I sincerely thank Physics department head, Prof. David Hofman and all my teachers at UIC for their help and encouragement in numerous ways.

Many thanks are due to our collaborator Prof. Phoebe Rice at the University of Chicago for her generous donation of IHF protein and it's mutants in large amounts whenever we needed it and for her help in interpreting our data. Her valuable comments, suggestions and expertise always directed us forward in the IHF project. I also would like 


\section{ACKNOWLEDGMENT (Continued)}

to thank Dr. Ying Z. Pigli at the University of Chicago for her help with expression and purification of IHF protein and its mutants.

I am ever grateful to our collaborator Prof. Jung-Hyun Min at the UIC Chemistry department, for her involvement in each and every step of the Rad4 project. Her thoughtful guidance, vibrant ideas, critical comments and positive approach when tackling challenges were invaluable assets and have helped me grow up as a researcher. None of the T-jump experiments would have been possible without the help and hard work of Prof. Min's graduate student Xuejing Chen. Thanks Xuejing for always preparing the samples for me with your busy schedule. I would also like to thank Phillip Slogoff-Sevilla for repeating the equilibrium experiments in $\operatorname{Rad} 4$ project over and over again until we got consistent results.

I sincerely thank our collaborator, Prof. Manju Hingorani at Wesleyan University, for her generous gift of MutS and Msh2-6 protein and for the insightful discussions and valuable advice in MutS/Msh2-6 projects.

I am also grateful to the former and current group members, for their various forms of support during my graduate studies: Dr. Paula Vivas, Dr. Ranjani Narayanan, Dr. Manas Kumar Sarangi, Jorjethe Roca, Sagnik Chakraborty, Mitchell Connolly and Viktoriya Zvoda. All of you have been part of this research at different points in time. Jorjethe Roca, Sagnik Chakraborty, Mitchell Connolly, and Viktoriya Zvoda, I appreciate your careful proofreading of this thesis 


\section{ACKNOWLEDGMENT (Continued)}

I also appreciate the efforts of many undergraduate students who did the preliminary equilibriums experiments for the Rad4 project: Akash Patel, Neilsen Little, Chris and Oluwapelumi Aderonke.

I owe a special thanks to the UIC Physics Department staff Melissa Mattingly Derrik Stanley, Brian Shim, James Nell and Melodie Shaw, Silvia Fortuna Dias and Chemeck Slowik and instrument makers in the physics workshop, Kevin, Rick, Rich, and Dave from physics workshop and all my classmates. All of you made this journey pleasant.

Many thanks to my friends whose support and care helped me overcome difficult times and stay focused on my studies. My sincere appreciation I send to friends and families back in my hometown, Jaffna, Sri Lanka. Their life, struggle and courage taught me valuable lessons in life. They are my inspiration and their stories always motivate me to rise again from setbacks. I am also grateful to the Sri Lankan families here in Chicago, they helped me in numerous ways.

Most importantly, none of this would have been possible without the support, strength and patience of my family. My family's hard work and sacrifice helped me to pursue graduate studies in USA. Thank you so much, Amma, Ambiga, Gnana, Jega, Kandee, Kiruba and Vimala, for always hiding the family difficulties from me and repeatedly using the mantra "Please focus on your work and we will take care of it". I also would like to thank my mother-in-law and father-in-law for their support and belief in me. 


\section{ACKNOWLEDGMENT (Continued)}

I must acknowledge my husband Sankar Poopalasingam whose love, constructive criticism and encouragement helped me in various ways to finish these studies. Finally I want to express my deepest appreciation to my lovely daughter Biranvi Ambigai for her great patience and for always being happy and cheering me up.

It's been a long journey. To those whom I may have forgotten to thank please accept my apology and my sincere thanks.

V.YOGA 


\section{TABLE OF CONTENTS}

CHAPTER

Page No.

I INTRODUCTION....................................

1.1 Protein-DNA interactions .............. 1

1.2 Sequence-dependent DNA deformability and its role in target recognition 3

1.2.1 Free energy cost for local deformation of DNA. .............

1.2.2 Sequence-dependent base-pair opening rate measured by NMR imino proton exchange .............. 8

1.2.3 How do site-specific proteins search for their target sites on genomic DNA? ..... 9

1.2.4 How do site-specific proteins recognize their target sites? ............. 11

1.2.5 Conformational capture or protein-induced DNA bending............. 14

1.2.6 Measurements of DNA binding and bending kinetics ............. 14

1.2.7 Competition between 1-D diffusion and binding-site recognition: the "speed-stability" paradox. $\quad$................ 16

$\begin{array}{lll}\text { 1.3 Experimental techniques to study dynamics of protein-DNA interactions... } & 17\end{array}$

$\begin{array}{ll}\text { 1.3.1 Laser temperature-jump spectroscopy. ......... } & 18\end{array}$

$1.4 \quad$ Thesis Overview.............. 20

II METHODS.................................................... 33

2.1 Equilibrium measurements............. 33

2.2 Laser Temperature Jump technique.............. 33

2.2.1 Laser Temperature jump spectrometer ......... 36 
2.2.2 Theoretical estimation of the size of the T-jump............. 38

2.2.3 Photo-acoustic effects and cavitation. .............. 39

2.2.4 Estimation of temperature jump using reference sample in a T-jump experiment............. 40

2.2.5 T-jump recovery kinetics............. 43

2.2.6 Discrete single- or double-exponential decay convoluted with T-jump recovery 46

2.2.7 Acquisition and matching of relaxation traces measured over different time-scales 46

2.2.8 Maximum entropy analysis............. 48

2.3 Equilibrium FRET measurements.............. 50

2.4 Nucleotide analog 2-Aminopurine (2AP) ............. 59

2.5 Fraction of Protein and DNA in complex at Equilibrium............. 61

$2.6 \mathrm{~K}_{\mathrm{D}}$ measurements from equilibrium FRET ........................ 62

2.6.1 Conventional titration experiments .......................... 62

2.6.2 Salt titration experiments.......................... 63

III Integration Host Factor (IHF)-DNA interaction..................... 67

$\begin{array}{lll}3.1 & \text { Introduction............... } & 67\end{array}$

3.1.1 Integration host factor (IHF) .............

3.1.2 IHF binds to the minor groove on DNA and recognizes its specific site via indirect readout.............. 68 
3.1.3 Structure of IHF-H' complex............. 69

3.1.4 Background of IHF/H’ interaction dynamics ............. 73

3.1.5 Binding site recognition versus protein diffusional search............. 78

$\begin{array}{lr}3.2 \text { Results............... } & 80\end{array}$

3.2.1 DNA bending kinetics in the IHF - H' complex are biphasic............. 80

3.2.2 The slow phase occurs on the same time scale as spontaneous bp

opening at a kink site. ............. $\quad 82$

3.2.3 Introducing mismatches at the site of the kinks affects the slow

phase but not the fast phase. ..............

3.2.4 DNA bending rates in the slow phase of IHF- TT8AT

complex reflect enhanced base-pair opening rates in mismatched DNA............. 90

3.2.5 DNA modifications away from the kink sites have no effect

on either of the two rates. .............. 92

3.2.6 Two plausible scenarios for biphasic relaxation kinetics.............. 95

3.2.7 Salt-dependence of the fast and slow components. .............. 95

3.2.8 Protein mutations distal to the kink sites affect affinity and

bending rate of slow phase............. 101

3.2.9 Control experiments to rule out contributions to the relaxation

kinetics from dye dynamics or dye interactions with protein or DNA............ 108 
3.3 Discussion

3.3.1. Nonspecific search and specific recognition by IHF

3.3.2. Nonspecific binding of DNA by IHF and its structurally

3.3.3 Nonspecific binding facilitates 1-D diffusion on DNA................

3.4 Concluding Remarks.............. 117

$\begin{array}{ll}\text { Statement of Authorship for chapter IV } & 123\end{array}$
IV LESION RECOGNITION BY XERODERMA PIGMENTOSUM C (XPC)

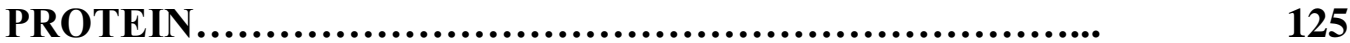

4.1 Introduction.............. 125

4.1.1 Nucleotide excision repair (NER) ............. 125

4.1.2 Experimental design.............. 132

4.2 Method............... 135

4.2.1 Preparation of double-stranded DNA substrates. .............. 135

4.2.2 Preparation of Rad4-Rad23 complexes. .............. 135

4.2.3 Duplex melting temperatures of mismatched and undamaged/matched DNA. .......................

4.2.4 Apparent binding affinities (Kd,app) determined by electrophoretic mobility shift assays....................................

4.2.5 Equilibrium FRET temperature scan experiments with $\mathrm{tCo} / \mathrm{tCnitro}$ probes. 
4.2.6 Acquisition and analyses of T-jump relaxation traces. .............. 139

4.3 Results .............. 145

4.3.1 Kinetics of Rad4 (wild type) induced DNA opening rate.............. 145

4.3.2 $\mathrm{tC}_{\mathrm{o}}$ and $\mathrm{tC}_{\text {nitro }}$ FRET pair as probes for sensing changes in

DNA helical structure. .............. 159

4.3.3 DNA bending dynamics measured with extrinsically attached FRET pair/ AN7 ............. 189

4.4 Discussion............................ 196

4.4.1 Rad4/XPC induced nucleotide flipping/ Open dynamics measured with 2AP probe............................... 196

4.4.2 Rad4/XPC induced helical distortion dynamics measured using tco/tcniro........ 198

4.4.3 Rad4/XPC induced DNA bending dynamics measured using TAMRA/Cy5 FRET pair.............. 204

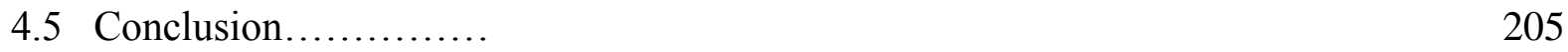

V DNA MISMATCH REPAIR...................................... $\quad 213$

5.1 Introduction............... 213

5.1.1 Structural Studies on MutS bound to mismatched DNA.............. 216

5.1.2 What role does the intrinsic flexibility of DNA play in the mismatch recognition and subsequent repair? .............. 218 
5.2 Results..............

5.2.1 Taq MutS binding to mismatch (T-bulge) DNA as probed by 2AP ............ 221

5.2.2 Taq MutS binding to mismatch (T-bulge) DNA as probed by FRET pair.

5.2.3 MutS $\alpha$ binding to mismatch (T-bulge) DNA as probed by $2 \mathrm{AP}$ (in DNA) and Trp (in MutS)

5.3 Discussion............... 238

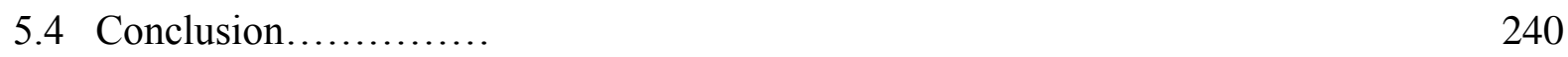

APPENDIX A...................................................... $\quad 244$

1.1 Energetic cost for bending DNA.............................. 244

1.2. DNA Geometry ............................ 245

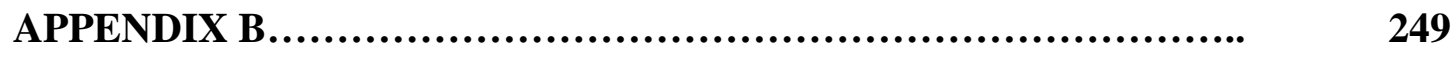

2.1 Equilibrium perturbation using temperature jump................................. 249

2.2 Temperature jump relaxation kinetics...................... 250

2.3 Fluorescence resonance energy transfer (FRET)........................ 252 


\section{LIST OF TABLES}

\section{$\begin{array}{llll}\text { Chapter } & \text { Table No } & \text { Table } & \text { Page No. }\end{array}$}

III I DNA sequence on $\lambda$ phage DNA that are............. 69

II Sequences of $\mathrm{H}^{\prime}$ substrate and its variants.............. 77

III Relaxation rates and dissociation constants.............. 83

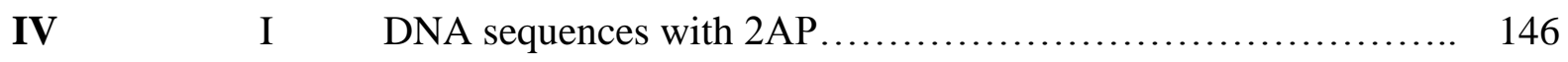

II $\quad \mathrm{K}_{\mathrm{d} \text {,app }}$ and $\mathrm{R}^{2}$ for different DNA constructs............... 150

III Diffusion constants (D) and residence times............... 156

IV Melting temperature of AN12 and AN12U................. 161

V Melting temperature of AN14 and AN14U................. 161

VI $\quad \mathrm{K}_{\mathrm{d}, \mathrm{app}}$ and $\mathrm{R}^{2}$ for different DNA constructs.................. 163

VII Relaxation times for Rad4-DNA complexes............ $\quad \ldots \quad 177$

VIII $\quad \mathrm{K}_{\mathrm{d}, \text { app }}$ and $\mathrm{R}^{2}$ for different DNA constructs............... 182

IX $\quad$ FRET mismatched DNA sequence......................... 189

V I DNA sequences with 2AP and FRET for MutS ........... 220

II DNA sequences with 2AP for Msh2-6 ................. 233 


\section{LIST OF FIGURES}

Figure

Page No

1.1 The crystal structure nucleosome.............................. 4

$1.2 \quad$ Nearest-neighbor(nn) stacking free energies...................... 6

1.3 Schematic representation of the stacked-to-unstacked conformation........... 7

$1.4 \quad$ Base pair breathing rates............................................ 9

1.5 The mechanism of facillitated diffusion................................... 10

$1.6 \quad$ Watson and Crick base pairs. ......................................... 12

1.7 NMR solution structure of the dimeric lac repressor........................ 13

1.8 Crystal structure IHF, Rad4, MutS proteins........................... 20

2.1 Schematic of laser temperature jump spectrometer set up................. 36

2.2 Fluorescence intensity variation as a function of temperature................. 42

2.3 The magnitude of the temperature jump in T-jump $\ldots \ldots \ldots \ldots \ldots \ldots \ldots \ldots \ldots . . . \ldots 3$

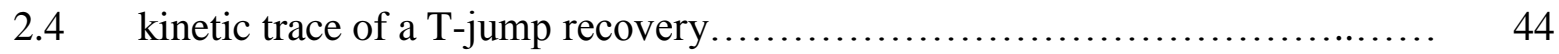

$2.5 \quad \mathrm{~T}$-jump recovery times during several T-jump experiments................. 45

2.6 Kinetics traces taken on two separate time-scales........................ 48

2.7 Analysis of the distribution of relaxation times............................... 50

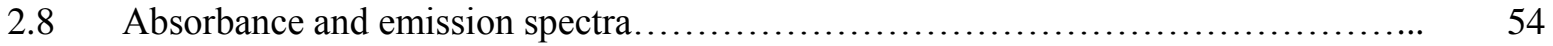




\section{LIST OF FIGURES (Continued)}

Figure

Page No

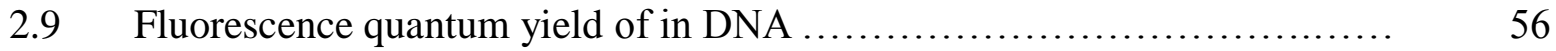

2.10 Normalized absorption and emission spectra of FRET donor and acceptor..... 57

2.11 Cytosine analogs $\left(\mathrm{tC}^{\mathrm{o}}\right.$ and $\left.\mathrm{tC}_{\text {nitro }}\right)$ FRET pair.......................... 59

2.12 chemical structure of $2 \mathrm{AP}$ and adenine.................................. 60

2.13 Example of binding affinity measurements (mutant IHF) ................... 64

3.1 Cocrystal structures of the IHF/HU family............................. 70

3.2 Cartoon figure of FRET dyes attached to DNA and illustration of T-jump......... 73

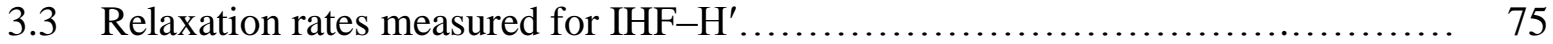

3.4 T-jump kinetics measurements and relaxation rates on $\mathrm{H}^{\prime}-\mathrm{IHF} \ldots \ldots \ldots \ldots \ldots . . . . .61$

3.5 Two phase kinetics rate of IHF-H' complex......................... 82

3.6 The scheme illustrates two possible pathways........................... 84

3.7 Equilibrium measurements on IHF-TT8AT. FRET ........................ 85

3.8 T-jump kinetics measurements on TT8AT loop-IHF $\ldots \ldots \ldots \ldots \ldots \ldots \ldots \ldots \ldots \ldots \ldots$

3.9 T-jump kinetics measurements on TT loop-IHF $\ldots \ldots \ldots \ldots \ldots \ldots \ldots \ldots \ldots \ldots \ldots$ 


\section{LIST OF FIGURES (Continued)}

Figure

Page No

3.10 T-jump kinetics measurements on AT loop-IHF

3.11 Biphasic rates of TT8AT loop-IHF ................................ 90

3.12 Cartoon of matched DNA base pair opening/closing rate..................... 91

3.13 A proposed mechanism for the IHF-DNA complex formation............... 92

3.14 T-jump kinetics measurements and relaxation rates on H'44A-IHF ........... 94

3.15 Equilibrium and kinetics measurements on IHF-H' ..................... 97

3.16 Kinetic traces of H' - IHF complex with $50 \mathrm{mM}$ salt..................... 99

3.17 Kinetic traces of H'- IHF complex with $100 \mathrm{mM}$ salt...................... 99

3.18 Kinetic traces of H'- IHF complex with $150 \mathrm{mM}$ salt..................... 100

3.19 Kinetic traces of H'- IHF complex with $200 \mathrm{mM}$ salt...................... 100

3.20 The rates and amplitudes for H'-IHF complex at salt $50 \mathrm{mM} \ldots \ldots \ldots \ldots \ldots \ldots \ldots . \ldots 1$

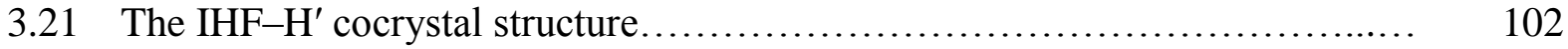

3.22 Equilibrium measurements on mutant IHF-H'. FRET $\ldots \ldots \ldots \ldots \ldots \ldots \ldots \ldots \ldots \ldots$

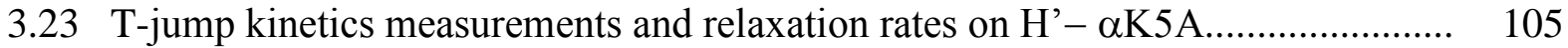

3.24 . T-jump kinetics measurements and relaxation rates on $\mathrm{H}^{\prime}-\beta \mathrm{K} 84 \mathrm{~A} \ldots \ldots \ldots \ldots \ldots . . . . .106$ 


\section{LIST OF FIGURES (Continued)}

Figure

Page No

3.25 T-jump kinetics measurements and relaxation rates on $H^{\prime}-\alpha \mathrm{R} 21 \mathrm{C}$

107

3.26 Control experiment to verify the fast phase

109

3.27 The rates and amplitudes of the two relaxation phases of control experiment $1 \ldots$

3.28 The rates and amplitudes of the two relaxation phases of control experiment $2 \ldots$

3.29 The rates and amplitudes of the two relaxation phases of control experiment $3 \ldots \quad 112$

$4.1 \quad$ NER repair pathway..................................................... 127

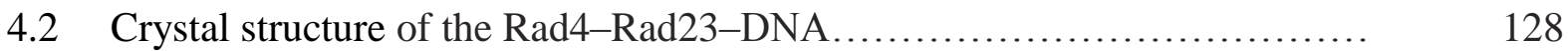

4.3 Crystal structure determination of $\operatorname{Rad} 4-\operatorname{Rad} 23$ bound to undamaged DNA.......

4.4 DNA conformational rearrangements during lesion recognition...................

4.5 Rad4-induced DNA-opening dynamics measured by T-jump.............. 147

4.6 Sequence-context dependence of Rad4-induced DNA-opening dynamics

4.7 2AP fluorescence and T-jump measurements to examine mutant Rad4-induced....

4.8 Relaxation rates measured on Rad4-mismatched DNA complexes.

4.9 Relaxation rates measured on full length Rad4 in complex with

mismatch DNA. 


\section{LIST OF FIGURES (Continued)}

Figure

Page No

4.10 Calculated free energy profiles for base pair opening matched/mismatched.....

4.11 Free energy profile for DNA opening by Rad4 at damaged and

undamaged sites

4.12 The DNA groove widths in Rad4-bound DNA structures

and the DNA construct sequences.

4.13 DNA conformational rearrangements during lesion

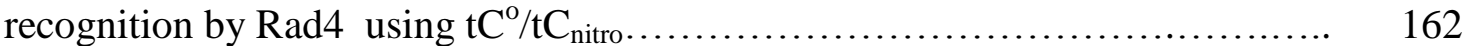

4.14 AN12 and AN12u DNA constructs........................ 164

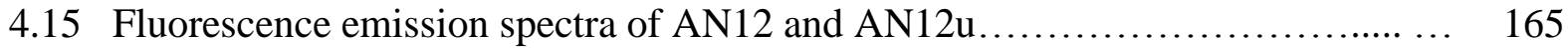

4.16 FRET efficiencies of free and protein-bound

AN12/AN12u under equilibrium

166

4.17 Equilibrium and T-jump measurements on

AN12 bound to Rad4

168

4.18 Equilibrium temperature scan and T-jump kinetics measurements

on $\mathrm{AN} 12 \mathrm{u}-\mathrm{Rad} 4$ complexes.

172 


\section{LIST OF FIGURES (Continued)}

Figure

Page No

4.19 Analysis of T-jump relaxation traces for AN12-(wild-type)

Rad4 complex

4.20 Rapid pre-melting kinetics on AN12-Rad4 complexes

174

4.21 Equilibrium and T-jump measurements on AN12 bound

to $\operatorname{Rad} 4 \beta$-hairpin mutants

175

4.22 Analysis of T-jump relaxation traces for AN12- $\Delta \beta$-hairpin3 complex

4.23 Analysis of T-jump relaxation traces for AN12- $\triangle \mathrm{BHD} 3$ complex

4.24 AN14 and AN14u DNA constructs.

4.25 Equilibrium and T-jump measurements on AN14 and

AN14u bound to wild-type Rad4

183

4.26 Equilibrium temperature scan and T-jump kinetics measurements

on AN14 and AN14u in the absence of Rad4.

184

4.27 Equilibrium temperature scan and T-jump kinetics measurements

on AN14 and AN14u in the absence of Rad4

4.28 Analysis of T-jump relaxation traces for AN14-Rad4 complex 


\section{LIST OF FIGURES (Continued)}

Figure

Page No

4.29 Analysis of T-jump relaxation traces for $\mathrm{AN} 14 \mathrm{u}-\mathrm{Rad} 4$ complex...........

188

4.30 Equilibrium and T-jump kinetics of AN7 in the presence

and absence of Rad4

191

4.31 Mem analysis of kinetics presented in figure 4.30.

192

4.32 Equilibrium and T-jump measurements on AN7 bound to

Rad4 $\beta$-hairpin mutants $(\Delta \mathrm{BHD} 3, \Delta \beta$-hairpin3)

4.33 Mem analysis of kinetics presented in figure 4.32 ....

195

4.34 'Kinetic gating' mechanism for DNA damage recognition by Rad4/XPC

197

4.35 Free energy schematic along the conformational trajectory of lesion

recognition by $\mathrm{Rad} 4$

201

5.1 The occurrence of errors in DNA during three main....

5.2 Mismatch recognition and subsequent repair in a "mismatch repair pathway ...

5.3 Co-crystal structure of Taq MutS protein

5.4 Equilibrium measurements on Taq MutS-T-bulge DNA

complex with 2 AP probe.... 


\section{LIST OF FIGURES (Continued)}

Figure

Page No

5.5 T-jump relaxation traces for Taq MutS-T-bulge DNA complex............. 225

5.6 Melting curve of 2AP labelled 23 base pair T-bulge.................... 226

5.7 Equilibrium FRET measurements on Taq MutS-T-bulge (FRET) .............. 228

5.8 Melting curve of FRET labelled 18 bp T-bulge (TFTDNA) ................... 229

5.9 Equilibrium FRET measurements on Taq MutS-TFTDNA complex............ 230

5.10 T-jump measurements on Taq MutS-T-bulge DNA (FRET) complex.......... 231

5.11 Relaxation rates of complexes, TDNA -Taq MutS....................... 233

5.12 Equilibrium measurements on yeast MutS $\alpha$-T-bulge DNA (37TDNA)......... 235

5.13 T-jump on yeast MutS $\alpha$-T-bulge DNA complex

with $2 \mathrm{AP}$ probe using UV laser.

237

5.14 Comparison of relaxation rates for all three different samples................. 238 


\section{LIST OF ABBREVIATIONS}

\begin{tabular}{|c|c|}
\hline IHF & Integration Host Factor \\
\hline XPC & Xeroderma pigmentosum, complementation group C \\
\hline $\operatorname{Rad} 4$ & Radiation-sensitive 4 (Rad4; yeast XPC ortholog) \\
\hline NER & Nucleotide excision repair pathway \\
\hline MMR & Mismatch repair pathway \\
\hline$T a q$ & Thermus aquaticus, A thermophilic bacteria \\
\hline T-jump & Temperature Jump \\
\hline DNA & Deoxyribonucleic Acid \\
\hline SS DNA & Single stranded DNA \\
\hline DS DNA & Double stranded DNA \\
\hline A & Adenine \\
\hline $\mathrm{T}$ & thymine \\
\hline $\mathrm{C}$ & Cytosine \\
\hline $\mathrm{D}$ & Guanine \\
\hline Int & $\lambda$ Integrase \\
\hline$\lambda$ DNA & $\lambda$ phage DNA \\
\hline $\mathrm{F}$ & Fluorescein-dT \\
\hline $\mathrm{R}$ & TAMRA-dT \\
\hline
\end{tabular}




\section{LIST OF ABBREVIATIONS (Continued)}

FRET

FCS

HU

MEM

$\mathrm{CD}$

NMR
Fluorescence energy transfer

Fluorescence correlation spectroscopy

Histone-like

Maximum entropy method

Circular dichroism

Nuclear Magnetic Resonance 


\section{SUMMARY}

Many cellular processes involve interactions between proteins and DNA in which proteins recognize and bind to specific sites on the DNA with thousand- or million-fold higher affinities than to random DNA sequences. How these proteins search for and find their specific sites in genomic DNA amidst a large excess ( $\sim 3$ billion) of nonspecific sites remains a puzzle. Many site-specific proteins kink, bend or twist DNA at that site, and undergo concerted conformational rearrangements to accommodate the deformed DNA ("induced-fit" mechanism). In many cases, the proteins discriminate between specific and nonspecific sites primarily by sensing differences in local DNA deformability ("indirect readout"), rather than by relying on direct interactions with target nucleotides. How rapidly the deformations occur during target recognition and how they compare with the time that a searching protein spends on a given DNA site before diffusing away remain largely unknown, obscuring our understanding of target recognition mechanisms. Direct observations of proteins undergoing one-dimensional diffusion on nonspecific DNA indicate "stepping" times (or "residence" times) per base pair (bp) ranging from $\sim 50 \mathrm{~ns}-500 \mu \mathrm{s}$. Dynamical interactions that lead to site recognition are expected to be fast or comparable to these "residence" times, thus on sub-millisecond time-scales.

Previous attempts to capture protein-DNA dynamics during target search and recognition have been limited due to lack of adequate time-resolution or sensitivity of the techniques used. In the studies presented in this thesis, we use laser temperature-jump (T-jump) perturbation approach in which $\sim 10 \mathrm{~ns}$ laser pulses at $1.55 \mu \mathrm{m}$ are absorbed by aqueous solution containing the biological samples to rapidly heat up a small volume of the sample, within the pulse-width of the laser. The T-jump perturbs the protein-DNA complexes, and the subsequent relaxation 


\section{SUMMARY (Continued)}

kinetics is monitored in the time-window of $\sim 20 \mu \mathrm{s}->50 \mathrm{~ms}$, before the T-jump decays back to the initial temperature, on time-scales of $\sim 200 \mathrm{~ms}$. Using a combination of unique sets of fluorescent probes, I have measured the dynamics of DNA distortions induced by site-specific proteins with high temporal resolution and sensitivity, and have uncovered previously unresolved DNA conformational dynamics during search and recognition for three protein-DNA complexes, described below. All three proteins rely primarily on indirect readout to recognize their DNA target site and therefore must have the ability to sense and discern sequence-dependent DNA deformability while searching DNA for their target sites.

The first system, discussed in Chapter III, is Integration Host Factor (IHF): a small ( 20 $\mathrm{kDa}$ ) prokaryotic architectural protein that recognizes and severely bends specific sites on $\lambda$ phage DNA into a U-turn. This sharp bend brings distal regions of DNA together to facilitate higher order protein-DNA complexes to be formed. IHF's small size and its ability to bend DNA to such a large degree make it an excellent candidate to study protein-DNA interactions, especially to examine the role that sequence-dependent DNA bendability/flexibility play in target recognition mechanisms. This study was carried out in collaboration with Prof. Phoebe Rice at the University of Chicago.

The crystal structure of IHF bound to a cognate DNA site shows that the sharp bend in DNA is introduced by kinking the DNA at two sites separated by $9 \mathrm{bp}$. IHF then wraps two long $\beta$-ribbon "arms" around the DNA and inserts conserved proline residues located on its arms into the gap created by the kinks to help stabilize this kinked/bent state. This specific complex is further stabilized by electrostatic interactions between the highly negatively charged DNA polymer and 


\section{SUMMARY (Continued)}

a stripe of positively charged residues on the contact interface of IHF. The kinetics of DNA bending/kinking in complex with IHF were first revealed as single-exponential decays in previous stopped-flow and laser T-jump measurements. The kinetics in these studies, as in this thesis, were monitored by time-resolved FRET measurements with fluorescein (donor) and TAMRA (acceptor) attached at either end of the DNA constructs, to probe changes in the "endto-end" distance. These studies showed that the DNA bending/kinking in binding site recognition by IHF occurred on time-scales similar to that for thermal disruption of a single A:T bp in canonical B-DNA, measured by NMR in the absence of any protein. These results suggested a "conformational capture" mechanism in which thermal fluctuations enable DNA to adopt "prebent" conformations, which, if they match the shape requirements of the DNA in the specific complex are then captured and stabilized by IHF. However, the time constants for these DNA kinking steps in the IHF-DNA complex, in the range of $\sim 1-10 \mathrm{~ms}$, were too slow in comparison with the range of reported one-dimensional diffusion times for various proteins on DNA. These results posed a puzzle as to how a rapidly diffusing protein can slow down enough at a potential binding site to be able to probe and recognize its binding site, and suggested that these previous studies were likely missing a faster "interrogation" step.

In this thesis, I present direct kinetic evidence for biphasic kinetics of DNA bending by IHF, with a rapid phase appearing on time-scales of $\sim 100 \mu \mathrm{s}-400 \mu \mathrm{s}$. Inserting mismatches in the DNA at the kink sites to make the DNA more flexible have no effect on this rapid phase while the slow phase speeds up. Introducing mutations in IHF protein that reduce the positive charge on its wrapping surface, thus destabilizing the specific complex, result in an increase in the 


\section{SUMMARY (Continued)}

relative amplitude of the fast phase. In contrast, increasing the salt concentrations in the buffer, which increases the specificity of IHF (i.e. increases its preference for specific sites versus nonspecific sites) diminishes the amplitude of the fast phase. Based on these results, we assign the fast component to nonspecific DNA bending by IHF prior to the formation of the specific complex and the slow component to the ultimate recognition step. An intriguing possibility is that this rapid phase represents conformational fluctuations in the protein as it switches from a nonspecific "search" mode to an "interrogation" mode, allowing the rapidly diffusing protein to slow down and examine a potential binding site. These sub-millisecond DNA deformation kinetics induced by a DNA bending protein bridge the gap between relatively slow (> ms) formation of specific complexes and relatively rapid $(<\mathrm{ms})$ one-dimensional diffusion times measured for proteins moving on DNA and enhance our understanding of target recognition mechanisms.

The second system, discussed in Chapter IV, is a DNA damage repair protein Rad4, a yeast ortholog of xeroderma pigmentosum $\mathrm{C}$ (XPC), which recognizes bulky lesions in DNA caused by several environmental factors such as UV rays, pollutants, cigarette smoke, food toxins, etc. Upon binding to a damage site, Rad4/XPC recruits downstream proteins to verify and repair the damage in the nucleotide excision repair (NER) pathway. This study was carried out in collaboration with Prof. Jung-Hyun Min and her group from the Department of Chemistry at UIC.

The crystal structures of Rad4 bound to UV-induced lesions showed that Rad4 inserts a $\beta$-hairpin into the lesion site, bends and unwinds the DNA at that site, and flips out two damage- 


\section{SUMMARY (Continued)}

containing nucleotides pairs. In this "open" recognition complex, Rad4 does not directly contact damaged nucleotides, but selectively accommodates the undamaged nucleotides on the complementary strand; such indirect binding enables Rad4/XPC to recognize extraordinarily diverse lesions. Intriguingly, the same "open" structure is also formed with Rad4 covalently tethered to undamaged DNA. These structural studies demonstrate that Rad4/XPC cannot discriminate between damaged and undamaged sites based on structural differences in the most stable states of the bound complexes; instead, lesion recognition must rely on the differences in the kinetics of recognition. However, how these distinct motions are orchestrated to lead to lesion discrimination and recognition has been unknown.

To map out the lesion recognition trajectory, I carried out T-jump measurements on Rad4 bound to model lesions (2- or 3-bp mismatch bubbles) that were shown to be recognized by Rad4 in a manner similar to a real lesion. The DNA distortion dynamics induced by Rad4 were monitored using three different spectroscopic probing strategies: (1) To measure nucleotide-flipping or "damage-opening" kinetics, we incorporated 2-aminopurine (2AP), a fluorescent analog of adenine, within the mismatch bubble. 2AP fluorescence within DNA depends on the extent of its stacking with nearest neighbors, making it a sensitive probe of local conformational changes in DNA; (2) To probe longer range motions that affect regions beyond the mismatched nucleotides, we incorporated a pair of recently developed FRET probes: cytosine analogs $\mathrm{tC}^{\circ}$ (donor) and $\mathrm{tC}_{\text {nitro }}$ (acceptor), on either side of the mismatch bubble. These probes are exquisitely sensitive to DNA helicity, making them highly suited to capture DNA unwinding dynamics; (3) To monitor DNA bending, we attached FRET pair: TAMRA (donor) and Cy5 (acceptor) at either end of the 


\section{SUMMARY (Continued)}

DNA constructs, to probe changes in the "end-to-end" distance upon Rad4 binding. Finally, to understand the role of $\beta$-hairpin of the protein in probing for lesions and/or stabilizing the recognition complex, we made measurements with mutants of $\operatorname{Rad} 4$ that do not have the $\beta$ hairpin.

T-jump measurements on the 2AP-labeled DNA constructs revealed that Rad4 can flip nucleotides within the mismatch lesions on time scales of $\sim 5-10 \mathrm{~ms}$. For the more stable, undamaged DNA, the nucleotide-flipping times are expected to be much longer and hence many orders of magnitude longer than the typical microseconds-regime "residence" times measured for many damage-repair proteins diffusing on DNA. These results suggest a "kinetic gating" mechanism for lesion discrimination whereby lesion selectivity arises from the kinetic competition between nucleotide flipping and diffusion times, and explain how Rad4/XPC avoids flipping "open" every undamaged site when searching DNA for a lesion, while having selectively higher probabilities to open helix-destabilizing NER lesion sites. For a tethered complex, the diffusion times are infinitely long, which explains why Rad4 can flip "open" even the undamaged nucleotides.

However, these results also posed a dilemma as to how Rad4 reliably recognizes damaged DNA if the recognition time $(\sim 5-10 \mathrm{~ms})$ is much slower than the diffusion times. Using the DNA constructs with the $\mathrm{tC}^{\mathrm{o}} / \mathrm{tC}$ nitro $\mathrm{FRET}$ probes, we uncovered new conformational dynamics occurring on the time-scales of $\sim 100-500 \mu$ s when Rad4 is nonspecifically bound to DNA. We interpret these dynamics as rapid unwinding ("twisting") of DNA by Rad4 resulting from the conformational interconversion between a rapidly scanning "search" mode and a momentarily 


\section{SUMMARY (Continued)}

stalled "interrogation" mode. We also uncovered a slower (>50 ms) DNA bending step to form the stable "recognition" conformation. Our results also showed that the $\beta$-hairpin is not required for the rapid unwinding but is essential for the nucleotide-flipping step and for the stability of the "recognition" conformation. These results mark the first observation of DNA distortion dynamics that reflect nonspecific "search-interrogation" process by a DNA repair protein that relies entirely on DNA deformability to recognize its lesions, and provides keys to understanding the protein's ability to rapidly search and yet also reliably recognize diverse lesions. These results have strong implications in our understanding of how Rad4/XPC discriminates between normal and damaged sites.

The third system, discussed in Chapter $\mathrm{V}$, is MutS, another DNA repair protein that recognizes mismatches in DNA, which occur as a result of misincorporation of nucleotides during replication and are repaired in the mismatch repair (MMR) pathway. MutS recognizes mispaired or unpaired bases, and subsequently recruits downstream proteins that rectify the mismatched site. Structural studies have shown that MutS-bound DNA is sharply kinked at the mismatch site. However, there are no studies reporting on the kinetics of mismatch recognition, leaving a big gap in our understanding of mismatch recognition mechanisms. This study was carried out in collaboration with Prof. Manju Hingorani and her group from Wesleyan University.

In this study, I measured the kinetics of mismatch recognition of a T-bulge (an unpaired thymine) by prokaryotic T. aquaticus (Taq) MutS and its eukaryotic homolog S. cerevisiae 


\section{SUMMARY (Continued)}

Mut $\mathrm{S}_{\alpha}$. The kinetics were measured by (1) incorporating a $2 \mathrm{AP}$ probe next to the T-bulge, to measure DNA kinking at that site; (2) FRET measurements on DNA “end-labeled" with TAMRA (donor) and Cy5 (acceptor), to measure DNA bending rates; and (3) Trp fluorescence measurements to probe protein conformational dynamics. Our results on Taq MutS reveal 2APreported nucleotide dynamics at the mismatch site at $\sim 8 \mathrm{~ms}$ and FRET-reported DNA bending dynamics at $\sim 70 \mathrm{~ms}$. Both these kinetic steps are considerably slower than the intrinsic bp opening dynamics at mismatched sites, as measured by NMR, indicating that spontaneous kinking at the mismatch sites, while perhaps serving to "stall" the scanning protein, is not the rate-limiting step to form the "recognition" complex. Our Trp fluorescence measurements on MutS $\mathrm{S}_{\alpha}$ uncover rapid kinetics that overlap with intrinsic bp opening rates on normal DNA; we attribute these dynamics to interactions in the nonspecifically bound mode, likely arising from protein conformational switching between "search" and "interrogation" modes, as discussed previously for both IHF and Rad4/XPC. Taken together, our study indicates at least three sequential events during mismatch (T-bulge) recognition: a rapid nonspecific DNA interrogation step, followed by local dynamics at the mismatch site, which we identify as a precursor to the recognition process, and a slower overall DNA bending step, attributed to the formation of the ultimate recognition complex. These studies represent the first observation of mismatch recognition dynamics by MMR protein MutS, and suggest some common themes in the indirect readout damage recognition mechanisms of MMR and NER proteins, such as the progressive, multi-step nature of the damage recognition process. 
To conclude, my studies have uncovered previously unresolved steps during the search and recognition of specific binding sites for three protein-DNA systems, including rapid (submillisecond) DNA bending and unwinding that are commensurate with rapid searching while nonspecifically bound, and slower specific recognition steps such as nucleotide flipping or severe DNA bending to form the specific complex. These kinetics measurements help illuminate how a searching protein interrogates DNA deformability and eventually "stumbles" upon its target site, revealing rich multi-step dynamics during this "search-interrogation-recognition" process. 


\section{CHAPTER I}

\section{INTRODUCTION}

\subsection{Protein-DNA interactions}

Deoxyribo-nucleic acids (DNA), which carry genetic information, and proteins which execute and regulate life process are two important biomolecules in any living organisms. The dynamical interactions between proteins and DNA play a central role in many biological processes such as DNA replication, transcription, recombination, gene regulation, repair and even the packaging of DNA into chromosomes. In order to interact with DNA and perform their designated functions proteins must first recognize and bind specifically to their target site on DNA. The study of the dynamics of protein-DNA interactions aimed at unraveling the mechanisms of binding-site recognition is a fascinating research field in biophysics and the central focus of this dissertation..

There are two classes of DNA-binding proteins: (1) proteins that bind to DNA in a sequence-independent manner (nonspecific binding), e.g.: wrapping of DNA along the histone cores in nucleosome; (2) proteins that bind to DNA in a sequence-dependent manner by recognizing specific sites in DNA (site-specific binding), e.g.: binding of transcription factors. Although in general, site-specific proteins are highly selective and bind to their target DNA sites with thousand- to million-fold higher affinities compared to random DNA sequences, some others, for example some DNA damage recognition proteins, recognize a broad range of target sites with only 10-100 fold higher affinity than non-target sites [1].

Two puzzles have particularly intrigued researchers in this field. The first seeks to understand how site-specific DNA binding proteins efficiently search for their binding sites in DNA among a sea of nonspecific sites. The second related puzzle is how this protein 
actually recognizes its target site on genomic DNA. This question is less well understood, and is the main topic of this thesis. The recognition problem can be separated from the search problem by focusing on short DNA oligomers that contain a target sequence for a specific DNA binding protein and by investigating the dynamics of the interactions that lead to the formation of the specific complex.

When proteins bind to DNA, they often distort the DNA at the binding site by bending, kinking, or twisting the DNA. As an example, genomic DNA is smoothly but severely bent when it is wrapped around the histone octamers to form nucleosomes for chromosome packaging [2]. In the semi-uniformly bent conformation of nucleosomal DNA these distortions are spread out over the length of the wrapped DNA. On the other hand, there are many examples of locally bent/kinked/unwound and otherwise severely distorted DNA when site-specific proteins bind to their specific target sites on DNA. These localized distortions in otherwise B-form DNA duplex have been revealed in several high-resolution structures and thermodynamic studies carried out on a wide range of protein-DNA complexes. Examples are: (1) bacterial nucleoid-associated architectural proteins such as the intergration host factor (IHF) that sharply bend DNA to bring distal regions of DNA spatially closer [3], (2) lac repressor, a tetrameric bacterial gene regulatory protein that simultaneously binds DNA at two sites and loops the intervening DNA [4], (3) (4) TATA binding protein (TBP), a transcription initiation protein that recognizes and sharply kinks a TATA element in the promoter sequnce of DNA [5], (5) restriction enzymes that bind to, kink, and cleave at a specific site in DNA [6], (6) DNA repair proteins that recognize and bind to lesions in DNA, typically flipping out the damaged nucleotides as a first step in the repair mechanism $[7,8]$. These examples illustrate that DNA deformability and concerted rearrangements in the bound protein to accommodate the deformed DNA is an essential aspect of nearly all biological 
phenomena involving protein-DNA interactions and likely play an essential role in the mechanism by which proteins recognize their target sites in genomic DNA.

While X-ray crystallography and NMR have been extraordinary in elucidating the 3dimensional structures of protein-DNA complexes and providing some insights into the recognition mechanisms, these structural studies don't reveal the time scales or the order in which these conformational changes occur, thus leaving a big gap in our understanding of the energetics of these interactions. Studying the dynamics of these conformational changes is important because the timescales on which these structural changes occur are inherently related to the biological function.

\subsection{Sequence-dependent DNA deformability and its role in target recognition}

It is well recognized that DNA has remarkable flexibility and can undergo considerable distortions in order to adopt many different conformations when bound to a protein. As discussed above, a classic example of distorted DNA is found in the building blocks of the chromosome: the nucleosomal core particle (NCP), in which 147 bp of DNA are wrapped approximately twice around the histone core (Fig. 1.1) [2]. To appreciate why this is quite remarkable, note that the persistence length of DNA is $\sim 150 \mathrm{bp}(\sim 50 \mathrm{~nm})$, which is comparable to the length of DNA in each NCP. We recall that the persistence length of a polymer is the characteristic length scale over which thermal fluctuations will bend the polymer by about 1 radian [9]; in the NCP, this length of the DNA polymer is bent by almost $4 \pi$ radians. The energetic cost of bending the DNA into tight loops as in the NCP is estimated to be remarkably high, $\sim 77 \mathrm{kcal} / \mathrm{mol}$, assuming a continuum elastic rod description for the DNA polymer (see Appendix A). 


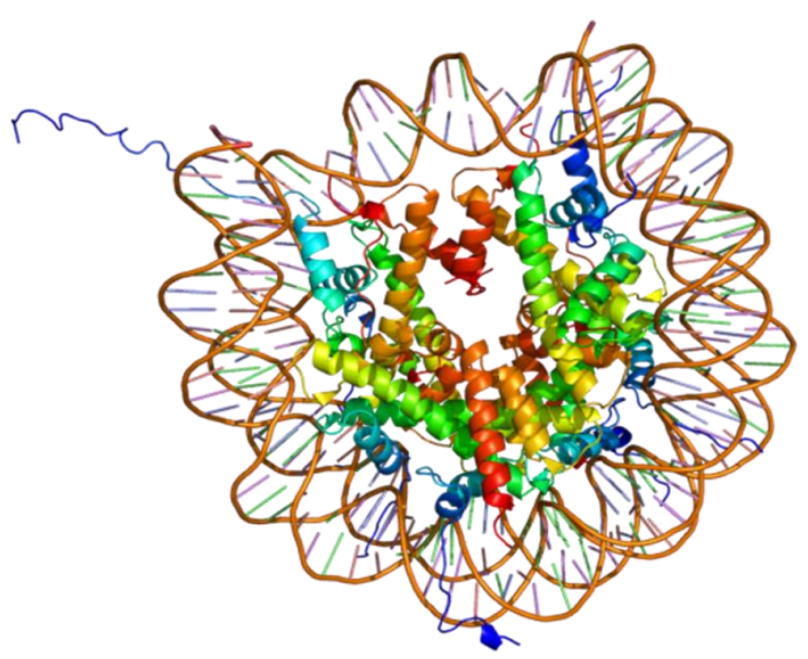

Fig.1.1. The crystal structure of DNA wrapped around the histone core to form nucleosome. Nucleosome consists of four core histones H2A (yellow) H2B (red), H3 (blue) and $\mathrm{H} 4$ (green). The view is from the top through the super helical axis. PDB:1AOI [10].

High resolution crystal structures of the NCP, first obtained by Richmond, Lugar and coworkers in 1997, showed that the DNA is not uniformly bent within the nucleosome but rather it is periodically kinked to adopt the donut-like surface of the histone octamer core [2, 11]; this kinked/bent confirmation is due to the combination of sequence dependent DNA flexibility and histone induced DNA bendability. Further evidence for sequence-dependent DNA flexibility and ability to adopt the wrapped conformation in the NCPs came from SELEX experiments by Widom and co-workers [12]. In SELEX experiments these researchers started with a large pool of chemically synthetic random DNA molecules to identify those sequences having the highest affinity for histone octamer. They found that a particular set of sequences, which obey certain sequence pattern, exhibited higher affinity, even higher than the previously known natural sequences $[12,13]$.

These and several other studies showed that the persistence length of DNA is not a constant for a given set of conditions but in fact depends on the sequence context. For example, alternating AT polymers is $20-30 \%$ more flexible than mixed sequence DNA [14, 
15]. Cyclization experiments carried out by Cloutier and Widom showed that a 94 bp DNA can be efficiently ligated into circles by thermal fluctuations without the aid of a DNA bending protein, and that the probability of formation of these minicircles is strongly dependent on its sequence context. Their measurements suggested that spontaneous formation of these DNA minicircles is up to $10^{5}$ times greater than expected from theoretical considerations of wormlike chain models [16]. While the experimental results of Cloutier and Widom are controversial [17-20], they were instrumental in the development of theoretical models that went beyond the continuum elastic polymer model (the wormlike chain) for DNA to include possibility of sharp kinking from local regions of weak stacking, flipped out bases, or locally denatured regions, thus reducing the energetic cost for sharply bending or kinking DNA [21-25].

These conclusions are consistent with the kinked DNA seen in NCP crystal structures, as discussed above, and imply that the sequence-dependent structural and mechanical properties of a DNA segment can strongly influence protein binding by reducing the energy cost of adopting a particular conformation suitable for protein binding.

\subsubsection{Free energy cost for local deformation of DNA.}

The structure and stability of DNA has contributions from both base stacking energy, which is from hydrophobic and van der Waals interactions between bases, and base-pairing energy, which is from hydrogen bonding between complementary bases. A smaller contribution to the helix stability also comes from phosphate - phosphate repulsions on the outside of the helix [11]. The major contribution to DNA stability is believed to be from the stacking interactions [26, 27], although it has been a challenge in the field to separately estimate the two: the free energy cost of breaking a stack and the free energy cost of breaking a Watson-Crick base pair. Traditionally, these estimates have come from thermal melting 
experiments performed with DNA oligomers with varying lengths and sequences, carried out under different salt conditions [28-33]. SantaLucia unified these experimental data from different thermal denaturation studies to obtain a consistent set of stacking free energy parameters of ten distinct dinucleotide steps [34]. The results of these analyses are summarized in Fig. 1.2 (vertical gray bars) that demonstrates the large uncertainties in the ability to accurately pinpoint these parameters from the thermal melting profiles [35].

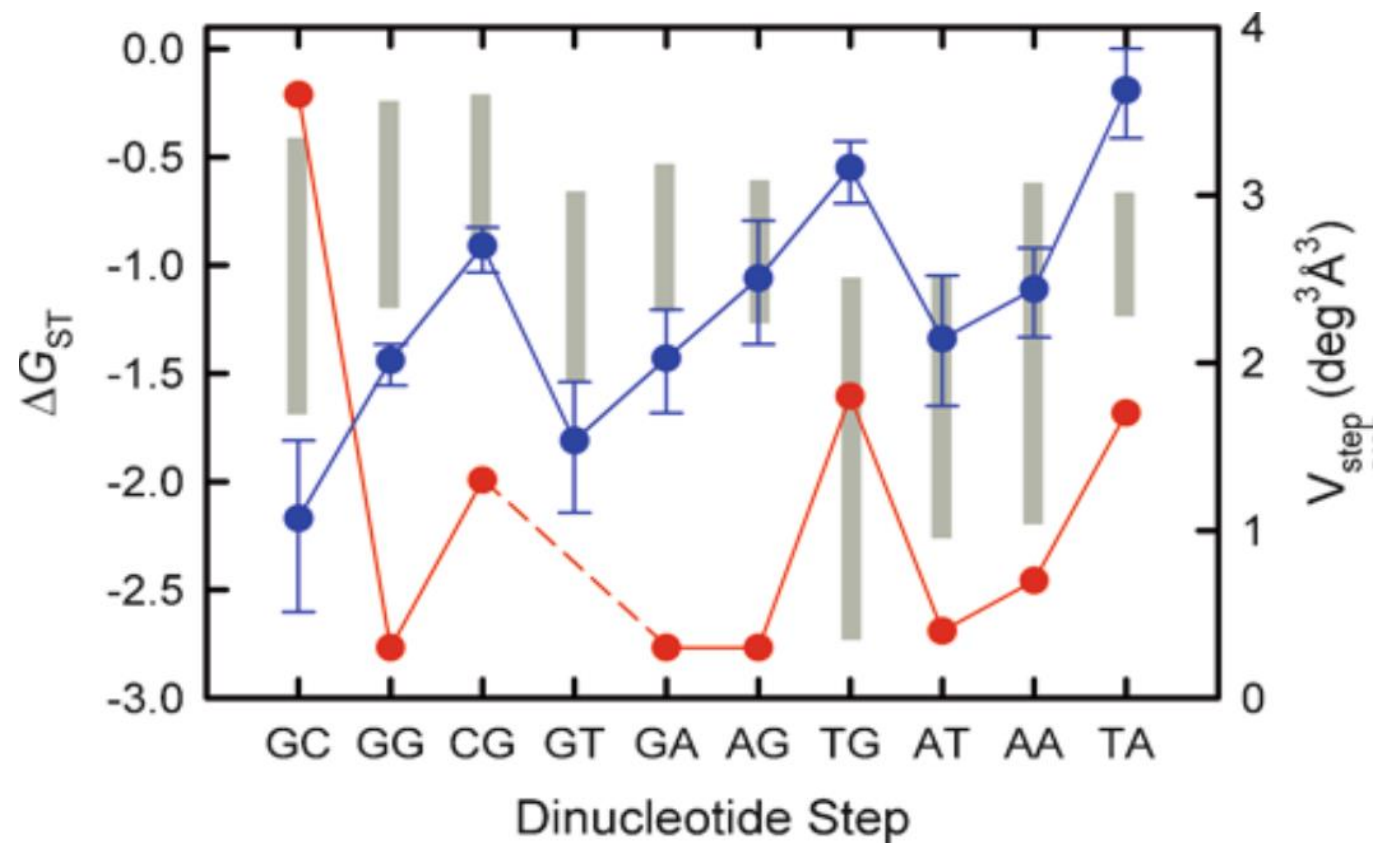

Fig.1.2. Nearest-neighbor(nn) stacking free energies $\left(\Delta \mathrm{G}_{\mathrm{ST}}\right)$ for the ten different dinucleotide steps of duplex DNA. The nn stacking free-energy parameters, extracted from thermal denaturation experiments on oligonucleotide duplexes, are shown as vertical gray bars. The length of each vertical bar indicates the rage of the stacking parameters from seven independent research groups, obtained under different salt conditions and for varying length of duplex DNA, and unified by SantaLucia. Stacking free energies from electrophoretic mobility measurements (filled blue circle) on DNA fragments containing a nick in the sugarphosphate backbone, between all possible combinations of dinucleotide steps are from FranKamenetskii's data. The volume of thermally accessible conformational space for each dinucleotide step (filled red circle), obtained from fluctuation and correlations of base step parameters in DNA- Protein crystal complexes. (Figure reproduce from [35] with permission).

In another study, Frank-Kamenetskii and co-workers used electrophoretic gel mobility measurements of a series of 300 base pair long DNA molecules nicked in the sugar phosphate backbone on one of the strands, with the nick sandwiched between all combinations of 
dinucleotide steps. The nick alters the equilibrium between stacked (straight) and unstacked at the nick (bent) conformations (Fig. 1.3) [36], which have different motilities on the gel. The relative populations of the two conformations as obtained from the gel studies then directly reveal the stacking free energy for that dinucleotide step. Results from this study are also shown in Fig. 1.2 (blue filled circle) for comparison [35].

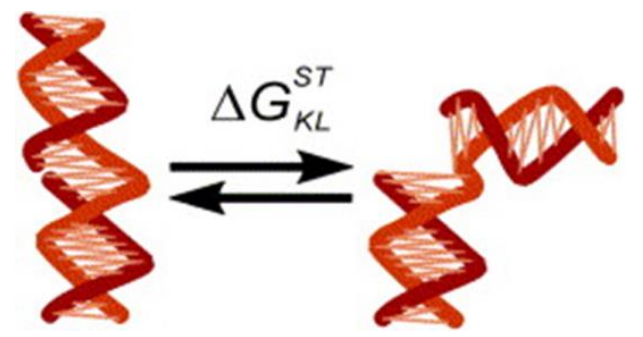

Fig.1. 3. Schematic representation of the stacked-to-unstacked conformational transition of the DNA fragment at the nick site described by the energy difference, $\boldsymbol{\Delta} \boldsymbol{G}_{\mathbf{S T}}^{\mathbf{K L}}$ (Figure reprinted from [37], copyright (2004), with permission from Elsevier.

In a separate study, Olson and coworkers analyzed the distorted DNA structures obtained from the data bank of crystal structures of 92 protein-DNA complexes, to extract the sequence dependent empirical energy functions for six parameters describing the local stacking geometry between each of the dinucleotide steps (see fig.1.1 in Appendix) [38, 39]. From the average values and deviations from the average of these six step parameters, they obtained an estimate of the volume of conformational space that is thermally accessible at each step (Fig. 1.2; red symbols) [35]. These various studies nicely illustrate that DNA deformability is sequence-dependent, and that this deformability is critical in the ability of proteins to make tight complexes with the correct target sequence, although obtaining accurate estimates of these sequence-dependent parameters remains a challenge in the field. 


\subsubsection{Sequence-dependent base-pair opening rate measured by NMR imino proton exchange}

Another dramatic illustration of sequence-dependent DNA properties comes from NMR measurements of imino proton exchange, which probe base-pair (bp) breathing dynamics and provide estimates for opening and closing rates at a single base-pair level inside a long DNA molecule [40]. These studies show a wide range of TA bp opening rates (39-360 $\mathrm{ms}$ at $25^{\circ} \mathrm{C}$ ), and a smaller range of CG bp opening rates $\left(36-58 \mathrm{~ms}\right.$ at $25^{\circ} \mathrm{C}$ ) (Fig. 1.4). This wide range of TA bp opening rates is consistent with the notion that the free energy cost of disrupting a TA bp within DNA has significant contributions from nearestneighbor (or even longer range) stacking interactions, which are sequence dependent; the narrower range for $\mathrm{CG}$ bp opening rates suggests that the primary contribution to the free energy cost of disrupting a CG bp is from the disruption of the 3 hydrogen bonds, with a relatively smaller contribution from base-stacking interactions. These sequence-dependent base pair breathing dynamics likely play a critical role in the thermal conformational fluctuations of DNA, including the propensity for spontaneous kinking, as discussed further in Chapter 3. 


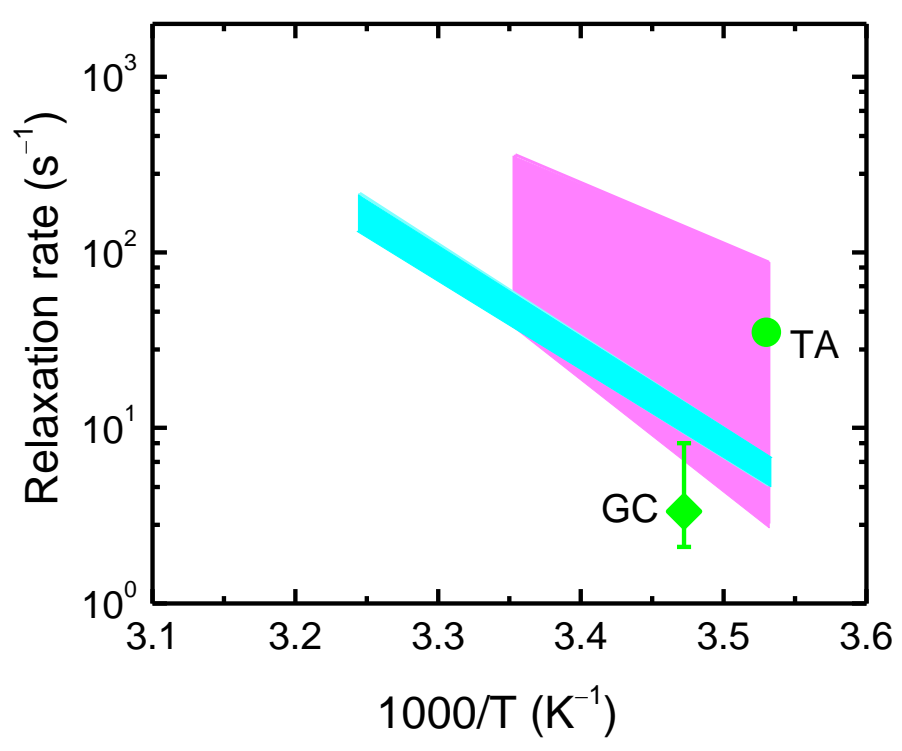

Fig.1.4. The pink (cyan) shaded regions represent the range of base-pair opening ('breathing') rates for $\mathrm{A} / \mathrm{T}(\mathrm{G} / \mathrm{C})$ base pairs, from NMR imino proton exchange measurements of Coman and Russu [40]. The opening rates and their ranges are also indicated from two other references, T/A base pair (green circle) [41], and G/C base pair (green diamond) [42].

\subsubsection{How do site-specific proteins search for their target sites on genomic DNA?}

Proteins that need to bind to specific target sites on DNA in order to perform their cellular function face an enormous challenge: they must first locate their target site, typically less than a few tens of base pairs long, buried amidst chromosomal DNA, which contains over a million bp of DNA. It is a puzzle how site specific DNA binding protein efficiently searches for their binding sites in DNA among a sea of nonspecific sites [35].

Efficient and reliable search mechanism is important in order to control biochemical process at the cellular level with remarkable speed and fidelity. All cellular activities depend on the timely expression of the information encoded in the cell's DNA. For example, gene expression is mediated through the direct interaction between transcription factors and their respective target sites. Surprisingly only relatively small number of transcription factors are 
involved in searching massive lengths of DNA. For example Escherichia coli has nearly 20 copies of lac repressor that must search its 4,600, 000 bp long genome [43].

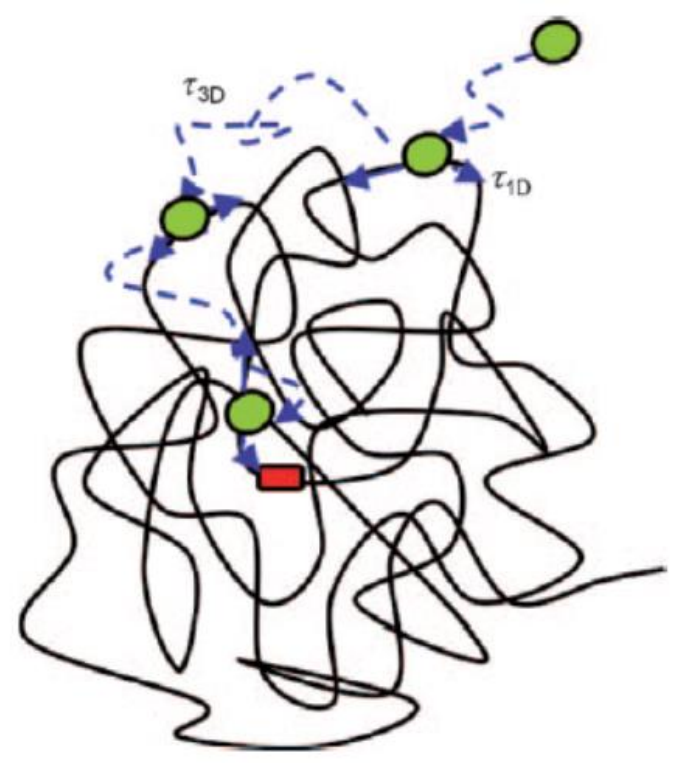

Fig.1. 5 . The mechanism of facillitated diffusion. The search process consists of alternative rounds of $3 \mathrm{D}$ and $1 \mathrm{D}$ diffuion, each with average duration $\tau_{3 \mathrm{D}}$ and $\tau_{1 \mathrm{D}}$ respectively. During the search for target site protein bind nonspecifically anywhere along the DNA, move along the DNA in 1D (slide or hop) then dissociates and diffuses $3 \mathrm{D}$ in solution and randomly collide with another segment of DNA to continue its search. Figure reproduced from reference [44] with permission.

In 1970, Riggs et al measured the association rate of the lac repressor and its target site on DNA as $10^{10} \mathrm{M}^{-1} \mathrm{~s}^{-1}$ [45]. However the diffusion limited rate of bimolecular association is estimated to be $\sim 10^{8} \mathrm{M}^{-1} \mathrm{~s}^{-1}$ [45]. Riggs et al showed that proteins can bind to their target sites on DNA apparently 100 times faster than the diffusion limited rates. These findings were interpreted in terms of a "facilitated diffusion" mechanism for target search, in which a combination of 3-D diffusion of proteins (jumping from one DNA site to another via diffusion through bulk solution) and an effective 1-D diffusion ("sliding" on DNA without dissociating from DNA, or "hopping" along DNA via a series of microscopic dissociation and re-association) could explain the observed acceleration of target site location (Fig. 1.5) [45-48]. During 1D sliding the protein is kept on DNA by the binding energy to nonspecific 
DNA. This energy has been measured for several DNA binding proteins and has a range of 10-15 $k_{\mathrm{B}} T$ (at physiological salt concentration), was shown to be driven primarily by screened electrostatic interactions between charged DNA and protein molecules, i.e. from the entropic gain of counterions released from the DNA upon association with the positively charged binding surface of the protein [49]. Several lines of evidence have since corroborated this 'facilitated diffusion' mechanism as a means by which proteins take advantage of nonspecific binding to efficiently search in genomic DNA for their targets [5053].

\subsubsection{How do site-specific proteins recognize their target sites?}

\subsubsection{Direct versus indirect readout}

Structural studies of several specific protein-DNA complexes have revealed that proteins discriminate between potential target sites and nonspecific sites of DNA either by direct interections, e,g. hydrogen bonds between specific bases within the target site and specific amino acids of the protein (“direct readout”) or by recognizing sequence dependent DNA shapes and sensing local DNA deformability ("indirect readout") [54]. Quite often proteins use a combination of both direct and indirect readout mechanism to recognize their binding site.

Generally proteins that bind to the major groove use direct readout mechanism: since the pattern of hydrogen bond donors and acceptors is distinct for each Watson-Crick base pair in the major groove (Fig. 1.6)[11], the protein can readily recognize the binding site by examining the hydrogen bonding pattern exposed in the major groove. 
(A)
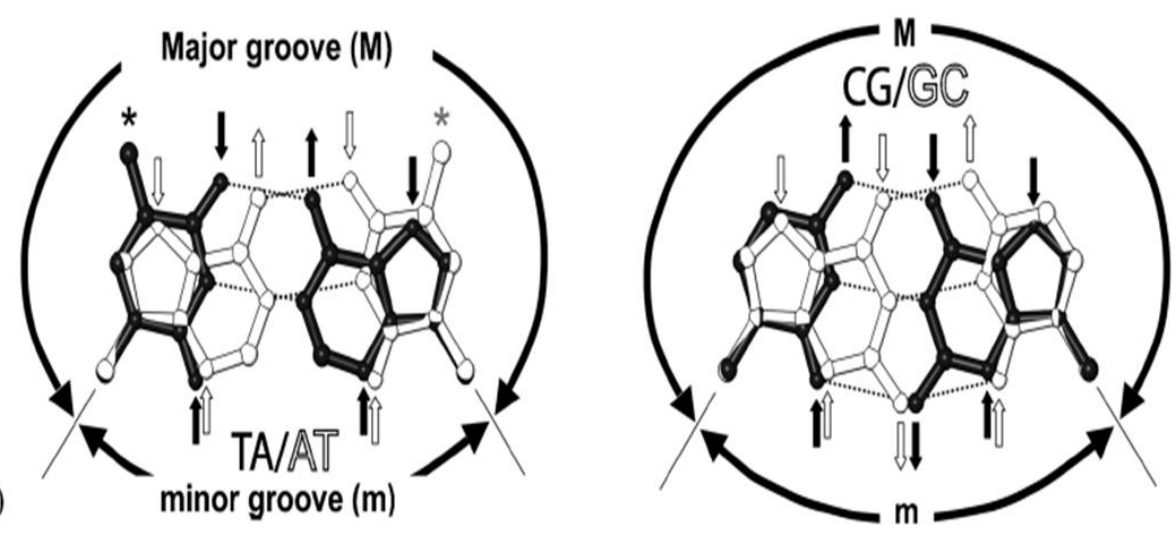

Fig.1. 6. Watson and Crick base pairs. (Left) AT (hollow drawing) and TA (solid drawing), (Right) GC (hollow drawing) and CG (Solid drawing) are superimposed. Hydrogen bond donors are indicated by arrows pointing away and acceptors by arrows pointing toward to the base. The methyl groups are represented by asterisks. It is diffecult for minor groove binding proteins to distinguish $\mathrm{A}$ from $\mathrm{T}$ and $\mathrm{G}$ from $\mathrm{C}$. In addition to this, a $\mathrm{G}: \mathrm{C}$ pair is distiguished from an A:T pair only by one additional centrally located donor group. Figure reproduced from Rice, P.A. and C.C. Correll, Protein-Nucleic Acid Interactions. 2008, Cambridge [11] with permission by The Royal Society of Chemistry.

Alternatively proteins that bind to minor groove rely primarily on indirect readout mechanism because in the minor groove the pattern of hydrogen bond donors and acceptors is similar for all Watson-Crick base pairs (Fig.1.6) [11]. For this reason the minor groove binding protein can't directly "read" the sequence information. These proteins need to rely on other sequence-dependent properties of DNA such as shape and mechanical deformability/flexibility. Thus, many indirect readout proteins interrogate potential binding sites by inspecting the ease of bending, twisting or deforming DNA sites in order to fit into the binding interface of the protein.

\subsubsection{Induced-fit mechanism}

Structural and thermodynamics studies of several protein-DNA complexes show that the bound protein also undergoes conformational changes in order to facilitate favorable 
interactions with the deformed DNA structure and these interactions stabilize the complex (Fig.1.7); this accommodation of distorted DNA by the protein is referred to as the "inducedfit mechanism" [55-58]. Thus, in addition to structural features within the recognition complex, the conformational dynamics of both binding partners, protein and DNA, is the key to the recognition mechanism. Induced-fit mechanism has emerged as a general principle of target recognition $[56,57]$.

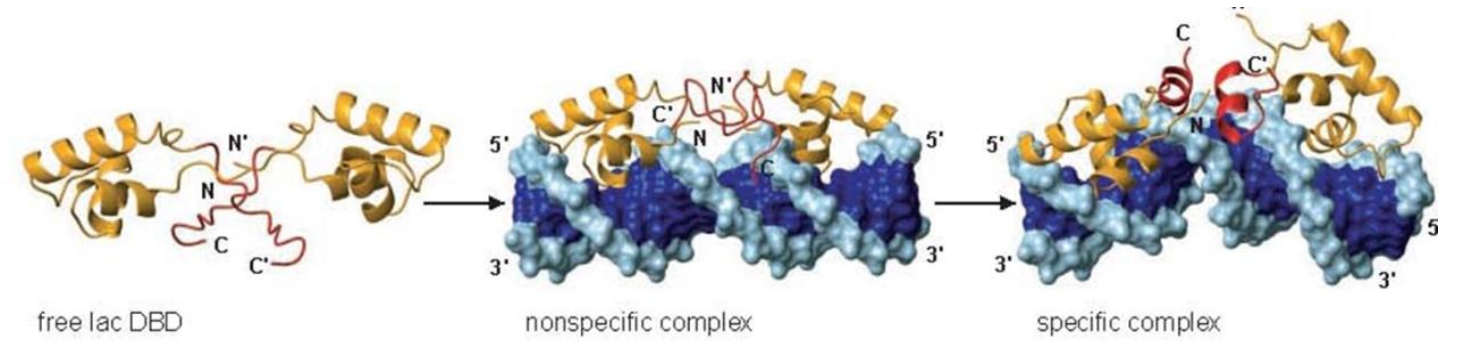

Fig.1. 7. NMR solution structure of the dimeric lac repressor bound to a nonspecific and a specific DNA sequence. The structures illustrate the process of protein binding to a $18 \mathrm{bp}$ long DNA. In the nonspecific complex the protein and the DNA structures are similar to their respective structures when not in complex. In the specific complex the protein adopts a different conformation; the unstructured region of the protein (Red) forms a secondary structure and the DNA is bent by $36^{\circ}$. The structure of the protein when free or bound nonspecifically is very different from the structure in the specific complex. Figure reproduced from [58] with permission from AAAS.

In an interesting study, Andrabi et al [59] analyzed the database of already known crystal structures of many DNA-binding proteins in the DNA-bound and unbound forms and showed that the extent of the conformational changes in the protein have direct implications for the stability and specificity of the complex. Their results indicated that proteins with larger conformational changes form more stable complexes and show greater specificity than their rigid counterpart. 


\subsubsection{Conformational capture or protein-induced DNA bending}

The mechanism that controls the conformational changes in both binding partners (protein and DNA) during binding-site recognition is a central question in molecular biophysics. There are two possibilities: (1) the protein first binds to straight DNA and then induce DNA distortions as a second step (protein induced conformational change); (2) the protein captures thermally accessible pre-distorted DNA conformation (conformational capture). An understanding of which of these mechanisms best describes a given interaction has enormous importance in drug design. For example if conformational capture is the mechanism governing protein/DNA interactions, it may be possible to design drugs to stabilize a particular conformation related to a desired biological activity from a possible spectrum of different conformational states [60]. The investigation of the relative contributions of these two extreme mechanisms in binding site recognition requires measurements of the conformational distribution of DNA in the absence of bound protein, and kinetics measurements of conformational rearrangements along the transition pathway from nonspecific to specific complex. These measurements have remained a challenge in the field.

\subsubsection{Measurements of DNA binding and bending kinetics}

Characterization of the rates at which proteins bind to and bend DNA is a natural starting point to probe the molecular mechanism of binding site recognition. These studies have been carried out on several DNA-bending proteins primarily using stopped-flow [61-67] or single-molecule [68-71] measurements that have yielded valuable information on the bimolecular association/dissociation kinetics, but were largely unable to capture the unimolecular DNA deformation steps that lead to the formation of the specific complex, owing to their limited time-resolution of a few milliseconds. 
Many attempts to resolve DNA bending kinetics during protein-DNA interactions have come from stopped-flow studies, which aimed to address the question: Are DNA binding and bending in these systems sequential or occur in a concerted manner? [61-64, 72]. In these studies DNA bending kinetics were measured using time-resolved FRET, with donor/acceptor fluorescent probes attached at either end of a short DNA oligomer; a change in FRET reported on a change in the end-to-end distance of the DNA oligomer, and hence on the dynamics of DNA bending in the complex. Additionally, relaxation kinetics of bimolecular association were monitored using fluorescence anisotropy decay of singlelabeled DNA oligomers. These studies showed that the time scales of bimolecular association and DNA bending were indistinguishable, either because DNA bending occurred simultaneously with protein binding or alternatively DNA bending was too fast to be resolved in stopped-flow measurements.

To date, the kinetics of binding site recognition has been resolved for only a handful of systems. The first such measurements were done for recognition of uracil in DNA by damage recognition protein UDG involved in base excision repair pathway [73]. In this system the kinetics of base-flipping was observed by measurements of $2 \mathrm{AP}$ fluorescence in stopped-flow measurements, and revealed base-flipping kinetics occurring on the $\sim 5-10 \mathrm{~ms}$ time-scale. In another set of measurements, Wuite and co-workers [68] probed the mechanism of sequence recognition and cleavage by EcoRV by monitoring the rate at which EcoRV cleaved a single molecule of DNA containing the specific site, stretched between two optical traps. At high values of the applied tension, the rate of DNA bending appeared to be the rate-limiting step in the cleavage reaction; extrapolation to zero force suggested DNA bending rates between 35 and $1000 \mathrm{~s}^{-1}$.

More recently, DNA bending kinetics upon specific binding of IHF to one of its cognate sites on lambda-phage DNA (the H' site) were directly visualized by time-resolved 
measurements of the change in FRET between donor and acceptor fluorophore molecules attached at either end of a short (35-bp) DNA oligomers containing the H' site [65, 72]. These measurements revealed DNA bending kinetics with time constants in the range of 1-10 ms.

\subsubsection{Competition between 1-D diffusion and binding-site recognition: the "speed- stability" paradox.}

Direct observations of proteins diffusing on nonspecific DNA have revealed onedimensional diffusion constants in the range of $\sim 2 \times 10^{3}-7 \times 10^{6} \mathrm{bp}^{2} / \mathrm{s}$ [74-79], which indicate stepping times (or residence time) per base pair ranging from $\sim 200 \mathrm{~ns}-500 \mu \mathrm{s}$ (Table I in Appendix A). Thus, the slow (> ms) recognition times reported in previous studies $[73,80$ 84] are slow in comparison with fast $(<\mathrm{ms})$ 1-D diffusion times measured for various DNAbinding proteins, as discussed above. This poses a puzzle, since the recognition step is expected to be fast or comparable to the time spent by the protein scanning the vicinity of a potential binding site.

It has been proposed that a conformational switch between a rapidly diffusing 'search' mode of the protein and a more tightly bound 'recognition' mode is needed to enable it to identify a potential target site without losing overall speed of searching [85-88]. Consistent with these arguments, alternative binding modes of various proteins interacting with nonspecific DNA have been observed [41, 58, 89-97], and have also been inferred from single-molecule studies of proteins diffusing on DNA [98-100]. Kalodimos et al. [58], using NMR studies, showed that lac repressor bound nonspecifically to a noncognate DNA sequence was bound in a loose and fuzzy way, and exhibited large scale conformational fluctuations in the microsecond-to-millisecond timescales. This study indicated that the 
nonspecific complex is highly dynamic and suggested that this dynamical flexibility is important for the efficient search of the specific binding site on genomic DNA.

While NMR studies have revealed microsecond-to-millisecond conformational fluctuations in proteins nonspecifically bound to DNA [58, 93, 97], the sequence of various conformational changes that takes the protein-DNA complex from its search conformation to its final target recognition conformation have remained largely elusive and require new approaches, as outlined in the next section.

\subsection{Experimental techniques to study dynamics of protein-DNA interactions}

As discussed above, the dynamics of protein-DNA interactions for several systems have been investigated with stopped-flow techniques $[61-64,72]$. With the exception of a few systems $[65,73]$, these studies have been unable to resolve DNA bending dynamics or other DNA conformational dynamics during target recognition, thus requiring the development of fast techniques.

In recent years, single molecule biophysical techniques have also emerged as powerful tools that allow novel measurements related to conformational dynamics of proteinDNA interactions [101]. These methods include atomic force microscopy [102], optical tweezers [103, 104], magnetic tweezers [105] and fluorescence microscopy [106], including single-molecule (sm) FRET [107] and fluorescence correlation spectroscopy (FCS) [108111]. These single molecule studies have yielded a wealth of dynamical information on DNA-bending site-specific proteins [68-71].

However both stopped-flow and single molecule techniques are limited in their timeresolution and sensitivity: stopped-flow and smFRET can only directly detect motions slower than a few milliseconds; both FCS and smFRET require high quantum-yield fluorescence labels, and can typically detect only large conformational changes, although FCS does enable 
macromolecular dynamics to be resolved with microseconds-time-resolution. The submillisecond conformational dynamics are amenable to NMR techniques, albeit only for smaller systems. Therefore experimental techniques with fast (sub-microsecond) timeresolution and enhanced sensitivity are needed that are capable of capturing rapid and often subtle conformational dynamics during the search and recognition steps of site-specific protein-DNA binding.

\subsubsection{Laser temperature-jump spectroscopy.}

In the last two decade, laser temperature-jump (T-jump) has emerged as a powerful complement to stopped-flow and single-molecule studies. Laser T-jump approach in combination with time-resolved optical spectroscopy enables kinetics measurements with high (sub-microsecond) temporal resolution (reviewed in [112]). The T-jump approach, together with exquisitely sensitive fluorescent probes such as tryptophan (in proteins) and nucleotide analogues (in DNA and RNA), have led to the characterization of otherwise unresolved conformational dynamics during protein folding [113-116], RNA folding [117], and protein-DNA [118-120] and protein-RNA [121] interactions. These studies, in addition to using conventional extrinsic fluorescent labels, also took advantage of (1) intrinsic fluorescence of proteins that can be monitored without interference from extrinsic labels, (2) a range of novel fluorescent analogues of DNA bases that can be incorporated within DNA with minimal disturbance of DNA structure, enabling measurements of subtle conformational changes in DNA upon protein binding.

Our group was the first to apply the T-jump approach to measure the dynamics of protein-DNA interactions. The first such study by Kuznetsov et al. probed the previously unresolved wrapping/unwrapping dynamics of single-stranded DNA (ssDNA) around a single-strand binding protein (SSB) [122]. This wrapping/unwrapping is an important step in 
all DNA metabolic processes. T-jump studies showed that this step occurs on a time scale of tens of microseconds, thus setting an upper limit on how fast SSB can translocate along DNA during replication, recombination and repair. The second such study also came from our group on resolving the bending/unbending dynamics of DNA in complex with IHF protein [72], as discussed in more detail in Chapter 3. These studies laid the groundwork for further studies on these and other protein-DNA systems, as reported here.

In this thesis I used our laser T-jump apparatus to probe the conformational dynamics of target recognition for three different DNA binding proteins (Fig. 1.8). A common feature of all these three protein-DNA systems, revealed from structural studies, is that these proteins rely primarily on indirect readout to recognize their DNA target site and therefore must sense sequence-dependent DNA deformability to discriminate between target and nontarget sites. The kinetics measurements reported here shed light on how a searching protein interrogates DNA deformability and eventually 'stumbles' upon its target site, revealing rich multi-step dynamics during this search-interrogation-recognition process. The salient findings on these different protein-DNA systems are briefly described below. 


\subsection{Thesis Overview}

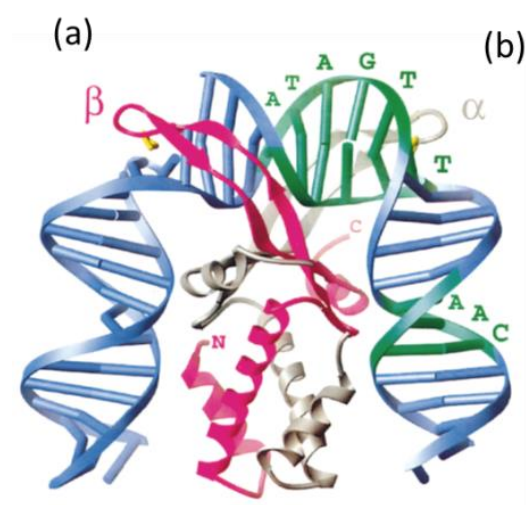

(b)

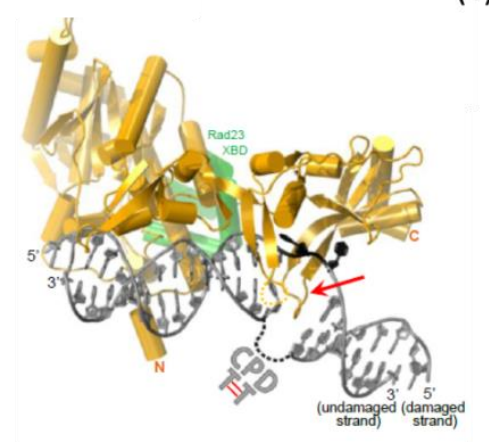

(c)

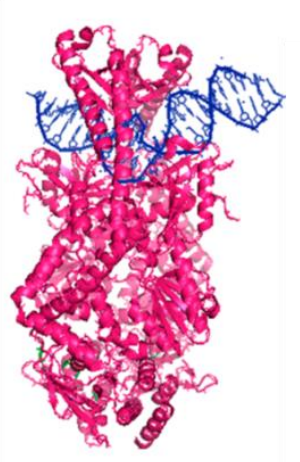

Fig.1. 8. (a) Crystal structure IHF (pink, while) bound to an H' site in 1 phage DNA (blue, green). The $\alpha$ and $\beta$ subunits are shown in white and pink respectively. The proline at the tip of each arm is marked in yellow and it intercalate between bases at the kink site. Reprinted from [3], Copyright (1996), with permission from Elsevier. (B) Crystal structures of XPC (yellow, green) bound to a CPD lesion in DNA (gray). The $\beta$ - hairpin that gets inserted into the damaged DNA is indicated by red arrows. Figure adapted by permission from Macmillan publishers Ltd (Nature) [126], copyright (2007) (C) Co-crystal structure of Taq MutS protein (pink), in complex with a T-bulge DNA (blue) (PDB: 1EWQ).

\section{(1) DNA bending dynamics IHF-DNA interaction}

The Escherichia coli integration host factor (IHF) is an architectural protein that acts as a host factor for lysogeny by bacteriophage $\lambda$. It recognizes several sites on phage $\lambda$ DNA, primarily by indirect readout, bending the DNA at its cognate site by nearly $180^{\circ}$ over $\sim 35$ bp, bringing distal regions of the DNA together to form higher-order nucleo-protein complexes [3]. The crystal structure of IHF bound to one such cognate site (the H' site) reveal that the DNA is sharply kinked at two sites $~ 9$-bp part, and this severely bent DNA is stabilized by intercalation of conserved proline residues located on two $\beta$-ribbon arms that wrap around the DNA (Fig. 1.8). IHF also bends nonspecific sites on DNA, which is important in DNA packaging in the cell and in regulating diverse cellular process. Because of the severe DNA bending and the indirect nature of site-specific recognition in this complex, IHF serves as an excellent model system for analyzing the mechanism by which DNA- 
bending proteins probe sequence-dependent DNA deformability to recognize their target sites [123-125].

Previous stopped-flow and laser T-jump measurements on IHF binding to its cognate H' site revealed that DNA bending in the complex occurs on $\sim 1-10 \mathrm{~ms}$, similar to the timescales for thermal disruption of a single base pair in B-DNA [72]. These rates for binding-site recognition were slow in comparison with the range of reported diffusion times for various proteins scanning DNA, leaving open the question whether the previous kinetics studies had missed a faster step more commensurate with the protein diffusion times. Evidence for a faster step in the DNA bending dynamics was reported earlier by our group [35, 127], although it was not completely clear whether this fast step was from dye dynamics (of the attached FRET labels) or conformational dynamics in the IHF-H' complex.

In this thesis, I present a series of measurements that confirm that this rapid phase, occurring on timescales of $\sim 100 \mu \mathrm{s}-500 \mu \mathrm{s}$ is indeed from IHF-H' dynamics. In contrast to the relaxation rates for the slow $(1-10 \mathrm{~ms})$ phase, which are affected by modifications in the DNA at the site of the kinks, the relaxation rates for the fast phase appear to be sequenceindependent. The amplitude of this fast phase diminishes with increasing [salt] and increases in IHF mutants for which the positive charge on the binding surface of the IHF protein is reduced. Based on these observations, we assign the fast component to nonspecific DNA bending by IHF prior to the formation of the specific complex and the slow component to the ultimate recognition step. These results represent the first observation of sub-millisecond DNA bending kinetics during binding-site recognition by a DNA bending protein that bridges the gap between relatively slow (> ms) formation of specific complexes and relatively rapid ( $<\mathrm{ms}$ ) one-dimensional diffusion times measured for proteins moving on DNA. We propose that this rapid phase in the IHF-DNA complex may correspond to the wrapping/unwrapping 
of the $\beta$-arms of the protein in a nonspecific binding mode, as IHF scans potential binding sites on genomic DNA.

This study was carried out in collaboration with Prof. Phoebe Rice and her group at the University of Chicago.

\section{(2) Lesion recognition in DNA by XPC protein}

The first step in DNA damage repair is the recognition of damaged sites by proteins that scan, interrogate and recognize DNA lesions from predominantly normal, genomic DNA in cells. In mammalian nucleotide excision repair (NER), this initial recognition step is carried out by the Xeroderma Pigmentosum C protein complex (XPC), which recognizes bulky helix distorting lesions caused by environmental factors such as UV, pollutants, cigarette smoke, food toxins, etc. Crystal structure of Rad4 (a yeast ortholog of XPC) bound to a damaged site showed that it inserts a $\beta$-hairpin into the damaged site and induces a series of conformational changes on DNA such as nucleotide flipping, unwinding and bending and form an "open" conformation (Fig. 1.8) [126]. In this 'open' recognition complex, Rad4 does not directly contact damaged nucleotides, but, instead, selectively accommodates the undamaged nucleotides on the complementary strand. This type of indirect binding explained how Rad4/XPC can recognize extraordinarily diverse lesions commonly accompanied by local helix destabilization. Recently, it was also observed that the same 'open' structure is also formed with Rad4 covalently tethered to undamaged DNA [120]. This demonstrated that the lesion recognition cannot be based on structural differences in the thermodynamically most stable states of the bound complexes, but rather must depend on the differences in the kinetics of recognition. However, how these distinct motions are orchestrated to lead to final damage recognition have been unknown. 
In this thesis, I report laser T-jump measurements on Rad4 bound to a model 3-bp mismatch lesion that mimics real lesions recognized by Rad4/XPC. Using a combination of unique sets of fluorescent probes, I have mapped the trajectory of distinct conformational changes during interrogation and recognition with high temporal resolution, spanning a window of $\sim 10 \mu$ s to $\sim 50 \mathrm{~ms}$. The results unveil that Rad4/XPC first unwinds and partially bends DNA while bound nonspecifically on a timescale of $\sim 500 \mu \mathrm{s}$; second, it flips out the damaged nucleotides in a slower $(\sim 10 \mathrm{~ms})$ step, and finally the DNA is fully bent on longer (>50 ms) timescale. Mutant Rad4 lacking the $\beta$-hairpin can nonspecifically unwind and also (partially) open the damaged site, but are unable to form the more stable "recognition" conformation. Altogether, we propose a "twist-open" model of lesion recognition, whereby Rad4/XPC attempts to twist/unwind DNA as it scans DNA for lesions; upon encountering a lesion, the unwinding promotes nucleotide flipping; once the duplex is transiently open, Rad4/XPC inserts its $\beta$-hairpin to stabilize the final fully-bent, flipped-out "recognition" conformation. We also propose a "kinetic gating" mechanism whereby the selectivity of Rad4/XPC for lesions is determined by the balance between the residence time of XPC/Rad4 at a site and the DNA opening times. The 'opening' times for more stable, undamaged/matched DNA are expected to be much longer than for mismatched DNA [128], thus many orders of magnitude longer than the microsecond-regime residence times per DNA site reported for various proteins [100, 129-134]. This, we proposed, explains how Rad4/XPC avoids flipping 'open' every undamaged site when searching DNA for a lesion, while having selectively higher probabilities to open helix-destabilizing NER lesion sites before diffusing away.

This study is carried out in collaboration with Prof. Jung-Hyun Min and her group from the Department of Chemistry at UIC. 


\section{(3) Recognition of mismatches in DNA by MutS protein}

Repair of defects in DNA is central to the preservation of DNA integrity. These defects include replication errors like mispaired bases and insertion-deletion loops (IDL) which distort DNA structure. If these errors are not corrected, they are carried forward as mutations implicated in diseases like cancer and atherosclerosis. This study focuses on prokaryotic T. aquaticus (Taq) MutS and its eukaryotic homolog S. cerevisiae $\mathrm{MutS}_{\alpha}$, both of which recognize mispaired bases with the wrong Watson-Crick partner or unpaired bases, and subsequently recruit downstream proteins that rectify the mismatched site. Structural studies have shown that MutS-bound DNA is bent/kinked at the mismatch site. However, the underlying mechanism of mismatch recognition, and, in particular, the role of DNA flexibility at the mismatch site remains unclear.

In this thesis, I present results on the dynamics of MutS-mismatch recognition using the laser T-jump approach, on microsecond-to-milliseconds time-scales relevant to the formation of the initial recognition complex that triggers DNA repair. The study focuses on a T-bulge mismatch (an extra T-insertion in an otherwise matched DNA oligomer). Mismatch recognition by MutS is monitored using time-resolved FRET, to monitor DNA bending/unbending dynamics, and 2AP placed adjacent to the T-bulge site, to monitor mismatch dynamics in the vicinity of the T-bulge. As in the case of damaged recognition by Rad4, we uncover conformational dynamics on three different time-scales which we interpret as: (1) rapid fluctuations in the complex on time-scales of protein-diffusion on DNA, likely corresponding to DNA bending during nonspecific 'probing', (2) initial mismatch recognition, as monitored by $2 \mathrm{AP}$ dynamics at the mismatch site, and (3) formation of the ultimate recognition complex with full kinking/bending of DNA. These studies represent the first observation of mismatch recognition dynamics by MMR protein MutS, and suggest 
some common themes in the indirect readout damage recognition mechanisms of MMR and NER proteins.

This study is carried out in collaboration with Prof. Manju Hingorani and her group from Wesleyan University.

The remainder of this thesis is organized as follows: Chapter 2 describes equilibrium and T-jump experimental techniques, data acquisition and analysis, and experimental design with different probes. Chapter 3 describes the results on DNA bending dynamics during sitespecific recognition by IHF for a cognate site in $\lambda$ DNA. Chapter 4 describes the results of the dynamics of DNA damage recognition by the NER protein XPC (Rad4). Chapter 5 describes the results on DNA mismatch recognition by the MMR repair protein MutS. 


\section{References}

1. Yang, W., Structure and mechanism for DNA lesion recognition. Cell Res, 2008. 18(1): p. 184-97.

2. Richmond, T.J. and C.A. Davey, The structure of DNA in the nucleosome core. Nature, 2003. 423(6936): p. 145-50.

3. Rice, P.A., et al., Crystal structure of an IHF-DNA complex: a protein-induced DNA U-turn. Cell, 1996. 87(7): p. 1295-1306.

4. Lewis, M., Response: DNA Looping and Lac Repressor--CAP Interaction. Science, 1996. 274(5294): p. 1931-1932.

5. Kim, Y., et al., Crystal structure of a yeast TBP/TATA-box complex. Nature, 1993. 365(6446): p. 512-520.

6. Winkler, F.K., et al., The crystal structure of EcoRV endonuclease and of its complexes with cognate and non-cognate DNA fragments. EMBO J., 1993. 12(5): p. 1781-1795.

7. Obmolova, G., et al., Crystal structures of mismatch repair protein MutS and its complex with a substrate DNA. Nature, 2000. 407(6805): p. 703-10.

8. Lamers, M.H., et al., The crystal structure of DNA mismatch repair protein MutS binding to a Gx T mismatch. Nature, 2000. 407(6805): p. 711-7.

9. Howard, J., Mechanics of motor proteins and the cytoskeleton. 2001, Sunderland: Sinauer Associates, Inc.

10. Luger, K., et al., Crystal structure of the nucleosome core particle at 2.8 A resolution. Nature, 1997. 389(6648): p. 251-60.

11. Rice, P.A. and C.C. Correll, Protein-Nucleic Acid Interactions. 2008, Cambridge: The Royal Society of Chemistry.

12. Widom, J., Role of DNA sequence in nucleosome stability and dynamics. Q. Rev. Biophys., 2001. 34(3): p. 269-324.

13. Lowary, P.T. and J. Widom, New DNA sequence rules for high affinity binding to histone octamer and sequence-directed nucleosome positioning. J. Mol. Biol., 1998. 276(1): p. 19-42.

14. Zhang, Y. and D.M. Crothers, High-throughput approach for detection of DNA bending and flexibility based on cyclization. Proc. Natl. Acad. Sci. USA, 2003. 100(6): p. 3161-6.

15. Okonogi, T.M., et al., Sequence-dependent dynamics in duplex DNA. Biophys J, 2000. 78(5): p. 2560-71.

16. Cloutier, T.E. and J. Widom, Spontaneous sharp bending of double-stranded DNA. Mol. Cell, 2004. 14(3): p. 355-62.

17. Vafabakhsh, R., Ha, T., Extreme bendability of DNA less than 100 base pairs long revealed by single-molecule cyclization. . Science., 2012. 337: p. 1097-101.

18. Du, Q., et al., Cyclization of short DNA fragments and bending fluctuations of the double helix. Proc. Natl. Acad. Sci. USA, 2005. 102(15): p. 5397-402.

19. Du, Q., A. Kotlyar, and A. Vologodskii, Kinking the double helix by bending deformation. Nucleic Acids Res., 2008. 36(4): p. 1120-8.

20. Alexander Vologodskii, Q.D., Maxim D. Frank-Kamenetskii, Bending of short DNA helices. Artif DNA PNA XNA., 2013. 4: p. 1-3.

21. Skoko, D., et al., Mechanism of chromosome compaction and looping by the Escherichia coli nucleoid protein Fis. J. Mol. Biol., 2006. 364(4): p. 777-98.

22. Wiggins, P.A., R. Phillips, and P.C. Nelson, Exact theory of kinkable elastic polymers. Phys. Rev. E., 2005. 71(2 Pt 1): p. 021909. 
23. Wiggins, P.A., et al., High flexibility of DNA on short length scales probed by atomic force microscopy. Nat Nanotechnol, 2006. 1(2): p. 137-41.

24. Travers, A., DNA dynamics: bubble ' $n$ ' flip for DNA cyclisation? Curr. Biol., 2005. 15(10): p. R377-9.

25. Yan, J. and J.F. Marko, Localized single-stranded bubble mechanism for cyclization of short double helix DNA. Phys. Rev. Lett., 2004. 93(10): p. 108108.

26. Mills, J.B. and P.J. Hagerman, Origin of the intrinsic rigidity of DNA. Nucleic Acids Res., 2004. 32(13): p. 4055-9.

27. Yakovchuk, P., E. Protozanova, and M.D. Frank-Kamenetskii, Base-stacking and base-pairing contributions into thermal stability of the DNA double helix. Nucleic Acids Res., 2006. 34(2): p. 564-74.

28. Gotoh, O.T., Y., Locations of frequently opening regions on natural DNAs and their relation to functional loci. Biopolymers, 1981. 20: p. 1033-1042.

29. Vologodskii, A.V., Amirikyan, B. R., Lyubchenko, Y. L. \& and M.D. FrankKamenetskii, Allowance for heterogeneous stacking in the DNA helix-coil transition theory. J. Biomol. Struct. Dyn. , 1984. 2: p. 131-148.

30. Delcourt, S.G., Blake, R. D., Stacking energies in DNA. J. Biol. Chem., 1991. 266: p. 15160-15169.

31. Doktycz, M.J., et al., Studies of DNA dumbbells. I. Melting curves of 17 DNA dumbbells with different duplex stem sequences linked by T4 endloops: evaluation of the nearest-neighbor stacking interactions in DNA. Biopolymers, 1992. 32(7): p. 84964.

32. SantaLucia, J., Jr., Allawi, H. \& Seneviratne, P. A. , Improved nearest-neighbor parameters for predicting DNA duplex stability. Biochemistry (Mosc). 1996. 35: p. 3555-3562.

33. Sugimoto, N., Nakano, S., Yoneyama, M. \& Honda, K. , Improved thermodynamic parameters and helix initiation factor to predict stability of DNA duplexes. Nucleic Acids Res., 1996. 24: p. 4501-4505.

34. SantaLucia, J., Jr., A unified view of polymer, dumbbell, and oligonucleotide DNA nearest- neighbor thermodynamics. Proc. Natl. Acad. Sci. USA, 1998. 95(4): p. 14601465.

35. Ansari, A. and S.V. Kuznetsov, Dynamics and mechanism of DNA-bending proteins in binding site recognition, in Biophysics of DNA-protein interactions, M.C.W.a.L.J.M. III, Editor. 2010, Springer: New York.

36. Arthanari, H., et al., Effects of $H U$ binding on the equilibrium cyclization of mismatched, curved, and normal DNA. Biophys J, 2004. 86(3): p. 1625-31.

37. Protozanova, E., P. Yakovchuk, and M.D. Frank-Kamenetskii, Stacked-Unstacked Equilibrium at the Nick Site of DNA. J. Mol. Biol., 2004. 342: p. 775-785.

38. Olson, W.K., et al., DNA sequence-dependent deformability deduced from proteinDNA crystal complexes. Proc. Natl. Acad. Sci. U S A, 1998. 95(19): p. 11163-8.

39. Olson, W.K. and V.B. Zhurkin, Modeling DNA deformations. Curr. Opin. Struct. Biol., 2000. 10(3): p. 286-97.

40. Coman, D. and I.M. Russu, A nuclear magnetic resonance investigation of the energetics of basepair opening pathways in DNA. Biophys. J., 2005. 89(5): p. 328592.

41. Parker, J.B., et al., Enzymatic capture of an extrahelical thymine in the search for uracil in DNA. Nature, 2007. 449(7161): p. 433-7.

42. Moe, J.G. and I.M. Russu, Kinetics and energetics of base-pair opening in 5'd(CGCGAATTCGCG)-3' and a substituted dodecamer containing G.T mismatches. Biochemistry, 1992. 31(36): p. 8421-8. 
43. Mario A. Di'az de la Rosa, E.F.K., Peter J. Mulligan, and Andrew J. Spakowitz, Dynamic Strategies for Target-Site Search by DNA-Binding Proteins. Biophysical Journal, 2010. 98: p. 2943-2953.

44. Tafvizi, A., L.A. Mirny, and A.M. van Oijen, Dancing on DNA: kinetic aspects of search processes on DNA. Chemphyschem, 2011. 12(8): p. 1481-9.

45. Riggs, A.D., S. Bourgeois, and M. Cohn, The lac repressor-operator interaction. 3. Kinetic studies. J. Mol. Biol., 1970. 53(3): p. 401-17.

46. Richter, P.H. and M. Eigen, Diffusion controlled reaction rates in spheroidal geometry. Application to repressor--operator association and membrane bound enzymes. Biophys Chem, 1974. 2(3): p. 255-63.

47. Winter, R.B., O.G. Berg, and P.H. von Hippel, Diffusion-driven mechanisms of protein translocation on nucleic acids. 3. The Escherichia coli lac repressor-operator interaction: kinetic measurements and conclusions. Biochemistry, 1981. 20(24): p. 6961-77.

48. Stanford, N.P., et al., One- and three-dimensional pathways for proteins to reach specific DNA sites. EMBO J., 2000. 19(23): p. 6546-57.

49. (ed.), R.A., The Biology of Nonspecific DNA Protein Interactions. Boca Raton, FL: CRC Press, 1990.

50. Halford, S.E. and J.F. Marko, How do site-specific DNA-binding proteins find their targets? Nucleic Acids Res., 2004. 32(10): p. 3040-52.

51. Gowers DM, W.G., \& Halford SE Measurement of the contributions of $1 D$ and $3 D$ pathways to the translocation of a protein along DNA. Proc. Natl. Acad. Sci. , 2005. 102: p. 15883-15888..

52. Widom, J., Target site localization by site-specific, DNA-binding proteins. Proc Natl Acad Sci U S A, 2005. 102(47): p. 16909-10.

53. JT, S.J.S., Timing facilitated site transfer of an enzyme on DNA. Nat. Chem. Biol., 2012. 8: p. 205-210.

54. Lawson, C.L. and H.M. Berman, Indirect readout of DNA sequence by proteins, in Protein-Nucleic Acid Interactions, P.A. Rice and C.C. Correll, Editors. 2008, Royal Society of Chemistry: Cambridge.

55. D.E.Jr, K., Application of a theory of enzyme specificity to protein synthesis. Proc.Natl. Acad. Sci. , 1958. 44: p. 98-104

56. Spolar, R.S. and M.T. Record, Jr., Coupling of local folding to site-specific binding of proteins to DNA. Science, 1994. 263(5148): p. 777-84.

57. Garvie, C.W. and C. Wolberger, Recognition of specific DNA sequences. Mol. Cell, 2001. 8(5): p. 937-46.

58. Kalodimos, C.G., et al., Structure and flexibility adaptation in nonspecific and specific protein-DNA complexes. Science, 2004. 305(5682): p. 386-9.

59. Munazah Andrabi, K.M., and Shandar Ahmad Conformational changes in DNAbinding proteins: Relationships with precomplex features and contributions to specificity and stability. Proteins, 2014. 82: p. 841-857

60. Jean-Pierre Changeux, S.E., Conformational selection or induced fit? 50 years of debate resolved. F1000 Biology Reports, 2011(3-19).

61. Perez-Howard, G.M., P.A. Weil, and J.M. Beechem, Yeast TATA binding protein interaction with DNA: fluorescence determination of oligomeric state, equilibrium binding, on-rate, and dissociation kinetics. Biochemistry, 1995. 34(25): p. 8005-8017.

62. Parkhurst, K.M., M. Brenowitz, and L.J. Parkhurst, Simultaneous binding and bending of promoter DNA by the TATA binding protein: real time kinetic measurements. Biochemistry, 1996. 35(23): p. 7459-7465. 
63. Dhavan, G.M., et al., Concerted binding and bending of DNA by Escherichia coli integration host factor. J. Mol. Biol., 2002. 315(5): p. 1027-1037.

64. Hiller, D.A., et al., Simultaneous DNA binding and bending by EcoRV endonuclease observed by real-time fluorescence. Biochemistry, 2003. 42(49): p. 14375-14385.

65. Sugimura, S. and D.M. Crothers, Stepwise binding and bending of DNA by Escherichia coli integration host factor. Proc. Natl. Acad. Sci. USA, 2006. 103(49): p. 18510-4.

66. Huang, S.N. and D.M. Crothers, The role of nucleotide cofactor binding in cooperativity and specificity of MutS recognition. J Mol Biol, 2008. 384(1): p. 31-47.

67. Hancock, S.P., et al., The energetic contribution of induced electrostatic asymmetry to DNA bending by a site-specific protein. J Mol Biol, 2011. 406(2): p. 285-312.

68. van den Broek, B., M.C. Noom, and G.J. Wuite, DNA-tension dependence of restriction enzyme activity reveals mechanochemical properties of the reaction pathway. Nucleic Acids Res., 2005. 33(8): p. 2676-2684.

69. Dixit, S., et al., Mechanics of binding of a single integration-host-factor protein to DNA. Phys. Rev. Lett., 2005. 94(11): p. 118101.

70. Tolic-Norrelykke, S.F., et al., Stepwise bending of DNA by a single TATA-box binding protein. Biophys. J., 2006. 90(10): p. 3694-703.

71. Reinhard, B.M., et al., Use of plasmon coupling to reveal the dynamics of DNA bending and cleavage by single EcoRV restriction enzymes. Proc. Natl. Acad. Sci. USA, 2007. 104(8): p. 2667-72.

72. Kuznetsov, S.V., et al., Direct observation of DNA bending/unbending kinetics in complex with DNA-bending protein IHF. Proc. Natl. Acad. Sci. USA, 2006. 103(49): p. $18515-20$.

73. Stivers, J.T., K.W. Pankiewicz, and K.A. Watanabe, Kinetic mechanism of damage site recognition and uracil flipping by Escherichia coli uracil DNA glycosylase. Biochemistry, 1999. 38(3): p. 952-63.

74. Blainey, P.C., et al., A base-excision DNA-repair protein finds intrahelical lesion bases by fast sliding in contact with DNA. Proc. Natl. Acad. Sci. USA, 2006. 103(15): p. 5752-7.

75. Wang, Y.M., R.H. Austin, and E.C. Cox, Single molecule measurements of repressor protein 1D diffusion on DNA. Phys. Rev. Lett., 2006. 97(4): p. 048302.

76. Tafvizi, A., et al., Tumor suppressor p53 slides on DNA with low friction and high stability. Biophys. J., 2008. 95(1): p. L01-3.

77. Bonnet, I., et al., Sliding and jumping of single EcoRV restriction enzymes on noncognate DNA. Nucleic Acids Res., 2008. 36(12): p. 4118-27.

78. Gorman, J., et al., Dynamic basis for one-dimensional DNA scanning by the mismatch repair complex Msh2-Msh6. Mol. Cell, 2007. 28(3): p. 359-70.

79. Barsky, D., T.A. Laurence, and C. Venclovas, How proteins slide on DNA, in Biophysics of DNA-protein interactions, M.C. Williams and L.J. Maher, Editors. 2010, Springer: New York p. 39-68.

80. van den Broek, B., M.C. Noom, and G.J. Wuite, DNA-tension dependence of restriction enzyme activity reveals mechanochemical properties of the reaction pathway. Nucleic Acids Res, 2005. 33(8): p. 2676-84.

81. Sugimura, S. and D.M. Crothers, Stepwise binding and bending of DNA by Escherichia coli integration host factor. Proc Natl Acad Sci U S A, 2006. 103(49): p. 18510-4.

82. Kuznetsov, S.V., et al., Direct observation of DNA bending/unbending kinetics in complex with DNA-bending protein IHF. Proc Natl Acad Sci U S A, 2006. 103(49): p. 18515-20. 
83. Koval, V.V., et al., Real-time studies of conformational dynamics of the repair enzyme E. coli formamidopyrimidine-DNA glycosylase and its DNA complexes during catalytic cycle. Mutat Res, 2010. 685(1-2): p. 3-10.

84. Kuznetsov, N.A., et al., Conformational Dynamics of DNA Repair by Escherichia coli Endonuclease III. J Biol Chem, 2015. 290(23): p. 14338-49.

85. Slutsky, M. and L.A. Mirny, Kinetics of protein-DNA interaction: facilitated target location in sequence-dependent potential. Biophys J, 2004. 87(6): p. 4021-35.

86. Savir, Y. and T. Tlusty, Conformational proofreading: the impact of conformational changes on the specificity of molecular recognition. PLoS One, 2007. 2(5): p. e468.

87. Friedman, J.I. and J.T. Stivers, Detection of damaged DNA bases by DNA glycosylase enzymes. Biochemistry, 2010. 49(24): p. 4957-67.

88. Zhou, R., et al., SSB functions as a sliding platform that migrates on DNA via reptation. Cell, 2011. 146(2): p. 222-32.

89. Wibley, J.E., et al., Structure and specificity of the vertebrate anti-mutator uracilDNA glycosylase SMUG1. Mol Cell, 2003. 11(6): p. 1647-59.

90. Banerjee, A., et al., Structure of a repair enzyme interrogating undamaged DNA elucidates recognition of damaged DNA. Nature, 2005. 434(7033): p. 612-8.

91. Banerjee, A., W.L. Santos, and G.L. Verdine, Structure of a DNA glycosylase searching for lesions. Science, 2006. 311(5764): p. 1153-7.

92. Banerjee, A. and G.L. Verdine, A nucleobase lesion remodels the interaction of its normal neighbor in a DNA glycosylase complex. Proc Natl Acad Sci U S A, 2006. 103(41): p. 15020-5.

93. Iwahara, J., M. Zweckstetter, and G.M. Clore, NMR structural and kinetic characterization of a homeodomain diffusing and hopping on nonspecific DNA. Proc Natl Acad Sci U S A, 2006. 103(41): p. 15062-7.

94. Iwahara, J. and G.M. Clore, Detecting transient intermediates in macromolecular binding by paramagnetic NMR. Nature, 2006. 440(7088): p. 1227-30.

95. Maiti, A., et al., Crystal structure of human thymine DNA glycosylase bound to DNA elucidates sequence-specific mismatch recognition. Proc Natl Acad Sci U S A, 2008. 105(26): p. 8890-5.

96. Qi, Y., et al., Encounter and extrusion of an intrahelical lesion by a DNA repair enzyme. Nature, 2009. 462(7274): p. 762-6.

97. Friedman, J.I., A. Majumdar, and J.T. Stivers, Nontarget DNA binding shapes the dynamic landscape for enzymatic recognition of DNA damage. Nucleic Acids Res, 2009. 37(11): p. 3493-500.

98. Leith, J.S., et al., Sequence-dependent sliding kinetics of p53. Proc Natl Acad Sci U S A, 2012. 109(41): p. 16552-7.

99. Ghodke, H., et al., Single-molecule analysis reveals human UV-damaged DNAbinding protein $(U V-D D B)$ dimerizes on DNA via multiple kinetic intermediates. Proc Natl Acad Sci U S A, 2014. 111(18): p. E1862-71.

100. Nelson, S.R., et al., Two glycosylase families diffusively scan DNA using a wedge residue to probe for and identify oxidatively damaged bases. Proc Natl Acad Sci U S A, 2014. 111(20): p. E2091-9.

101. Gell, C., Brockwell, D., Handbook of single molecule fluorescence spectroscopy. Oxford University press, 2006. Oxford University Press.

102. Jalili, N., Laxminarayana, K., A review of atomic force microscopy imaging systems: application to molecular metrology and biological sciences. Mechatronics, 2004. 14(907-945). 
103. Iddo Heller, T.P.H., Graeme A. King, Erwin J. G. Peterman, and Gijs J. L. Wuite, Optical Tweezers Analysis of DNA-Protein Complexes. Chem. Rev., 2014. 114: p. 3087-3119

104. Jeffrey R. Moffitt, Y.R.C., Steven B. Smith, Carlos Bustamante, Recent Advances in Optical Tweezers. Annu. Rev. Biochem., 2008. 77: p. 205-28

105. Dekker, I.D.V.a.C., Recent Advances in Magnetic Tweezers. Annu. Rev. Biophys. , 2012. 41: p. 453-472

106. Van Orden, A., K. Fogarty, and J. Jung, Fluorescence fluctuation spectroscopy: a coming of age story. Appl. Spectrosc., 2004. 58(5): p. 122A-137A.

107. Sass, L.E., et al., Single-molecule FRET TACKLE reveals highly dynamic mismatched DNA-MutS complexes. Biochemistry, 2010. 49(14): p. 3174-90.

108. Bonnet, G., et al., Thermodynamic basis of the enhanced specificity of structured DNA probes. Proc. Natl. Acad. Sci. USA, 1999. 96(11): p. 6171-6176.

109. Kim, H.D., et al., Mg2+-dependent conformational change of RNA studied by fluorescence correlation and FRET on immobilized single molecules. Proc. Natl. Acad. Sci. USA, 2002. 99(7): p. 4284-4289.

110. Altan-Bonnet, G., A. Libchaber, and O. Krichevsky, Bubble dynamics in doublestranded DNA. Phys. Rev. Lett., 2003. 90(13): p. 138101.

111. Li, G., et al., Rapid spontaneous accessibility of nucleosomal DNA. Nat Struct Mol Biol, 2005. 12(1): p. 46-53.

112. Kubelka, J., Time-resolved methods in biophysics. 9. Laser temperature-jump methods for investigating biomolecular dynamics. Photochem Photobiol Sci, 2009. 8(4): p. 499-512.

113. Thompson, P.A., W.A. Eaton, and J. Hofrichter, Laser temperature jump study of the helix-coil kinetics of an alanine peptide interpreted with a 'kinetic zipper' model. Biochemistry, 1997. 36(30): p. 9200-10.

114. Qiu, L., et al., Smaller and faster: the 20-residue Trp-cage protein folds in 4 micros. J Am Chem Soc, 2002. 124(44): p. 12952-3.

115. Yang, W.Y. and M. Gruebele, Folding at the speed limit. Nature, 2003. 423(6936): p. 193-7.

116. Kubelka, J., et al., Chemical, physical, and theoretical kinetics of an ultrafast folding protein. Proc Natl Acad Sci U S A, 2008. 105(48): p. 18655-62.

117. Narayanan, R., et al., Fast folding of RNA pseudoknots initiated by laser temperaturejump. J Am Chem Soc, 2011. 133(46): p. 18767-74.

118. Kuznetsov, S.V., et al., Microsecond dynamics of protein-DNA interactions: direct observation of the wrapping/unwrapping kinetics of single-stranded DNA around the E. coli SSB tetramer. J Mol Biol, 2006. 359(1): p. 55-65.

119. Vivas, P., et al., Mapping the transition state for DNA bending by IHF. J Mol Biol, 2012. 418(5): p. 300-15.

120. Chen, X., et al., Kinetic gating mechanism of DNA damage recognition by Rad4/XPC. Nat Commun, 2015. 6: p. 5849.

121. Anunciado, D., et al., Multistep kinetics of the U1A-SL2 RNA complex dissociation. J Mol Biol, 2011. 408(5): p. 896-908.

122. Kuznetsov, S.V., et al., Microsecond Dynamics of Protein-DNA Interactions: Direct Observation of the Wrapping/Unwrapping Kinetics of Single-Stranded DNA around the E. coli SSB Tetramer. J. Mol. Biol., 2006. 359: p. 55-65.

123. Yang, C.C. and H.A. Nash, The interaction of E. coli IHF protein with its specific binding sites. Cell, 1989. 57(5): p. 869-880.

124. Swinger, K.K. and P.A. Rice, IHF and HU: flexible architects of bent DNA. Curr. Opin. Struct. Biol., 2004. 14(1): p. 28-35. 
125. Khrapunov, S., et al., Binding then bending: A mechanism for wrapping DNA. Proc. Natl. Acad. Sci. USA, 2006. 103(51): p. 19217-8.

126. Min, J.H. and N.P. Pavletich, Recognition of DNA damage by the Rad4 nucleotide excision repair protein. Nature, 2007. 449(7162): p. 570-5.

127. Vivas, P., Mechanism of integration host factor, a DNA-bending protein, probed with laser temperature-jump. PhD thesis, 2009.

128. Zheng, H., et al., Base flipping free energy profiles for damaged and undamaged DNA. Chem Res Toxicol, 2010. 23(12): p. 1868-70.

129. Wang, Y.M., R.H. Austin, and E.C. Cox, Single molecule measurements of repressor protein 1D diffusion on DNA. Phys Rev Lett, 2006. 97(4): p. 048302.

130. Blainey, P.C., et al., A base-excision DNA-repair protein finds intrahelical lesion bases by fast sliding in contact with DNA. Proc Natl Acad Sci U S A, 2006. 103(15): p. $5752-7$.

131. Gorman, J., et al., Dynamic basis for one-dimensional DNA scanning by the mismatch repair complex Msh2-Msh6. Mol Cell, 2007. 28(3): p. 359-70.

132. Tafvizi, A., et al., Tumor suppressor p53 slides on DNA with low friction and high stability. Biophys J, 2008. 95(1): p. L01-3.

133. Bonnet, I., et al., Sliding and jumping of single EcoRV restriction enzymes on noncognate DNA. Nucleic Acids Res, 2008. 36(12): p. 4118-27.

134. Schonhoft, J.D. and J.T. Stivers, Timing facilitated site transfer of an enzyme on DNA. Nat Chem Biol, 2012. 8(2): p. 205-10. 


\section{CHAPTER II}

\section{METHODS}

\subsection{Equilibrium measurements}

To examine protein binding to DNA, first the steady-state fluorescence emission spectra were measured using a FluoroMax-4 spectrofluorimeter (81005, Horiba Scientific) on fluorescence labelled DNA samples in the presence and absence of protein. The spectral differences between free DNA and protein-DNA complex can be correlated both with the extent of protein binding and with the protein induced conformational changes in DNA at equilibrium. To examine the thermal dissociation of the complex, and to determine the temperatures at which to carry out laser T-jump studies, equilibrium measurements were done as a function of temperature for all protein DNA complexes studied in this thesis.

\subsection{Laser Temperature Jump technique}

Laser induced temperature jump relaxation spectroscopy has emerged as a very useful technique to probe the conformational dynamics of biomolecules (protein, DNA, RNA) and the dynamics of the interactions between them. The basic idea behind this approach is that the equilibrium of interconverting chemical species is suddenly perturbed with a temperature jump, forcing the system to establish a new equilibrium point at a higher temperature. The change in the populations of the interconverting species as they evolve in response to the Tjump perturbation can be monitored with a suitable spectroscopic probe attached to the biomolecule. This time-resolved spectroscopy enables us to follow the conformational dynamics of the biomolecules over an enormous time window from few nanoseconds to several milliseconds. This T-jump perturbation must be fast in comparison with the 
conformational dynamics of interest. Dynamical interactions relevant to the function of a biomolecule can take place on time scales from sub-microseconds to several seconds or longer. Using a pulsed laser excitation with pulses in the near IR range, where water absorbs, it is possible to induce a fast $\mathrm{T}$ - jump perturbation in aqueous samples. Since vibrational relaxation takes place on the picosecond time scale both in water and in biomolecules, complete thermalization of solvent and solute occurs in 10-20 ps time scale [1]. However, since we use a laser pulse of $10 \mathrm{~ns}$, the temperature of the sample volume reaches its maximum value within $10-20 \mathrm{~ns}$. In this case, the dead time of the T-jump apparatus is determined by the IR laser pulse width convoluted with the response time of the detection electronics.

In 1964 Eigen and Demayer designed the first T-jump technique based on the electrical discharge of a capacitor through a conducting solution. The heating time in this setup is determined by the RC (R-resistance, C- capacitance) of the capacitor circuit and is typically on the order of microseconds for a temperature jump of a few degrees. The discovery of pulsed lasers in the 1960's led to an alternative way of directly heating the solution by an intense light pulse. Since the emission wavelengths of then available ruby (694 $\mathrm{nm})$ and Nd:glass (1060 nm) lasers are not absorbed by water, the earlier studies were done in the presence of strongly absorbing dyes in the sample solution to produce heating [2-4]. Adding the dyes in samples may pose some problems as the dyes may interfere with the reaction process of the samples and it can also interfere with the spectroscopic probing of the reaction by overlapping the spectral signals. Therefore, the desirable method is directly heating the solvent. Direct heating of the aqueous solvent required near infrared pulses with several $\mathrm{mJ}$ of energy between $1300 \mathrm{~nm}$ and $2100 \mathrm{~nm}$ to excite the overtone of the $\mathrm{OH}$ stretching band [5]. 
The laser we have been using in our T-jump set up is a Nd:Yag laser with a fundamental wavelength of $1064 \mathrm{~nm}$. Laser pulses at the desirable wavelength are obtained by Raman conversion (Fig. 2.1). Raman scattering is the elastic light scattering where the energy (and hence the frequency) of the scattered photon is shifted with respect to that of incident photon by the vibrational (or rotational) frequency of the gas medium [5]. The frequency shift occurs when some of the energy of the scattered photon is taken up by a molecule, which is excited into vibrational motion. The frequency can decrease (giving rise to Stokes lines) or increase (anti-Stokes lines) by integer multiples of the vibrational frequency of the medium, depending on whether the molecule start in the ground state or an excited state. Initially, most of the molecules would be in the ground state, with some molecules in the excited states due to thermal fluctuations. Since the initial population of the excited states is usually very small, the anti-Stokes lines are much weaker than the Stokes lines. When the incident laser beam power is sufficiently high, the intensity of the Stokes radiation reaches the threshold. At this point the Raman Effect becomes stimulated. The stimulated Stokes beam behaves like a laser emission (coherent and propagates in the direction of the pump beam). This is a relatively simple and useful method to extend the wavelength range of the existing laser source. In our apparatus, we use a $1 \mathrm{~m}$ long Raman cell containing high pressure (400 psi) methane gas, which shifts the Nd:Yag fundamental (1064 $\mathrm{nm})$ to $1542 \mathrm{~nm}, 2800 \mathrm{~nm}$,etc., and anti-Stoke lines to $812 \mathrm{~nm}, 656 \mathrm{~nm}$ and $474 \mathrm{~nm}$. The first Stoke's line at $1542 \mathrm{~nm}$ is the desired wavelength to heat the aqueous samples and is separated from the other lines that emerge from the Raman cell by a Pellin-Broca prism. The conversion efficiency of the Raman cell is $15 \%$. Hence in our T-jump setup we get energy of $\sim 50 \mathrm{~mJ} /$ pulse at the sample position, if we start with $\sim 300 \mathrm{~mJ} /$ pulse laser energy at $1064 \mathrm{~nm}$, incident on the Raman cell. 


\subsubsection{Laser Temperature jump spectrometer}

(a)

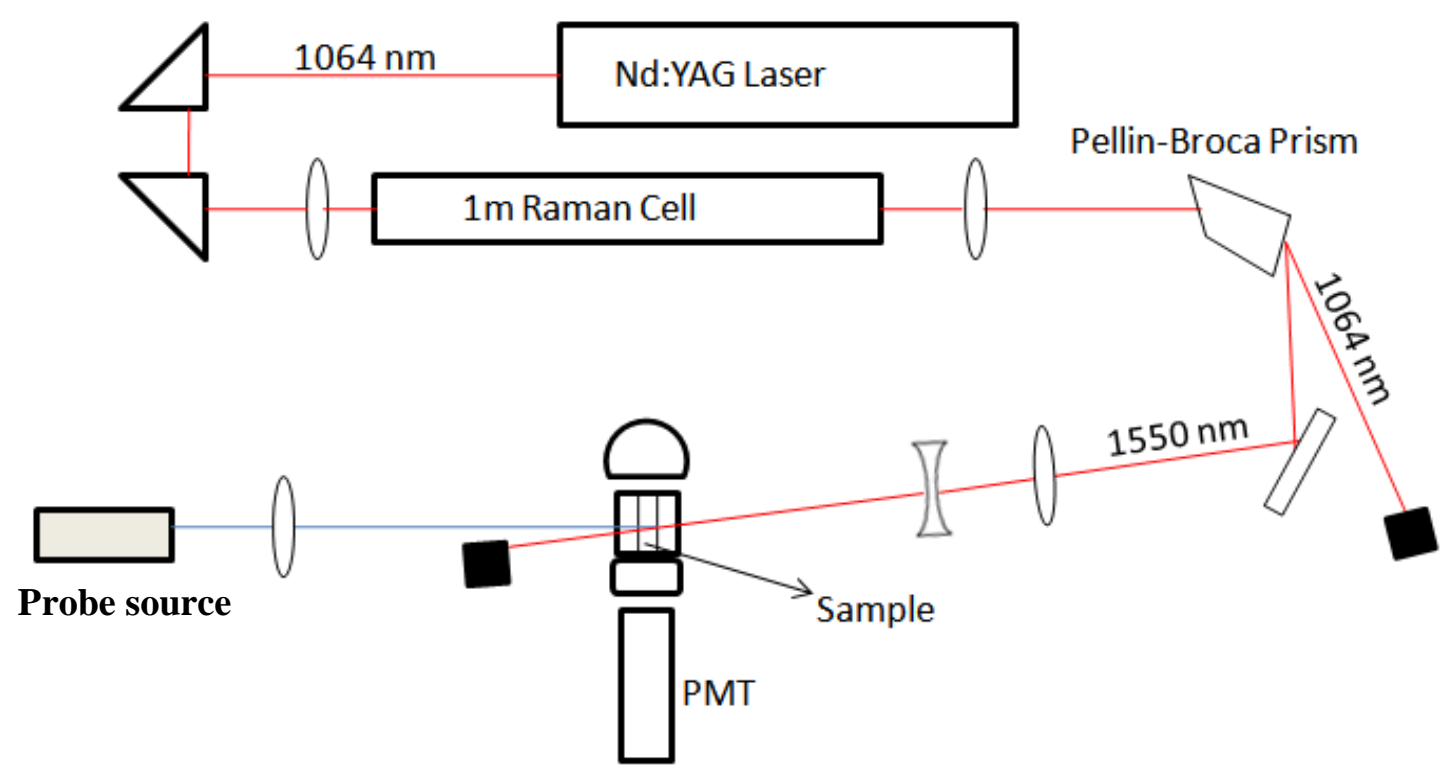

(b)

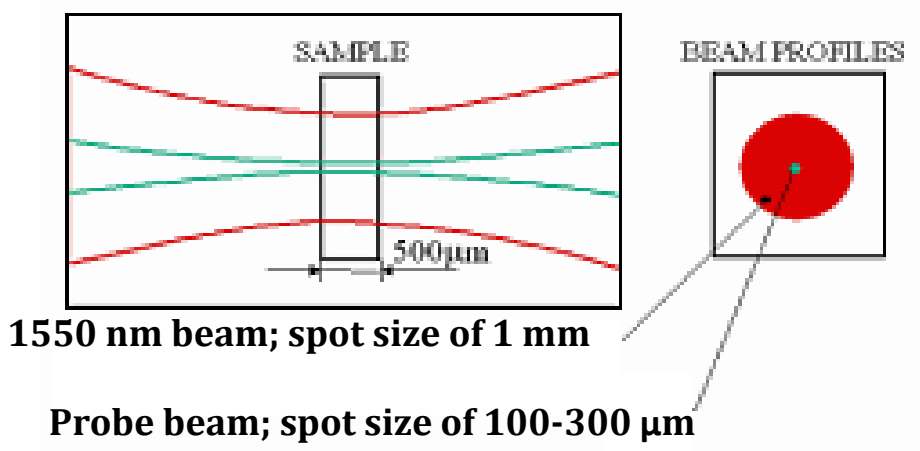

Fig.2.1. (a) Schematic of laser temperature jump spectrometer set up. (b) IR beam profile (red) (spot size $1 \mathrm{~mm}$ ) and probe beam profile (green) (spot size 100-300 $\mu \mathrm{m}$ ) at the sample position. Three different probe beam sources were used in this thesis (1) Xe-Hg lamp, (2) $488 \mathrm{~nm}$ diode laser, (3) $532 \mathrm{~nm}$ diode laser.

For all kinetic measurements on fluorescein dye-labeled DNA, the probe source to monitor the fluorescence intensity was a $20 \mathrm{~mW}$ CW diode laser at $488 \mathrm{~nm}$ (Newport PC13589) or a $50 \mathrm{~mW} 532 \mathrm{~nm}$ diode laser (Crystal Laser LC, GCL-050-L). The diode laser beam was focused directly on to the sample, to a spot size of $100 \mu \mathrm{m}$, and aligned to fall at the center of the $1 \mathrm{~mm}$ diameter heated spot produced by the IR beam. For kinetic measurements that probed fluorescent nucleotide analogs incorporated in DNA (e.g. 2AP or $\mathrm{tC}^{\mathrm{o}}$ probes, as described in Sec. 2.3 and 2.4), the probe source was a $200 \mathrm{~W}-\mathrm{Xe} / \mathrm{Hg}$ lamp. 
The light from the $\mathrm{Xe} / \mathrm{Hg}$ lamp was first focused on to a $300 \mu \mathrm{m}$ aperture, and the aperture was then reimaged on to the sample at the center of the $1 \mathrm{~mm}$ diameter heated spot. In the case of lasers we were able to directly focus the beam at sample position with the focal-spot diameter of $\sim 100 \mu \mathrm{m}$ as the beam profile from the laser was a well-defined collimated beam with the diameter of $\sim 1 \mathrm{~cm}$ after it passes through a beam expansion lens. However, in the case of the UV lamp, we needed an aperture, as the beam output from the lamp was much wider $(\sim 2 \mathrm{~cm})$ and hence, we used a $300 \mu \mathrm{m}$ aperture to reimage it at the sample position. The sample cuvettes of rectangular cross-sections of $0.5 \mathrm{~mm}$ by $2 \mathrm{~mm}$ and height $20 \mathrm{~cm}$ were designed from Quartz glass tubes, which were then cut to a smaller pieces (lengths of 3 cm's) and sealed at one end (at the UIC Chemistry Department glass shop). The samples were oriented such that the path length through which the IR beam and the probe beam traversed was $0.5 \mathrm{~mm}$, while the surface facing the IR beam (on one side) and the probe beam (on the other side) had dimensions $2 \mathrm{~mm}$ across and $3 \mathrm{~cm}$ high. The typical volume of the sample required in these cuvettes was $\sim 40 \mu \mathrm{L}$. The equilibrium temperature of the sample was controlled by a heat bath (TRE 111 from Neslab). The transient temperature of the sample after the heater pulse was measured as described in Sec. 2.1.4.

The fluorescence emission is collected $90^{\circ}$ to the excitation direction and focused on a photomultiplier (Hamamatsu 12928) after passing through band pass filters designed to transmit around the fluorescence emission maximum, while rejecting any stray fundamental or Stokes/anti-Stokes lines. However complete elimination of the stray light has been one of the challenges we face in our spectrometer, and during kinetics measurement this stray light saturates the PMT tube and basically limits the time-resolution of the T-jump apparatus to 10$20 \mu$ s, even though in principle we should be able to get the time resolution of $200 \mathrm{~ns}$ with the slow amplifier and $10 \mathrm{~ns}$ with the fast amplifier. The photomultiplier tube is coupled to either a $5 \mathrm{MHz}$ preamplifier (C1053-51, Hamamatsu) when the probe source was the $\mathrm{Hg} / \mathrm{Xe}$ 
lamp, or a $300 \mathrm{MHZ}$ preamplifier (SR445A, Stanford Research) when the probe source was the diode laser. The signal from the PMT was digitized using a $500 \mathrm{MHZ}$ transient digitizer (Hewlett Packard 54825A), which was recently replaced by a new digitizer from Tektronix (DPO 4054B). For each kinetic trace at a given initial temperature, typically over 500 shots (each with 20,000 data points from the HP digitizer or 1 million data points from the Tektronix digitizer) were averaged together. In order to minimize photo damage to the dye molecules, a fast mechanical shutter (UniBLITZ, VS14) was employed to block the probe beam between acquisitions of laser pulses. For each laser shot, the shutter was kept open for 60-450 ms depending on the kinetics timescale of a particular interaction. The averaged kinetics traces from the digitizer were transferred to a computer for further analysis.

\subsubsection{Theoretical estimation of the size of the T-jump}

The temperature increase upon the absorption of energy from the infrared laser pulse by the sample is given by

$$
\Delta T=\frac{I_{a}}{\rho V C_{w}}
$$

In Eq.2.1, $I_{a}$ is the absorbed intensity by the sample, $\rho$ is the density of the absorbing medium, $C_{w}$ is the specific heat, and $V$ is the volume of sample which absorbed the energy. In writing this equation, we have assumed that the incident energy is absorbed uniformly throughout the volume.

If an incident radiation $I_{o}$ passes through a substance of thickness $l$ and molar concentration $\mathrm{C}$, then the transmitted intensity is given by the Beer-Lambert law

$$
I_{t}=I_{o} 10^{-O D}
$$

where $O D=\in l C=\alpha l$ and $\alpha=\in C$

The absorbed intensity $I_{a}$ is thus given by 


$$
\begin{aligned}
& I_{a}=I_{o}-I_{t} \\
& I_{a}=I_{o}\left(1-10^{-\alpha l}\right)
\end{aligned}
$$

which yields

$$
\Delta T=\frac{I_{o}\left(1-10^{-a l}\right)}{\rho V C_{w}}
$$

In our experimental setup $I_{o} \approx 50 \mathrm{~mJ}$ at $1554 \mathrm{~nm}$ and this energy is absorbed by the aqueous solution of $\alpha \approx 0.52 \mathrm{~mm}^{-1}$ [6], $\boldsymbol{\rho}=1 \mathrm{~g} / \mathrm{cm}^{2}$ and $\mathrm{C}_{\mathrm{w}}=4.186 \mathrm{~J} /\left(\mathrm{g}{ }^{\circ} \mathrm{C}\right)$. If we assumed that the heated volume is a cylinder with radius $\sim 0.5 \mathrm{~mm}$, ignoring the Gaussian beam profile of the focused IR spot and use the path length of the sample $l=0.5 \mathrm{~mm}$, we obtain $\Delta \mathrm{T} \approx 12^{\circ} \mathrm{C}$. This T-jump agrees reasonably well with the $\mathrm{T}$-jump of $\sim 5-10^{\circ} \mathrm{C}$ that we obtain during our experiments, measured using a reference sample, as described in Sec. 2.2.4. The primary reasons for the lower than expected T-jump under our experimental conditions most probably due to imperfect alignment between the IR beam and the probe beam, fluctuations in beam alignment due to lamp (probe source) drifting or unstable IR beam over time.

In principle, we can increase the size of the T-jump by increasing the incident laser beam energy or by focusing the beam more sharply on the sample. However, large T-jumps $\left(>20{ }^{\circ} \mathrm{C}\right)$ also create undesirable effects such as photo-acoustic effects and cavitation, as discussed below.

\subsubsection{Photo-acoustic effects and cavitation.}

A rapid temperature jump can produce large pressure within the heated volume. This pressure can reach well over 100 bar [7]. The shock wave (photo-acoustic) produced by the rapid increase in the pressure propagates outwards and reflects back from the walls of the sample cell and produce large oscillations of the density of refractive index in the probed 
volume. In aqueous solution the timescale of these events is given by the speed of the sound and the cell geometry [5]. The photo-acoustic effect in the observed signal intensity can be seen as damped periodic oscillations. If these oscillations were present in the kinetic traces during the T-jump experiment then the focusing of the infrared beam with respect to probe beam was realigned until the oscillations disappeared from the trace.

Another problem caused by large T-jumps is cavitation. When the short laser pulse produces local heating, the pressurized liquid expands outward, the pressure within the heated volume drops to negative (tensile) values. When the tensile pressure reaches 5-10 bar, it exceeds the cavitation threshold, which in turn results in nucleation and growth of bubbles in the fluid medium [5]. The initial negative pressure peaks around $100 \mathrm{~ns}$ after the pulse and cavitation effects are usually observed for several microseconds. Bubbles with maximum diameters up to $100 \mu \mathrm{m}$ can be generated [5]. These bubbles can defocus the incident probe beam or deflect it away from the PMT, resulting in a dramatic decrease in the detected signal, which appears as an additional transient in the measured kinetic trace. Cavitation effects can be reduced by filtering the sample and thoroughly degassing it, which helps to eliminate possible nucleation sites.

Both photo-acoustic oscillations and cavitation effects, if they persist, typically last for about $20-30 \mu$ s, thus hampering the time-resolution of our measurements.

\subsubsection{Estimation of temperature jump using reference sample in a $\mathrm{T}$-jump experiment}

The magnitude of the T-jump in our apparatus, for a given alignment, is estimated using a reference sample (e.g. donor-only-labelled single-stranded (ss) or duplex DNA or free fluorescence dye). Ideally, free dye samples are preferable since they exhibit only a temperature-dependent change in quantum yield as the temperature of the sample is raised, which can be used to estimate the size of the T-jump as described below. In contrast, ssDNA 
may form hairpin structures that can unfold as a result of the T-jump perturbation, thus exhibiting unwanted relaxation kinetics in the control experiments. Similarly, duplex DNA may exhibit DNA melting/pre-melting kinetics at high enough temperatures. In chapter 4, we used probes incorporated double stranded DNA samples as a control, since this particular dye in free form was not commercially available. However the DNA melting temperatures for these DNA constructs are much higher $\left(>70^{\circ} \mathrm{C}\right)$ compare to our T-jump temperature window and therefore these samples behave well during the T-jump measurements.

As illustrated in Fig. 2.2 A, first the donor fluorescence intensity of the control sample as a function of temperature is obtained under equilibrium conditions, as described in Sec. 2.1 The fluorescence intensity typically decreases linearly with increasing temperature. As an example, for free fluorescein, the fluorescence decreases by $\sim 1 \%$ for every degree increase in temperature (Fig. 2.2 A). T-jump experiment on free fluorescein (Fig. 2.2 B) shows the fluorescence intensity level $I\left(0^{-}\right)$measured at the initial (equilibrium) temperature of the sample before the arrival of the heater pulse (the pre-laser level), followed by a rapid drop in the fluorescence intensity immediately after the arrival of the heater pulse to a post-pulse level $I\left(0^{+}\right)$, as a result of the rapid increase in the temperature of the sample. The fluorescence intensity then stays approximately the same up to about $20 \mathrm{~ms}$, after which it slowly recovers to the pre-heater-pulse level with a time constant of $~ 200$ ms (Fig. 2.4), concurrent with the recovery of the sample temperature back to its pre-T-jump equilibrium value. 

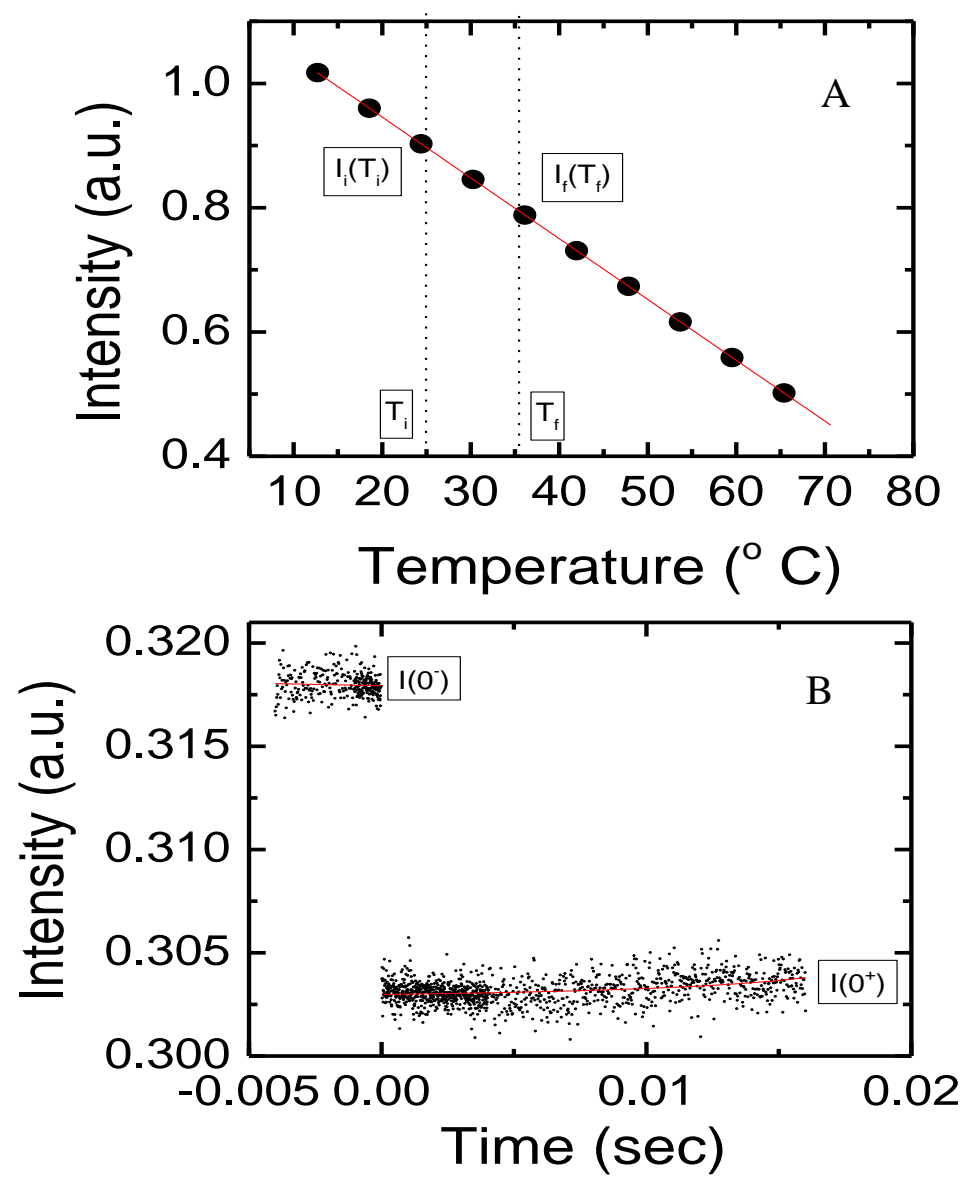

Fig.2.2. (A). Fluorescence intensity as a function of temperature of a free fluorescein dye sample under equilibrium conditions (B). Intensity as a function of time as a result of a $\sim 5 \mathrm{C} \mathrm{T}$-jump

To estimate the size of the T-jump, we compare the fluorescence intensities at the initial $\left(T_{i}\right)$ and final $\left(T_{f}\right)$ temperatures, denoted by $I_{i}\left(T_{i}\right)$ and $I_{f}\left(T_{f}\right)$ in equilibrium experiment, Fig. 2.2 A, with the intensities before and after the t-jump, $I\left(0^{-}\right)$and $I\left(0^{+}\right)$, respectively, in the $\mathrm{T}$-jump experiment. The relationship between the intensities from the equilibrium and T-jump measurements, given by:

$$
\frac{I_{f}\left(T_{f}\right)}{I_{i}\left(T_{i}\right)}=\frac{I\left(0^{+}\right)}{I\left(0^{-}\right)}
$$

is used to estimate the value of $I_{f}\left(T_{f}\right)$ in terms of the experimentally measured $I_{i}\left(T_{i}\right), I\left(0^{-}\right)$ and $I\left(0^{+}\right)$, and interpolated on the equilibrium fluorescence intensity versus temperature plot 
of Fig. 2.2 A to obtain the final temperature $T_{f}$. Fig. 2.3 shows the fluctuations in T-jump values measured on a $2 \mathrm{AP}$-labeled control sample during a 12 hour experimental period using an old IR laser (A) and new IR laser (B) and demonstrates the stability of the alignment in the T-jump apparatus over the course of a typical experiment.
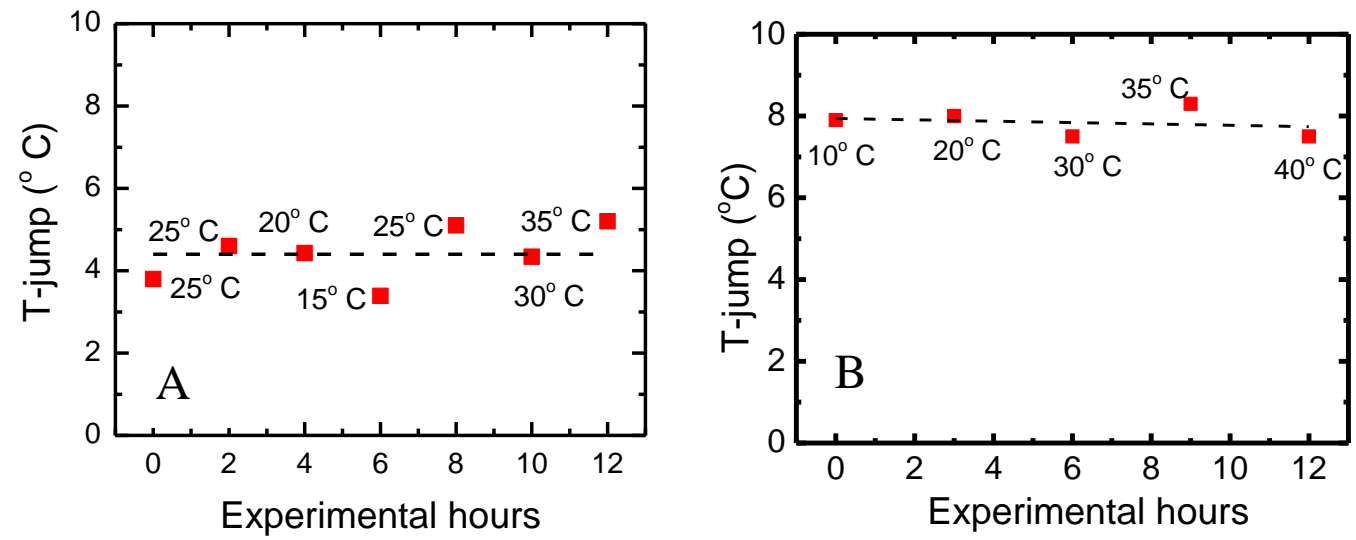

Fig.2.3. (A) The magnitude of the T-jump measured on a control sample consisting of a short 3-nt long DNA oligomer containing 2-aminopurine (TXA with 2AP at the position labeled $\mathrm{X})$. The measurements were done every two hours over a period of 12 hours. The initial equilibrium temperature before the T-jump was varied between $15{ }^{\circ} \mathrm{C}$ and $35{ }^{\circ} \mathrm{C}$ as shown in the figure (Heating pulse is from an old IR laser). (B) The same experiment as in (A) was repeated with a new IR laser, which shows very stable IR beam energy and hence reflects as a stable high temperature jump (measured with a free 2AP reference sample).

\subsubsection{T-jump recovery kinetics}

Measurements on control samples taken over a longer time-window (up to about 800 ms) show the "T-jump recovery kinetics", which is the relaxation of the temperature of the heated volume of the sample back to its pre-laser equilibrium level. A representative recovery trace (shown in Fig. 2.4) reveals that the temperature stays high up to about 10-20 ms before it starts to recover. 


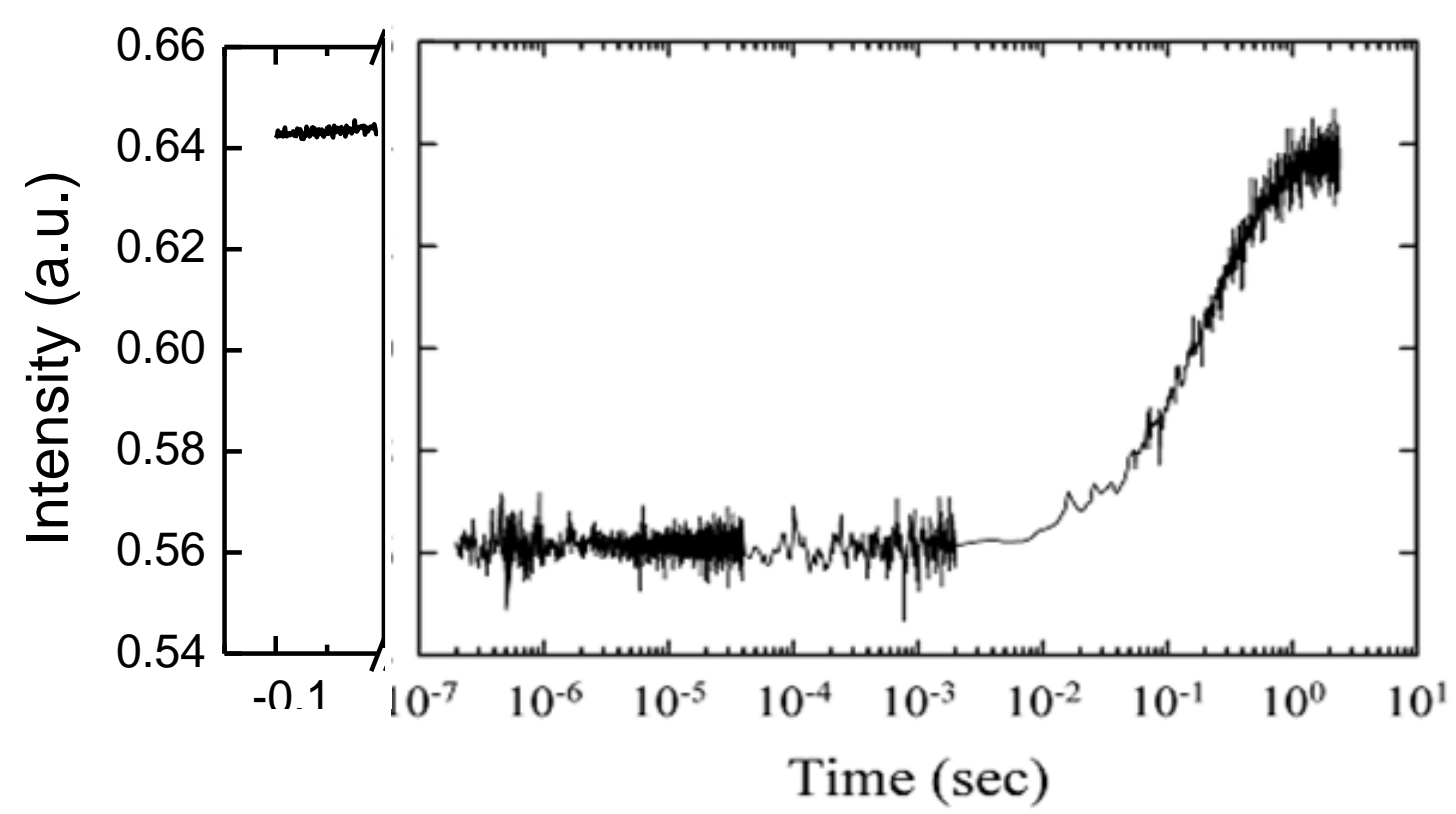

Fig.2.4. Kinetics trace from a control experiment (free fluorescent dye sample) plotted on logarithmic time scale, to illustrate the recovery of the temperature to the initial $\mathrm{T}_{\mathrm{i}}$.

The recovery kinetics is well described by the following recovery function:

$$
I(t)=\left[I\left(0^{+}\right)-I\left(0^{-}\right)\right] f_{r e c}(t)+I\left(0^{-}\right)
$$

with $f_{\text {rec }}(t)=\left(1+t / \tau_{r e c}\right)^{-1}$, where $\tau_{\text {rec }}$ is a characteristic time for the decay of the T-jump. This functional form of the recovery function is an empirical function that was found to fit the T-jump recovery kinetics slightly better than a single-exponential decay function. $I\left(0^{+}\right)$and $\tau_{r e c}$ were varied as free parameters in a non-linear least-squares fit to the T-jump recovery traces obtained on control samples. The recovery time constant $\tau_{\text {rec }}$ was determined for each sample from the average of several such measurements. Figure 2.5 shows a range of recovery times obtained from measurements on several different reference samples, over the course of 72 months. The average value of the recovery time constant from these set of measurements is found to be $\tau_{\text {rec }}=215 \pm 105 \mathrm{~ms}$. 


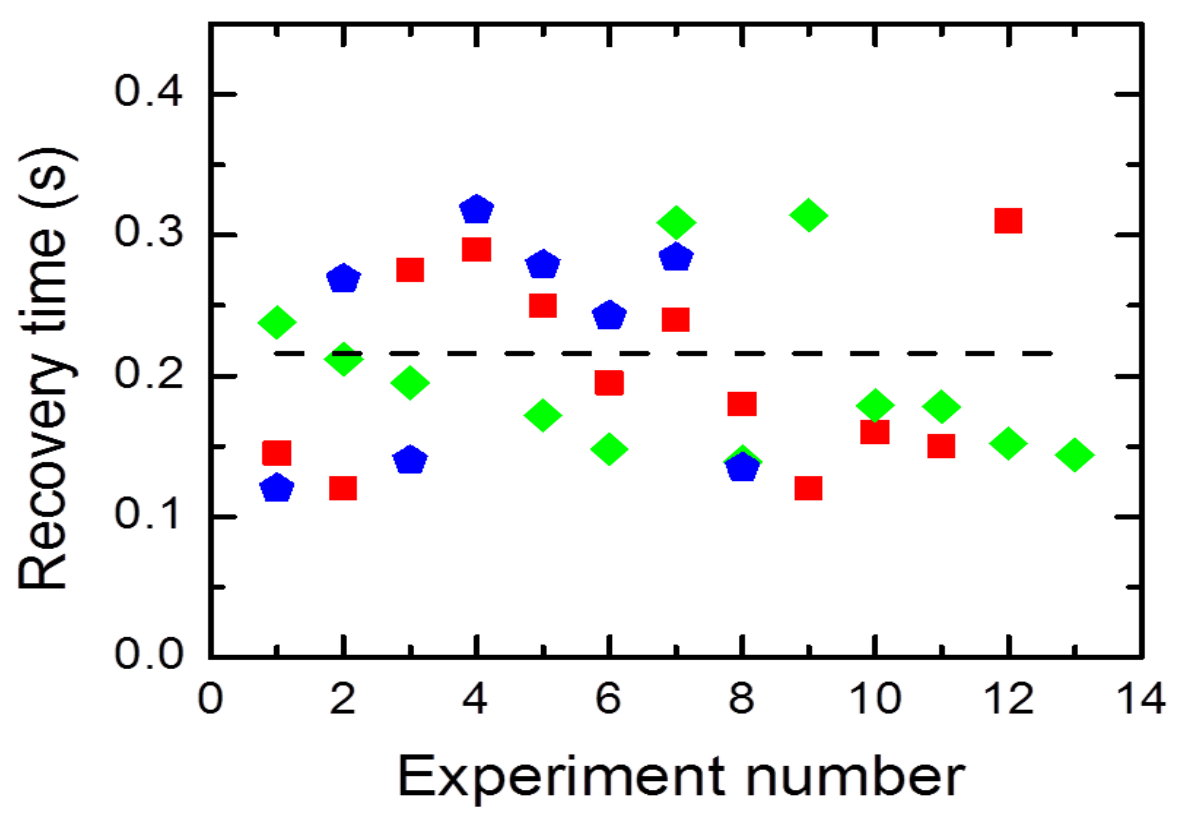

Fig.2.5. T-jump recovery times measured with different reference samples (Red- fluorescence labeled ss DNA (2008), Blue- Cy3 labeled SS DNA (2010) and Green-TAAMRA labeled ss DNA (2014)) at different points in time.

In general, kinetic processes that occur with relaxation times smaller than $\sim 20 \mathrm{~ms}$ are not significantly disturbed by the T-jump recovery response of the apparatus. However if the amplitude change in fluorescence from the relaxation kinetics of interest is significantly smaller than the change in fluorescence from the T-jump itself (which is proportional to the temperature-dependent quantum yield change of a particular dye), then the T-jump recovery kinetics can have an appreciable interference with the relaxation kinetics even below $20 \mathrm{~ms}$,

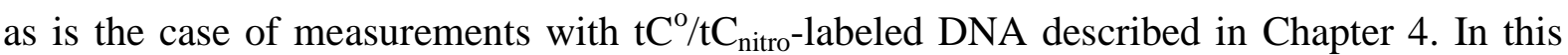
case, it is necessary to deconvolute the T-jump recovery from the observed relaxation kinetics. This deconvolution is also necessary when kinetic processes occur on time scales that exceed $\sim 20 \mathrm{~ms}$, as is the case of FRET labelled samples (TAMRA and Cy5 labels) described in Chapters 4 and 5. The deconvolution is described below. 


\subsubsection{Discrete single- or double-exponential decay convoluted with $\mathrm{T}$-jump recovery}

Relaxation kinetics traces $I(t)$ measured as a function of time after the T-jump were analyzed in terms of a single-exponential decay (Eq.2. 8, with relaxation rate $k_{\mathrm{r}}$ ) or doubleexponential decay (Eq. 2.9, with relaxation rates $k_{\text {fast }}$ and $k_{\text {slow }}$ ), convoluted with the recovery of the fluorescence intensity back to the pre-laser levels characteristic of the initial (equilibrium) temperature. The functional form used to fit the relaxation traces were:

$I(t)=\left(I\left(0^{+}\right)-I_{a p p}(\infty)\right) \times \exp \left(-k_{r} t\right)+\left(I_{a p p}(\infty)-I\left(0^{-}\right)\right) f_{r e c}(t)+I\left(0^{-}\right)$

or:

$I(t)=\left[I\left(0^{+}\right)-I_{a p p}(\infty)\right]\left[f_{1} \exp \left(-k_{f a s t} t\right)+\left(1-f_{1}\right) \exp \left(-k_{\text {slow }} t\right]+\left[I_{\text {app }}(\infty)-\right.\right.$ $\left.I\left(0^{-}\right)\right] f_{\text {rec }}(t)+I\left(0^{-}\right)$

In Eqs. 2.8 and 2.9, $I\left(0^{+}\right)$and $I\left(0^{-}\right)$are as defined earlier, and $I_{\text {app }}(\infty)$ is the fluorescence intensity at the end of the observed relaxation process (at $t>k_{\mathrm{r}}^{-1}$ in Eq.2. 8 or $t$ $>k_{\text {slow }}{ }^{-1}$ in Eq. 2.9). The parameters that were varied in a single-exponential fit are $I\left(0^{+}\right)$, $I_{\text {app }}(\infty)$, and $k_{\mathrm{r}}$, and in a double-exponential fit are $I\left(0^{+}\right), I_{\text {app }}(\infty), k_{\text {fast }}, k_{\text {slow }}$, and $f_{1}$, the fractional amplitude in the fast component.

\subsubsection{Acquisition and matching of relaxation traces measured over different time- scales}

To acquire data with the highest temporal resolution and be able to span several decades in time, it is necessary to measure the T-jump kinetics traces over different timescales and then combine the different traces. We typically acquired the kinetics traces on at least two time scales. Most of the kinetics traces reported in Chapters 3 and 5 were obtained 
using the Infinium digitizer from Hewlett Packard (500 MHz, $2 \mathrm{GSa} / \mathrm{s}$ ), which was eventually damaged and replaced by a new digitizer brought from Tektronix (DPO-4054B, $500 \mathrm{MHz}, 2.5 \mathrm{GS} / \mathrm{s})$. With the HP digitizer, typically 20,000 points were collected at each time scale such that the short timescale covered kinetics up to $2 \mathrm{~ms}$, with a time resolution of $100 \mathrm{~ns}$, while the longer time-scale covered kinetics up to $20 \mathrm{~ms}$, with a time-resolution of 1 $\mu \mathrm{s}$. All the kinetic traces reported in Chapter 4 were obtained with the Tektronix digitizer, which enabled us to collect $1 \mathrm{M}$ points at each time scale, thus significantly enhancing resolution and signal-to-noise in our kinetics traces. With this digitizer, the short time-scale covered kinetics up to $1.6 \mathrm{~ms}$, with a time-resolution of $1.6 \mathrm{~ns}$, while the longer time-scale covered kinetics up to $32 \mathrm{~ms}$, with a time-resolution of $32 \mathrm{~ns}$. The data acquired in the short time-scale were reduced to 2,000 points by averaging 500 points together; the data in the long time-scale were reduced to 10,000 points by averaging 100 points. After averaging, the time interval between data points was $800 \mathrm{~ns}$ and $3.2 \mu$ s for the short and long time-scale data, respectively. Prior to any further analysis, data acquired below $\sim 10-20 \mu$ s in each trace were discarded because of artifacts either from scattered IR laser light into the photomultiplier tube, or due to cavitation effects or from microbubbles in the samples.

In order to combine the data acquired over the two different time-scales, the two traces were fitted simultaneously with a double-exponential plus T-jump recovery function (Eq.2.9), with an additional fitting parameter that served as a multiplicative scale factor and was applied to one of the traces to account for any systematic difference in the measured intensities for the two traces. Once appropriately scaled, the two data sets were combined into a single kinetic trace that covered the time range from $\sim 10-20 \mu$ s to tens of ms. These matched and combined traces were then used for all subsequent analyses. Figure 2.6 A shows the kinetic trace obtained on the short time-scale $\left(I_{1}\left(T_{1}\right)\right)$, and the kinetic trace on the longer 
time scale $\left(I_{2}\left(T_{2}\right)\right)$, prior to any matching. Fig.2.6. B shows the kinetic traces that have been matched by scaling $\left(\mathrm{I}_{1}\left(\mathrm{~T}_{1}\right)\right.$ by a factor of 1.0065 .

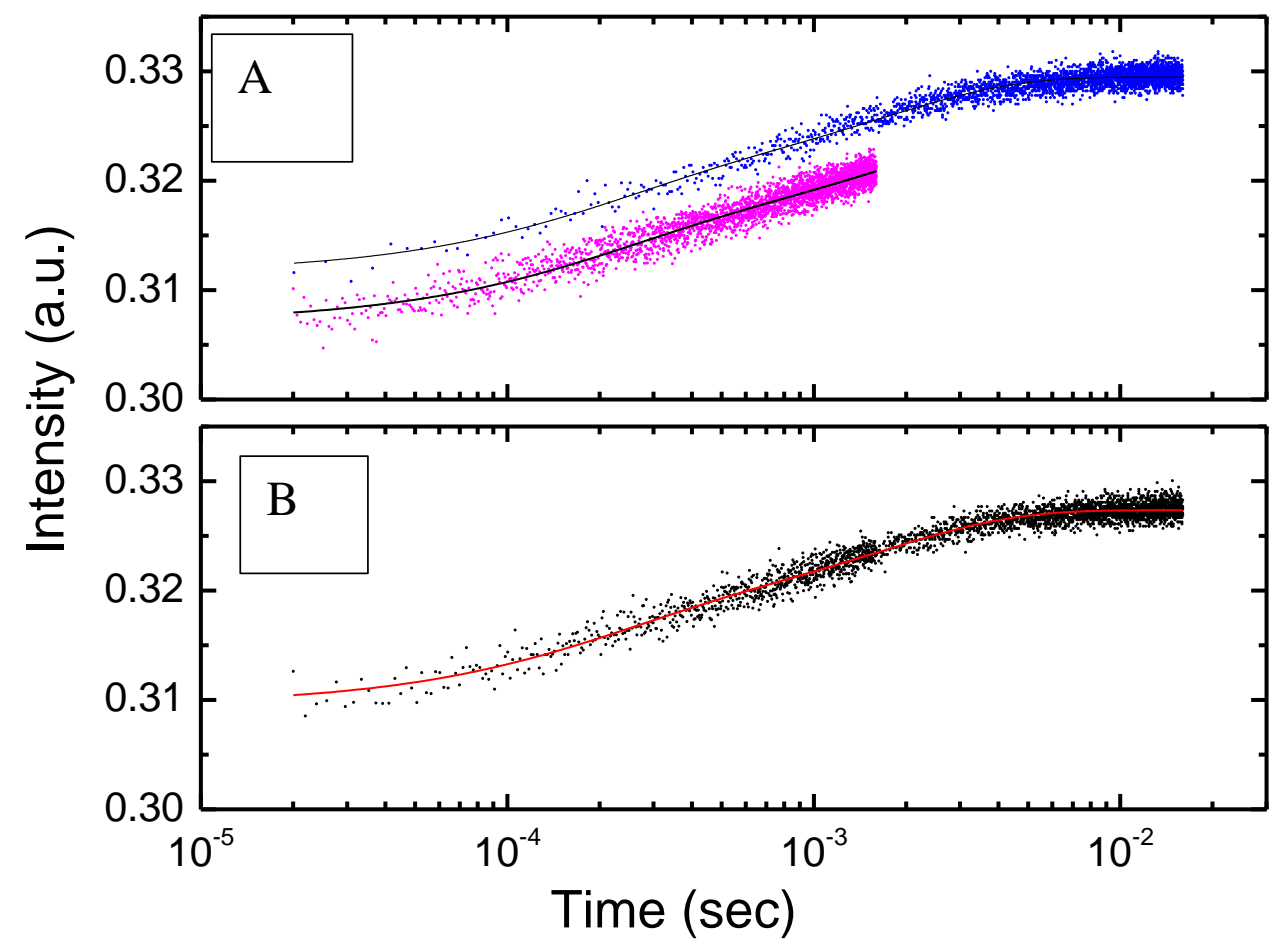

Fig.2.6. (A) Kinetics traces taken on two separate time-scales: $1.6 \mathrm{~ms}$ full time-scale (magenta) and $16 \mathrm{~ms}$ full time-scale (blue) and the black line is a two exponential fit before matching the data. (B) The combined kinetic trace obtained after scaling on data set to match the intensity of the other, as described in the text. The red solid line is a two exponential fit to the combined trace using equation 2.9.

\subsubsection{Maximum entropy analysis}

Maximum entropy method (mem) is used to obtain distribution of relaxation rates. For many relaxation traces, the kinetics showed deviations from a single-exponential behavior. However, adding an additional exponential decay, while it improved the fit, gave unreliable estimates of the relaxation times and amplitudes for the two exponential components. Note that for purposes of obtaining a scale factor to match data on different time-scales, a two-exponential fit was adequate. However, to overcome the limitations of such discrete exponential analysis, we chose the maximum entropy method (mem) that yields a model-independent distribution of rate constants. The mem algorithm was provided to us by 
Dr. Pete Steinbach of the National Institutes of Health, and is described in refs. [8-10]. The mem analysis yields a distribution $f(\log \tau)$ of relaxation times $\tau$ that maximizes the entropy $S$, defined below, while constraining the normalized residual sum of squares to be equal to one. The entropy function is defined as:

$$
S(f, F)=\sum_{j=1}^{M}\left[f_{j}-F_{j}-f_{j} \ln \left(f_{j} / F_{j}\right)\right]
$$

where $f_{j}$ are the discretized values of the distribution $f(\log \tau)$ and $F$ is a model distribution that is the default distribution in case of noisy data and is assumed to be a uniform, flat distribution.

The mem analyses on our data typically reveal two distinct peaks, which is either a combination of one relaxation phase and a slower $\mathrm{T}$-jump recovery phase, or two relaxation phases, with the T-jump phase unresolved. In most cases, the two peaks were reasonably well separated, enabling us to compute an average relaxation time $\tau_{\text {ave }}=10^{<\log \tau>}$ for each phase, where $\langle\log \tau\rangle$ is computed from the distribution of relaxation times within each peak, and defined as:

$$
<\log \tau>=\frac{\sum_{j=1}^{M} \log \tau_{j} f\left(\log \tau_{\mathfrak{j}}\right) \log \tau_{\mathrm{j}}}{\sum_{j=1}^{M} f\left(\log \tau_{\mathrm{j}}\right) \log \tau_{\mathrm{j}}}
$$

If there was some overlap between the peaks, or an asymmetric peak indicating a "shoulder" suggesting two phases in the relaxation kinetics as in Figure 2.7, the distribution $f(\log \tau)$ was fitted to the sum of two Gaussian distributions, and the average $<\log \tau>$ was obtained from the peak position of each Gaussian distribution. The area under each Gaussian curve was used to obtain the relative amplitude in each kinetic phase.

To determine whether the observed peaks are from real relaxation phases or T-jump recovery, we also carried out mem analysis on control experiments measured on identical 
time-scales as the relaxation kinetics, as well as on control experiments measured on a longer time-scale.
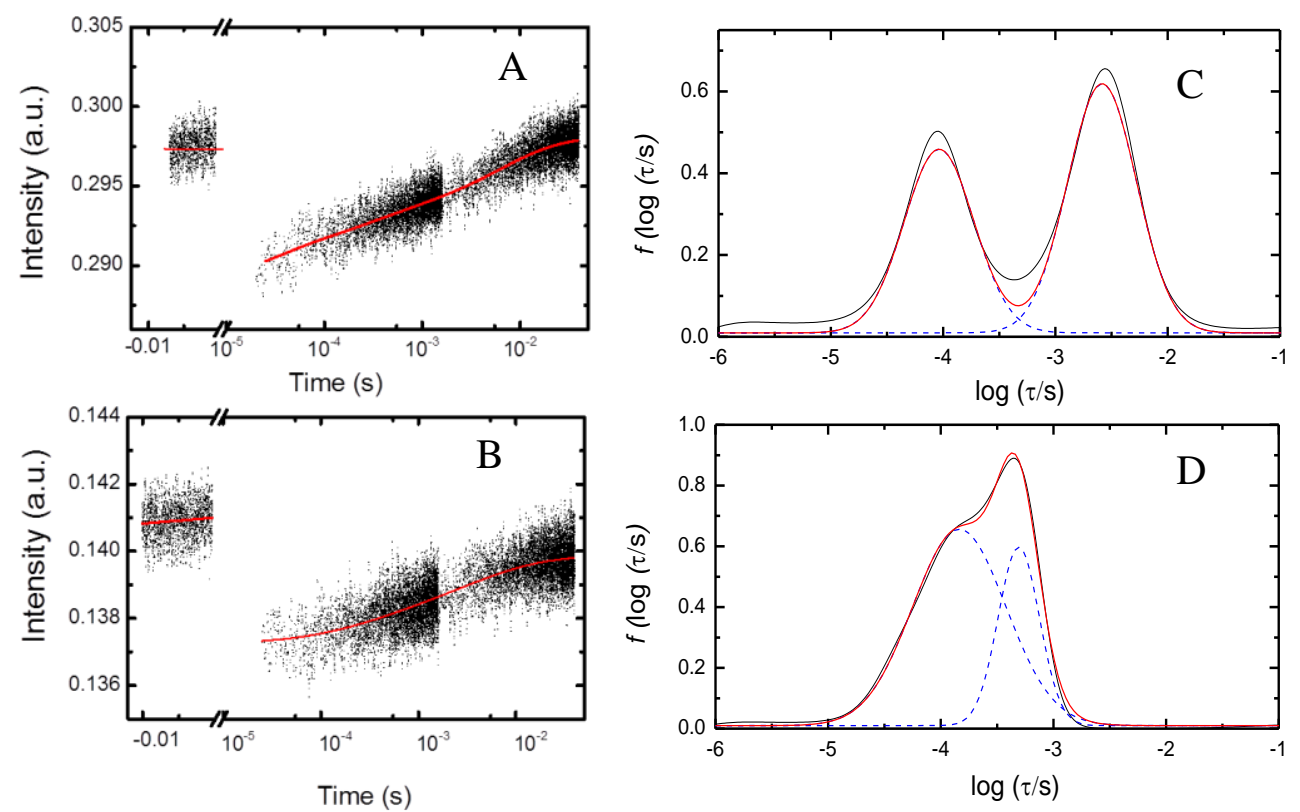

Fig.2.7. Analysis of the distribution of relaxation times from the maximum entropy method. (A,B) Kinetics of a protein- DNA complex at two different temperatures. (C,D)The distribution of relaxation times $f(\log (\tau / s)$ is plotted versus $\log (\tau / s)$ (solid black line) in two different situations, (C) when the two peaks are not well separated (corresponding to the kinetics trace in A), (D) the second peak appears as a shoulder (corresponding to the kinetics trace in B). The two Gaussian functions that fit the distribution are shown (dashed blue line). The overall fit is also shown (red line).

\subsection{Equilibrium FRET measurements}

Several methods can be used to determine the FRET efficiency on samples labelled with a donor-acceptor pair [11].

(1) Measurements of lifetime of donor in the excited state, in the presence and absence of an acceptor,

(2) Measurements of the donor emission intensity in the presence and absence of an acceptor.

(3) Acceptor emission intensity as results of energy transfer in comparison with acceptor emission intensity from direct excitation of the acceptor. 
In this study we have used the second and third method to determine FRET efficiency values.

\subsubsection{FRET determination using the donor emission}

In this method FRET efficiency in the free DNA or in complex with protein is determined by using the donor emission intensity in the presence $\left(\mathrm{I}_{\mathrm{DA}}\right)$ and in the absence $\left(\mathrm{I}_{\mathrm{D}}\right)$ of an acceptor. $\mathrm{I}_{\mathrm{D}}$ is obtained from the donor fluorescence intensity of single-labeled (donor only) substrate, and $\mathrm{I}_{\mathrm{DA}}$ is obtained from the donor fluorescence intensity of the doublelabeled (donor and acceptor) substrate, under identical conditions (e.g to find the FRET of free DNA we used donor only free DNA and donor and acceptor labeled free DNA and in the case of complex it is donor only labeled DNA+protein and donor and acceptor labeled DNA +protein). The FRET efficiency value $\mathrm{E}$ is calculated from:

$$
E=1-\frac{I_{D A}}{I_{D}}
$$

\subsubsection{FRET determination from Acceptor emission}

FRET efficiency can also be measured as the increase in acceptor fluorescence intensity due to the presence of donor fluorophore for both free and protein bound DNA substrates. To make this measurement, the donor fluorophore was excited at donor excitation wavelength, and the emission spectra were collected covering the donor and acceptor emission. A normalized emission spectrum of a donor-only labeled sample, excited at donor excitation wavelength, was subtracted from the emission spectrum to isolate the acceptor emission. The acceptor fluorophore was directly excited at acceptor excitation wavelength and emission spectra were collected. The area under the corrected spectrum of acceptor emission was divided by the area under the directly excited emission spectrum to obtain the value of the acceptor ratio $r_{\mathrm{A}}$. 


$$
E=\left[\frac{\varepsilon_{\lambda_{A}}^{A}}{\varepsilon_{\lambda_{D}}^{D}}\right]\left[r_{A}-\frac{\varepsilon_{\lambda_{A}}^{A}}{\varepsilon_{\lambda_{D}}^{A}}\right]
$$

where $\varepsilon_{\lambda_{D}}^{D}$ and $\varepsilon_{\lambda_{A}}^{A}$ are the extinction coefficients at excitation wavelengths for donor and acceptor, respectively. The acceptor ratio method to determine the FRET efficiency is preferable over the donor fluorescence method, because the latter requires ratio of fluorescence intensities from measurements on two separate samples, single-labeled and double-labeled DNA samples. This method for determining FRET efficiency best compensates for uncertainties in the degree of labeling of the acceptor, quantum yield of the acceptor, and the concentration of DNA used [11].

To convert acceptor ratio into real FRET efficiency requires knowledge of the extinction coefficients of the donor and acceptor fluorophores at the two different excitation wavelengths, and accurate estimates of labeling efficiency. If we are only interested in variation of FRET with protein binding or variation of FRET with salt or with varying temperature, then it is not necessary to determine absolute FRET efficiency for each sample.

\subsubsection{Following are the FRET pairs used in this thesis}

(1) Conventional FRET pairs (Fluorescein and TAMRA or TAMRA and Cy5)

(2) Nucleotide analog FRET pair $\mathrm{tC}^{\mathrm{o}}$ and $\mathrm{tC}_{\text {nitro }}$

\section{(1) Conventional FRET pairs (Fluorescein and TAMRA, TAMRA and Cy5)}

Fluorescein (6-carboxylicfluorescein (6-FAM)) (donor)/TAMRA

(6-carboxytetramethylrhodamine) (acceptor) is a common donor acceptor pair used in FRET fluorescein studies due to their good spectral overlap (Fig.2.8. A, B). Experiments reported in 
this thesis with FAM/TAMRA FRET pair, the donor 6-FAM is excited at $488 \mathrm{~nm}$ which is the probe laser wavelength used in the T-jump set up and the energy is transferred to TAMRA, which then emits light centered at $580 \mathrm{~nm}$. The Föster distance for this donor acceptor pair is $R_{o}=50 \AA$ when the donor and/or acceptor molecules sample all possible orientations within the fluorescence lifetime of the donor, and for which $\kappa_{2}=2 / 3$ (see appendix B). Another commonly used FRET pair is TAMRA/Cy5 (Fig.2.8. B,C) and in this case TAMRA's excitation wavelength was kept as $532 \mathrm{~nm}$, which is the probe laser beam wavelength used in the T-jump set up to excite TAMRA. TAMRA emission wavelength is centered at $580 \mathrm{~nm}$ and then due to FRET energy transfer Cy5 emits with a peak position at $662 \mathrm{~nm}$. The Föster distance for TAMRA and Cy5 is $R_{o}=65 \AA$ [12]. In these FRET pairs, there are emission wavelengths where only donor emission is observed with no contribution from the acceptor. Therefore for this FRET pairs, the FRET can be measured using donor peak. For each sample, the characteristic emission intensities $I_{\mathrm{D}}$ and $I_{\mathrm{DA}}$ were computed as the area under the corresponding emission curves in the range $\pm 50 \mathrm{~nm}$ around the maximum peak position. However when determining FRET using donor method, as we need two separate samples, single labeled (donor only) and double labeled (donor and acceptor), the accuracy of the concentration of the samples are crucial. The dye molecules fluorescein, TAMRA and Cy5 are attached to oligo nucleotides via an amino C6 linker. 

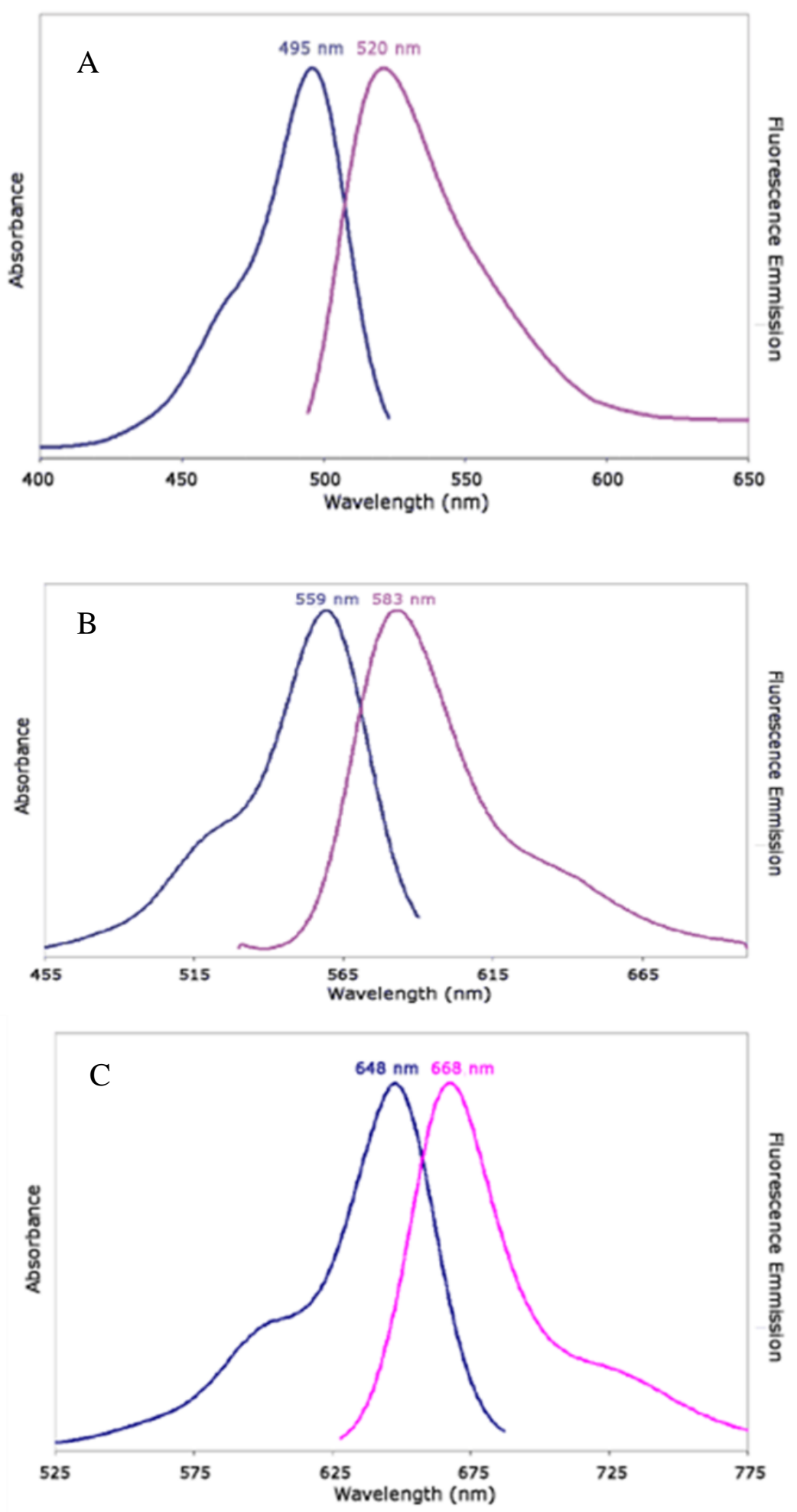

Fig.2.8. Absorbance and emission spectra of (A) 6-FAM, (B) TAMRA and (C) Cy5. Figures reproduced from the Integrated DNA technology (IDT) web page: https://www.idtdna.com/site 


\section{(2) Nucleotide analog FRET pair $\mathrm{tC}^{\mathrm{o}}$ and tCnitro.}

The tricyclic cytosine analog tC (1,3-diaza-2-olophnothiazine) and its oxo-homologue $\mathrm{tC}^{\mathrm{o}}$ (1,3-diaza-2-oxo-phenoxazine) was originally developed by Matteucci and co-workers in 1995 [13]. In recent years, after the elaborate characterization of these fluorescent nucleic acid bases mainly by Wilhelmsson group [14], these base analogues have emerged as novel probes with very attractive features for the study of nucleic acid structure or for investigating nucleic-acid-protein interactions. One of the striking features of these new tC products in contrast to the previously existing base analogs is that they are insensitive to their immediate surrounding bases. For example, tC is insensitive to the microenvironment in single $\left(\varphi_{f}=\right.$ $0.17-0.24)$ and double $\left(\varphi_{f}=0.16-0.21\right)$ stranded $\mathrm{DNA}$ and $\mathrm{tC}^{\mathrm{o}}$ is insensitive to the micro environment of double stranded DNA $\left(\varphi_{f}=0.17-0.27\right)$ (Fig. 2.9) [14]. The quantum yield of $\mathrm{tC}^{0}$ in double stranded DNA is 0.22 on average. In combination with its high molar absorptivity $\left(\varepsilon=9000 \mathrm{M}^{-1} \mathrm{~cm}^{-1}\right), \mathrm{tC}^{\mathrm{o}}$ on average is the brightest (Brightness $\propto$ $\left.\left(\varphi_{f} * \varepsilon\right)\right)$ base analog in duplex DNA context among the currently available base analogs [14]. However $\mathrm{tC}^{\mathrm{o}}$ is sensitive to its surrounding bases in single stranded DNA $\left(\left(\varphi_{f}=\right.\right.$ $0.14-0.41)$. Though the quantum yield of $\mathrm{tC}^{\mathrm{o}}$ changes approximately by a factor of 2 between single and double stranded DNA, it preserves more of its high fluorescence quantum yield upon hybridization than any other base analogs [15]. 


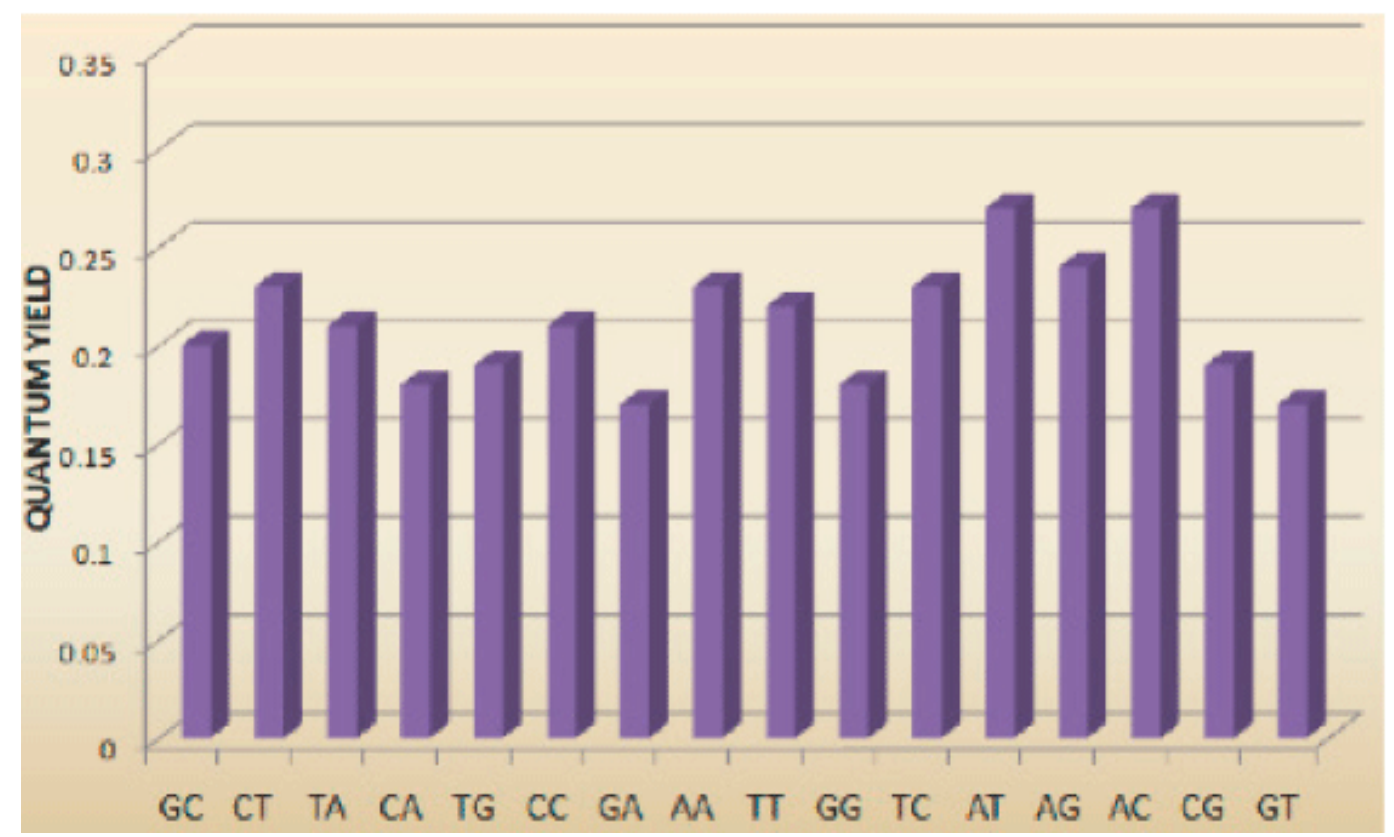

Fig.2.9. Fluorescence quantum yield of in DNA double-stranded systems. Letters denote bases surrounding $\mathrm{tC}\left(5^{\prime}-\ldots \mathrm{X}(\mathrm{tC}(\mathrm{O})) \mathrm{Y} \ldots-. .3^{\prime}\right)$. Figure reproduced from [16].

Wilhelmsson et al developed a new FRET pair consisting of $\mathrm{tC}^{\mathrm{o}}$ and another cytosine analog $\mathrm{tC}_{\text {nitro }}$ (7-nitro-1, 3-diaza-2-oxophenothiazine) in order to achieve the highest possible control of donor/acceptor orientation [17]. They showed that both $\mathrm{tC}^{\mathrm{o}}$ and $\mathrm{tC}_{\mathrm{nitro}}$ are rigidly stacked within the duplex and therefore are excellent candidates for FRET measurements aimed to probe the duplex conformation. Moreover, they incorporated these analogs in different sequences and with all 4 different base pairings and showed that the melting temperature of $\mathrm{tC}^{\mathrm{o}} / \mathrm{tC}_{\text {nitro }}-\mathrm{G}$ is much higher than the $\mathrm{tC}^{\mathrm{o}} / \mathrm{tC}_{\text {nitro }}-\mathrm{A}, \mathrm{T}, \mathrm{C}$ base pairs $\left(\mathrm{tC}^{\mathrm{o}}-\mathrm{A}, \mathrm{T}, \mathrm{C}\right.$ melting temperature is $12-26^{\circ} \mathrm{C}$ lower than $\mathrm{tC}^{\mathrm{o}}-\mathrm{G}$ base pair and $\mathrm{tC}_{\mathrm{nitro}}-\mathrm{A}, \mathrm{T}, \mathrm{C}$ base pair melting temperature is $13-19^{\circ} \mathrm{C}$ lower than $\mathrm{tC}_{\text {nitro }}-\mathrm{G}$ base pair) $[17,18]$. These results show that $\mathrm{tC}^{\mathrm{o}}$ and $\mathrm{tC}_{\text {nitro }}$ are highly selective for base - paring with guanine and retain Watson-Crick pairing with guanine. In addition both $\mathrm{tC}^{\mathrm{o}}$ and $\mathrm{tC}_{\text {nitro }}$ slightly increase the duplex stability, melting experiments on duplex DNA with either $\mathrm{tC}^{\mathrm{o}}$ or $\mathrm{tC}_{\text {nitro }}$ showed that on average both of these analogs increase the melting temperature by $2^{\circ} \mathrm{C}$ compare to $\mathrm{C}$. [17]. The enhanced stability most likely comes from the increased $\Pi-\Pi$ overlap between the extended ring system of $\mathrm{tC}^{\mathrm{o}}$ 
and the neighboring bases. Studies on secondary structure of $\mathrm{tC}^{\mathrm{o}}$ and $\mathrm{tC}_{\text {nitro }}$ incorporated duplex DNA using circular dichroism (CD) measurements showed that both $\mathrm{tC}^{\mathrm{o}}$ and $\mathrm{tC}_{\text {nitro }}$ sequences exhibit typical characterization of B-form DNA [16, 17]. In addition, fluorescence anisotropy measurements showed further evidence that $\mathrm{tC}^{\mathrm{o}}$ makes proper base-pairing and is firmly stacked in the DNA duplex [16]. In these measurements, it was shown that the emission of $\mathrm{tC}^{\mathrm{O}}$ reports on the overall mobility of the DNA duplex without any interference from intrinsic mobility of the base analog itself [16]. All the previous studies on $\mathrm{tC}^{\mathrm{o}}$ and $\mathrm{tC}_{\text {nitro }}$ confirm that incorporation of $\mathrm{tC}^{\mathrm{o}}$ or $\mathrm{tC}_{\text {nitro }}$ has no significant effect on the overall conformation of duplex DNA [14, 16-18].

$\mathrm{tC}^{\mathrm{O}}$ can easily be selectively excited. It has the lowest energy absorption band centered $~ 365 \mathrm{~nm}$ in duplex DNA as well as in ssDNA and the emission maximum is at 465 $\mathrm{nm}$ (Fig.2.10). The lowest energy absorption maximum of the virtually non-fluorescent $\mathrm{tC}_{\mathrm{nitro}}$ in dsDNA is centered at $\sim 440 \mathrm{~nm}$ with an excitation coefficient of $5400 \mathrm{M}^{-1} \mathrm{~cm}^{-1}$. This gives a very good overlap with the emission of $\mathrm{tC}^{\mathrm{o}}[17]$.

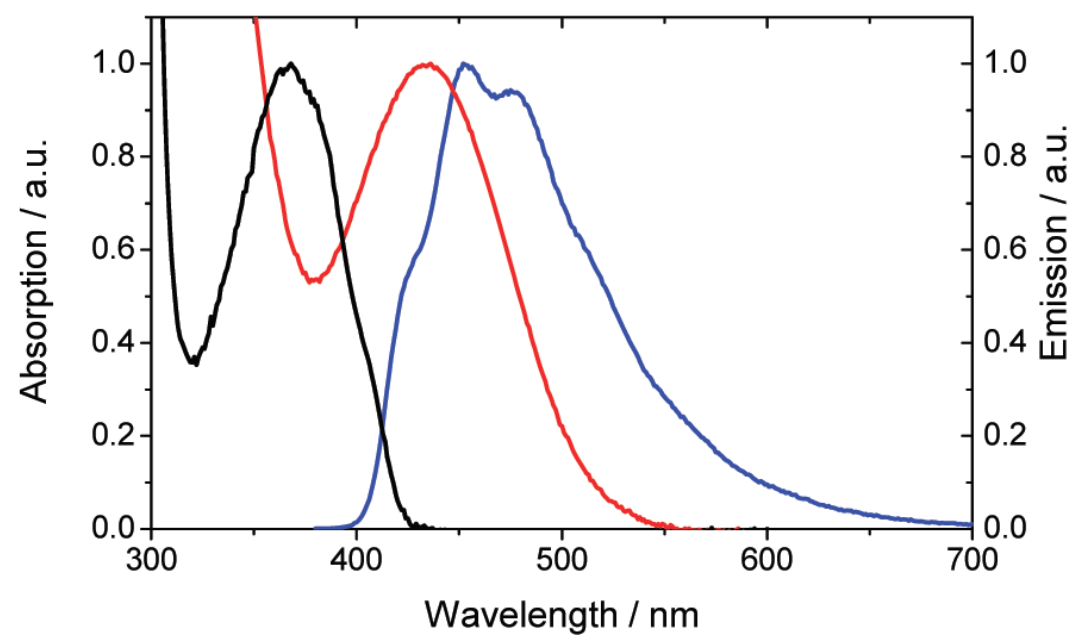

Fig.2. 10. Normalized absorption (black) and emission (blue) spectra of FRET donor $\mathrm{tC}^{\mathrm{o}}$ and absorption spectrum (red) of virtually nonfluorescent acceptor $\mathrm{tC}_{\text {nitro }}$ within dsDNA showing the donor/acceptor spectral overlap. Measurements performed at $22{ }^{\circ} \mathrm{C}$ in $25 \mathrm{mM}$ phosphate buffer (pH 7.5) and $100 \mathrm{mM}[\mathrm{Na}+]$. Figure adapted from [17] with permission. Copyright (2009) American Chemical Society. 
Wilhelmsson group studied the FRET efficiency of 12 different DNA sequences, in which the FRET labels were placed starting from 2 base pair apart to 13 base pair apart, both using steady state measurements and time- resolved fluorescence measurements. The results showed that the efficiency is highly dependent on both distance and orientation. This is because when the distance changes the orientation of the dipole changes as well due to DNA helicity. Fig.2.11 shows the variation of FRET as a function of base pair separation. In addition to the conventional behavior of distance dependent FRET, where FRET decreases sharply with distance, here they observed a stepwise function which oscillates between a local maxima and minima as the orientation of the transition dipole of the donor and acceptor changes between more parallel to more perpendicular.

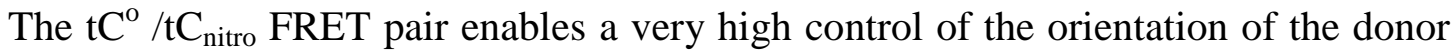
and acceptor (hence the Förster distance) due to the fact that both the analogues are rigidly located within the base stack as well as due to a very stable donor quantum yield. If the FRET pairs are carefully chosen at the positions where the slopes are steep (Fig.2.11) then it is possible to accurately distinguish distance from the orientation changes using FRET.

Attracted by the promising features of this newly discovered $\mathrm{tC}^{\mathrm{o}} / \mathrm{tCnitro}$ FRET pair, we used it to measure conformational changes in DNA upon protein binding and took advantage of a detectable change in the FRET efficiency of this pair as a consequence of a change in their separation and relative orientation upon DNA unwinding. 


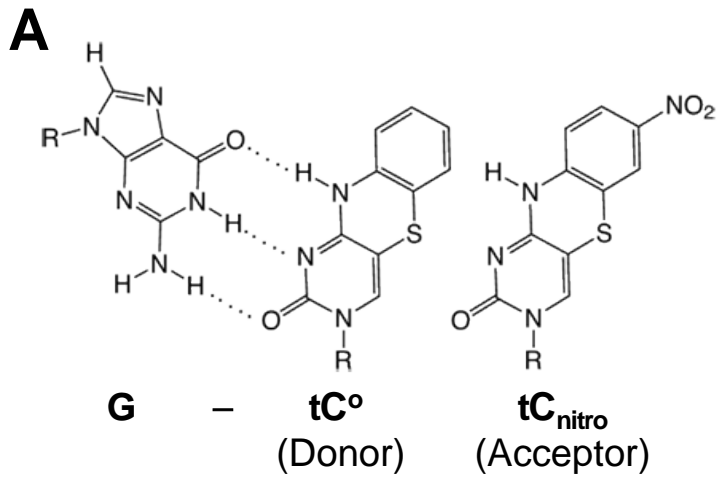

B

Fig.2.11. Cytosine analogs $\left(\mathrm{tC}^{\mathrm{O}}\right.$ and $\left.\mathrm{tC}_{\mathrm{nitro}}\right)$ act as a FRET pair and provide distance and relative orientation information in DNA. (A) Chemical structures of (left) $\mathrm{tC}^{\mathrm{o}}$ and (right) $\mathrm{tC}_{\text {nitro }}$ and Watson-Crick type base pairing of $\mathrm{tC}^{\mathrm{O}}$ with a guanine. (B) FRET efficiency between the $\mathrm{tC}^{\mathrm{O}}$ (donor) and $\mathrm{tC}_{\text {nitro }}$ (acceptor) incorporated within normal B-DNA is plotted as a function of the number of base-pairs between the two probes. FRET measurements were done as described in Borjesson et al. [17]. The FRET efficiency generally decreases as the distance increases, but additionally depends on the relative orientations of the absorption and emission dipoles of the fluorophores, which changes by $2 \pi$ over the helical pitch $(\sim 34 \AA)$ of DNA. (Figures reproduced from ref. [17] with permission. Figure reproduced from [17] with permission. Copyright (2009) American Chemical Society.

\subsection{Nucleotide analog 2-Aminopurine (2AP)}

2-Aminopurine is a fluorescence analog of adenine nucleotide which forms hydrogen bonds with thymine very similar to those of adenine. 2AP is the most widely used fluorescent probe of DNA structure and the perturbation of that structure by interaction with enzymes and other molecules, since its potential as a fluorescence base analogue was first recognized 50 years ago. Although 2AP differs from the natural base (adenine: 6-aminopurine) only in the position of the exocyclic amine group, its fluorescence intensity is one thousand times greater [19] (Fig.2.12). 
(a)

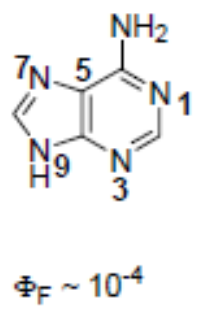

(b)

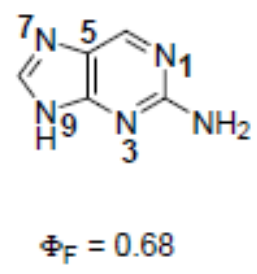

Fig.2. 12 (a) $2 \mathrm{AP}$ and (b) adenine and their fluorescence quantum yields, $\varphi_{\mathrm{f}}$.

The substitution of $2 \mathrm{AP}$ at a specific position of a DNA sequence preserves the structure of the double helix as 2AP hydrogen bonds and stacks with the natural bases in DNA. 2AP incorporated in DNA can be excited at $314 \mathrm{~nm}$ and the maximum emission occurs approximately at $370 \mathrm{~nm}$. At this excitation wavelength either natural bases or the fluorescent amino acids tyrosine and tryptophan when the DNA is bound to protein will not get excited.

2AP fluoresce decreases dramatically when it is placed inside DNA (it is quenched 100 times in duplex DNA compare to its free form). The main cause of this fluorescence quenching is charge transfer to the neighboring natural bases and it is mediated by base stacking interactions and base dynamics[20]. For this reason 2AP is very sensitive to the protein induced local conformations and dynamics when incorporated in DNA [21-24].

The fluorescence intensity of an extrahelical 2AP is high whereas the intensity of an intrahelical 2AP is low, since 2AP is strongly fluorescent when free in solution but very weakly fluorescent when inside the DNA. Allan and Reich [21] first used this fact to monitor the methyltransferece induced base flipping of DNA. They showed that binding of M.EcoRI to 2AP labelled DNA as such 2AP is placed at the target position methylation, induced a 14fold increase in the fluorescence intensity of the 2AP. In recent years $2 \mathrm{AP}$ is used to study nucleotide flipping induced by DNA repair proteins such as MutS and $\operatorname{Rad} 4[25,26]$. 


\subsection{Fraction of Protein and DNA in complex at Equilibrium}

Association and dissociation of protein in a simple bimolecular scheme is shown below,

$$
\text { Protein }+ \text { DNA } \rightleftarrows \text { complex } 2.14
$$

The equilibrium association constant $\mathrm{K}_{\mathrm{a}}$ can be written as:

$$
K_{A}=\frac{f_{x}}{P_{e q} D_{e q}}
$$

where $\boldsymbol{f}_{\boldsymbol{x}}$ is the equilibrium concentration of the complex and $\mathrm{P}_{\mathrm{eq}}$ and $\mathrm{D}_{\mathrm{eq}}$ are the concentrations of free protein and DNA that are not formed as complex. Expressing $P_{e q}$ and $D_{e q}$ in terms of the total protein and DNA concentrations ( $P_{o}$ and $D_{o}$, respectively) as $P_{e q}=$ $P_{o}-\boldsymbol{f}_{\boldsymbol{x}}$ and $D_{e q}=D_{o}-\boldsymbol{f}_{\boldsymbol{x}}$, we can write the association constant in terms of the total concentrations as:

$$
\begin{aligned}
& \boldsymbol{K}_{A}=\frac{\boldsymbol{f}_{X}}{\left(\boldsymbol{P}_{o}-\boldsymbol{X}\right)\left(D_{o}-\boldsymbol{X}\right)} \\
& \boldsymbol{K}_{\boldsymbol{A}}\left[\boldsymbol{f}_{\boldsymbol{X}}-\left(\boldsymbol{P}_{\boldsymbol{o}}+\boldsymbol{D}_{o}\right) \boldsymbol{f}_{X}+\boldsymbol{P}_{o} \boldsymbol{D}_{o}\right]-\boldsymbol{X}=\mathbf{0}
\end{aligned}
$$

or, in terms if the dissociation constant $K_{D}=\frac{1}{K_{A}}$, we get,

$$
f_{X}^{2}-\left(P_{o}+D_{o}+K_{D}\right) f_{X}+P_{o} D_{o}=0
$$

Thus, for a given value of $P_{o}, D_{o}$ and $K_{D}$, we obtain the concentration in complex as:

$$
f_{X}=\left(P_{o}+D_{o}+K_{D}\right) \pm \frac{\sqrt{\left(P_{o}+D_{o}+K_{D}\right)^{2}-4 P_{o} D_{o}}}{2}
$$

Only the solution with minus sign in equation (2.19) gives physically meaningful values for $f_{X}$, which cannot be larger than $D_{o}$

$$
f_{X}=\left(P_{o}+D_{o}+K_{D}\right)-\frac{\sqrt{\left(P_{o}+D_{o}+K_{D}\right)^{2}-4 P_{o} D_{o}}}{2}
$$




\section{6 $K_{D}$ measurements from equilibrium FRET}

\subsubsection{Conventional titration experiments}

Assuming only two states for the protein and DNA, free and in complex, the measured FRET efficiency $E$ or acceptor ratio $r_{\mathrm{A}}$ depend on the fraction of DNA in complex $\boldsymbol{f}_{\boldsymbol{x}}$ as follows:

$$
\begin{array}{r}
E=E_{X} f_{X}-E_{\text {free }}\left(1-f_{X}\right) \\
r_{A}=r_{A}^{X} f_{X}-r_{A}^{\text {free }}\left(1-f_{X}\right)
\end{array}
$$

where $E_{X}\left(r_{A}^{X}\right)$ is the FRET efficiency (acceptor ratio) for the complex, $E_{\text {free }}\left(r_{A}^{\text {free }}\right)$ is the FRET efficiency (acceptor ratio) for free DNA substrate, and $f_{X}$ is obtained from equation (20) with the condition $P_{0} \geq D_{0}$.

$\mathrm{K}_{\mathrm{D}}$ of protein-DNA complex can be determined from equilibrium titration measurements. Typically the FRET versus [protein] was measured in about 15 to 20 samples containing a fixed concentration of DNA $(\sim 1-10 \mathrm{nM})$ and varying concentration of protein. These binding isotherms were obtained for $[\mathrm{KCl}]$ ranging from $100 \mathrm{mM}$ to $500 \mathrm{mM}$ and the corresponding $\mathrm{K}_{\mathrm{D}}$ values were obtained from nonlinear least- squares fit to the data using equation 2.20 and 2.21 with $\mathrm{K}_{\mathrm{D}}$ and $\mathrm{E}_{\mathrm{x}}$ as free parameters and with $\mathrm{E}_{\text {free }}$ is determined with free DNA sample. However this method is inaccurate in determining $K_{D}$ values smaller than about $2 \mathrm{nM}$ as we need to keep the fixed DNA concentration during titration less than the $\mathrm{K}_{\mathrm{D}}$ value of the complex we are aiming to determine and since the signal from DNA concentrations below $2 \mathrm{nM}$ is comparable to the background noise (instrument limitation), a high level of fluctuations observed in the measured signal. 


\subsubsection{Salt titration experiments [27]}

To obtain reliable $K_{D}$ values in the sub-nanomolar range from equilibrium measurements, we have used an alternative approach in which we exploit the expected linear dependence of $\log \left(K_{\mathrm{D}}\right)$ versus $\log ([\mathrm{KCl}])$. We measured the acceptor ratio in about 20-25 samples containing a fixed protein-DNA concentration, but with $[\mathrm{KCl}]$ ranging from $\sim 10 \mathrm{mM}$ to $700 \mathrm{mM}$. Increasing the salt concentration destabilizes the complex, thus decreasing the fraction of DNA in complex, and the acceptor ratio drops from a value characteristic of complex at low $[\mathrm{KCl}]\left(f_{\mathrm{X}} \approx 1\right)$ to a value characteristic of the fraction in complex at high $[\mathrm{KCl}]\left(f_{\mathrm{X}} \approx 0\right)$. To simulate $f_{\mathrm{X}}$ versus $[\mathrm{KCl}]$, we use Eq. 2.20, and parameterize the salt dependence of $K \mathrm{~d}$ as follows:

$$
K_{D}=K_{D o}\left(\frac{[K C l]}{[K C l]_{o}}\right)^{S K_{d}}
$$

where $K_{\mathrm{Do}}$ is the dissociation constant at a reference salt concentration $[\mathrm{KCl}]_{\mathrm{o}}$ and $S K_{\mathrm{d}}$ is the expected slope on a $\log \left(K_{\mathrm{D}}\right)$ versus $\log ([\mathrm{KCl}])$ plot. The measured acceptor ratio versus $[\mathrm{KCl}]$ data is then described in terms of Eqs. (2.20-2.22), with four free parameters: $K_{\text {Do, }} S K_{\mathrm{d}}, r_{A}^{X}$, and $r_{A}^{\text {free }}$, and the best fit parameters that minimize the residuals are obtained from a Monte Carlo search in parameter space [28]. For a fixed value of the reference $[\mathrm{KCl}]_{0}$, we carried out 20 independent Monte Carlo searches in parameter space for each set of measurements to ensure a global minimum in the residual chi-squares. A typical plot of salt dependence titration is shown in Fig. 2.13. The $K_{\mathrm{D}}$ values at different [KCl], and the slope parameter $S K_{\mathrm{d}}$, reported in Table 3.4 in chapter 3 , are the mean values obtained from the best fits of two independent sets of measurements and the errors are the statistical variations obtained from the separate fits. The dominant source of error in determining $K_{\mathrm{D}}$ at a given salt concentration comes from the uncertainty in the determination of the slope $S K_{\mathrm{D}}$. The results of Table 3.4 are 
independent of our choice of the reference $[\mathrm{KCl}]_{0}$, which was varied between 100 to $300 \mathrm{mM}$ $\mathrm{KCl}$, to check for consistency.

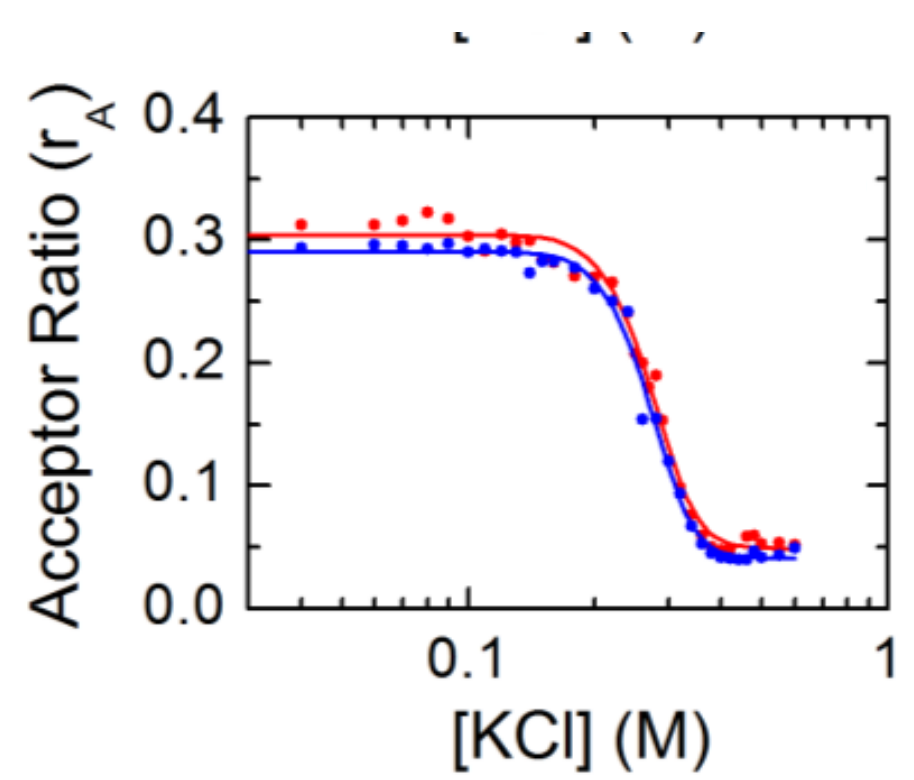

Fig.2.13. Binding affinity measurements on mutant IHF ( $\alpha \mathrm{R} 21 \mathrm{C})-\mathrm{H}$ ' complex by salt titration binding isotherms. The acceptor ratio of the complexes is plotted as a function of $[\mathrm{KCl}]$ at $25^{\circ} \mathrm{C}$. 


\section{References}

1. Bernasconi, C.F., Relaxation Kinetics. 1976, New York Academic Press.

2. Staerk, H., and Czerlinski, G.,, Nanosecond heating of aqueous systems by giant laser pulses. Nature, 1965. 205: p. 63-64.

3. Rigler, R., A. Jost, A.,and Demaeyer, L., , Chemical kinetics at micro level - a laser micro temperature jump apparatus for relaxation studies in micro samples. Exp. Cell Res., 1970. 62: p. 197-203.

4. C. M. Phillips, Y.M.a.R.M.H., Ultrafast thermally-induced unfolding of RNAse-A. Proc. Natl. Acad. Sci., 1995: p. 7292-7296.

5. Kubelka, J., Time-resolved methods in biophysics. 9. Laser temperature-jump methods for investigating biomolecular dynamics. Photochem. Photobiol. Sci., 2009. 8(4): p. 499-512.

6. Thompson, P.A., W.A. Eaton, and J. Hofrichter, Laser temperature jump study of the helix-coil kinetics of an alanine peptide interpreted with a 'kinetic zipper' model. Biochemistry, 1997. 36(30): p. 9200-10.

7. Wray, W.O., T. Aida, and R.B. Dyer, Photoacoustic cavitation and heat transfer effects in the laser-induced temperature jump in water. Appl. Phys. B, 2002. 74: p. 57-66.

8. Livesey, A.K. and J.C. Brochon, Analyzing the Distribution of Decay Constants in Pulse-Fluorimetry Using the Maximum Entropy Method. Biophys J, 1987. 52(5): p. 693-706.

9. Steinbach, P.J., R. Ionescu, and C.R. Matthews, Analysis of kinetics using a hybrid maximum-entropy/nonlinear-least-squares method: application to protein folding. Biophys. J., 2002. 82: p. 2244-2255.

10. Steinbach, P.J., Inferring lifetime distributions from kinetics by maximizing entropy using a bootstrapped model. J Chem Inf Comput Sci, 2002. 42(6): p. 1476-8.

11. Clegg, R.M., Fluorescence resonance energy transfer and nucleic acids. Methods Enzymol., 1992. 211: p. 353-388.

12. Sass, L.E., et al., Single-molecule FRET TACKLE reveals highly dynamic mismatched DNA-MutS complexes. Biochemistry, 2010. 49(14): p. 3174-90.

13. Lin, K.-Y., Jones, R.J., Matteucci, M., Tricyclic-2'-deoxycytidine analogs:synthesis and incorporation into oligodeoxynudeotides which have enhanced binding to complementary RNA, . J.Am.Chem.Soc., 1995. 117: p. 3873-3874.

14. Wilhelmsson, L.M., Fluorescent nucleic acid base analogues. Quarterly Reviews of Biophysics, 2010. 43: p. 159-183.

15. Sandin, P., et al., Fluorescent properties of DNA base analogue tC upon incorporation into DNA--negligible influence of neighbouring bases on fluorescence quantum yield. Nucleic Acids Res, 2005. 33(16): p. 5019-25.

16. Sandin, P., et al., Characterization and use of an unprecedentedly bright and structurally non-perturbing fluorescent DNA base analogue. Nucleic Acids Res, 2008. 36(1): p. 157-67.

17. Borjesson, K., et al., Nucleic acid base analog FRET-pair facilitating detailed structural measurements in nucleic acid containing systems. J Am Chem Soc, 2009. 131(12): p. 4288-93.

18. Borjesson, K., Sandin, P., Wilhelmsson, L.M.,, Nucleic acid structure and sequence probing using fluorescent base analogue $t C^{o}$. Biophysical Chemistry, 2009. 139: p. 24-28.

19. Jones, A., Neely, RK.,, 2-aminopurine as a fluorecent probe on DNA conformation and the DNA-enzyme interface. Quarterly Reviews of Biophysics, 2015. 48: p. 244279. 
20. Rachofsky, E., Osman, R., Ross, JB., Probing structure and dynamics of DNA with 2-aminopurine: effects of local environment on fluorescence. Biochemistry 2001. 40: p. $946-956$

21. Reich, N.O., Allan, B.W.,, Targeted base stacking disruption by the EcoRI DNA methyltransferase. Biochemstry, 1996. 35: p. 14757-14762

22. Frey, M.W., sowers, L.C, Millar, D.P, Benkovic, S.J.,, The nucleotide analog 2aminopurine as a spectroscopic probe of nucleotide incorporation by the Klenow fragment of Escherichia coli polymerase I and bacteriophage T4 DNA polymerase. Biochemstry 1995,. 34: p. 9185-9192.

23. Krosky, D.J., Song, F.H., Stivers, J. T. , , The origins of high-affinity enzyme binding to an extrahelical DNA base. Biochemstry, 2005. 44: p. 5949-5959.

24. Neely, R.K., daujotyte, D. Grazuili, S., Magennis, S. W., Dryden, D. T. F., Klimasauskas, S. Jones, A.C., , Time-resolved fluorescence of 2-aminopurine as a probe of base flipping in M.HhaI-DNA complexes. Nucleic Acids Research, 2005. 33: p. 6953-6960

25. Jacobs-Palmer, E. and M.M. Hingorani, The effects of nucleotides on MutS-DNA binding kinetics clarify the role of MutS ATPase activity in mismatch repair. J. Mol. Biol., 2007. 366(4): p. 1087-98.

26. Chen, X., et al., Kinetic gating mechanism of DNA damage recognition by Rad4/XPC. Nat Commun, 2015. 6: p. 5849.

27. Vivas, P., et al., Mapping the transition state for DNA bending by IHF. J Mol Biol, 2012. 418(5): p. 300-15.

28. Kuznetsov, S.V., et al., Loop dependence of the stability and dynamics of nucleic acid hairpins. Nucleic Acids Res., 2008. 36: p. 1098-1112. 


\section{Chapter III}

\section{Integration Host Factor (IHF)-DNA interaction}

\subsection{Introduction}

\subsubsection{Integration host factor (IHF)}

Integration host factor (IHF) is a small hetero dimeric protein $(\sim 20 \mathrm{kDa})$, ubiquitous in eubacteria. It binds to DNA in a sequence-specific manner and causes $\sim 35$-bp long cognate site of DNA to bend by $>160^{\circ}$ [1]. Even though IHF was first discovered as a host factor for bacteriophage $\lambda$ integration, where $\lambda$ phage cleverly facilitates its E.coli host's protein IHF to infest its target, IHF also aids in chromosomal compaction as well as in the assembly of higher-order nucleoprotein complexes necessary for replication initiation, some-site specific recombination and transcriptional regulation of certain genes [2, 3].

IHF's primary function is architectural; it induces a large bend in DNA, which allows distant sites on the DNA to be in close proximity. Rice and co-workers first proposed that the role of IHF in integration is perhaps to bring distant DNA sites together such that they align with each other in order to form higher order nucleo-protein complexes [1]. Goodman and co-workers observed that the requirement of IHF for recombination can be omitted by either using a pre-bent DNA or by replacing IHF by another small bacterial histone-like, DNA bending protein called HU. These results support possible architectural role of IHF in its interaction with DNA $[4,5]$. IHF is also known to bind non-specifically to DNA, although with $10^{3}-10^{4}$ lower affinity than for specific binding sites [6-8]. In its nonspecific binding mode, IHF plays a role in chromatin compaction [9]. The ability of IHF and HU to compact DNA has been investigated using micromanipulation techniques as well as with atomic force microscopy [10-13]. It is interesting to note that the intracellular concentration of IHF is much higher $(12-55 \mu \mathrm{M})$ in exponential growth phase to early stationary phase than the 
concentration required to site-specific interactions $\left(K_{D}=2-20 \mathrm{nM}\right)$. This implies that nonspecific binding of IHF to DNA plays a role in the physical organization of bacterial chromatin [14].

IHF's small size and its ability to bend DNA to such a large degree make it an excellent candidate to study protein-DNA interactions, especially to examine the role that sequence-dependent DNA bendability/flexibility play in the mechanism by which proteins recognize their specific binding sites on DNA [15].

\subsubsection{IHF binds to the minor groove on DNA and recognizes its specific site via indirect readout}

Yang and co-workers found three IHF binding sites on $\lambda$ phage DNA: $\mathrm{H}_{1}, \mathrm{H}_{2}, \mathrm{H}^{\prime}$ (Table I) [16]. The DNA sequences for these three binding sites are shown in Fig 3.1. All three IHF binding sites consist of a 13-bp long consensus sequence WATCAANNNNTTR (W is A or T, $\mathrm{R}$ is $\mathrm{A}$ or $\mathrm{G}, \mathrm{N}$ is any nucleotide) as shown in bold in Fig 3.1. In $\mathrm{H}_{2}$ and $\mathrm{H}^{\prime}$ sequence there is an A-tract region approximately one helical turn away from the consensus region; this Atract region in absent in the $\mathrm{H}_{1}$ sequence. In $\mathrm{H}^{\prime}$ sequence this A-tract region is important for specific IHF binding; its removal impairs the ability of IHF to bind specifically to DNA [17]. However, the ability of the A-tract sequence to adopt the required conformation rather than direct interactions of the protein with the nucleotides themselves appears to be the important determinant here [18]. IHF is a member of the class of DNA bending proteins that interact with the minor groove, where the ability of the protein to distinguish between different nucleotides is limited. IHF binding was blocked when the minor groove was modified [16]. All the contacts of IHF are either in the minor groove or are part of an extensive network of electrostatic interaction with the phosphate backbone [18]. The sequence dependent conformation of DNA rather than base-specific contacts determines the specific binding of 
IHF to its target site [18]. IHF interacts with DNA mainly by inspecting the structural constrains of the minor groove of DNA and by indirectly reading the local shape and deformability of DNA [16].

Table I. DNA sequence on $\lambda$ phage DNA that are protected from the hydroxyl radical attack. Atracks and consensus sequences are shown in bold, italics and bold, gray respectively

\section{H1: AGTCACTATGAATCAACTACTTAGATGGTATTAG \\ H2: GAAACGTAAAATGATATAAATATCAATATATTAAATTAGATTTTGCA \\ $\mathrm{H}^{\prime}$ : $\quad$ TGgCATTATAAAAAAGCATTGCTTATCAATTTGTTGCAACGAACAGGT}

\subsubsection{Structure of IHF-H' complex}

The X-ray crystal structure of IHF bound to 35-bp long H' binding site from phage $\lambda$ was solved by Rice et al [1] (Fig. 3.1). The crystal structure reveals that within two and a half turns of the double helix, the DNA executes a U-turn with an overall bend angle of at least $160^{\circ}$ [1]. It is possible that the overall bend angle exceeds $180^{\circ}$ in the absence of crystal packaging effects in the X-ray crystallography study [1].

The crystal structure revealed that the two subunits of IHF ( $\alpha$ and $\beta$ ) heterodimer are intertwined to form a compact core $[1,18]$. Each subunit of IHF consists of three $\alpha$-helical segments that form the lower half of the protein; the upper half of the protein consists of $\beta$-sheets that extend into two $\beta$-ribbon "arms" that are disordered in the absence of DNA (Fig. 3.1D,E) [1, 19-23]. Upon binding to DNA the $\beta$-ribbon arms of IHF wrap around the DNA and lie in the minor groove (Fig. 3.1D,E). In turn, the DNA is severely kinked at two sites separated by 9 bp, which results in a large "U-turn" bend, with the DNA wrapped around the protein. 

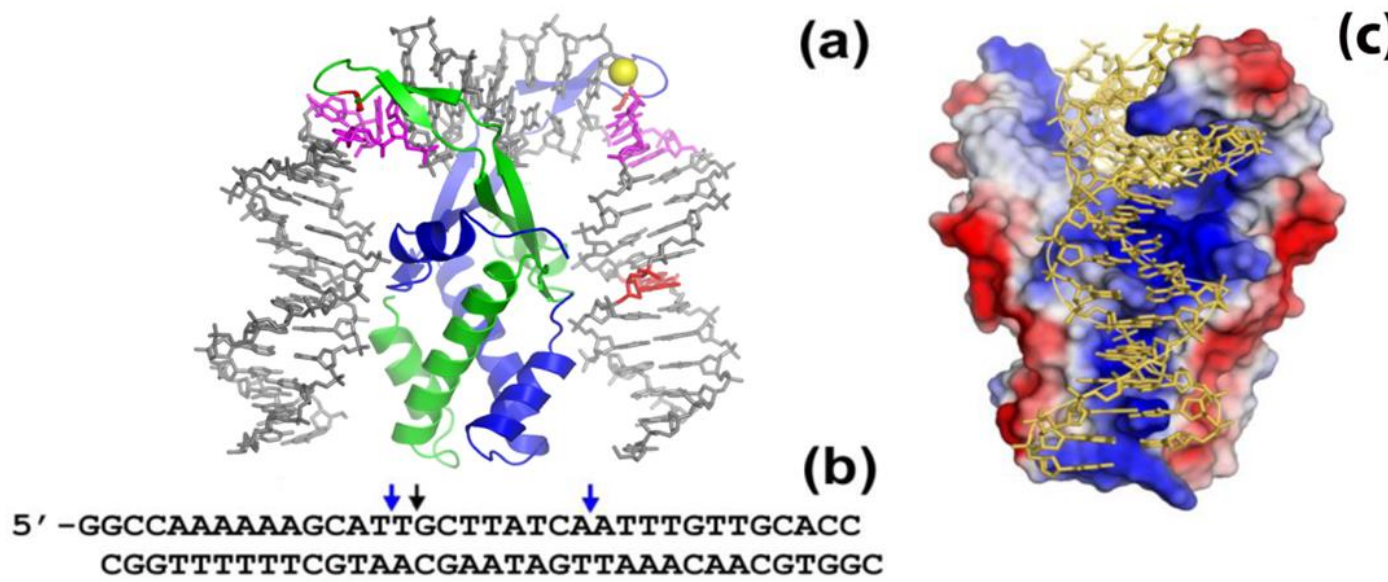

(c)
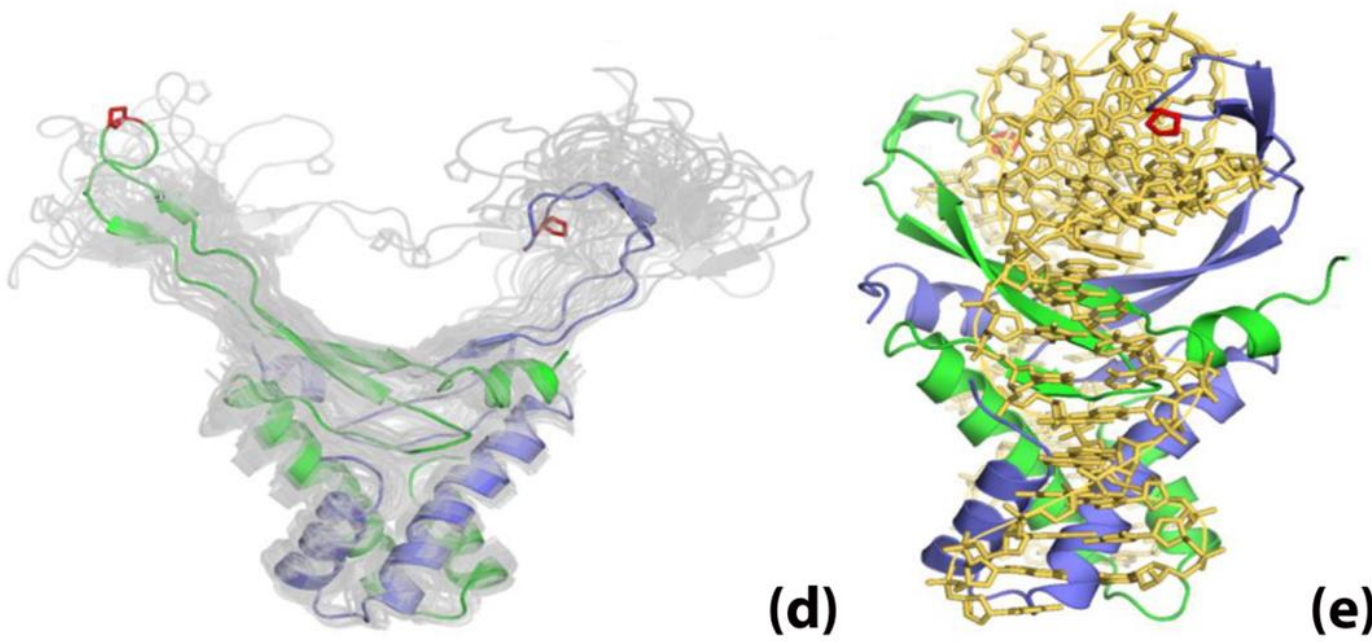

Fig.3. 1. Cocrystal structures of the IHF/HU family of DNA-bending proteins. (a) IHF-DNA cocrystal structure [Protein Data Bank (PDB) code: 1IHF]. The $\alpha$ - and $\beta$-chains of the IHF protein are shown in blue and green, respectively. The DNA is shown in gray, with locations of modifications in the DNA sequences used in this study highlighted in color: yellow sphere, site of a nick in the sugarphosphate backbone in the NickC substrate; pink, mismatches introduced in the TT8AT, TT-loop, and AT-loop substrates; red, $\mathrm{T} \rightarrow \mathrm{A}$ mutation in the $\mathrm{H}^{\prime} 44 \mathrm{~A}$ substrate. (b) Sequence of the DNA substrate in the cocrystal structure with IHF, which contains the $\mathrm{H}^{\prime}$ binding site from bacteriophage $\lambda$. The DNA was nicked at the position indicated by the black arrow, to facilitate crystal packing. In the complex, the DNA is sharply kinked at the two sites indicated by the blue arrows. (c) Side view of solvent accessible surface representation of IHF. Regions of positive and negative electrostatic potential are shown in blue and red, respectively. (d) The ensemble of 25 NMR structures of the homodimeric HU protein from Bacillus Stearothermophilus (PDB code: 1HUE) is shown in gray. One of these structures is highlighted, in blue and green with conserved proline residues in red. (e) The structure of the IHF- $\mathrm{H}^{\prime}$ complex (1IHF), rotated by $90^{\circ}$ relative to the view in (a). The $\beta$-ribbon arms of the proteins of this family are more flexible in the absence of a bound DNA. Upon binding, they wrap around the DNA and lie in the minor groove, and the prolines intercalate into the kinks in the DNA. (Figure adapted with permission from [24]). 
In order to bend the DNA, IHF must overcome the forces that keep DNA straight, such as the repulsive force between negatively charged phosphate backbone and the energy from base stacking. The DNA segment on either side of the kink closely "hugs" the protein and makes several contacts with the positively charged residues on the surface of the protein $[1,25]$. IHF is able to asymmetrically neutralize the double helix by placing a large positive surface on the inside of the bent DNA. The crystal structure showed that $\sim 26$ positively charged residues on the surface of the protein make ionic interactions with DNA in the wrapped complex $[1,26]$. To counteract the energetic cost of disrupting base stacking at the kink sites, a highly conserved hydrophobic proline residue at the tip of each $\beta$ arm intercalates between thymine bases, one at the consensus binding site and the other at the non-consensus binding site, at the site of the kinks [1] (Table II and Fig. 3.1). The thymine bases buckle as a result of the intercalation and the minor grooves in the vicinity become shallow and wide. On the outside of the bend the hydrophobic intercalation stabilizes the opening of the minor groove and on the inside, charge neutralization counteracts the enhanced repulsion between the phosphates on the opposite sides of the narrowed grooves. These combined effects trigger a "push-pull" mechanism and stabilize the bend in the complex [18]. The bend does not cause unwinding or melting of the DNA double helix [1,27].

The free energy cost of disrupting stacking interactions range from $\sim 0.2-2.7 \mathrm{kcal} / \mathrm{mol}$ [15] as measured from thermal melting experiments. This value is much smaller than the energy cost of bending DNA into a U-turn (estimated to be $\sim 13 \mathrm{kcal} / \mathrm{mole}$ for a 35 -bp long DNA sequence assuming an elastic rod description of DNA, as discussed in Appendix 1). Thus it is very likely that proteins take advantage of the much lower cost of disrupting stacking interactions to introduce kinking in the DNA, as seen in the co-crystal structure of the IHF-H' complex (Fig. 3.1 A). 
IHF is closely related to HU, another architectural protein of E.coli. IHF and HU have $40 \%$ sequence identity and are similar in their composition, size and shape $[1,27]$. Though HU does not bind specifically to any particular sequence, it recognizes and binds with high affinity to DNA with inserted distortions $[19,25]$. The distribution of positive charge is conserved in both proteins as are the prolines at the tip of the $\beta$-sheet arms. Both HU and IHF are used when DNA bending is required and serve as architectural factors. IHF and HU are functionally analogous to both the histones and HMG box proteins of eukaryotes and aid in chromosome compaction as well as play specific architectural roles [19, 28]. All prokaryotes appear to have at least one HU/IHF homolog [25]. 


\subsubsection{Background of IHF/H' interaction dynamics}

(A)

Laser temperature-jump to measure DNA-bending rates

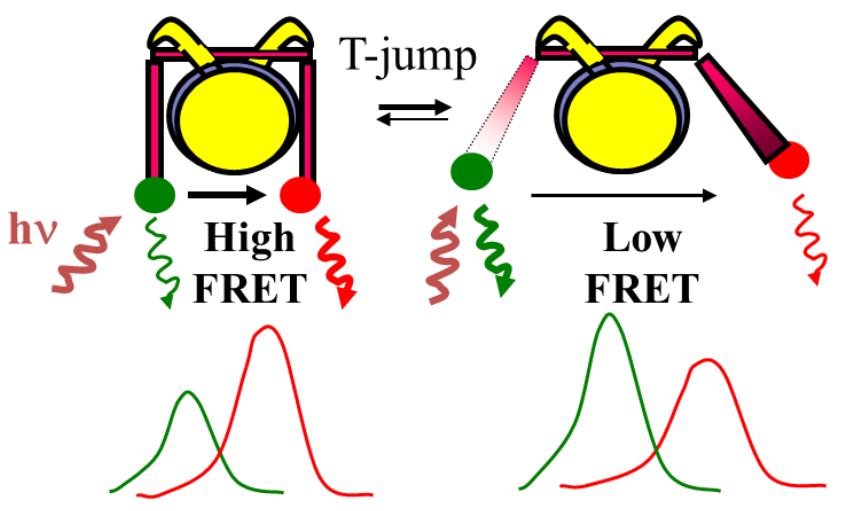

(B)

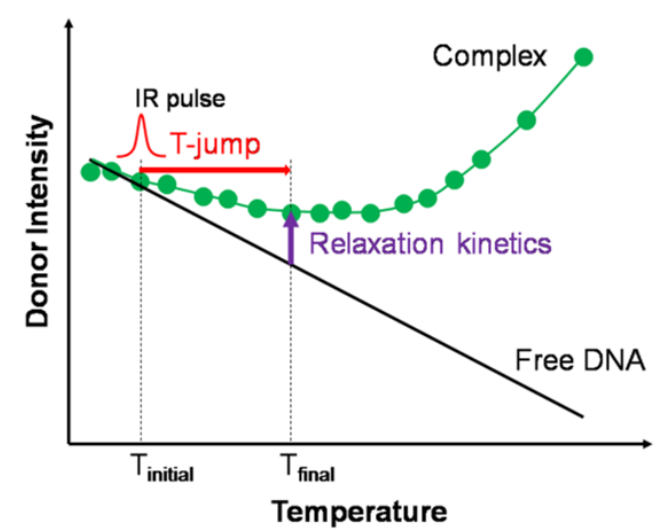

Fig.3.2. (A) Cartoon figure of FRET dyes attached to a long DNA, (B) Correlation between equilibrium and T-jump experiment, illustrative figure.

The kinetics of DNA bending in complex with IHF were first revealed as singleexponential decays in stopped-flow measurements on the IHF-H' complex by the Crothers group [29] and laser T-jump measurements by the Ansari group [30]. In these studies, the relaxation kinetics was monitored using time-resolved FRET. Briefly, fluorescent labels (fluorescein and TAMRA) attached at either end of a short (35-bp) DNA oligomer containing the H' site of IHF were used as a FRET pair to measure the end-to-end distance and hence the extent of DNA bending in the IHF-H' complex [31] (Fig. 3.2). Upon binding of IHF, the end to end distance of 35 -bp long DNA containing the $\mathrm{H}^{\prime}$ site is shortened from $\sim 100 \AA$ to $\sim 50^{\circ}$ 
$\AA$ [31]. Thus, FRET measurements on end-labeled H' substrates are very sensitive to the distance change and suitable for direct measurement of DNA bending in solution.

In stopped-flow and T-jump measurements, the fluorescence of the donor (fluorescein) molecule, with excitation at $488 \mathrm{~nm}$, was measured as a function of time. These studies showed that the DNA bending step in binding site recognition by IHF was relatively slow, and occurred on time scales of a few milliseconds [24, 30]. Most notably, the rates and activation energy for the DNA bending step in the IHF-H' complex were found to be similar to the time scales measured for single $\mathrm{A} / \mathrm{T}$ bp opening dynamics in unbound duplex B-DNA (Fig. 3.3). Note that the A/T base pair opening rate show a wide range of opening times depending on the sequence context (pink shaded region in Fig. 3.3, data from [32]). The energetic cost for disrupting base pair interactions between bases is sequence dependent and ranges from $\sim 6-9 \mathrm{kcal} / \mathrm{mol}$ [32] as measured by NMR imino proton exchange method. If disrupting the stacking interactions is the dominant contributor to the energetic cost of basepair disruption, we expect to see a wide range of base-pair opening rates, consistent with the data. In contrast $\mathrm{C} / \mathrm{G}$ base pair opening times are less sequence dependent (blue shaded region in Fig. 3.3), likely because in this case the dominant contribution to disrupting the stacking interactions is from breaking the three hydrogen bonds between a $\mathrm{C} / \mathrm{G}$ base pair. Remarkably, the A/T base pair opening rate for $\mathrm{H}^{\prime}$ sequence at one of the kink sites, measured by NMR [33], falls well within the DNA bending rates measured on the IHF-H' complex (H' bp opening rate shown in red bar in Fig. 3.3). 


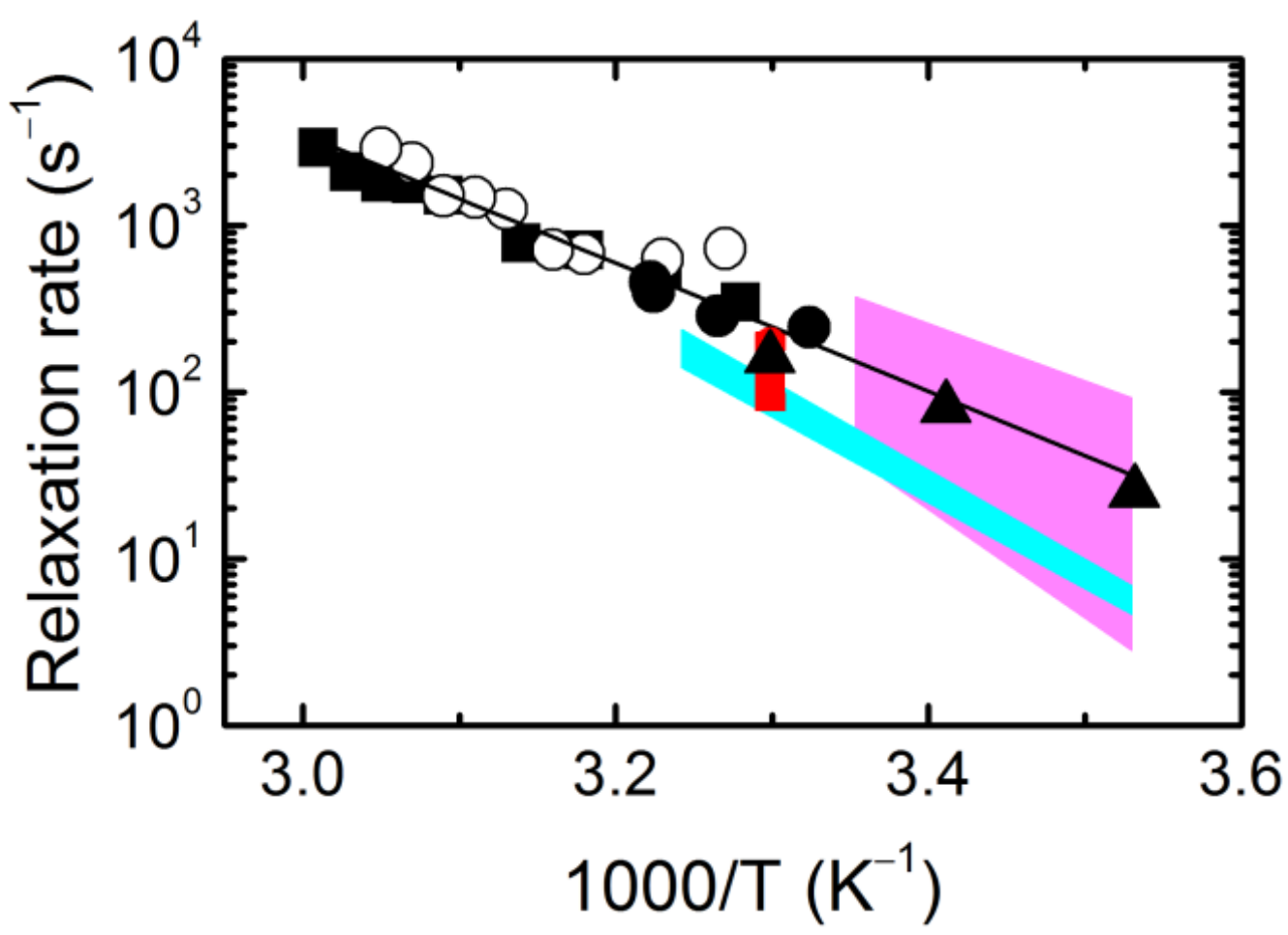

Fig.3. 3. Relaxation rates measured for $\mathrm{IHF}-\mathrm{H}^{\prime}$. The relaxation rates, $\mathrm{k}_{\mathrm{r}}$, for the $\mathrm{IHF}-\mathrm{H}^{\prime}$ complex are plotted versus inverse (final) temperature $\left(\mathrm{T}_{\mathrm{f}}\right)$ as obtained from three sets of $\mathrm{T}$-jump measurements $(\bullet, \bullet, \circ)$ under conditions where the T-jump perturbation does not dissociate the IHF- $\mathrm{H}^{\prime}$ complex. The plateau values of the stopped-flow relaxation rates at high $[\mathrm{IHF}]$ are also shown ( $\boldsymbol{\Delta}$; data from Sugimura and Crothers [30]). The shaded areas represent the range of base-pair opening rates from imino proton exchange measurements, from Coman and Russu [32] (pink, A:T; blue, C:G). The red vertical bar is the A:T base-pair opening rate for the $\mathrm{H}^{\prime}$ sequence, at the site of one of the kinks, from Dhavan et al. [33]. (Figure reproduced with permission from [24]).

We also note here that the cocrystal structure of $\mathrm{IHF}-\mathrm{H}^{\prime}$ and structures of other members of this family (HU and $\mathrm{Hbb}$ ) bound to their cognate sites show complete disruption of base stacking at the kinks but no disruption of the Watson-Crick pairs [1, 25, 34]. Thus, our observation that IHF-H' DNA bending rates occur on the same time scales as NMR bp opening rates suggested that severe kinking of DNA, as seen in the IHF-H' complex, disrupts the same stacking interactions as those needed to "open" a base-pair and make the iminoprotons solvent accessible. Taken together, these results suggested that local distortions of the DNA structure, as a consequence of thermal fluctuations in base pairing nucleated at regions of weak stacking, may be sufficient to overcome the free energy barrier needed to partially bend/kink DNA prior to forming a tight complex with $\operatorname{IHF}[24,30]$. Based on this study, 
Ansari and co-workers proposed a conformational capture mechanism for binding site recognition by IHF, in which thermal fluctuations enable DNA to adopt "pre-bent" conformations; for cognate sites these pre-bent conformations match the shape requirements of the DNA in the specific complex and hence can be subsequently captured and stabilized by IHF.

In a separate study, Vivas et al found that the DNA bending rate is independent of the salt concentration. These results indicate that any conformational rearrangements in the complex that are accompanied by ion release occur after the rate limiting step [35]. These studies pointed to a picture of the transition state ensemble as one in which the DNA is partially bent/kinked, but stabilizing interaction between IHF and DNA have not yet been made.

Next to examine how the sequence-dependent DNA flexibility influences IHF's ability to recognize its cognate site and to further probe the nature of the transition state ensemble we used modified DNA substrates with nicks and mismatches at the kink sites, designed to enhance the local flexibility of the DNA at these sites (Table II). We also investigated the effect of sequence modification in the recognition site far from the kinked regions and the effect of mutating charged residues on the surface of the IHF protein, designed to destabilize the complex by perturbing the electrostatic interactions between IHF and the bound DNA [24]. 
Table II. Sequences of $\mathrm{H}^{\prime}$ substrate and its variants. The intact $\mathrm{H}^{\prime}$ substrate is identical with the sequence shown in Fig. 3.1b and contains no nicks. The 5'-end of the top strand is fluorescein-dT (yellow), and the 5'-end of the bottom (complementary) strand is TAMRA-dT (dark pink). The consensus region is shown in gray. The blue arrows indicate locations of kinks in the DNA when in complex with IHF. In $\mathrm{H}^{\prime} 44 \mathrm{~A}$, modification of a base pair in the TTR consensus region is shown in red. In TT8AT, TT-loop, and AT-loop, mismatches inserted at or near the site of the kinks are shown in pink. The locations of these modifications are also indicated in Fig. 3.1a. DNA sequences used in control experiments are also shown in this table. In the short $14 \mathrm{bp}$ sequence the four nucleotides next to the dyes (green) are similar to the s nucleotides closer to dyes in intact H' sequence, the $55 \mathrm{bp}$ and $130 \mathrm{bp}$ sequences contains the intact H' sequence in the middle and in $130 \mathrm{bp}$ the dyes are attached internally. In 35_spb sequence the dyes are attached on the sugar phosphate backbone.

\section{Intact}

$\mathrm{H}^{\prime}$ 44A

TT8AT

AT-10op

TT-100p

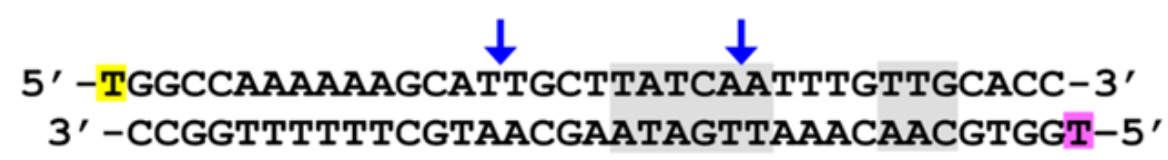

5' -TGGCCAAAAAAGCATTGCTTATCAATTTGTAGCACC-3' 3' -CCGGTTTTTTCGTAACGAATAGTTAAACATCGTGGT-5'

5' -TGGCCAAAAAAGCATTGCTTATCAATTTGTTGCACC-3' 3' -CCGGTTTTTTCGTTTCGAATAGTATAACAACGTGGT-5

5' -TGGCCAAAAAAGCATTGCTTATCAATTTGTTGCACC-3' $3^{\prime}$-CCGGTTTTTTCGTAACGAATAGTATAACAACGTGGT-5'

5' -TGGCCAAAAAAGCATTGCTTATCAATTTGTTGCACC-3' 3' -CCGGTTTTTTCGTTTCGAATAGTTAAACAACGTGGT-5

$\mathrm{H}^{\prime} \_\mathrm{Spb}$

5' - GGCCAAAAAAGCATTGCTTATCÄATTTGTTGCACC-3'

3'-CCGGTTTTTTCGTAACGAATAGTTAAACAACGTGG_-5'

$14 \mathrm{bp}$

5' -TGGCCAGCATTCACC-3'

CCGGTCGTAAGTGGT-5'

$\mathrm{H}^{\prime} 55 \mathrm{bp}$

5' -TC̄CCACGGCAGGCCAAAAAAGCATTGCTTATCAATTTGTTGCACCGAACAGGTCGG GGGTGCCGTCCGGTTTTTTCGTAACGAATAGTTAAACAACGTGGCTTGTCCAGCCT-5'

$\mathrm{H}^{\prime} \_130 \mathrm{bp}$

5' -GAG'TATGAGTCAGTAAGAGAGCAGCTGGCACATTCGATCAGAGTAGCTGGCCAAAAAAGCATTG

3' -CTCATACTCAGTCATTCTCTCGTCGACCGTGTAAGCTAGTCTCATCGACCGGTTTTTTCGTAAC... CTTATCAATTTGTTGCACCAGCGATCAGTGCATCCAGTAAGAGAATCCACTAGTGTCTCGCGGTTC -3' GAATAGTTAAACAACGTGGTCGCTAGTCACGTAGGTCATTCTCTTAGGTGATCACAGAGCGCCAAG -5' 


\subsubsection{Binding site recognition versus protein diffusional search}

An intriguing observation from the kinetics measurements carried out thus far with different proteins: UDG [36] or EcoRV [37] or IHF [30], is that the binding site recognition appears to be on time scales of a few milliseconds or longer. In contrast, experimental estimates of one-dimensional diffusion constants measured for proteins "sliding" or "hopping" on DNA, for a wide range of proteins, indicate base-pair stepping times that are considerably shorter, and range from $\sim 200 \mathrm{~ns}$ to $500 \mu \mathrm{s}$ [38-43]. In order for a protein to reside at its target site long enough to recognize it (that is, long enough for conformational fluctuations that lead to the formation of a tight complex to occur), either these fluctuations must be sufficiently fast or the diffusion times sufficiently slow. It cannot be ruled out that the systems that exhibit millisecond recognition times fall in the category of slow one-dimensional diffusers. Alternatively, there may be unresolved fast events in the recognition mechanism pathway occurring on submillisecond timescales, commensurate with rapid diffusion of proteins on DNA.

In this chapter, I will present direct kinetic evidence for a fast phase in the kinetics of DNA bending during binding site recognition by IHF. This rapid phase, occurring on timescales of $\sim 400 \mu \mathrm{s}$, appears at low salt concentrations (100 $\mathrm{mM} \mathrm{KCl}$ or lower), and is found to be sequence nonspecific. An intriguing possibility is that this rapid phase represents conformational fluctuations in the protein as it switches from a nonspecific (passive) "search" to a specific (active) "recognition" binding mode, as has been suggested in theoretical studies that have attempted to reconcile "speed" of diffusion versus "stability" of the final (recognition) complex [44, 45]. 


\section{Materials and method}

All DNA oligonucleotides except H'_Spb were synthesized and PAGE purified by the W. M. Keck facility at Yale University. These oligonucleotides were labeled with fluorescein (F) and TAMRA (T) by incorporating F-dT and R-dT (Glen Research, Sterling, VA) at the 5' ends of the top and bottom strands via a C6 linker, respectively, as indicated in table II. The H'_Spb DNA oligonucleotide was ordered from Oligo.Etc (Wilsonville, USA) and in this sequence the dyes are attached on sugar phosphate backbone via a C6 linker. The concentrations of each DNA strand were determined by measuring the UV absorbance at 260 $\mathrm{nm}$ using the extinction coefficients $4.00 \times 105 \mathrm{M}^{-1} \mathrm{~cm}^{-1}$ for the fluorescein-labeled top strand and $4.15 \times 105 \mathrm{M}^{-1} \mathrm{~cm}^{-1}$ for the TAMRA-labeled bottom strand. For determination of the amount of labeled DNA in solution, fluorescein and TAMRA concentrations were also determined in the labeled samples by measuring the absorbance of F-labeled strands at 494 $\mathrm{nm}$ and R-labeled strands at $555 \mathrm{~nm}$ and compared with the concentrations of the oligomers obtained from measurements at $260 \mathrm{~nm}$. For the dyes, the molar extinction coefficients were $75,000 \mathrm{M}^{-1} \mathrm{~cm}^{-1}$ for (Molecular Probes§). The percentage of unlabeled DNA in solution was estimated to be $\sim 5-10 \%$.

Wild type IHF and its mutant were gifts from the laboratory of Prof. Phoebe Rice at university of Chicago. IHF proteins were stored at $454 \mu \mathrm{M}$ or $\sim 800 \mu \mathrm{M}$ in a storage buffer of $25 \mathrm{mM}$ Hepes (pH 7.5), $200 \mathrm{mM} \mathrm{NaCl}, 20 \%$ glycerol, and $1 \mathrm{mM}$ ethylenediaminetetraacetic acid (EDTA). Droplets of proteins were first flash-frozen in liquid nitrogen prior to storage in cryogenic tubes at $-80{ }^{\circ} \mathrm{C}$. Individual frozen droplets were diluted into binding buffer [20 $\mathrm{mM}$ Tris- $\mathrm{HCl}, 1 \mathrm{mM}$ EDTA (pH 8), plus $2 \mathrm{mM}$ DTT in $\alpha \mathrm{R} 21 \mathrm{C}$ samples, and $\mathrm{KCl}$ concentrations from $100 \mathrm{mM}$ to $500 \mathrm{mM}$ ]. IHF concentrations were measured by absorbance at $276 \mathrm{~nm}$, with an extinction coefficient of $5800 \mathrm{M}^{-1} \mathrm{~cm}^{-1}$ [24]. 


\subsection{Results}

\subsubsection{DNA bending kinetics in the IHF - H' complex are biphasic}

The ability to resolve a rapid phase in the T-jump kinetics of the IHF-H' complex, occurring on time scales of $\sim 400 \mu \mathrm{s}$, was the result of a series of improvements in the sensitivity of the T-jump spectrometer, the data acquisition protocol, and the use of maximum entropy analysis (MEM) to describe the relaxation kinetics, as described in Methods. The advantage of the maximum entropy analysis is that each of the kinetics traces can be described in a model independent manner in terms of a distribution of relaxation times that describe the kinetics, without any a priori assumptions as to the number of discrete exponentials needed to describe the kinetics [46].

T-jump measurements on the IHF-H' complex in $100 \mathrm{mM} \mathrm{KCl}$ are summarized in Fig. 3.4. Representative kinetics traces at a final temperature of $38{ }^{\circ} \mathrm{C}$, in response to a $5{ }^{\circ} \mathrm{C}$ T-jump perturbation, are shown in Fig. 3.4A. The corresponding distribution of relaxation times obtained from MEM analysis revealed at least two distinct phases in the observed relaxation kinetics (Fig. 3.4B). A control experiment, on donor-only-labeled H'-DNA, measured under similar conditions, showed no relaxation kinetics other than slow T-jump recovery (Fig. 3.4C). The relaxation kinetics traces were measured for a series of initial temperatures, with the final temperatures in the range $28-59{ }^{\circ} \mathrm{C}$. An Arrhenius plot of the relaxation rates for the two phases, obtained from the maximum entropy analysis, shows that the fast phase occurs at around $100 \mu$ s and is weakly dependent on the temperature, while the slow phase appears to be activated (Fig. 3.4D). The amplitude of the slow phase is considerably larger at lower temperatures $\left(>70 \%\right.$ at $\left.30{ }^{\circ} \mathrm{C}\right)$, and decreases to $\sim 50 \%$ at $53{ }^{\circ} \mathrm{C}$ (Fig. 3.4E). 

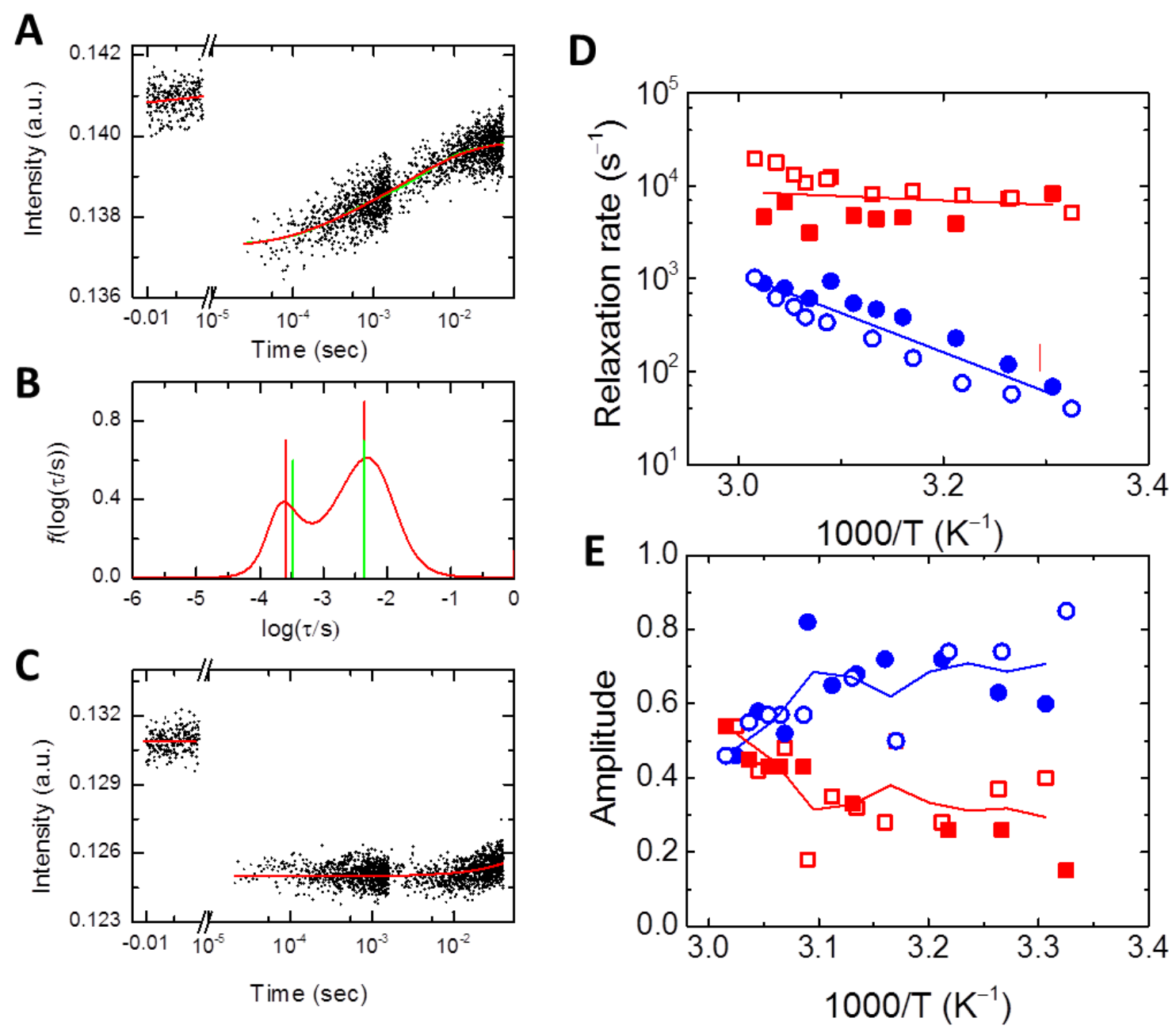

Fig.3. 4. T-jump kinetics measurements and relaxation rates on H'-IHF complexes.

(A) Donor fluorescence emission intensities of double-labeled H' samples in the presence of IHF measured in response to a T-jump perturbation are plotted as a function of time. Relaxation traces show two phases for a T-jump perturbation from $34.3{ }^{\circ} \mathrm{C}$ to $38.2{ }^{\circ} \mathrm{C}$. Red continuous lines are mem fits to the relaxation traces, with the corresponding distributions of relaxation times shown in panels (B). The green continuous line is from a double-exponential relaxation convoluted with T-jump recovery. The average time constants describing the best fit parameters from the two analyses are shown as vertical lines, red (mem) and green (two exponential + recovery function), $\tau_{\text {fast }}=255 \mu \mathrm{s}$ (red), $\tau_{\text {slow }}=4.4 \mathrm{~ms}$ (red) and $\tau_{\text {fast }}=329 \mu \mathrm{s}$ (green), $\tau_{\text {slow }}=4.3 \mathrm{~ms}$ (green). (C) Control T-jump measurements on donor-only labeled single $H^{\prime}$ top is shown. T-jump measurements on these samples do not exhibit any relaxation kinetics, instead they show only the much slower T-jump recovery kinetics. The rates and amplitudes of the two relaxation phases in the H'-IHF complex obtained from the mem analysis are plotted in (D, E) for two different data set (open and closed symbol) as a function of inverse temperature. (D) The relaxation rates $k_{\text {fast }}$ (red square) and $k_{\text {slow }}$ (blue circle) and (E) the corresponding amplitudes. The red and blue lines in (D) are Arrhenius fits to the fast and slow relaxation rates and the red and blue lines in $(\mathbf{E})$ represent the average change in amplitude as function of inverse temperature. The distribution of rate constants obtained from the maximum entropy analysis was fitted to two Gaussian functions. The relaxation rates for the two phases, $\mathrm{k}_{\text {fast }}$ and $\mathrm{k}_{\text {slow }}$, were obtained from the peak position of the Gaussian fits, and the amplitudes for the two phases were obtained from the area under the Gaussian curves, normalized to yield a total amplitude of one. 


\subsubsection{The slow phase occurs on the same time scale as spontaneous bp opening at a}

\section{kink site.}

Next, we compared the fast and the slow relaxation rates with bp opening rates measured by NMR (Fig. 3.5). We find that the relaxation rate for the slow phase at $30{ }^{\circ} \mathrm{C}$ is remarkably similar to A/T base-pair opening rate measured at that temperature for the $\mathrm{H}^{\prime}$ sequence at one of the kink sites, from NMR imino-proton exchange measurements (Fig 3.5). Previously we had proposed that spontaneous DNA kinking from thermal fluctuations presented the primary bottleneck in forming the fully bent recognition complex in IHF [24, 30]. The results presented here indicate that recognition occurs in two steps, with the slower ( 10-20 ms $)$ step corresponding to the rate-limiting spontaneous kinking event occurring on the same timescale as $\mathrm{A} / \mathrm{T}$ base pair opening rate for the $\mathrm{H}^{\prime}$ sequence at one of its kink sites.

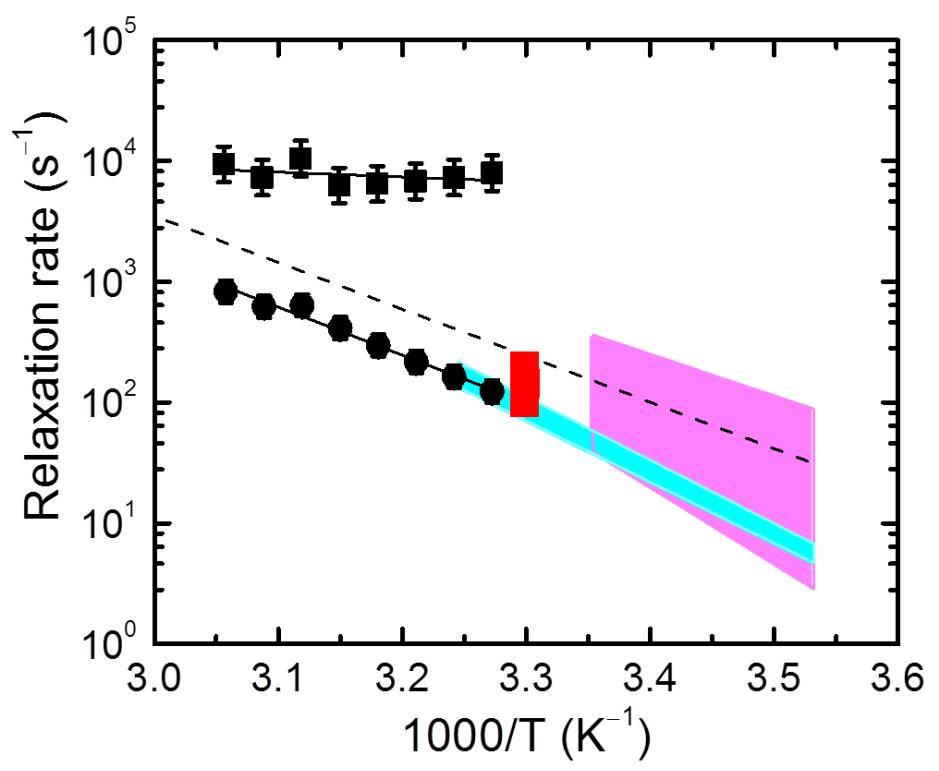

Fig.3. 5. Relaxation rates measured for $\mathrm{IHF}-\mathrm{H}^{\prime}$. The relaxation rates, $\mathrm{k}_{\text {slow }}$ and $\mathrm{k}_{\text {fast }}$, for the $\mathrm{IHF}-\mathrm{H}^{\prime}$ complex are plotted versus inverse (final) temperature $\left(\mathrm{T}_{\mathrm{f}}\right)$. The continuous line is obtained from the average of two sets of T-jump measurements under conditions where the T-jump perturbation does not dissociate IHF $-\mathrm{H}^{\prime}$ complex, the kinetics shows biphasic behavior, fast and slow rates. The dashed line is obtained from three sets of T-jump measurements and from the plateau values of the stopped-flow relaxation rates at high [IHF] (Vivas JMB, 2012 [24] and Sugimura and Crothers [29]). Here the kinetics is treated as single phase kinetics. The shaded areas represent the range of base-pair opening rates from imino proton exchange measurements, from Coman and Russu [32] (pink, A:T; blue, $\mathrm{C}: \mathrm{G})$. The red vertical bar is the A:T base-pair opening rate for the $\mathrm{H}^{\prime}$ sequence, at the site of one of the kinks, from Dhavan et al.[33]. 
Table III. Relaxation rates and dissociation constants

\begin{tabular}{|c|c|c|c|c|c|c|}
\hline$\frac{3}{0}$ & $\begin{array}{l}\infty \\
+\infty \\
\infty \\
+1 \\
0 \\
\dot{+1} \\
\stackrel{m}{-1}\end{array}$ & $\begin{array}{l}\stackrel{+}{\sigma} \\
\stackrel{+}{+} \\
+1 \\
+ \\
\stackrel{8}{\circ}\end{array}$ & 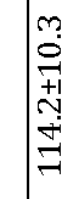 & 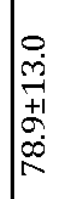 & 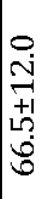 & बू. \\
\hline 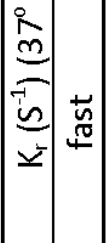 & 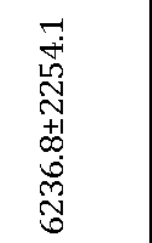 & 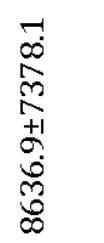 & 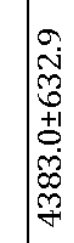 & 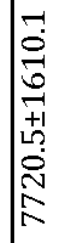 & 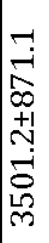 & 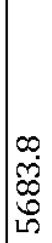 \\
\hline$-\frac{3}{n}$ & $\begin{array}{l}0 \\
\stackrel{N}{ } \\
+1 \\
+\infty \\
\infty \\
\infty \\
m\end{array}$ & $\begin{array}{l}\infty \\
\stackrel{\infty}{o} \\
\stackrel{0}{0} \\
+1 \\
\stackrel{+1}{a} \\
\stackrel{\sigma}{m}\end{array}$ & 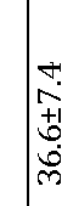 & 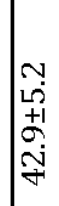 & 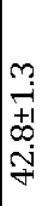 & $\stackrel{m}{\stackrel{m}{m}}$ \\
\hline 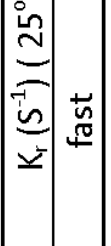 & 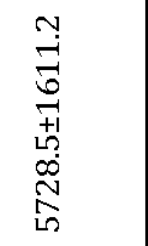 & 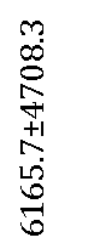 & 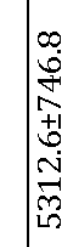 & 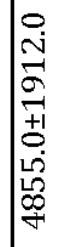 & 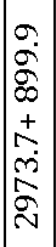 & $\begin{array}{l} \\
\sigma \\
0 \\
00 \\
0 \\
\text { Lิ }\end{array}$ \\
\hline 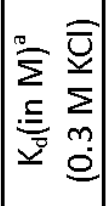 & 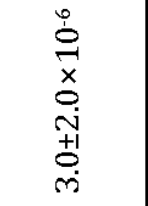 & 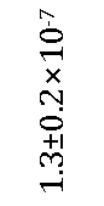 & 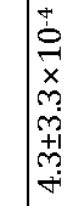 & |ll & 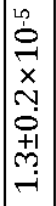 & 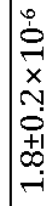 \\
\hline 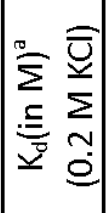 & 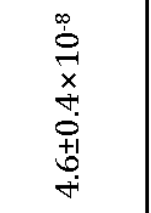 & $\begin{array}{l}\hat{\sigma} \\
\hat{0} \\
\dot{x} \\
\hat{0} \\
0 \\
+1 \\
\dot{0}\end{array}$ & 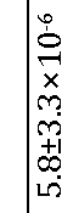 & 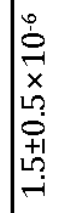 & 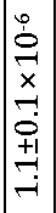 & 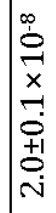 \\
\hline 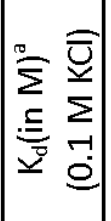 & 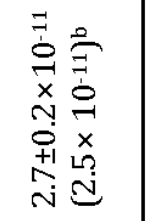 & 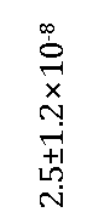 & 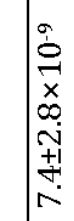 & 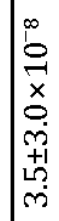 & 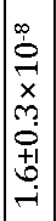 & 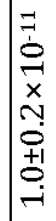 \\
\hline $\begin{array}{l}\frac{x}{0} \\
\frac{0}{\varrho} \\
\grave{\jmath}\end{array}$ & 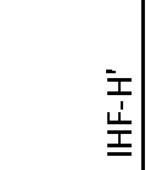 & 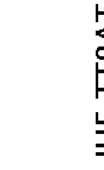 & 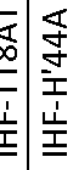 & $\frac{1}{\frac{1}{4}}$ & $\begin{array}{l}\frac{1}{1} \\
\frac{\dot{x}}{q} \\
\infty \\
\frac{0}{0} \\
0\end{array}$ & 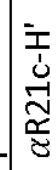 \\
\hline
\end{tabular}

${ }^{a}$ All Kd values are obtained at $25^{\circ} \mathrm{C}$ in buffer: $20 \mathrm{mM}$ Tris-Cl (pH 8.0), $100 \mathrm{mM} \mathrm{KCl}, 1 \mathrm{mM}$ EDTA, and $0.01 \%$ NP-40.

${ }^{\mathrm{b}}$ Values in parenthesis are from ratio of association and dissociation rates measured in stopped flow at $20^{\circ} \mathrm{C}[30]$. 


\subsubsection{Introducing mismatches at the site of the kinks affects the slow phase but not the}

\section{fast phase.}

A plausible explanation for the two relaxation phases is the sequential bending of first one flanking arm of the DNA and then the other (Fig. 3.6). To examine this possibility, we designed a series of modified H' constructs in which 2-bp mismatch bubbles were introduced at (i) both the kink sites (TT8AT), (ii) one side (TTloop) or the other (ATloop), as shown in Table. II.

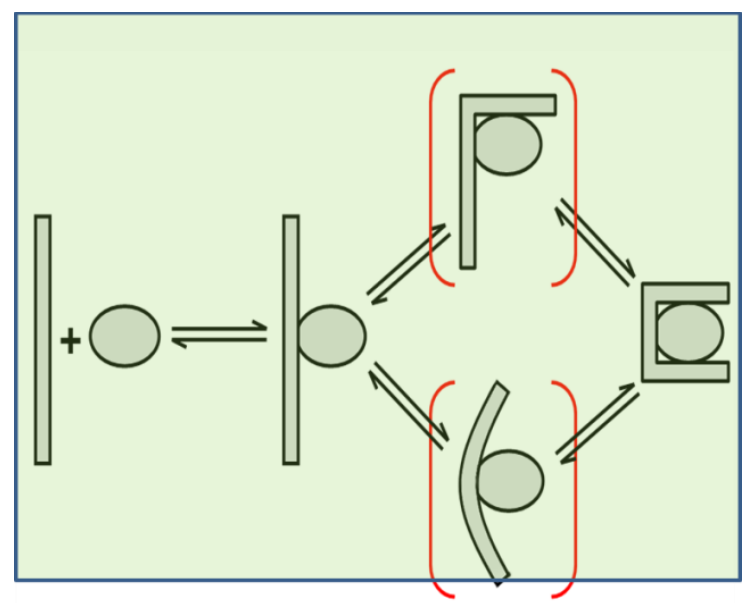

Fig.3. 6. The scheme illustrates two plausible pathways for the bending step with an intermediate partially bent state: (i) bending of first one arm of the DNA and then the other arm (top); or (ii) a partial bending of DNA, perhaps from the wrapping of the $\beta$-arms of IHF around DNA.

Binding studies on IHF-H' complex, carried out by us and others [24, 47] showed that the $K_{\mathrm{d}}$ for the IHF-TT8AT complex is smaller by a factor of 11-15 in comparison with that for the IHF-H' complex (Table III). These results indicate that introducing mismatches at the site of the kinks likely lowers the energetic cost of bending/kinking DNA, thus leading to a tighter final complex. Furthermore, equilibrium FRET measurements done on the IHFTT8AT complex as a function of temperature showed that the change in FRET with increasing temperature is independent of the IHF and DNA concentrations (at $10 \mu \mathrm{M}$ or greater) in the range of $20{ }^{\circ} \mathrm{C}$ to $50{ }^{\circ} \mathrm{C}$ (Fig 3.7, [24]). These measurements indicate that at 
concentrations used in the T-jump measurements $(5 \mu \mathrm{M}-20 \mu \mathrm{M})$, the change in FRET is primarily from unimolecular bending/unbending of DNA while still bound to the protein.

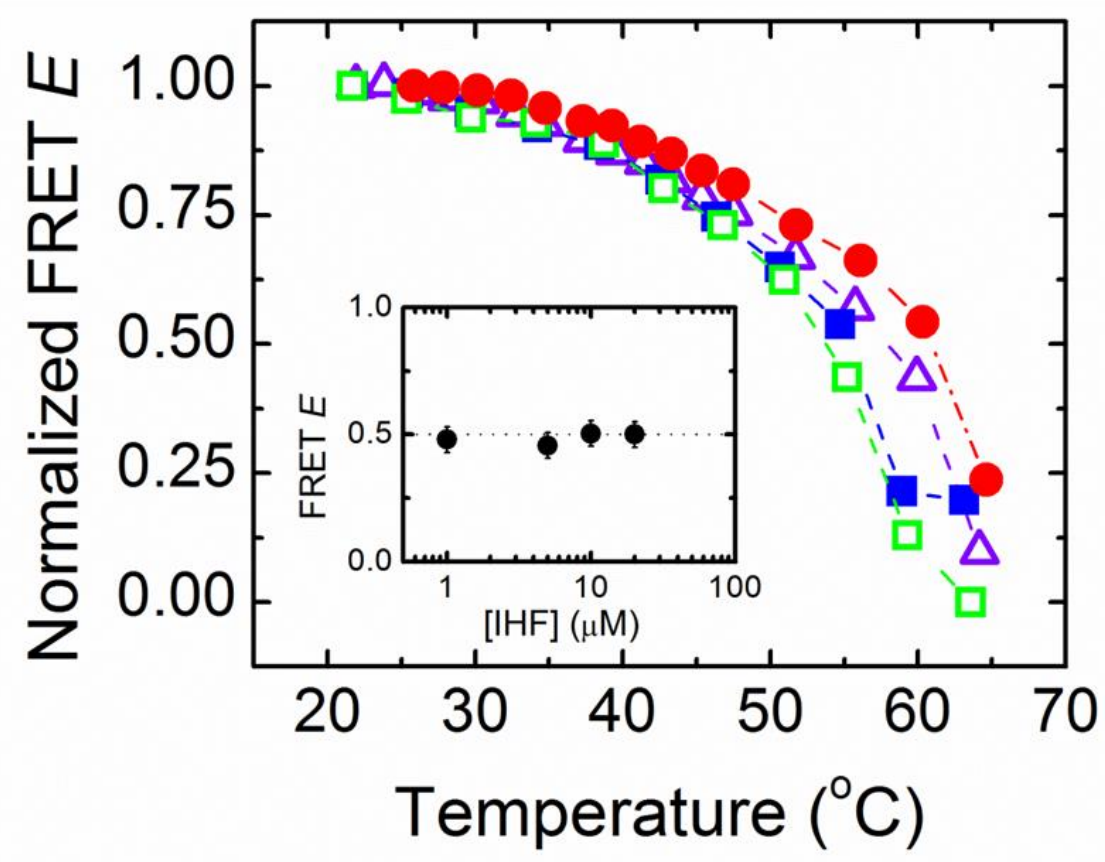

Fig.3. 7. Equilibrium measurements on IHF-TT8AT. FRET efficiency versus temperature at four different concentrations of IHF-TT8AT: (•) $20 \mu \mathrm{M}: 20 \mu \mathrm{M},(\Delta) 12.5 \mu \mathrm{M}: 10 \mu \mathrm{M},(\boldsymbol{\square}) 5 \mu \mathrm{M}: 5 \mu \mathrm{M}$ and $(\square) 1 \mu \mathrm{M}: 1 \mu \mathrm{M}$. (Inset) The actual FRET values for each sample at $20{ }^{\circ} \mathrm{C}$ are plotted as a function of the IHF concentration in that sample. (Figure reproduced with permission from [24]).

T-jump measurements on the IHF-TT8AT complex also showed biphasic relaxation kinetics (Fig. 3.8A, B), as was the case for the IHF-H' complex. The relaxation rates of the fast phase overlapped for the two complexes (Fig. 3.8D); the slow phase, on the other hand, was found to be $\sim 5$-fold faster in the IHF-TT8AT complex than in the IHF-H' complex (Fig. 3.8D). Thus, mismatches introduced at the kink sites accelerate DNA bending rates although the enhanced DNA bending rates do not fully account for the 11- fold increase in binding affinity of IHF for TT8AT. 

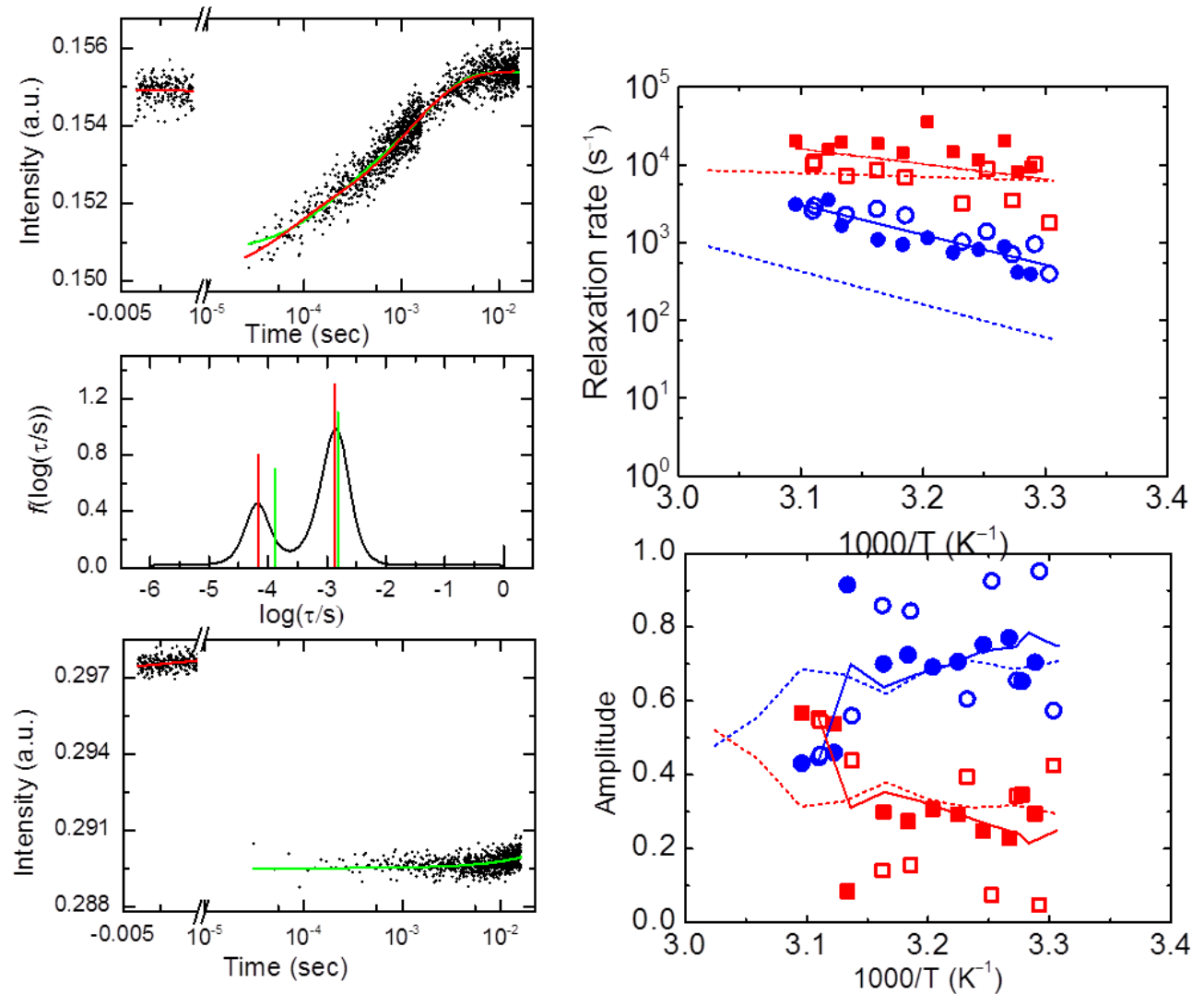

Fig.3. 8. T-jump kinetics measurements and relaxation rates on TT8AT loop-IHF complexes. (A) Donor fluorescence emission intensities of double-labeled TT8AT samples in the presence of IHF measured in response to a T-jump perturbation are plotted as a function of time. Relaxation traces show two phases for a T-jump perturbation from $34{ }^{\circ} \mathrm{C}$ to $37{ }^{\circ} \mathrm{C}$. Red continuous lines are mem fits to the relaxation traces, with the corresponding distributions of relaxation times shown in panels (B). The green continuous line is from double-exponential relaxation convoluted with T-jump recovery. (B) The average time constants describing the best fit parameters from the two analyses are shown as vertical lines, red (mem) and green (two exponential +recovery function), $\tau_{\text {fast }}=68.7 \mu \mathrm{s}$ (red), $\tau_{\text {slow }}=1.4 \mathrm{~ms}$ (red) and $\tau_{\text {fast }}=138.7 \mu \mathrm{s}$ (green), $\tau_{\text {slow }}=1.5 \mathrm{~ms}$ (green). (C) Control T-jump measurements on free fluorescein sample is shown. T-jump measurements on these samples do not exhibit any relaxation kinetics, instead they show only the much slower T-jump recovery kinetics. The rates and amplitudes of the two relaxation phases in the H'-IHF complex obtained from the mem analysis are plotted in (D, E) for two different data set (open and closed symbol) as a function of inverse temperature. (D) The relaxation rates $k_{\text {fast }}$ (red square) and $k_{\text {slow }}$ (blue circle) and (E) the corresponding amplitudes. The red and blue continuous lines in (D) are Arrhenius fits to the fast and slow relaxation rates and the red and blue dashed lines are corresponding average Arrhenius fits to the H'-IHF complex. The Red and blue dashed lines in (E) are average amplitudes of H'-IHF complex.

Kinetics measurement on IHF-ATloop and IHF-TTloop again reveal biphasic relaxation rates. As seen in the IHF-TT8AT complex, the fast phase appears unchanged in comparison with the fast phase of IHF-H', while the slow phase is 2-4 times faster than the corresponding slow phase of IHF-H' (Fig. 3.9D and 3.10D). The enhancement in the slow 
relaxation rates measured for all three mismatched sequences are attributed to the increased flexibility of the DNA substrates at the mismatch sites. In all three variants of the $\mathrm{H}^{\prime}$ substrate, only the slower phase was affected by the insertion of the 2-bp mismatch bubble loop, whether on one kink site or the other or both, while the fast phase remained unchanged [48]. These results demonstrate that the two steps do not reflect the sequential bending of the two flanking arms of DNA; rather, the fast step, which appears to be sequence-independent, or at least insensitive to the sequence at the kink site, may correspond to the formation of a partially bent intermediate "interrogation" (nonspecific) complex, while the slow phase, which is responsive to the ease with which one or the other kink sites can be distorted, would then be the formation of the ultimate "recognition" complex (fig 3.13). 


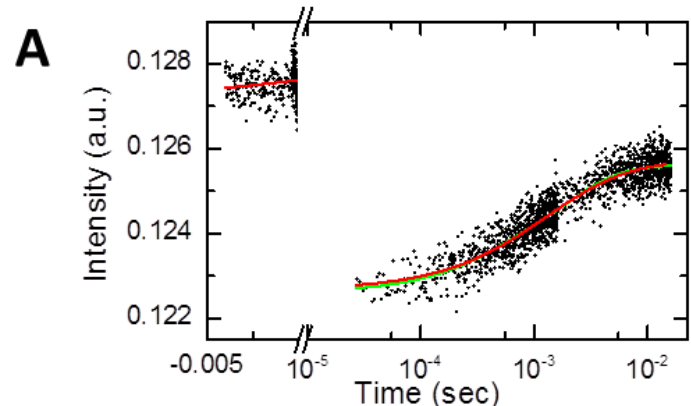

B
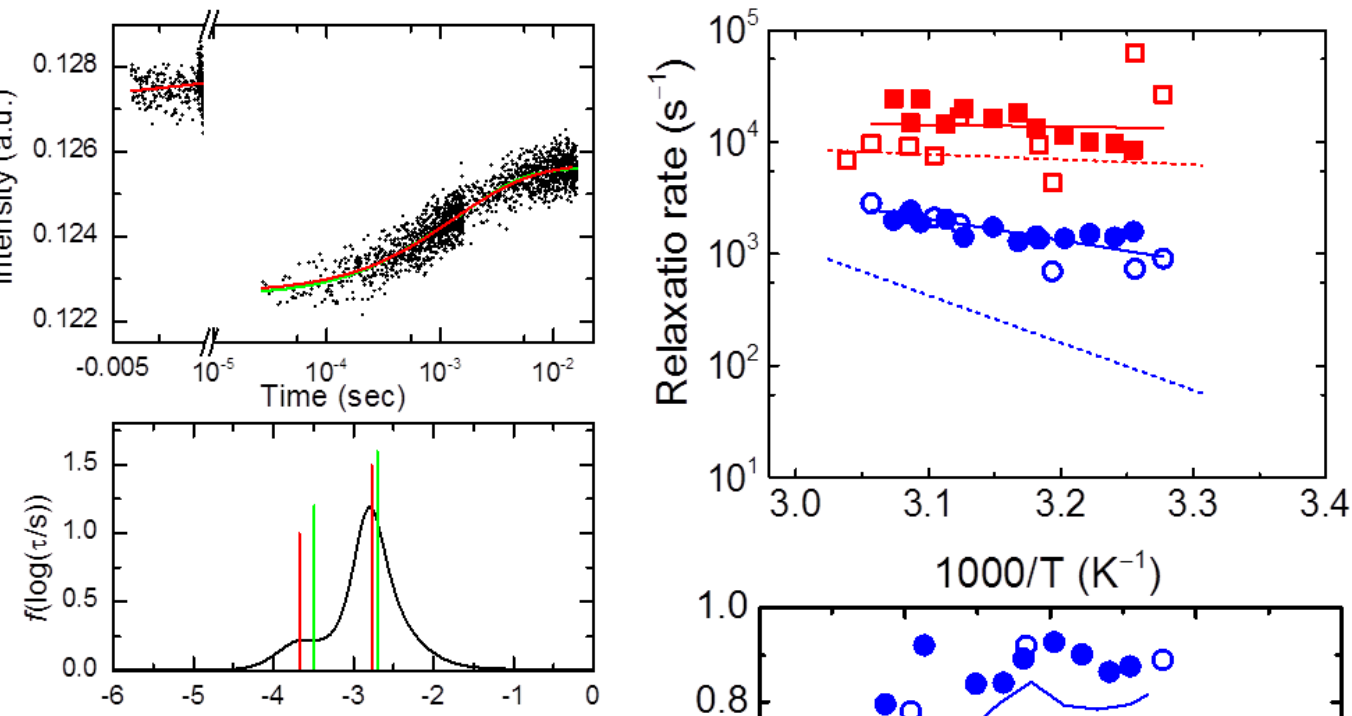

C
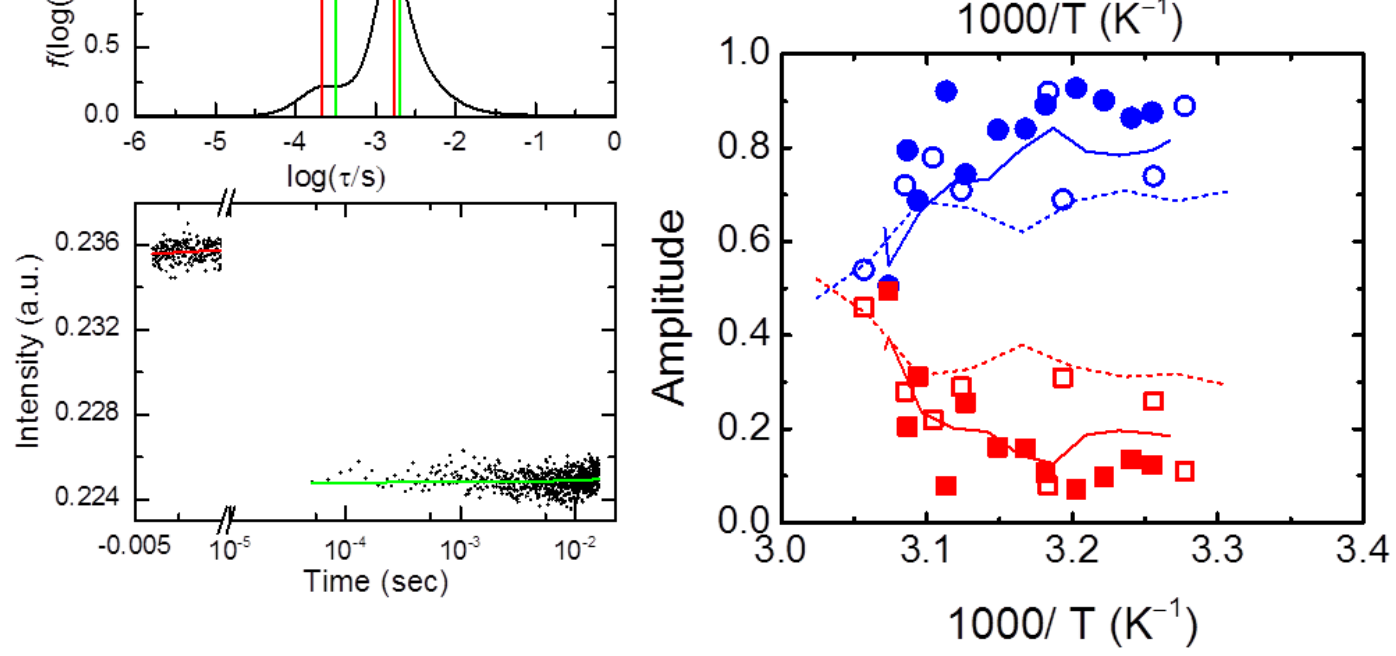

Fig.3. 9. T-jump kinetics measurements and relaxation rates on TT loop-IHF complexes.

(A) Donor fluorescence emission intensities of double-labelled TT samples in the presence of IHF measured in response to a T-jump perturbation are plotted as a function of time. Relaxation traces show two phases for a T-jump perturbation from $36.6{ }^{\circ} \mathrm{C}$ to $40{ }^{\circ} \mathrm{C}$. Red continuous lines are mem fits to the relaxation traces, with the corresponding distributions of relaxation times shown in panels (B). The green continuous line is from double-exponential relaxation convoluted with T-jump recovery. (B) The average time constants describing the best fit parameters from the two analyses are shown as vertical lines, red (mem) and green (two exponential + recovery function), $\tau_{\text {fast }}=212 \mu \mathrm{s}$ (red), $\tau_{\text {slow }}=1.7 \mathrm{~ms}$ (red) and $\tau_{\text {fast }}=324 \mu \mathrm{s}$ (green), $\tau_{\text {slow }}=2 \mathrm{~ms}$ (green). (C) Control T-jump measurements on free fluorescein sample are shown. T-jump measurements on these samples do not exhibit any relaxation kinetics; instead they show only the much slower T-jump recovery kinetics. The rates and amplitudes of the two relaxation phases in the H'-IHF complex obtained from the mem analysis are plotted in (D, E) for two different data set (open and closed symbol) as a function of inverse temperature. (D) The relaxation rates $k_{\text {fast }}$ (red square) and $k_{\text {slow }}$ (blue circle) and (E) the corresponding amplitudes. The blue and red line in (D) are Arrhenius fits to the fast and slow relaxation rates and the red and blue dashed lines are corresponding average Arrhenius fits to the H'IHF complex. The Red and blue dashed lines in (E) are average amplitudes of H'-IHF complex. 

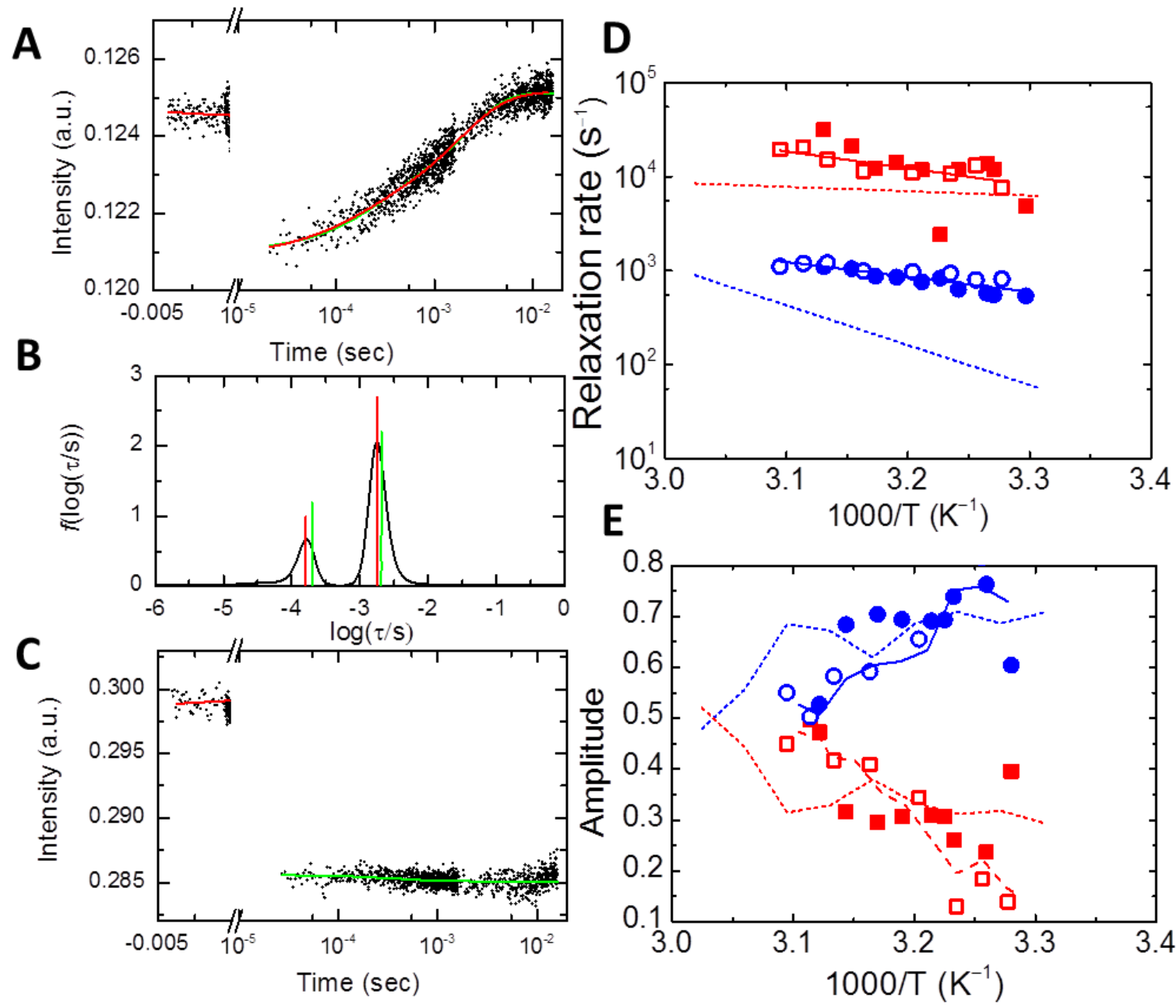

Fig.3. 10. T-jump kinetics measurements and relaxation rates on AT loop-IHF complexes.

(A) Donor fluorescence emission intensities of double-labelled AT samples in the presence of IHF measured in response to a T-jump perturbation are plotted as a function of time. Relaxation traces show two phases for a T-jump perturbation from $34{ }^{\circ} \mathrm{C}$ to $37{ }^{\circ} \mathrm{C}$. Red continuous lines are mem fits to the relaxation traces, with the corresponding distributions of relaxation times shown in panels (B). The green continuous line is from double-exponential relaxation convoluted with T-jump recovery. (B) The average time constants describing the best fit parameters from the two analyses are shown as vertical lines, red (mem) and green (two exponential + recovery function), $\tau_{\text {fast }}=161 \mu \mathrm{s}$ (red), $\tau_{\text {slow }}=1.8 \mathrm{~ms}$ (red) and $\tau_{\text {fast }}=199 \mu \mathrm{s}$ (green), $\tau_{\text {slow }}=2.1 \mathrm{~ms}$ (green). (C) Control T-jump measurements on donor-only labeled single $H^{\prime}$ top are shown. T-jump measurements on these samples do not exhibit any relaxation kinetics, instead they show only the much slower T-jump recovery kinetics. The rates and amplitudes of the two relaxation phases in the H'-IHF complex obtained from the mem analysis are plotted in (D, E) for two different data set (open and closed symbol) as a function of inverse temperature. (D) The relaxation rates $k_{\text {fast }}$ (red square) and $k_{\text {slow }}$ (blue circle) and (E) the corresponding amplitudes. The red and blue continuous lines in (D) are Arrhenius fits to the fast and slow relaxation rates and the red and blue dashed lines are corresponding Arrhenius fits to the H'-IHF complex. The red and blue dashed lines in (E) are the average amplitudes of fast and slow phase of H'-IHF complex. 


\subsubsection{DNA bending rates in the slow phase of IHF- TT8AT complex reflect enhanced base-pair opening rates in mismatched DNA}

Previously several researchers showed that the base pair opening rate of a mismatch in DNA is much faster than the corresponding rates in normal Watson-Crick matched base pairs [4951], since mismatched base pairs are kinetically and energetically destabilized relative to normal base pairs (Fig. 3.11). For example the opening rate of a G/T mismatch is 166 fold faster than that of a GC base pair, for the same sequence context [49]. Another study showed 6-fold increase in opening rate for a U/A pair in comparison with a T/A pair [52]. It should be noted here that the extent of increase in the bp-opening rate between a normal and a mismatched bp strongly depends on the sequence contest [49-51].

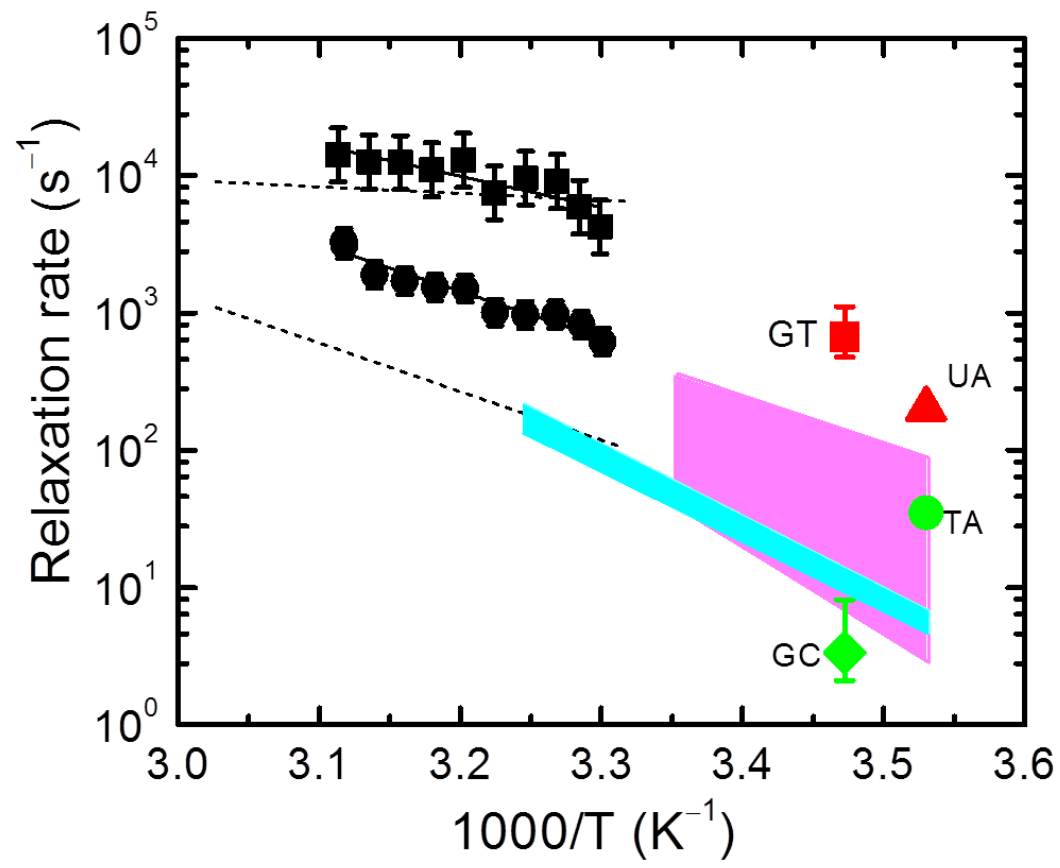

Fig.3. 11. The average biphasic rates obtained from two sets of T-jump measurements on TT8AT IHF (black continuous lines) and H'-IHF (black dashed lines) are plotted versus inverse of the final temperature after T-jump. The pink (cyan) shaded regions represent the range of base pair opening ("breathing") rates for $\mathrm{A} / \mathrm{T}(\mathrm{G} / \mathrm{C})$ base pairs, from NMR imino proton exchange measurements of Comon and Russu [32]. The opening rates and their ranges are also indicated for G/T mismatch (red square) from Moe and Russu [49] and U/A mismatch (red triangle) from Parker [52], T/A base pair (green circle) from Parker [52] and G/C base pair (green diamond) from Moe and Russu [49]. 
Our T-jump data reveal that the slow rates of the biphasic DNA bending kinetics for the TT8AT-IHF complex, when extrapolated to low temperatures where the NMR measurements are done, agree remarkably well with the U/A bp opening rate measured at $\sim 5$ ${ }^{\circ} \mathrm{C}$ (Fig.3.11). This result is consistent with the conformational capture mechanism proposed for binding site recognition by IHF, in which the rate-limiting step in forming the specific complex is the spontaneous kinking of DNA at the kink sites, which in turn is rate-limited by the thermal disruption of the stacking interactions at that site.
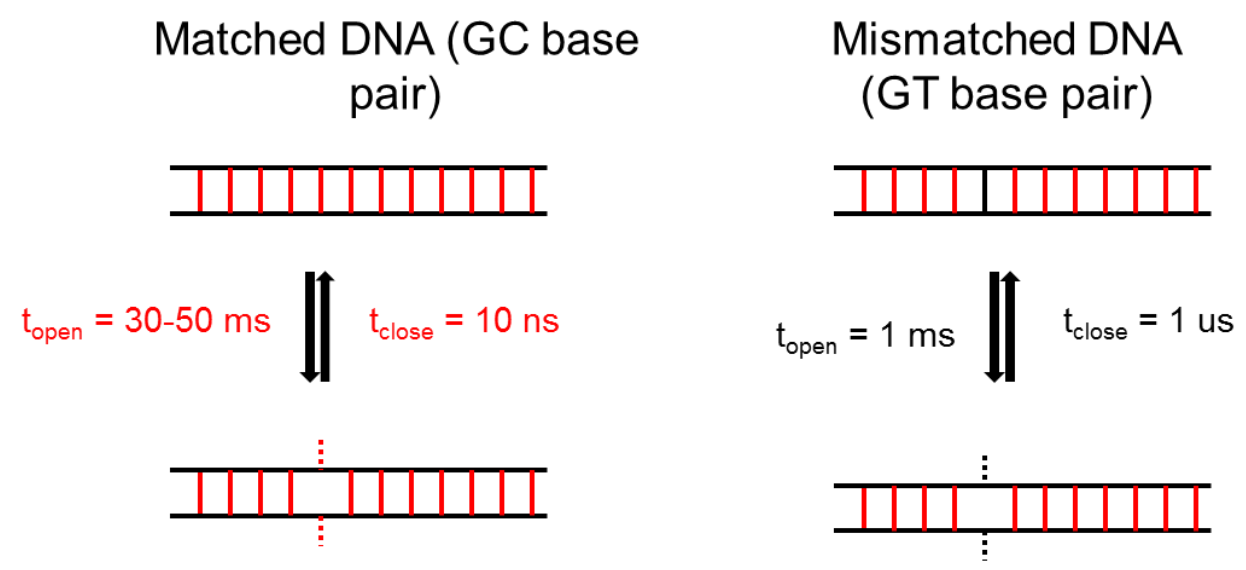

Ref: Pratip k.Bhattacharya et al,2002, Danial Coman et al 2005, James G. Moe et al, 1992

Fig.3. 12. Cartoon of matched DNA base pair opening/closing rate versus mismatch base pair opening/closing rate as measured by NMR imino proton exchange method from references [32, 49, 50]. DNA opening rate is faster (100 fold) in mismatched DNA compare to matched DNA. 
(1)

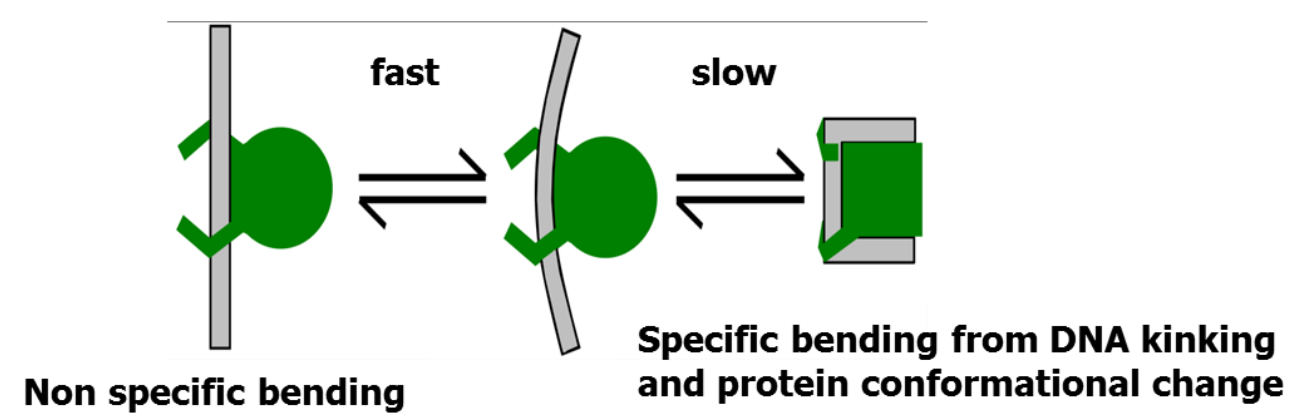

(2)

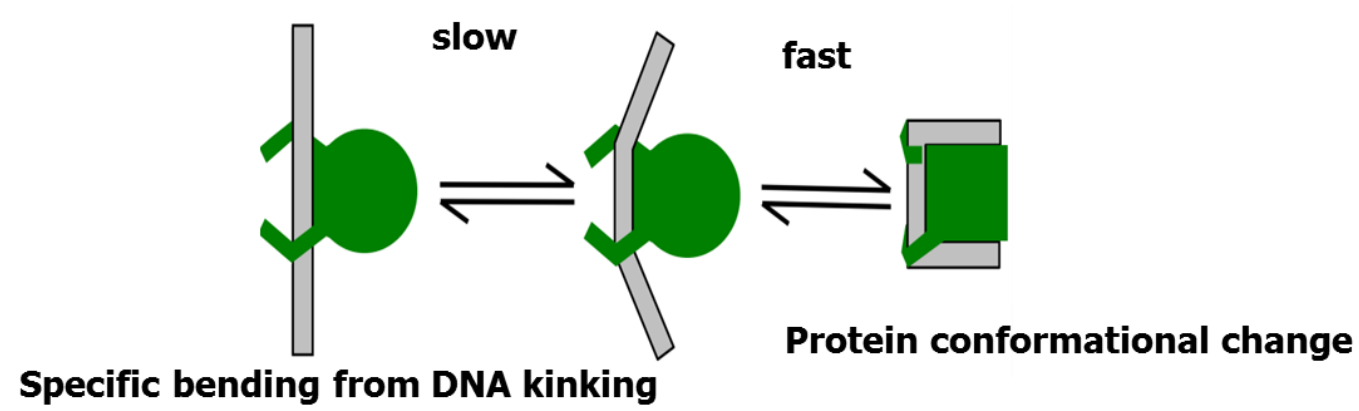

Fig.3. 13. A proposed mechanism for the IHF-DNA complex formation. Plausible alternative pathways for the formation of the specific complex, after bimolecular association, are shown for binding site recognition by IHF.

\subsubsection{DNA modifications away from the kink sites have no effect on either of the two rates.}

Next, we carried out equilibrium and T-jump studies with the H'44A sequence, which has a single $\mathrm{T} \rightarrow \mathrm{A}$ mutation in the TTR consensus region of the $\mathrm{H}^{\prime}$ sequence (Table. II). Rice and co-workers used gel mobility shift assays to show that this single mutation results in $\sim 100$ fold decrease in the binding affinity for $\mathrm{H}^{\prime} 44 \mathrm{~A}$ relative to $\mathrm{H}^{\prime}$ [53]. We carried out equilibrium FRET titration studies and found a 250 -fold decrease in the binding affinity in comparison with H' (Table III). A comparison of the crystal structures of IHF-H' and IHF-H'44A showed that a very high twist in the TG step of the TTG trinucleotide in the original $\mathrm{H}^{\prime}$ consensus sequence allows formation of a chain of salt bridges (ionic interactions) involving three amino acid residues of IHF and the DNA itself [53]. In contrast, in the H'44A sequence the 
AG step of the TAG sequence is less flexible, and, in complex with IHF, the twist is spread out more evenly among the dinucleotide steps, thus disrupting the specific ionic interactions and destabilizing the complex. Thus, the modification introduced in the $\mathrm{H}^{\prime}$ sequence in the TTR consensus region alters the "twistability" of DNA whereby disrupting stabilizing ionic interactions between IHF and DNA and illustrates another indirect readout feature that IHF exploits to recognize a part of its binding site $[53,54]$. Our FRET measurements show that the FRET efficiency for the IHF- $\mathrm{H}^{\prime} 44 \mathrm{~A}$ complex is $\sim 0.45$ at $25{ }^{\circ} \mathrm{C}$, significantly smaller than the $\sim 0.55$ FRET efficiency obtained for the IHF- $\mathrm{H}^{\prime}$ complex under identical solvent conditions [24]. These results suggest that, despite the very similar crystal structures of IHF$\mathrm{H}^{\prime}$ and IHF- $\mathrm{H}^{\prime} 44 \mathrm{~A}$ complexes [53], the average conformation of $\mathrm{H}^{\prime} 44 \mathrm{~A}$ in the complex is less bent under solution conditions, consistent with weaker interactions between IHF and one of the flanking "arms" of the bent DNA.

Thus, the IHF-H'44A complex provides a useful control that enables us to examine the DNA bending kinetics in a complex that is considerably weakened in comparison with the IHF-H' complex, but one in which the bendability of DNA at the kink sites is left undisturbed [24]. Note that the modification site in H'44A is separated from the location of the nearest kink by 6 bp (Table. II). T-jump measurements on the IHF-H'44A complex also reveal biphasic kinetics (Fig. 3.14). Notably, the relaxation rates of both phase overlap with the corresponding rates measured in the IHF- $\mathrm{H}^{\prime}$ complex, over the entire temperature range of these measurements (Fig. 3.14D). These results reaffirm that the slow phase kinetics remain unaltered unless the kink site is modified, despite the much weaker IHF-H'44A complex. 
A
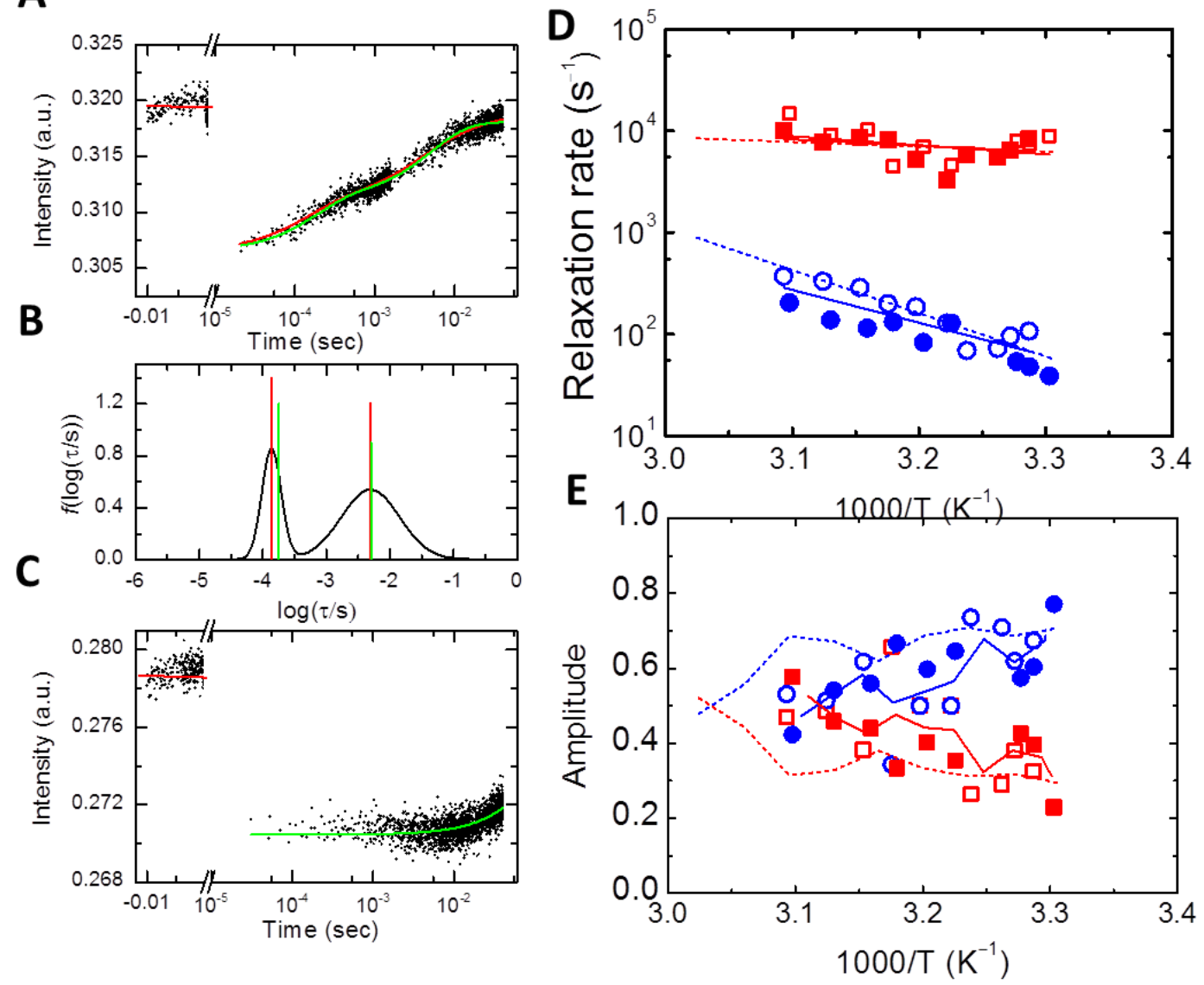

Fig.3. 14. T-jump kinetics measurements and relaxation rates on H'44A-IHF complexes.

(A) Donor fluorescence emission intensities of double-labelled H'44A samples in the presence of IHF measured in response to a T-jump perturbation are plotted as a function of time. Relaxation traces show two phases for a T-jump perturbation from $34.2{ }^{\circ} \mathrm{C}$ to $38.2{ }^{\circ} \mathrm{C}$. Red continuous lines are mem fits to the relaxation traces, with the corresponding distributions of relaxation times shown in panels (B). The green continuous line is from double-exponential relaxation convoluted with T-jump recovery. (B) The average time constants describing the best fit parameters from the two analyses are shown as vertical lines, red (mem) and green (two exponential + recovery function), $\tau_{\text {fast }}=138 \mu \mathrm{s}$ (red), $\tau_{\text {slow }}=5.0 \mathrm{~ms}$ (red) and $\tau_{\text {fast }}=180 \mu \mathrm{s}$ (green), $\tau_{\text {slow }}=5.1 \mathrm{~ms}$ (green). (C) Control T-jump measurements on free fluorescein sample are shown. T-jump measurements on these samples do not exhibit any relaxation kinetics, instead they show only the much slower T-jump recovery kinetics. The rates and amplitudes of the two relaxation phases in the H'-IHF complex obtained from the mem analysis are plotted in (D, E) for two different data set (open and closed symbol) as a function of inverse temperature. (D) The relaxation rates $k_{\text {fast }}$ (red square) and $k_{\text {slow }}$ (blue circle) and (E) the corresponding amplitudes. The red and blue continuous lines in (D) are Arrhenius fits to the fast and slow relaxation rates and the red and blue dashed lines are corresponding Arrhenius fits to the H'IHF complex. The red and blue dashed lines in (E) are the average amplitudes of fast and slow components of H'-IHF complex 


\subsubsection{Two plausible scenarios for biphasic relaxation kinetics}

Based on the results thus far, we propose two alternative scenarios that could explain the biphasic kinetics observed for binding-site recognition by IHF. In the first scenario, shown as scheme 1 in Fig. 3.13, the fast phase corresponds to nonspecific bending of DNA by IHF, followed by the slow recognition step that is rate-limited by intrinsic fluctuations in DNA at the kink sites. In the second scenario, shown as scheme 2 in Fig. 3.13, the slow phase is still the rate-limiting DNA kinking step, followed by a rapid reorganization of the protein and the DNA to form the stable recognition complex. In both cases, the fast step is predicted to remain unaffected by changes in the DNA sequence at the kink sites. To further explore the origin of the two phases, we carried out T-jump measurements as a function of the ionic strength and for different IHF mutants, as discussed in the sections below.

\subsubsection{Salt-dependence of the fast and slow components.}

The binding of proteins to DNA depends strongly on the ionic environment in solution, due to the polyelectrolyte nature of DNA, which results in the condensation of high concentration of cation in the vicinity of DNA. Numerous previous publications have reported that DNA-binding specificity typically improves with increasing salt concentration [55]. For example, in the case of EcoRV endonuclease, an increase in $\mathrm{NaCl}$ concentration from $0.1 \mathrm{M}$ to $0.2 \mathrm{M}$ in vitro increases the specificity ratio by about a factor of 3 [55]. This is because nonspecific binding is primarily mediated by contacts to the DNA backbone via electrostatic interactions between basic residues in the protein and phosphate groups in the DNA backbone; the strength of this interactions decreases with increasing salt concentrations. On the other hand, sequence specific interactions are primarily mediated by contacts to the individual bases and typically involve hydrogen bonds or van der Waals interactions between amino acid side chains and specific DNA sequences. Hence in general, these interactions are expected to be substantially less sensitive to salt concentration. This is reflected in a generally 
larger slope of $\log (\mathrm{ka})$ versus $\log [\mathrm{salt}]$ observed for nonspecific than for specific binding [5661], as expected if protein-phosphate interactions are proportionally more important to nonspecific binding.

Thermodynamics and kinetics measurements on the IHF-H' complex are consistent with these notions. ITC measurements on the IHF-H' complex carried out by Record and coworkers showed that the overall $K_{\mathrm{d}}$ for the complex decreases with increasing [salt] with a slope of $\sim 8-9$ on a $\log \left(K_{\mathrm{d}}\right)$ versus $\log ([$ salt $])$ plot, indicating that $\sim 8-9$ counterions are released when this complex is formed (cite Saecker and Record). They further showed that at low to moderate $\left[\mathrm{K}^{+}\right](60-100 \mathrm{mM})$, there is a strong competition between specific and nonspecific binding of IHF due to low specificity ratio $\left(\mathrm{K}_{\mathrm{s}} / \mathrm{K}_{\mathrm{ns}} \sim 10^{2}\right)$ and a very small nonspecific site size ( 10 bp) [62]. In our group, we previously carried out a comprehensive study of the saltdependence of binding and relaxation kinetics using FRET measurements on IHF bound to end-labeled H' DNA[35, 63]. These studies showed that at 100 and $200 \mathrm{mM} \mathrm{KCl}$, raising the temperature up to $\sim 60^{\circ} \mathrm{C}$ resulted in weakening of the IHF-H' complex and straightening of the DNA in the complex but without any bimolecular dissociation of the complex, as indicated by the lack of IHF:DNA concentration dependence in the FRET versus temperature profiles, in the concentration range $200 \mathrm{nM}-5 \mu \mathrm{M}$ (Fig. 3.15A,B). In contrast, at $400 \mathrm{mM}$ $\mathrm{KCl}$, the FRET versus temperature profiles varied with IHF:DNA concentrations, indicative of bimolecular dissociation of the complex as the temperature was raised (Fig. 3.15C). These and further equilibrium and T-jump relaxation kinetics measurements on the IHF-H' complex for $\mathrm{KCl}$ concentrations ranging from 100 to $450 \mathrm{mM}$ showed that relaxation kinetics at $[\mathrm{KCl}]$ below $\sim 250 \mathrm{mM}$ were from unimolecular bending/unbending processes, while relaxation 

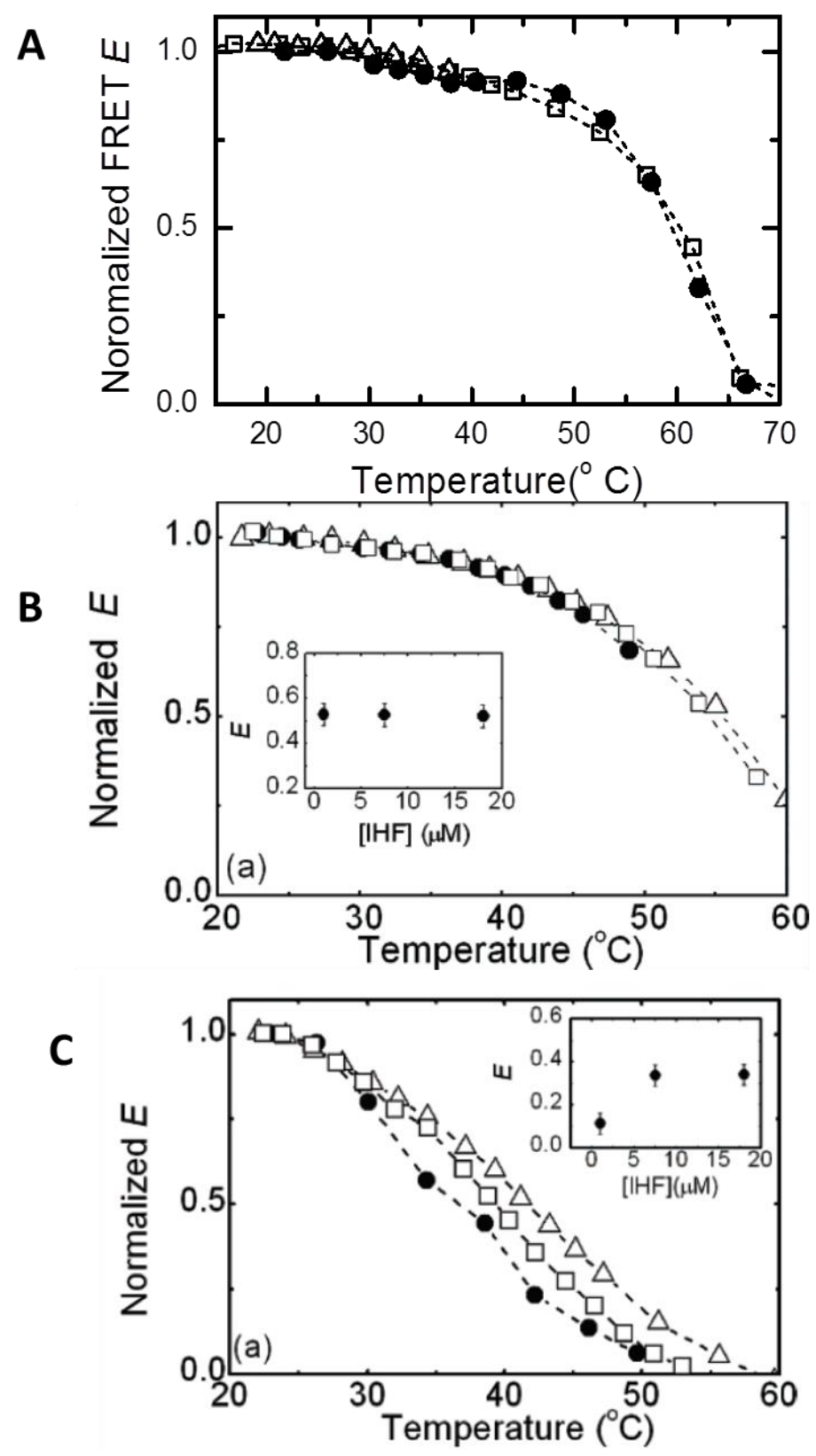

Fig.3. 15. Equilibrium measurements on $\mathrm{H}^{\prime}-\mathrm{IHF}$ at three different salt concentrations. (A) FRET efficiency (E) as a function of temperature in $100 \mathrm{mM} \mathrm{KCl}$, normalized at $25{ }^{\circ} \mathrm{C}$ is plotted for the IHF- $\mathrm{H}^{\prime}$ complex at three different concentrations of IHF and $\mathrm{H}^{\prime}:(\Delta)(5 \mu \mathrm{M}: 5 \mu \mathrm{M}),(\square)(1 \mu \mathrm{M}: 1 \mu \mathrm{M})$ and (•) (200 nM:200 nM). (B). The FRET efficiency (E) in $200 \mathrm{mM} \mathrm{KCl}$, normalized at $25^{\circ} \mathrm{C}$, is plotted as a function of temperature for IHF-H' concentrations of $[1 \mu \mathrm{M}: 1 \mu \mathrm{M}](\bullet),[7.5 \mu \mathrm{M}: 5 \mu \mathrm{M}]$ $(\square)$, and $[18 \mu \mathrm{M}: 18 \mu \mathrm{M}](\Delta)$. The inset shows the actual FRET efficiency E versus the [IHF] concentration at $25^{\circ} \mathrm{C}$. (C) This figure is identical to (B) for data obtained on the IHF-H' complex in $400 \mathrm{mM} \mathrm{KCl}$. (Fig. B.C reprinted with permission from [35]. Copyright (2008) American Chemical Society)

kinetics measured at $[\mathrm{KCl}]$ above $\sim 250 \mathrm{mM}$ had contributions from bimolecular dissociation $[24,64]$. Furthermore, by carrying out a global analysis of the salt-dependence of the 
measured $K_{\mathrm{d}}$ and the measured relaxation rates $k_{\mathrm{r}}$ at each [salt], we showed that nearly all the counter-ions released when IHF binds to $\mathrm{H}^{\prime}$ are released in the formation of the nonspecific complex [63], consistent with the findings of Record and co-workers that [salt] has a larger effect on nonspecific than specific binding.

Against the backdrop of these previous results, and to gain further insights into the origin of the fast and slow components of the unimolecular kinetics, we carried out T-jump experiments on IHF-H' at four different salt concentrations: $50 \mathrm{mM}, 100 \mathrm{mM}, 150 \mathrm{mM}$ and $200 \mathrm{mM} \mathrm{KCl}$, at conditions where we have shown that there is no bimolecular dissociation when the temperature is raised, and hence the T-jump relaxation kinetics are from unimolecular processes. Our hypothesis is that if the fast phase in the observed kinetics is from bending/unbending between nonspecific binding modes, then it should diminish with increasing [salt].

Results of T-jump measurements at the different salt concentrations and for two different temperatures at each salt condition are shown in Figs. 3.16-3.19. MEM analysis of the relaxation traces measured at $50 \mathrm{mM} \mathrm{KCl}$ (Fig. 3. 16) and $100 \mathrm{mM} \mathrm{KCl}$ (Fig. 3.17) reveals two distinct peaks in the distribution of relaxation times, clearly indicating two kinetics phases. In the case of $150 \mathrm{mM} \mathrm{KCl}$, a predominantly single phase is observed at low temperature with barely detectable amplitude in the fast phase; at high temperature the amplitude in the fast phase increases slightly and appears as a shoulder in the distribution of the relaxation times (Fig. 3.18). At $200 \mathrm{mM}$ salt, the amplitude of the fast phase remains small at all temperatures, and only a predominantly single distribution is observed in the MEM analysis, albeit with a broad distribution at higher temperatures. The salient result here is that biphasic kinetics are observed at low [salt], with the relaxation rates and 

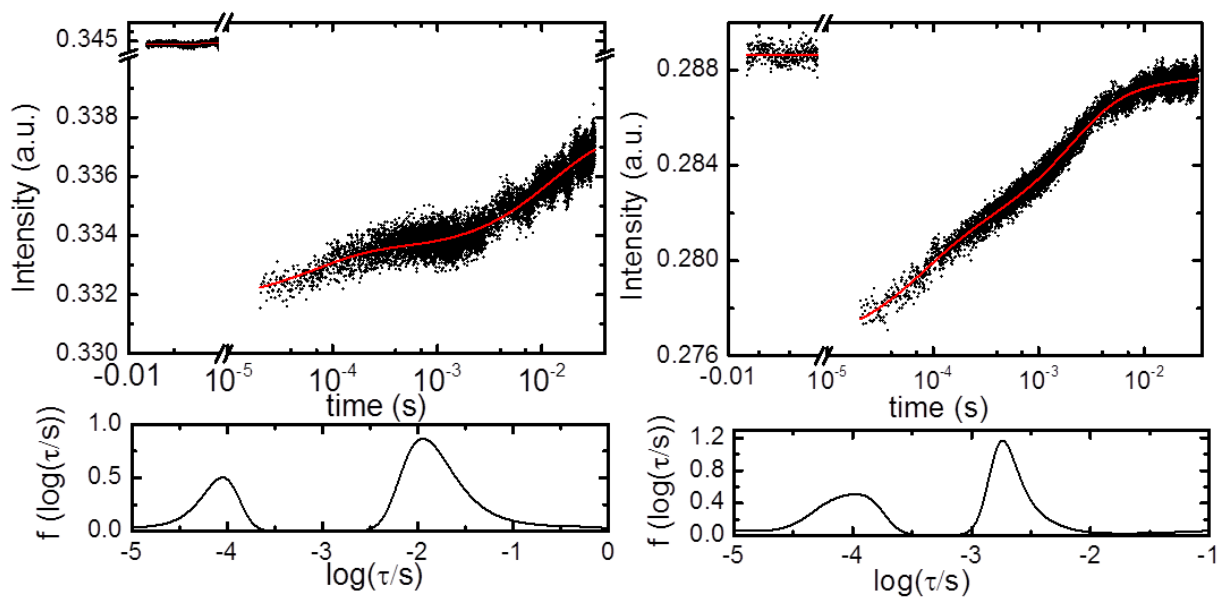

Fig.3. 16. Kinetic traces of $\mathrm{H}^{\prime}$ - IHF complex with $50 \mathrm{mM}$ salt condition and at two different temperatures are shown

$33^{\circ} \mathrm{C}$

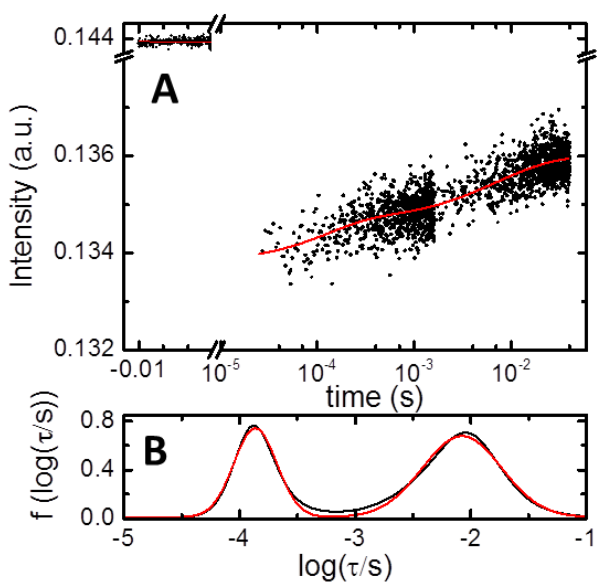

$50^{\circ} \mathrm{C}$

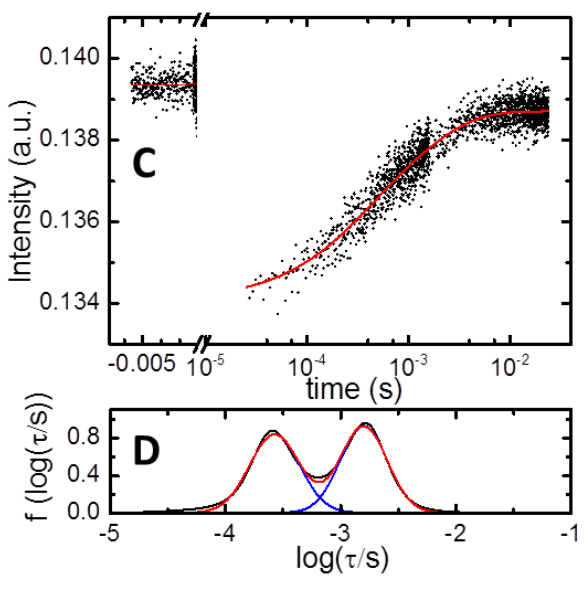

Fig.3. 17. Kinetic traces of H'- IHF complex with $100 \mathrm{mM}$ salt conditions and at two different temperatures are shown. Since the two peaks are not well separated, it was fit to two Gaussian functions (red line)

relative amplitudes of the fast and the slow phases observed at $50 \mathrm{mM} \mathrm{KCl}$ virtually indistinguishable from those at $100 \mathrm{mM} \mathrm{KCl}$ (Fig 3.20); at higher [salt] (150-200 mM KCl), the amplitude of the fast phase diminishes significantly. This implies that the fractional population that contributes to the fast phase decreases significantly at high salt.

These findings suggest that the fast phase corresponds to the step where IHF binds nonspecifically and partially bends the DNA, while the slow phase corresponds to the 
conversion of nonspecific complex to the final specific complex through further bending and subsequent wrapping of DNA (scheme 1 in Fig. 3.13)
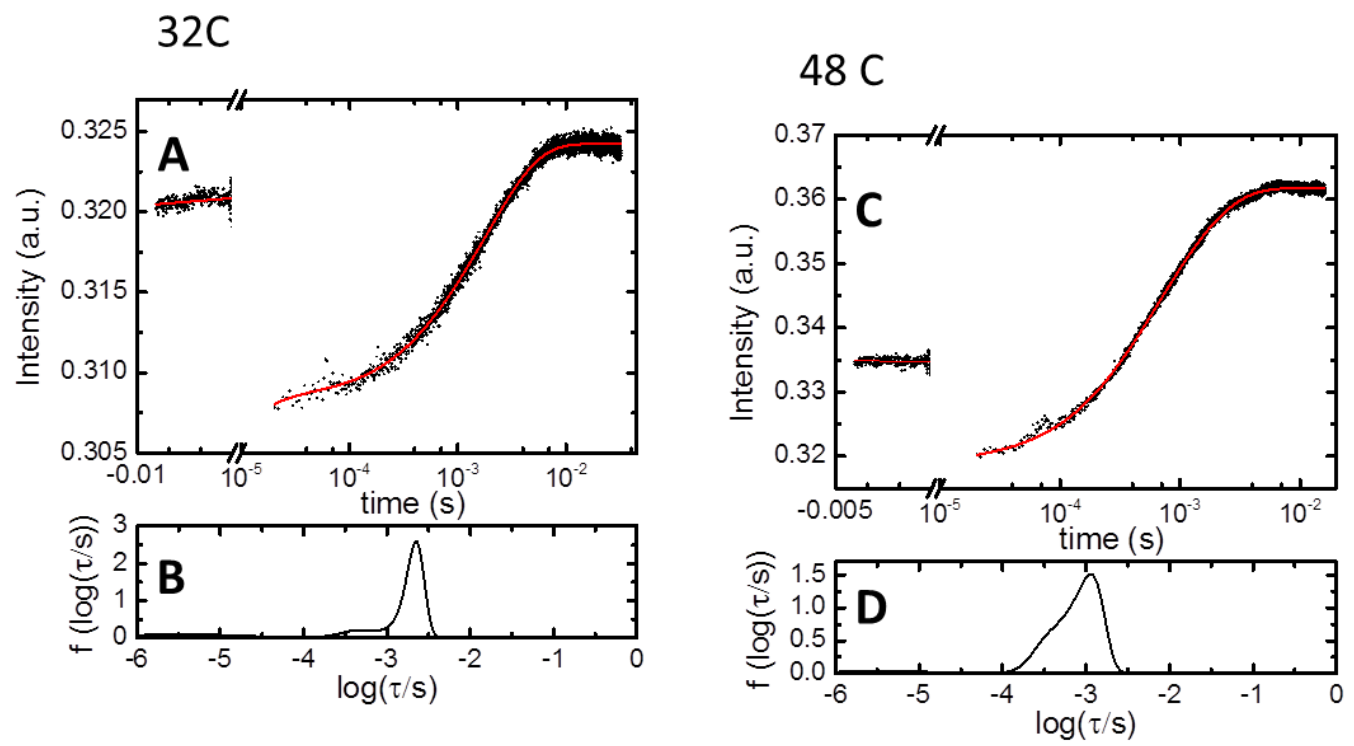

Fig.3. 18. Kinetic traces of H'- IHF complex with $150 \mathrm{mM}$ salt condition and at two different temperatures are shown

$37^{\circ} \mathrm{C}$
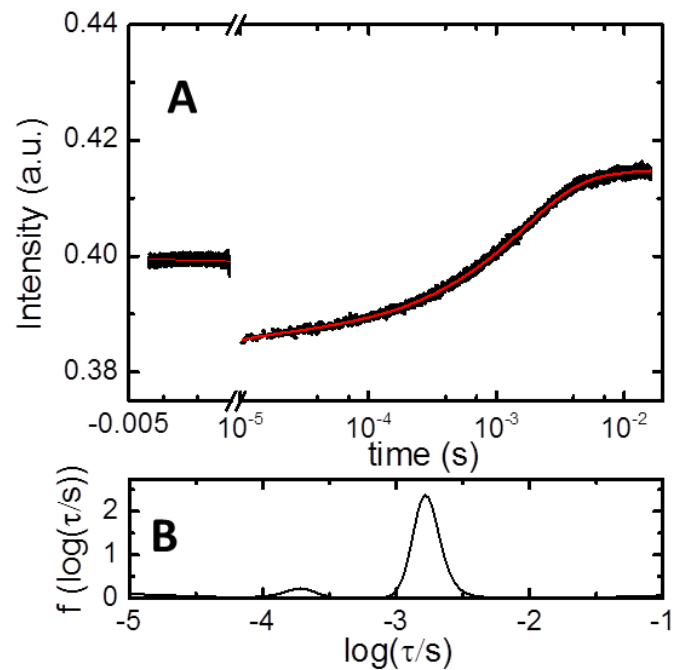

$43^{\circ} \mathrm{C}$
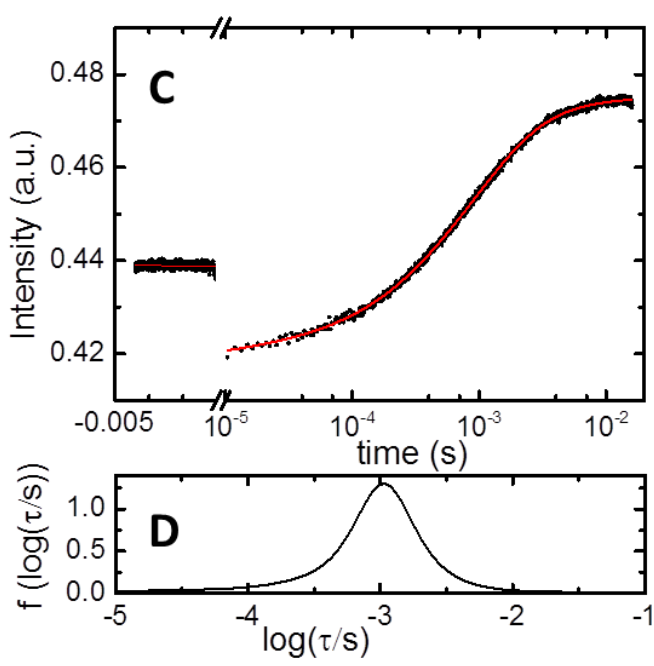

Fig.3. 19. Kinetic traces of H'- IHF complex with $200 \mathrm{mM}$ salt condition and at two different temperatures are shown 

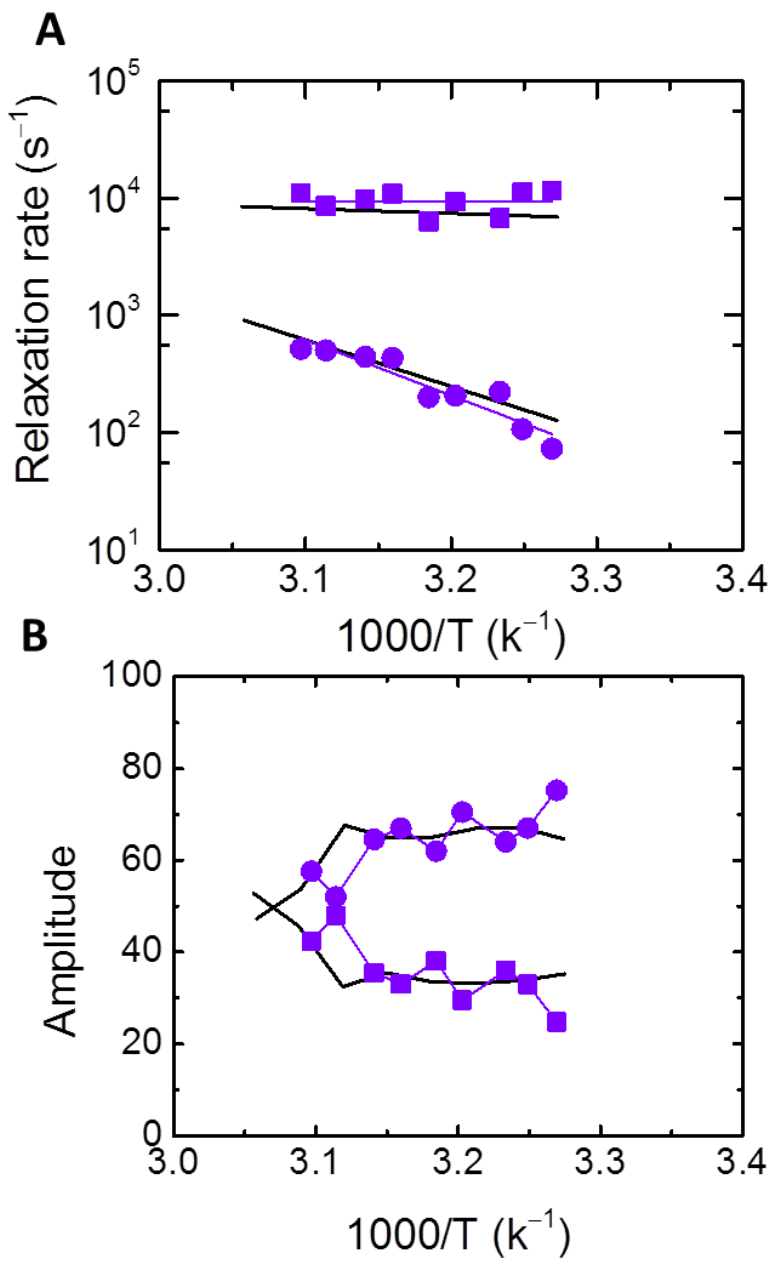

Fig.3. 20. The rates and amplitudes of $H^{\prime}-\mathrm{IHF}$ complex at a salt concentration of $50 \mathrm{mM}$. (A) The relaxation rates $k_{\text {fast }}$ (purple square) and $k_{\text {slow }}$ (purple circle) and (B) the corresponding amplitudes are plotted versus inverse temperature. The purple lines in panel (A) are Arrhenius fits to the relaxation rates and the black line is the Arrhenius fit to the H'-IHF complex at the salt condition $100 \mathrm{mM}$.

\subsubsection{Protein mutations distal to the kink sites affect affinity and bending rate of slow}

\section{phase}

We next examined the effect of IHF mutants, designed to perturb the complex, on the DNA bending rates. Three IHF mutants were studied, in which positively charged residues (Lys or Arg) at increasing distances from the kink sites were replaced with neutral residues. In $\alpha \mathrm{K} 5 \mathrm{~A}$, a positively charged lysine $(\mathrm{K})$ residue at position 5 of the $\alpha$-chain of the IHF heterodimer was substituted for a neutral alanine (A) residue; in $\beta \mathrm{K} 84 \mathrm{~A}$, a similar substitution was made at position 84 of the $\beta$-chain; in $\alpha \mathrm{R} 21 \mathrm{C}$, a positively charged arginine (R) residue at position 
21 of the $\alpha$-chain is replaced by a cysteine (C) residue (Fig. 3.21). For the complexes with these three mutants, $\alpha \mathrm{K} 5 \mathrm{~A}-\mathrm{H}^{\prime}, \beta \mathrm{K} 84 \mathrm{~A}-\mathrm{H}^{\prime}$, and $\alpha \mathrm{R} 21 \mathrm{C}-\mathrm{H}^{\prime}$, the dissociation constants $\mathrm{K}_{\mathrm{d}}$ were determined to be $35 \pm 30 \mathrm{nM}, 16 \pm 3 \mathrm{nM}$, and $10 \pm 2 \mathrm{pM}$, respectively, in comparison with $27 \pm 2 \mathrm{pM}$ for the wt IHF- $\mathrm{H}^{\prime}$ complex (Table III). Thus, two of the mutants ( $\alpha \mathrm{K} 5 \mathrm{~A}$ and $\beta \mathrm{K} 84 \mathrm{~A}$ ) exhibited significant loss of stabilizing interactions with bound $\mathrm{H}^{\prime}$ substrate, while no loss of stability was detected for the $\alpha \mathrm{R} 21 \mathrm{C}-\mathrm{H}^{\prime}$ complex.

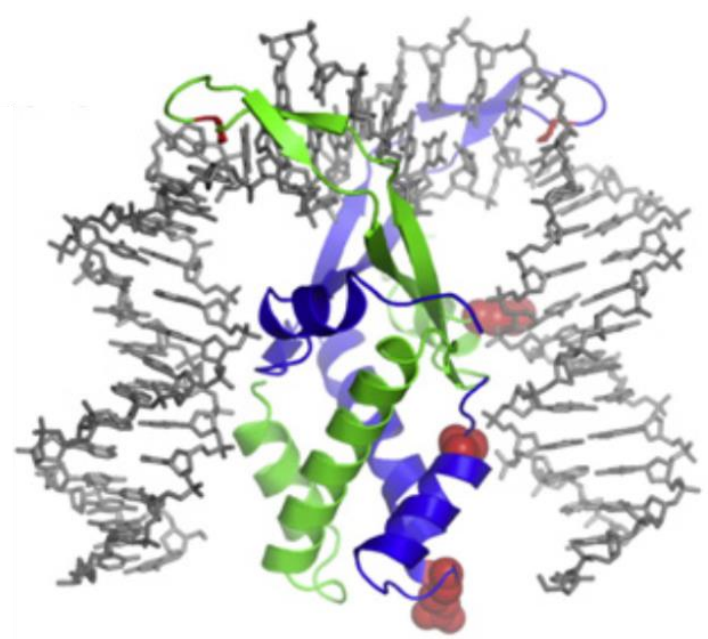

Fig.3. 21. The IHF- $\mathrm{H}^{\prime}$ cocrystal structure (PDB code: $1 \mathrm{IHF}$ ) is shown with the locations of three positively charged residues, shown in red, that were substituted for a neutral residue: (top) $\beta \mathrm{K} 84 \mathrm{~A}$, (middle) $\alpha \mathrm{K} 5 \mathrm{~A}$, and (bottom) $\alpha \mathrm{R} 21 \mathrm{C}$ (figure reproduced with permission from [24]).

Equilibrium FRET efficiency values were determined as a function of temperature for $\alpha \mathrm{K} 5 \mathrm{~A}-\mathrm{H}^{\prime}$ and $\beta \mathrm{K} 84 \mathrm{~A}-\mathrm{H}^{\prime}$ complexes at two different IHF-DNA concentrations of $1 \mu \mathrm{M}: 1$ $\mu \mathrm{M}$ and $20 \mu \mathrm{M}: 20 \mu \mathrm{M}$. For both complexes, the decrease in FRET efficiency with increasing temperature was independent of concentration, indicating that this decrease was primarily from unbending of DNA within the complex and not from any significant bimolecular disruption (Fig. 3.22). For $\alpha \mathrm{K} 5 \mathrm{~A}-\mathrm{H}^{\prime}$, the FRET value at $20{ }^{\circ} \mathrm{C}$ is $0.25 \pm 0.04$, in comparison with $0.55 \pm 0.05$ for the wild-type wild type(wt) $\mathrm{IHF}-\mathrm{H}^{\prime}$ complex, indicating that a mutation at this site has a significant effect on the ability of the protein to keep the DNA in a fully bent conformation. For $\beta \mathrm{K} 84 \mathrm{~A}-\mathrm{H}^{\prime}$, the FRET value at $20{ }^{\circ} \mathrm{C}$ is $0.45 \pm 0.05$. The FRET value of $\alpha \mathrm{R} 21 \mathrm{C}-\mathrm{H}^{\prime}$ complex is similar to that of the wt IHF-H' complex (data not shown). 


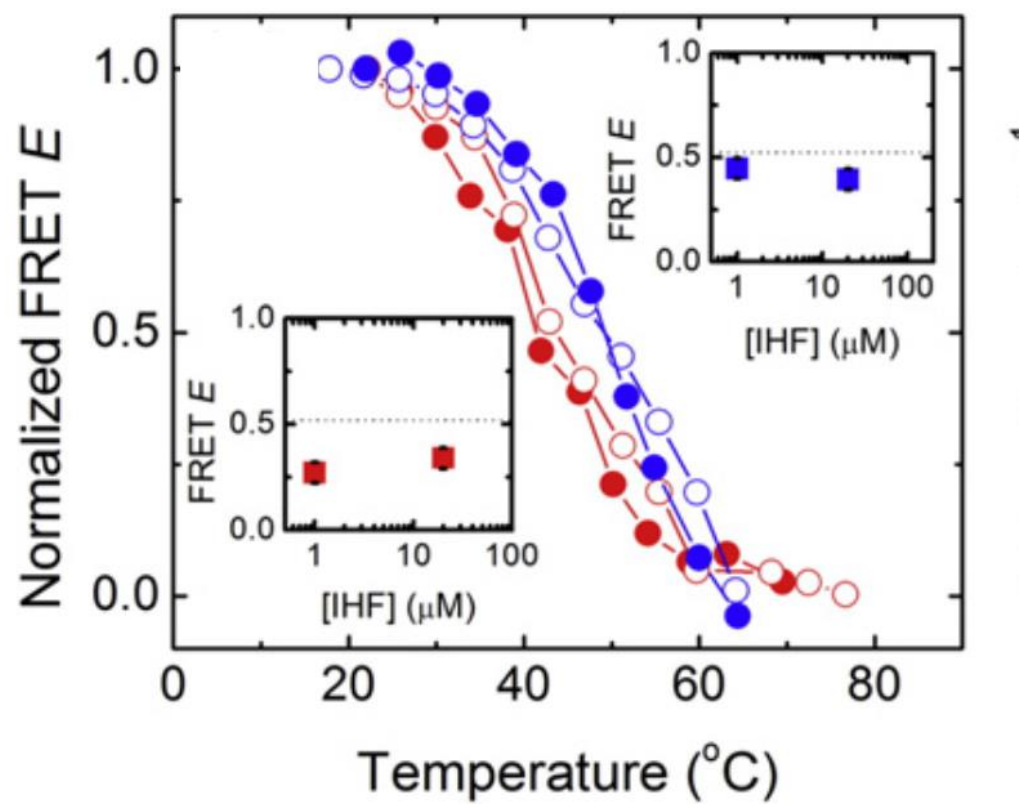

Fig.3. 22. Equilibrium measurements on mutant $\mathrm{IHF}-\mathrm{H}^{\prime}$. FRET efficiency versus temperature for the $\alpha \mathrm{K} 5 \mathrm{~A}-\mathrm{H}^{\prime}$ complex $(\bullet, \mathrm{o})$ and the $\beta \mathrm{K} 84 \mathrm{~A}-\mathrm{H}^{\prime}$ complex $(\bullet, \mathrm{o})$, obtained at two different proteinDNA concentrations: $20 \mu \mathrm{M}: 20 \mu \mathrm{M}$ (open circles) and $1 \mu \mathrm{M}: 1 \mu \mathrm{M}$ (filled circles). The data are normalized to match at the lowest temperature. The insets show the actual FRET values for each sample at $20^{\circ} \mathrm{C}$; the broken line represents the average FRET value for the IHF-H' complex (figure reproduced with permission from [24]).

T-jump measurements on $\alpha \mathrm{K} 5 \mathrm{~A}-\mathrm{H}^{\prime}$ and $\beta \mathrm{K} 84 \mathrm{~A}-\mathrm{H}^{\prime}$ also yielded biphasic kinetics (Fig. 3.23 and 3.25). The fast phase observed for both mutants occurred on time-scales similar to the fast phase of the wt IHF-H' complex, over the entire temperature range of the measurements $\left(33-55{ }^{\circ} \mathrm{C}\right)$. The slow phase on the other hand, while exhibiting similar rates as the wt at the lower temperatures near $\sim 30^{\circ} \mathrm{C}$, was nearly 10 -fold slower at the highest temperature of $\sim 50^{\circ} \mathrm{C}$. The activation energy of the slow phase for both these mutant complexes was found to be $8.0 \pm 2.1$ and $7.6 \pm 0.9 \mathrm{kcal} / \mathrm{mol}$, respectively, significantly smaller than the $19.4 \pm 1.6 \mathrm{kcal} / \mathrm{mol}$ activation energy of slow step in the wt IHF-H'. Interestingly, for both mutants, the relative amplitudes of the fast phase exceed that of the slow phase at high temperatures. These results are consistent with scheme 1 of Fig. 3.13 and indicate that, as the temperature is raised, a larger fraction of the bound mutant proteins is in the nonspecific binding modes. In the case of $\alpha \mathrm{R} 21 \mathrm{C}-\mathrm{H}^{\prime}$ both the relaxation rates and their relative amplitudes remain the same as those of wt IHF-H'(Fig. 3.26). This result is not surprising 
since the mutation site in this protein variant is at the edge of the DNA wrapping surface and hence neither the thermodynamics nor the kinetics are affected by the loss of the positive charge at the location of this mutation. 

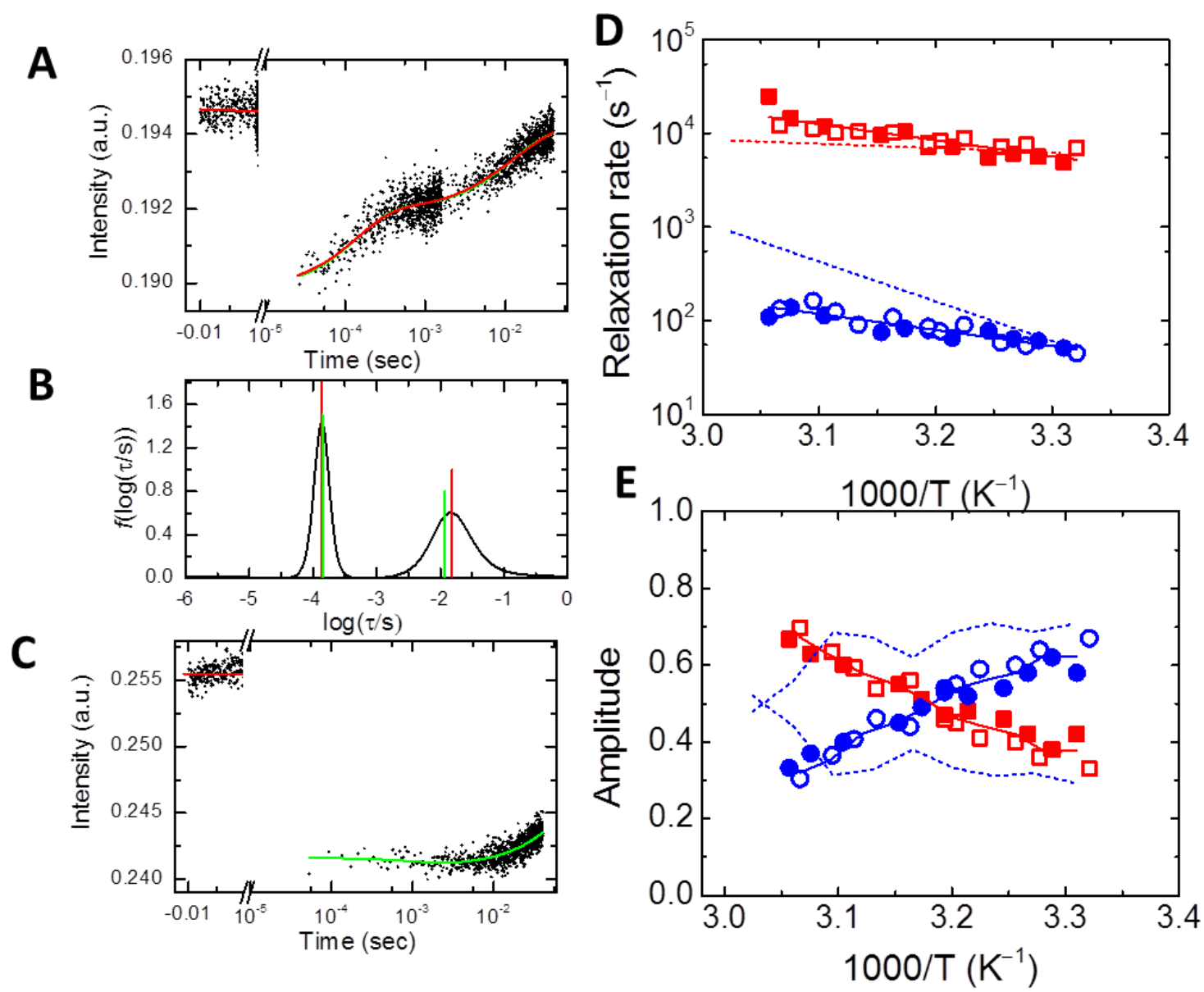

Fig.3. 23. T-jump kinetics measurements and relaxation rates on $H^{\prime}$ - mutant IHF ( $\alpha$ K5A) complexes. (A) Donor fluorescence emission intensities of double-labeled H' samples in the presence of $\alpha \kappa 5 \mathrm{~A}$ measured in response to a T-jump perturbation are plotted as a function of time. Relaxation traces show two phases for a T-jump perturbation from $33.8^{\circ} \mathrm{C}$ to $37.6^{\circ} \mathrm{C}$. Red continuous lines are mem fits to the relaxation traces, with the corresponding distributions of relaxation times shown in panels (B). The green continuous line is from double-exponential relaxation convoluted with T-jump recovery. (B )The average time constants describing the best fit parameters from the two analyses are shown as vertical lines, red (mem) and green (two exponential + recovery function), $\tau_{\text {fast }}=137 \mu \mathrm{s}$ (red), $\tau_{\text {slow }}=15.4 \mathrm{~ms}$ (red) and $\tau_{\text {fast }}=140 \mu \mathrm{s}$ (green), $\tau_{\text {slow }}=$ $11.8 \mathrm{~ms}$ (green). (C) Control T-jump measurements on free fluorescein sample are shown. T-jump measurements on these samples do not exhibit any relaxation kinetics, instead they show only the much slower T-jump recovery kinetics. The rates and amplitudes of the two relaxation phases in the H'-IHF complex obtained from the mem analysis are plotted in (D, E) for two different data set (open and closed symbol) as a function of inverse temperature. (D) The relaxation rates $k_{\text {fast }}$ (red square) and $k_{\text {slow }}$ (blue circle) and (E) the corresponding amplitudes. The red and blue continuous lines in (D) are Arrhenius fits to the fast and slow relaxation rates and the red and blue dashed lines are corresponding Arrhenius fits to the H'-IHF complex. The Red and blue dashed lines in (E) are the fast and slow phase amplitudes of H'-IHF complex. 
A

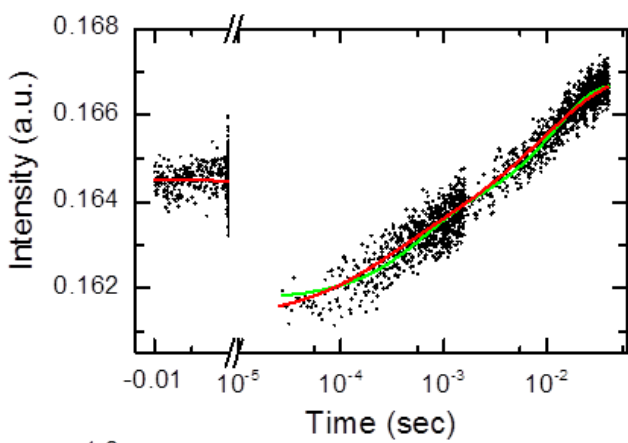

B

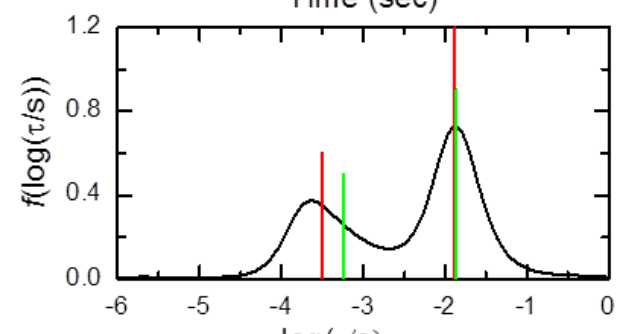

C

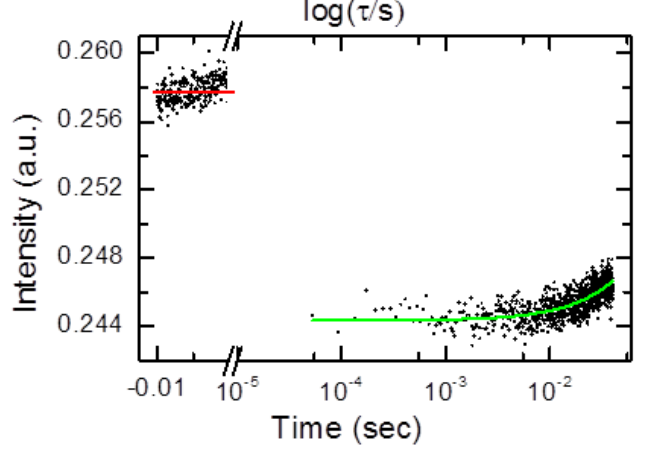

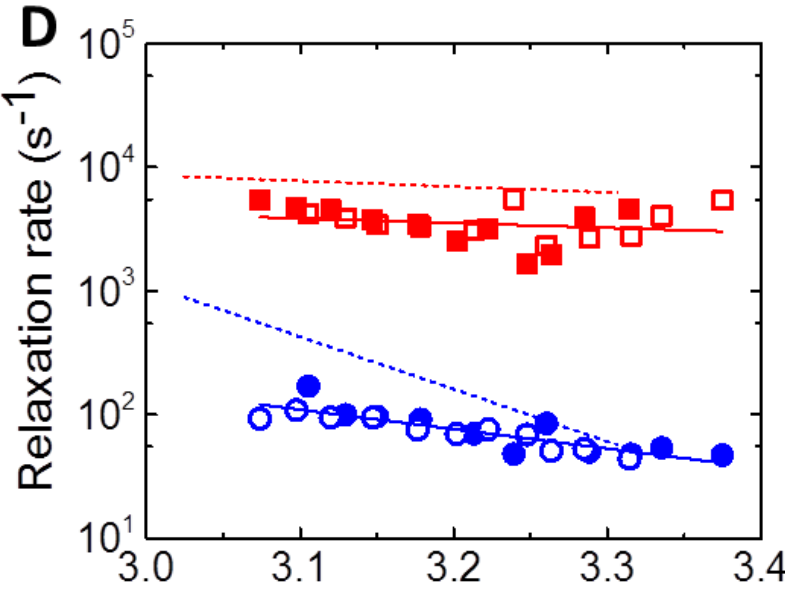

E

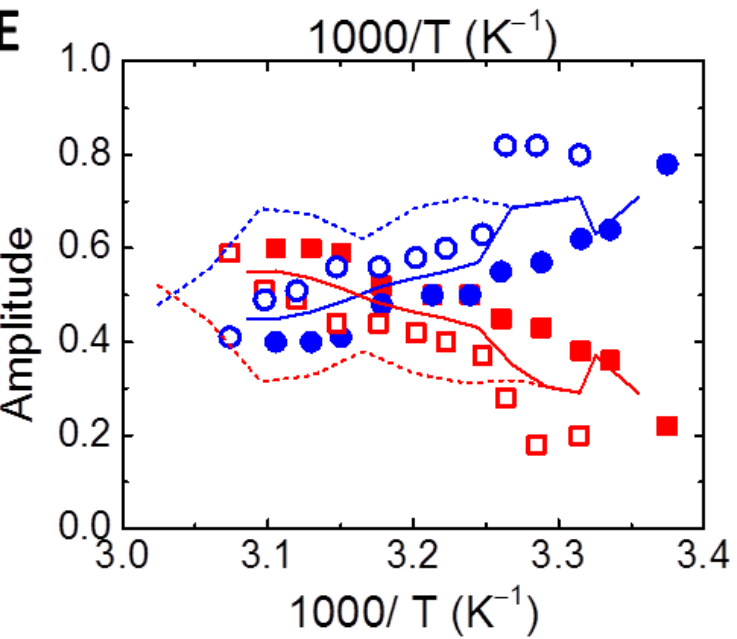

Fig.3.24. T-jump kinetics measurements and relaxation rates on H'-mutant IHF ( $\beta$ K84A) complexes. (A) Donor fluorescence emission intensities of double-labeled H' samples in the presence of $\beta \kappa 84 \mathrm{~A}$ measured in response to a T-jump perturbation are plotted as a function of time. Relaxation traces show two phases for a T-jump perturbation from $34.6^{\circ} \mathrm{C}$ to $37.2^{\circ} \mathrm{C}$. Red continuous lines are mem fits to the relaxation traces, with the corresponding distributions of relaxation times shown in panels (B). The green continuous line is from double-exponential relaxation convoluted with T-jump recovery. (B) The average time constants describing the best fit parameters from the two analyses are shown as vertical lines, red (mem) and green (two exponential + recovery function), $\tau_{\text {fast }}=315.4 \mu \mathrm{s}$ (red), $\tau_{\text {slow }}=13.2 \mathrm{~ms}$ (red) and $\tau_{\text {fast }}=578.1 \mu \mathrm{s}$ (green), $\tau_{\text {slow }}=13.6 \mathrm{~ms}$ (green). (C) Control T-jump measurements on free fluorescein sample are shown. Tjump measurements on these samples do not exhibit any relaxation kinetics, instead they show only the much slower T-jump recovery kinetics. The rates and amplitudes of the two relaxation phases in the H'-IHF complex obtained from the mem analysis are plotted in (D, E) for two different data set (open and closed symbol) as a function of inverse temperature. (D) The relaxation rates $k_{\text {fast }}$ (red square) and $k_{\text {slow }}$ (blue circle) and (E) the corresponding amplitudes. The red and blue continuous line in (D) are Arrhenius fits to the fast and slow relaxation rates and the red and blue dashed lines are corresponding Arrhenius fits to the H'-IHF complex. The red and blue dashed lines in (E) are the average fast and slow phase amplitudes of H'-IHF complex. 
A

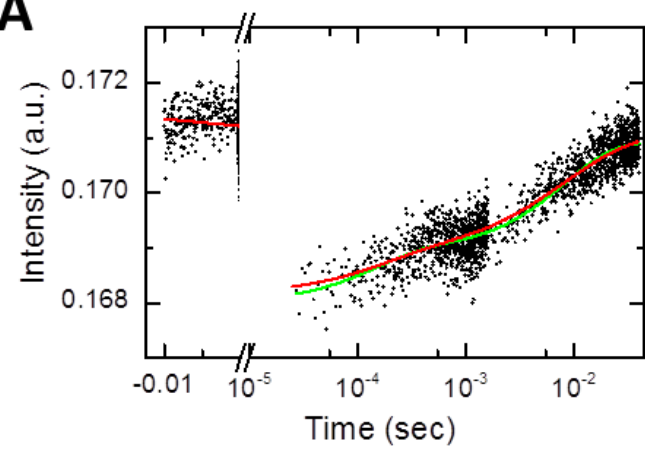

B
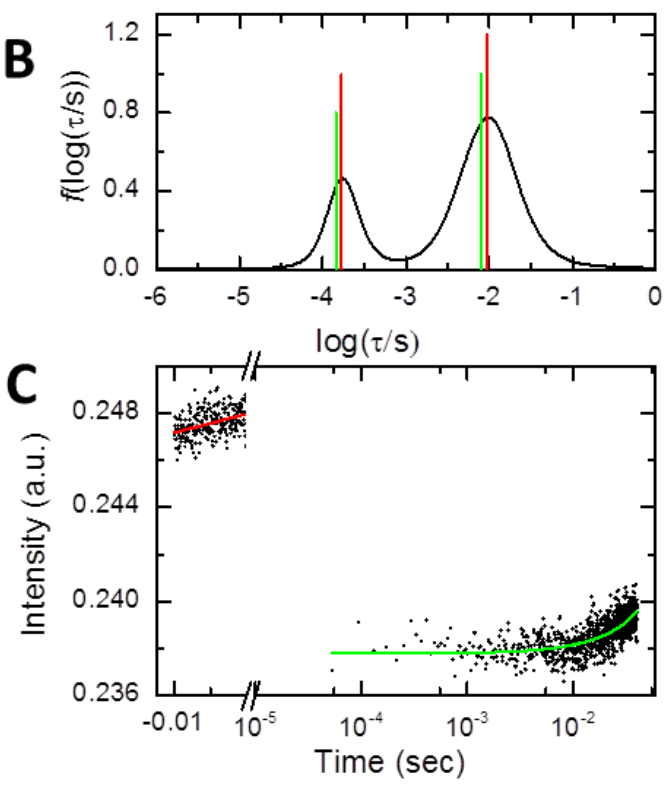
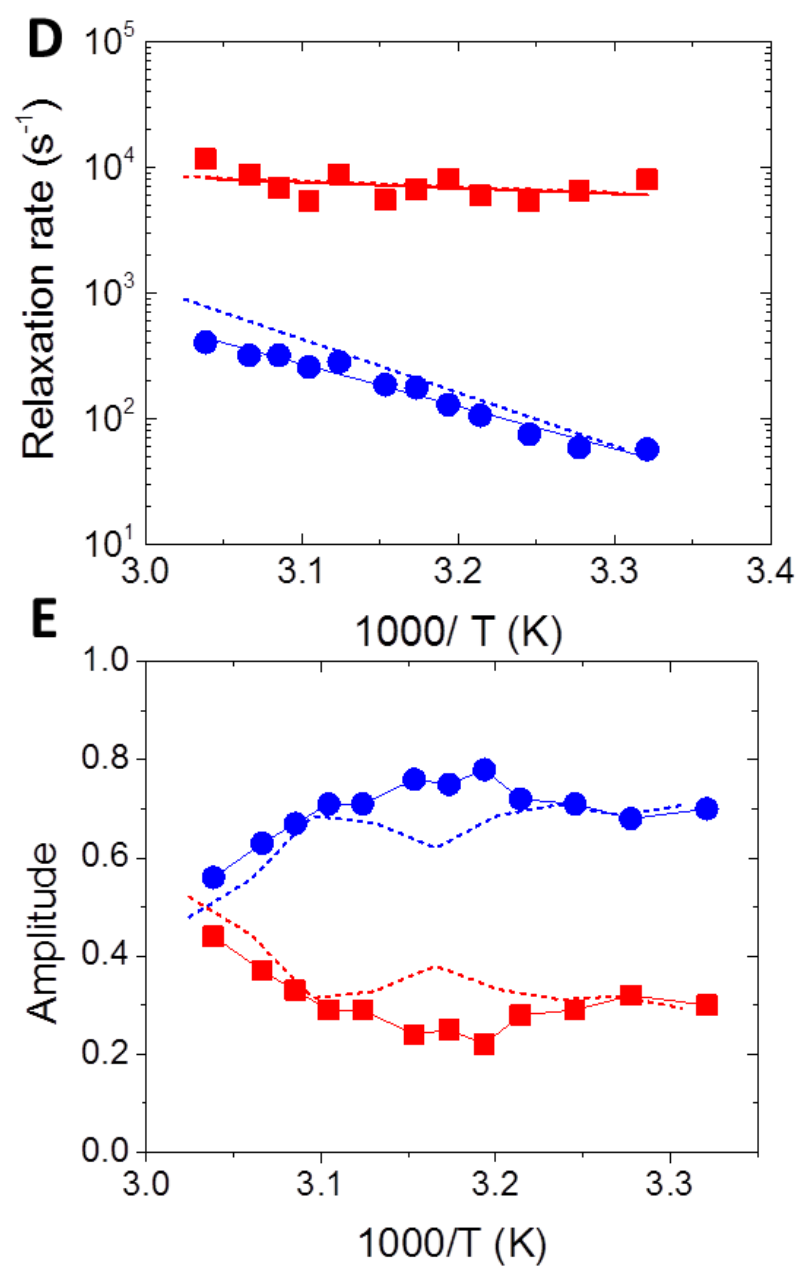

Fig.3. 25. T-jump kinetics measurements and relaxation rates on H'- mutant IHF $(\alpha \mathrm{R} 21 \mathrm{C})$ complexes. (A) Donor fluorescence emission intensities of double-labeled H' samples in the presence of $\boldsymbol{\alpha} \mathbf{R} 21 \mathrm{C}$ measured in response to a T-jump perturbation are plotted as a function of time. Relaxation traces show two phases for a T-jump perturbation from $34.7{ }^{\circ} \mathrm{C}$ to $38{ }^{\circ} \mathrm{C}$. Red continuous lines are mem fits to the relaxation traces, with the corresponding distributions of relaxation times shown in panels (B). The green continuous line is from double-exponential relaxation convoluted with T-jump recovery. (B )The average time constants describing the best fit parameters from the two analyses are shown as vertical lines, red (mem) and green (two exponential + recovery function), $\tau_{\text {fast }}=169.4 \mu \mathrm{s}$ (red), $\tau_{\text {slow }}=9.4 \mathrm{~ms}$ (red) and $\tau_{\text {fast }}=145.8 \mu \mathrm{s}$ (green), $\tau_{\text {slow }}=8.0 \mathrm{~ms}$ (green). $(\mathbf{C})$ Control T-jump measurements on free fluorescein sample are shown. T-jump measurements on these samples do not exhibit any relaxation kinetics, instead they show only the much slower T-jump recovery kinetics. The rates and amplitudes of the two relaxation phases in the H'-IHF complex obtained from the mem analysis are plotted in $(\mathbf{D}, \mathbf{E})$ as a function of inverse temperature. (D) The relaxation rates $k_{\text {fast }}$ (red square) and $k_{\text {slow }}$ (blue circle) and (E) the corresponding amplitudes. The red and blue continuous line in (D) are Arrhenius fits to the fast and slow relaxation rates and the red and blue dashed lines are corresponding Arrhenius fits to the H'-IHF complex. The Red and blue dashed lines in (E) are the fast and slow phase amplitudes of H'-IHF complex. 


\subsubsection{Control experiments to rule out contributions to the relaxation kinetics from dye dynamics or dye interactions with protein or DNA.}

A series of control experiments were carried out to rule out any contributions from dye interactions and/or dye dynamics to the observed relaxation kinetics. First, for any FRETlabeled sample, corresponding measurements were done on donor-only labeled samples to ensure that the observed kinetics were from FRET changes and not from interactions of the donor (fluorescein) dye with either the DNA or the protein. No kinetics were observed on any of the donor-only samples other than the slow T-jump recovery kinetics (Fig. 3.2A), similar to those observed on control measurements done using either a fluorescein-labeled single strand of the H' DNA or with free fluorescein dye (see control experiments shown in all Tjump kinetics figures). These measurements also confirmed the absence of any photo bleaching of the fluorescein dye in the window of the T-jump measurements.

Second, to rule out any contribution to the observed kinetics from interactions of the acceptor (TAMRA) dye, T-jump experiments were carried out on FRET-labelled IHF-H' complex by directly exciting TAMRA (at $532 \mathrm{~nm}$, where fluorescein does not absorb) and measuring the temporal response of the fluorescence emission of TAMRA. Again, no relaxation kinetics were observed (Fig. 3.26B), thus confirming that interactions of the acceptor dye with the DNA or the protein do not interfere with the observed relaxation kinetics.

Third, to examine whether there were any T-jump induced changes in the relative orientation of the dyes attached to the DNA that were not related to an overall change in the DNA conformation, we carried out T-jump measurements on FRET-labeled DNA only samples. For these measurements we designed a shorter 14 bp DNA oligomer labelled with fluorescein and TAMRA, with the sequence context of each of the dyes identical to that in the longer H' sequence (see Table II note that in $14 \mathrm{bp}$ sequence (14bp) the four nucleotides 
at the ends, next to the dyes, are the same as in the original H' sequence). The reason for this shorter sequence is that the end-to-end distance in the 35-bp H' sequence is too long for there to be any FRET between the dyes without the bound protein. By design, the FRET value in the 14-bp DNA is 0.5, close to the FRET of $\mathrm{H}^{\prime}$ in the presence of IHF, and in a region where small changes in the distance or relative orientation of the dyes would result in a detectable FRET change. Once again, T-jump measurements on this DNA sample did not result in kinetics other than the T-jump recovery kinetics (Fig. 3.2C). Thus, we see no evidence of dye reorientational dynamics in DNA only samples contributing to the observed relaxation kinetics.

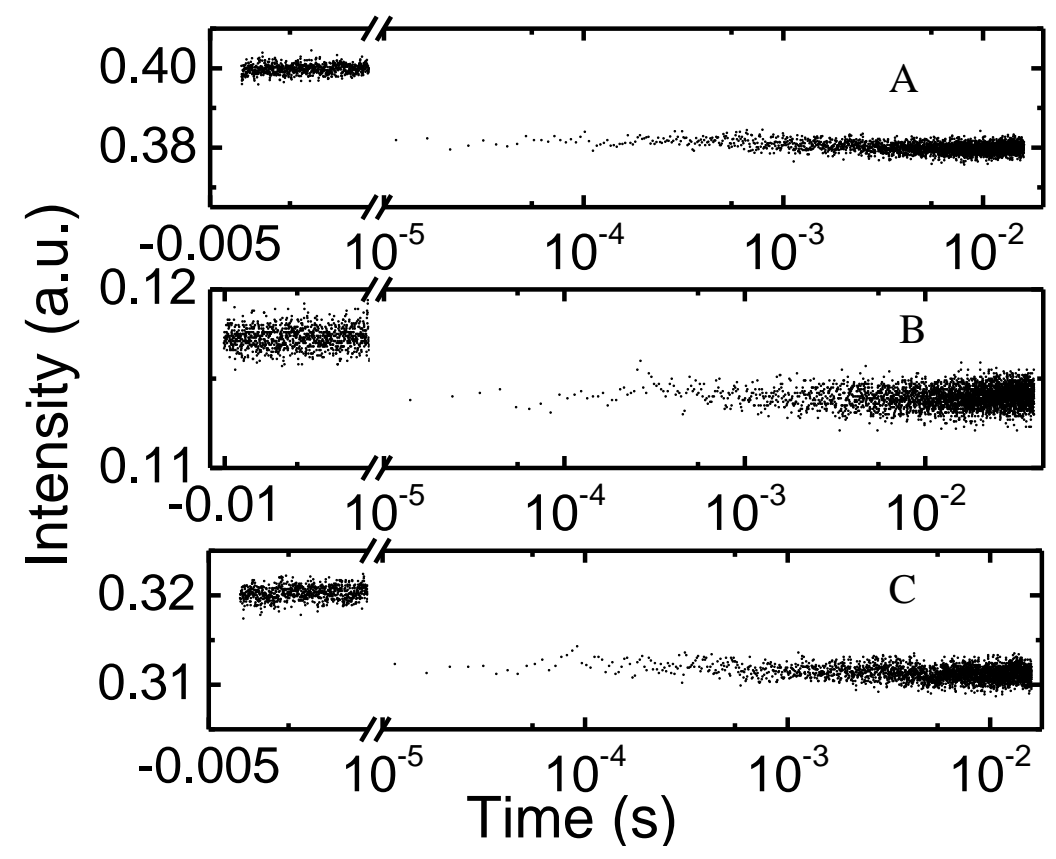

Fig.3. 26. Control experiments to verify the fast phase is not coming from artifact. (A) Donor only H'-IHF complex was excited at donor wavelength and monitored the donor (B) H'-IHF complex was excited at acceptor wavelength and monitored the acceptor. (C) Short 14 bp DNA with fluorescein and TAMRA (the four nucleotides next to the dyes are similar to that of H' sequence (marked in green, in table II)) was excited at donor wavelength and monitored the donor.

Finally, to examine whether the relaxation rates and the relative amplitudes in the fast and slow phases of the IHF-H' kinetics were affected by the location of the dyes or how they were attached to the DNA sequence or even by the length of the DNA sequence, we conducted control experiments with a few different DNA lengths and with dyes attached at 
different positions along the DNA sequence: (1) dyes were attached directly to the sugar phosphate backbone via a $\mathrm{C}_{6}$ linker at the end of the $35 \mathrm{bp} \mathrm{H}$ ' duplex as opposed to the dyes attached to the thymine bases at the ends, as in the original design of the FRET-labeled H' sequence; (2) dyes were attached to the thymine bases at the end of a longer (55 bp) DNA sequence that contained the original $\mathrm{H}^{\prime}$ sequence in the middle. This sequence was designed to test whether close proximity of the dyes to the protein as in the 35-bp H' DNA wrapped around IHF, and their subsequent perturbation, could be contributing in part to the fast kinetics; the 55-bp long sequence construct resulted in the dyes being moved further away from the protein; (3) dyes were attached internally (not at the ends) but still 35 bp apart on thymine bases within a much longer (130 bp) DNA sequence. In all these complexes, the Tjump experiments yielded very similar relaxation rates for the fast and slow components, respectively, as in the original H'-IHF complex (Fig. 3.28, 3.29, 3.30). The relative amplitudes in the two phases were, however, affected by the dye attachment position or the DNA length. For the 35-bp H' sequence, altering the dye-attachment method from the thymine bases to the sugar-phosphate backbone yielded very similar trends in the temperature dependence of the fast and slow amplitudes, but with slightly larger amplitudes seen in the slow phase. This is not surprising, because changing the chemistry of attachment can potentially alter the fluorescence levels in each of the kinetic species and hence affect the relative amplitudes in each phase. For the longer (55- and 130-bp) sequences, the relative amplitudes measured at low temperatures matched those measured for the 35-bp sequence but showed significant deviations at the higher temperatures, with larger amplitude in the slow phase for the longer sequences. The reason for this deviation is not immediately apparent, but may have some contributions from a multiplicity of nonspecific binding modes accessible to the protein in the longer sequences with unpredictable effect on the FRET levels for these altered binding modes. 

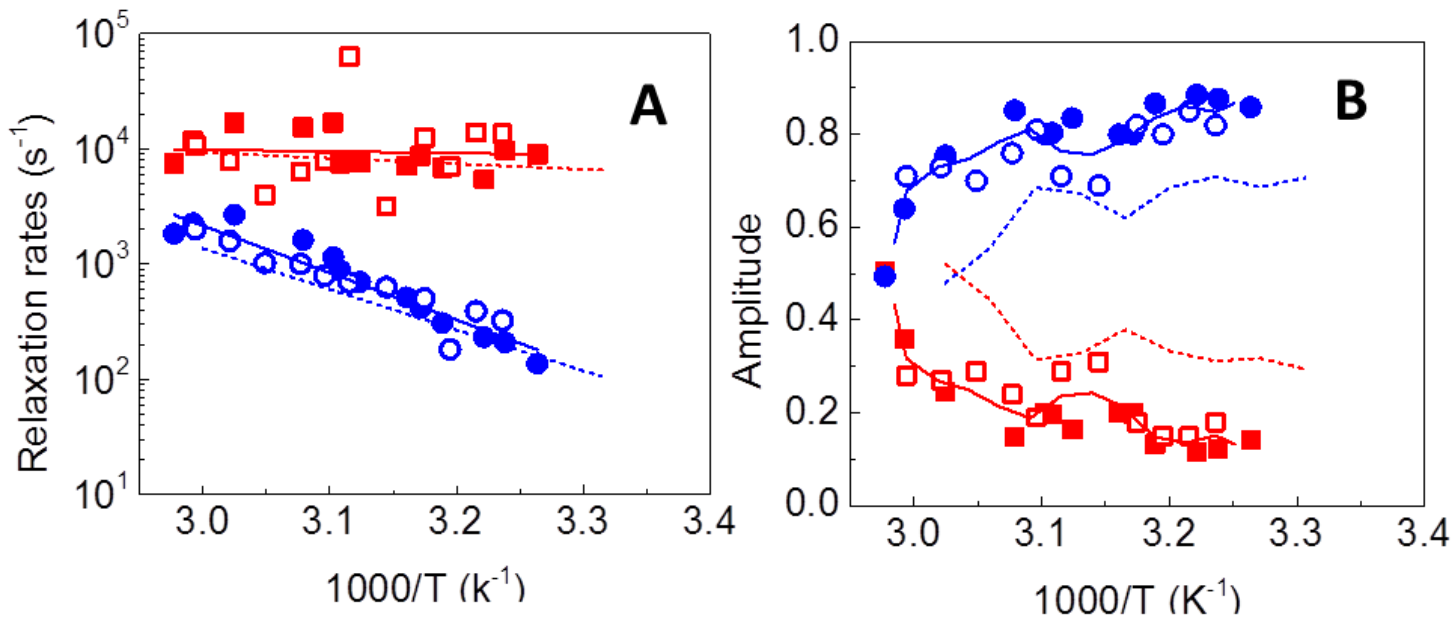

Fig.3. 27. The rates and amplitudes of the two relaxation phases in the H'_Spb (dyes are attached on the sugar phosphate backbone via a $\mathrm{C}_{6}$ linker) - IHF complex obtained from the mem analysis are plotted in (A, B) for two different data set (open and closed symbol) as a function of inverse temperature. (A) The relaxation rates $\mathrm{k}_{\text {fast }}$ (red square) and $\mathrm{k}_{\text {slow }}$ (blue circle) and (B) the corresponding amplitudes. The red and blue continuous line in (A) are Arrhenius fits to the fast and slow relaxation rates and the red and blue dashed lines are corresponding Arrhenius fits to the H'IHF complex.
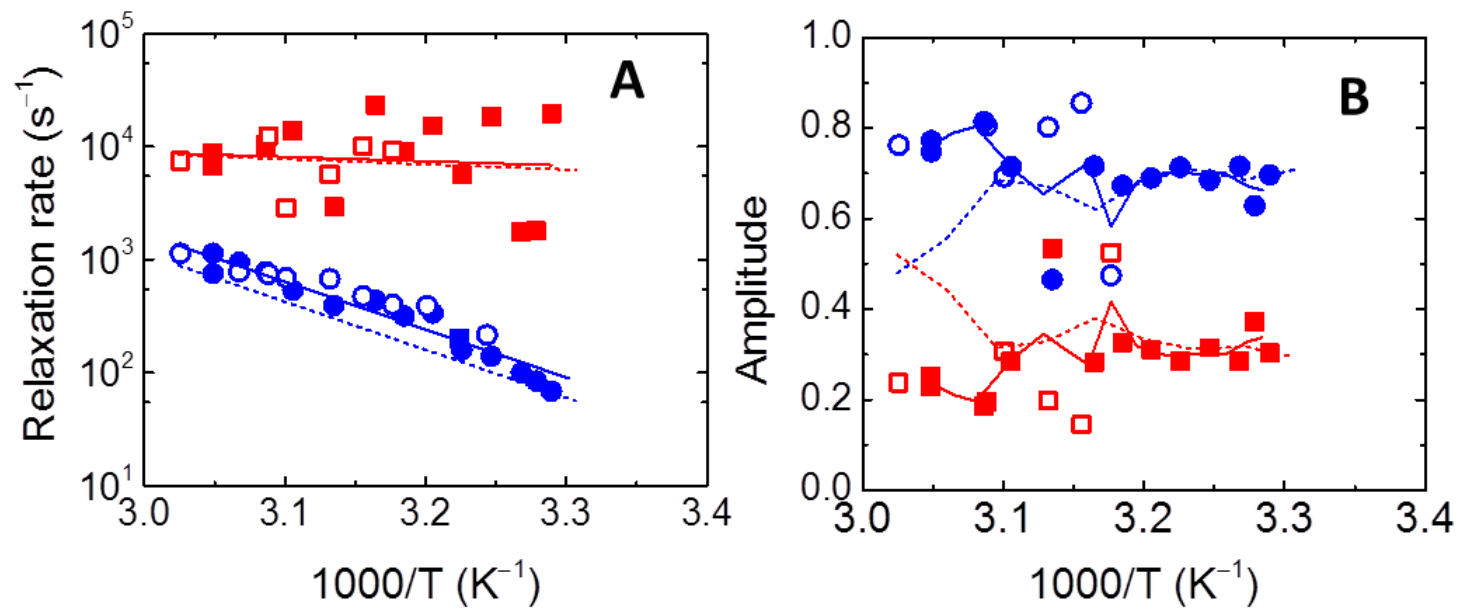

Fig.3. 28. The rates and amplitudes of the two relaxation phases in the $\mathrm{H}_{-}$55bp (dyes are attached at the end of a 55 bp DNA on T-nucleotide via $\mathrm{C}_{6}$ linker as in the original H') -IHF complex obtained from the mem analysis are plotted in (A, B) for two different data set (open and closed symbol) as a function of inverse temperature. (A) The relaxation rates $\mathrm{k}_{\text {fast }}$ (red square) and $\mathrm{k}_{\text {slow }}$ (blue circle) and (B) the corresponding amplitudes. The blue and red continuous line in (A) are Arrhenius fits to the fast and slow relaxation rates and the red and blue dashed lines are corresponding Arrhenius fits to the H'-IHF complex. 

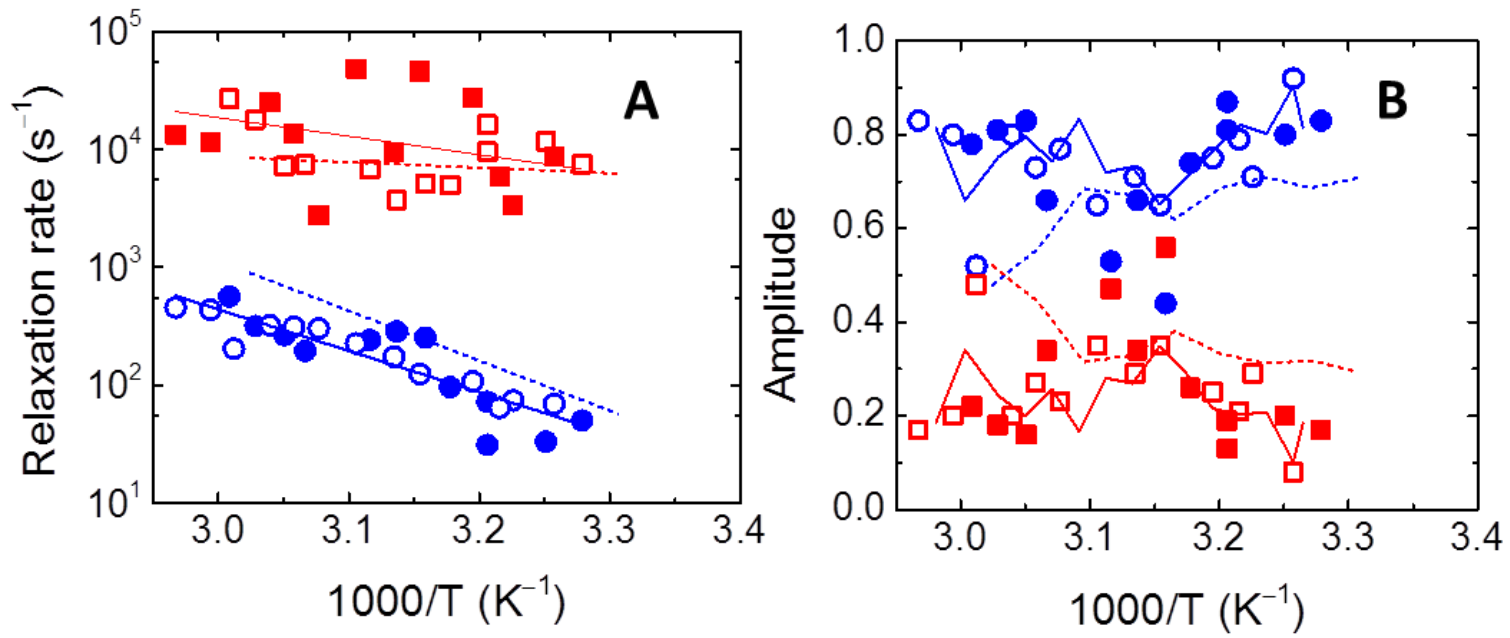

Fig.3. 29. The rates and amplitudes of the two relaxation phases in the $\mathrm{H}^{\prime}{ }_{-} 130 \mathrm{bp}$ (dyes are attached internally at $35 \mathrm{bp}$ apart on a $130 \mathrm{bp}$ sequence via $\mathrm{C}_{6}$ linker) - IHF complex obtained from the mem analysis are plotted in (A, B) for two different data set (open and closed symbol) as a function of inverse temperature. (A) The relaxation rates $\mathrm{k}_{\text {fast }}$ (red square) and $\mathrm{k}_{\text {slow }}$ (blue circle) and (B) the corresponding amplitudes. The red and blue continuous lines in (A) are Arrhenius fits to the fast and slow relaxation rates and red and blue dashed lines are corresponding Arrhenius fits to the H'-IHF complex.

\subsection{Discussion}

\subsubsection{Nonspecific search and specific recognition by IHF}

The microsecond time-resolution and enhanced sensitivity of our T-jump apparatus has enabled us to reveal two distinct phases in the kinetics of binding-site recognition in the IHFH' system, with a previously undetected fast phase appearing at $\sim 100 \mu \mathrm{s}$, and a slower phase between $\sim 1-10 \mathrm{~ms}$ (Fig. 3.5). These biphasic kinetics are observed under conditions where Tjump perturbation does not dissociate the complex and therefore reflect unimolecular conformational changes in the IHF-H' complex. Thus, the mechanism by which IHF recognizes and binds tightly to its cognate site appears to involve at least two steps (Fig. 3.13). Kinetics measurements on the TT8AT substrate (Fig. 3.11), together with two additional substrates, the TT-loop (with the 4-nt loop located on only one kink site), and the AT-loop (with the 4-nt loop located on the other kink site), provide a clue. All three variants of the H' substrate showed two phases. However, in each case, only the slower phase was 
accelerated by the insertion of the 4-nt loop, whether on one kink site or the other or both, while the fast phase remained unchanged. These results suggest that the two steps do not reflect sequential bending of the two flanking arms of DNA but, instead, suggest a rapid partial bending followed by a slow recognition step in which the DNA is fully bent. The relaxation rates measured for the fast step appear to be independent of DNA flexibility at the kink sites. In H'44A, where the modification in the TTR consensus site of the H' sequence is removed from the site of the kinks, neither of the two components in the bending kinetics are significantly affected (Fig.3.14).

One plausible explanation for the biphasic kinetics is illustrated in scheme 1 of Fig. 3.13 , in which the rapid phase corresponds to sequence-nonspecific bending of DNA, due to asymmetric neutralization of the phosphate charges on one face of the DNA [65-67], prior to the sequence-specific kinking that leads to recognition. Maher and co-workers demonstrated spontaneous bending of DNA when the charge on one helical face was modified, either by incorporation of neutral phosphate analogs [68] or by tethering cations on bases to form ions pairs with phosphates [69], resulting in a DNA bend in the direction toward the reduced charge. Nonspecific bending of DNA has also been observed in protein-DNA complexes, in which the charges on the protein binding surface are modified by substitution of uncharged residues by charged residues, with the DNA bending toward the protein if the excess charges are positive, and away from the protein if the excess charges are negative [65, 70-72]. In the case of IHF, both the $\beta$-arms of the protein as well as the protein surface between the arms are lined with positive charges, and it is likely that the protein partially bends DNA even in its nonspecific binding mode, with perhaps the $\beta$-arms of IHF weakly wrapped around DNA. An alternative scenario is the one illustrated in scheme 2 of Fig. 3.13, with the slow sequence-dependent kinking step occurring first followed by rapid reorganization of the protein and other parts of the DNA to form the stable complex. Our T-jump studies, together 
with evidence from prior studies of nonspecific DNA bending by IHF, support scheme 1 as the more likely explanation for the observed biphasic kinetics.

\subsubsection{Nonspecific binding of DNA by IHF and its structurally homologous cousin HU}

Previous in vitro studies suggest that IHF interacts with DNA both specifically and non-specifically and that even in the nonspecific binding mode IHF bends the DNA to some extent. There is ample experimental evidence that nonspecific binding of IHF and other histone like proteins serve to compact nucleoid structure by a series of partial bends $[62,73]$. The first evidence for nonspecific DNA bending by IHF came from mechanical single $\lambda$ DNA stretching experiment in the presence of IHF [10]. These studies demonstrated that addition of IHF shortened the contour length of $\lambda$-DNA, and showed that the effect of IHF binding on the force response of $\lambda$-DNA was similar to that predicted for DNA bending proteins $[74,75]$. Thus, this study provided evidence that nonspecific IHF binding altered DNA structure, although to a smaller extent than that expected from specific IHF binding.

Saecker, Record and co-workers carried out extensive ITC and equilibrium FRET measurements on IHF and HU, which closely resembles the structure of IHF. It is interesting to note here that during the cell transition from exponential growth to stationary phase E.coli replaces large amount of HU with its structural homolog IHF. This exchange appears to conserve the total amount of these homologs in the cell semi-quantitatively [73]. Unlike IHF, HU exhibits no sequence-specificity but binds specifically to DNA with inserted distortions such as mismatched base pairs and extra helical bases $[25,73]$, and severely bends the DNA at the site of these distortions. In these highly-bent HU-DNA structures, as in the IHF-H' complex, two invariant proline residues on the $\beta$ arm of $\mathrm{HU}$ insert between base pairs, stabilizing the sharply bent DNA $[1,25]$. In ITC studies carried out on E.coli $\mathrm{HU}_{\alpha \beta}$, the protein bound to DNA was found to exhibit at least three different nonspecific binding modes 
with site sizes of $\sim 34 \mathrm{bp}, 10 \mathrm{bp}$ and $6 \mathrm{bp}$. These nonspecific binding modes co-exist on the same DNA molecule and interconversion between the modes occurs as a function of salt concentration, salt type, $\mathrm{pH}$, temperature and binding density ([HU]/DNA) [73]. FRET measurements on these complexes provide evidence that the 34-bp nonspecific binding mode of $\mathrm{HU}$ appear to be highly bent (by $\sim 143^{\circ}$ ), similar to the specific complexes seen with $\mathrm{HU}$ and IHF. The implications from these studies is that similar multiple nonspecific binding modes are also accessible to IHF. In fact, ITC studies that showed that nonspecific IHF binding is favoured at low $\mathrm{KCl}$ concentration and high IHF-DNA stoichiometry also showed that a smaller occluded size of DNA ( 10 bp) was observed in the nonspecific binding mode compared to the specific complex $[62,76]$.

\subsubsection{Nonspecific binding facilitates 1-D diffusion on DNA}

While nonspecific binding of IHF and partial DNA bending is important for DNA compaction in the cells, it also likely plays a critical role in the mechanism by which IHF scans DNA in search of its target site, as suggested by the "facilitated diffusion" models of the search process. The few existing crystal structures of proteins bound nonspecifically to DNA show that the protein-DNA interfaces are less intimately apposed than when they are in the specific complex, due to lack of specific close-fitting interactions with the DNA, leaving room for retention of substantial interfacial water between them. This relatively loose structure allows the protein-DNA to remain mobile and enables rapid scanning of the DNA while bound nonspecifically [55].

The hydration differences between specific and nonspecific complex imply that an increased concentration of cosolute enhances the specificity. In a similar manner, variations in salt concentration affect the specific and nonspecific binding differently. In various protein-DNA systems, there is a marked departure from the proposed linear dependence of 
$\log (\mathrm{ka})$ on $\log [\mathrm{salt}]$, with a change in slope observed at low salt concentrations [55]. This slope change at low salt $(<0.1 \mathrm{M})$ is attributed to the effects of facilitated diffusion ("sliding") of the protein along nonspecific DNA sequence if the DNA segment is sufficiently large [55]. In our T-jump experiments, the amplitude of the fast phase increases when we increase the length of the DNA, and diminishes considerably when the salt concentration is increased above $100 \mathrm{mM}$. These results strongly indicate that the fast phase is from nonspecific binding of IHF that induces a partial bend in the DNA.

Measurements on the IHF mutants also support scheme 1 over scheme 2. In scheme 2, the slow phase is the rate-limiting DNA kinking step followed by rapid reorganization or "induced-fit" dynamics of the IHF-DNA complex to form the stabilizing interactions in the specific complex. In this scenario, making mutations in the protein wrapping surface that stabilize or destabilize the complex are expected to alter the rates for the fast phase. In IHF mutants $\alpha \mathrm{K} 5 \mathrm{~A}$ and $\beta \mathrm{K} 84 \mathrm{~A}$, in which positively charged residues in the DNA-wrapping surface are mutated to neutral residues, these specific interactions are destabilized by $>500$ fold. However, the fast phase relaxation rates remain unchanged while the slow phase relaxation rates decrease by less than 10 -fold, mostly at high temperatures. However, a significant increase in the amplitude of the fast phase is observed with these mutants at higher temperatures. This observation is consistent with scheme 1 and indicates that a reduction of positively charged residues in the DNA wrapping surface of IHF tilts the equilibrium towards the nonspecific binding modes, thus increasing the amplitude of the relaxation phase that is attributed to the interconversion between these nonspecific binding modes. 


\subsection{Concluding Remarks}

Our finding of the fast phase in the IHF-H' complex dynamics is an exciting discovery in the kinetics of target recognition by a DNA-bending protein, and the first observation of the kinetics of nonspecific DNA bending, enabled by the fast time-resolution of laser T-jump technique. These rapid kinetics occurring at $\sim 400 \mu \mathrm{s}$ at $25^{\circ} \mathrm{C}$ bridge the gap between the slow (> ms) target-recognition time-scales and the fast $(<\mathrm{ms})$ time-scales for protein diffusion on DNA, as discussed earlier (see Table III) and shed light on the fundamental question in the field of protein-DNA interactions: how fast must the recognition step be in comparison with the time that a nonspecifically bound protein spends in the vicinity of a potential binding site? Our results indicate that binding-site recognition by IHF is, at a minimum, a two-step process, with rapid, nonspecific DNA bending by IHF in its search mode, on time-scales that likely overlap with the residence time of the protein per site as it rapidly scans the DNA; the slow phase would then be the interconversion from the search to the recognition mode.

We envision the scan/search mode of the protein as one in which the relatively unstructured $\beta$-arms of IHF wrap and unwrap around the DNA, testing each site for conformability. The measurements reported here are blind to the dynamics of the protein, and only detect the bending/unbending of the bound DNA via the FRET labels attached to the DNA ends. Direct measurements of the wrapping/unwrapping dynamics of the $\beta$-arms of the protein, for example, by introducing Trp residues at suitable positions on the arms, could be a key experiment to further examine the origin of the fast phase. These measurements are currently underway in our group. 


\section{References}

1. Rice, P.A., et al., Crystal structure of an IHF-DNA complex: a protein-induced DNA U-turn. Cell, 1996. 87(7): p. 1295-1306.

2. Dhavan, G.M., et al., Concerted binding and bending of DNA by Escherichia coli integration host factor. J. Mol. Biol., 2002. 315(5): p. 1027-1037.

3. Perez-Howard, G.M., P.A. Weil, and J.M. Beechem, Yeast TATA binding protein interaction with DNA: fluorescence determination of oligomeric state, equilibrium binding, on-rate, and dissociation kinetics. Biochemistry, 1995. 34(25): p. 8005-8017.

4. Goodman SD, N.S., Nash HA., Deformation of DNA during site-specific recombination of bacteriophage lambda: replacement of IHF protein by HU protein or sequence-directed bends. Proc Natl Acad Sci 1992. 89: p. 11910-11914.

5. Lorenz, M., et al., Global structure similarities of intact and nicked DNA complexed with IHF measured in solution by fluorescence resonance energy transfer. Nucleic Acids Res., 1999. 27(23): p. 4619-4625.

6. Yang, S.W. and H.A. Nash, Comparison of protein binding to DNA in vivo and in vitro: defining an effective intracellular target. Embo. J., 1995. 14(24): p. 6292-300.

7. Wang, S., et al., The specific binding of Escherichia coli integration host factor involves both major and minor grooves of DNA. Biochemistry, 1995. 34(40): p. 13082-90.

8. Murtin, C., et al., A quantitative UV laser footprinting analysis of the interaction of IHF with specific binding sites: re-evaluation of the effective concentration of IHF in the cell. J Mol Biol, 1998. 284(4): p. 949-61.

9. Johnson, J.B., S. Stella, and J.K. Heiss, Bending and compaction of DNA by proteins, in Protein-Nucleic Acid Interactions, P.A. Rice and C.C. Correll, Editors. 2008, Royal Society of Chemistry: Cambridge.

10. Ali, B.M., et al., Compaction of single DNA molecules induced by binding of integration host factor (IHF). Proc. Natl. Acad. Sci. U S A, 2001. 98(19): p. 1065810663.

11. Skoko, D., et al., Micromechanical analysis of the binding of DNA-bending proteins $H M G B 1$, NHP6A, and HU reveals their ability to form highly stable DNA-protein complexes. Biochemistry, 2004. 43(43): p. 13867-13874.

12. van Noort, J., et al., Dual architectural roles of $H U$ : formation of flexible hinges and rigid filaments. Proc. Natl. Acad. Sci. USA, 2004. 101(18): p. 6969-74.

13. Sagi, D., et al., Modulation of DNA conformations through the formation of alternative high-order HU-DNA complexes. J. Mol. Biol., 2004. 341(2): p. 419-28.

14. Lin, J., Chen, H., Droge, P., Yan, J, Physical organization of DNA by multiple nonspecific DNA bending modes of Integration Host Factor (IHF). PLOS ONE, 2012. 7: p. e49885.

15. Ansari, A. and S.V. Kuznetsov, Dynamics and mechanism of DNA-bending proteins in binding site recognition, in Biophysics of DNA-protein interactions, M.C.W.a.L.J.M. III, Editor. 2010, Springer: New York.

16. Yang, C.C. and H.A. Nash, The interaction of E. coli IHF protein with its specific binding sites. Cell, 1989. 57(5): p. 869-880.

17. Hales, L.M., R.I. Gumport, and J.F. Gardner, Examining the contribution of a $d A+d T$ element to the conformation of Escherichia coli integration host factor-DNA complexes. Nucleic Acids Res., 1996. 24(9): p. 1780-6.

18. Travers, A., DNA-protein interactions: IHF--the master bender. Curr. Biol., 1997. 7(4): p. R252-4.

19. Swinger, K.K. and P.A. Rice, IHF and HU: flexible architects of bent DNA. Curr. Opin. Struct. Biol., 2004. 14(1): p. 28-35. 
20. Tanaka, I., et al., 3-A resolution structure of a protein with histone-like properties in prokaryotes. Nature, 1984. 310(5976): p. 376-81.

21. Vis, H., et al., Solution structure of the HU protein from Bacillus stearothermophilus. J. Mol. Biol., 1995. 254(4): p. 692-703.

22. Boelens, R., et al., Structure and dynamics of the DNA binding protein HU from Bacillus stearothermophilus by NMR spectroscopy. Biopolymers, 1996. 40(5): p. 5539.

23. White, S.W., et al., The high-resolution structure of DNA-binding protein HU from Bacillus stearothermophilus. Acta Crystallogr. D, 1999. 55(Pt 4): p. 801-9.

24. Vivas, P., et al., Mapping the Transition State for DNA Bending by IHF. J Mol Biol, 2012. 418(5): p. 300-15.

25. Swinger, K.K., et al., Flexible DNA bending in HU-DNA cocrystal structures. EMBO J., 2003. 22(14): p. 3749-3760.

26. Saecker, R.M. and M.T. Record, Jr., Protein surface salt bridges and paths for DNA wrapping. Curr. Opin. Struct. Biol., 2002. 12: p. 311-9.

27. Rice, P.A., Making DNA do a U-turn: IHF and related proteins. Current Opinion in Structural Biology 1997. 7: p. 86-93

28. Swinger, K.K. and P.A. Rice, Structure-based Analysis of HU-DNA Binding. J. Mol. Biol., 2007. 365(4): p. 1005-16.

29. Sugimura, S. and D.M. Crothers, Stepwise binding and bending of DNA by Escherichia coli integration host factor. Proc. Natl. Acad. Sci. USA, 2006. 103(49): p. 18510-4.

30. Kuznetsov, S.V., et al., Direct observation of DNA bending/unbending kinetics in complex with DNA-bending protein IHF. Proc. Natl. Acad. Sci. USA, 2006. 103(49): p. $18515-20$.

31. Hillisch, A., M. Lorenz, and S. Diekmann, Recent advances in FRET: distance determination in protein-DNA complexes. Curr. Opin. Struct. Biol., 2001. 11(2): p. 201-207.

32. Coman, D. and I.M. Russu, A nuclear magnetic resonance investigation of the energetics of basepair opening pathways in DNA. Biophys. J., 2005. 89(5): p. 328592.

33. Dhavan, G.M., et al., Decreased imino proton exchange and base-pair opening in the IHF-DNA complex measured by NMR. J. Mol. Biol., 1999. 288(4): p. 659-71.

34. Mouw, K.M. and P.A. Rice, Shaping the Borrelia burgdorferi genome: crystal structure and binding properties of the DNA-bending Hbb. Molecular Microbiology, 2007. 63: p. 1319-1330.

35. Vivas, P., S.V. Kuznetsov, and A. Ansari, New insights into the transition pathway from nonspecific to specific complex of DNA with Escherichia coli integration host factor. J. Phys. Chem. B, 2008. 112: p. 5997-6007.

36. Stivers, J.T., K.W. Pankiewicz, and K.A. Watanabe, Kinetic mechanism of damage site recognition and uracil flipping by Escherichia coli uracil DNA glycosylase. Biochemistry, 1999. 38(3): p. 952-63.

37. van den Broek, B., M.C. Noom, and G.J. Wuite, DNA-tension dependence of restriction enzyme activity reveals mechanochemical properties of the reaction pathway. Nucleic Acids Res., 2005. 33(8): p. 2676-2684.

38. Blainey, P.C., et al., A base-excision DNA-repair protein finds intrahelical lesion bases by fast sliding in contact with DNA. Proc. Natl. Acad. Sci. USA, 2006. 103(15): p. 5752-7.

39. Wang, Y.M., R.H. Austin, and E.C. Cox, Single molecule measurements of repressor protein 1D diffusion on DNA. Phys. Rev. Lett., 2006. 97(4): p. 048302. 
40. Tafvizi, A., et al., Tumor suppressor p53 slides on DNA with low friction and high stability. Biophys. J., 2008. 95(1): p. L01-3.

41. Bonnet, I., et al., Sliding and jumping of single EcoRV restriction enzymes on noncognate DNA. Nucleic Acids Res., 2008. 36(12): p. 4118-27.

42. Gorman, J., et al., Dynamic basis for one-dimensional DNA scanning by the mismatch repair complex Msh2-Msh6. Mol. Cell, 2007. 28(3): p. 359-70.

43. Barsky, D., T.A. Laurence, and C. Venclovas, How proteins slide on DNA, in Biophysics of DNA-protein interactions, M.C. Williams and L.J. Maher, Editors. 2010, Springer: New York p. 39-68.

44. Slutsky, M. and L.A. Mirny, Kinetics of protein-DNA interaction: facilitated target location in sequence-dependent potential. Biophys. J., 2004. 87(6): p. 4021-35.

45. Zhou, H.X., Rapid search for specific sites on DNA through conformational switch of nonspecifically bound proteins. Proc Natl Acad Sci U S A, 2011. 108(21): p. 8651-6.

46. Steinbach, P.J., R. Ionescu, and C.R. Matthews, Analysis of kinetics using a hybrid maximum-entropy/nonlinear-least-squares method: application to protein folding. Biophys. J., 2002. 82: p. 2244-2255.

47. Grove, A., et al., Localized DNA flexibility contributes to target site selection by DNA-bending proteins. J. Mol. Biol., 1996. 260(2): p. 120-125.

48. Vivas, P., Mechanism of integration host factor, a DNA-bending protein, probed with laser temperature-jump, in Physics. 2009, University of Illinois at Chicago: Chicago.

49. Moe, J.G. and I.M. Russu, Kinetics and energetics of base-pair opening in 5'd(CGCGAATTCGCG)-3' and a substituted dodecamer containing G.T mismatches. Biochemistry, 1992. 31(36): p. 8421-8.

50. Bhattacharya, P.K., J. Cha, and J.K. Barton, $1 H$ NMR determination of base-pair lifetimes in oligonucleotides containing single base mismatches. Nucleic Acids Res, 2002. 30(21): p. 4740-50.

51. Cao, C., et al., Dynamic opening of DNA during the enzymatic search for a damaged base. Nat Struct Mol Biol, 2004. 11(12): p. 1230-6.

52. Parker, J.B., et al., Enzymatic capture of an extrahelical thymine in the search for uracil in DNA. Nature, 2007. 449(7161): p. 433-7.

53. Lynch, T.W., et al., Integration host factor: putting a twist on protein-DNA recognition. J. Mol. Biol., 2003. 330(3): p. 493-502.

54. Read, E.K., R.I. Gumport, and J.F. Gardner, Specific recognition of DNA by integration host factor. Glutamic acid 44 of the beta-subunit specifies the discrimination of a T:A from an A:T base pair without directly contacting the DNA. J Biol Chem, 2000. 275(43): p. 33759-64.

55. Rice, P.A. and C.C. Correll, Protein-Nucleic Acid Interactions. 2008, Cambridge: The Royal Society of Chemistry.

56. Kalodimos, C.G., et al., Structure and flexibility adaptation in nonspecific and specific protein-DNA complexes. Science, 2004. 305(5682): p. 386-9.

57. Engler, L.E., et al., The energetics of the interaction of BamHI endonuclease with its recognition site GGATCC. J Mol Biol, 2001. 307(2): p. 619-36.

58. Engler, L.E., K.K. Welch, and L. Jen-Jacobson, Specific binding by EcoRV endonuclease to its DNA recognition site GATATC. J Mol Biol, 1997. 269(1): p. 82101.

59. Lesser, D.R., Kurpiewski, M.R., Jen-Jacobson, Science, 1990. 250: p. 776.

60. Ha, J.H., Capp, M.W., Hohenwalter, M.D., Baskerville, M., Record Jr, M.T.,, Mol. Biol., 1992. 228.

61. Record, M.T., Anderson, C.F., Mills, P., Mossing, M.C., Roe, J.H.,, Adv. Biophys., 1985. 20. 
62. Holbrook, J.A., et al., Specific and non-specific interactions of integration host factor with DNA: thermodynamic evidence for disruption of multiple IHF surface saltbridges coupled to DNA binding. J. Mol. Biol., 2001. 310: p. 379-401.

63. Vivas, P., et al., Global analysis of ion dependence unveils hidden steps in DNA binding and bending by integration host factor. J Chem Phys, 2013. 139(12): p. 121927.

64. Vivas, P., S.V. Kuznetsov, and A. Ansari, New insights into the transition pathway from nonspecific to specific complex of DNA with Escherichia coli integration host factor. J Phys Chem B, 2008. 112(19): p. 5997-6007.

65. Mirzabekov, A.D. and A. Rich, Asymmetric lateral distribution of unshielded phosphate groups in nucleosomal DNA and its role in DNA bending. Proc. Natl. Acad. Sci. USA, 1979. 76(3): p. 1118-21.

66. Manning, G.S., et al., An estimate of the extent of folding of nucleosomal DNA by laterally asymmetric neutralization of phosphate groups. J. Biomol. Struct. Dyn., 1989. 6(5): p. 877-89.

67. Williams, L.D. and L.J. Maher, 3rd, Electrostatic mechanisms of DNA deformation. Annu. Rev. Biophys. Biomol. Struct., 2000. 29: p. 497-521.

68. Ramge, K., Mayaan, E., Maher, L.J. 3rd,, York, D.M., , The contribution of phosphate-phosphate repulsions to the free energy of DNA bending. Nucleic Acids Res., 2005. 33: p. 1257.

69. Rouzina, I., Boomfield, V.A.,, Biophysical Journal, 1998. 74: p. 3152.

70. Baumann, C.G., et al., Ionic effects on the elasticity of single DNA molecules. Proc. Natl. Acad. Sci. U S A, 1997. 94(12): p. 6185-90.

71. Baumann, C.G., et al., Stretching of single collapsed DNA molecules. Biophys J, 2000. 78(4): p. 1965-78.

72. Wang, M.D., Ying, H.,Landick, R.,Gelles, J., Block, S.M.,, biophysical Journal, 1997. 73: p. 1335.

73. Koh, J., R.M. Saecker, and M.T. Record, Jr., DNA binding mode transitions of Escherichia coli HU(alphabeta): evidence for formation of a bent DNA--protein complex on intact, linear duplex DNA. J Mol Biol, 2008. 383(2): p. 324-46.

74. Yan, J. and J.F. Marko, Effects of DNA-distorting proteins on DNA elastic response. Phys Rev E Stat Nonlin Soft Matter Phys, 2003. 68(1 Pt 1): p. 011905.

75. Yan, J., R. Kawamura, and J.F. Marko, Statistics of loop formation along double helix DNAs. Phys. Rev. E., 2005. 71(6 Pt 1): p. 061905.

76. Vander Meulen, K.A., R.M. Saecker, and M.T. Record, Jr., Formation of a wrapped DNA-protein interface: experimental characterization and analysis of the large contributions of ions and water to the thermodynamics of binding IHF to $H^{\prime}$ DNA. J. Mol. Biol., 2008. 377(1): p. 9-27. 


\section{Statement of Authorship}

Major part of chapter 4 contains materials from the following two papers: "Kinetic gating mechanism of DNA damage recognition by Rad4/XPC”, published in Nature Communications, Jan. 2015 [1] and "Twist-open mechanism of DNA damage recognition by Rad4/XPC nucleotide excision repair complex", accepted for publication in PNAS, Feb. 2016 [2].

1. Xuejing Chen*, Yogambigai Velmurugu*, Guanqun Zheng, Beom Seok Park, Yoonjung Shim, Youngchang Kim, Lili Liu, Bennett Van Houten, Chuan He, Anjum Ansari and Jung-Hyun Min Kinetic gating mechanism of DNA damage recognition by Rad4/XPC. Nat Commun, 2015. 6: p. 5849.

2. Yogambigai Velmurugu*, Xuejing Chen*, Phillip Slogoff-Sevilla, Jung-Hyun Min, and Anjum Ansari, Twist-open mechanism of DNA damage recognition by Rad4/XPC nucleotide excision repair complex. Accepted for publication in Proc. Natl. Acad. Sci., 2016.

\section{* Co-first authors}

Author contributions for publication [1]: X.C carried out protein engineering and purifications, protein-DNA crosslinking and crystallization experiments with contributions from B.P and Y.S. G.Z. and C.H. synthesized modified oligonucleotides for crosslinking experiments. X.C., Y. K., and J.H.M. collected and analyzed crystallographic data. J.H.M and Y.K. did model building and refinement. Y.V. and A.A. designed the fluorescence measurements on complexes with 2APlabeled DNA substrates with contributions from X.C and J.H.M. Y.V. carried out the equilibrium and T-jump experiments, and analyzed the relaxation traces to obtain the DNA opening times. 
L.L. and B.V.H. carried out the AFM studies. A.A and J.M. wrote the manuscript with contributions from all authors.

Author contributions for publication [2]: X.C carried out protein engineering and purifications of Rad4 protein and its mutants. Y.V. and A.A. designed the fluorescence measurements on complexes with $\mathrm{tC}^{\mathrm{o}}-\mathrm{tC}_{\text {nitro }}$-labeled DNA substrates with contributions from X.C and J.H.M. Y.V., X.C., and P. S-S carried out the equilibrium temperature scan measurements. X.C measured the melting temperatures of all DNA constructs reported in this chapter, and also measured the binding affinities of the different DNA constructs in complex with wild type and mutant Rad4 proteins. Y.V. carried out all the T-jump experiments, and analyzed the relaxation traces to obtain the DNA dynamics in the presence of Rad4. A.A and J.M. wrote the manuscript with contributions from all authors. 


\section{Chapter IV}

\section{Lesion recognition by XPC $(\operatorname{Rad} 4)$ protein}

\subsection{Introduction}

\subsubsection{Nucleotide excision repair (NER)}

DNA contains the blueprint for the proper development, functioning and reproduction of every organism. DNA in cells is continuously being damaged by a wide variety of environmental sources such as UV rays, pollutants, cigarette smoke, food toxins, etc [1]. The lesions, if not repaired, can hamper critical cellular functions such as replication and transcription and lead to cell death or turn into genomic instability (mutagenesis) [1-5]. Nucleotide excision repair (NER) is a highly versatile and sophisticated repair pathway that has been conserved from yeast to humans to counter these diverse lesions and keep the genome integrity. NER removes primarily bulky, helix distorting damages induced by environmental sources that include intra-strand crosslinks such as (6-4) photo product and cyclobutane pyrimidine dimer (CPD) generated by UV light, a variety of adducts formed by environmental pollutants such as polycylic aromatic hydrocarbons (PAH) (induced by components in cigarette smoke) or aromatic amines, interstrand crosslinks created by chemotherapeutic agents such as cisplatin, and endogenous metabolites including reactive oxygen species $([1,6,7]$. NER in human cell is a complex biochemical process that requires several proteins [7-15]).

Two modes of NER can be distinguished: (1) Repair of lesions over the entire genome - Global genome NER (GG-NER) and (2) repair of transcription blocking lesions present in the transcribed DNA strands-Transcription coupled NER (TC-NER). Genetic impairment in GG-NER leads to extreme sun sensitivity in cells and can cause xeroderma pigmentosum (XP) cancer predisposition syndrome in humans. XP cells, similar to their yeast counterparts, 
are highly sensitive to UV and exhibit over 2000- fold increased risk of skin cancer [16]), underscoring the conserved importance of NER in protecting cells against genotoxic attacks by UV. Additionally there are two other disorders: cockayne syndrome and trichothiodystrophy (TTD) ascribed to a defect in TC-NER genes. Patients with these two syndromes do not have skin cancer predisposition, but rather a host of developmental and neurological abnormalities [17].

The lesion recognition task by NER is a particularly formidable challenge as it recognizes a virtually unlimited number of "bulky" lesions with diverse shapes and sizes. Since these lesions do not share a common chemical structure, they would not lend themselves to a common lesion binding pocket. However a common feature of good NER substrates is that they thermodynamically destabilize the DNA duplex and are bulky [18]. Intriguingly, the recognition efficiency of these lesions can vary by several orders of magnitude [19-24]. Even though experiments on structurally different adducts showed that factors such as disruptive base pairing, bending and flexibility can contribute to repair efficiencies ([25-28], how these diverse lesions are recognized and what determines the varying efficiency is still not clear. In cells, these lesions exist among a vast excess of undamaged nucleotides. For example, UV radiation in strong sunlight can induce $\sim 100,000$ lesions per cell per hour, but even in this extreme case, the number of damaged nucleotides makes up only $0.002 \%$ of total nucleotides in the genome [2]. This is a "needle in the haystack" problem and how these diverse NER lesions are recognized from the majority of undamaged nucleotides has been a major mystery.

In mammalian GG-NER, xeroderma pigmentosum C (XPC) (125 kD) -Rad23B (58kD) complex (hereafter XPC) has been identified as a damage sensor and repair recruitment factor. To initiate NER, XPC needs to recognize an extraordinarily wide variety of lesions based on thermal fluctuation alone. Conserved from yeast to humans, XPC (i) 
searches the genome for damage, (ii) recognizes extraordinarily diverse DNA lesions embedded within, and (iii) recruits the general transcription factor TFIIH (Fig. 4.1). TFIIH in turn verifies the lesion and leads to the recruitment of the downstream factors. This in turn leads to the excision of a lesion-containing, 24-32 nt single-stranded DNA and gap-filling repair synthesis occurs subsequently $[1,29]$. Although the lesion recognition step by XPC is considered the rate-limiting step of NER $[28,30]$ much remains unknown as to how XPC achieves its specific binding efficiently and reliably.

\section{Nucleotide Excision Repair (NER)}

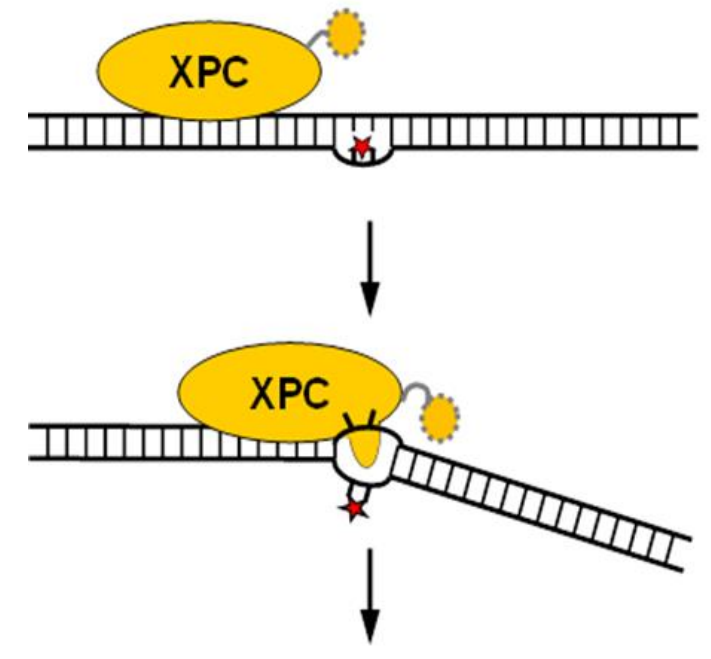

\section{Search}

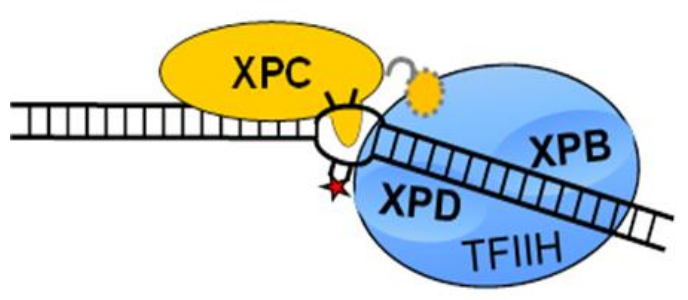

\section{$\underline{\text { TFIIH recruitment }}$}

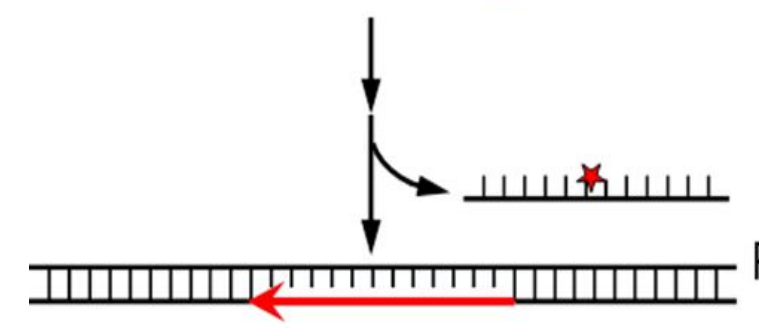

$\underline{\text { Recognition }}$<smiles>[3H]C</smiles>

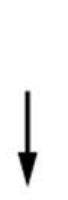

Unwinding \&

Verification

\section{Excision \&} Repair synthesis

Fig 4. 1. NER repair pathway. XPC (1) scans the genome, (2) recognizes diverse lesions, and (3) recruits the multi-subunit TFIIH complex. TFIIH unwinds and verifies the lesion, which ultimately leads to excision and repair. 
XPC is an indispensable factor for the lesion recognition and initiation of NER. In vitro, XPC distinguishes and binds specifically to a wide range of NER lesions but it also has nanomolar affinity towards undamaged DNA [19-24, 31]. Notably, XPC resides in DNA-rich chromatin in undamaged cells [31], which is unique among other mammalian NER proteins $[32,33]$. In UV-irradiated cells, XPC localizes to lesions and is strictly required for the recruitment of TFIIH and all the other subsequent factors needed for lesion repair [34, 35]. It has been shown in in vitro studies that XPC can also recognize artificially destabilized DNA such as 2- or 3-base-pair (bp) mismatch bubble [36-38], although such mismatches fail the verification step by TFIIH and thus are not excised by NER.

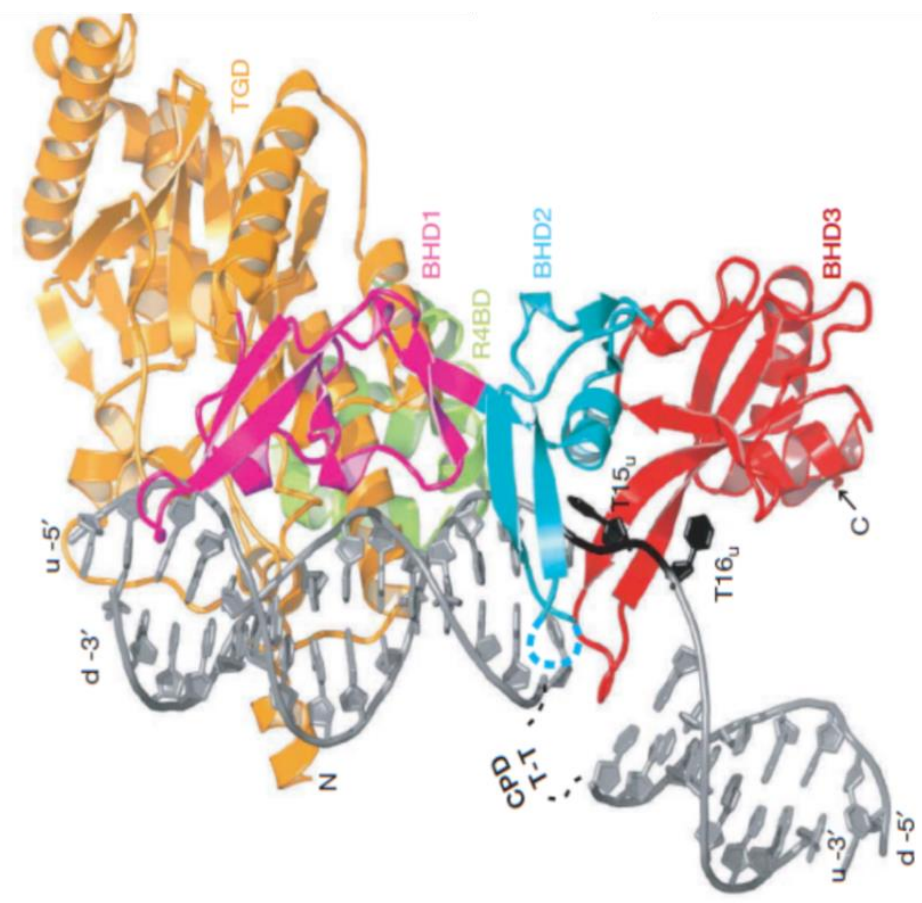

Fig. 4. 2. Crystal structure of the Rad4-Rad23-DNA complex: TGD, gold; BHD1, magenta; BHD2, cyan; BHD3, red; R4BD, green; and DNA, grey. The complex has two main functional domains: (1) a nonspecific DNA binding domain made up of a trnasglutamase homology domain and a $\beta$-hairpin (BHD1, magenta ) anchors the protein on the DNA; (2) a double $\beta$-hairpin domain (BHD2, cyan and BHD3, red ) binds the undamaged strand of the DNA without making direct contact with the lesion. The two disordered CPD-linked thymidines are indicated schematically (dotted lines), and the two flipped-out thymidines bound to Rad4 are colored black. Figure reproduced from [36]. 
The crystal structure of the yeast XPC orthologue, Rad4-Rad23 complex (hereafter referred to as Rad4), bound to model lesions was solved by Min et al [36]. The crystal structure revealed that Rad4 and, by analogy, XPC recognizes the damaged DNA site by inserting a $\beta$-hairpin into DNA duplex and flipping out damage-containing nucleotide pairs to form an 'open' conformation [36] (Fig.4.2). In this conformation, Rad4 interacts exclusively with the two nucleotides on the undamaged strand without making specific contacts with the two damaged residues of a UV lesion. This indirect recognition mode [36, 39] explained how $\operatorname{Rad} 4 / \mathrm{XPC}$ is capable of binding to a variety of lesions. However, it also posed a paradox regarding the mechanism of lesion recognition since the protein can no longer "see" the difference between damaged versus undamaged DNA once such conformation is formed. It has thus been posited that Rad4/XPC may be limited in its ability to fully 'open' undamaged DNA in the same way as "opens" damaged DNA [36].

To address the question as to how XPC/Rad4 can discriminate between lesions sites and normal sites during the "search" process, our collaborators Prof. Min and her group recently solved the crystal structure of Rad4 bound to undamaged DNA [38]. To overcome the intrinsic heterogeneity of nonspecific complexes, they captured Rad4 on a single register of undamaged DNA using disulphide tethering. The structure they obtained of this nonspecifically bound Rad4 on undamaged DNA came as a surprise: the structure revealed that tethered Rad4 flips out normal nucleotide pairs and bends the DNA by $42^{\circ}$ to form the same 'open' conformation as with damaged DNA (Fig. 4.3). Consistent with this result, an atomic force microscopy (AFM) study of $\operatorname{Rad} 4$ bound (but not tethered) to a $514 \mathrm{bp}$ undamaged DNA also showed that the DNA is bent $\left(48.4 \pm 34.2^{\circ}\right)$ [38]. These results agree remarkably with a pervious AFM study of human XPC bound to undamaged DNA segments that also revealed bent DNA $\left(49 \pm 36^{\circ}\right)[40]$. 
The crystal structures pose an intriguing question: Does XPC flip out each and every site in the genome during its search for the damaged site? If this is the case then XPC would need to recruit TFIIH every time to verify the damage and such a scenario is not compatible with an efficient search mechanism. In contrast these results indicate that structural discrimination between normal and damaged DNA cannot be the basis for lesion recognition by $\mathrm{Rad} 4 / \mathrm{XPC}$; instead, the speed and temporal order of conformational deformations seen in the crystal structures must be important determinants of the target recognition mechanisms. Therefore, to obtain a complete understanding of lesion recognition, especially in this case where the crystal structures of $\operatorname{Rad} 4$ bound to damaged or undamaged DNA have identical features, it is necessary to characterize the dynamics of the interactions between Rad4/XPC and DNA. 

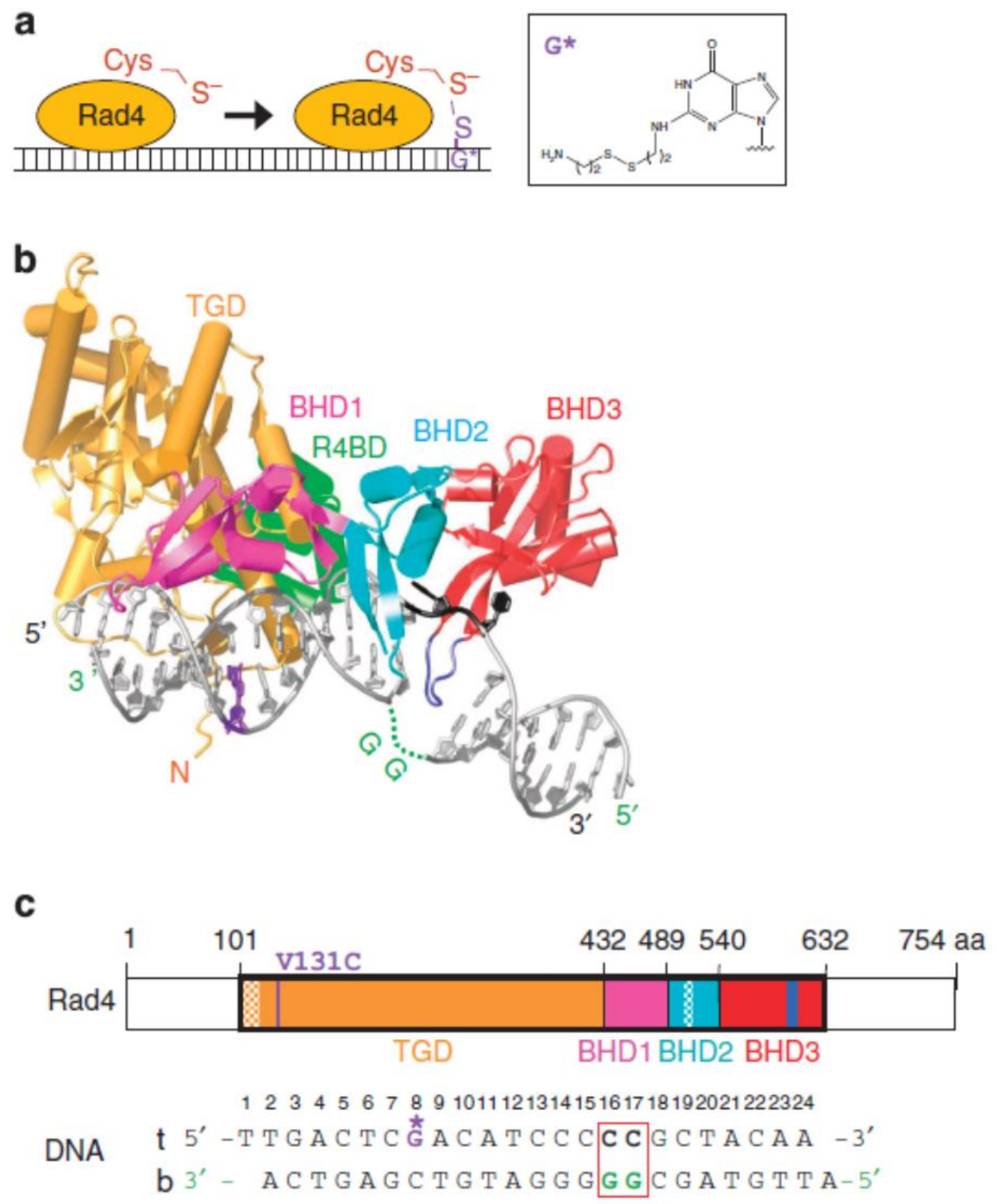

Fig. 4. 3. Crystal structure determination of Rad4-Rad23 bound to undamaged DNA using disulphide crosslinking. (a) Crosslinking scheme to tether Rad4 on DNA. A disulphide crosslink between cysteine 131 in $\operatorname{Rad} 4$ and a modified nucleotide $\left(\mathrm{G}^{*}\right)$ on DNA was introduced to capture the protein-DNA complex in a defined register of binding. The chemical structure of $\mathrm{G}^{*}$ is shown on the right. (b) Structure of Rad4-Rad23 tethered to undamaged DNA (PDB code 4U29). The transglutaminase domain (TGD, orange) and $\beta$-hairpin domain 1 (BHD1, magenta) of Rad4 bind to an 11-bp duplex segment of the DNA (silver), while BHD2 (cyan) and BHD3 (red) of Rad4 bind to a 4bp segment in which two nucleotide pairs are flipped out. The tip of the long $\beta$-hairpin in BHD3 (residues 599-605, blue) is inserted into the DNA duplex and fills the gap created by the flipped-out nucleotides. The crosslinked, G*8 in DNA and Cys131 in TGD are in purple. The two flipped-out cytidines (black) are bound by BHD2-BHD3, while the complementary guanosines flipped out away from the protein are disordered in the crystal (green dotted line). Rad23 binds to TGD through its Rad4-binding domain (R4BD, light green). ' $\mathrm{N}$ ' indicates the $\mathrm{N}$ terminus of Rad4. (c; top)Domain arrangements and boundaries of $\operatorname{Rad} 4$ used in this study. The colour scheme is the same as in $b$. The crystallized Rad4 construct spans residues 101-632 as before [36] and contains point mutations 
V131C (purple) and C132S. These residues are at the end of the $\mathrm{N}$ terminus of TGD, away from the BHD2-BHD3 bound to flipped-out nucleotides. The disordered regions in the crystal are checkered. (The) Rad23 construct is the same as [36]. (Bottom) DNA duplex sequence used for the structure determination. The nucleotide pairs flipped out by Rad4 are boxed in red. The disulphide-modified nucleotide, $\mathrm{G}^{*} 8$, was at a position 8-bp away from the flipped-out (flipped out) ones (CC/GG at 16 and 17). Top strand (' $t$ ') corresponds to the undamaged strand and the bottom ('b') the damaged strand in the lesion-bound structure [36] (PDB 2QSG). (Figure reproduced from [38]).

Accordingly, we have carried out kinetics studies of DNA deformations induced by Rad4 using the laser T-jump approach, and, with insights gained from the crystallographic snapshots we have identified the intermediates and the bottlenecks along the lesion recognition trajectory. Such kinetics measurements, together with the high resolution structures, are needed to unveil the underlying energetic landscape.

\subsubsection{Experimental design}

Crystal structure of Rad4 complexed with damaged site or with Rad4 tethered to an undamaged site showed severe distortions at the binding site. Here, we used three different spectroscopic probing strategies to monitor the series of Rad4 induced conformational dynamics in DNA such as nucleotide flipping, unwinding and bending.

1. To measure nucleotide-flipping or "damage-opening" kinetics, we incorporated 2aminopurine (2AP), a fluorescent analog of adenine, into the DNA. 2AP fluorescence within DNA depends on the extent of its stacking with nearest neighbors and on the extent of its exposure to the solvent, making it a sensitive probe of local conformational changes in DNA in the vicinity of the probe.

2. To probe longer range motions that affect regions beyond the mismatched nucleotides such as local unwinding around the lesion, as observed in the 'open' structures (Figs. $4.2 \& 4.3$ ), we adopted a pair of recently developed FRET probes: $\mathrm{tC}^{\mathrm{o}}$ (1,3-diaza-2-oxophenoxazine as donor) and $\mathrm{tC}_{\text {nitro }}$ (7-nitro-1,3-diaza-2oxophenothiazine as acceptor) (Fig. 4.4B) [41, 42]. These FRET probes are tricyclic 
cytosine analogs that retain Watson-Crick-type pairing with guanines in duplex

DNA; they also allow favorable base-stacking interactions with neighboring bases.

A

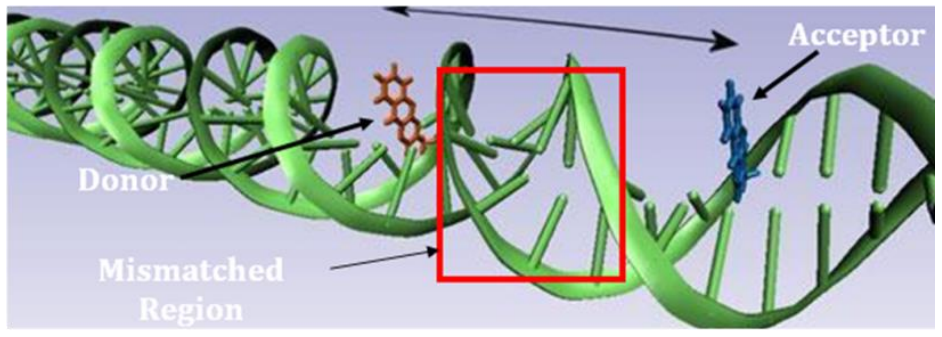

B

C

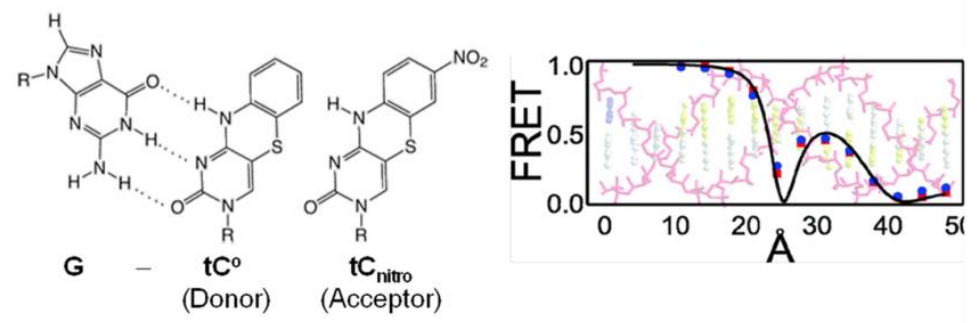

Fig. 4. 4. DNA conformational rearrangements during lesion recognition by Rad4 probed by $\mathbf{t} \mathbf{C}^{\mathbf{0}} / \mathbf{t} \mathbf{C}_{\text {nitro }}$ (A) Structural model of a DNA helix with $\mathrm{tC}^{\circ}$ (donor) and $\mathrm{tC}_{\text {nitro }}$ (acceptor) incorporated in the vicinity of a mismatch site. (B) Chemical structures of $\mathrm{tC}^{\mathrm{o}}$ and $\mathrm{tC}_{\text {nitro }}$ cytosine analogs and Watson-Crick type base pairing of $\mathrm{tC}^{\mathrm{o}}$ with a guanine (G). (C) FRET efficiency between the $\mathrm{tC}^{\mathrm{o}}$ (donor) and $\mathrm{tC}_{\text {nitro }}$ (acceptor) incorporated within normal $\mathrm{B}$-DNA is plotted as a function of the distance between the two probes. FRET generally decreases as the distance increases, but additionally depends on the relative orientations of the absorption and emission dipoles of the fluorophores. Figures in (B,C) are adapted with permission from Borjesson et al.[41]; copyright (2009) American Chemical Society.

3. To monitor DNA bending, we attached fluorescent labels (TAMRA/Cy5 (donor/acceptor) FRET pair) on the DNA to probe changes in the "end-to-end" distance upon Rad4 binding.

To understand the role of $\beta$-hairpin of the protein in probing for lesions and/or stabilizing the recognition complex, we made measurements with mutants of Rad4 that do not have the $\beta$-hairpin. The wild type and mutant Rad4 proteins as well as all the Rad4/DNA complexes 
for the equilibrium and kinetics experiments reported in this chapter were prepared by Xuejing Chen in Prof. Min's group. 


\subsection{Method}

\subsubsection{Preparation of double-stranded DNA substrates.}

The concentrations of each single-stranded oligonucleotide were determined by UV absorbances at $260 \mathrm{~nm}$ using the extinction coefficients provided by TriLink Biotechnologies, based on the nearest neighbor method [43, 44]. The errors in the measured DNA concentrations were estimated to be less than $\sim 10 \%$. To prepare duplex DNA, two complementary oligonucleotides, each at $100 \mu \mathrm{M}$, were mixed in PBS buffer in a microcentrifuge tube. The tube was then immersed in $2 \mathrm{~L}$ of $\sim 100{ }^{\circ} \mathrm{C}$ water bath on a hot plate. After $10 \mathrm{~min}$, the hot plate was turned off and the oligonucleotides were annealed as the water bath was cooled down slowly to room temperature over 5 to $6 \mathrm{~h}$.

\subsubsection{Preparation of Rad4-Rad23 complexes.}

All Rad4-Rad23 complexes (hereafter Rad4) were prepared as previously described [36, 38]. Briefly, Hi5 insect cells co-expressing the proteins were harvested 2 days after infection. After lysis, the proteins were purified by affinity chromatography (His-Select Nickel agarose, Sigma), anion-exchange (Source Q, GE healthcare) and cation exchange (Source S, GE healthcare) chromatography followed by gel-filtration (Superdex200, GE healthcare). Final sample was concentrated by ultrafiltration to $\sim 13-14 \mathrm{mg} \mathrm{ml}^{-1}(135-150 \mu \mathrm{M})$ in $5 \mathrm{mM}$ bis-tris propane- $\mathrm{HCl}(\mathrm{BTP}-\mathrm{HCl}), 800 \mathrm{mM} \mathrm{NaCl}$ and $5 \mathrm{mM}$ DTT, pH 6.8, for all constructs used in this study.

\subsubsection{Duplex melting temperatures of mismatched and undamaged/matched DNA.}

To determine the effects of mismatch bubbles and the $\mathrm{tC}^{\mathrm{o}} / \mathrm{tC}_{\text {nitro }}$ labels on the overall thermal stabilities of the DNA duplexes, we determined the melting temperatures of the unlabeled (DNA_NL), donor-only (DNA_D) and donor-acceptor (DNA_DA) duplex DNA samples as follows: The absorbances at $260 \mathrm{~nm}$ of each duplex DNA at $1.5 \mu \mathrm{M}$ (per strand) were 
measured at every $0.5{ }^{\circ} \mathrm{C}$ interval, using Cary 300 Bio UV-Visible spectrophotometer and temperature controller (Varian) with sample cuvettes of path length $1 \mathrm{~cm}$ as the temperature was increased from 25 to $90{ }^{\circ} \mathrm{C}$. Prior to calculating the derivative, the absorbance data were smoothed (averaged) over a $2.5^{\circ} \mathrm{C}$ (5 data points) window. Melting temperatures were then determined from the melting profiles using two methods described below.

\subsubsection{Method 1}

All melting profiles were analyzed in terms of a two-state van't Hoff transition: $\boldsymbol{A}(\boldsymbol{T})=$ $\boldsymbol{A}_{L}(\boldsymbol{T})+\left(\boldsymbol{A}_{U}(\boldsymbol{T})-\boldsymbol{A}_{L}(\boldsymbol{T})\right) \boldsymbol{f}$, where $\boldsymbol{A}(\boldsymbol{T})$ is the absorbance of the sample, $\mathrm{A}_{\mathrm{U}}$ and $\mathrm{A}_{\mathrm{L}}$ are the upper and lower baselines, parameterized as linear functions of temperature with the slopes either constrained to be the same or varied independently, and $\mathrm{f}$ is the fraction unfolded as a function of temperature. The fraction $\mathrm{f}$ is described in terms of two parameters: the van't Hoff enthalpy change $\boldsymbol{\Delta H}$ and melting temperature $\mathrm{T}_{\mathrm{m}}$ as follows:

$$
f=\left(1+\exp \left[-\frac{\Delta H}{R}\left(\frac{1}{T}-\frac{1}{T_{m}}\right)\right]\right)^{-1}
$$

Therefore, the total number of parameters are six when choosing baselines with independent slopes (4 parameters for the two baselines and 2 parameters for the unfolding transition) and five when the two baselines are constrained to have the same slope). 


\subsubsection{Method 2}

Alternatively, the derivative of the melting profiles $\boldsymbol{\delta} \boldsymbol{A} / \boldsymbol{\delta}$ can be obtained numerically from the measured $\boldsymbol{A}(\boldsymbol{T})$, and described in terms of the van't Hoff two-state system. For example, if the upper and lower baselines are assumed to have the same slope, we can write:

$$
A_{L}=m T+\beta ; A_{U}-A_{L}=\alpha ; \text { and } A(T)=\alpha f+m T+\beta,
$$

which yields $: \frac{\partial A}{\partial T}=\alpha \frac{\partial f}{\partial T}+m$

$$
\text { and } \frac{\partial f}{\partial T}=-\left(\frac{\Delta H}{R T^{2}}\right) \frac{\exp \left(-\frac{\Delta H}{R}\left(\frac{1}{T}-\frac{1}{T_{m}}\right)\right)}{\left[1+\exp \left(-\frac{\Delta H}{R}\left(\frac{1}{T}-\frac{1}{T_{m}}\right)\right)\right]^{2}}
$$

Thus, the experimental data $\frac{\boldsymbol{\delta} \boldsymbol{A}}{\boldsymbol{\delta} \boldsymbol{T}}$ can be described in terms of the two van't Hoff parameters $\left(\boldsymbol{\Delta H}\right.$ and $T_{\mathrm{m}}$ ) and two additional parameters: $\boldsymbol{\alpha}$ and $\boldsymbol{m}$. While this method is analogous to fitting the absorbance data $\boldsymbol{A}(\boldsymbol{T})$ directly, the number of parameters is reduced by one.

\subsubsection{Apparent binding affinities $\left(\mathbf{K}_{\mathbf{d}, \text { app }}\right)$ determined by electrophoretic mobility shift assays.}

Electrophoteric mobility shift assays or gel-shift assays were carried out essentially as previously described $[36,38]$. Various concentrations of the wild-type Rad4-Rad23 complex were mixed with $1 \mathrm{nM}{ }^{32} \mathrm{P}$-labelled DNA of interest (undamaged/matched or mismatched) in the presence of $1000 \mathrm{nM}$ cold, undamaged DNA, CH7_NX (Table VI and VII) in a binding assay buffer (5 mM BTP-HCl, $75 \mathrm{mM} \mathrm{NaCl,} 5 \mathrm{mM}$ DTT, 5\% glycerol, $0.74 \mathrm{mM}$ 3-[(3cholamidopropyl)dimethylammonio]-1-propanesulfonate (CHAPS), $500 \mu \mathrm{g} \mathrm{m} \mathrm{m}^{-1}$ bovine serum albumin, $\mathrm{pH}$ 6.8). Mixed samples were subsequently incubated at room temperature for $20 \mathrm{~min}$ and separated on $4.8 \%$ non-denaturing polyacrylamide gels in 1x TBE buffer (89 
$\mathrm{mM}$ Tris- $\mathrm{HCl}, 89 \mathrm{mM}$ Boric acid, $2 \mathrm{mM}$ EDTA, $\mathrm{pH}$ 8.0) for $15 \mathrm{~min}$ at $4{ }^{\circ} \mathrm{C}$. The gels were quantitated by autoradiography using Personal Molecular Imager ${ }^{\mathrm{TM}}$ and Quantity One software (Bio-Rad). Apparent dissociation constants $\left(\mathrm{K}_{\mathrm{d}, \mathrm{app}}\right)$ of the DNA were obtained as described [36, 38], against the nonspecific binding of the matched CH7_NX. The nonlinear regression was done using Origin software (OriginLab).

\subsubsection{Equilibrium FRET temperature scan experiments with $\mathbf{t C}^{\mathbf{0}} / \mathrm{tC}_{\text {nitro }}$ probes.}

The fluorescence measurements under equilibrium conditions were carried out on $\mathrm{tC}^{\mathrm{o}} / \mathrm{tC}_{\mathrm{nitro}}{ }^{-}$ labeled DNA in the presence and absence of proteins, using a FluoroMax4 spectrofluorometer (JobinYvon, Inc., NJ). The raw data for each sample consists of fluorescence emission spectra measured in the wavelength range $375-550 \mathrm{~nm}$, with excitation of the donor $\left(\mathrm{tC}^{\circ}\right)$ at $365 \mathrm{~nm}$; the spectra were measured over the temperature range starting from $10{ }^{\circ} \mathrm{C}$ up to $40{ }^{\circ} \mathrm{C}$ (or $30{ }^{\circ} \mathrm{C}$, as indicated in the text) in $2.5{ }^{\circ} \mathrm{C}$ increments. Corresponding measurements of buffer-only control samples were done under identical conditions. To ensure the integrity of each sample after heating, fluorescence intensities were measured again at $25{ }^{\circ} \mathrm{C}$ after a heating phase was complete. Only data with reproducible signals after the heat-cool cycle were taken into consideration.

As the acceptor $\mathrm{tC}_{\text {nitro }}$ does not fluoresce under aqueous conditions $[42,45]$, an acceptor fluorescence-based method could not be used to obtain the FRET efficiency for the

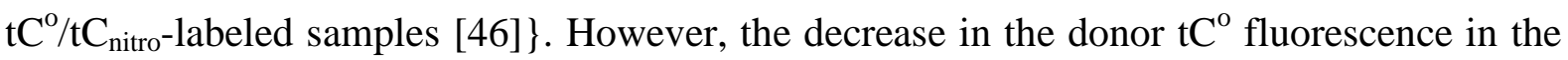
presence of $\mathrm{tC}_{\text {nitro }}$ compared with its absence indicates the Förster energy transfer between the FRET pair [42], and the FRET efficiency $(E)$ can be obtained from a 'donor fluorescence ratio' method [46]. In this method, the donor fluorescence emission intensities are measured in two separate samples: one $\left(I_{\mathrm{D}}\right)$ from the donor-only sample ('DNA construct name'_D) 
and the other $\left(I_{\mathrm{DA}}\right)$ from the donor-acceptor sample ('DNA construct name'_DA), and the FRET efficiency is calculated using the equation: $\boldsymbol{E}=\mathbf{1}-\frac{\boldsymbol{I}_{\boldsymbol{D} A}}{\boldsymbol{I}_{\boldsymbol{D}}}$. For each sample, the characteristic emission intensities $I_{\mathrm{D}}$ and $I_{\mathrm{DA}}$ were computed as the area under the corresponding emission curves in the range of $425-515 \mathrm{~nm}$. Since the 'donor ratio' method requires ratio of fluorescence intensities from two separate samples, any errors in matching the concentrations of the two samples get amplified in the calculation of the FRET efficiencies. The FRET values reported in the manuscript are the average from 2-15 independent measurements, and the error bars represent the corresponding standard error of the mean (s.e.m.) values.

\subsubsection{Acquisition and analyses of $\mathbf{T}$-jump relaxation traces.}

To acquire data with the highest temporal resolution and be able to span several orders of magnitudes in time scale, we typically acquired the kinetics traces on at least two time scales, with one million data points in each trace. In order to combine the data acquired over the two different time-scales, the two traces were fitted simultaneously with a double-exponential plus T-jump recovery function, with an additional fitting parameter (see method section below entitled 'Double-exponential decay convoluted with T-jump recovery'). The additional parameter served as a multiplicative scale factor and was applied to one of the traces to account for any systematic difference in the measured intensities for the two traces. Once appropriately scaled, the two data sets were combined into a single kinetic trace that covered the time range from $\sim 20 \mu \mathrm{s}$ to $32 \mathrm{~ms}$. These combined traces were then used for all subsequent analyses, using either a single- or double-exponential decay convoluted with a predetermined function for the $\mathrm{T}$-jump recovery kinetics, or a maximum entropy analysis, as described below. 


\subsubsection{T-jump recovery kinetics.}

The T-jump recovery kinetics were obtained from measurements on control (donor-only samples) that were shown to exhibit only the T-jump recovery kinetics, with measurements carried out over a longer time-window (up to about $320 \mathrm{~ms}$ ). The recovery kinetics was fitted to the recovery function, equation 2.7 shown in chapter 2 . The recovery time constant $\tau_{\text {rec }}$ was determined for each sample as the average of several control measurements. Representative recovery traces are shown in Figs. 4.19C, 4.28C, \& 4.29C). The recovery times for all samples were found to be in the range of $133-320 \mathrm{~ms}$ with an average value of $192.3 \pm 82.8$ ms.

\subsubsection{Maximum entropy method (mem) for analyzing $T$-jump relaxation rates.}

For many relaxation traces, the kinetics showed deviations from a single-exponential behavior. However, adding an additional exponential decay, while it improved the fit, gave unreliable estimates of the relaxation times and amplitudes for the two exponential components. Note that for purposes of obtaining a scale factor to match data on different time-scales, a two-exponential fit was adequate. However, to overcome the limitations of such discrete exponential analysis, we chose the maximum entropy method (mem) that yields a model-independent distribution of rate constants. A detailed explanation of the maximum entropy analysis is presented in chapter 2. If there was some overlap between the mem analysis output peaks, as in Fig. 4.23, the distribution $f(\log \tau)$ was fitted to the sum of two Gaussian distributions, and the average $\langle\log \tau\rangle$ was obtained from the peak position of each Gaussian distribution.

To determine whether the observed peaks are from real relaxation phases or T-jump recovery, we also carried out mem analysis on control experiments measured on identical 
time-scales as the relaxation kinetics, as well as on control experiments measured on a longer time-scale. For example, for the relaxation traces measured on double-labelled samples shown in Fig. 4.29A, we observed two relaxation peaks in the mem analysis (Fig.4. 29D), one centered at $\log (\tau / \mathrm{s}) \approx-3.2(\tau \approx 631 \mu \mathrm{s})$, and the other at $\log (\tau / \mathrm{s}) \approx-1.36(\tau \approx 44 \mathrm{~ms})$. For control measurements on donor-only samples (Fig. 29B) carried out over the same timewindow as in Fig.4. 29A (spanning up to $32 \mathrm{~ms}$ ), we observed a single-peak distribution in our mem analysis (Fig.4. 29E), centered at $\log (\tau / \mathrm{s}) \approx-1.47(\tau \approx 34 \mathrm{~ms})$. The same control experiment was also done over a longer time-window (spanning up to $320 \mathrm{~ms}$ ) and combined with the shorter time scale data (Fig. 4.29C), as described above. Mem analysis on the combined data set for the control sample also yields a single-peak distribution (Fig. 4.29F), but this time the peak is centered at $\log (\tau / \mathrm{s}) \approx-0.69(\tau \approx 204 \mathrm{~ms})$. This analysis indicates that, while the donor only samples exhibit only the T-jump recovery kinetics, the mem analysis yields reasonable T-jump recovery times only when the kinetics are measured over a longer time-window, as is to be expected. Nevertheless, we can correctly assign the slow phase appearing at $\sim 44 \mathrm{~ms}$ in Fig. 4.29 A, D as arising from partial T-jump recovery, since a similar peak is also observed in the mem analysis on the control sample (Fig. 4.29 B, E) when restricted to the shorter time scales. To cover the time-span from $20 \mu$ s to $>320 \mathrm{~ms}$ and get more complete information about relaxation kinetics and $\mathrm{T}$-jump recovery in one combined trace, it would be ideal to acquire all kinetics traces over three different time-windows. However, such practice makes the kinetics measurements unmanageably long. We therefore use measurements on control (donor-only) samples to guide our interpretations of the relaxation phases observed, as explained above.

The mem analyses gave the most reproducible results for the relaxation rates obtained for all samples over the temperature range measured, and are the rates shown in the main manuscript. However, the mem analysis has two caveats. First, as described above, we do 
not have the ability to constrain the T-jump recovery kinetics from measurements on control samples taken over longer time scales; hence the mem analyses on data acquired over the shorter time-scales do not reproduce the correct T-jump recovery times as evidenced from the analyses using longer time-scale data. Second, the $f_{\mathrm{j}}$ in equation 2.10 (Chpater 2) that describe the distribution of relaxation times can only take positive values in the maximum entropy algorithm we have used. Thus, for relaxation traces in which the amplitude of the relaxation phases and the amplitude of the recovery phase have opposite signs, as in AN12Rad4 (Fig. 4.19A) and AN12-mutant Rad4 (Figs. 4.22A and 4.23A), the mem algorithm is unable to simultaneously describe the relaxation and the recovery kinetics. To confirm that the average rates that we report from mem analysis of the relaxation traces are not unduly influenced by incorrect attributions of the T-jump recovery kinetics, we also analysed the relaxation traces using discrete (single- or double-) exponential decays explicitly convoluted with T-jump recovery kinetics, as described below.

\subsubsection{Single-exponential decay convoluted with $T$-jump recovery.}

Relaxation kinetics traces $I(t)$ measured as a function of time after the T-jump were also analyzed in terms of a single-exponential decay with relaxation rate $k_{\mathrm{r}}$. The overall decay curve was convoluted with the recovery of the donor intensity back to the pre-laser levels characteristic of the initial temperature. The fitting function for the relaxation traces was written as in equation 2.8 in chapter 2.

\subsubsection{Double-exponential decay convoluted with $T$-jump recovery.}

As needed, the decay traces were also analyzed in terms of a two-exponential decay convoluted with the T-jump recovery kinetics as described in Eq. 2.9 in chapter 2.

We also used this two-exponential decay convoluted with T-jump recovery function when combining the raw data acquired over the two different time-scales (See the section on 
'acquisition and analyses of relaxation traces'). Two-exponential fits provided an improvement over single-exponential fits in almost all cases. However, the observations of a single peak in the mem analysis for the same data sets led us to interpret the relaxation traces on all complexes (with the exception of AN12- $\triangle \mathrm{BHD} 3$ ) in terms of a single relaxation phase (plus T-jump recovery); it is likely that each relaxation phase in fact contains a distribution of relaxation times that are clustered in a narrow range.

\subsubsection{MEM versus discrete-exponential analysis.}

Comparisons of the relaxation rates obtained from the mem analysis and that from discreteexponential fits are summarized for AN12-Rad4 (Fig. 4.19), AN12- $\Delta$ BHD3 (Fig. 4.23), AN14-Rad4 (Fig. 4.28), and AN14u-Rad4 (Fig. 4.29). The two analyses methods are in good agreement in most cases. In the case of AN12- $\triangle$ BHD3 exhibiting two relaxation phases, the rates obtained from a two-exponential fit (Fig. 4.23 E) showed larger variations than those from mem analysis (Fig. 4.21G), but the Arrhenius fits remain similar. For data on AN14u-Rad4, the very small amplitudes of the relaxation kinetics contributed to a small but systematic discrepancy between the mem and the single-exponential results (Fig. 4.29G).

\subsubsection{Amplitude analysis.}

The relative amplitudes of the relaxation traces $\left(A_{\mathrm{r}}\right)$ for each of the samples were obtained from the discrete-exponential fits (Eqs. 2,8 and 2.9 in Chapter 2) as follows:

$$
A_{r}(T)=\left(I_{a p p}(\infty)-I\left(0^{+}\right)\right) / I\left(0^{+}\right)
$$

To compare these amplitudes with those 'expected' from equilibrium measurements of $I_{\mathrm{D}}$ and $I_{\mathrm{DA}}$, we assumed that any difference in $I_{\mathrm{D}}$ and $I_{\mathrm{DA}}$ at the final temperature $\left(T_{\mathrm{f}}\right)$, after normalizing the two intensities at the initial temperature $\left(T_{\mathrm{i}}\right)$, must appear in a relaxation phase in a T-jump measurement. This 'expected' amplitude $\left(A_{\mathrm{e}}\right)$ was calculated from the measured $I_{\mathrm{D}}(T)$ and $I_{\mathrm{DA}}(T)$ equilibrium thermal profiles using the following expression: 


$$
A_{e}=\frac{I_{D A}\left(T_{f}\right)-\propto I_{D}\left(T_{f}\right)}{\propto I_{D}\left(T_{f}\right)}
$$

where $\propto=I_{D A}\left(T_{i}\right) / I_{D}\left(T_{i}\right)$ matches the intensities of the two measurements at the initial temperature. This analysis assumes that the initial drop in intensity occurring on T-jump time-scale should be proportional to $I_{D}\left(T_{f}\right)-I_{D}\left(T_{i}\right)$, reflecting a change in the quantum yield of the donor as a result of the T-jump, prior to any conformational change. This change in the intrinsic quantum yield appears as a monotonic decrease in $I_{\mathrm{D}}(T)$ in equilibrium measurements.

For AN12 samples, the T-jump amplitudes $A_{\mathrm{r}}$ were significantly smaller than the 'expected' amplitudes $A_{\mathrm{e}}$. We interpret this disparity as arising from a 'missing amplitude' in the T-jump relaxation traces as a result of rapid acceptor unstacking kinetics, on time-scales faster than the $20 \mu$ s resolution of our T-jump measurements. Consistent with this picture, we observe an initial increase in intensity immediately after T-jump, on AN12_DA DNA only samples (Fig. 4.17D) as well as AN12_DA-Rad4, at higher temperatures (Fig. 4.20), and AN12_DA-mutant Rad4 samples (Fig. 4.21C, D and Figs. 4.22 \& 23).

For AN14/AN14u samples, the amplitudes predicted from equilibrium measurements are smaller than the noise in these measurements (Figs. $4.28 \mathrm{H} \& 29 \mathrm{H}$ ), as $I_{\mathrm{D}}(T)$ and $I_{\mathrm{DA}}(T)$ are nearly overlapping in these samples (Fig. 4.25A, B). Consistent with these, the T-jump measurements show relaxation kinetics with small amplitudes (Figs.4.28 \& 29). 


\subsection{Results}

\subsubsection{Kinetics of Rad4 (wild type) induced DNA opening rate}

The crystal structure of nonspecific Rad4-DNA and the fact that DNA repair protein relies exclusively on indirect read-out and makes no direct contacts with the damaged nucleotides in the specific recognition complex suggests kinetic read-out. The nonspecific complex is structurally identical to the specific complex was unexpected in light of previous studies on multiple other proteins that had shown clear differences between the two structures [47-52], including those that used similar crosslinking approaches [49-51]. These earlier studies have been on sequence-specific DNA binding proteins or damage-repair enzymes. For these proteins, target recognition lies, at least in part, in the ability of the proteins to directly accommodate target DNA sites and reject non-target sites as structural misfits. In contrast, the XPC crystal structures reveal that such a direct structural distinction cannot be the basis for the lesion recognition by Rad4. Instead, these results suggest that the key to Rad4's recognition mechanism lies in the differential probability that Rad4 can open up a damaged versus undamaged DNA site before it diffuses away. A key determinant of the 'opening' probability is the free energy barrier encountered by Rad4 to fully open DNA to form the recognition complex, which, in turn, determines the Rad4-induced opening rate.

Using T-jump spectroscopy we have measured the Rad4 induced DNA opening rate. We used the Rad4 construct identical to the one used in crystallography studies [36]. Rad4was bound to model lesions (2- or 3-bp mismatch bubbles in two different sequence contexts) in which $2 \mathrm{AP}$ was incorporated as a fluorescent probe sensitive to DNA-opening events [53] (Table I). All equilibrium and kinetics measurements reported here were carried out on untethered Rad4-DNA Complexes. 
Table I: DNA sequences

\begin{tabular}{|l|l|}
\hline Name & Sequence \\
\hline AN3 & 5'-CGTGACTCAACATCCTATGCTACAACTCAGTGC - 3' \\
& 3'-GCACTGAGTTGTAGGTXTCGATGTTGAGTCACG - 5' \\
\hline AN4 & 5'-CGTGACTCAACATCCATAGCTACAACTCAGTGC - 3' \\
& 3'-GCACTGAGTTGTAGGTXTCGATGTTGAGTCACG - 5' \\
\hline AN21* & 5'-TTGACTCGACATCCCCCGCTACAA -3' \\
& 3'- ACTGAGCTGTAGGGCXCGATGTTA -5' \\
\hline
\end{tabular}

$\mathrm{X}$ denotes 2-aminopurine (2AP). Red indicates mismatched base pairs; green indicates the corresponding matched ones.

* The length and sequence of AN21 was chosen to match those used for structural studies (Min et al, 2007). Note that AN21 has a 2-bp mismatch rather than a 3-bp mismatch as in AN3.

Fig 4.5 and 4.6 show equilibrium and T-jump measurements on AN3 (3-bp bubble) (Fig 4.5, left) and corresponding matched DNA, AN4 (Fig 4.5, right) and AN21 (2-bp bubble) samples (Fig. 4.6). Specific binding of Rad4 to the mismatch model lesions resulted in a greater than fourfold increase in 2AP fluorescence of the DNA at equilibrium (Fig. 4.5A, left; Fig. 4.6A). While small (less than approximately twofold) increase in 2AP fluorescence can come from unstacking of 2AP from its neighbors, for example, from partial duplex melting or unwinding, we attribute the much larger increase in the lesion-bound, specific complexes as arising from Rad4-induced DNA 'opening' that results in the complete flipping out of two nucleotide pairs as seen in the crystal structures [36]. A temperature scan from 10 to $40^{\circ} \mathrm{C}$ showed that $2 \mathrm{AP}$ fluorescence of the specific, Rad4-mismatch DNA complexes increases as temperature is raised (Fig. $4.5 \mathrm{~B}$, left red), indicating that the extent of $2 \mathrm{AP}$ unstacking and/or the population of fully flipped-out conformations increases at higher temperatures. In contrast, the 2AP fluorescence intensity in mismatch DNA alone (Fig. 4.5B, left black) or in undamaged, matched DNA with or without Rad4 (Fig. 4.5B, right) decreases monotonically with increasing temperature, which reflects a temperature-dependent decrease in 2AP quantum yield. 
a
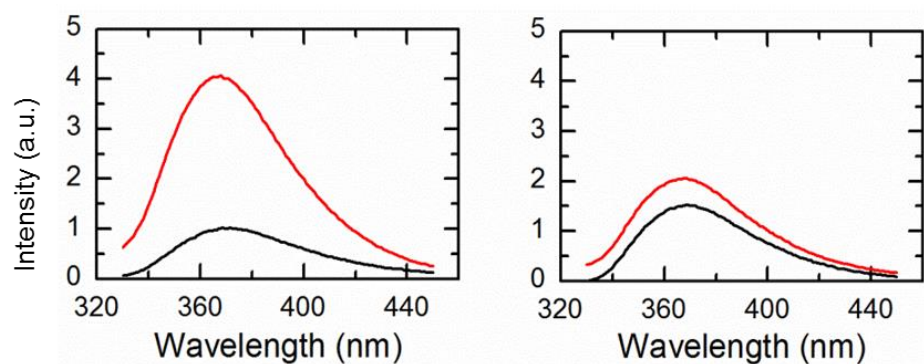

$b$
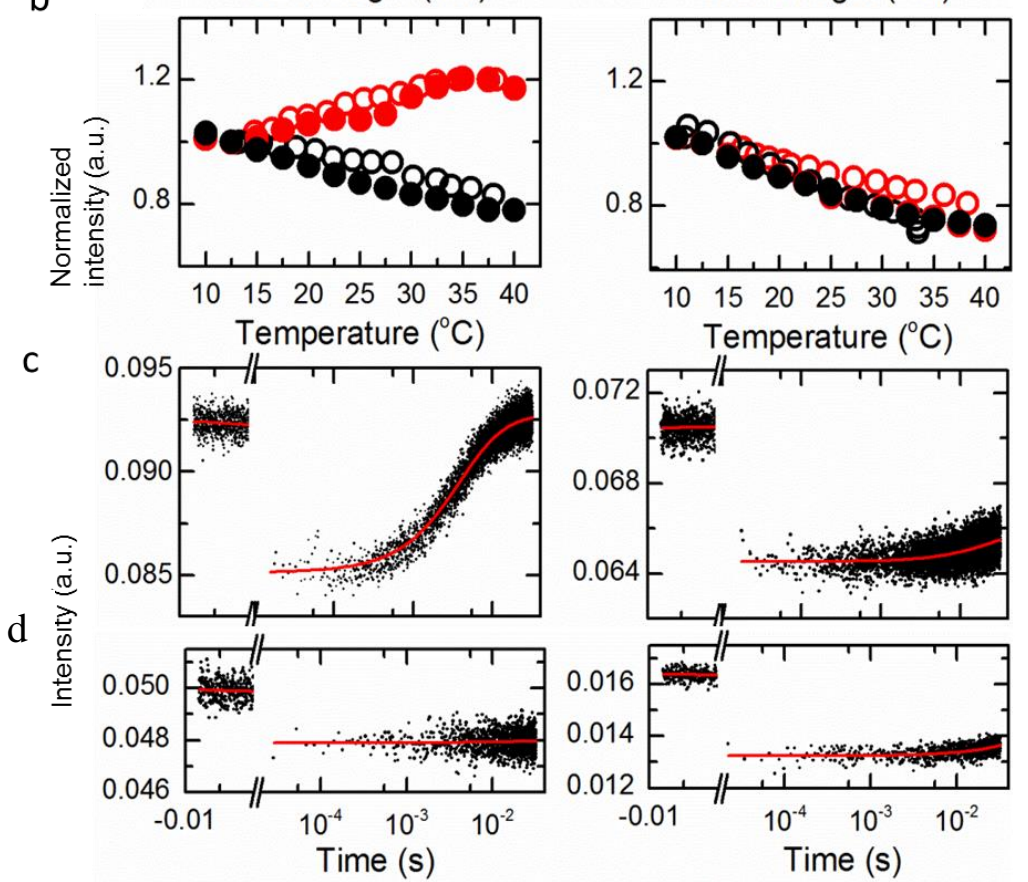

Fig. 4. 5. Rad4-induced DNA-opening dynamics measured by T-jump spectroscopy using $2 \mathrm{AP}$ fluorescence as a probe. (a) $2 \mathrm{AP}$ ( $\mathrm{X}$ in the schematic representation of DNA substrates) was placed within 3-bp mismatch DNA (AN3) and matched DNA (AN4; Table I). The 2AP fluorescence emission spectra were measured for DNA alone (black) and Rad4-DNA complexes (red) with excitation at $314 \mathrm{~nm}$ at $25^{\circ} \mathrm{C}$ (left: AN3; right: AN4). The 2AP fluorescence emission intensities increase $4.0( \pm 0.7)$-fold and $1.4( \pm 0.1)$-fold on Rad4 binding to mismatch and matched DNA, respectively. All measurements were done with untethered Rad4. Protein and DNA concentrations were $10 \mu \mathrm{M}$ each. (b) The maxima of the equilibrium 2AP fluorescence emission, measured at 365 $\mathrm{nm}$, are plotted as a function of temperature for DNA alone (black) and Rad4-DNA complexes (red; left:AN3; right: AN4). Open and filled symbols are for two independent sets of measurements on each sample. In each panel, the intensities for free DNA and DNA in complex have been normalized to match at the lowest temperature. (c) Relaxation kinetic traces measured in response to a $7^{\circ} \mathrm{C}$ T-jump show (left) single-exponential kinetics, with relaxation time $5.1 \pm 0.5 \mathrm{~ms}$ (at final temperature $26^{\circ} \mathrm{C}$ ) for Rad4-mismatch DNA, and (right) much slower kinetics, with relaxation time $190 \pm 42 \mathrm{~ms}$ (at final temperature $29^{\circ} \mathrm{C}$ ) for Rad4-matched DNA. The uncertainties in the relaxation times are sample s.d. from two sets of measurements. Note that the Kd values of Rad4 bound to mismatch or matched DNA are in nanomolar range, well below the $60 \mu \mathrm{M}$ concentrations used in the T-jump experiments. (d) Relaxation kinetics measured on the DNA-only samples: AN3 alone, in response to a $6^{\circ} \mathrm{C}$ T-jump (left), and AN4 alone, in response to a $10^{\circ} \mathrm{C}$ T-jump (right), exhibit much slower kinetics, with relaxation times $341 \pm 70$ and $240 \pm 35 \mathrm{~ms}$, respectively, consistent with the T-jump recovery kinetics. The relaxation times for samples that exhibit only the slow kinetics were determined by making measurements over a longer time window, up to $80-320 \mathrm{~ms}$. 


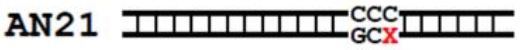

A

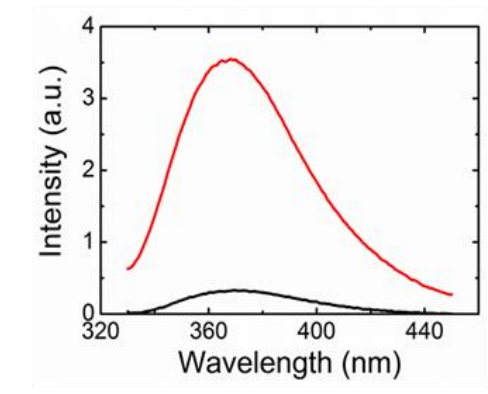

B

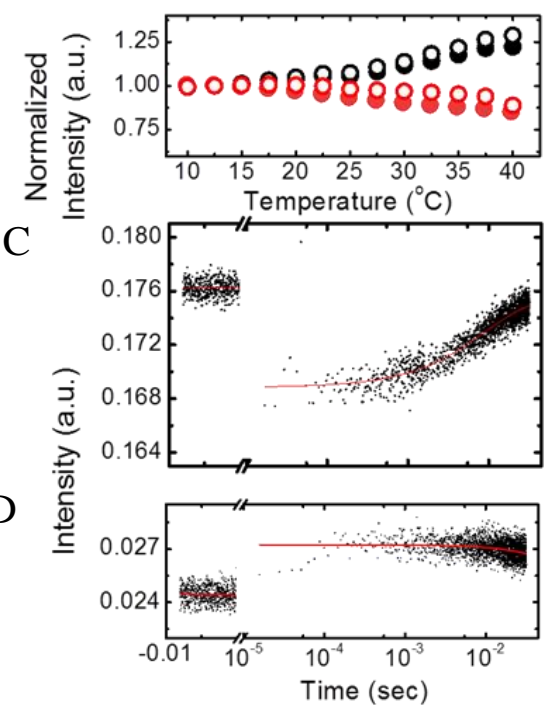

Fig. 4. 6. Sequence-context dependence of Rad4-induced DNA-opening dynamics. 2AP fluorescence measurements similar to those reported for AN3 (Fig. 4.5) were carried out for another mismatched sequence, AN21 (Table 1). AN21 contains a 2-bp mismatch and more closely resembles the sequence used in crystallographic studies. (A). The 2AP fluorescence emission spectra measured for DNA alone (black) and Rad4-DNA complex (red), with excitation at $314 \mathrm{~nm}$ at $25{ }^{\circ} \mathrm{C}$. The fluorescence emission intensities for AN21 increased 10-fold upon Rad4 binding. (B). The maxima of the 2AP fluorescence emission spectra, measured at $365 \mathrm{~nm}$ as a function of temperature, for DNA alone (black) and Rad4-DNA complex (red); the intensities are normalized to match at the lowest temperature. Open and filled symbols are for two independent sets of measurements on each sample. Notably, 2AP fluorescence in AN21 alone increases with increasing temperature, in contrast to the behavior observed in AN3, which showed a decrease in 2AP fluorescence with increasing temperature (Fig. $4.5 \mathrm{~b}$, left). Thus, in the AN21 context, 2AP in free DNA has an increased propensity to unstack as the temperature is raised. (C). Relaxation traces measured on the Rad4-DNA complex, in response to a $4{ }^{\circ} \mathrm{C}$ T-jump, show single-exponential kinetics, with relaxation time $8.0 \pm 1.9 \mathrm{~ms}$ (at final temperature $21^{\circ} \mathrm{C}$ ). The relaxation rates measured on the Rad4-AN21 and Rad4-AN3 complexes overlap (Fig.4. 8), indicating that the rate-limiting step in forming the "open" conformation is not sequence-context dependent. (D). Relaxation kinetics measured on the AN21 DNA-only sample exhibited much slower kinetics, with relaxation time $169 \pm 98 \mathrm{~ms}$, similar to T-jump recovery kinetics back to the initial temperature. The uncertainty is the sample standard deviation obtained from two independent measurements. Note that the intensity immediately after the T-jump increases for this sample, consistent with its equilibrium temperature-dependence (panel $\mathrm{B}$ ), indicating that $2 \mathrm{AP}$ unstacking is too fast to be resolved on the $\sim 5 \mu$ s time-resolution of our T-jump instrument. In contrast, for the Rad4-AN21 complex, the intensity immediately after T-jump drops (panel c), indicating that rapid $2 \mathrm{AP}$ unstacking is suppressed in the complex. 
T-jump measurements on the ensemble of Rad4-mismatch DNA complexes revealed relaxation kinetics with time constants of $5.5 \pm 0.5$ and $7.7 \pm 2.0 \mathrm{~ms}$ for the 3-bp and 2-bp mismatch constructs, respectively (interpolated at $25^{\circ} \mathrm{C}$ ), indicating that the kinetics were also sequence context independent (Fig. 4.5C, left; Fig. 4.6C). We attribute these to the Rad4induced DNA 'opening' kinetics. In contrast, measurements on Rad4 nonspecifically bound (but not tethered) to undamaged, matched DNA showed a much smaller increase in $2 \mathrm{AP}$ fluorescence on Rad4 binding and no relaxation kinetics on the millisecond timescale (Fig. 4.5 , right). Instead, the complex showed much slower kinetics on timescales characteristic of the re-equilibration of the heated sample temperature back to the initial (bath) temperature (T-jump recovery), similar to the control samples, matched and mismatch DNA in the absence of Rad4 (Fig. 4.5D; Fig. 4.6D). The apparent absence of conformational relaxation kinetics on the untethered complex with undamaged DNA was somewhat unexpected in view of the 'open' crystal structure of the tethered complex. The absence of kinetics can arise from heterogeneous binding registers (since Rad4-induced opening of DNA at a site some distance away from the fixed 2AP site will not be reported by 2AP fluorescence change) and/or slow relaxation kinetics not resolved in the T-jump time window.

\subsubsection{Interaction between Rad4 mutant and DNA}

Structural studies have shown that the $\beta$-hairpin in the $\beta$-hairpin domain 3 (BHD3) inserts into the DNA duplex on forming the 'open' conformation (Fig. 4.3; [36]); this $\beta$-hairpin is also shown to be important in the ability of $\operatorname{Rad} 4 / \mathrm{XPC}$ to recognize DNA lesions in biochemical and cellular studies (Table II, [54] [55]). 
Table II: $\mathrm{K}_{\mathrm{d} \text {,app }}$ and $\mathrm{R}^{2}$ for different DNA constructs. (From Xuejing Chen)

\begin{tabular}{|c|c|c|c|c|}
\hline & $\begin{array}{c}\text { Full-length } \\
\text { Rad4 }\end{array}$ & $\begin{array}{c}\text { Truncated } \\
\text { V131C/C132S }\end{array}$ & $\Delta \beta$-hairpin3 & $\Delta$ BHD3 \\
\hline $\mathrm{K}_{\mathrm{s}}(\mathrm{nM})$ & $45 \pm 4$ & $31 \pm 3$ & $280 \pm 8$ & $293 \pm 8$ \\
\hline $\mathrm{R}^{2}$ & 0.954 & 0.958 & 0.987 & 0.986 \\
\hline $\mathrm{K}_{\mathrm{ns}}(\mathrm{nM})$ & $430 \pm 45$ & $363 \pm 45$ & $626 \pm 57$ & $650 \pm 56$ \\
\hline $\mathrm{R}^{2}$ & 0.956 & 0.964 & 0.967 & 0.965 \\
\hline
\end{tabular}

To check whether the double mutations V131C/C132S introduced for crosslinking affects the intrinsic DNA binding of the Rad4-Rad23 complex used for crystallization, the DNA binding of this mutant was tested and compared with the full-length Rad4-Rad23 complex using electrophoretic mobility shift assays (EMSA) by our collaborator Xuejing Chen/ Prof. Min lab( Min et al, 2007). The DNA-binding activities of the $\beta$-hairpin mutants used in T-jump studies ( $\triangle$ BHD3 and $\Delta \beta$-hairpin3) were also tested and compared. While the full-length Rad4-Rad23 complex contained full-length $\operatorname{Rad} 23$, all other $\operatorname{Rad} 4$ constructs were expressed with Rad23 truncated as described (Min et al, 2007).

Kd values for specific binding to mismatch DNA (Ks) and for nonspecific binding for undamaged DNA (Kns) were obtained as described1. Solid lines indicate the fit curves of the data points of the same color. Note that the presence of single 5'-T/A overhangs in the DNA duplexes used in this study did not significantly affect the Ks or Kns compared with those of analogous DNA constructs with blunt-ends used previously (Min et al, 2007), also consistent with other studies (Sugasawa K, Shimizu Y, Iwai S, Hanaoka F. (2002) A molecular mechanism for DNA damage recognition by the xeroderma pigmentosum group $\mathrm{C}$ protein complex. DNA Repair 1, 95-107 ). The data show that V131C/C132S mutations did not affect DNA binding of Rad4. The $\triangle$ BHD3 and $\Delta \beta$-hairpin3 mutants, however, exhibit significantly weakened binding to mismatch DNA while retaining affinities to undamaged DNA, resulting in significant loss in lesion recognition specificity.

To probe what role the $\beta$-hairpin plays in the observed increase in 2AP fluorescence in the mismatched DNA on Rad4 binding, and in the relaxation kinetics when perturbed by T-jump, we have performed corresponding 2AP fluorescence measurements with mutant Rad4 (Fig. 4.7) that lack either the $\beta$-hairpin in BHD3 ( $\Delta \beta$ hairpin3, missing residues $599-605$ indicated in blue in Fig. 4.3B,C) or the entire BHD3 domain ( $\triangle \mathrm{BHD} 3$, missing residues $541-632$ indicated in red in Fig. 4.3B,C). The 2AP fluorescence of the mismatched DNA in complex with either of the Rad4 mutants shows temperature dependence very similar to that of free 
DNA (Fig. 4.7B). These results contrast with the behavior of the wild- type Rad4mismatched DNA complex, which showed an increase in fluorescence emission intensity with increasing temperature (Fig. 4.5B, left). Furthermore, no relaxation kinetics (other than the T-jump recovery kinetics) was observed with these $\beta$-hairpin mutants (Fig. 4.7C). The results indicate that the $\beta$-hairpin is indeed critical for forming and stabilizing the 'open' conformation, consistent with other studies (Table II; [36, 54]).

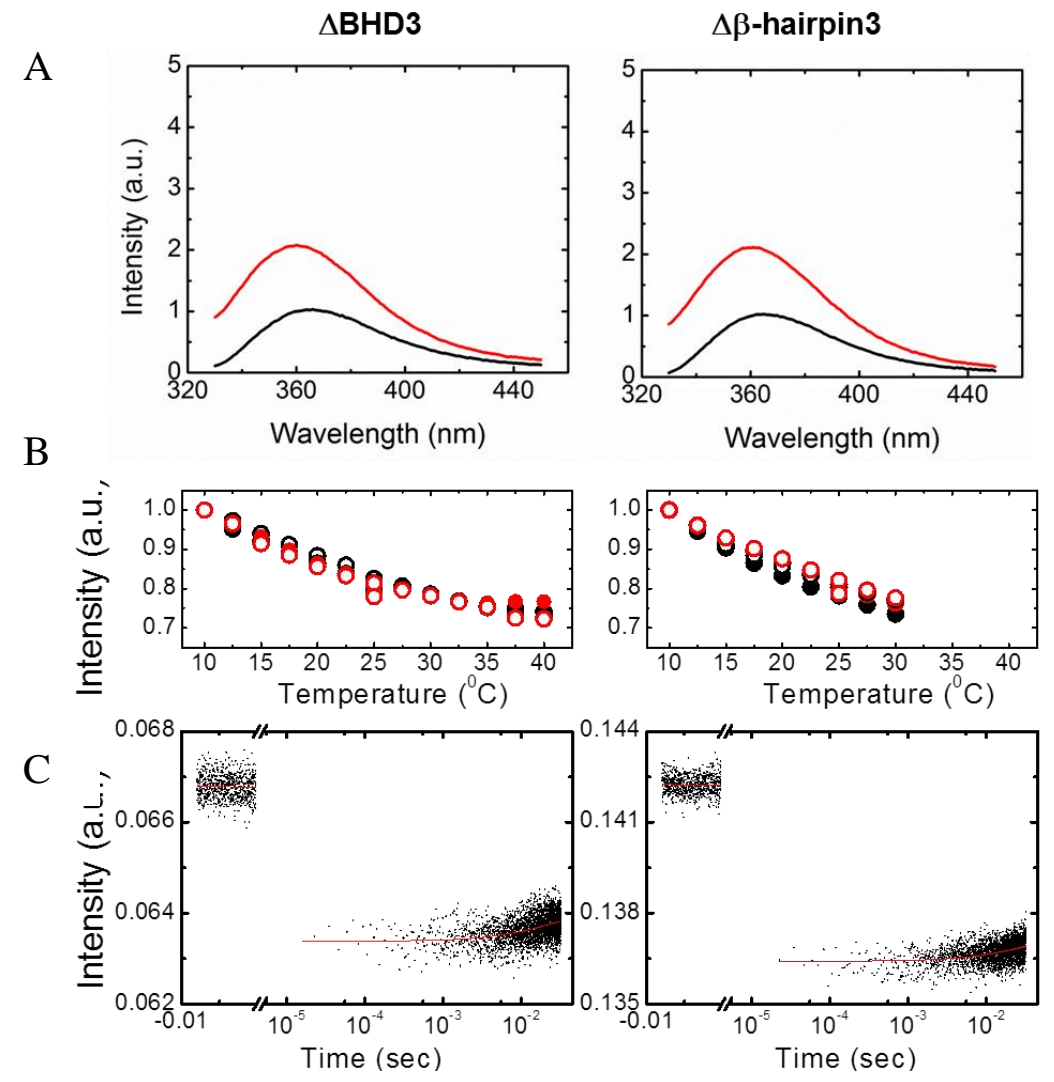

Fig. 4.7. 2AP fluorescence and T-jump measurements to examine Rad4-induced DNA-opening dynamics with Rad4 $\beta$-hairpin mutants. Equilibrium and relaxation kinetics measurements were carried out on 3-bp mismatch DNA (AN3) bound to two $\beta$-hairpin mutants of Rad4, $\triangle$ BHD3 (left) and $\Delta \beta$-hairpin3 (right). (a) The 2AP fluorescence emission spectra measured for DNA alone (black) and Rad4-DNA complexes (red) with excitation at $314 \mathrm{~nm}$ at $25^{\circ} \mathrm{C}$. The 2AP fluorescence emission intensities of AN3 DNA increase $2.1( \pm 0.1)$-fold and $2.3( \pm 0.1)$-fold on binding of $\triangle \mathrm{BHD} 3$ and $\Delta \beta$ hairpin3, respectively, compared with DNA alone. (b) The maxima of the equilibrium 2AP fluorescence emission measured at $365 \mathrm{~nm}$ are plotted as a function of temperature for DNA alone (black) and Rad4-DNA complexes (red). The open and filled symbols are for two independent sets of measurements on each sample. The intensities are normalized to match at the lowest temperature. (c) T-jump measurements on the $\beta$-hairpin mutants of Rad4-DNA complexes do not show any relaxation kinetics other than the slow recovery of the T-jump itself. 


\subsubsection{Comparison of DNA opening rate/ Nucleotide flipping rate with Iminio -Proton exchange measurements of basepair opening rate.}

The observed Rad4-induced DNA-opening kinetics of $\sim 7 \mathrm{~ms}$ on mismatched DNA are almost tenfold slower than the spontaneous base-pair 'breathing' measured even for a single (GT) mismatch (Fig. 4.8) [56].

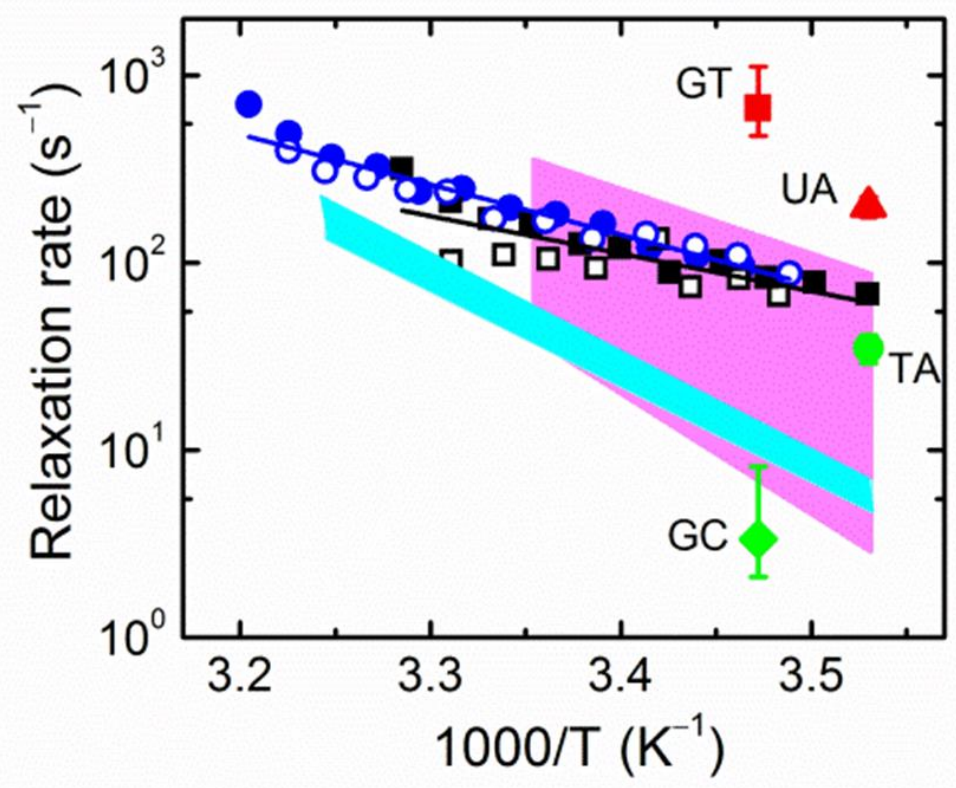

Fig. 4. 8. Arrhenius plots of relaxation rates measured on Rad4-mismatched DNA complexes. Relaxation rates obtained from T-jump measurements on Rad4-mismatch DNA (AN3 in blue circles and AN21 in black squares) are plotted versus the inverse of the final temperature after the T-jump. The open and filled symbols are for two independent sets of measurements on each sample. The continuous (blue and black) lines are an Arrhenius fit to the relaxation rates for each sample; the activation enthalpies obtained from the Arrhenius fit are $12.1 \pm 2.5$ and $7.9 \pm 4.7 \mathrm{kcal} \mathrm{mol}^{-1}$, respectively, for the two sequences, and the relaxation times interpolated at $25^{\circ} \mathrm{C}$ are $5.5 \pm 0.5$ and $7.7 \pm 2.0 \mathrm{~ms}$, respectively. The uncertainties in the activation enthalpies and relaxation times are sample s.d. obtained from two independent sets of measurements. The pink (cyan) shaded regions represent the range of base-pair opening ('breathing') rates for $A / T(G / C)$ base pairs, from NMR imino proton exchange measurements of Coman and Russu [57]. The opening rates and their ranges are also indicated for G/T mismatch (red square) [56], U/A mismatch (red triangle) [48], T/A base pair (green circle) [48], and G/C base pair (green diamond) [56](figure reproduced from [38].

Similar Rad4-induced DNA-opening rates were also observed with full-length Rad4 complex on the 3-bp mismatched DNA (Fig. 4.9). These results show that the free energy barrier for forming the 'open' structure is higher than for the smaller 'breathing' motions, and thus indicate that the rate-limiting transition state for 'opening' entails extensive structural 
deformation around the lesion. Notably, NER lesions often weaken base-pair hydrogenbonding and stacking interactions and render the DNA more deformable with increased propensity for local bending/unwinding/nucleotide flipping [1]. Thus, the free energy barrier for Rad4-induced opening of undamaged DNA is expected to be even higher compared with damaged DNA. A recent simulations study estimated that the free energy barrier for flipping out normal DNA could be 5-8 $\mathrm{kcal} \mathrm{mol}^{-1}$ higher than for a bulky lesion [58] (fig. 4.10). Assuming that this entire difference appears in the free energy barrier for Rad4-induced DNA-opening kinetics, the opening times for normal DNA bases could well be 4,000-fold longer than the $\sim 7 \mathrm{~ms}$ opening time observed for our model lesion. Taken together, our present study strongly suggests that the kinetic barriers for forming the 'open' conformation are key to distinguishing damaged versus undamaged DNA, although the final 'open' structures appear to be nearly the same for both DNA. Figure 4. 11A illustrates this notion. In this schematic, the free energies of the final 'open' structures, as well as the corresponding transition states for forming such structures, are assumed to be very similar for the damaged and undamaged DNA. However, the free energies when Rad4/XPC loosely and nonspecifically interacts with the DNA in the 'search' mode [59] are assumed to differ by an amount equal to the free energy difference between undamaged and damaged free DNA. Although an experimental structure of Rad4/XPC in an untethered, bona fide search mode is lacking, one can anticipate that the DNA duplex in this mode remains largely in the conformation of free DNA (as modelled in Fig. 4. 11B). Consequently, the higher free energy barrier for Rad4/XPC 'opening' undamaged versus damaged DNA mainly arises from the lower free energy of the Rad4/XPC-normal DNA complex in the search mode that reflects the higher stability of normal DNA than that of damaged DNA. 


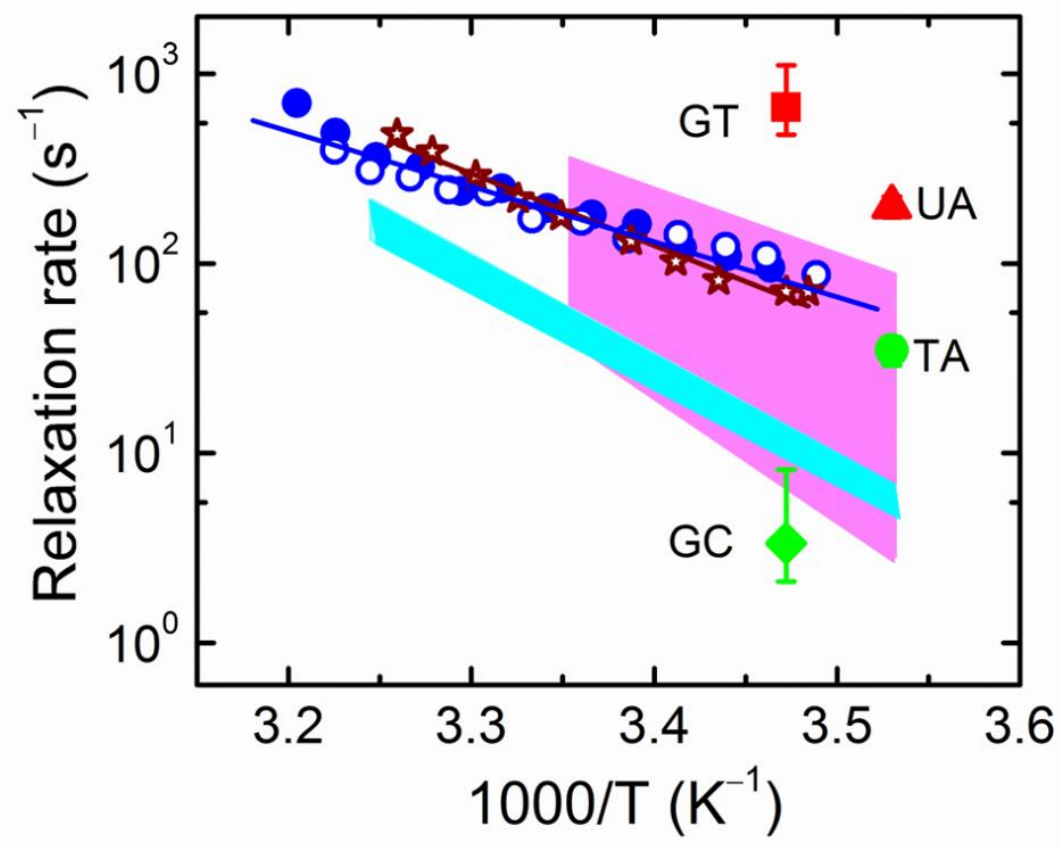

Fig. 4. 9. Arrhenius plots of relaxation rates measured on full length Rad4 in complex with mismatch DNA. Relaxation rates obtained from T-jump measurements carried out with full-length Rad4 bound to mismatch DNA substrate (AN3; brown stars) are plotted versus the inverse of the final temperature (after the T-jump). The blue circles are the relaxation rates measured with the truncated $\operatorname{Rad} 4$ construct [36] as shown in Fig.4.8, with the open and filled circles indicating two independent sets of measurements. The continuous (brown and blue) lines are an Arrhenius fit to the relaxation rates for each sample. The pink (cyan) shaded regions represent the range of base-pair opening ("breathing") rates for $\mathrm{A} / \mathrm{T}(\mathrm{G} / \mathrm{C})$ base pairs, from NMR imino proton exchange measurements of Coman and Russu [57]. The opening rates and their ranges are also indicated for G/T mismatch (red square) [56]4 and U/A mismatch (red triangle) [48], T/A base pair (green circle) [48] and G/C base pair (green diamond) [56]. These descriptions are the same as in the legend to Fig.4. 8. 

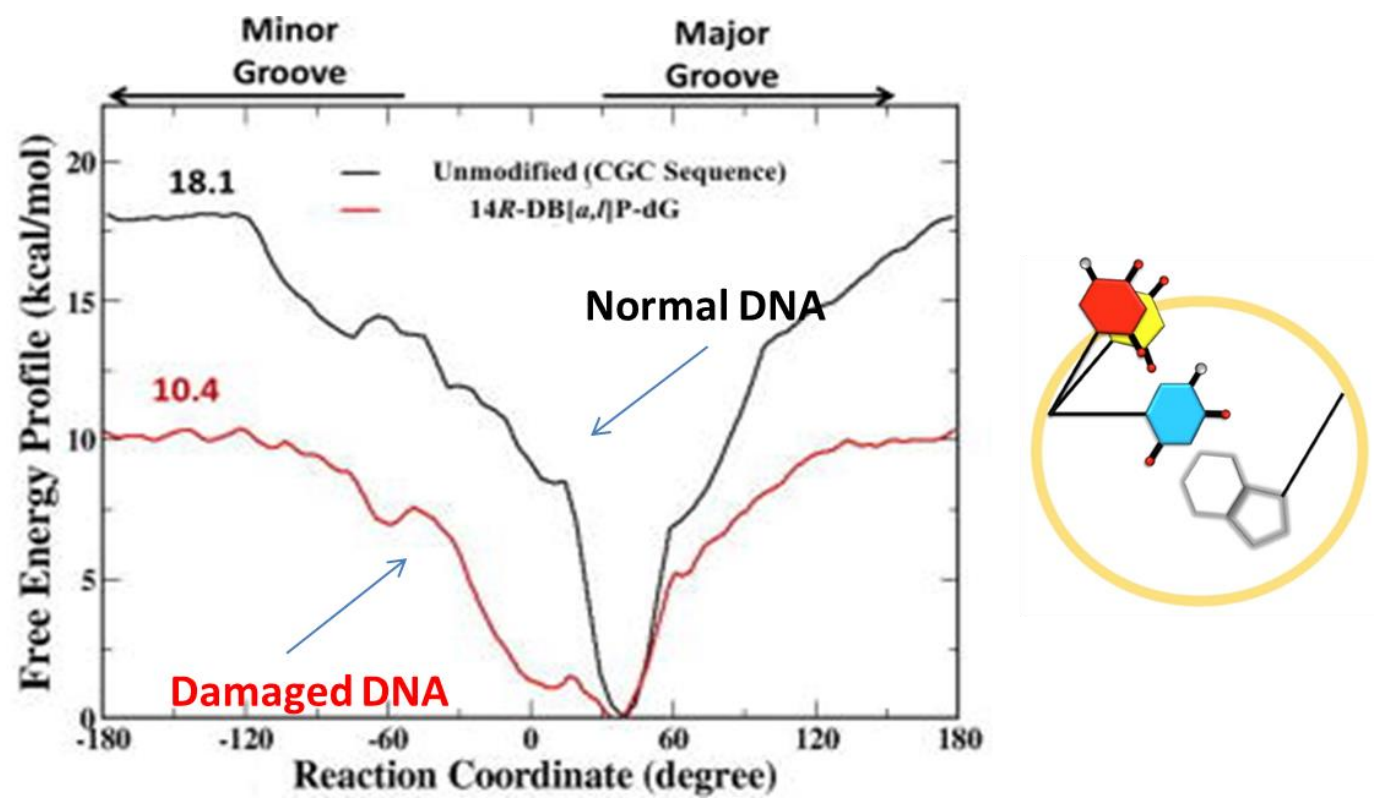

Fig. 4. 10. Calculated free energy profiles for base pair opening of a damaged base (red) and for an unmodified base. The barrier difference is $7.7 \mathrm{kcal} \mathrm{mol}^{-1}$. Figure reprinted with permission from Zheng, H. et al. (2010) Chem. Res. Toxicol 23, 1868-1870; copyright (2010) American Chemical Society.

\subsubsection{Interplay between residence time and opening time.}

Given a free energy barrier to open a DNA site, the overall probability of Rad4 opening the site also depends on whether this barrier can be overcome by Rad4 before it steps to the next site or falls off, that is, the residence time of $\operatorname{Rad} 4$ at the site. The opening probability per site then can be expressed as the ratio of the opening time $\left(t_{o p}\right)$ and the residence time $\left(t_{r e s}\right)$, and this probability increases exponentially as the ratio decreases: $P_{o p e n}=1-\exp \left(t_{r e s} / t_{o p}\right)$. In the structural study, chemical tethering of Rad4 on DNA prolonged Rad4's residence time indefinitely, thus allowing even undamaged DNA to be fully opened. However, under untethered conditions, the protein is free to diffuse away from a given site, thus decreasing the residence time per site. If $t_{r e s} \ll t_{o p}$, this opening probability is likely to be small. While there are no direct measurements of the residence time for Rad4/XPC on DNA, several measurements have characterized diffusion of DNA repair proteins on undamaged, nonspecific DNA. These measurements yield a wide range of residence times per base pair, 
$0.1 \mathrm{~ms}$ to $0.3 \mathrm{~ms}$ (Table III) [60-62], all of which are significantly shorter than the Rad4induced opening time expected for undamaged DNA. This estimate for opening normal DNA is based on the $7 \mathrm{~ms}$ opening time that we measured for mismatch DNA at $25^{\circ} \mathrm{C}$ (Fig. 4.8) and the simulations study [58] that suggests significantly larger free energy barrier for flipping out normal bases in matched DNA (as illustrated in Fig. 4.10 \& 11). Assuming that the residence time of Rad4 on undamaged DNA falls in the submillisecond range, the probability that Rad4 will open a normal site is expected to be exceedingly small. Although it remains to be determined, we speculate that the presence of an NER lesion that distorts or destabilizes DNA not only decreases the opening time, but may also increase the residence time of Rad4/XPC on the lesion compared with an undamaged site, further contributing to the selective opening and thus recognition of NER lesions. On the other hand, certain carcinogen adducts resistant to NER $[1,63]$, may evade detection in part because they present very high free energy barriers for being opened by $\operatorname{Rad} 4$ compared with its residence time, and thus their opening probability may be similar to or worse than that of undamaged DNA.

Table III : Diffusion constants (D) and residence times per base of various DNA-repair proteins on normal DNA duplexes.

\begin{tabular}{|l|l|l|l|}
\hline Protein & $\mathrm{D}\left(\mathrm{m}^{2} \mathrm{~s}^{-1}\right)$ & $\mathrm{D}\left(\mathrm{bp}^{2} \mathrm{~s}^{-1}\right)$ & Residence time $(\mu \mathrm{s})$ \\
\hline HUNG $^{1}$ & & $6 \times 10^{3}$ & 83 \\
\hline hOGG1 $^{2}$ & $6 \times 10^{-13}$ & $5 \times 10^{6}$ & 0.1 \\
\hline MutM $^{2}$ & $5 \times 10^{-14}$ & $4 \times 10^{5}$ & 1.4 \\
\hline Msh2-Msh6 $^{3}$ & $9 \times 10^{-14}-2 \times 10^{-16}$ & $8 \times 10^{5}-2 \times 10^{3}$ & $0.6-289$ \\
\hline
\end{tabular}

${ }^{1}$ Schonhoft JD, Stivers JT. (2012) Timing facilitated site transfer of an enzyme on DNA. Nat Chem Biol 8, 205-210

${ }^{2}$ Blainey PC, van Oijen AM, Banerjee A, Verdine GL, Xie XS. (2006) A base-excision DNArepair protein finds intrahelical lesion bases by fast sliding in contact with DNA. Proc Natl Acad Sci U S A 103, 5752-5757

${ }^{3}$ Gorman J, et al. (2007) Dynamic basis for one-dimensional DNA scanning by the mismatch repair complex Msh2-Msh6. Mol Cell 28, 359-370 
As a first step to characterize Rad4/XPC recognition dynamics, we measured the Rad4-induced 'opening' of mismatched DNA using T-jump perturbation approach and showed that it occurred on time scales of $\sim 5-10 \mathrm{~ms}$. The 'opening' times for more stable, undamaged/matched DNA are expected to be much longer than for mismatched DNA, thus many orders of magnitude longer than the microsecond-regime residence times per DNA site reported for various proteins [60-62, 64-67]. This, we proposed, explains how Rad4/XPC avoids flipping 'open' every undamaged site when searching DNA for a lesion, while having selectively higher probabilities to open helix-destabilizing NER lesion sites. However, this 'kinetic gating' also posed a dilemma as to how Rad4 reliably recognizes damaged DNA if the recognition $(\sim 5-10 \mathrm{~ms})$ is much slower than the typical diffusion of proteins on DNA during nonspecific 'search'. 
a
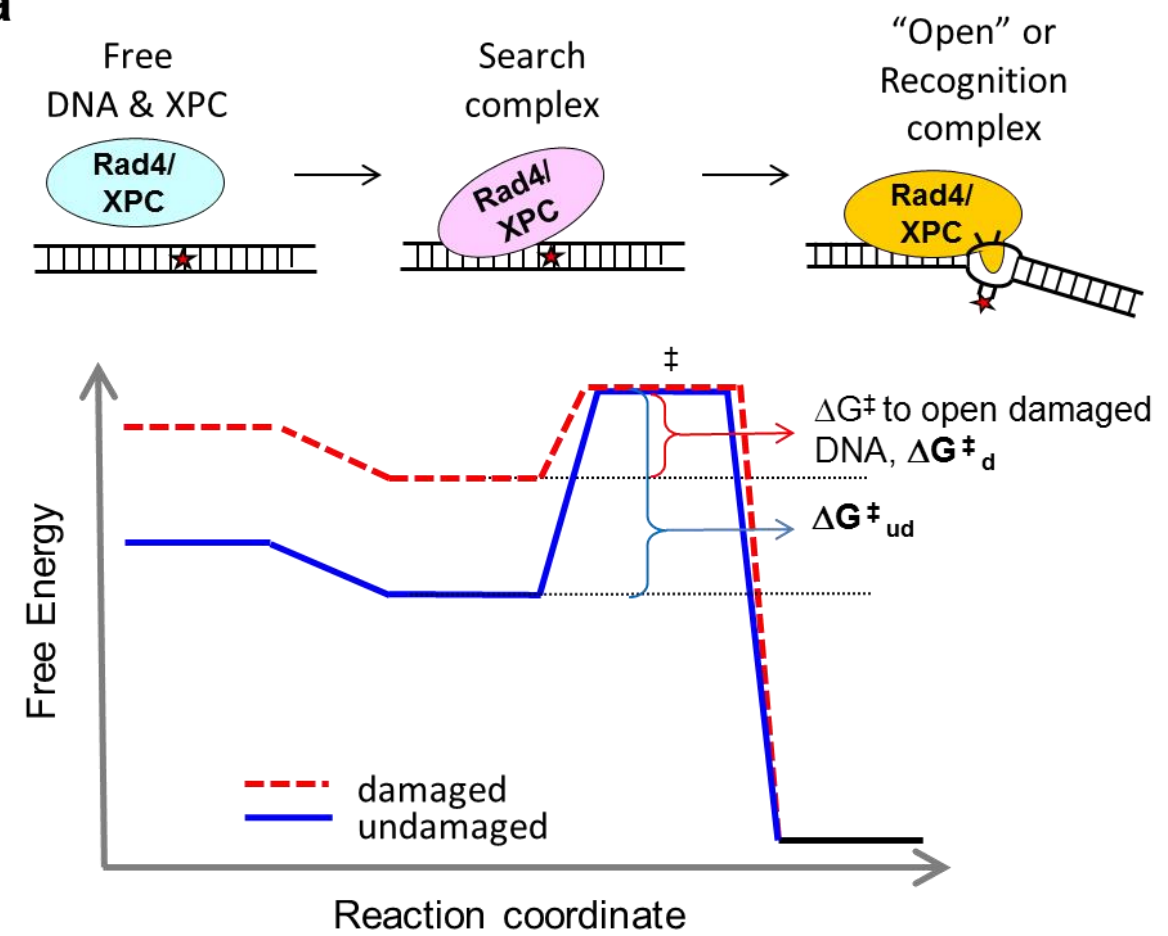

b
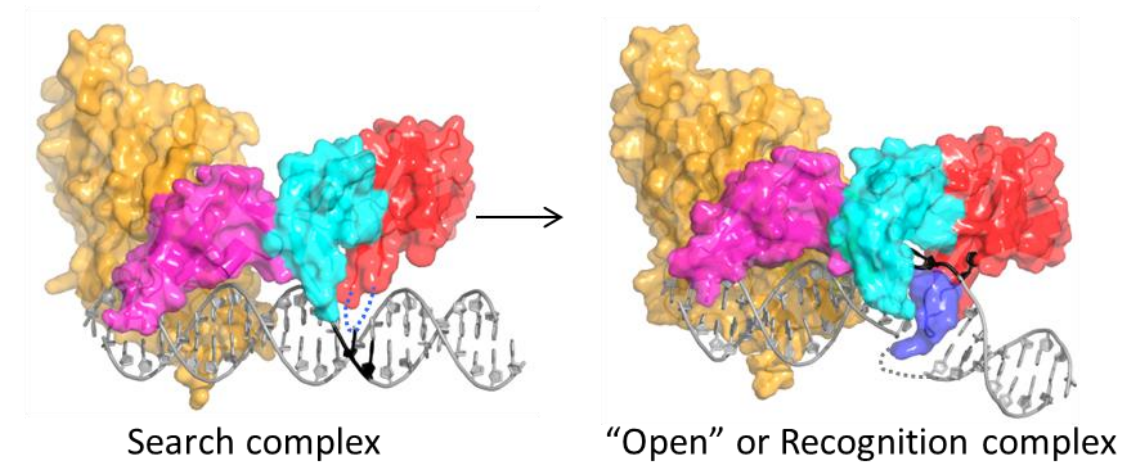

Fig. 4. 11. Free energy profile for DNA opening by Rad4/XPC at damaged and undamaged sites. (a) A schematic representation of the free energy profile of the Rad4/XPC-induced DNA-opening process is shown: free Rad4/XPC is indicated in cyan, Rad4/XPC in the 'search' mode in pink and in the 'open' recognition complex in orange. The free energy surface for opening damaged DNA sites is in red and that for undamaged sites is in blue. $\Delta \mathrm{G}_{\mathrm{d}}^{\ddagger}$ and $\Delta \mathrm{G}_{\text {ud }}^{\ddagger}$ denote the free energy barriers to open damaged and undamaged sites in the complex, respectively. (b) Structural models for Rad4 in a 'search' mode (left) and in a recognition complex (right). Here we used the apo-Rad4-Rad23 structure (PDB code 2QSF) [36] to model Rad4 nonspecifically bound to DNA in a search mode. The $\beta$-hairpin in BHD3 in this conformation can be flexible (blue dotted line), while the DNA duplex largely retains the B-DNA conformation. On the contrary, the $\beta$-hairpin in the recognition complex is inserted into the DNA duplex (blue surface representation) and stabilizes the 'open' structure of the DNA. The 'open' complex is formed with damaged DNA [36] and also with undamaged DNA when Rad4's residence time is prolonged. 


\subsection{2 $\mathbf{t C}^{\circ}$ and $\mathbf{t C}_{\text {nitro }}$ FRET pair as probes for sensing changes in DNA helical structure.}

Next we tried on capturing kinetic intermediates that could bridge the gap between the fast, nonspecific search and the slow recognition of the specific damaged site. Taking advantage of the T-jump approach with the unique sensitivity of novel Förster resonance energy transfer (FRET) pair (donor, $\mathrm{tC}^{\mathrm{o}}$, acceptor $\mathrm{tC}_{\text {nitro }}$ ) to DNA helicity $[41,68,69]$ here, we aimed at probing motions that affect regions beyond the mismatched nucleotides such as local unwinding around the lesion, as observed in the 'open' structures (Figs. 4. 13A \& 14A). To monitor these longer range motions, we adopted a pair of recently developed FRET probes, $\mathrm{tC}^{\mathrm{o}}$ and $\mathrm{tC}_{\text {nitro }}$ (Fig. 4.13B) $[41,42]$. These FRET probes are tricyclic cytosine analogs that retain Watson-Crick-type pairing with guanines in duplex DNA; they also allow favourable base-stacking interactions with neighbouring bases. The melting temperatures of labeled DNA duplexes show that the probes do not compromise the overall DNA duplex stability [47]. Importantly, the FRET efficiency of the probes depends on their relative orientation as well as the distance between them; this feature is exhibited by an oscillatory trend as a function of separation of the probes within a DNA duplex, which reflects the helical periodicity of B-DNA [47] (Fig. 4. 13C). Such unique characteristics of these probes distinguish them from most conventional FRET probes typically tethered to DNA through flexible linkers. Though it has been shown that some of these linker-attached FRET probes can be responsive to DNA helicity when stacked at the ends of DNA duplexes [70], these probes are not readily incorporated as rigid internal labels within duplex DNA without disrupting the DNA backbone/structure [71], as is possible with the $\mathrm{tC}^{\mathrm{o}} / \mathrm{tC}_{\text {nitro }}$ probes $[41,42]$. 


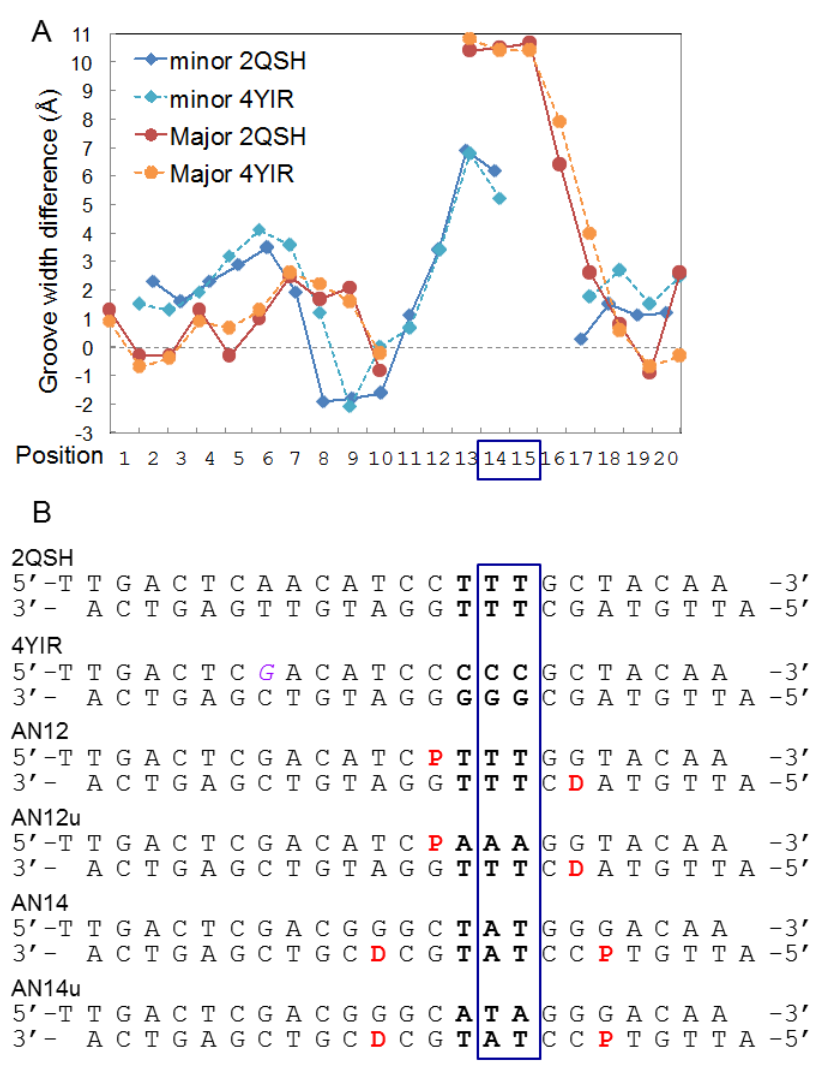

Fig. 4. 12. The DNA groove widths in Rad4-bound DNA structures and the DNA construct sequences. (A) The widths of major and minor grooves of the DNA in the 'open' recognition complex structures (2QSH [36], 4YIR [38]) were determined using DNA backbone phosphate-tophosphate distances, as described in ref. [72]. The differences between the measured value and an average value from idealized B-DNA (11.5 ̊ for minor groove, $17.2 \AA$ for major groove) are plotted. The flipped-out nucleotide pairs in the 'open' structure correspond to positions 14 and 15 in this plot (rectangular outline); the area flanking these nucleotide pairs show the largest positive deviations, indicating unwinding of the duplex DNA. (B) The sequences of the DNA constructs used in the structural studies (2QSH, 4YIR) and AN12/AN12u and AN14/AN14u used in this study. Bold letters indicate mismatch nucleotide pairs or the corresponding positions in matched DNA. The positions of the flipped-out nucleotide pairs seen in the 'open' structures are indicated with rectangular outline as in panel A. ' $G$ ' indicates the position of disulfide-modified nucleotide used for chemical tethering in 4YIR(4). 'D' in AN12/AN12u and AN14/AN14u indicates the position of the donor (tCo) and ' $\mathrm{P}$ ' the position of the acceptor $\left(\mathrm{tC}_{\text {nitro }}\right)$.

These characteristics make these probes a suitable choice to capture protein-induced DNA motions involving changes in its helicity, such as local unwinding. Accordingly, we incorporated the $\mathrm{tC}^{\mathrm{o}} / \mathrm{tC}_{\text {nitro }}$ probes on either side of the mismatch site, spanning the region expected to undergo most dramatic unwinding upon Rad4-binding (Fig. 4.12). These probes are not recognized by Rad4 as DNA lesions (see below). 


\subsubsection{1 $t C^{o} / t C_{n i t r o}-l a b e l e d$ TTT/TTT-mismatched DNA (AN12) as a model lesion for specific binding.}

First, we tested a 24-bp DNA duplex sequence containing a TTT/TTT mismatch (AN12) as a model lesion for Rad4, and its undamaged/matched counterpart (AN12u). TTT/TTTmismatch has been shown to form an 'open' structure in crystals when bound to Rad4 (Fig. 4.12, 4.13) [36]. In these constructs, tCnitro was placed immediately abutting the mismatch site while tCo was placed one base pair away from the other side of the mismatch bubble (Fig. 4.14A, B). The melting temperatures (Tm) of the mismatched AN12 DNA were $8-11{ }^{\circ} \mathrm{C}$ lower than those of the corresponding undamaged AN12u counterparts, as expected from the destabilization caused by the mismatched bases (Fig. 4.12 and table IV). Introducing FRET labels affected the $\mathrm{T}_{\mathrm{m}}$ for each construct by less than $2{ }^{\circ} \mathrm{C}$, compared with unlabelled DNA (containing cytosines in place of the labels), indicating that the FRET probes do not significantly alter the thermodynamic stability of AN12 or AN12u ( Methods, section 4.2.3; Table IV\&V).

Table IV: Melting temperature of AN12 and AN12U, (From Xuejing Chen)

\begin{tabular}{|l|c|c|c|}
\hline & NL & D & DA \\
\hline AN12 & $62.5 \pm 0.4$ & $60.7 \pm 0.4$ & $61.7 \pm 0.2$ \\
\hline AN12u & $70.2 \pm 0.1$ & $70.6 \pm 0.2$ & $72.5 \pm 0.3$ \\
\hline
\end{tabular}

Table V: Melting temperature of AN14 and AN14U (From Xuejing Chen)

\begin{tabular}{|l|c|c|c|}
\hline & NL & D & DA \\
\hline AN14 & $68.8 \pm 0.3$ & $71.8 \pm 0.1$ & $73.4 \pm 0.5$ \\
\hline AN14u & $75.3 \pm 0.3$ & $78.3 \pm 0.4$ & $81.0 \pm 0.1$ \\
\hline
\end{tabular}




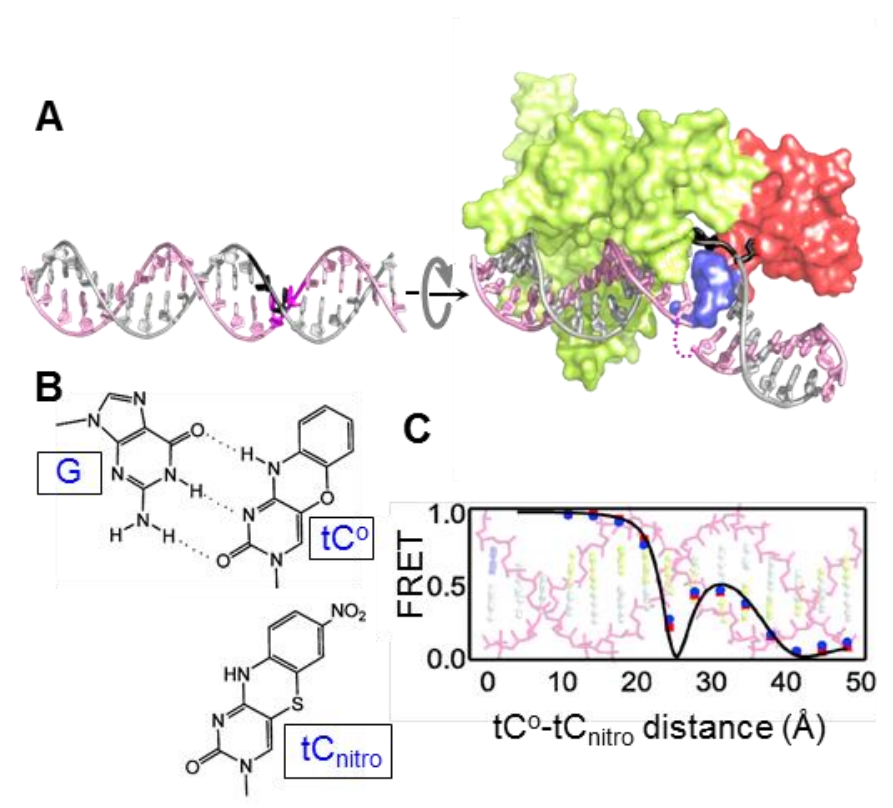

Fig. 4. 13. DNA conformational rearrangements during lesion recognition by Rad4 probed by $\mathbf{t} \mathbf{C}^{\mathbf{0}} / \mathbf{t C}_{\text {nitro }}$. (A) Structural models are shown for an ideal B-form DNA (left) and Rad4-bound specific, lesion recognition ('open') complex (right, PDB code: 2QSH) [36]). The gray rotation arrow indicates the direction of DNA unwinding upon Rad4-induced 'opening'. The DNA-binding domains of Rad4 are shown: TGD-BHD1-BHD2 in lime green; BHD3 in red and the $\beta$-hairpin within BHD3 in blue. In the 'open' complex, the flipped-out nucleotides on the undamaged strand (black) make specific contacts with Rad4, while those on the damaged strand (magenta) do not contact Rad4 and become disordered (dotted magenta line). (B) Chemical structures of tCo and tCnitro cytosine analogs and Watson-Crick type base pairing of tCo with a guanine (G). (C) FRET efficiency between the tCo (donor) and tCnitro (acceptor) incorporated within normal B-DNA is plotted as a function of the distance between the two probes. FRET generally decreases as the distance increases, but additionally depends on the relative orientations of the absorption and emission dipoles of the fluorophores. Figures in (B,C) are adapted with permission from permission from Borjesson et al. [41] ; copyright (2009) American Chemical Society

Next, the relative binding affinities of $\mathrm{AN} 12$ and $\mathrm{AN} 12 \mathrm{u}$ to Rad4 using gel--shift (or electrophoretic mobility shift) assays (Methods, section 4.2.4 \& table VI) were measured by Xuejing Chen/ Prof.Min's lab. The wild-type Rad4 construct used in this study spans residues 101-632 and contains all four domains involved in DNA-binding. This construct has previously been shown to exhibit the same DNA binding characteristics as the fulllength protein [36]. The presence of $\mathrm{tC}^{\mathrm{O}} / \mathrm{tC}_{\text {nitro }}$ labels in the $\mathrm{AN} 12 / \mathrm{AN} 12 \mathrm{u}$ did not affect the Rad4-binding affinities of each DNA compared with unlabeled DNA. However, regardless of the labels, the mismatch-containing AN12 constructs showed about 4-fold lower 
apparent dissociation constant $\left(\mathrm{K}_{\mathrm{d}, \mathrm{app}}\right)$ than the corresponding undamaged $\mathrm{AN} 12 \mathrm{u}$ or other undamaged DNA constructs. Although this specificity of AN12 is slightly lower than the 7-fold specificity exhibited by another DNA construct containing CCC/CCC mismatches (CH10_NX, Table VI) [38], the results indicate that the TTT/TTT mismatch in AN12 is specifically recognized by Rad4 (as also expected from the crystal structure) while AN12u is bound nonspecifically. The small differences in the apparent binding affinities between damaged and undamaged DNA are consistent with other studies for human XPC protein when using short duplex oligonucleotides as substrates, with likely contributions from end-binding $[73,74]$.

Table VI: $\mathrm{K}_{\mathrm{d} \text {,app }}$ and $\mathrm{R}^{2}$ for different DNA constructs. (From Xuejing Chen)

\begin{tabular}{|l|c|c|}
\hline & $\mathrm{K}_{\mathrm{d} \text { app }}(\mathrm{nM})$ & $\mathrm{R}^{2}$ \\
\hline $\mathrm{CH} 10 \_\mathrm{NX}$ & $54 \pm 1$ & 0.996 \\
\hline $\mathrm{CH} 7 \_\mathrm{NX}$ & $367 \pm 19$ & 0.992 \\
\hline AN12_NL & $114 \pm 4$ & 0.980 \\
\hline AN12_D & $101 \pm 3$ & 0.987 \\
\hline AN12_DA & $106 \pm 3$ & 0.988 \\
\hline AN12u_NL & $411 \pm 17$ & 0.964 \\
\hline AN12u_D & $384 \pm 11$ & 0.983 \\
\hline AN12u_DA & $358 \pm 10$ & 0.985 \\
\hline
\end{tabular}

\begin{tabular}{|c|c|}
\hline CH10_NX: & $\begin{array}{l}5^{\prime}-\text { TTGACTCGACATCCCCCGCTACAA - }-3^{\prime} \\
3^{\prime}-\text { ACTGAGCTGTAGGCCCCATGTTA }-5^{\prime}\end{array}$ \\
\hline \multirow{2}{*}{ CH7_NX: } & 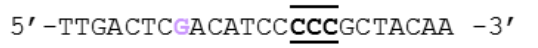 \\
\hline & $3^{\prime}-$ ACTGAGCTGTAGGGGGCGATGTTA $-5^{\prime}$ \\
\hline \multirow{2}{*}{ AN12_NL: } & $5^{\prime}$-TTGACTCGACATCCTTTGGTACAA $-3^{\prime}$ \\
\hline & 3'- ACTGAGCTGTAGGTTTCCATGTTA - -5' \\
\hline \multirow{2}{*}{ AN12_D: } & $5^{\prime}-$ TTGACTCGACATCCTTTGGTACAA $-3^{\prime}$ \\
\hline & $3^{\prime}-$ ACTGAGCTGTAGGTTTCDATGTTA -5' \\
\hline \multirow{2}{*}{ AN12_DA: } & 5'-TTGACTCGACATCPTTTGGTACAA -3' \\
\hline & 3'- ACTGAGCTGTAGGTTTCDATGTTA - -5' \\
\hline \multirow{2}{*}{ AN12u_NL: } & 5'-TTGACTCGACATCCTTTGGTACAA - -3' \\
\hline & $3^{\prime}-$ ACTGAGCTGTAGGTTTCCATGTTA -5' \\
\hline \multirow{2}{*}{ AN12u_D: } & $5^{\prime}$-TTGACTCGACATCCAAA \\
\hline & 3'- ACTGAGCTGTAGGTTTCDATGTTA -5' \\
\hline \multirow{2}{*}{ AN12u_DA: } & 5'-TTGACTCGACATCPAAAGGTACAA - -3' \\
\hline & 3'- ACTGAGCTGTAGGTTTCDATGTTA -5' \\
\hline
\end{tabular}

The errors indicate the errors of the nonlinear regression fit. AN12 shows significant specificity against the matched DNA ( 4-fold) albeit slightly lower than $\mathrm{CH} 10$ ( 7-fold). The results also show that the presence of $\mathrm{tC}^{\circ} / \mathrm{tC}_{\text {nitro }}$ probes in $\mathrm{AN} 12 / \mathrm{AN} 12 \mathrm{u}$ has little effect on binding specificity (compare_NL vs_D and_DA). 


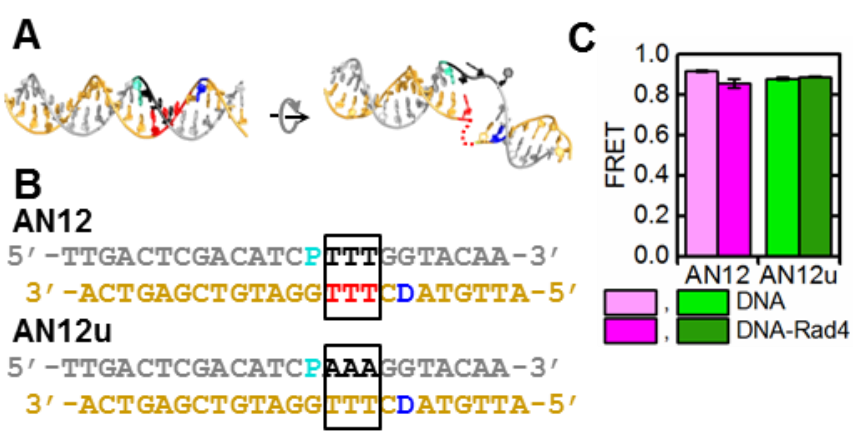

Fig. 4. 14. AN12 and AN12u DNA constructs. (A) Models of DNA duplexes when in B-DNA conformation (left) and in Rad4-bound 'open' conformation (right), in the same orientations as in Fig. 13A. The positions are marked for the mismatched nucleotides (black and red) and the FRET probes (cyan for tCo (D, donor); blue for $\mathrm{t}_{\text {Cnitro }}(\mathrm{P}$, acceptor)) of AN12. (B) The DNA sequences of AN12 and AN12u. Colors are the same as in (A). (C) FRET measured in free AN12 (pink), AN12-Rad4 (magenta), free AN12u (lime) and AN12u-Rad4 (green).

Next, we measured the FRET efficiency for each DNA at equilibrium in the absence and presence of Rad4 (Methods, section 4.2.5). We first acquired two donor fluorescence emission spectra (Fig. 4.15), one from the DNA containing only the donor (denoted as 'AN12_D', 'AN12u_D') and the other from the DNA containing both the donor and acceptor probes ('AN12_DA, 'AN12u_DA'), and calculated the FRET efficiencies from the measured spectra (Fig. 4.14C). The experimental FRET value for the undamaged $\mathrm{AN} 12 \mathrm{u}$ in the absence of $\operatorname{Rad} 4$ was in reasonable agreement with predictions based on the relative orientations and distances between the probes in a canonical B-DNA structure [41, 75] (Fig. 4.16A). Furthermore, the mismatched AN12 yielded FRET values very similar to $\mathrm{AN} 12 \mathrm{u}$, which indicates that the DNA largely retains the B-DNA conformation despite the helix-destabilizing mismatches, or at least any changes in the average DNA conformation due to mismatches are not detected in these bulk FRET measurements.

In the presence of Rad4, the FRET of AN12 decreased in comparison with protein-free DNA, whereas that of AN12u did not show a measurable difference (Fig. 4.14C and Fig. 
4.16A). As described above, AN12 is a specific substrate for Rad4 and is expected to undergo lesion-specific 'opening' when bound to Rad4 under the experimental conditions. A decrease in FRET upon Rad4-binding is expected based on the changes in DNA conformation from B-DNA to the 'open' conformation seen in the crystal structures [36]. However, the observed FRET value $(0.87 \pm 0.02)$ differed significantly from the value calculated (0.04) based on the crystal structures; various factors may contribute to the deviations between the theoretical estimates and the observed FRET in the protein-bound samples, which is discussed below in detail.
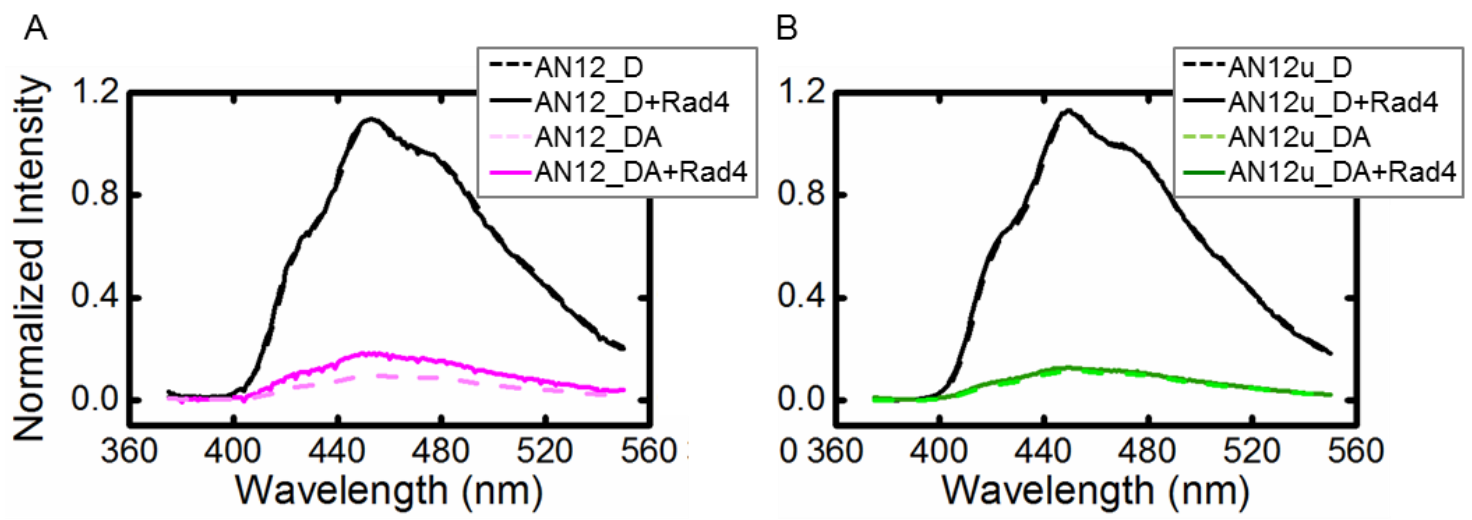

Fig. 4. 15. Fluorescence emission spectra of $\mathrm{AN12}$ and $\mathrm{AN12u}$ in the absence and presence of Rad4. (A,B) Fluorescence emission intensities of tCo (donor) with excitation at $365 \mathrm{~nm}$, are plotted as a function of the wavelength at $25^{\circ} \mathrm{C}$, for (A) AN12_D (black, dotted) and AN12_D-Rad4 (black), AN12_DA (pink, dotted), and AN12_DA-Rad4 (magenta); (B) AN12u_D (black, dotted) and AN12u_D-Rad4 (black), AN12u_DA (lime, dotted), and AN12u_DA-Rad4 (green). For each sample, the intensities are normalized such that the donor-only spectra overlap. 
A
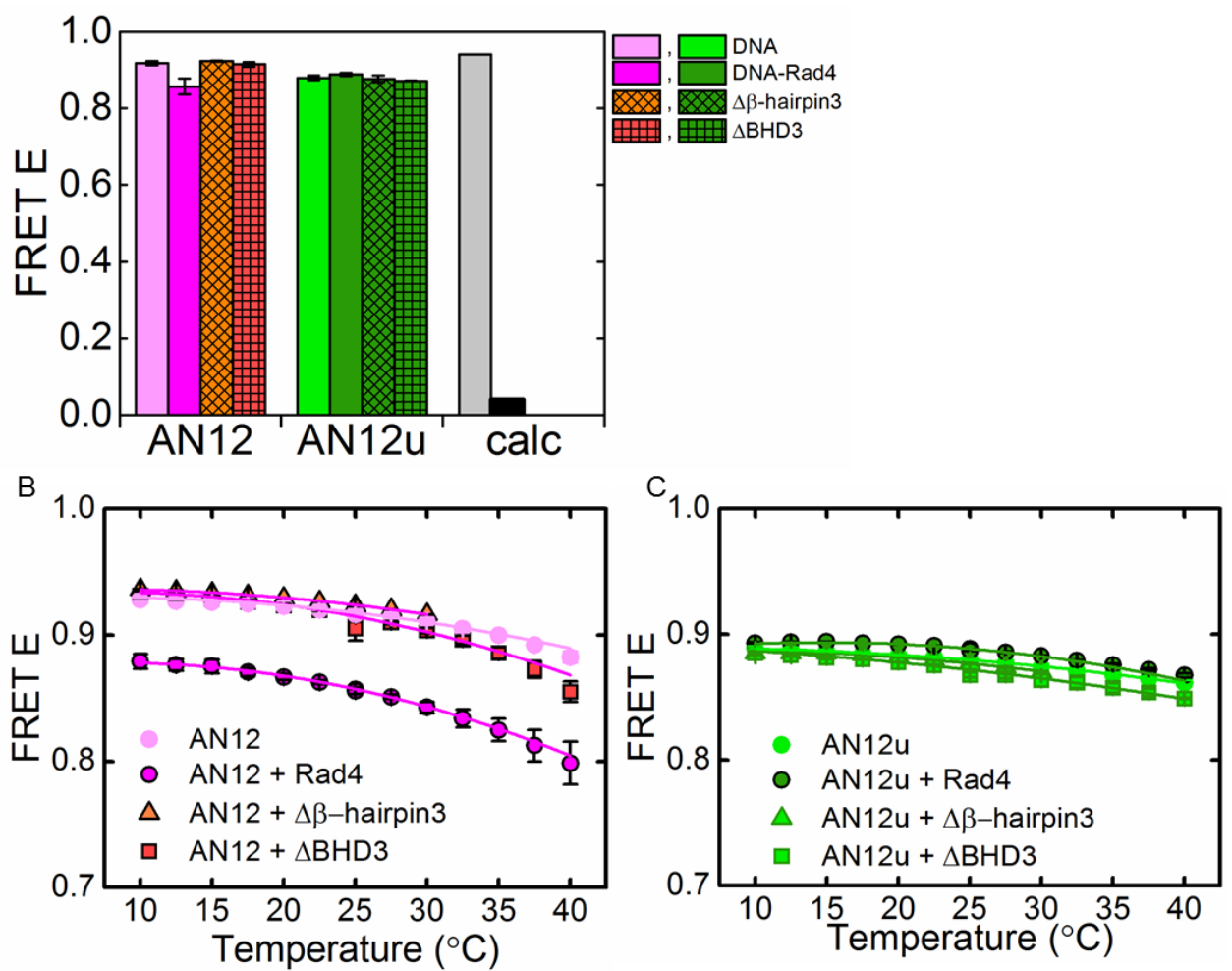

Fig. 4.16. FRET efficiencies of free and protein-bound AN12/AN12u under equilibrium (A) FRET efficiencies of free and protein-bound AN12 and AN12u measured at $25{ }^{\circ} \mathrm{C}$. Gray and black bars indicate the calculated FRET values based on B-DNA and Rad4-bound 'open' structures (PDB 2QSH), respectively, using methods described in ref. [41, 75]. (B) FRET efficiency versus temperature for free and bound AN12. Each scan was normalized to the mean value of FRET of the sample at $25^{\circ} \mathrm{C}$. In free AN12_DA, the increase in $\mathrm{I}_{\mathrm{DA}}$ with temperature (Fig. 17B) translates to a decrease in FRET from $0.928 \pm 0.004$ to $0.874 \pm 0.008$ in the temperature range 10 to $40{ }^{\circ} \mathrm{C}$. The AN12 bound to wild-type Rad4 complex (Fig. 17A) shows a larger decrease in FRET from $0.88 \pm$ 0.02 to $0.80 \pm 0.04$ in the same temperature range than the DNA alone. (C) FRET efficiency normalized at $25^{\circ}$ ' $\mathrm{C}$ (as in (B)) versus temperature for free and bound AN12u. AN12u (see also Fig. $18 \mathrm{~A}, \mathrm{~B})$ exhibited nearly the same change in FRET $(0.889 \pm 0.005-0.861 \pm 0.008$ and $0.893 \pm 0.003$ $-0.867 \pm 0.005$ ) in the temperature range 10 to $40{ }^{\circ} \mathrm{C}$ for both free and Rad4-bound DNA.

\section{Differences between measured and calculated FRET in the AN12-Rad4 complex.}

Possible reasons for the discrepancy in the measured FRET versus the calculated FRET (based on the 'open' crystal structure) for AN12 bound to wild-type Rad4 (Fig 4.16 A) include (i) disparity between the relative distances and orientations between the probes in the Rad4-bound DNA in solution compared with those in crystal conditions and potential heterogeneity in binding registers for a 3-bp mismatch bubble in solution, (ii) a change in the photophysical properties of the fluorescent probes in the presence of the protein, and (iii) 
breakdown of the ideal dipole approximation used in FRET calculations as has been proposed when fluctuating dipoles are closely separated $(<20 \AA)(36,37)$; in AN12/AN12u, the $\mathrm{tC}^{\circ} / \mathrm{tC}_{\text {nitro }}$ are $\sim 17 \AA$ apart. Some of these factors may also have contributions from the increased dynamics of the $\mathrm{tC}_{\text {nitro, }}$, placed adjacent to the mismatch bubble and its propensity to pre-melt as observed in the FRET changes measured with increasing temperature and rapid (unresolved) kinetics seen in T-jump experiments.

Nevertheless, the distinct decrease in FRET in AN12 but not in AN12u upon Rad4 binding indicates that the probes detect the change in average conformations in AN12 mismatched DNA induced upon specific binding to Rad4.

\subsubsection{Increase in temperature amplifies Rad4-induced opening sensed by the probes in} AN12.

We next monitored the extent to which temperature may alter the population distribution of DNA conformations probed by $\mathrm{tC}^{\circ} / \mathrm{tC}_{\text {nitro }}$ by measuring equilibrium $\mathrm{FRET}$ at different temperatures. The donor emission intensities $\left(I_{\mathrm{D}}\right)$ measured in AN12_D containing only the donor decreased monotonically with increasing temperature from 10 to $40{ }^{\circ} \mathrm{C}$ for both free and Rad4-bound DNA (Fig. 4.17A, B, black), corresponding to the quantum yield decrease of the donor with increasing temperature $[45,76]$. In contrast, the donor emission intensity in the presence of the acceptor $\left(I_{\mathrm{DA}}\right)$ increased with increasing temperature for both free and Rad4-bound AN12_DA (Fig. 4.17A, B, magenta/pink). The increase in $I_{\mathrm{DA}}$ with respect to $I_{\mathrm{D}}$ translates to a decrease in FRET by $0.05 \pm 0.01$ (mean \pm S.E.M; $n=2$ ) in free AN12 and by $0.08 \pm 0.02$ (mean \pm S.E.M $n=2$ ) in the Rad4-bound AN12 in the 10 to $40{ }^{\circ} \mathrm{C}$ temperature range ( Fig. 4.16B). While the equilibrium measurements show similar trends for free and Rad4-bound AN12_DA, the decrease in FRET over this temperature range is slightly larger in the presence of the protein. 


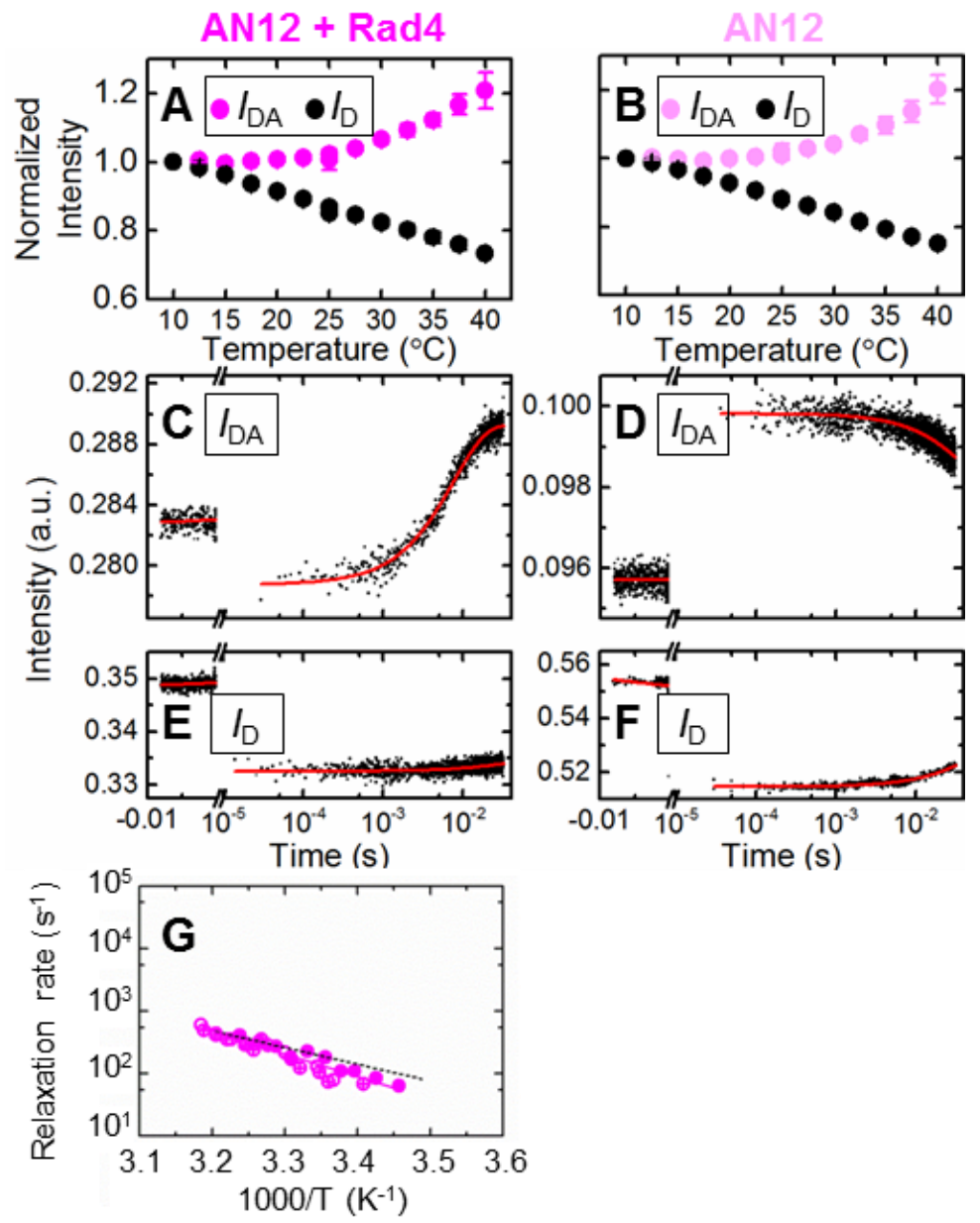

Fig. 4. 17. Equilibrium and T-jump measurements on AN12 bound to Rad4. (A, B) The fluorescence emission intensities $I_{D A}$ as a function of temperature for AN12-Rad4 (magenta) and AN12 (pink); the corresponding $I_{D}$ in each panel is in black. The intensities $I_{D}$ and $I_{D A}$ have been normalized to match at the lowest temperature. The emission intensities at $25{ }^{\circ} \mathrm{C}$ were measured before and after the heating/cooling cycle to check for reversibility and both are indicated. (C-F) T-jump relaxation traces are shown for donor/acceptor-labeled AN12_DA in the presence/absence of Rad4 (C/D), and for donor-only labeled AN12_D in the presence/absence of Rad4 (E/F). Only AN12_DA-Rad4 exhibit relaxation kinetics in the T-jump time-window with a characteristic relaxation time $7.7 \pm 0.5 \mathrm{~ms}$ in response to a T-jump from $19{ }^{\circ} \mathrm{C}$ to $26^{\circ} \mathrm{C}(\mathrm{C})$; with similar T-jump as in (C), relaxation traces in (DF) exhibit only the slow T-jump recovery. (G) Relaxation rates of AN12-Rad4 (pink) versus the inverse of the final temperature (after the T-jump). The different symbols indicate three independent sets of measurements. The continuous pink line is an Arrhenius fit to the relaxation rates, yielding an activation enthalpy of $18.5 \pm 1.1 \mathrm{kcal} / \mathrm{mol}$. The dashed black line is an Arrhenius fit to the relaxation rates on 2AP-labeled mismatch DNA (AN3) bound to Rad4, from ref. [38].

These results indicate that, while temperature increase alters the ensemble of conformations in free $\mathrm{AN} 12$, it also amplifies the extent and/or population of the $\mathrm{tC}^{\mathrm{o}} / \mathrm{tC}_{\mathrm{nitro}}$-sensed $\mathrm{Rad} 4$ induced conformational changes (Fig. 4.16B), as observed previously on 2AP-sensed conformational changes at mismatched sites [38] (Fig. 4.5). Furthermore, T-jump 
perturbation kinetics measured on free and Rad4-bound AN12 samples are distinctly different (discussed below). Guided by the observed kinetics, we attribute the temperature-dependent FRET decrease in free DNA to the enhanced DNA distortions sensed by $\mathrm{tC}_{\text {nitro }}$ acceptor placed adjacent to the mismatch as the region 'pre-melts' (and presumably unwinds) at the higher temperatures. This pre-melting is also found to be partially suppressed in the presence of Rad4 (discussed below). Similar bubble pre-melting and its partial suppression by Rad4 have also been observed at the site of a mismatch using 2AP as a probe [38]. AN12u, on the other hand, exhibited smaller changes in FRET with increasing temperature, which are almost the same for free $(0.03 \pm 0.004)$ (mean \pm S.E.M; $n=4)$ and Rad4-bound DNA $(0.03 \pm 0.002)$ (mean \pm S.E.M; $n=2$ ) (Figs. 4.16C, Fig 4.18A-B). Thus, temperature increases cause smaller changes in the average conformations of the undamaged/matched AN12u than of mismatched AN12, regardless of the presence of the protein.

\subsubsection{3 $t C^{o} / t C_{n i t r o}$-sensed dynamics in Rad4-bound AN12 are concurrent with $2 A P$-sensed,}

\section{Rad4-induced lesion 'opening' dynamics.}

To measure the dynamics of conformational changes in AN12 upon binding to Rad4, we performed T-jump measurements on AN12 with and without Rad4. The relaxation traces in response to a $\sim 5-10{ }^{\circ} \mathrm{C} \mathrm{T}$-jump perturbation were monitored by recording the donor fluorescence intensities $\left(I_{D}\right.$ or $\left.I_{D A}\right)$ over time, for a series of initial temperatures with final temperatures in the range of $16-41^{\circ} \mathrm{C}$. First, $I_{D}$ from AN12_D showed a rapid initial drop (much faster than our earliest observable time of $\sim 10 \mu \mathrm{s}$ ), followed by a slow relaxation of $200 \pm 60 \mathrm{~ms}$, characteristic of T-jump recovery kinetics (Fig. 4.17F). The initial rapid drop in AN12_D is due to the decrease in the quantum yield of the donor probe in response to the T-jump to a higher temperature, as also observed in the equilibrium measurements (Fig. 4.17B, black). The presence of bound Rad4 did not change the observed kinetics of 
AN12_D (Fig. 4.17E). Thus, the donor-only AN12_D samples do not exhibit any conformational dynamics for either free or Rad4-bound DNA.

On the other hand, $I_{D A}$ from free AN12_DA revealed an immediate increase after the T-jump perturbation, on a time-scale faster than the $\sim 10 \mu$ s resolution limit of our $\mathrm{T}$ jump apparatus (Fig. 4.17D), followed by the slow T-jump recovery kinetics (Fig. 4.20B, C). The rapid increase in AN12_DA fluorescence is opposite of the rapid decrease seen in AN12_D; this increase in $I_{D A}$, however, mirrors the increase in $I_{D A}$ seen in equilibrium measurements on the AN12_DA sample (Fig. 4.17B, pink), and is consistent with rapid kinetics of mismatch unstacking ('pre-melting') observed in AN21 using 2AP as a probe at a mismatch site [38]). We thus propose that the rapid increase in AN12's $I_{D A}$ after the Tjump reflects similar rapid pre-melting kinetics, as sensed by the $\mathrm{tC}_{\text {nitro }}$ acceptor adjoining the mismatch bubble.

Notably, AN12_DA, when bound to Rad4, showed additional relaxation kinetics in the T-jump time-window of $20 \mu \mathrm{s}-32 \mathrm{~ms}$, distinct from the rapid pre-melting $(<10 \mu \mathrm{s})$ and slow T-jump recovery ( $200 \mathrm{~ms}$ ) (Fig. 4.17C \& Figs. 4.20). The additional kinetics are detected only in the presence of the protein and require both the donor and the acceptor in AN12; they were not detected in AN12_D-Rad4 (Fig. 4.17E) or in the absence of the protein (Fig. 4.17D). The undamaged counterpart, AN12u_D and AN12u_DA, also did not show any relaxation kinetics in the same time-window either in the presence or absence of Rad4 (Fig. 4.18C-F). Thus, the kinetics uniquely observed in Rad4-bound mismatch AN12_DA reflect DNA motions specifically induced by Rad4, as they alter the FRET between the $\mathrm{tC}^{\mathrm{o}} / \mathrm{tC}_{\text {nitro }}$ probes in the vicinity of the TTT/TTT mismatch. Considering the sensitivity of the probes to DNA helical conformations, our results also indicate that the bubble pre-melting and the Rad4-induced 'open' conformation sensed by the FRET probes in AN12 may share similar directionality of structural distortions (unwinding). 
To determine the minimum number of relaxation phases to describe the new kinetics observed within the T-jump time window in the AN12_DA-Rad4 complex, we analyzed the relaxation traces using maximum entropy analysis ( Methods, section 4.2.6.2). The analysis revealed monophasic kinetics with average relaxation times ranging from $15.9-1.6 \mathrm{~ms}\left(7.7 \pm 0.8 \mathrm{~ms}\right.$ at $\left.25{ }^{\circ} \mathrm{C}\right)$ in the temperature range $16-41{ }^{\circ} \mathrm{C}$ (final temperature) (Fig. 4.20, Fig. $4.17 \mathrm{G}$ ). These $\sim 8 \mathrm{~ms}$ relaxation kinetics are on similar timescales as the Rad4-induced DNA 'opening' dynamics probed by 2AP [38] (Fig. 4.5). Since the $2 \mathrm{AP}$ probes were placed as one of the mismatched nucleotides that are flipped out when Rad4 binds specifically, 2AP likely senses the kinetics of nucleotide-flipping. Our

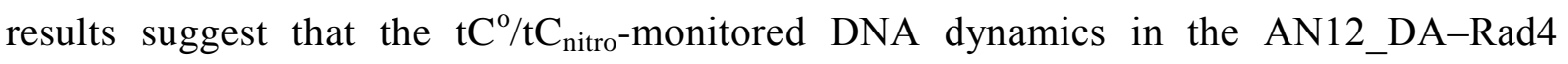
complex involve the same rate-limiting distortions as in the nucleotide-flipping during specific 'opening'. However, 2AP labelled mismatched DNA (AN3, AN21) and $\mathrm{tC}^{\mathrm{o}} / \mathrm{tC}_{\text {nitro }}$ labelled mismatched DNA (AN12) do not necessarily probe identical motions during 'opening', as evidenced by the measurements with Rad4 mutants described below. 

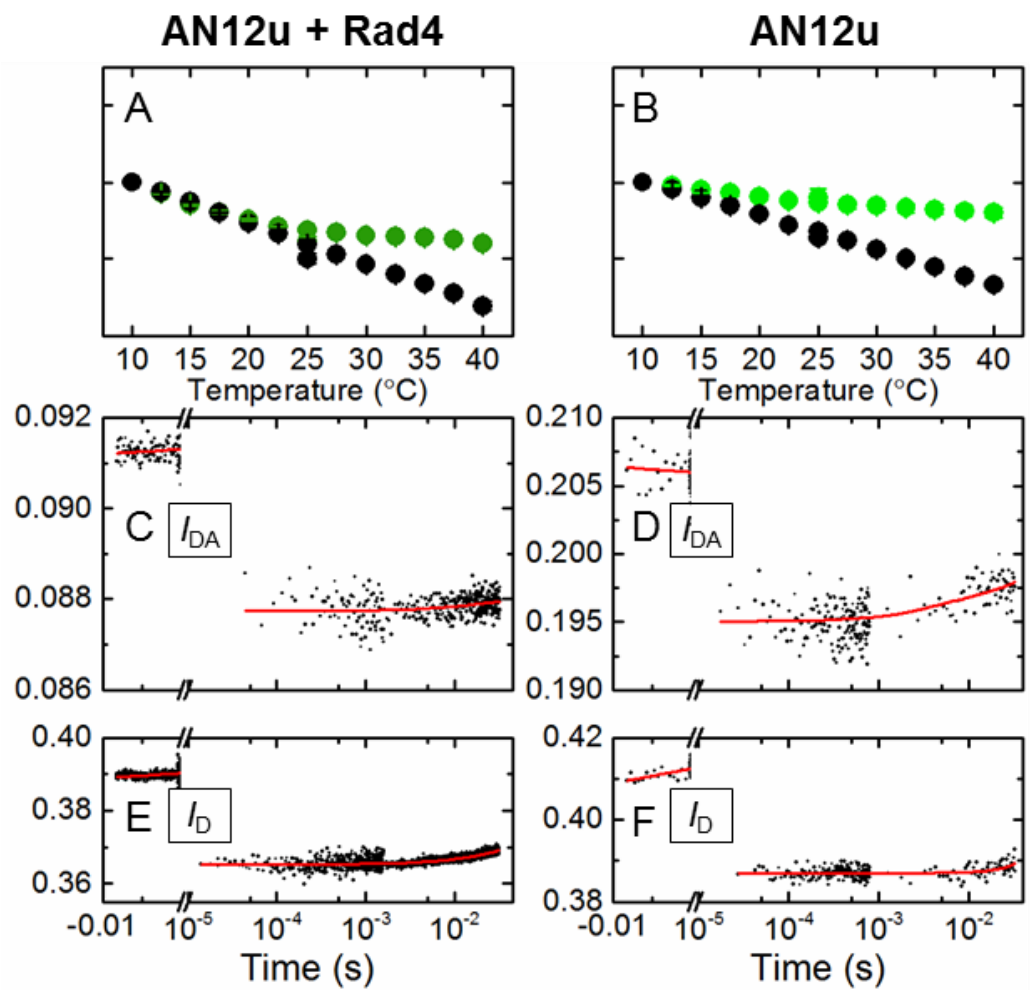

Fig. 4.18. Equilibrium temperature scan and T-jump kinetics measurements on AN12u-Rad4 complexes. (A, B) The fluorescence emission intensities $I_{D}$ (black) and $I_{D A}$ (green) calculated as described in the text from the emission spectra measured at different temperatures, are plotted as a function of temperature, for AN12u-bound to wild-type Rad4 (A) and for free AN12u (B). In each panel, the intensities $I_{D}$ and $I_{D A}$ have been normalized to match at the lowest temperature. $(C, D)$ Donor fluorescence emission intensities of double-labeled AN12u_DA samples in the presence (C) and absence (D) of Rad4, measured in response to a T-jump perturbation, are plotted as a function of time. (E, F) Control T-jump measurements on donor-only labeled samples are shown in the presence (E) and absence (F) of Rad4. T-jump measurements on these samples do not exhibit any relaxation kinetics; instead they show only the much slower T-jump recovery kinetics. 

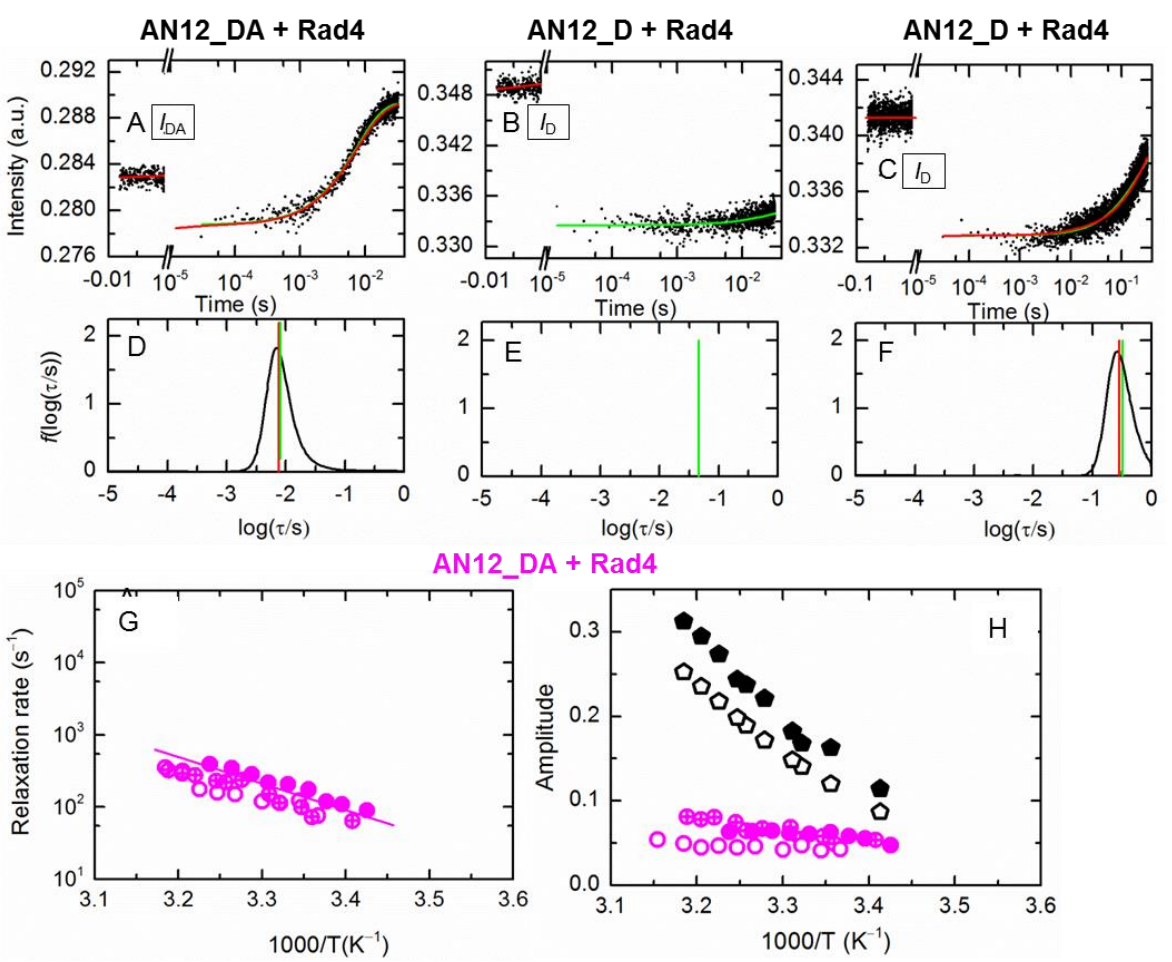

Fig. 4. 19. Analysis of T-jump relaxation traces for AN12-(wild-type) Rad4 complex. Relaxation traces in response to a T-jump perturbation are shown for (A) double-labeled AN12_DA-Rad4 complex, and (B, C) donor-only AN12_D-Rad4 complex. In panels (A, B), the time window after Tjump is $20 \mu \mathrm{s}-32 \mathrm{~ms}$, while it is longer in (C), from $20 \mu \mathrm{s}-320 \mathrm{~ms}$. The continuous red lines are mem fits (say what is mem) to the relaxation traces, with the corresponding distributions of relaxation times shown in panels (D,F). Mem analysis did not converge for control experiment on AN12_DRad4 shown in panel (B), likely due to very small amplitude of T-jump recovery in the shorter timewindow. The green continuous lines in (A-C) are from single-exponential relaxation convoluted with T-jump recovery (Eq. e6 in panel $\mathrm{A}$, with $\tau_{\text {rec }}$ as a fixed parameter), and T-jump recovery function (Eq. S3 in panels B \& C, with trec a variable parameter). The average time constants describing the best fit parameters from the two analyses are shown as vertical red (mem) and green (from Eq. e3 or e6), and are (A): $\tau_{\mathrm{ave}}=7.7 \mathrm{~ms}$ (red) and $\tau_{\mathrm{r}}=\mathrm{k}_{\mathrm{r}}^{-1}=8.2 \mathrm{~ms}$ (green, with $\tau$ rec fixed at $325 \mathrm{~ms}$ ); (B) $\tau$ rec $=45.6 \mathrm{~ms}$ (green); and (C) $\tau \mathrm{rec}=288 \mathrm{~ms}$ (red) and $325 \mathrm{~ms}$ (green). $(\mathrm{G})$ Relaxation rates obtained from single-exponential kinetics convoluted with T-jump recovery (as described for panel A) are plotted versus the inverse of the final temperature (after the T-jump) (pink symbols). The pink continuous line (reproduced from Fig. 17G ) is the Arrhenius fit to the mem relaxation rates obtained for the same data sets. $(\mathrm{H})$ The relative amplitudes in the relaxation traces (Ar, calculated from Eq. S8) are plotted as a function of inverse temperature (pink symbols), together with the amplitudes expected from the equilibrium measurements $\left(\mathrm{A}_{\mathrm{e}}\right.$, calculated from Eq. e9) (black symbols). These data show that in AN12-Rad4 complex, the amplitudes observed within the T-jump time-window are significantly smaller than the total amplitude expected, consistent with our interpretation that there are unresolved 'missing' kinetics at short times $(<20 \mu \mathrm{s})$ corresponding to rapid unstacking of the FRET labels. 


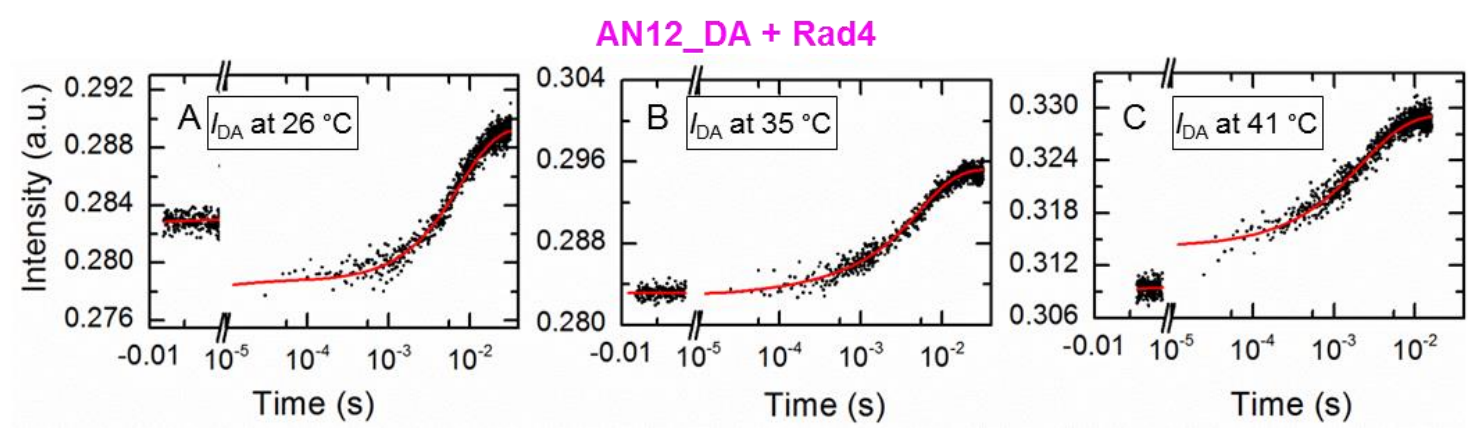

Fig. 4. 20. Rapid pre-melting kinetics on AN12-Rad4 complexes. Relaxation traces in response to a T-jump perturbation are shown for AN12_DA-Rad4 complex (IDA), for T-jump from 19 to $26{ }^{\circ} \mathrm{C}$ (A), 28 to $35^{\circ} \mathrm{C}(\mathrm{B})$, and 34 to $41^{\circ} \mathrm{C}(\mathrm{C})$. Red continuous lines are mem fits to the relaxation traces, with average relaxation times $\tau_{\text {ave }}=7.7 \mathrm{~ms}, 3.3 \mathrm{~ms}$, and $1.6 \mathrm{~ms}$, respectively. The measurements show that the drop in the donor intensity immediately after the T-jump (panel A) decreases in amplitude as the temperature is raised, and in fact changes signs at the higher temperatures, with the donor intensity increasing after the T-jump, as in panel (C). This initial change in donor intensity has contributions from an intrinsic temperature-dependent decrease in the quantum yield of the donor (as observed in equilibrium measurements on donor-only samples as a function of temperature), and an increase in donor intensity as a result of a rapid decrease in FRET, which we attribute to the premelting involving unwinding/unstacking of the acceptor $\left(\mathrm{tC}_{\text {nitro }}\right)$ placed adjacent to the mismatch bubble in AN12_DA.

\subsubsection{4 $\beta$-hairpin mutants exhibit novel sub-millisecond kinetics and help reveal the multi-step nature of lesion recognition by Rad4.}

Previous studies have shown that the $\beta$-hairpin from the $\beta$-hairpin domain 3 (BHD3) plays an important role in Rad4's ability to recognize lesions [36, 38, 54]. In the 'open' conformation, the $\beta$-hairpin is shown to be inserted into the damaged DNA site, filling the gap in the DNA duplex resulting from the flipped-out nucleotide $[36,38]$. The mutant Rad4 lacking either the $\beta$-hairpin in BHD3 (residues 599-605, blue in Fig. 4.13A, denoted as $\Delta \beta$-hairpin3) or the entire BHD3 domain (residues 541-632, blue and red in Fig. 4.13A, denoted as $\triangle \mathrm{BHD} 3$ ) retain nanomolar affinities to undamaged DNA duplex, but have significantly reduced specificity for DNA damage compared with the wild type Rad4 in gel shift assays (table II) [38]. Importantly, neither mutant revealed any Rad4-induced 'opening' kinetics detected by 2AP [38]. 


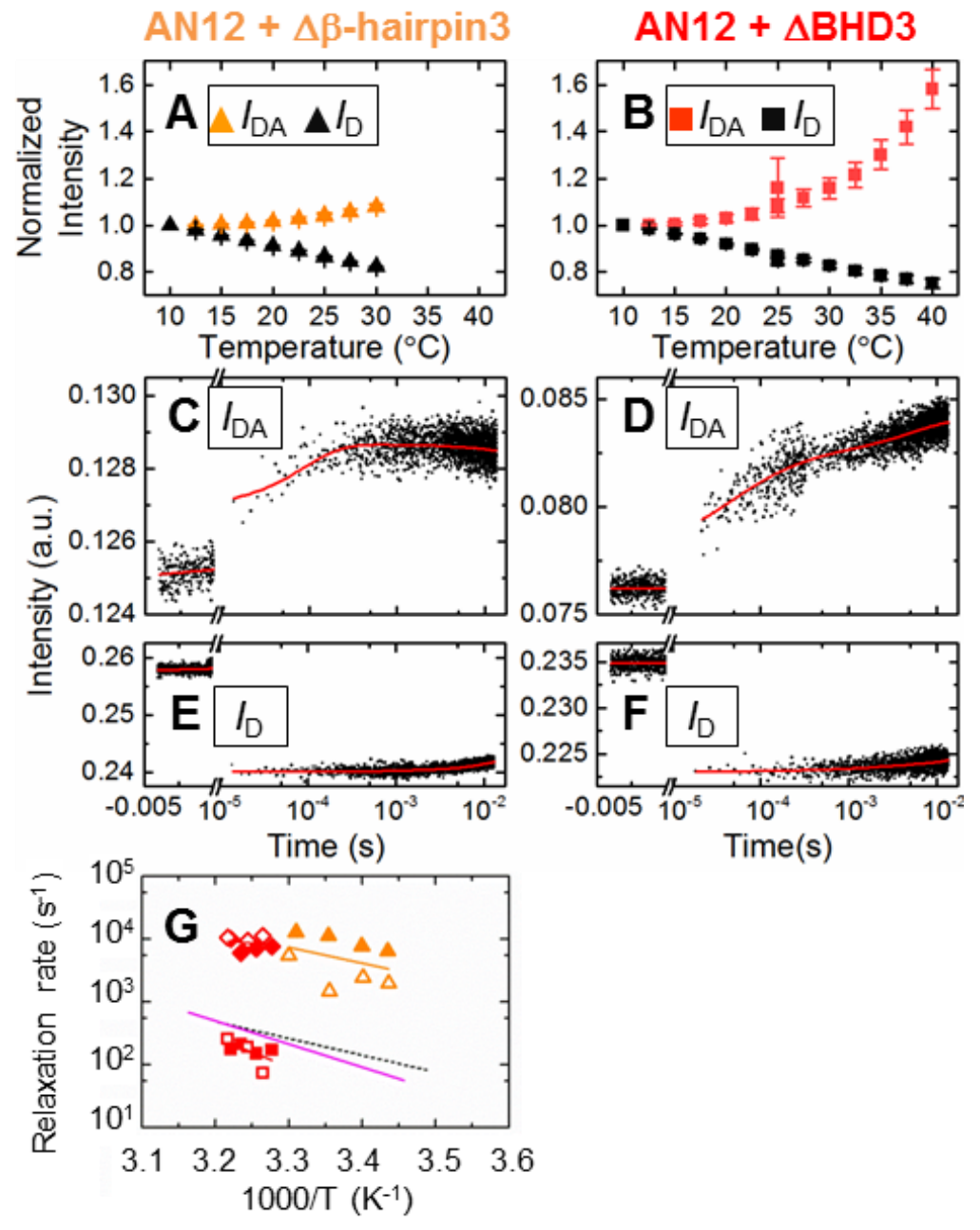

Fig. 4. 21 . Equilibrium and T-jump measurements on AN12 bound to Rad4 $\boldsymbol{\beta}$-hairpin mutants. The experiments describing each panel (A-G) are similar to those for Fig. 4.17. (A, B) $\mathrm{I}_{\mathrm{DA}}$ for AN12$\Delta \beta$-hairpin3 (orange) and for AN12- $\Delta$ BHD3 (red) with the corresponding $\mathrm{I}_{\mathrm{D}}$ in black. (C, D) T-jump relaxation traces with donor/acceptor-labeled DNA are shown: (C) AN12- $\Delta \beta$-hairpin3 shows a single phase with relaxation time $89 \pm 7.4 \mu \mathrm{s}$ (at $25{ }^{\circ} \mathrm{C}$ ) after a T-jump from $18{ }^{\circ} \mathrm{C}$ to $25^{\circ} \mathrm{C}$. (D) AN12$\triangle \mathrm{BHD} 3$ shows two phases with relaxation times $131 \pm 12 \mu \mathrm{s}$ and $5.8 \pm 0.3 \mathrm{~ms}\left(\right.$ at $32{ }^{\circ} \mathrm{C}$ ) after a Tjump from $26{ }^{\circ} \mathrm{C}$ to $32{ }^{\circ} \mathrm{C}$. (E, F) Control measurements on donor-only AN12_D with either $\Delta \beta$ hairpin3 (E) or $\triangle \mathrm{BHD} 3(\mathrm{~F})$ exhibit only the slow T-jump recovery kinetics. (G) Relaxation rates of AN12- $\Delta \beta$-hairpin3 (orange triangles) and AN12- $\Delta$ BHD3 (red diamonds for fast phase; red squares for slow phase) versus inverse temperature from two independent sets of measurements (open/filled symbols). The continuous lines are Arrhenius fits to the relaxation rates, with activation enthalpies $11.9 \pm 0.3 \mathrm{kcal} / \mathrm{mol}$ for AN12- $\Delta \beta$-hairpin3; $3.3 \pm 2.4 \mathrm{kcal} / \mathrm{mol}$ and $27.5 \pm 32.4 \mathrm{kcal} / \mathrm{mol}$ for the fast and slow phases, respectively, for AN12- $\triangle$ BHD3. The pink and dashed black lines are reproduced from Fig. 17G.

Here, we examine the role of the $\beta$-hairpin in inducing the conformational changes monitored in AN12. The FRET changes in AN12 upon binding to the mutants, $\Delta \beta$ hairpin3 and $\triangle \mathrm{BHD} 3$, were barely detectable in equilibrium measurements at $25{ }^{\circ} \mathrm{C}$, 
consistent with the impaired specificity of the mutants (Fig 4.16 A). The FRET measured on these samples as the temperatures were raised from 10 to $30{ }^{\circ} \mathrm{C}$ essentially overlapped with the FRET measured on free AN12 ( Fig. 4.16B; see also Fig. 4.21A, B). Thus, the FRET in the AN12-mutant complexes in this temperature range predominantly indicate enhanced pre-melting of the mismatch region sensed by the tCnitro probe at the higher temperatures, as in free AN12. For $\Delta \beta$-hairpin3, the temperature range was limited to up to $\sim 30{ }^{\circ} \mathrm{C}$ because the protein-DNA sample heated above $\sim 35{ }^{\circ} \mathrm{C}$ showed irreversible behaviour in fluorescence measurements indicating early denaturation/aggregation of the protein [38]. For $\triangle \mathrm{BHD} 3$, the measurements were carried out up to $40{ }^{\circ} \mathrm{C}$ as with the wildtype Rad4. Though the FRET versus temperature trends in AN12- $\triangle \mathrm{BHD} 3$ resembled that of the free DNA below $30{ }^{\circ} \mathrm{C}$, small deviations were observed at temperatures above $\sim 30$ ${ }^{\circ} \mathrm{C}$ (Fig. 4.16B), indicating a contribution from the $\triangle \mathrm{BHD} 3$-induced conformational changes at these higher temperatures. The FRET versus temperature measurements on the undamaged AN12u with either of the two mutants essentially overlapped with those on free AN12u and on the AN12u-wild-type Rad4 complex (Fig. 4.16C).

T-jump measurements with the mutants detected no kinetics with the mismatched AN12 in the absence of the acceptor probe ( $I_{D}$ from AN12_D) (Fig. 4.21 E, F) nor with the undamaged $\mathrm{AN} 12 \mathrm{u}$, as were the cases with wild-type Rad4. However, $I_{D A}$ in mutant-bound AN12_DA showed not only the rapid $(<20 \mu$ s) pre-melting kinetics but also unique, protein-induced kinetics in the T-jump window of $20 \mu \mathrm{s}-32 \mathrm{~ms}$ : First, $\Delta \beta$-hairpin3-bound AN12_DA revealed monophasic relaxation kinetics, $374.0-174.0 \mu$ s in the range from $18-29{ }^{\circ} \mathrm{C}$, the highest temperature testable with this mutant (Fig. 4.21C and Fig. 4.22 A,E). This kinetics $\left(227.5 \pm 18.2 \mu\right.$ s at $\left.25^{\circ} \mathrm{C}\right)$ are about 30 -fold faster than the $5-10$-ms kinetics observed with the same DNA bound to the wild-type or the 2AP-monitored 
nucleotide-flipping kinetics. We note that these kinetics were observed despite the lack of detectible differences between the bound and the free AN12_DA in equilibrium fluorescence measurements (Fig. 4.16 A, B), underscoring the sensitivity of the $\mathrm{tC}^{\mathrm{o}} / \mathrm{tC}_{\text {nitro- }}$ based T-jump approach to detect relaxation kinetics with small (less than $2 \%$ change) amplitudes ( Methods, section 4.2.6.6; Fig. 4.23F). Second, $\triangle$ BHD3-bound AN12_DA showed biphasic relaxation kinetics in the temperature range $30-40{ }^{\circ} \mathrm{C}$ (final temperature), but no kinetics below $\sim 30{ }^{\circ} \mathrm{C}$. The fast component of the biphasic behaviour had 110.6$118.2 \mu$ s relaxation times, and overlapped those of AN12- $\Delta \beta$-hairpin3 (Table VII), whereas the slow component was 4.4-11.1 ms. similar to the 5-10 ms relaxation kinetics discussed above (Fig. 4.21D, G; Fig. 4.23).

Table VII. Relaxation times for Rad4-DNA complexes at $25^{\circ} \mathrm{C}$ and $32^{\circ} \mathrm{C}$

\begin{tabular}{|c|c|c|c|c|}
\hline Sample & $\mathrm{T}=25^{\circ} \mathrm{C}$ (fast) & $\mathrm{T}=25^{\circ} \mathrm{C}$ (slow) & $\mathrm{T}=32{ }^{\circ} \mathrm{C}$ (fast) & $\mathrm{T}=32{ }^{\circ} \mathrm{C}$ (slow) \\
\hline AN12-Rad4 & --- & $7.7 \pm 0.8 \mathrm{~ms}$ & --- & $4.1 \pm 0.6 \mathrm{~ms}$ \\
\hline AN12- $\Delta$ BHD3 & $113.1 \pm 24.0 \mu \mathrm{s}$ & $\mathrm{nd}^{\mathrm{a}}$ & $118.2 \pm 35.2 \mu \mathrm{s}$ & $11.1 \pm 7.1 \mathrm{~ms}$ \\
\hline AN12- $\Delta \beta$-hairpin3 & $227.5 \pm 18.2 \mu \mathrm{s}$ & --- & $142.7 \pm 115 \mu \mathrm{s}$ & --- \\
\hline AN14-Rad4 & $448.4 \pm 98.3 \mu \mathrm{s}$ & --- & $228.1 \pm 66.7 \mu \mathrm{s}$ & --- \\
\hline AN14u-Rad4 & $292.4 \pm 173.9 \mu \mathrm{s}$ & --- & $136.0 \pm 25.8 \mu \mathrm{s}$ & --- \\
\hline
\end{tabular}

The sub-millisecond kinetics observed for both $\Delta \beta$-hairpin3 and $\Delta \mathrm{BHD} 3$ had similar relaxation times $\left(113.1\right.$ and $227.5 \mu$ s at $25^{\circ} \mathrm{C}$ ) with closely overlapping Arrhenius trends, which are distinct from that observed with the wild type (Table VII and Fig. 4.21G). The low specificity of these mutants to the mismatch lesion in comparison with wild-type [38] raises the possibility that the main conformations in the AN12-mutant Rad4 complexes are of nonspecific modes that lie along the trajectory towards the final recognition conformation, and the fast phase observed in T-jump kinetics with these mutants primarily result from the motions in the nonspecific modes (further discussed next pages). 

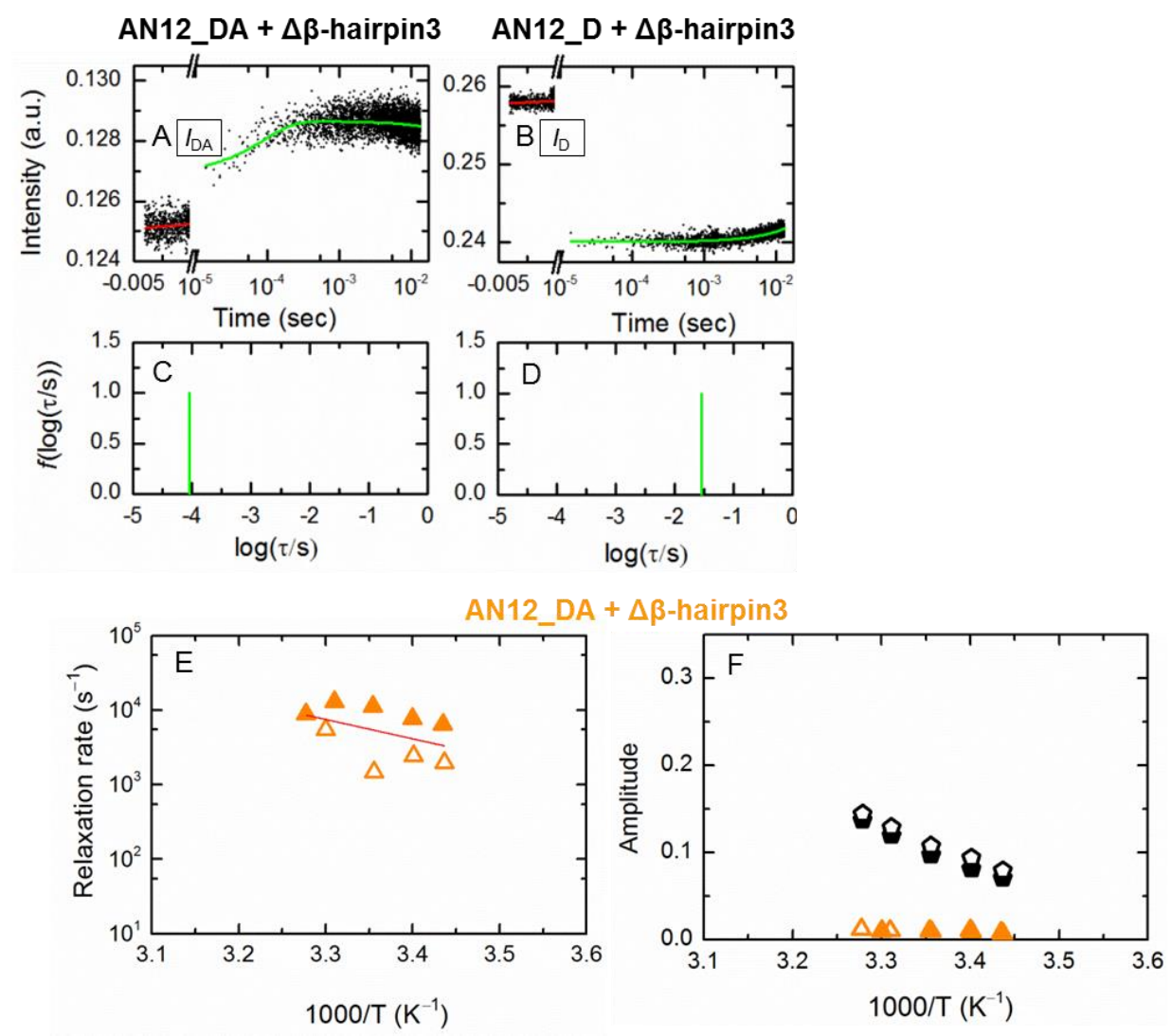

Fig. 4. 22. Analysis of $T$-jump relaxation traces for $A N 12-\Delta \beta$-hairpin3 complex. Relaxation traces in response to a T-jump perturbation are shown for (A) double-labeled AN12_DA- $\Delta \beta$-hairpin3 complex, and (B) donor-only AN12_D- $\Delta \beta$-hairpin3 complex, and were analyzed only in terms of single-exponential relaxation kinetics convoluted with T-jump recovery. The mem analysis did not converge for these samples, likely because of the small amplitudes in the relaxation traces compounded with the added complication that the amplitudes of the relaxation and T-jump recovery in this data set have opposite signs. Characteristic relaxation times from the single-exponential analysis (Eq. 6) or T-jump recovery function (Eq. 3) are shown as vertical green lines, and are (C): $\tau_{\mathrm{r}}=\mathrm{k}_{\mathrm{r}}^{-1}=88.8 \mu \mathrm{s}$ (with $\tau_{\text {rec }}$ fixed at $150 \mathrm{~ms}$ ); and (D) $\tau_{\mathrm{rec}}=28.5 \mathrm{~ms}$ (green). Control measurements on donor-only AN12_D- $\Delta \beta$-hairpin3 complex over longer time-scales were not carried out for these samples. Instead, the T-jump recovery time constant was fixed from measurements on free AN12_D samples. E. Relaxation rates obtained from single-exponential kinetics convoluted with T-jump recovery (as described for panel A) are plotted versus the inverse of the final temperature (after the Tjump) (orange symbols). The symbols (and Arrhenius lines) are identical to the data shown in Fig. 4.21. (F) The relative amplitudes in the relaxation traces (orange symbols) and from equilibrium measurements (black symbols) are plotted as a function of inverse temperature. These amplitudes for AN12- $\Delta \beta$-hairpin3 complex are significantly smaller than those for AN12-Rad4 (Fig. $4.19 \mathrm{H}$ ) and AN12- $\triangle$ BHD3 (Fig. 4.23F) complexes, and correspond to about $1 \%$ change in the donor intensities. 

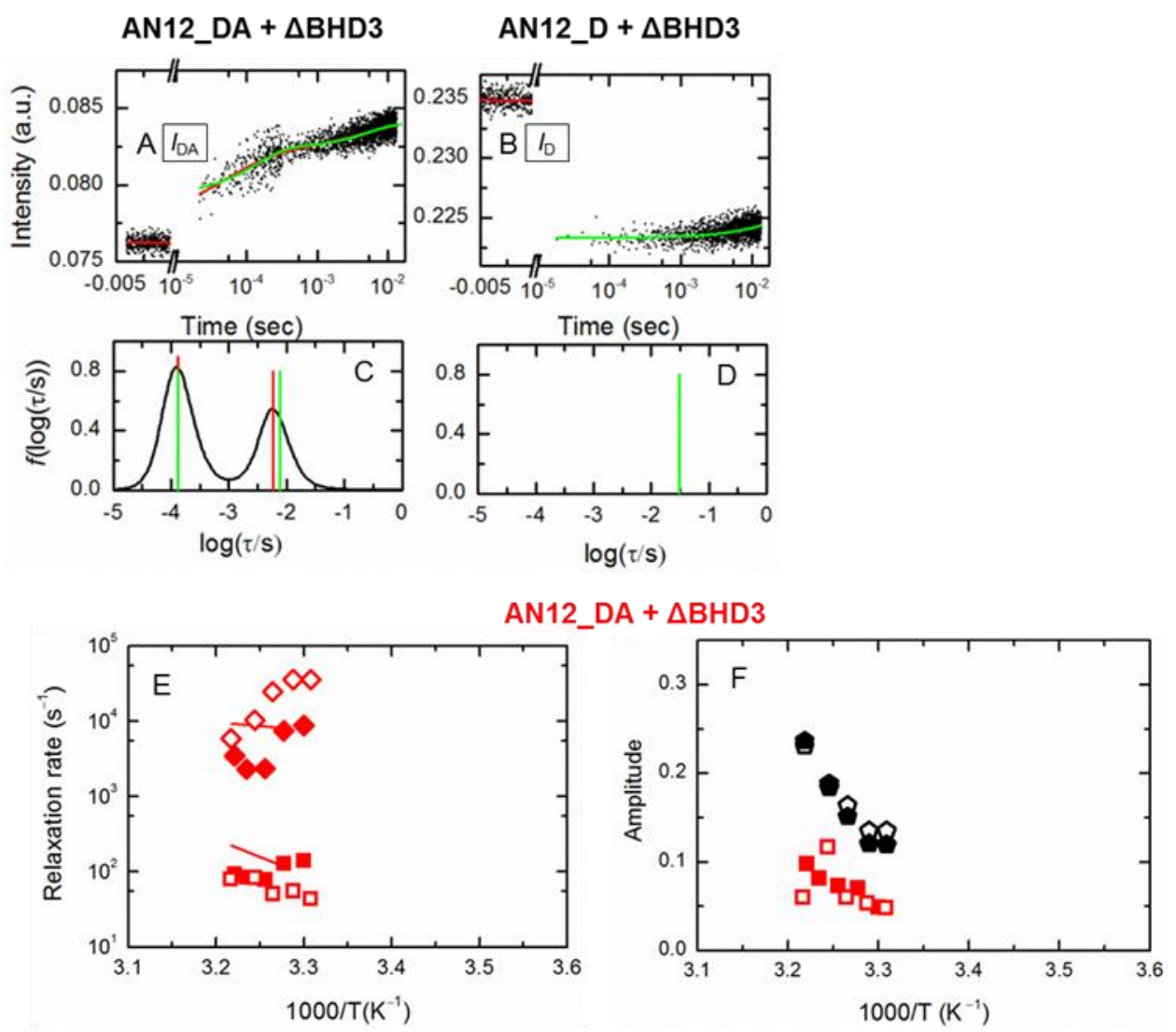

Fig. 4. 23. Analysis of $T$-jump relaxation traces for $A N 12-\triangle B H D 3$ complex. Relaxation traces in response to a T-jump perturbation are shown for (A) double-labeled AN12_DA- $\Delta$ BHD3 complex, and (B) donor-only AN12_D- $\triangle$ BHD3 complex. The panels are as described in Fig. 4.22. The average time constants describing the best fit parameters from the two analyses are shown as vertical red (mem) and green (from Eq. 3 or 7), and are (C): $\tau_{-}$ave $=131 \mu$ s and $5.84 \mathrm{~ms}$ (red; fast and slow components, respectively), and $\tau_{\text {fast }}=\mathrm{k}_{\text {fast }}{ }^{-1}=134 \mu \mathrm{s}$ and $\tau_{\text {slow }}=\mathrm{k}_{\text {slow }}{ }^{-1}=7.8 \mathrm{~ms}$ (green, with $\tau_{\text {rec }}$ fixed at $190 \mathrm{~ms}$ ); (D) $\tau_{\text {rec }}=30.5 \mathrm{~ms}$ (green); mem analysis did not converge for this data set. Control measurements on donor-only AN12_D- $\triangle$ BHD3 complex over longer time scales were not carried out for these samples. Instead, the T-jump recovery time constant was fixed from measurements on AN12_D DNA only samples. (E) Relaxation rates obtained from double-exponential kinetics convoluted with T-jump recovery (as described for panel A) are plotted versus the inverse of the final temperature (after the T-jump) (fast component: red diamonds; slow component: red squares). The red continuous lines (reproduced from Fig. 4.21G are the Arrhenius fit to the two components obtained from the mem analysis on the same data sets. (F) The total relative amplitudes (sum of both components) in the relaxation traces (pink symbols) and from equilibrium measurements (black symbols) are plotted as a function of inverse temperature. The total amplitudes for AN12- $\triangle \mathrm{BHD} 3$ complex, for both components combined, are comparable to those for AN12-Rad4, and correspond to less than $10 \%$ change in the donor intensities.

It is also noteworthy that the $\triangle \mathrm{BHD} 3$-bound AN12 showed the slow phase matching the kinetics of DNA 'opening' induced by wild-type Rad4 in addition to the fast phase,. The 
relative amplitudes of the fast and slow phases were similar ( 60\% in the fast phase at 30 ${ }^{\circ} \mathrm{C}$ and $\sim 50 \%$ at $40{ }^{\circ} \mathrm{C}$ ), while the overall amplitude in the two phases combined was similar to that with wild type Rad4 ( Fig. 4.23F). We recall that neither $\triangle$ BHD3 nor $\Delta \beta$-hairpin3 exhibited 2AP-based nucleotide-flipping kinetics [38]. Thus, the results suggest that the slow phase in $\mathrm{AN} 12-\triangle \mathrm{BHD} 3$ captures the rate-limiting structural distortion along the recognition trajectory, and that this distortion does not require the BHD3 domain at least at the higher temperatures at which it was observed $\left(>30{ }^{\circ} \mathrm{C}\right)$. However, the formation of the fully flipped-out 'open' conformation as monitored by $2 \mathrm{AP}$ must still require the insertion of the $\beta$-hairpin, which is lacking in these mutants.

\subsubsection{Fast sub-millisecond kinetics are also observed with wild-type Rad4 on nonspecific DNA substrates:}

AN14/AN14u. As discussed above, the fast sub-millisecond kinetics observed with AN12 bound to $\beta$-hairpin mutants may indicate kinetics resulting from nonspecific binding modes of the mutants. We thus further investigated whether these nonspecific-mode kinetics could be captured with nonspecifically bound wild-type Rad4, particularly if the probes were positioned further away from the mismatch to avoid interference coming from the mismatch dynamics (Rad4-induced or pre-melting). Thus, in the new DNA substrates, AN14 and AN14u, we placed both FRET probes two base pairs away from either side of the 3-bp mismatch (Fig. 4.24A, B, and Fig. 4.12). 


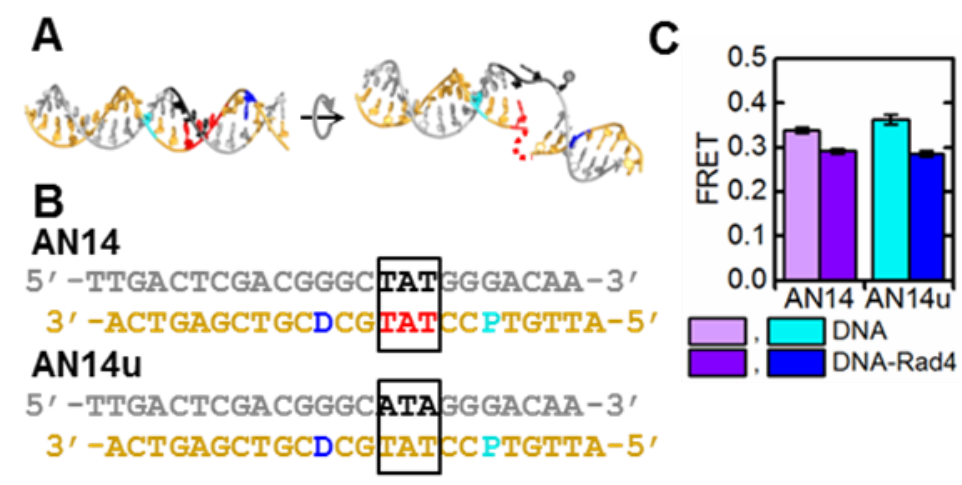

Fig. 4. 24. AN14 and AN14u DNA constructs. (A) Models of DNA duplexes as in Fig. 14A . The positions are marked for the mismatched nucleotides (black and red) and the FRET probes (cyan for tCo; blue for tCnitro) of AN14. (B) The DNA sequences of AN14 and AN14u. Colors are the same as in (A). (C) FRET measured in free AN14 (light purple), AN14-Rad4 (purple), free AN14u (cyan) and AN14u-Rad4 (blue).

In AN14, we also incorporated TAT/TAT mismatch instead of the TTT/TTT mismatch in AN12: The TAT/TAT mismatch lowers the melting temperatures of the DNA duplexes by $\sim 7-8{ }^{\circ} \mathrm{C}$ than the corresponding undamaged DNA (Table V)); this effect is slightly less than but still comparable to the $8-11{ }^{\circ} \mathrm{C}$ destabilization caused by TTT/TTT mismatch of AN12. However, contrary to AN12, AN14 does not show specific binding to Rad4, when compared with other undamaged DNA constructs including its own matched version, AN14u (Table VIII)). We further confirm that AN14's low specificity is due to its mismatch sequence rather than the presence of the FRET labels: $\mathrm{tC}^{\mathrm{o}} / \mathrm{tC}_{\mathrm{nitro}}$-labeled AN14/AN14u exhibited Rad4-binding affinities similar to those of unlabeled constructs, as was also the case with AN12/AN12u (Table VIII (Xuejing Chen/ Prof.Min's group)). We posit that TAT/TAT mismatch could perhaps mimic lesions such as cyclobutane pyrimidine dimer (CPD): a CPD lesion also lowers the melting temperature of the duplex DNA [77], but is not specifically recognized by XPC in vitro [37]. 
Table VIII: $\mathrm{K}_{\mathrm{d} \text {,app }}$ and $\mathrm{R}^{2}$ for different DNA constructs. (From Xuejing Chen)

\begin{tabular}{|l|c|c|}
\hline & $\mathrm{K}_{\mathrm{d}, \text { app }}(\mathrm{nM})$ & $\mathrm{R}^{2}$ \\
\hline CH10_NX & $70 \pm 7$ & 0.923 \\
\hline CH7_NX & $503 \pm 24$ & 0.991 \\
\hline AN14_NL & $504 \pm 14$ & 0.983 \\
\hline AN14_D & $599 \pm 12$ & 0.990 \\
\hline AN14_DA & $492 \pm 7$ & 0.995 \\
\hline AN14u_NL & $554 \pm 16$ & 0.978 \\
\hline AN14u_D & $573 \pm 10$ & 0.992 \\
\hline AN14u_DA & $468 \pm 7$ & 0.995 \\
\hline
\end{tabular}

\begin{tabular}{|c|c|}
\hline CH10_NX: & $\begin{array}{l}5^{\prime}-\text { TTGACTCGACATCCCCCGCTACAA }-3^{\prime} \\
3^{\prime}-\text { ACTGAGCTGTAGGCCCGATGTA }-5^{\prime}\end{array}$ \\
\hline \multirow{2}{*}{ CH7_NX: } & 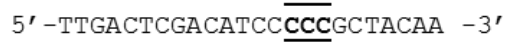 \\
\hline & $3^{\prime}-$ ACTGAGCTGTAGGGGGCGATGTTA $-5^{\prime}$ \\
\hline \multirow{2}{*}{ AN14_NL: } & $5^{\prime}-$ TTGACTCGACGGGCTATGGGACAA $-3^{\prime}$ \\
\hline & $3^{\prime}-$ ACTGAGCTGCCCGTATCCCTGTTA $-5^{\prime}$ \\
\hline \multirow{2}{*}{ AN14_D: } & 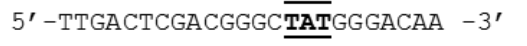 \\
\hline & $3^{\prime}-$ ACTGAGCTGCDCGTATCCCTGTTA - -5' \\
\hline \multirow{2}{*}{ AN14_DA: } & $5^{\prime}-$ TTGACTCGACGGGCTATGGGACAA $-3^{\prime}$ \\
\hline & $3^{\prime}-$ ACTGAGCTGCDCGTATCCPTGTTA $-5^{\prime}$ \\
\hline \multirow{2}{*}{ AN14u_NL: } & $5^{\prime}-$ TTGACTCGACGGGCATAGGGACAA $-3^{\prime}$ \\
\hline & $3^{\prime}-$ ACTGAGCTGCCCGTATCCCTGTTA -5' \\
\hline \multirow{2}{*}{ AN14u_D: } & $5^{\prime}-$ TTGACTCGACGGGCATAGGGACAA $-3^{\prime}$ \\
\hline & $3^{\prime}-$ ACTGAGCTGCDCGTATCCCTGTTA $-5^{\prime}$ \\
\hline \multirow[t]{2}{*}{ AN14u_DA: } & $5^{\prime}$-TTGACTCGACGGGCATAGGGACAA $-3^{\prime}$ \\
\hline & $3^{\prime}-$ ACTGAGCTGCDCGTATCCPTGTTA $-5^{\prime}$ \\
\hline
\end{tabular}

The FRET of free AN14 and AN14u in equilibrium were very close to the calculated values assuming B-DNA conformations, again indicating average B-DNA conformations of these constructs (Fig. 4.25C, Fig. 4.26A). The FRET of AN14 decreased upon Rad4binding $\left(0.28 \pm 0.03\right.$ at $\left.25{ }^{\circ} \mathrm{C}\right)$ compared with free DNA $(0.32 \pm 0.04)$. Interestingly, AN14u also showed a decrease in FRET upon Rad4 binding similar to that seen with AN14 (Fig. 4.24C, Fig. 4.26A), suggesting that Rad4 induces similar conformational changes in AN14 and AN14u. As Rad4 exhibits little specificity towards these DNA constructs, this decrease in FRET is primarily ascribed to nonspecific interactions of the protein with DNA. Additionally, these decreases in FRET upon Rad4 binding are consistent with the direction of FRET change calculated for conversion from B-DNA conformation to that of the specific 'open' complex (Fig. 4.26A); this suggests that even in these nonspecific complexes, the bound DNA may be distorted in a manner that share structural resemblances to the 'open' conformation, for example, by unwinding partway to the full 'open' complex.

Equilibrium fluorescence measurements as a function of temperature showed nearly identical decrease in both $I_{D}$ and $I_{D A}$ in both AN14 and AN14u, with and without Rad4, as the temperature was increased from 10 to $40{ }^{\circ} \mathrm{C}$, (Fig. 4.25A, B; Fig 4.27A, B). These data 
correspond to a much smaller change in FRET as the temperature is raised, thus indicating little change in the equilibrium population distribution of DNA conformations sensed by the probes over this temperature range (Fig. 4.26B, C). However, the outstanding sensitivity of the T-jump approach, as also demonstrated with AN12 bound to $\Delta \beta$-hairpin3, enabled us to observe the relaxation kinetics in both AN14 and AN14u when bound to $\operatorname{Rad} 4$.

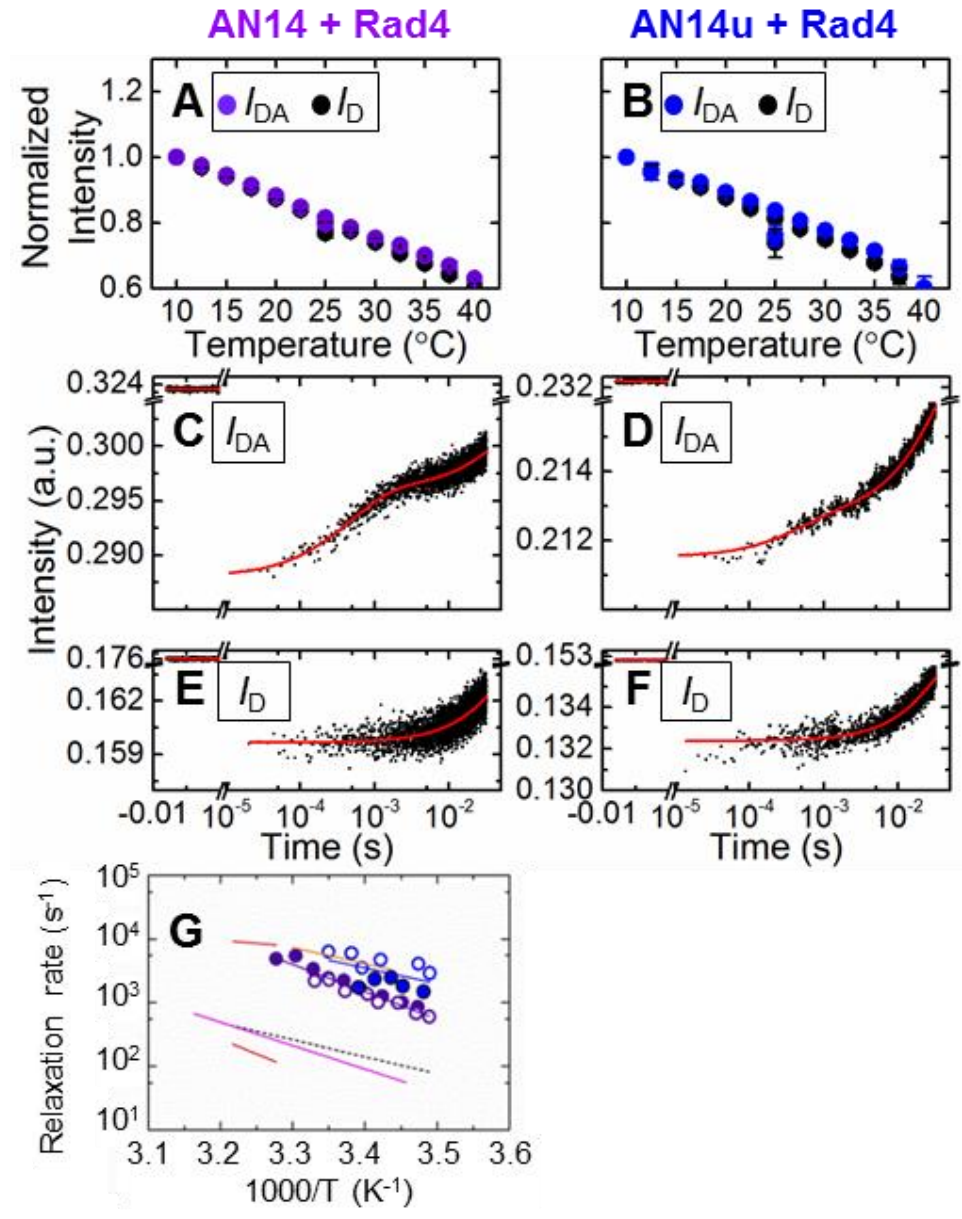

Fig. 4. 25. Equilibrium and T-jump measurements on AN14 and AN14u bound to wild-type Rad4. The experiments describing each panel (A-G) are similar to those for Figs. 4.17 \& 4.21. (A, B) IDA for AN14-Rad4 is in purple and IDA for AN14u-Rad4 in blue; the corresponding ID is in black. (C, D) T-jump relaxation traces with donor/acceptor-labeled DNA are shown: (C) AN14-Rad4 shows relaxation time $603 \pm 35 \mu$ s (at $22{ }^{\circ} \mathrm{C}$ ) after a T-jump from $15{ }^{\circ} \mathrm{C}$ to $22{ }^{\circ} \mathrm{C}$, (D) AN14u-Rad4 shows relaxation time $575 \pm 114 \mu$ s (at $22{ }^{\circ} \mathrm{C}$ ) after the same T-jump. (E, F) Control measurements on the Rad4-bound, donor-only DNA (AN14_D and AN14u_D) exhibit only the slow T-jump recovery kinetics. (G) Relaxation rates of AN14-Rad4 (purple) and AN14u-Rad4 (blue) versus inverse temperature from two independent sets of measurements (open/filled symbols). The continuous lines are Arrhenius fits to the relaxation rates, with activation enthalpies $18.2 \pm 2.2 \mathrm{kcal} / \mathrm{mol}$ and $11.6 \pm 3.3$ 
$\mathrm{kcal} / \mathrm{mol}$ for AN14-Rad4 and AN14u-Rad4, respectively. The pink, orange, red and dashed black lines are reproduced from Fig. 21G.
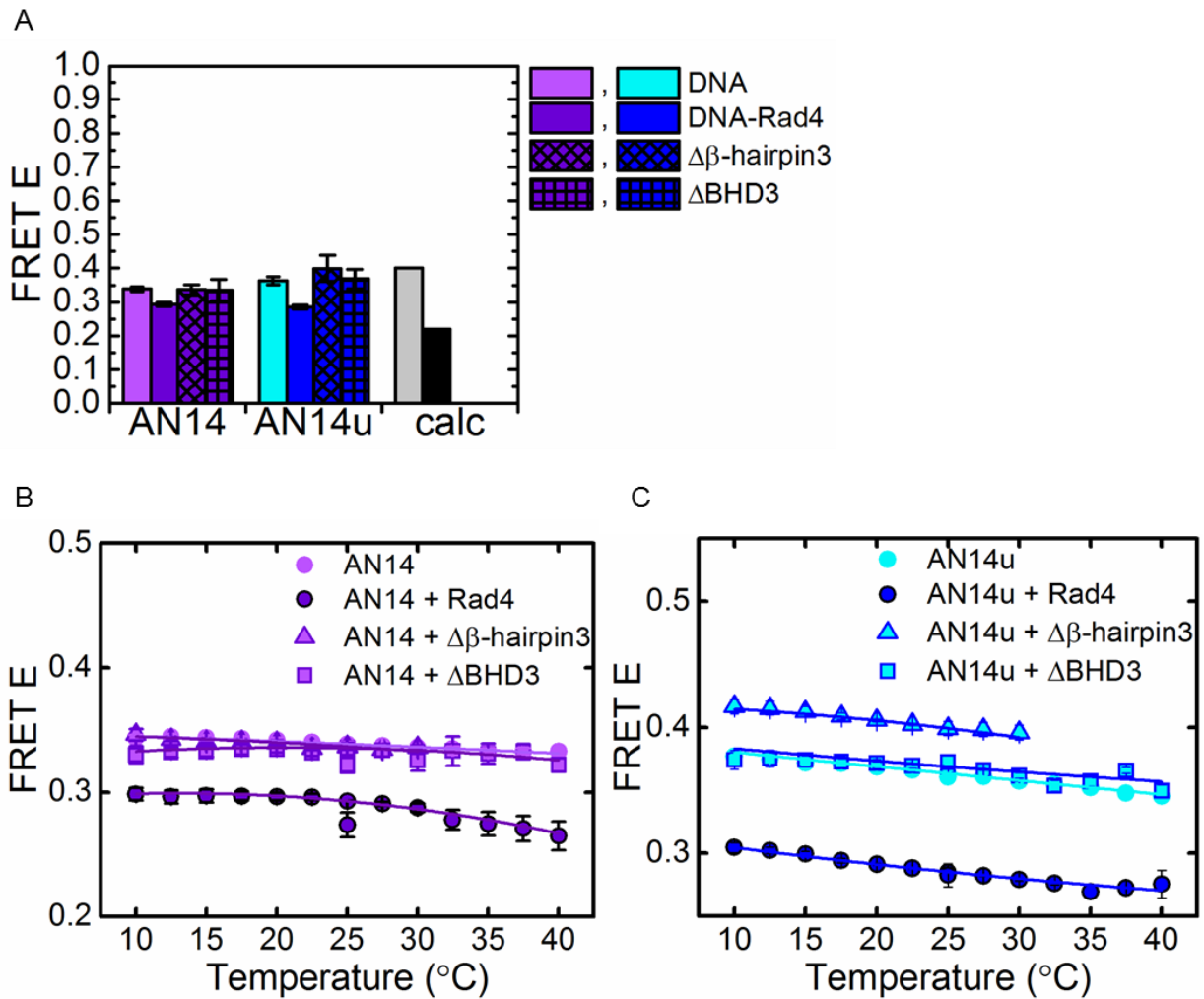

Fig. 4. 26. Equilibrium temperature scan and T-jump kinetics measurements on AN14 and AN14u in the absence of Rad4. Equilibrium (A, B) and T-jump data (C-F) for AN14 (mismatched) and AN14u (matched) DNA are shown, as control experiments for those done in the presence of Rad4 (Fig. 4.25A-F). Relaxation kinetics shown in (C, E) are for AN14 in response to a $\sim 7^{\circ} \mathrm{C}$ T-jump (from $15{ }^{\circ} \mathrm{C}$ to $22{ }^{\circ} \mathrm{C}$ ), and (D, F) is for AN14u in response to the same T-jump. (E, F) Control measurements on donor-only samples (AN14_D).

In T-jump experiments with AN14, relaxation kinetics (besides the typical, initial rapid drop and the T-jump recovery) were observed only with AN14_DA bound to Rad4 (Fig. 4. 25C, Fig. 4.29), but not in the absence of the acceptor or the protein (Fig. 4.25E; Fig. 4.27C, E). These results therefore capture the conformational dynamics of AN14 induced by Rad4. Interestingly, the relaxation kinetics on AN14_DA bound to Rad4 occurred with a characteristic time constant of $448.4 \pm 98.8 \mu$ s at $25^{\circ} \mathrm{C}$ (Fig. $4.25 \mathrm{G}$ ), similar to the submillisecond kinetic phase previously observed with AN12 bound to $\Delta \beta$-hairpin3 and $\triangle$ BHD3 (Fig. 4.21G). Notably, similar relaxation kinetics $\left(292.4 \pm 173.9 \mu\right.$ s at $\left.25^{\circ} \mathrm{C}\right)$ were 
also observed with undamaged AN14u_DA bound to Rad4 (Fig. 4.25D, G, Fig. 4.29A,G), although the amplitudes for AN14u-Rad4 were smaller than for AN14-Rad4 (Figs. 4.28H \& Fig, 4.29H). Again, no relaxation kinetics other than the initial rapid drop and slow Tjump recovery were observed in the absence of the acceptor or the protein (Fig. 4.25F; Fig. 4.27D, F). These results suggest that the $292-448 \mu$ s kinetics observed with wild-type Rad4 on the AN14/AN14u DNA substrates (see Table VII) reflect the same nonspecific mode dynamics that underlie the 113-228 $\mu$ s kinetics observed when the low-specificity Rad4 mutants were bound to a specific substrate, AN12.

Finally, we note that no relaxation kinetics with $\Delta \beta$-hairpin3 or $\Delta$ BHD3 were observed on AN14 or AN14u. The amplitudes of the relaxation kinetics on AN14/AN14u with wildtype Rad4 were already significantly smaller than the amplitudes on AN12 with wild-type Rad4, and at the limit of detection of the current set-up (see Fig. 4.20H for AN12; Figs. $4.28 \mathrm{H} \& 4.29 \mathrm{H}$ for AN14/AN14u). Any conformational changes induced by the mutant Rad4 in AN14/AN14u, if any, were thus likely to be below detection limit.

We uncovered new conformational dynamics occurring on the time-scales of $\sim 100$ $500 \mu$ s when Rad4 is nonspecifically bound to DNA. We interpret these dynamics as rapid unwinding ('twisting') of DNA by Rad4 resulting from the conformational interconversion between a rapidly scanning 'search' mode and a momentarily stalled 'interrogation' mode, en route to damage recognition. We also characterized the transition state (bottleneck) for lesion-specific 'opening' and demonstrated that the $\beta$-hairpin that stabilizes the 'open' conformation is inserted after the rate-limiting step. The progressive, multi-step nature of target recognition uncovered for this DNA repair protein provides critical insights into the speed-and-stability paradox of specific DNA recognition. 
AN14
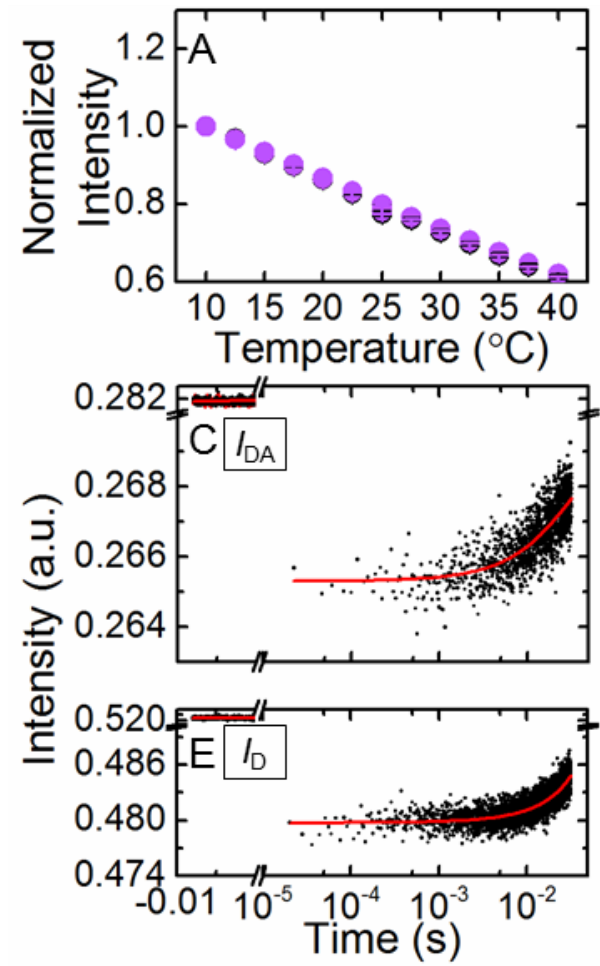

AN14u
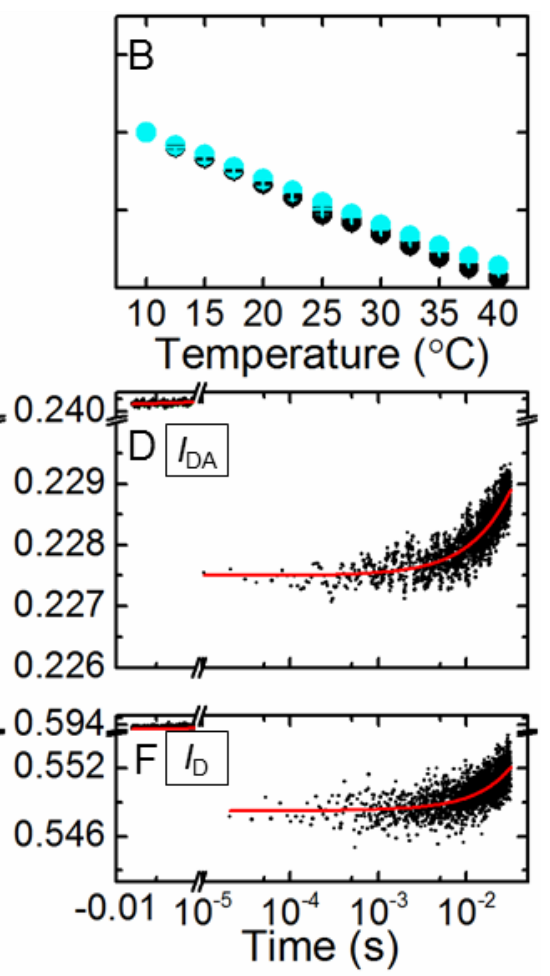

Fig. 4. 27. Equilibrium temperature scan and T-jump kinetics measurements on AN14 and AN14u in the absence of Rad4. Equilibrium (A, B) and T-jump data (C-F) for AN14 (mismatched) and AN14u (matched) DNA are shown, as control experiments for those done in the presence of Rad4 (Fig.4. 25A-F). Relaxation kinetics shown in (C, E) are for AN14 in response to a $\sim 7^{\circ} \mathrm{C}$ T-jump (from $15{ }^{\circ} \mathrm{C}$ to $22{ }^{\circ} \mathrm{C}$ ), and (D, F) is for AN14u in response to the same T-jump. (E, F) Control measurements on donor-only samples (AN14_D). 

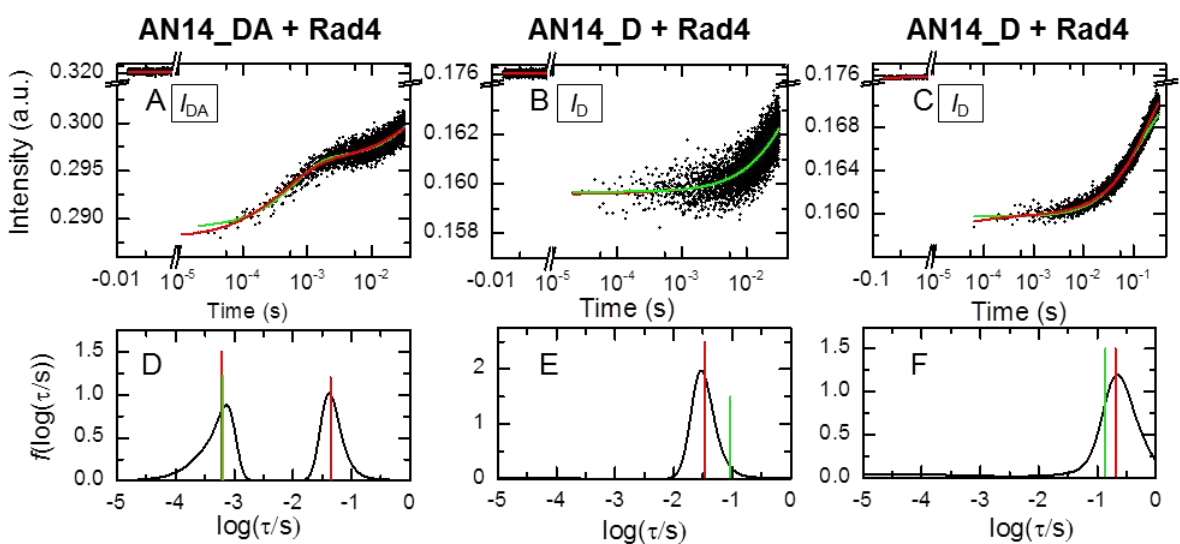

AN14_DA + Rad4
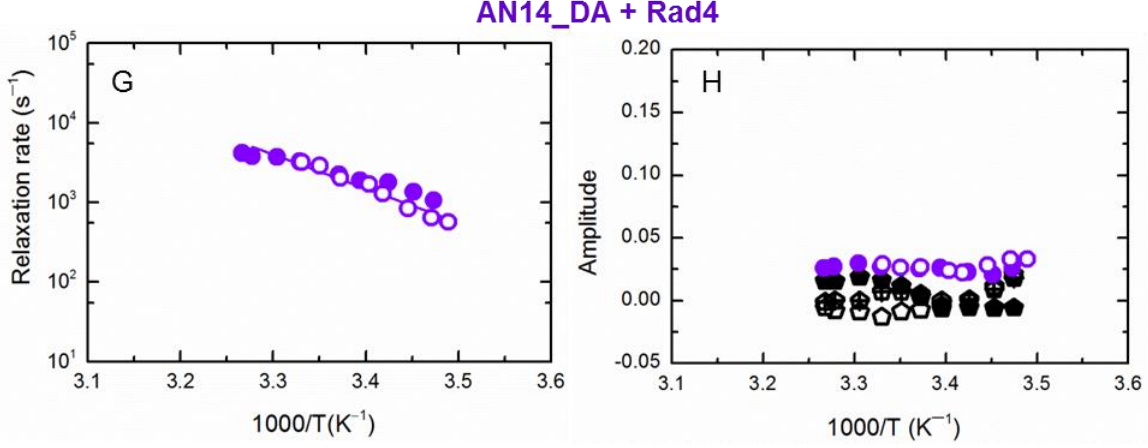

Fig. 4. 28. Analysis of T-jump relaxation traces for AN14-Rad4 complex. Relaxation traces in response to a T-jump perturbation are shown for (A) double-labeled AN14_DA-Rad4 complex, and (B, C) donor-only AN14_D-Rad4 complex. The panels are as described in Fig. 4.19. The average time constants describing the best fit parameters from the two analyses are shown as vertical red (mem) and green (from Eq. 3 or 6), and are (A) $\tau_{\text {ave }}=603 \mu$ s and $43.8 \mathrm{~ms}$ (red; fast and slow components, respectively) and $\tau \mathrm{r}=\mathrm{k}_{\mathrm{r}}^{-1}=638 \mu \mathrm{s}$ (green, with $\tau_{\text {rec }}$ fixed at $135 \mathrm{~ms}$ ); (B) $\tau_{\text {rec }}=30 \mathrm{~ms}$ (red) and $92.6 \mathrm{~ms}$ (green); and (C) $\tau_{\text {rec }}=200 \mathrm{~ms}$ (red) and $135 \mathrm{~ms}$ (green). (G) Relaxation rates obtained from single-exponential kinetics convoluted with T-jump recovery (as described for panel A) are plotted versus the inverse of the final temperature (after the T-jump) (purple symbols). The continuous purple line (reproduced from Fig.4. 25G) is the Arrhenius fit to the mem relaxation rates obtained for the same data sets. $(\mathrm{H})$ The relative amplitude in the relaxation traces (purple symbols) and from equilibrium measurements (black symbols) are plotted as a function of inverse temperature. These data highlight that the T-jump amplitudes for AN14-Rad4 are more than 2-fold smaller than those for AN12-Rad4, and correspond to approximately 3\% change in the donor intensities. The amplitudes predicted from equilibrium experiments are also small and reflect the noise in the measured donor intensities in these experiments. 

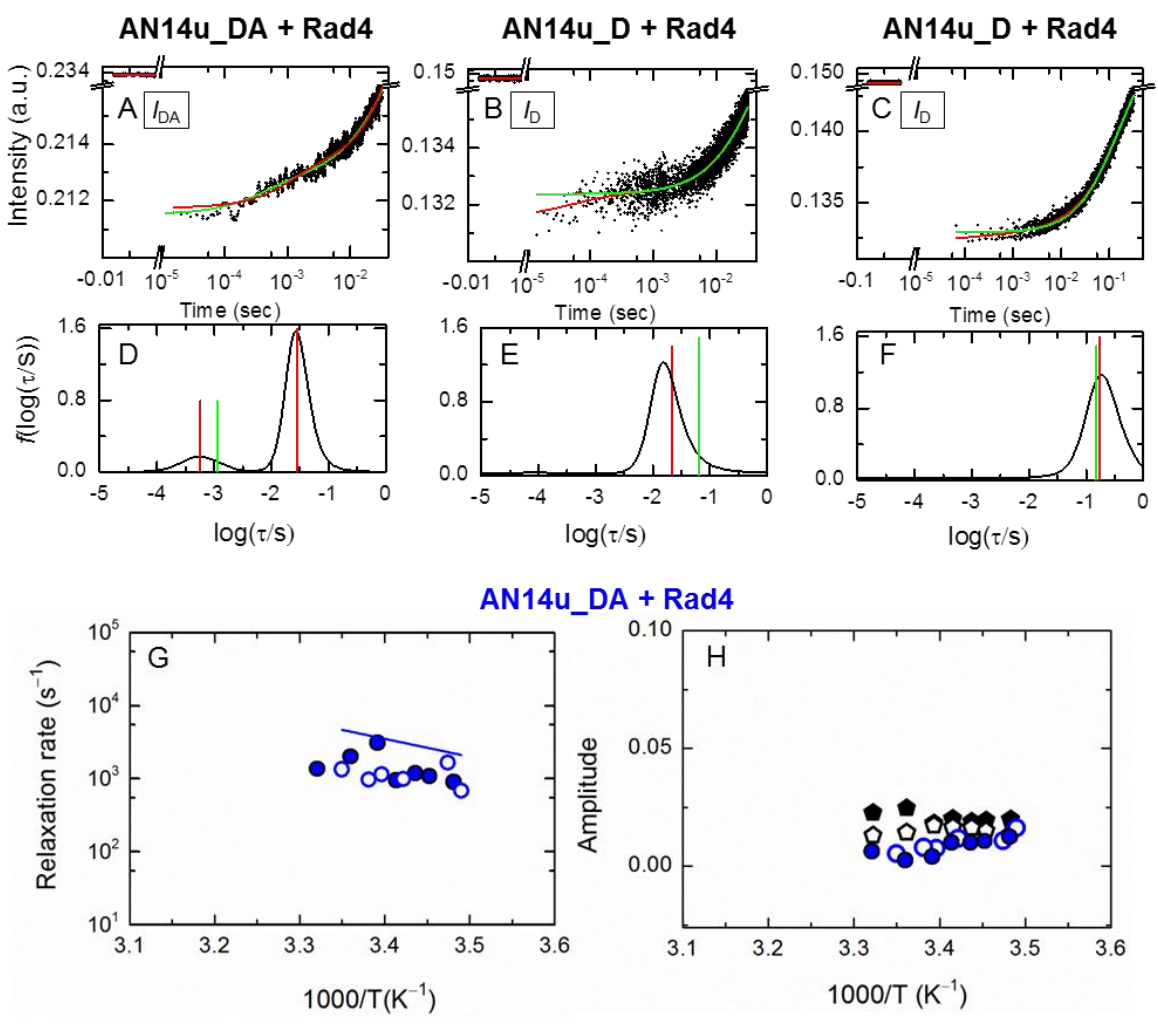

Fig. 4. 29. Analysis of $\mathrm{T}$-jump relaxation traces for $\mathrm{AN} 14 \mathrm{u}-\mathrm{Rad} 4$ complex. Relaxation traces in response to a T-jump perturbation are shown for (A) double-labeled AN14u_DA-Rad4 complex, and (B, C) donor-only AN14u_D-Rad4 complex. The panels are as described in Fig. 4.19 and 4.28. The average time constants describing the best fit parameters from the two analyses are shown as vertical red (mem) and green (from Eq. 3 or 6), and are (A): $\tau_{\text {ave }}=575 \mu$ s and $27.8 \mathrm{~ms}$ (red; fast and slow components, respectively) and $\tau_{\mathrm{r}}=\mathrm{kr}^{-1}=1.1 \mathrm{~ms}$ (green, with $\tau_{\mathrm{rec}}$ fixed at $151 \mathrm{~ms}$ ); (B) $\tau \mathrm{rec}=21 \mathrm{~ms}$ (red) and $64.9 \mathrm{~ms}$ (green); and (C) $\tau \mathrm{rec}=172 \mathrm{~ms}$ (red) and $151 \mathrm{~ms}$ (green). (G) Relaxation rates obtained from single-exponential kinetics convoluted with T-jump recovery, as described for Fig.4.28A, are plotted versus the inverse of the final temperature (after the T-jump) (blue symbols). The continuous blue line (reproduced from Fig. 4.25G) is the Arrhenius fit to the mem relaxation rates obtained for the same data sets. $(\mathrm{H})$ The relative amplitude in the relaxation traces (blue symbols) and from equilibrium measurements (black symbols) are plotted as a function of inverse temperature. These amplitudes for AN14u-Rad4 are even smaller than those for AN14-Rad4, and correspond to less than $1 \%$ change in the donor intensities. 


\subsubsection{DNA bending dynamics measured with extrinsically attached FRET pair/ AN7}

To measure directly the kinetics of DNA bending/unbending we used FRET label DNA construct (AN7). We attached FRET pairs (TAMRA and Cy5) to a 33-mer long DNA, with the FRET labels attached internally to thymine bases on either side of the protein binding site (ATA mismatch), approx. 18 base-pair apart, to measure changes in the distance between the labels as the DNA bends (Table IX). The design of this DNA construct was based on previous constructs used in equilibrium and single-molecule FRET studies of DNA bending in complex with MutS, another DNA repair protein that recognizes mismatches in DNA [78, 79].

Table IX: FRET mismatched DNA sequence

\begin{tabular}{|l|l|}
\hline AN7 & $\begin{array}{l}\text { ATTTCCTTCAGCAGATAATAACCATACYGATTCACAT } \\
\text { TAAAGGAAGXCGTCTATATATGGTATGACTAAGTGTA }\end{array}$ \\
\hline
\end{tabular}

X-TAMRA-dT

Y- Cy5 - dT

The dyes are attached on the T nucleotide

The fluorescence emission spectra of donor-acceptor labeled AN7 and donor only labelled AN7 was measured in the presence and absence of Rad4 (Fig. 4.30 A,B) and from these spectrum the ratio (which is proportional to the actual FRET value: see method, chapter 2) of the mismatch AN7 DNA while in free or bound to Rad4 was calculated. The ratio increases by $\sim 0.15$ when AN7 is complexed with the Rad4; for free AN7 the ratio is $\sim 0.2\left(25^{\circ} \mathrm{C}\right)$ and in the presence of Rad4 it increased to $\sim 0.35\left(25^{\circ} \mathrm{C}\right)$. This increase in ratio/FRET can be 
attributed to the Rad4 induced DNA bending; which is seen in the crystal structure of lesion bound Rad4.

Next we measured the T-jump kinetics of the free AN7 and AN7 bound to Rad4. We expanded the time scale up to $320 \mathrm{~ms}$, since the kinetics of this complex is slow and the 32 ms time scale which we used earlier to capture the DNA opening dynamics or the DNA unwinding was not enough to completely monitor this dynamics. We observed a very slow DNA bending rate in the presence of Rad4 (Fig. 4.30G) whereas free AN7 DNA and the donor AN7 in the presence or in the absence of Rad4 showed only the typical tjump recovery kinetics in this time scale (Fig. $4.30 \mathrm{H}, \mathrm{I}, \mathrm{J})$. However since the bending dynamics is slow, it is pretty much convoluted with the tjump recovery time. The mem analysis only gave the average rate both from the actual bending kinetics and tjump recovery, which is faster ( 56 $\mathrm{ms}$ ) than if there is only tjump recovery ( $200 \mathrm{~ms}$ ) present in the kinetics (Fig. $4.30 \mathrm{G}, \mathrm{H}, \mathrm{I}, \mathrm{J}$ : Fig. 4.31 ). 

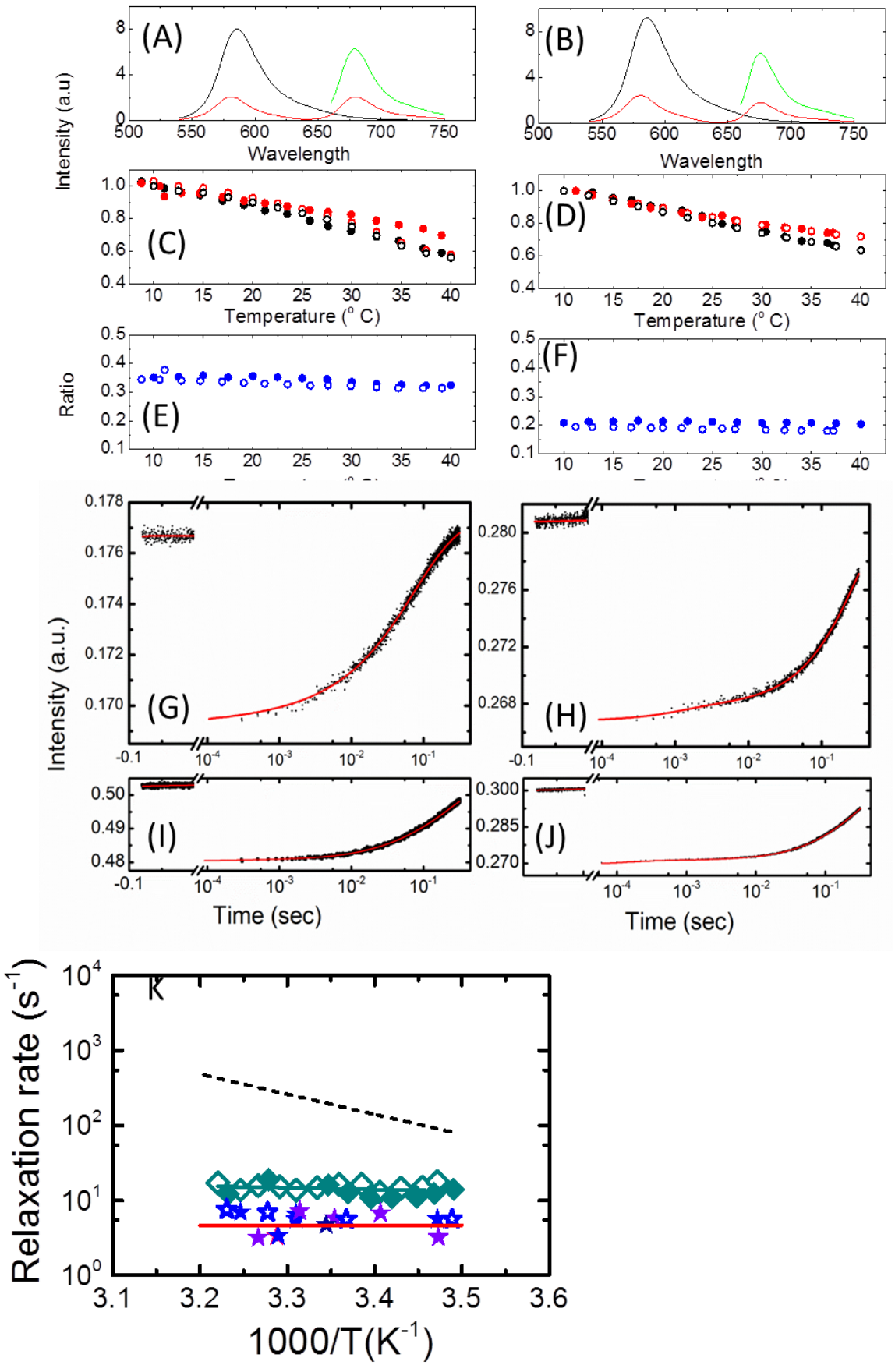

Fig. 4. 30. Equilibrium and T-jump kinetics of AN7 in the presence and absence of Rad4. (A,B): Fluorescence emission spectra of AN7 in the presence and in the absence of Rad4. Fluorescence emission intensities of TAMRA (donor) with excitation at $536 \mathrm{~nm}$, are plotted as a function of the wavelength at $25^{\circ} \mathrm{C}$, AN7_D—Rad4 (black), AN12_DA-Rad4 (red) (A), and AN7_D (black), AN7_DA (red) (B). Fluorescence emission intensities of Cy5 (acceptor) with direct excitation of Cy5 at $565 \mathrm{~nm}$ are also plotted (green). (C, D) $\mathrm{I}_{\mathrm{DA}}$ (red) and $\mathrm{I}_{\mathrm{D}}$ (black) in the presence $(\mathrm{C})$ and in the absence of Rad4 (D). (E,F) Ratio (FRET efficiency) versus temperature for bound (E) and free (F) AN7. (G,H) T-jump relaxation traces of AN7_DA in the presence and in the absence of Rad4: AN7_DA-Rad4 shows relaxation time $56 \mathrm{~ms}\left(\right.$ at $15{ }^{\circ} \mathrm{C}$ ) after a T-jump from $8{ }^{\circ} \mathrm{C}$ to $15{ }^{\circ} \mathrm{C}$ (the relaxation rate is the average rate of actual kinetics plus T-jump recovery, since the kinetics is slow 
hence convoluted with the recovery kinetics and either mem or single exp+recovery function can't separate completely the kinetics from the recovery) (G). Free AN_DA shows relaxation time $310 \mathrm{~ms}$ (at $22{ }^{\circ} \mathrm{C}$ ) after the same T-jump (H). (I, J) Control measurements on the AN7_D in the presence (I) and in the absence (J) of Rad4 exhibit only the slow T-jump recovery kinetics (I: $177 \mathrm{~ms}, \mathrm{~J}: 260 \mathrm{~ms}$ ). (K) Relaxation rates of AN7_DA-Rad4 (dark cyan), and T-jump recovery of AN7_DA (blue) versus inverse temperature from two independent sets of measurements (open/filled symbols). The continuous dark cyan lines is Arrhenius fits to the relaxation rates, the red continuous line is the average recovery time constant from nearly sixty control measurements and the black dashed line is DNA opening rate measured with $2 \mathrm{AP}$ probe.
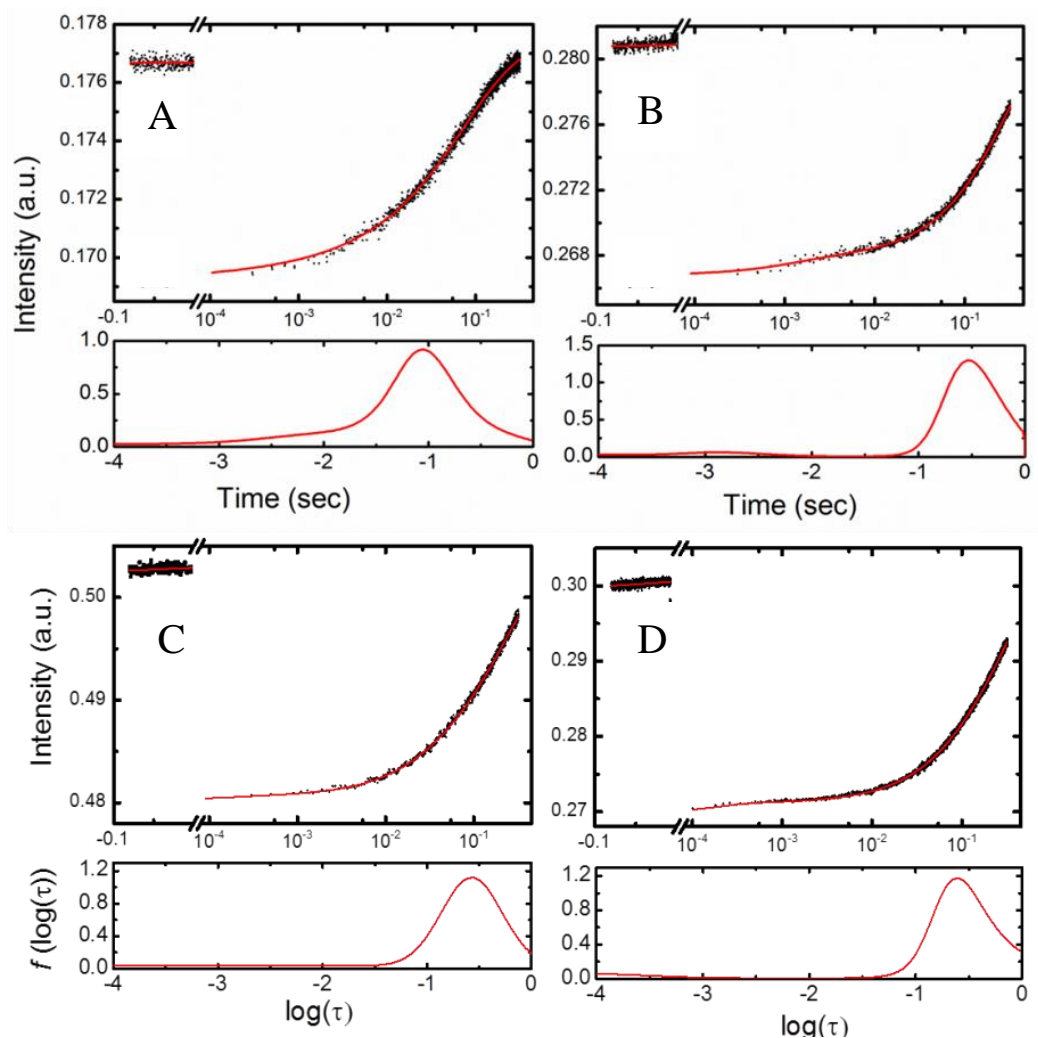

Fig. 4. 31. Mem analysis of kinetics presented in figure 30 E,F,G\&H are shown in A,B,C\&D (Top: mem fit, bottom: Mem peak distribution) respectively. 


\subsubsection{DNA bending rate in the presence of mutant Rad4}

The equilibrium measurements showed that in the presence of both mutants the ratio of AN7 increased: AN7- $\Delta \beta$-hairpin3 gives a ratio of $0.28\left(25^{\circ} \mathrm{C}\right)$ and AN7- $\Delta \mathrm{BHD} 3$ gives a ratio of $0.32\left(25^{\circ} \mathrm{C}\right)$ compare to the ratio $0.2\left(25^{\circ} \mathrm{C}\right)$ of free AN7.

T-jump measurements with the mutants detected no kinetics with AN7 in the absence of the acceptor probe (Fig. 4.32) as in the case of wild type Rad4. Also in the presence of acceptor, $\Delta \beta$-hairpin3-bound AN7 revealed no kinetics other than tjump recovery in the temperature window we measured from 10-40 C. However, $\triangle \mathrm{BHD} 3$-bound AN7 showed biphasic relaxation kinetics in the temperature range $10-40{ }^{\circ} \mathrm{C}$ (final temperature). The fast component of the biphasic behaviour had 197-552 $\mu$ s relaxation times, and overlapped those of AN14-Rad4 or AN12- mutant Rad4 (Table VII), whereas the slow component was $309-660 \mathrm{~ms}$, which is similar to the recovery at low temperatures but it deviates (slower) from the recovery timescale at higher temperatures. Though it is possible that there is a slower kinetics, which is slower than what we observed with wild type $\mathrm{Rad} 4$, it is impossible to precisely determine this kinetics due to the limitation imposed by the temperature recovery of the tjump apparatus.

The sub-millisecond kinetics observed for $\triangle \mathrm{BHD} 3$ had relaxation which is distinct from that observed with the wild type (Fig. 4.32G) but similar to the nonspecific conformational changes that we observe with other probes discussed earlier in this chapter. The fast phase observed in T-jump kinetics with this mutant can be primarily due to the motions in the nonspecific modes. 

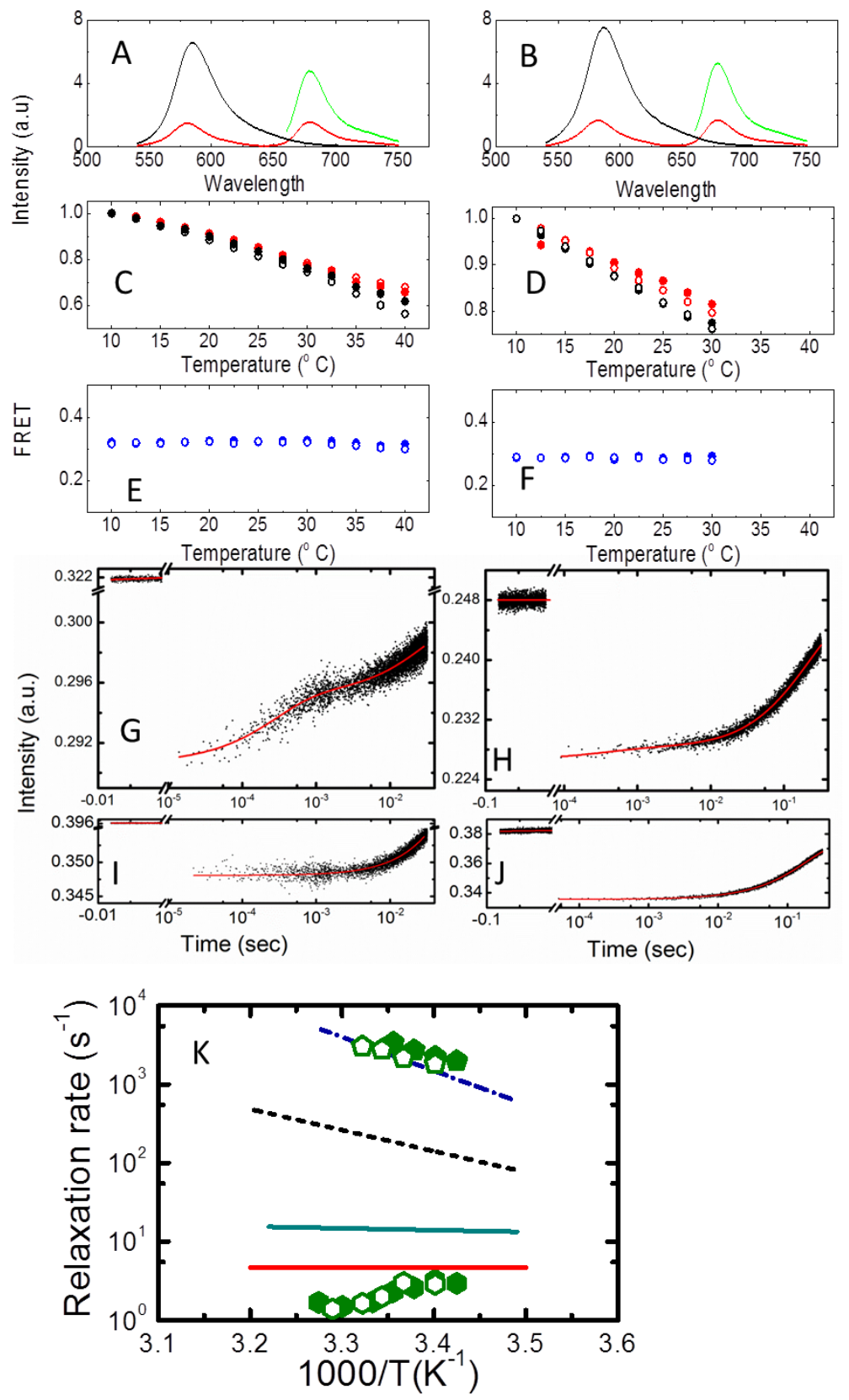

Fig. 4. 32. Equilibrium and T-jump measurements on AN7 bound to Rad4 $\beta$-hairpin mutants $(\Delta \mathrm{BHD} 3, \Delta \beta$-hairpin3). The experiments describing each panel (A-J) are similar to those for Fig.4. 30. (A, B) Equilibrium spectrum for AN7- $\triangle B$ HD3 (A) and AN7- $\Delta \beta$-hairpin3 (B). (C,D) $\mathrm{I}_{\mathrm{DA}}$ for AN7- $\triangle$ BHD3 (red) (C) and AN7- $\Delta \beta$-hairpin3 (red) (D) with the corresponding $\mathrm{I}_{\mathrm{D}}$ in black. $(\mathrm{E}, \mathrm{F})$ Ratio (FRET efficiency) versus temperature for AN7- $\mathrm{BHD} 3(\mathrm{E})$ and AN7- $\Delta \beta$-hairpin3 (F). $(\mathrm{G}, \mathrm{H})$ T-jump relaxation traces of AN7_DA in the presence of $\triangle B H D 3(\mathrm{G})$ and in the presence of $\Delta \beta$ hairpin3 (H) : (G) AN7_DA- $\triangle$ BHD3 shows fast relaxation time $362 \mu \mathrm{s}$ (at $23{ }^{\circ} \mathrm{C}$ ) after a T-jump from $14{ }^{\circ} \mathrm{C}$ to $23{ }^{\circ} \mathrm{C},(\mathrm{H}) \mathrm{AN} 7-\Delta \beta$-hairpin 3 shows only the temperature recovery kinetics with a relaxation time $238 \mathrm{~ms} \mathrm{(at} 22{ }^{\circ} \mathrm{C}$ ) after the same T-jump. (I, J) Control measurements on the donoronly DNA (AN7_D) in the presence of $\triangle \mathrm{BHD} 3$ (I) and in the presence of $\Delta \beta$-hairpin3 (J) exhibit only the slow T-jump recovery kinetics (I: $294 \mathrm{~ms}, \mathrm{~J}: 208 \mathrm{~ms}$ ). (K) Fast relaxation rates of AN7 DA$\triangle \mathrm{BHD} 3$ (olive) versus inverse temperature from two independent sets of measurements (open/filled symbols). The continuous dark olive line is Arrhenius fits to the relaxation rates, the red continuous 
line is the average recovery time constant from sixty control measurements, the continuous dark cyan line is AN7 relaxation rate with wild type Rad4 and the black dashed line is DNA opening rate measured with $2 \mathrm{AP}$ probe. Note the fast relaxation rate was captured by zooming the data within 32 $\mathrm{ms}(\mathrm{G})$ and the control experiment (I) also done with this same time window.
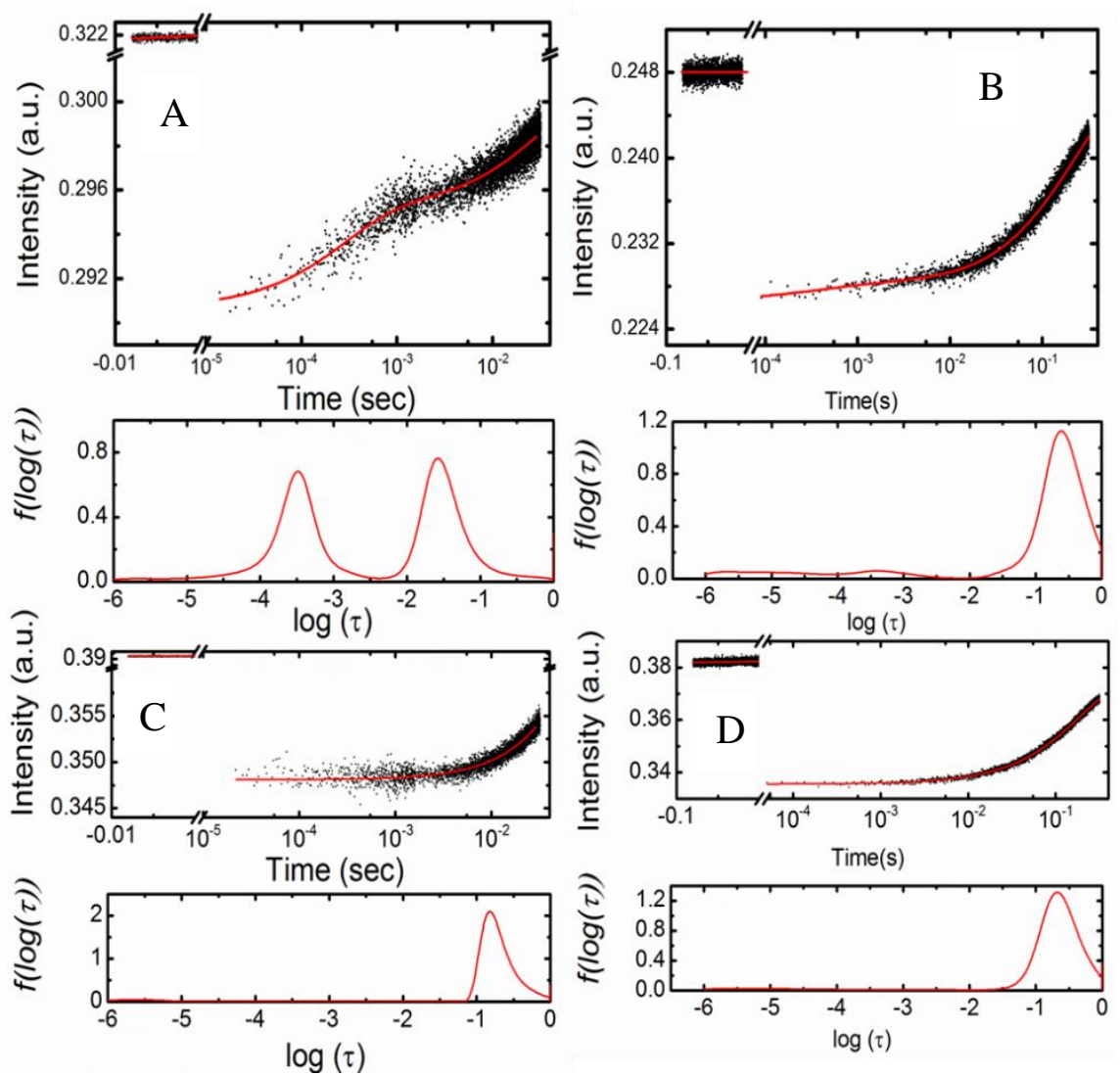

Fig. 4. 33. Mem analysis of kinetics presented in figure $4.32 \mathrm{E}, \mathrm{F}, \mathrm{G} \& \mathrm{H}$ are shown in $\mathrm{A}, \mathrm{B}, \mathrm{C}, \& \mathrm{D}$ (Top: Mem fit, bottom: Mem peak distribution) respectively. 


\subsection{Discussion}

\subsubsection{Rad4/XPC induced nucleotide flipping/ Open dynamics measured with 2AP probe}

Rad4/XPC does not make any direct contacts with damaged nucleotides in the final recognition ('open') complex and thus relies exclusively on 'indirect readout' to recognize diverse DNA lesions. Structural studies have provided static snapshots of $\operatorname{Rad} 4$ bound to DNA, but the sequence of structural changes and their dynamics in the Rad4-DNA complex that leads to efficient and reliable recognition have remained elusive.

We propose that Rad4's lesion recognition relies on 'kinetic gating' mechanism to achieve selectively higher probability of 'opening' damaged DNA than normal DNA. In this mechanism, lesion recognition by Rad4/XPC leading to the 'open' conformation is controlled ('gated') by the competition between two kinetic parameters, as illustrated in Fig. 4.34: (1) opening time to form the thermodynamically stable, 'open' or recognition complex and (2) residence time per site as determined by the one dimensional diffusion such as sliding and hopping of protein on DNA when it is in the loosely bound, nonspecific 'search' mode [60]. The opening rate for damaged DNA (red arrows) is expected to be larger than the opening rate for undamaged DNA (blue arrows), because of, for instance, the weakened base stacking and hydrogen bonding within a lesion. The residence time, on the other hand, may well be shorter for undamaged than for damaged DNA. The balance between these two factors together results in a higher net probability of $\operatorname{Rad} 4 / \mathrm{XPC}$ opening damaged rather than undamaged DNA. This difference in the opening probabilities forms the basis for lesion recognition despite little difference in the thermodynamically most stable structures.

How proteins find their specific targets with a large number of closely related nonspecific molecules around is not limited to DNA-binding proteins, but is prevalent in biology. It is inevitable that erroneous bindings occur, but most systems are tolerant of such 
thermodynamic noise owing to intermediate 'proofreading' before proceeding down a pathway. 'Kinetic proofreading' originally proposed by $[80,81]$ is one such mechanism that detects and aborts incorrect bindings using chemical energy (for example, ATP hydrolysis). Our model showcases, for the first time, that 'kinetic proofreading' can be accomplished with neither chemical energy consumption nor difference in the thermodynamically most preferred states of the protein-DNA complexes [82].

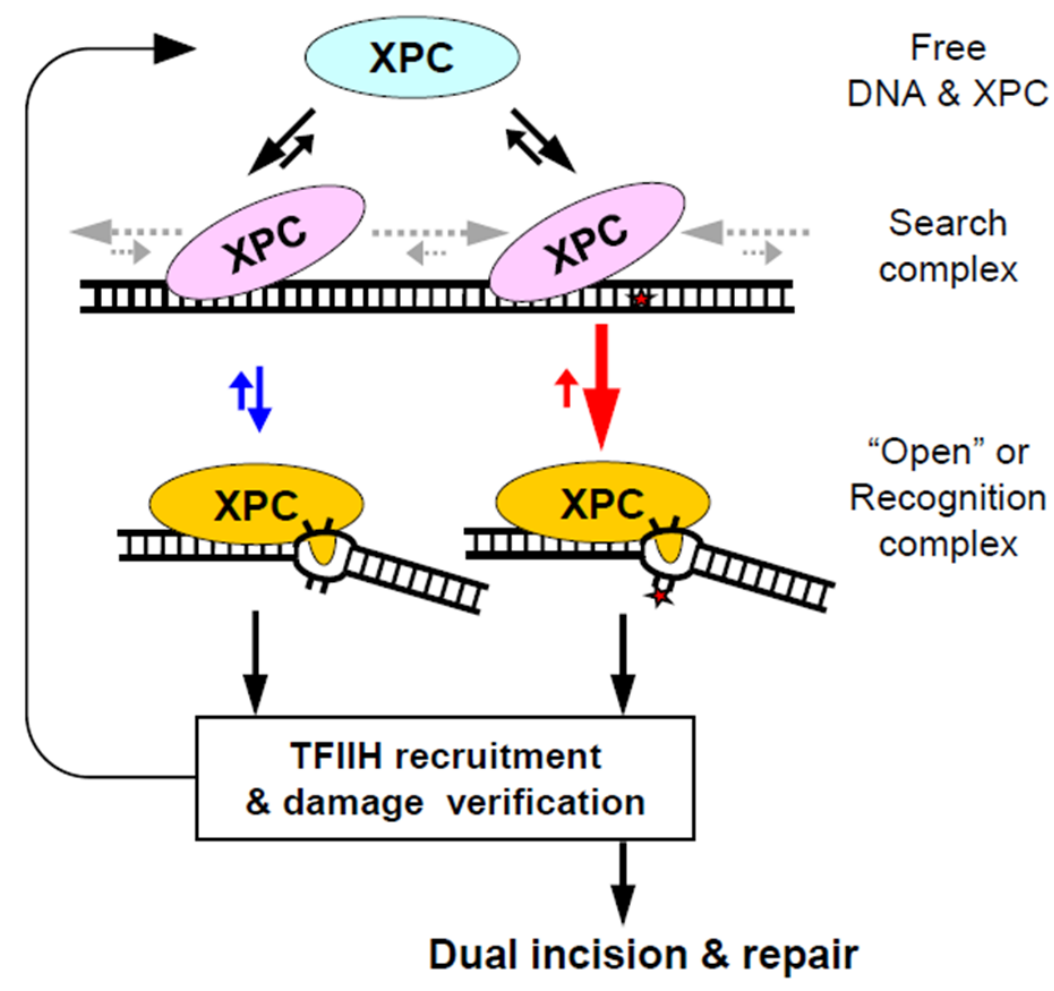

Fig. 4. 34. 'Kinetic gating' mechanism for DNA damage recognition by Rad4/XPC. This schematic illustrates that the lesion recognition by Rad4/XPC is controlled by the competition between Rad4's residence time per site when it is in the 'search' mode (pink) and its opening time to form the 'open' or recognition complex (orange). The diffusion rates that control the residence time are indicated by the grey arrows. The opening (and its reverse) rates are depicted in red and blue arrows for damaged and undamaged DNA, respectively.

During NER, lesion recognition by XPC is also followed by a bona fide, ATP-dependent damage verification by TFIIH, which further augments NER accuracy [83]. Finally, the DNA 'opening' induced by prolonged residence time may help explain the mechanisms of XPC within and beyond NER that cannot be explained solely by lesion-binding preferences of 
XPC $[5,84-86]$. In these cases, XPC-interacting proteins (for example, UV-damaged DNAbinding protein complex or DNA glycosylases) and/or post-translational modifications on XPC can help 'stall' the protein on DNA and induce opening of otherwise non-cognizant DNA. We also posit that 'kinetic gating' is a general target-recognition mechanism even for DNA-binding proteins that ultimately rely on direct structural discrimination, as a way of reducing wasteful interrogation at each and every site. Our results may have broad implications in genome maintenance, gene regulation, and cancer biology.

\subsubsection{Rad4/XPC induced helical distortion dynamics measured using tco/tcniro}

By combining recently developed $\mathrm{tC}^{\mathrm{O}} / \mathrm{t} \mathrm{C}_{\text {nitro }}$ FRET pair with microsecond-resolved $\mathrm{T}$-jump spectroscopy, we have uncovered novel conformational dynamics in duplex DNA during damage search and recognition. We observe two distinct kinetic steps along the lesion recognition trajectory of $\operatorname{Rad} 4:$ (i) a slow $(\sim 5-10 \mathrm{~ms})$ phase comparable to the previously reported DNA 'opening' kinetics and (ii) a novel, fast $(\sim 100-500 \mu \mathrm{s})$ phase that represents nonspecific DNA unwinding. The relative amplitudes of the two phases vary depending on the location of the probes and on the presence/absence of the $\beta$-hairpin or the BHD3 domain of Rad4. This work provides missing keys to constructing the lesion recognition trajectory of $\operatorname{Rad} 4$.

I. Slow phase - The rate-limiting step towards forming the 'open' recognition conformation does not require $\beta$-hairpin insertion. The mismatched AN12 DNA that exhibited specific binding to $\operatorname{Rad} 4$ showed $\sim 8 \mathrm{~ms}$ kinetics when bound to wild-type $\operatorname{Rad} 4$. This slow phase is concurrent with the $\sim 5-10 \mathrm{~ms}$ kinetics of the Rad4-induced DNA 'opening', probed at specific mismatch sites by $2 \mathrm{AP}$ Thus, we propose that the slow phase reflects the kinetics of overcoming the rate-limiting transition state ensemble (bottleneck) during the DNA 'opening' process. However, the structural distortions captured by $\mathrm{tC}^{\mathrm{o}} / \mathrm{tC}_{\text {nitro }}$ in $\mathrm{AN} 12$ are not 
necessarily the same as those reported by the $2 \mathrm{AP}$ probe, though they may share a common bottleneck: the 2AP kinetics were completely absent in either of the $\beta$-hairpin deletion mutants, while the slow phase probed by $\mathrm{tC}^{\mathrm{o}} / \mathrm{tC}_{\text {nitro }}$ in $\mathrm{AN} 12$ could be observed at least with $\triangle \mathrm{BHD} 3$ mutant at higher temperatures $\left(>30{ }^{\circ} \mathrm{C}\right)$. Considering the placement of the $2 \mathrm{AP}$ probe at the flipped-out nucleotide site and the high sensitivity of its fluorescence to solvent exposure, we assign the $2 \mathrm{AP}$ probed motions to full nucleotide flipping of the mismatched nucleotides; our results indicate that the $\beta$-hairpin is required to execute this step, for instance, by lowering the energetic barrier for the full-flipping and/or stabilizing the final complex conformation. On the other hand, the $\mathrm{tC}^{\mathrm{o}} / \mathrm{tC}$ nitro $\mathrm{FRET}$ probes, especially in $\mathrm{AN} 12$ where the acceptor flanks the mismatch site, may be sensing distortions/unwinding around the mismatch nucleotides even with the damaged/mismatch nucleotides still intra-helical. The $\triangle$ BHD3 mutant, despite the lack of the entire $\beta$-hairpin domain, could induce the ratelimiting distortions sensed by $\mathrm{tC}^{\mathrm{o}} / \mathrm{tC}_{\text {nitro }}$ probes, though still incapable of the $2 \mathrm{AP}$-sensed, full nucleotide-flipping. (Whether $\Delta \beta$-hairpin3 could also induce the bottleneck distortions at these higher temperatures could not be tested because of instability of this mutant above 30 $\left.{ }^{\circ} \mathrm{C}\right)$. We conclude that full nucleotide flipping that engages the $\beta$-hairpin occurs after the ratelimiting distortion, or partial 'opening' on path to the fully 'open' conformation. We envision this partially 'open' conformation as one that entails a significantly unwound DNA structure, potentially with disrupted hydrogen bonding and unstacking, although the nucleotides at the lesion site may still be intra-helical. The late involvement of the $\beta$-hairpin during Rad4induced DNA opening is also consistent with our observation that the preliminary interrogation step also does not require the $\beta$-hairpin, as further discussed below.

II. Fast phase - Preliminary interrogation prior to slow, specific 'opening' of the damaged site. The $\sim 100-500 \mu$ s fast phase is more than 10 -fold faster than the slow phase. It was observed with both $\beta$-hairpin deletion mutants on AN12, and with wild-type protein bound to 
AN14 and AN14u. These protein-DNA complexes share nonspecific binding as a common feature, either due to a low-specificity mutant protein or a low-specificity DNA construct. We therefore interpret the rapid dynamics as arising from conformational fluctuations underlying nonspecific interactions. Remarkably, all these fast-phase kinetics exhibited approximately the same Arrhenius behavior, largely independent of the location or neighboring sequence context of the probes, and all required the presence of Rad4 (Fig. 4.25G); this indicates that the observed dynamics involve protein-assisted conformational changes and that the intrinsic energetic differences in distorting these DNA by the protein must have been removed upon formation of the complex, apparently by using the binding energy. Based on the nonspecific nature of the complexes, the sensitivity of the FRET probes to DNA helicity, and a comparison of the Rad4-bound DNA and B-form structures [36], we attribute the fast dynamics primarily to the dynamics of DNA unwinding/rewinding fluctuations ('twisting') during nonspecific interactions. These nonspecific twisting motions should involve smaller energetic barrier than the distortions sensed by the slower rate-limiting step. The $\beta$-hairpin or BHD3 were also not required for these fast unwinding; the nonspecifically unwound structures are most likely to retain intra-helical nucleotides at putative lesion sites.

Considering the progressive nature of lesion 'opening' trajectory, we propose that the fast nonspecific unwinding depicts a preliminary step en route to full DNA 'opening' in Rad4 lesion recognition trajectory (further discussed below) (Fig. 4.35). Consistent with this, the FRET changes induced by Rad4 binding to AN14/AN14u in equilibrium were in the same direction as the FRET changes expected in a conformational change from 'straight' B-DNA to the 'open' conformation. We also note that both the fast and the slow phases were observable simultaneously in AN12- $\triangle \mathrm{BHD} 3$, supporting the sequential multistep nature of recognition. Our results mark the first direct observation of DNA conformational fluctuations captured during nonspecific interrogation with a DNA repair protein that relies on indirect 
readout to recognize its target lesions, and also the first report of DNA dynamics attributed to unwinding distinct from other motions such as nucleotide flipping or bending.

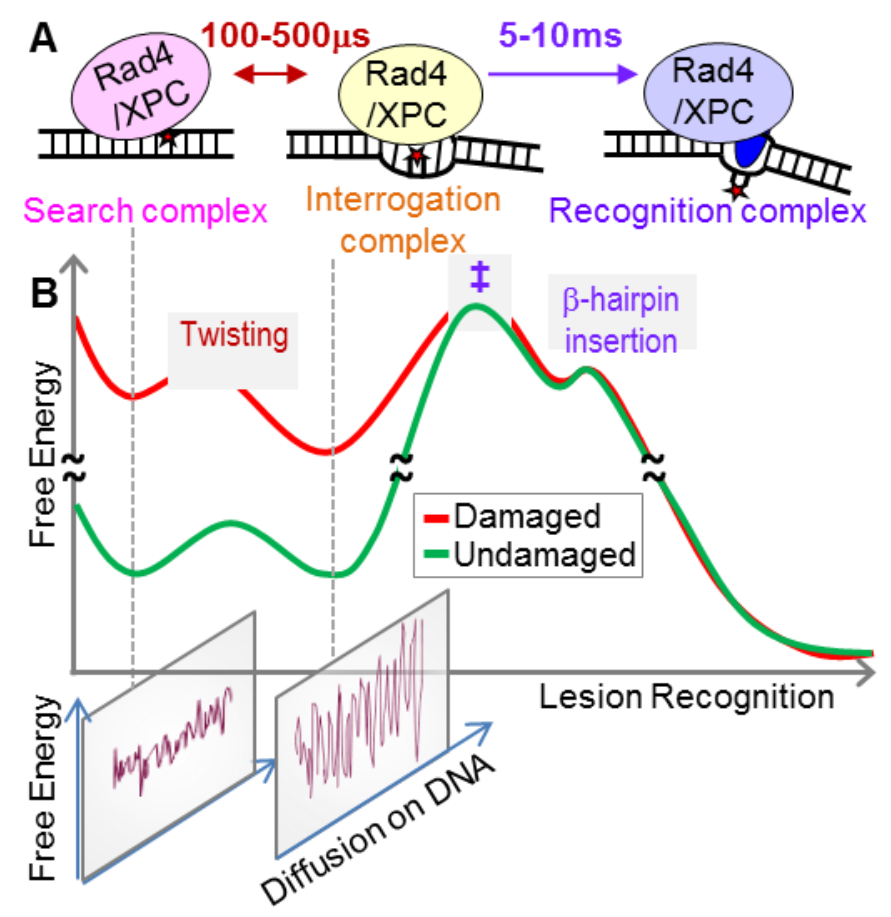

Fig. 4. 35. Free energy schematic along the conformational trajectory of lesion recognition by Rad4. (A) The three distinct binding modes for Rad4/XPC as it searches for, interrogates, and recognizes a damaged site are illustrated, and the time-scales indicated for interconversion between these modes. (B) (Top) A free energy profile that may underlie the observed kinetics along the recognition trajectory. Damaged DNA in nonspecific binding modes is shown with inherently higher free-energy than undamaged DNA due to the helical destabilization induced by DNA lesion. The 100-500 $\mu \mathrm{s}$ 'twisting' interrogation step is depicted with a smaller energetic barrier than the 5-10 ms rate-limiting distortion step ( $\$$, which is followed by rapid $\beta$-hairpin insertion and full-nucleotide flipping. (Bottom) Putative diffusional landscapes of the protein along DNA are illustrated along a coordinate orthogonal to the lesion recognition trajectory in (B). As the recognition proceeds, the diffusional landscape gets increasingly rough; once the $\beta$-hairpin is inserted, the protein is practically obstructed from diffusing away, thus 'recognition'.

Fast nonspecific unwinding may represent a conformational switch between 'search' and 'interrogation' modes. How site-specific DNA binding proteins are able to rapidly scan the genome while reliably locating and recognizing their respective binding sites have been dubbed as 'search-speed stability' paradox [87]. Previous studies to resolve the problem have invoked two modes of protein-DNA binding: the 'search' mode and the 'recognition' mode 
$[59,87-89]$. The search mode is presumed to have few stabilizing protein-DNA interactions, allowing rapid 1-D diffusion of protein on DNA in a relatively smooth energy landscape and/or dissociation from DNA. However, the search mode comes at a price that the protein has limited ability to sense/probe the presence of the target site, owing to having only a few specific interactions with DNA in this mode. In the recognition mode, on the other hand, the protein assumes a conformation that resembles its conformation in the final 'recognition' complex; in this mode it can make more extensive protein-DNA contacts, allowing the protein to test the targets with increased accuracy/specificity. It has been proposed that an efficient, stochastic interchange between the two modes enables the resolution of the speedstability paradox in searching and recognition.

Consistent with these notions, various studies have shown evidences of multiple binding modes in nonspecific protein-DNA complexes [47, 49, 50, 90-99]. The structures of DNA repair glycosylases captured on nonspecific DNA showed severely kinked conformations of DNA even on those non-target sites [49, 50, 91, 92, 94, 95]. Though enabling specific probing of a potential target lesion, such distortions seem incompatible with fast sliding/scanning of the protein on DNA, as anticipated in the 'search' mode. These complexes have been termed as 'interrogation' complexes distinct from a search complex but lying along the conformational trajectory towards forming the lesion-specific, recognition complex [100]. NMR analyses of lac repressor and uracil DNA glycosylase bound to nonspecific DNA have revealed large-scale, sub-milliseconds conformational fluctuations in the proteins that are potential candidates for such search/interrogation conformational switches [96, 101]. However, direct measurements of corresponding DNA dynamics to account for such switches in the sub-millisecond regime have been limited to a few NMR imino-proton exchange experiments that detect base-pair 'breathing' $[97,102]$. While previous stopped-flow studies on DNA glycosylases have indicated multiple kinetic steps for 
damage recognition and repair [103, 104], these observed kinetics are too slow to capture interrogation compatible with 1-D diffusion and are likely probing additional steps leading to recognition and catalysis.

Our study directly revealed that there are at least two distinct kinetic phases (fast winding/unwinding \& slow 'opening'). We attribute the fast phase to conformational interconversions between a rapidly diffusing 'search' and a slowly diffusing or momentarily stalled 'interrogation' mode, and the slow phase to the 'recognition' step, i.e., the conversion from the search/interrogation to the final 'open' conformation. Our T-jump approach that also took advantage of novel probes sensitive to unwinding ('twisting') of DNA was critical in unveiling the sub-millisecond search-interrogation dynamics. A rapid ( 100 $\mu$ s time-scale) nonspecific interrogation preceding a slow ( 10 ms time-scale) target recognition has previously also been observed in T-jump studies of the integration host factor (IHF), a DNAbending bacterial architectural protein (chapter 3). Thus, interrogation on time-scales of hundreds of microseconds may well be a common feature during target recognition, compatible with rapid diffusion of proteins on DNA.

Interrogation may promote 'kinetic gating' by stalling the protein preferentially at damaged sites. In this model, discrimination between normal and damaged DNA sites is determined by the differential probabilities of the Rad4-induced DNA 'opening' of a DNA site before it diffuses away from the site. Residence times reported for damage repair proteins on normal, nonspecific DNA range from $0.1 \mu \mathrm{s}$ to $300 \mu \mathrm{s}[60,62,90,105]$. Thus, as previously argued, it has not been clear how Rad4 can efficiently 'open' to recognize damaged DNA, which takes 5-10 ms, if indeed Rad4 should exhibit the microsecondregime residence times per DNA site during the nonspecific search. The interrogation mode of $\mathrm{Rad} 4$ revealed by $\mathrm{tC}^{\mathrm{O}}, \mathrm{tC}_{\text {nitro }}$ probes provides critical insights into bridging this gap between fast diffusion and slow recognition: the increased interactions between the protein 
and the DNA in the interrogation mode may work to slow down the diffusion of XPC/Rad4 on DNA, thus increasing the probability that it can open a given site, according to the 'kinetic gating' mechanism (Fig. 4.35). It is tempting to speculate that abnormal DNA structures and dynamics induced by NER lesions may promote the conversion from search to interrogation in comparison with normal DNA, and also further stall XPC/Rad4 when in the interrogation mode, ultimately to allow residence times in the milliseconds regime. Our kinetics data show slightly faster relaxation rates for the undamaged (AN14u) construct than for the mismatched AN14 (Fig. 4.25G), consistent with the notion that Rad4 may engage more tightly with the mismatch site than normal sites, though this difference is not detectible on a gel-shift assay. Additionally, it is also conceivable that some DNA-stabilizing lesions may present a significantly high barrier even for 'twisting', leading to a perpetual 'search' mode at the lesion site that impairs recognition and thus repair.

\subsubsection{Rad4/XPC induced DNA bending dynamics measured using TAMRA/Cy5 FRET pair}

Our equilibrium data suggests that both wild type and mutant Rad4 were able to bend the DNA in solution. AN7 upon Rad4 binding exhibit relaxation rates that are $\sim 10$-fold slower $\left(>50 \mathrm{~ms}\right.$ at $25^{\circ} \mathrm{C}$ ) than DNA opening / nucleotide-flipping kinetics. We interpret this as monitoring XPC-induced DNA bending kinetics. While AN7 - Rad4 shows a slow bending dynamics, the AN7-mutant Rad4 shows a fast bending kinetics which is similar to the fast nonspecific DNA conformational changes observed with tCo/tCnitro probe. These results indicate that the preliminary probing/unwinding of DNA by a nonspecifically bound protein is accompanied by partial DNA bending. 


\subsection{Conclusion}

The present study reveals relaxation dynamics in the Rad4-DNA complexes on three distinct timescales: a rapid ( $200 \mu \mathrm{s})$ phase observed on both damaged and undamaged DNA is attributed to nonspecific DNA unwinding (an initial interrogation step), while two slower phases ( 5-10 ms and >50 ms) observed only with damaged DNA are identified with more severe distortions at the lesion site as Rad4 opens the DNA and flips out the damaged nucleotides, followed by DNA bending to form the ultimate recognition complex. Altogether, these results suggest that the initial unwinding of DNA may be the result of a conformational switch in the protein while bound nonspecifically, from a "search" mode in which it can rapidly scan DNA to an "interrogation" mode that perhaps momentarily stalls the protein, allowing it more time to investigate the DNA site for lesions.

We propose that Rad4's lesion recognition relies on 'kinetic gating' mechanism to achieve selectively higher probability of 'opening' damaged DNA than normal DNA. We also conclude that Rad4 interrogates DNA by twisting DNA on 100--500 $\mu$ s time-scales. This provides a compelling account for how $\operatorname{Rad} 4$ may intermittently examine DNA if also rapidly diffusing on DNA at a comparable rate, and thus reconcile rapid searching with reliable recognition. In general, the multi-step recognition process through dynamic conformational changes would allow the protein to span a wide range of residence times on DNA that can be fine-tuned at each successive step. Similar multi-step, multi-conformation mechanism may be applicable to diverse DNA-binding proteins. The actual diffusion landscapes of XPC/Rad4 on DNA in each binding mode, and the structural and kinetic parameters that control each step along the recognition trajectory, remain as fascinating problems to be further explored. 
This study reveals, for the first time, the structural and temporal features of a distinct interrogation step for a damage recognition protein that relies exclusively on indirect readout to recognize damage sites in DNA. 


\section{References}

1. Gillet, L.C.a.S., O.D. , Molecular mechanisms of mammalian global genome nucleotide excision repair. Chem Rev. , 2006. 106: p. 253-276

2. Jackson, S.P. and J. Bartek, The DNA-damage response in human biology and disease. Nature, 2009. 461(7267): p. 1071-8.

3. Cleaver, J.E., Cancer in Xeroderma pigmentosum and related disorders of DNA repair. Nature Reviews:Cancer. , 2005. 5: p. 564-573

4. Cleaver, J.E., Lam., E.T. and Revet, I, Disorders of nucleotide excision repair: the genetic and molecular basis of heterogeneity. Nat Rev Genet., 2009. 10: p. 756-768.

5. Pascucci, B., D’Errico, M., Parlanti, E., Giovannini, S. and Dogliotti,E. , Role of nucleotide excision repair proteins in oxidative DNA damage repair: an updating. Biochemistry (Mosc). 2011. 76: p. 4-15

6. Kemileri, I., Karakasilioti, I., Garinis, G.,A. (2012) Nucleotide excision repair: new tricks with old bricks. Cell. 28, 566-572, Nucleotide excision repair: new tricks with old bricks. . Cell., 2012. 28: p. 566-572.

7. de Boer, J.H., J. H. J. , Nucleotide excision repair and human syndromes. Carcinogenesis, 2000. 21: p. 453-460

8. Friedberg, E.C., Walker, G. C. \& Siede, W. , DNA Repairand Mutagenesis. ASM Press, Washington., 1995.

9. $\quad$ Lindahl, T.W., R. D., Quality control by DNA repair. Science 1999. 286: p. 18971905

10. Hoeijmakers, J.H., Genome maintenance mechanisms for preventing cancer. Nature, 2001. 411(6835): p. 366-74.

11. Sancar, A.D.e.r.A.R.B., 43-81 DNA excision repair. Annu. Rev. Biochem. Annu. Rev. Biochem. , 1996. 65: p. 43-81.

12. Prakash, S., Prakash, L., Nucleotide excision repair in yeast. Mutat. Res., 2000. 451: p. 13-24.

13. de Laat, W.L., Jaspers, N. G. J. \& Hoeijmakers, J. H. J., Molecular mechanism of nucleotide excision repair. Genes Dev., 1999. 13: p. 768-785

14. Petit, C.S., A., Nucleotide excision repair: from E. coli to man. Biochimie., 1999. 81: p. 15-25

15. Wood, R.D., Nucleotide excision repair in mammalian cells. J. Biol. Chem. , 1997. 272: p. 23465-23468

16. Achter, E.K. and G. Felsenfeld, The conformation of single-strand polynucleotides in solution: sedimentation studies of apurinic acid. Biopolymers, 1971. 10(9): p. 162534.

17. Lahmann, A., DNA repair-deficient disseases, xeroderma pigmentosum, cockayne syndrome and trichothiodystrophy. Biochimie., 2003. 85: p. 1101-111118. Hess MT, S.U., Petretta M, Giese B, Naegeli H. , Bipartite substrate discrimination by human nucleotide excision repair. Proc Natl Acad Sci., 1997. 94: p. 6664-6669.

19. Batty, D., Rapic-Otrin, V., Levine, A.S., Wood,R.D stable binding of human XPC complex to irradiated DNA confers strong discrimination for damaged sites. . 2000. J.Mol.Biol. : p. 275-290

20. Hey, T., Lipps, G., Sugasawa, K,. Iwai, S., Hanaoka, F., Krauss, G., The XPC-HR23B complex displays high affinity and specificity for damaged DNA in a true-equilibrium fluorescence assay. Biochemsitry (Mosc), 2002. 41: p. 6583-6587 
21. Kusumoto, R., Masutani,C., Sugasawa, K., Iwai, S., Araki, M., Uchida,A.,Mizukoshi, T. and Hanaoka, F., Diversity of the damage recognition step in the global genomic nucleotide excision repair in vitro. . Mutat.Res. , 2001. 485: p. 219-227

22. Reardon, J.T., Mu, D., Sancar, A., Overproduction, purification and characterization of the XPC subunit of the human DNA repair excision nuclease. J.Biol.Chem. , 1996. 271: p. 19451-19456

23. Bunick, C.G., Miller, M.R., Fuller, B.E.,Fanning, E., Chazin, W.J., Biochemical and structural domain analysis of xeroderma pigmentosum complementation group $C$ protein. Bichemistry (Mosc),, 2006. 45: p. 14965-14979

24. Tergo, K.S., Turchi,J.J., Pre-steady state binding of damaged DNA by XPC-hHR23B reveals a kinetic mechanism for damage discrimination. Biochemistry (Mosc). , 2006. 45: p. 1961-1969

25. Mocquet V, K.K., Kolbanovskiy M, Kolbanovskiy A, Tapias A, Cai Y, Broyde S, Geacintov NE, Egly JM., The human DNA repair factor XPC-HR23B distinguishes stereoisomeric benzo[a]pyrenyl-DNA lesions. . Embo J. , 2007. 26: p. 2923-2932.

26. Cai Y, K.K., Xu R, Tang Y, Kolbanovskii M, Kolbanovskii A, Amin S, Patel DJ, Broyde S, Geacintov NE., Distant neighbor base sequence context effects in human nucleotide excision repair of a benzo[a]pyrenederived DNA lesion. J Mol Biol 2010. 399: p. 397-409.

27. $\mathrm{Mu}, \mathrm{H}$., et al., Nucleotide excision repair of 2-acetylaminofluorene- and 2aminofluorene-(C8)-guanine adducts: molecular dynamics simulations elucidate how lesion structure and base sequence context impact repair efficiencies. Nucleic acids research, 2012. 40(19): p. 9675-90.

28. Yeo, J.-E., et al., The efficiencies of damage recognition and excision correlate with duplex destabilization induced by acetylaminofluorene adducts in human nucleotide excision repair. Chemical research in toxicology, 2012. 25(11): p. 2462-8.

29. Riedl, T., F. Hanaoka, and J.-M. Egly, The comings and goings of nucleotide excision repair factors on damaged DNA. The EMBO journal, 2003. 22(19): p. 5293-303.

30. Luijsterburg, M.S., et al., Stochastic and reversible assembly of a multiprotein DNA repair complex ensures accurate target site recognition and efficient repair. The Journal of cell biology, 2010. 189(3): p. 445-63.

31. Hoogstraten, D., et al., Versatile DNA damage detection by the global genome nucleotide excision repair protein XPC. Journal of cell science, 2008. 121(Pt 17): p. 2850-9.

32. Luijsterburg, M.S., et al., Dynamic in vivo interaction of DDB2 E3 ubiquitin ligase with $U V$-damaged DNA is independent of damage-recognition protein XPC. Journal of cell science, 2007. 120(Pt 15): p. 2706-16.

33. Vermeulen, W., Dynamics of mammalian NER proteins. DNA repair, 2011. 10(7): p. 760-71.

34. Uchida, A., et al., The carboxy-terminal domain of the XPC protein plays a crucial role in nucleotide excision repair through interactions with transcription factor IIH. DNA repair, 2002. 1(6): p. 449-61.

35. Yokoi, M., et al., The xeroderma pigmentosum group C protein complex XPC-HR23B plays an important role in the recruitment of transcription factor IIH to damaged DNA. The Journal of biological chemistry, 2000. 275(13): p. 9870-5.

36. Min, J.H. and N.P. Pavletich, Recognition of DNA damage by the Rad4 nucleotide excision repair protein. Nature, 2007. 449(7162): p. 570-5.

37. Sugasawa, K., et al., A multistep damage recognition mechanism for global genomic nucleotide excision repair. Genes Dev, 2001. 15(5): p. 507-21. 
38. Chen, X., et al., Kinetic gating mechanism of DNA damage recognition by Rad4/XPC. Nat Commun, 2015. 6: p. 5849.

39. Buterin, T., et al., DNA quality control by conformational readout on the undamaged strand of the double helix. Chem Biol, 2005. 12(8): p. 913-22.

40. Janicijevic, A. and D.N. A., DNA bending by the human damage recognition complex. XPCHR23B Amst, 2003. 2 SRC - GoogleScholar: p. 325-336.

41. Borjesson, K., et al., Nucleic acid base analog FRET-pair facilitating detailed structural measurements in nucleic acid containing systems. J Am Chem Soc, 2009. 131(12): p. 4288-93.

42. Preus, S., et al., Characterization of nucleobase analogue FRET acceptor tCnitro. J Phys Chem B, 2010. 114(2): p. 1050-6.

43. Cantor, C.R., M.M. Warshaw, and H. Shapiro, Oligonucleotide interactions. 3. Circular dichroism studies of the conformation of deoxyoligonucleotides. Biopolymers, 1970. 9(9): p. 1059-77.

44. Cavaluzzi, M.J. and P.N. Borer, Revised UV extinction coefficients for nucleoside-5'monophosphates and unpaired DNA and RNA. Nucleic Acids Res, 2004. 32(1): p. e13.

45. Preus, S., et al., Photophysical and structural properties of the fluorescent nucleobase analogues of the tricyclic cytosine ( $t C$ ) family. Phys Chem Chem Phys, 2010. 12(31): p. 8881-92.

46. Clegg, R.M., Fluorescence resonance energy transfer and nucleic acids. Methods Enzymol, 1992. 211: p. 353-88.

47. Kalodimos, C.G., et al., Structure and flexibility adaptation in nonspecific and specific protein-DNA complexes. Science, 2004. 305(5682): p. 386-9.

48. Parker, J.B., et al., Enzymatic capture of an extrahelical thymine in the search for uracil in DNA. Nature, 2007. 449(7161): p. 433-7.

49. Banerjee, A., et al., Structure of a repair enzyme interrogating undamaged DNA elucidates recognition of damaged DNA. Nature, 2005. 434(7033): p. 612-8.

50. Banerjee, A. and G.L. Verdine, A nucleobase lesion remodels the interaction of its normal neighbor in a DNA glycosylase complex. Proc Natl Acad Sci U S A, 2006. 103(41): p. 15020-5.

51. Yi, C., et al., Duplex interrogation by a direct DNA repair protein in search of base damage. Nature structural \& molecular biology, 2012. 19(7): p. 671-6.

52. Viadiu, H. and A.K. Aggarwal, Structure of BamHI bound to nonspecific DNA: a model for DNA sliding. Mol Cell, 2000. 5(5): p. 889-95.

53. Stivers, J.T., K.W. Pankiewicz, and K.A. Watanabe, Kinetic mechanism of damage site recognition and uracil flipping by Escherichia coli uracil DNA glycosylase. Biochemistry, 1999. 38(3): p. 952-63.

54. Fei, J., et al., Regulation of nucleotide excision repair by UV-DDB: prioritization of damage recognition to internucleosomal DNA. PLoS biology, 2011. 9(10): p. e1001183.

55. Camenisch, U., et al., Two-stage dynamic DNA quality check by xeroderma pigmentosum group C protein. The EMBO journal, 2009. 28(16): p. 2387-99.

56. Moe, J.G. and I.M. Russu, Kinetics and energetics of base-pair opening in 5'd(CGCGAATTCGCG)-3' and a substituted dodecamer containing G.T mismatches. Biochemistry, 1992. 31(36): p. 8421-8.

57. Coman, D. and I.M. Russu, A nuclear magnetic resonance investigation of the energetics of basepair opening pathways in DNA. Biophys. J., 2005. 89(5): p. 328592. 
58. Zheng, H., et al., Base flipping free energy profiles for damaged and undamaged DNA. Chemical research in toxicology, 2010. 23(12): p. 1868-70.

59. Friedman, J.I. and J.T. Stivers, Detection of damaged DNA bases by DNA glycosylase enzymes. Biochemistry, 2010. 49(24): p. 4957-67.

60. Schonhoft, J.D. and J.T. Stivers, Timing facilitated site transfer of an enzyme on DNA. Nat Chem Biol, 2012. 8(2): p. 205-10.

61. Blainey, P.C., et al., A base-excision DNA-repair protein finds intrahelical lesion bases by fast sliding in contact with DNA. Proc. Natl. Acad. Sci. USA, 2006. 103(15): p. 5752-7.

62. Gorman, J., et al., Dynamic basis for one-dimensional DNA scanning by the mismatch repair complex Msh2-Msh6. Mol. Cell, 2007. 28(3): p. 359-70.

63. Kropachev, K., et al., Adenine-DNA adducts derived from the highly tumorigenic Dibenzo[a,l]pyrene are resistant to nucleotide excision repair while guanine adducts are not. Chemical research in toxicology, 2013. 26(5): p. 783-93.

64. Wang, Y.M., R.H. Austin, and E.C. Cox, Single molecule measurements of repressor protein 1D diffusion on DNA. Phys. Rev. Lett., 2006. 97(4): p. 048302.

65. Bonnet, I., et al., Sliding and jumping of single EcoRV restriction enzymes on noncognate DNA. Nucleic Acids Res., 2008. 36(12): p. 4118-27.

66. Tafvizi, A., et al., Tumor suppressor p53 slides on DNA with low friction and high stability. Biophys. J., 2008. 95(1): p. L01-3.

67. Nelson, S.R., et al., Two glycosylase families diffusively scan DNA using a wedge residue to probe for and identify oxidatively damaged bases. Proc Natl Acad Sci U S A, 2014. 111(20): p. E2091-9.

68. Shi, Y., et al., Mammalian transcription factor $A$ is a core component of the mitochondrial transcription machinery. Proceedings of the National Academy of Sciences of the United States of America, 2012. 109(41): p. 16510-5.

69. Posse, V., et al., The amino terminal extension of mammalian mitochondrial RNA polymerase ensures promoter specific transcription initiation. Nucleic acids research, 2014. 42(6): p. 3638-47.

70. Iqbal, A., et al., Orientation dependence in fluorescent energy transfer between Cy3 and Cy5 terminally attached to double-stranded nucleic acids. Proc Natl Acad Sci U S A, 2008. 105(32): p. 11176-81.

71. Ranjit, S., K. Gurunathan, and M. Levitus, Photophysics of backbone fluorescent DNA modifications: reducing uncertainties in FRET. J Phys Chem B, 2009. 113(22): p. 7861-6.

72. El Hassan, M.A. and C.R. Calladine, Two distinct modes of protein-induced bending in DNA. J Mol Biol, 1998. 282(2): p. 331-43.

73. Hey, T., et al., The XPC-HR23B complex displays high affinity and specificity for damaged DNA in a true-equilibrium fluorescence assay. Biochemistry, 2002. 41(21): p. 6583-7.

74. Trego, K.S. and J.J. Turchi, Pre-steady-state binding of damaged DNA by XPChHR23B reveals a kinetic mechanism for damage discrimination. Biochemistry, 2006. 45(6): p. 1961-9.

75. Preus, S., et al., FRETmatrix: a general methodology for the simulation and analysis of FRET in nucleic acids. Nucleic Acids Res, 2013. 41(1): p. e18.

76. Sandin, P., et al., Characterization and use of an unprecedentedly bright and structurally non-perturbing fluorescent DNA base analogue. Nucleic Acids Res, 2008. 36(1): p. 157-67. 
77. Jing, Y., J.F. Kao, and J.S. Taylor, Thermodynamic and base-pairing studies of matched and mismatched DNA dodecamer duplexes containing cis-syn, (6-4) and Dewar photoproducts of TT. Nucleic Acids Res, 1998. 26(16): p. 3845-53.

78. Huang, S.N. and D.M. Crothers, The role of nucleotide cofactor binding in cooperativity and specificity of MutS recognition. J Mol Biol, 2008. 384(1): p. 31-47.

79. Sass, L.E., et al., Single-molecule FRET TACKLE reveals highly dynamic mismatched DNA-MutS complexes. Biochemistry, 2010. 49(14): p. 3174-90.

80. Hopfield, J.J., Kinetic proofreading: a new mechanism for reducing errors in biosynthetic processes requiring high specificity. Proc Natl Acad Sci U S A, 1974. 71(10): p. 4135-9.

81. Ninio, J., Kinetic amplification of enzyme discrimination. Biochimie 1975. 57: p. 587-595.

82. Savir, Y.T., T., RecA-mediated homology search as a nearly optimal signal detection system. Mol. Cell., 2010. 40: p. 388-396.

83. Naegeli, H.S., K., The xeroderma pigmentosum pathway: decision tree analysis of DNA quality. . DNA Repair (Amst), 2011. 10: p. 673-683.

84. Sugasawa, K.e.a., UV-induced ubiquitylation of XPC protein mediated by UV-DDBubiquitin ligase complex. . Cell 2005. 121: p. 387-400

85. Le May, N., Egly, J. M. \& Coin, F. , rue lies: the double life of the nucleotide excision repair factors in transcription and DNA repair. . J. Nucleic Acids, 2010.

86. Fong, Y.W.e.a., DNA repair complex functions as an oct4/sox2 coactivator in embryonic stem cells. Cell 2011. 147: p. 120-131.

87. Slutsky, M. and L.A. Mirny, Kinetics of protein-DNA interaction: facilitated target location in sequence-dependent potential. Biophys. J., 2004. 87(6): p. 4021-35.

88. Savir, Y. and T. Tlusty, Conformational proofreading: the impact of conformational changes on the specificity of molecular recognition. PLoS One, 2007. 2(5): p. e468.

89. Zhou, R., et al., SSB functions as a sliding platform that migrates on DNA via reptation. Cell, 2011. 146(2): p. 222-32.

90. Nelson, S.R., et al., Two glycosylase families diffusively scan DNA using a wedge residue to probe for and identify oxidatively damaged bases. Proceedings of the National Academy of Sciences of the United States of America, 2014. 111(20): p. E2091-9.

91. Wibley JE, W.T., Haushalter K, Verdine GL, \& Pearl LH, Structure and specificity of the vertebrate anti-mutator uracil-DNA glycosylase SMUG1. . Mol. Cell 2003. 6: p. 1647-1659.

92. Banerjee, A., W.L. Santos, and G.L. Verdine, Structure of a DNA glycosylase searching for lesions. Science, 2006. 311(5764): p. 1153-7.

93. Iwahara, J., M. Zweckstetter, and G.M. Clore, NMR structural and kinetic characterization of a homeodomain diffusing and hopping on nonspecific DNA. Proc Natl Acad Sci U S A, 2006. 103(41): p. 15062-7.

94. Maiti, A., et al., Crystal structure of human thymine DNA glycosylase bound to DNA elucidates sequence-specific mismatch recognition. Proc Natl Acad Sci U S A, 2008. 105(26): p. 8890-5.

95. Qi, Y., et al., Encounter and extrusion of an intrahelical lesion by a DNA repair enzyme. Nature, 2009. 462(7274): p. 762-6.

96. Friedman JI, M.A., \& Stivers JT Nontarget DNA binding shapes the dynamic landscape for enzymatic recognition of DNA damage. Nucleic Acids Res., 2009. 37: p. 3493-3500. 
97. Parker JB, e.a., Enzymatic capture of an extrahelical thymine in the search for uracil in DNA. Nature 2007. 449: p. 433-437

98. Leith JS, e.a., Sequence-dependent sliding kinetics of p53. Proc. Natl. Acad. Sci. , 2012. 109: p. 16552-16557

99. Ghodke, H., et al., Single-molecule analysis reveals human UV-damaged DNAbinding protein $(U V-D D B)$ dimerizes on DNA via multiple kinetic intermediates. Proc Natl Acad Sci U S A, 2014. 111(18): p. E1862-71.

100. Friedman JI, M.A., \& Stivers JT, Detection of damaged DNA bases by DNA glycosylase enzymes. . Biochemistry (Mosc). 2010. 49: p. 4957-4967.

101. Kalodimos CG, e.a., Structure and flexibility adaptation in nonspecific and specific protein-DNA complexes. . Science 2004. 305: p. 386-389.

102. Cao, C., et al., Dynamic opening of DNA during the enzymatic search for a damaged base. Nat Struct Mol Biol, 2004. 11(12): p. 1230-6.

103. Koval VV, K.N., Ishchenko AA, Saparbaev MK, \& Fedorova OS eal-time studies of conformational dynamics of the repair enzyme E. coli formamidopyrimidine-DNA glycosylase and its DNA complexes during catalytic cycle. Mutat. Res., 2010. 685: p. 3-10.

104. Kuznetsov, N.A., et al., Conformational Dynamics of DNA Repair by Escherichia coli Endonuclease III. J Biol Chem, 2015. 290(23): p. 14338-49.

105. Blainey PC, v.O.A., Banerjee A, Verdine GL, \& Xie XS, A base-excision DNA-repair protein finds intrahelical lesion bases by fast sliding in contact with DNA. . Proc. Natl. Acad. Sci. , 2006. 103: p. 5752-5757. 


\section{CHAPTER V}

\section{DNA Mismatch repair}

\subsection{Introduction}

The DNA replication machinery synthesizes 500 DNA base pairs every second in bacteria and $\sim 50$ base pairs every second in eukaryotes [1]. Mismatches can occur in a newly synthesized DNA due to misincorporation of nucleotides by DNA polymerase or slippage during DNA replication. Mismatches in DNA can also occur during genetic recombination or chemical modifications due to metabolic processes and environmental factors such as various types of ionizing radiation. The cells have evolved a complex network of repair pathway called mismatch repair pathway (MMR) to identify and correct the mistakes that escape DNA polymerase proof reading.

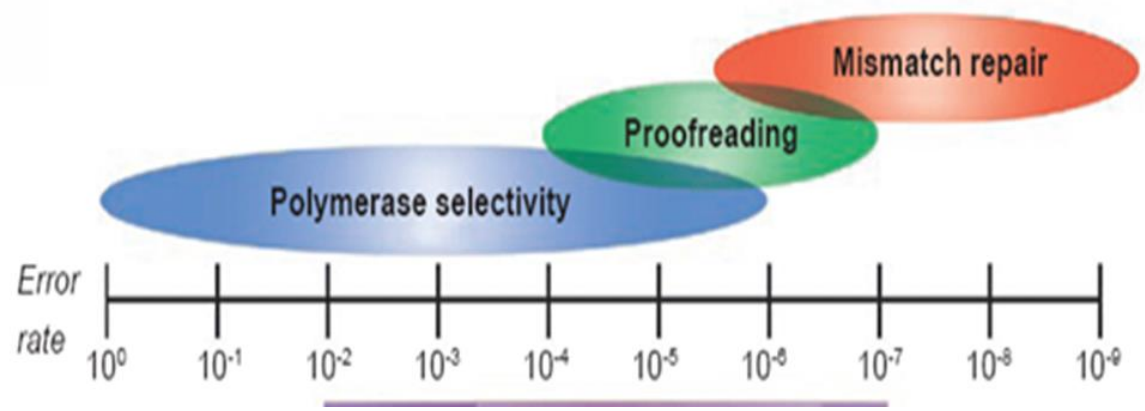

Fig.5.1. The relative contribution levels of the three main components of replication fidelity estimated from mutation rates of systems defective in one or more of the components are shown. The overlapping ovals represent the fact that there is a range of possible increases in the level of fidelity that each mechanism provides depending on many factors [2].

Fig. 5.1 shows the rate of occurrence of errors starting from polymerase activity to DNA repair. Efficient and reliable recognition of mismatches in DNA is critical for the effective MMR. DNA mismatch repair is a highly conserved repair pathway essential to the maintenance of genomic stability and the proteins that initiate MMR are conserved in both 
prokaryotes and eukaryotes $[3,4]$. When the repair machinery fails, errors in DNA are carried forward as mutations implicated in many diseases [5-7]. How DNA mismatch repair proteins recognize mismatches in DNA and signal the repair machinery remains a puzzle [8-12].

The MMR protein, MutS, recognizes normal nucleotides that are either mispaired with the wrong Watson-Crick partner, or are unpaired (insertion-deletion loops). Mutations in MutS homologs in humans are implicated in hereditary nonpolyposis colorectal carcinoma $[13,14]$ as well as other genetic disorders including fragile $\mathrm{X}$ syndrome and Huntington's disease [15-18]. Mismatch recognition is a challenging process as MutS needs to recognize a wide range of mismatches, but, unlike damage recognition proteins in the NER and BER pathway, the recognition is not based on altered or damaged bases [5]. The specificity of MutS to a heteroduplex (mismatched) DNA is $10^{2}-10^{3}$ fold higher compared to homoduplex DNA [19], which implies that MutS can bind to normal (matched) DNA with a probability of 1 in $10^{2}-10^{3}$ base pairs. Considering the rate of mismatch occurrence during replication, which is 1 in $10^{6}-10^{8}$, this implies that MMR could accidently signal for the repair of a normal base pair. To avoid this scenario, a further ATP-dependent step is required in the MMR pathway, in which MutS utilizes ATP hydrolysis and kinetic proofreading to verify the presence of a mismatch. This process is often accompanied by an induced-fit conformational change in the protein-DNA complex upon association with a mismatch [5]. When MutS binds to matched DNA, it hydrolyzes ATP rapidly; upon encountering a mismatched site in DNA, ATP hydrolysis is suppressed, which allows the formation of a MutS-DNA-ATP complex [20]. Only when associated with both ATP and a mismatch, MutS is able to recruit a downstream repair protein MutL and activate nucleases (Fig. 5.2) [1, 21]. 


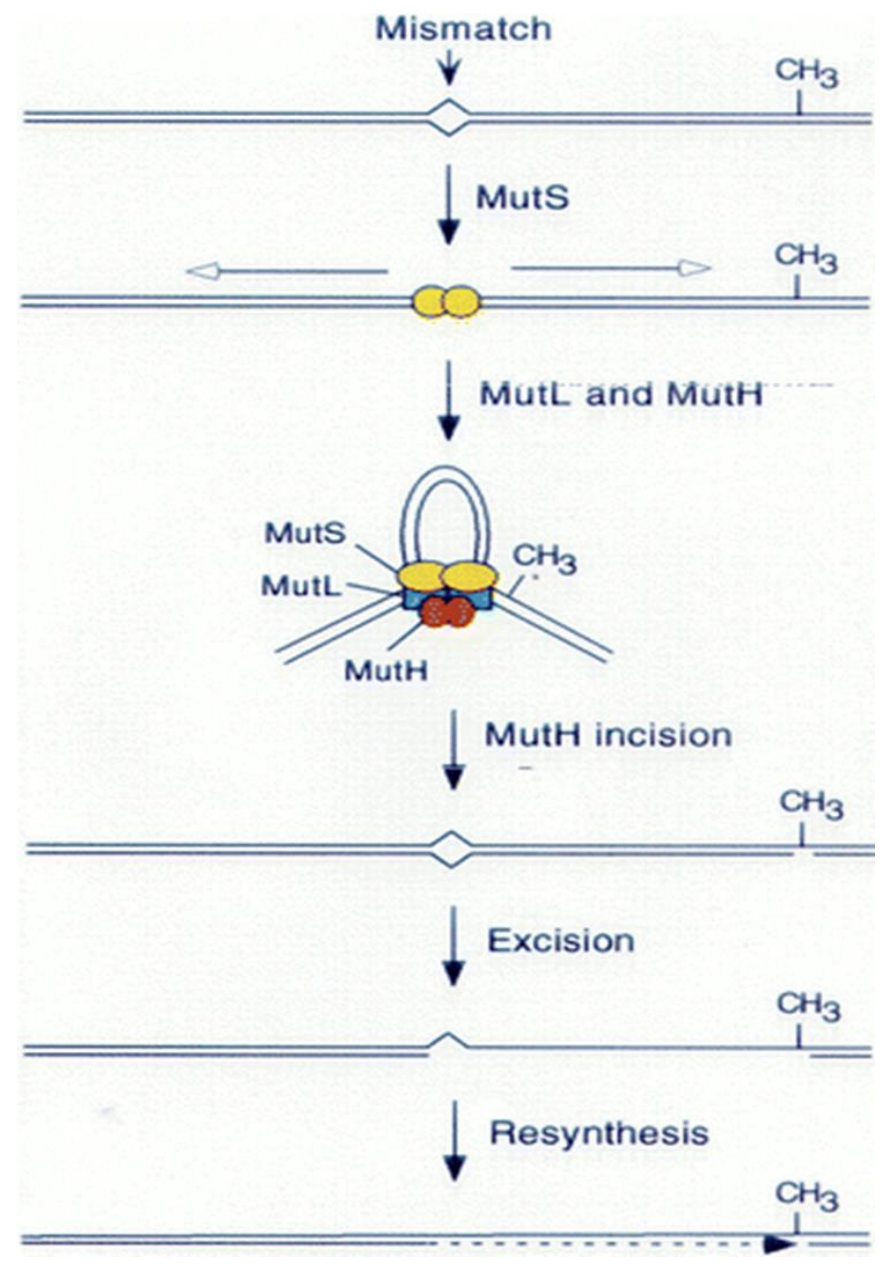

Fig.5.2. Mismatch recognition and subsequent repair in a "mismatch repair pathway (MMR. MutS has an intrinsic ATPase activity for kinetic proofreading and often undergoes an induced fit conformational change upon association with mismatch. The steps involved in MMR are: mismatch recognition $\rightarrow$ ATP binding \&sliding $\rightarrow$ incision $\rightarrow$ excision $\rightarrow$ gap filling $\rightarrow$ ligation. .

Crystal structures of prokaryotic and human MutS homologs complexed with DNA containing a variety of mismatch sites reveal a common recognition mode, with mismatchindependent interactions between MutS and DNA [22-25], although mismatches are repaired with varying efficiency in vivo [26-28]. This poses a puzzle as to what are the common features at the mismatch site that initiate the recognition and binding by MutS, and what are the differences that result in variations in repair efficiencies. 


\subsubsection{Structural Studies on MutS bound to mismatched DNA}

MutS proteins recognize and bind to a wide range of mismatches. Most studies to date have focused on the structural aspects of the recognition complex [9, 22, 23, 25, 29, 30], and have yielded detailed information on the bent conformations adopted by DNA in complex with repair proteins. Fig. 5.3 shows a crystal structure of Thermus aquaticus MutS (Taq MutS) bound to DNA containing a T-bulge (an extra Thymine inserted in one strand). In all MutS-DNA complexes for which structures have been obtained, the DNA is kinked at the mismatch site, a feature also observed in AFM studies [9], with significant disruption of the stacking between the unpaired or mispaired base and its neighbours. Instead, the mismatched base is stacked with a conserved Phe side-chain (Fig.5.3b). The only other specific interaction is a hydrogen-bond between another conserved Glu side-chain and the mismatched base. This Phe-X-Glu motif in MutS is highly conserved [22-24]. All other MutS-DNA interactions involve solely the DNA backbone. Thus, MutS relies primarily on recognizing changes in structural and/or dynamical features of DNA (indirect readout), rather than specific interactions between amino acids of the protein and bases of DNA (direct readout), to find the mismatch embedded in a sea of normal Watson-Crick pairs.

DNA bending by MutS has also been visualized by Erie and co-workers using AFM $[9,10,31]$. These studies demonstrated that MutS can bend even normal (matched) DNA. However, with MutS bound to matched DNA (nonspecific complex), the AFM images showed a single distribution of bent conformations, while with MutS bound to mismatch sites (specific complexes), AFM surprisingly showed two populations of 


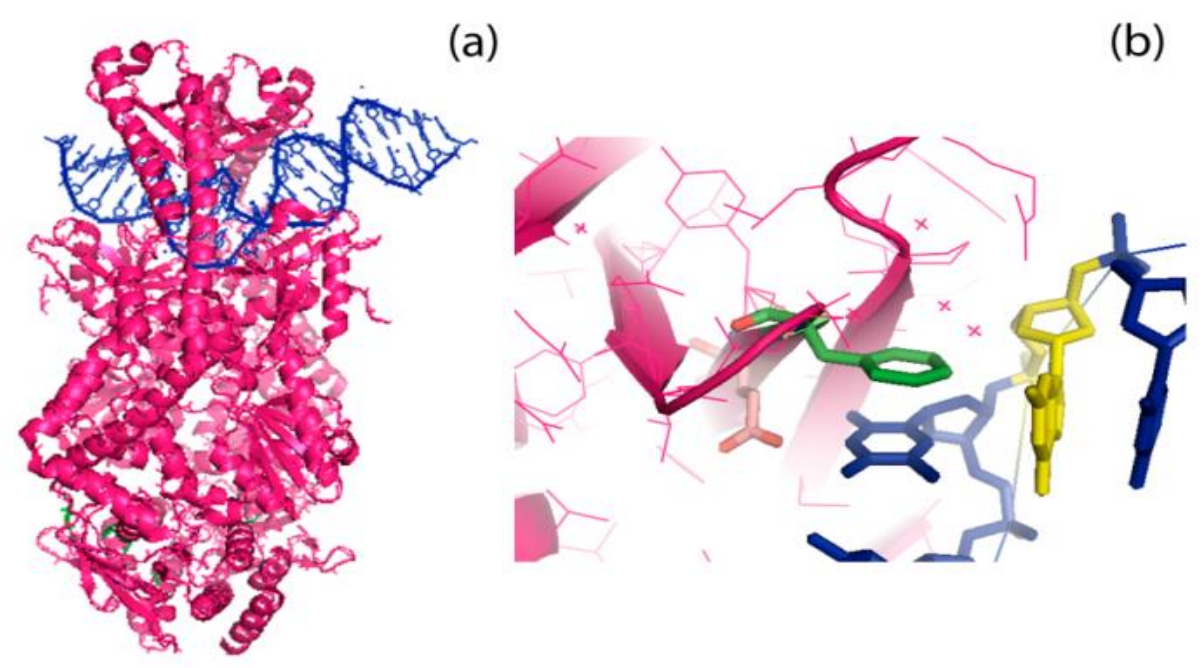

Fig.5.3. (a) Co-crystal structure of Taq MutS protein (pink) in complex with a 23-mer DNA (blue) containing a T-bulge (PDB: 1EWQ). The DNA is bent at the mismatch site by $\sim 60^{\circ}$. (b) A close-up of the mismatch site shows conserved residues Phe39 (green) and Glu41 (orange) making contact with the T-insertion (T-bulge) (blue). The position adjacent to the T-insertion (shown in yellow) is selected for $2 \mathrm{AP}$ substitution.

conformations, bent and unbent [9]. This study revealed an unusual finding that indicated that the thermodynamically stable unbent conformation in the specific complex may be the result of a unique interaction between MutS and the mismatched base, and suggested that the bent confirmation, as seen in the crystal structure, may be an intermediate in the formation of the specific complex [32]. Their results also showed a broader distribution of bend angles when MutS was bound to matched sites, and a narrower distribution of the bent conformation when bound to mismatched sites, suggesting that in the absence of a mismatch it is likely more energetically favourable to spread the bend over several base pairs rather than localize it to a kink, as in the specific complex with a locally destabilized mismatch site [32]. Based on these and further dynamical studies using single-molecule (sm) FRET, Erie and co-workers argued that the kinked confirmation observed in the crystal structure represents an initial recognition complex (IRC) which is an intermediate state in the ultimate recognition complex (URC) that signals repair $[9,31,32]$. The main conclusion from the (sm) FRET studies is that MutS- 
DNA complex is highly dynamic and rapidly interconverts between different conformational states and suggest that the dynamics of these complexes may be important for signalling DNA repair [32].

\subsubsection{What role does the intrinsic flexibility of DNA play in the mismatch recognition and subsequent repair?}

The features that distinguish mismatched from matched DNA include enhanced flexibility of DNA at the mismatch site due to disrupted base-pairing/stacking in DNA. The energetic difference between a normal and mismatched base pair cannot be more than $2-3 \mathrm{kcal} / \mathrm{mol}$, the total free energy contributed by a base pair to a DNA duplex [33]. This difference is in good agreement with the experimentally measured specificity of MutS binding to heteroduplex DNA [34]. A hypothesis has been put forward in the literature that DNA repair proteins recognize weakened base stacking, and thus a "flexible hinge" at the DNA lesion site [35]. This hypothesis is based in part on measurements that have reported enhanced dynamics of bases in the vicinity of mismatches. NMR measurements of imino-proton exchange for basepairs adjacent to various mismatches show faster bp opening rates in comparison with normal Watson-Crick pairs [8, 36-41]. Fluorescence measurements of DNA monitored with 2AP placed next to a mismatch site have also reported enhanced DNA dynamics near the mismatch compared to normal DNA [11]. Of particular interest is the observation that 2AP dynamics is maximum when positioned adjacent to a GT mismatch or bulges, and minimal near a CC mismatch [11]. Notably, GT mismatches and T-bulges are repaired with high efficiency while CC and TT mismatches are repaired with low efficiency in vivo [42, 43]. Thermodynamic studies of MutS-DNA complexes also indicate a partial correlation of binding affinities with repair efficiencies, with high binding affinity of MutS for GT and Tbulge errors and a very low binding affinity for $\mathrm{CC}$ and TT mismatches [44, 45]. 
Measurements of the rates at which MutS can recognize various mismatch sites and whether the rates of recognition play a role in the efficiency with which the sites are repaired remains an open question in the field.

\subsubsection{Dynamics of DNA binding and bending by MutS.}

Previous measurements on the dynamics of DNA binding and bending with MutS, aimed at understanding the dynamics of mismatch recognition and the role of ATP in these processes, have been carried out with stopped-flow techniques [44, 46]. Though these studies helped to unravel crucial steps in the repair mechanism: how MutS ATPase activity enhance the specificity of mismatch repair, as MutS:ATP form a stable complex with DNA only when mismatch is present and releases the mismatch slowly which allows interactions with downstream repair proteins, they failed to capture the initial recognition event, presumably because of the longer than a few milliseconds time-resolution of the stopped-flow measurements. The only measurements of DNA bending kinetics in complex with MutS have come from smFRET measurements on immobilized DNA samples [32]. Again, the $~ 100 \mathrm{~ms}$ time-resolution of this study indicates that these measurements cannot be observing the kinetics of the initial recognition step, but instead, slower events in the recognition pathway.

Here, I report on laser T-jump measurements of mismatch recognition by MutS. These studies, carried out in collaboration with Prof. Manju Hingorani at Wesleyan University, represent the first measurements on the rates of mismatch recognition by MutS, and set the stage for a deeper understanding of the underlying mechanism. 


\subsection{Results}

The study of the dynamics of MutS-mismatch interaction were carried out using Taq MutS. Taq MutS is a thermostable MutS homolog from thermus aquaticus, a bacterium which lives at high temperatures. It thrives at $70^{\circ} \mathrm{C}$, but can survive at temperatures of $50^{\circ} \mathrm{C}$ to $80^{\circ}$ C. Taq MutS (89.3 $\left.\mathrm{kDa}\right)$ shares extensive amino acid sequence homology with MutS homologs from both prokaryotes and eukaryotes [47]. We also carried out experiments with eukaryotic MutS homolog from yeast S. cerevisae, Msh2-Msh6 (MutSa), which is highly homologous to the human Msh2-Msh6.

To monitor the local conformational dynamics of DNA near the vicinity of the mismatch, we used 2AP adjacent to a T-bulge on a 23-mer DNA (+1 base insertion) (TDNA) (table I). The Hingorani group first showed that 2AP placed adjacent to a mismatch site is sensitive to conformational changes induced by MutS at the mismatch site and successfully used this probe to measure the association/dissociation kinetics of MutS to DNA with mismatches [46]. To monitor MutS-induced global conformational changes in DNA, such as DNA bending, we used FRET pairs (TAMRA and Cy5) placed 19-bp apart, on either end of the DNA substrate. The design of the DNA constructs with FRET labels was motivated by previous FRET studies by the Erie group [32] and by the Crothers group [44]. 
Table I. DNA sequence used in Taq MutS - T-bulge study

T-bulge DNA sequence with 2AP (marked as A ) (TDNA)

5'- GCG CGA CGG TAT A ${ }^{*}$ TA GCT GCC GG-3'

$3^{\prime}-$ CGC GCT GCC AT TAT CGA CGG CC-5'

T-bulge DNA sequence with FRET labels (TFT)

5' - GCG CGA CGG TAT ATA GCT GCC GG- TAMRA dT-3'

$3^{\prime}-$-Cy5 dT- CGC GCT GCC AT TAT CGA CGG CC-5'

\subsubsection{Taq MutS binding to mismatch (T-bulge) DNA as probed by 2AP}

Equilibrium measurements were carried out on Taq MutS bound to a T-bulge DNA containing 2AP (TDNA sequence in Table I) as described in the Methods. The emission spectra of TDNA in the presence and absence of MutS are shown in Fig. 5.4A and demonstrate that when Taq MutS binds to a T-bulge DNA, the 2AP fluorescence increases by 3-fold. Thus, as reported previously by the Hingorani group [46], 2AP adjacent to a T-bulge is a sensitive probe for DNA conformational changes at the mismatch site. This increase in 2AP fluorescence may arise from a kinked DNA and/or base flipped DNA conformation as these disrupt the base-stacking and base-pairing interactions adjacent to $2 \mathrm{AP}$ and make $2 \mathrm{AP}$ more solvent accessible [22, 32, 48]. Previous measurements with a mutant MutS in which Phenylalanine (Phe39) is replaced with Alanine did not detect any increase in 2AP intensity [46]. Furthermore, although AFM studies showed that MutS smoothly bends homoduplex (matched) DNA, no 2AP increase was observed when MutS was bound to homoduplex DNA $[10,31,32]$. These results confirmed that $2 \mathrm{AP}$ fluorescence reports selective MutS binding to mismatched DNA and that the signal depends on interaction with Phe39 [48]. 


\subsubsection{Equilibrium temperature dependent change in 2AP intensity for TDNA-Taq MutS} complex

To examine whether we could perturb the complex with T-jump and utilize the 2AP fluorescence to monitor the conformational relaxation dynamics, we next investigated how the 2AP fluorescence in the MutS-mismatch complex changes with temperature, under equilibrium conditions, and compared that with corresponding 2AP fluorescence measurements in TDNA alone. For the DNA only sample, the 2AP fluorescence first decreases, as a result of the temperature-dependent decrease in the quantum yield of $2 \mathrm{AP}$ in the TDNA context, and then starts to increase above $\sim 50{ }^{\circ} \mathrm{C}$, which we attribute to DNA melting (Fig. 5.4B and Sup. Fig.5.5A). In contrast, the 2AP fluorescence in the complex decreases monotonically as the sample is heated from $12^{\circ} \mathrm{C}$ to $70^{\circ} \mathrm{C}$, and shows reversible behaviour when the temperature is cooled back from $70^{\circ} \mathrm{C}$ to $12^{\circ} \mathrm{C}$ (Fig. 5.4B). There are three important conclusions from these results. First, that the binding of MutS to the DNA stabilizes the DNA against thermal denaturation; second, that the MutS-TDNA complex is stable up to at least $70^{\circ} \mathrm{C}$; third; that the relative change in $2 \mathrm{AP}$ fluorescence in the complex, although similar to that of free DNA up to $\sim 30^{\circ} \mathrm{C}$, starts to deviate from the DNA alone signal above $\sim 30^{\circ} \mathrm{C}$, but still is well below the temperatures at which DNA starts to melt. We interpret this deviation as indicative of conformational changes at the T-bulge site in DNA bound to MutS as the complex is heated up.

Next to examine whether these 2AP-detected conformational changes in the MutSTDNA complex arise from unimolecular or bimolecular processes, we measured the temperature-dependent $2 \mathrm{AP}$ changes for different concentrations of the complex (MutS:TDNA $=500 \mathrm{nM}: 500 \mathrm{nM}, 1 \mu \mathrm{M}: 1 \mu \mathrm{M}, 40 \mu \mathrm{M}: 40 \mu \mathrm{M}$ ). The relative change in 2AP intensity as a function of temperature for these three set of measurements are nearly identical (Fig. 5.4C). 

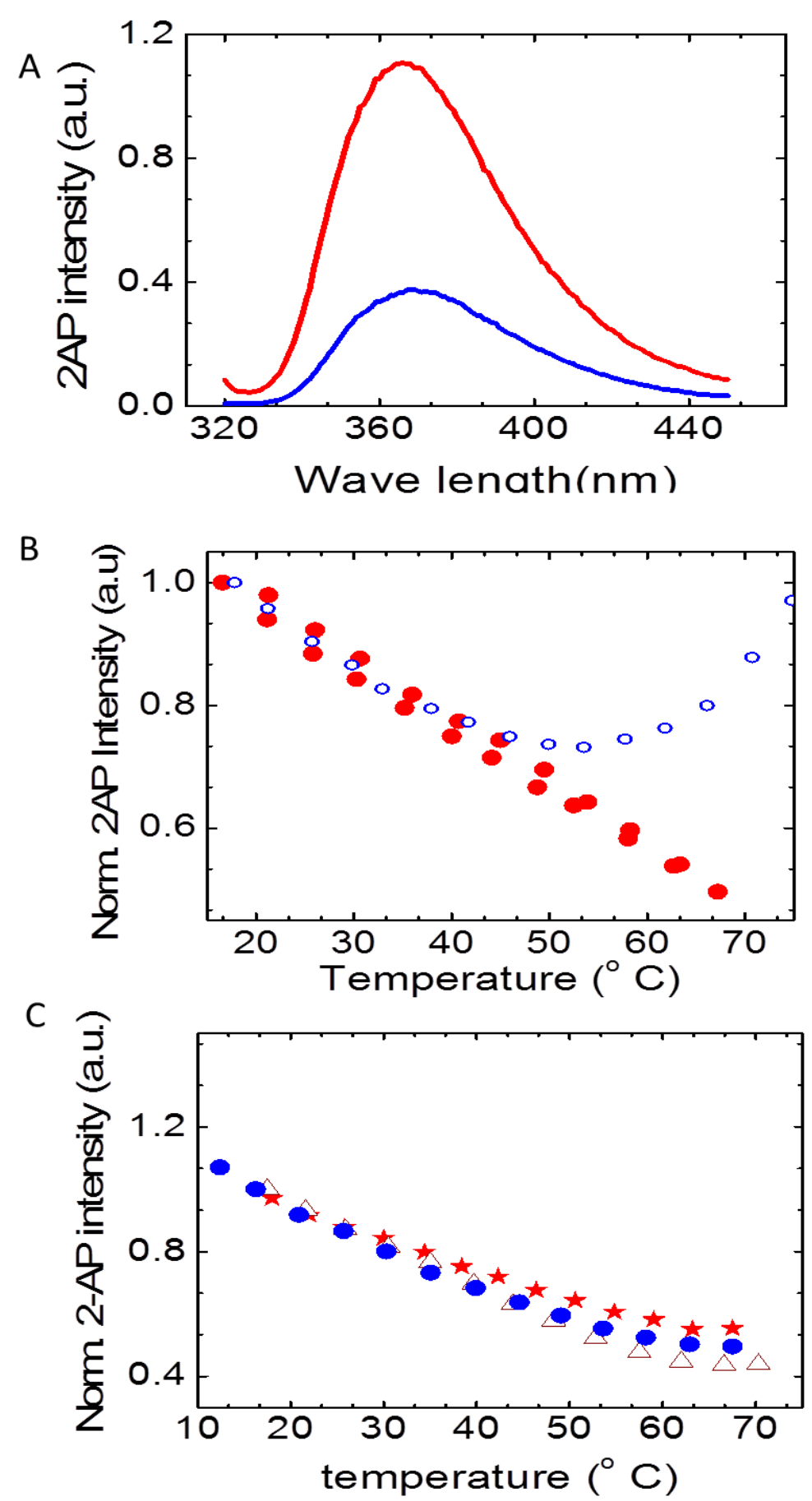

Fig.5.4. Equilibrium measurements on Taq MutS-T-bulge DNA complex with 2AP probe. (A) Fluorescence emission spectra of DNA in the absence (blue) and presence of MutS (red). (B) Normalized 2AP intensity as a function of temperature in the absence (blue) and presence of MutS (red). To check the reproducibility of the complex, the temperature was first increased slowly (by $2.5^{\circ}$ $\mathrm{C}$ steps) up to $70^{\circ} \mathrm{C}$ and then slowly cooled down back to the initial temperature (both data are marked in red closed symbol). (C). Normalized intensity of MutS-T-bulge complexes with different concentrations: $40 \mu \mathrm{M}: 40 \mu \mathrm{M}$ (red), $1 \mu \mathrm{M}: 1 \mu \mathrm{M}$ (brown), $500 \mathrm{nM}: 500 \mathrm{nM}$ (blue). 
Although some deviations are observed at the higher temperatures, the trends observed in the experiments do not correlate with increasing MutS or TDNA concentrations, and are likely from experimental errors in these measurements. Thus, these results indicate that $2 \mathrm{AP}$ fluorescence changes in the complex with increasing temperature, over and above the intrinsic quantum yield change, are from unimolecular processes and not from the thermal dissociation of the complex. We interpret these changes as arising from MutS induced conformational changes in the vicinity of the T-bulge in the MutS-TDNA complex.

Our results that the MutS-TDNA complex is stable up to at least $70^{\circ} \mathrm{C}$ is consistent with previous studies on Taq MutS by the Hsieh group, who investigated Taq MutS binding to a heteroduplex DNA over a broad temperature range from $0-70{ }^{\circ} \mathrm{C}$ [49]. Above $\sim 70{ }^{\circ} \mathrm{C}$, they could not accurately measure the binding of MutS as their DNA also started to melt. However, they showed that the ATPase activity of Taq MutS is optimum at $80^{\circ} \mathrm{C}$ [49]. We therefore carried out T-jump studies on the 2AP-labeled MutS-TDNA complex in a temperature range where the protein remains bound to its specific site and is known to still be its active form, 


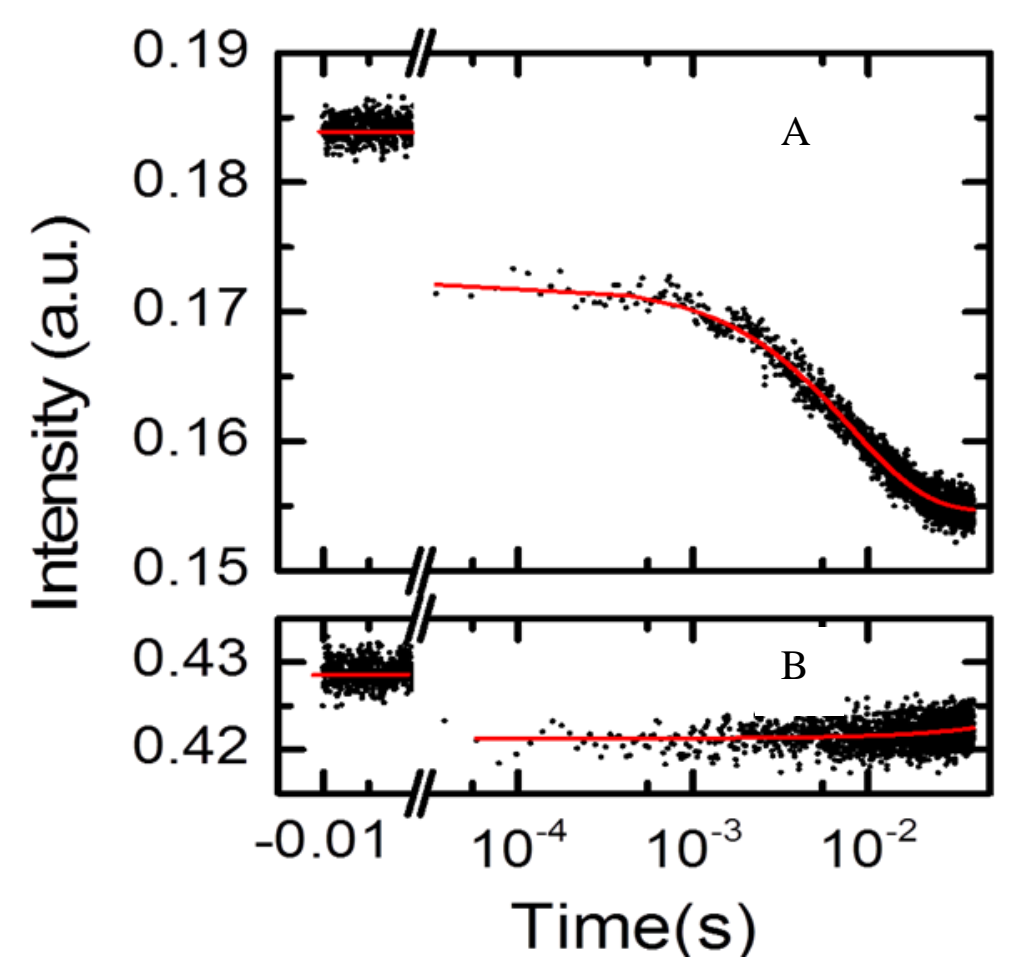

Fig.5.5. T-jump relaxation traces for Taq MutS-T-bulge DNA complex with 2AP probe are shown in response to a $9^{\circ} \mathrm{C}$ T-jump, from $61^{\circ}$ to $70^{\circ} \mathrm{C}$, with relaxation time $\sim 8 \mathrm{~ms}$. Sample concentration for the complex is $40 \mu \mathrm{M}: 40 \mu \mathrm{M}$. (B). Control experiment on free $2 \mathrm{AP}$ shows a slow T-jump recovery with a characteristic time constant $\sim 220 \mathrm{~ms}$.

Relaxation kinetics in response to laser T-jump perturbation were carried out on MutS-TDNA complex at different protein:DNA concentrations: $20 \mu \mathrm{M}: 20 \mu \mathrm{M}, 30 \mu \mathrm{M}: 30 \mu \mathrm{M}$ and $40 \mu \mathrm{M}: 40 \mu \mathrm{M}$, using $2 \mathrm{AP}$ fluorescence as a probe of the dynamics of conformational changes. The $\mathrm{K}_{\mathrm{D}}$ for the MutS-TDNA complex was previously measured by the Hingorani group, and as $15-25 \mathrm{nM}$ at $50 \mathrm{mM}$ salt, the same salt condition used in our studies [46]. Thus, at concentrations used in the T-jump measurements, at greater than $5 \mu \mathrm{M}$ for both MutS and TDNA, we have $100 \%$ complex at room temperature. 2AP-probed relaxation kinetics in response to a T-jump perturbation were observed between $67^{\circ} \mathrm{C}-72^{\circ} \mathrm{C}$ (final temperature). A representative kinetics trace measured for the MutS-TDNA complex together with the corresponding control measurement on free 2AP are shown in Fig. 5.5. The 2AP fluorescence 
in the complex drops immediately after the T-jump, due to the intrinsic thermal response of the $2 \mathrm{AP}$ probe, and then continues to decrease as a function of time, exhibiting relaxation kinetics in the T-jump time-window that report on the dynamics of the complex (Fig. 5.5A). The decrease in 2AP as the system evolves from the conformations populated at the initial temperature to those populated at the final (higher) temperature after the $\mathrm{T}$-jump indicate that the conformational changes in the DNA correspond to a decrease in the extent of kinked/nucleotide-flipped-out when the temperature in the sample is increased. In contrast, the free 2AP sample shows only the initial drop due to the T-jump perturbation, and no relaxation kinetics, as to be expected (Fig. 5.5B). The relaxation kinetics traces measured for the complex are well described in terms of single-exponential kinetics convoluted with Tjump recovery, as described in Chapter 2. The relaxation rates obtained from these analyses are only weakly dependent on temperature in the measured range, with an average relaxation time constant of $9 \pm 5 \mathrm{~ms}$.

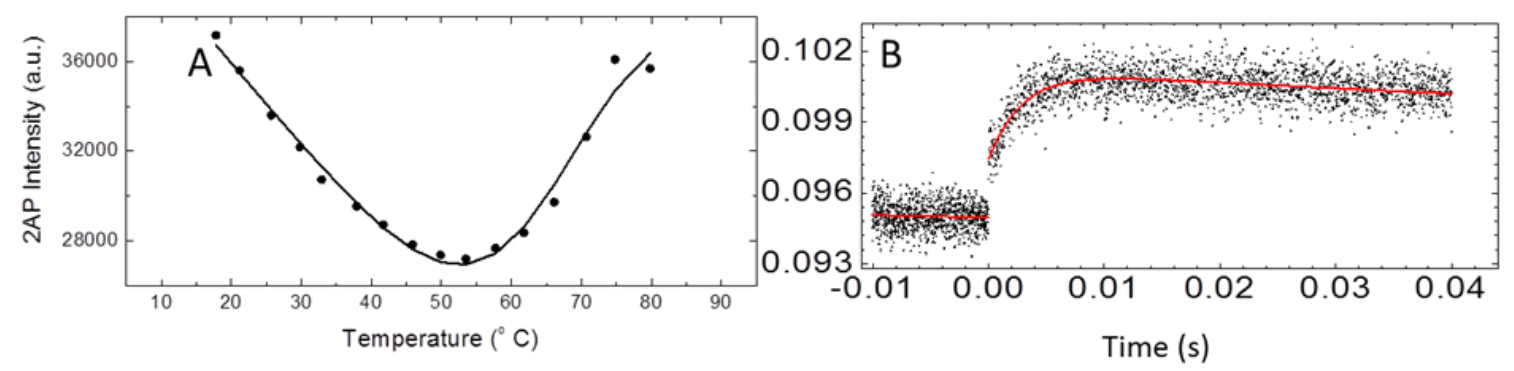

Fig.5.6. (A) Melting curve of $2 \mathrm{AP}$ labelled 23 base pair T-bulge (TDNA). The melting temperature is determined as $\sim 72^{\circ} \mathrm{C}$ from the fit (see Methods). (B) T-jump relaxation traces are shown for TDNA. The relaxation rate of DNA melting is $1.8 \mathrm{~ms}$ in response to a T-jump from $67^{\circ} \mathrm{C}$ to $70^{\circ} \mathrm{C}$.

We also carried out control measurements on free TDNA. No relaxation kinetics were seen on these samples up to $67^{\circ} \mathrm{C}$. Above $67^{\circ} \mathrm{C}$, TDNA samples exhibited DNA melting kinetics reported by an increase in 2AP fluorescence after the initial drop, consistent with the 
equilibrium 2AP fluorescence measurements on these samples (Fig. 5.6). At $70^{\circ} \mathrm{C}$ the DNA melting kinetics showed a relaxation time of $\sim 1.8 \mathrm{~ms}$.

\subsubsection{Taq MutS binding to mismatch (T-bulge) DNA as probed by FRET pair}

\subsubsection{Temperature dependent FRET change in T-bulge (FRET)(TFT) - Taq MutS complex}

To examine overall DNA bending induced by MutS, we carried out equilibrium and kinetics measurements on an 18-bp long DNA containing a T-bulge and labelled with FRET pair: TAMRA (donor) and Cy5 (acceptor); the DNA sequence is shown in Table I The fluorescence emission spectra of MutS-TFT DNA complex and free TFT DNA, for donor only (D) and donor-acceptor (DA) labelled samples, were measured as a function of temperature with excitation of the donor at $532 \mathrm{~nm}$. A typical set of fluorescence emission spectra at $25^{\circ} \mathrm{C}$ are shown in Fig. 5.7A; these data demonstrate that there is energy transfer between donor and acceptor in DA labelled free DNA and that the extent of the energy transfer increases when MutS binds, indicating that the DNA is bent in the presence of MutS. The FRET efficiency in each of the DA_labelled samples were obtained from the donormethod, as described in Chapter 2. The FRET efficiency measured at $25^{\circ} \mathrm{C}$ increases from $\sim 0.57$ in free DNA to $\sim 0.66$ when MutS binds (Fig. 5.7C), consistent with the extent of DNA bending seen in the crystal structure. Within the complex, the FRET efficiency decreases from $\sim 0.66$ to $\sim 0.37$ as the sample is heated from $15^{\circ} \mathrm{C}$ to $70^{\circ} \mathrm{C}$, indicating that the DNA unbends slightly, on average as the complex is heated up (Fig. 5.7C). Measurements on DNA alone also showed a decrease in FRET over the same temperature range, from $\sim 0.57$ to $\sim 0.33$, which we attribute to DNA melting (see Fig. 5.8, which shows a DNA melting profile for TFT DNA, with a melting temperature of $\sim 73^{\circ} \mathrm{C}$ ). However, based on our studies on the 2AP-labeled TDNA that showed that MutS binding inhibits DNA melting up to $\sim 70{ }^{\circ} \mathrm{C}$, as 
well as the lack of any dependence of protein:DNA concentrations on the FRET changes measured for the complex (discussed below), we attribute the change in FRET in the complex as mainly coming from DNA unbending in the complex as the temperature is raised and not from DNA melting.
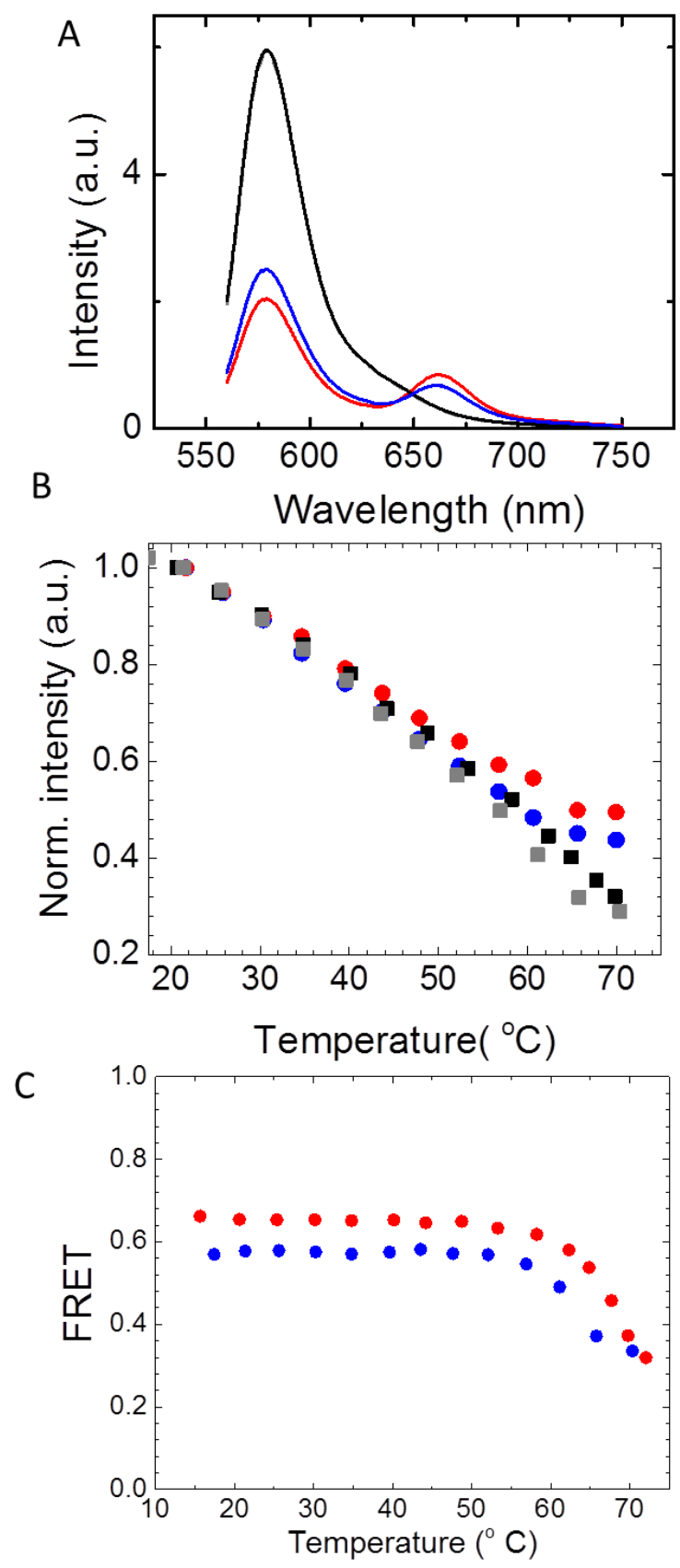

Fig.5.7. Equilibrium FRET measurements on Taq MutS-T-bulge (FRET)(TFT) complex. (A) Fluorescence emission spectra of T-bulge containing DNA: labeled with donor (TAMRA) in the absence (gray) and presence (black) of MutS; labeled with donor (TAMRA) and acceptor (Cy5) in the 
absence (blue) and presence (red) of MutS at $25^{\circ} \mathrm{C}$. The concentrations are $10 \mu \mathrm{m}: 10 \mu \mathrm{M}$ complex. (B) The changes in donor intensity as a function of temperature is plotted for all four samples described in (A) with the same color scheme. (C) Variation in FRET as a function of temperature for free TFTDNA (blue) and for TFTDNA-MutS complex (red).
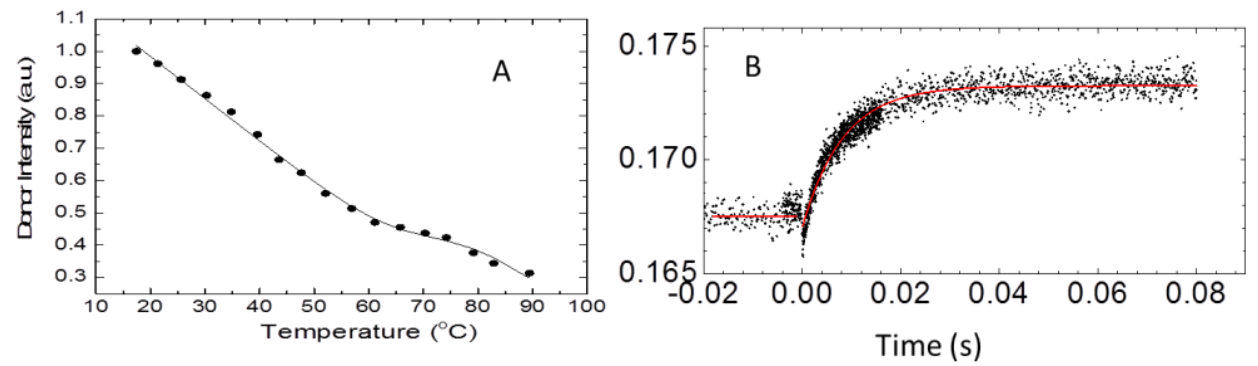

Fig.5.8. (A) Melting curve of FRET labelled 18 bp T-bulge (TFTDNA). The melting temperature is determined as $\sim 73^{\circ} \mathrm{C}$ from the fit (see Methods). (B) T-jump relaxation for TFTDNA: the relaxation rate of DNA melting is $8 \mathrm{~ms}$ in response to a T-jump from $55^{\circ} \mathrm{C}$ to $60{ }^{\circ} \mathrm{C}$.

To verify that indeed the FRET changes in the complex as the temperature is raised are from unimolecular unbending of DNA, we carried out these equilibrium studies for three different MutS:DNA concentrations: $1 \mu \mathrm{M}: 1 \mu \mathrm{m}, 2 \mu \mathrm{M}: 2 \mu \mathrm{M}$ and $20 \mu \mathrm{M}: 20 \mu \mathrm{M}$. Although the absolute FRET values, obtained at $20^{\circ} \mathrm{C}$, exhibit variations of \pm 0.04 between the different samples, the relative change in FRET for all three sets of measurements are independent of MutS or DNA concentrations (Fig.5.9A). Thus, in $50 \mathrm{mM}$ salt conditions of our buffer and for MutS:DNA concentrations used in both equilibrium and T-jump $(>1 \mu \mathrm{M})$, the decrease in FRET efficiency with increasing temperature are from unimolecular processes and not from thermal dissociation of the complex and subsequent melting of DNA.

Finally, to verify thermal stability of the MutS-DNA complex under the conditions at which T-jump measurements are carried out, we checked the reversibility of the complex by FRET measurements as the temperature of the complex was raised from $15^{\circ} \mathrm{C}$ to $70^{\circ} \mathrm{C}$ and 
back to $15^{\circ} \mathrm{C}$. The results show that the FRET versus temperature profile for the complex is reversible up to $70^{\circ} \mathrm{C}$ (Fig. 5.9B).
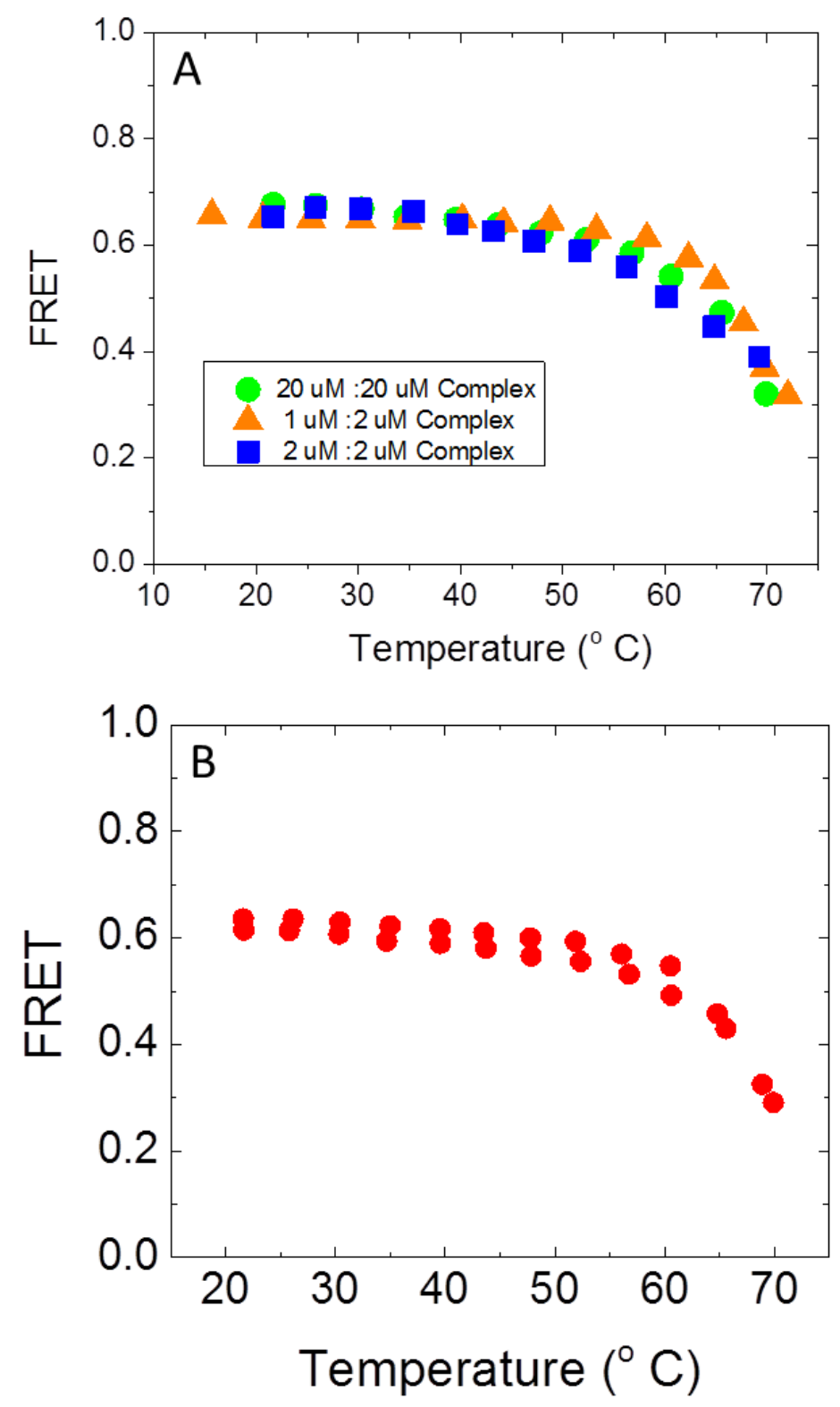

Fig.5.9. Equilibrium FRET measurements on Taq MutS-TFTDNA complex. (A) FRET efficiency as a function of temperature for different complex concentrations, $20 \mu \mathrm{M}: 20 \mu \mathrm{M}$ (green), $2 \mu \mathrm{M}: 2 \mu \mathrm{M}$ (blue) and $1 \mu \mathrm{M}: 1 \mu \mathrm{M}$ (orange). (B) To check the reversibility of the sample as a function of temperature, the emission intensities were measured while both heating the sample up to $70^{\circ} \mathrm{C}$ and then slowly cooling down back to the initial temperature (red). 


\subsubsection{T-jump measurements on T-bulge (FRET)-Taq MutS}

T-jump measurements were carried out on the MutS-TFTDNA complex at concentrations $20 \mu \mathrm{M}: 20 \mu \mathrm{M}$. The relaxation kinetics traces in response to the T-jump perturbation were monitored by time-resolved changes in the fluorescence emission of the donor (TAMRA), with the $532 \mathrm{~nm}$ diode laser as the probe beam source, as described in Chapter 2. A representative relaxation trace measured on donor/acceptor (DA)-labelled MutS-TFTDNA complex shows a rapid decrease in the donor intensity, as a result of the Tjump induced quantum-yield change of the donor, followed by an increase in the donor fluorescence, consistent with a change in the population from more bent (high FRET) to less bent (low FRET) DNA conformations as the temperature in the sample is suddenly raised (Fig.5.10).

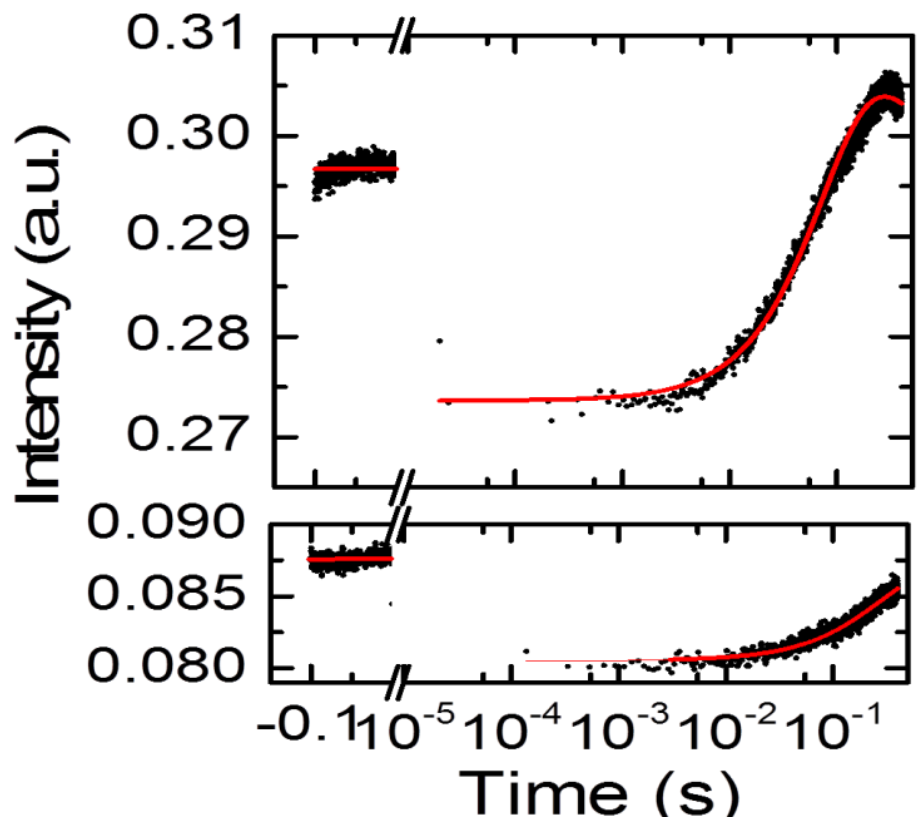

Fig.5.10. T-jump measurements on Taq MutS-T-bulge DNA (FRET) complex. (A) Relaxation kinetics in response to a T-jump from $65^{\circ} \mathrm{C}$ to $69^{\circ} \mathrm{C}$ with relaxation time $\sim 70 \mathrm{~ms}$. (B) Control experiment on donor labelled single stranded DNA shows the slow T-jump recovery with a characteristic time constant $\sim 222 \mathrm{~ms}$.

As in the case of the 2AP-probed kinetics, the relaxation kinetics are well described in terms of single-exponential decay convoluted with T-jump recovery (Fig.5.10A). The 
relaxation times thus obtained range from $70-120 \mathrm{~ms}$ in the temperature range from $56^{\circ} \mathrm{C}$ $68^{\circ} \mathrm{C}$ (final temperature). Control measurements carried out on donor-only labelled singlestrand of TFT DNA showed only the T-jump recovery kinetics (Fig.5.10B). Relaxation kinetics were also observed on DA-labelled DNA only samples, with relaxation time constants of 5-8 ms which we attribute to DNA melting kinetics (Fig. 5.8.B). No such kinetics arising from DNA melting were observed in the MutS-TFT DNA complex.

\subsubsection{Taq MutS-induced DNA kinking/bending rates compared with intrinsic bp opening dynamics of mismatched DNA}

In Fig. 5.11, we compare the MutS-induced DNA conformational dynamics in the specific complex with previous bp opening dynamics on matched and mismatched DNA, from NMR imino-proton measurements. Some trends are remarkably similar to 2AP-probed nucleotide flipping and FRET-probed DNA kinking/bending seen in the case of another damage recognition protein, $\mathrm{Rad} 4$, bound to a 3-bp mismatch bubble (discussed in Chapter 4). As in the case of Rad4-induced DNA dynamics, the 2AP-probed kinetics in the vicinity of the mismatch site are $~ 10$-fold faster than the overall DNA bending, which appears to be a later step in the recognition process. Also, the MutS-induced DNA conformational dynamics in the specific complex are significantly slower than the intrinsic bp opening dynamics of mismatched DNA, as was the case in Rad4. These results point to a protein-induced rather than conformational capture mechanism for DNA damage recognition, at least in the MMR and NER pathways. 


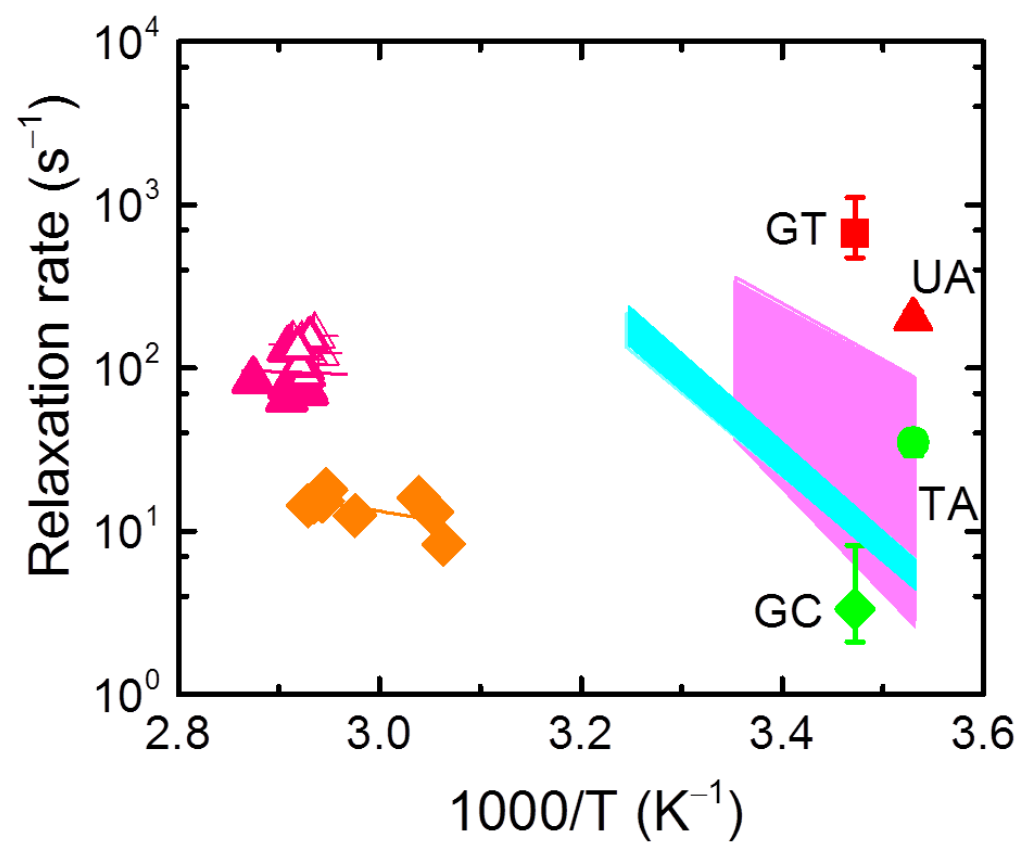

Fig.5.11. Relaxation rates of complexes, TDNA -Taq MutS (pink) and TFTDNA -Taq MutS (orange) as a function of the inverse final temperature (after the T-jump). For TDNA -Taq MutS, the different symbols indicate three independent sets of measurements. The continuous pink and orange lines are Arrhenius fit to the relaxation rates. The Cyan and pink bar data is described in fig.1.4, Chapter I.

\subsubsection{MutS $\alpha$ binding to mismatch (T-bulge) DNA as probed by 2AP (in DNA) and Trp} (in MutS)

Next we carried out equilibrium and T-jump studies with eukaryotic MutS homolog from S. cerevisae Msh2-Msh6 (MutS $\alpha$ ), which is highly homologous to the human Msh2Msh6 mismatch repair protein. For these studies, we used a 37-mer duplex DNA with 2AP probe next to an inserted T-bulge (37TDNA in Table II). The design of this DNA construct is identical to the one used by the Hingorani group in their stopped-flow studies [50].

Table II. 37 bp, 2AP incorporated DNA for MutS $\alpha$ (37TDNA)

5'-ATG TGA ATC AGT ATG GTA TA T ATC TGC TGA AGG AAA T-3'

$3^{\prime}$-TAC ACT TAG TCA TAC CAT TA TAG ACG ACT TCC TTT A-5' 
Fluorescence emission spectra of 37TDNA in the presence and absence of MutS $\alpha$ measured at $25^{\circ} \mathrm{C}$ are shown in Fig. 5.12A. The 2AP fluorescence increases 1.6-fold upon MutS $\alpha$ binding to the 37TDNA. We attribute this increase to the change in the stacking interactions of the 2AP probe in the vicinity of the T-bulge upon DNA kinking in the specific complex, similar to the Taq MutS-TDNA complex, although the 2AP increase in the Taq MutS complex was larger.

Next, we examined how the temperature dependent changes in 2AP fluorescence in the complex compared with corresponding changes in free DNA. The fluorescence emissions spectra for the complex and DNA only samples were measured in the temperature range $15-40^{\circ} \mathrm{C}$. The intensities of the fluorescence maxima (measured at $370 \mathrm{~nm}$ ) for the complex and for the DNA, normalized to match at the lowest temperature, are plotted as a function of temperature in Fig. 5.12B. The data show that, unlike in the case of the Taq MutS-TDNA complex, the 2AP fluorescence intensities of the complex overlap with those of free DNA, indicating that any perturbation of the complex with increasing temperature is likely small and hard to detect by the $2 \mathrm{AP}$ probe under equilibrium conditions. However, as illustrated in the Rad4 studies discussed in Chapter 4, our T-jump apparatus has sufficient sensitivity to detect relaxation kinetics even under conditions where equilibrium data do not detect any conformational changes.

Prior to carrying out the T-jump studies, we checked for reversibility of the complex against temperature changes, by first heating the sample up to $40^{\circ} \mathrm{C}$ and then cooling it back to $20^{\circ} \mathrm{C}$. These data (shown in Fig. 5.12B) demonstrate good reversibility under these conditions. All measurements on these samples were carried out at temperatures below $40^{\circ} \mathrm{C}$; above this temperature we did observe irreversible behaviour, presumably from protein dissociation and subsequent aggregation. We also compared the temperature dependent 2AP 
changes for two different complex concentrations $(10 \mu \mathrm{M}: 10 \mu \mathrm{M}$ and $1 \mu \mathrm{M}: 1 \mu \mathrm{M})$. These data are shown in Fig. 5.12C and show that the relative changes in 2AP intensity for these two sets of measurements are independent of MutS $\alpha$ or 37TDNA concentrations. Thus, we do not detect any significant bimolecular dissociation of the complex in this temperature range.

A

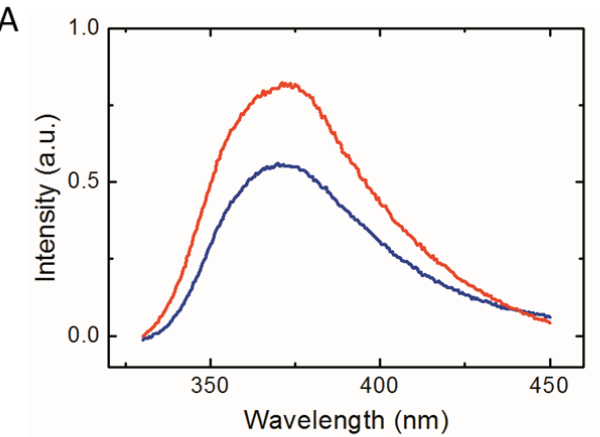

B
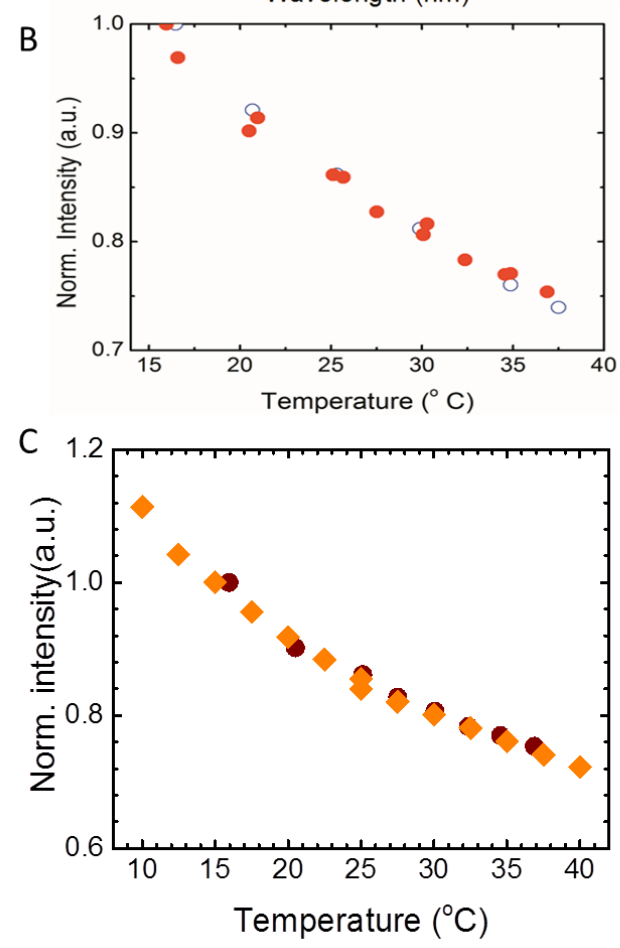

Fig.5.12. Equilibrium measurements on yeast MutS $\alpha$-T-bulge DNA (37TDNA) complex with 2AP probe. (A) Fluorescence emission spectra of DNA in the absence (blue) and presence of MutS (red). (B) Normalized 2AP intensity as a function of temperature in the absence (blue) and presence of MutS (red). To check the reproducibility of the complex, temperature was first increased slowly by $2.5^{\circ} \mathrm{C}$ steps up to $40^{\circ} \mathrm{C}$ and then it was cooled down back to the initial temperature (red). (C) Normalized intensity of complexes at two different concentrations, $10 \mu \mathrm{M}: 10 \mu \mathrm{M}$ (orange), $1 \mu \mathrm{M}: 1$ $\mu \mathrm{M}$ (brown). 
Next we carried out T-jump measurements on MutS $\alpha-37$ TDNA complex at concentrations of $8 \mu \mathrm{M}: 8 \mu \mathrm{M}$. Note that these concentrations are $>2$-fold smaller than the $20 \mu \mathrm{M}: 20 \mu \mathrm{M}$ concentrations used in the 2AP studies of the Taq MutS-TDNA complex. While higher concentrations of 2AP-labeled samples are needed to get sufficient signals in our Tjump apparatus, when we use $\mathrm{Hg} / \mathrm{Xe}$ lamp with $314 \mathrm{~nm}$ excitation as our probe source (see Chapter 2), we were unable to increase the concentrations of the MutS $\alpha$ protein due to concerns of protein aggregation above $\sim 10 \mu \mathrm{M}$. Therefore, for these measurements, we opted to use a $280 \mathrm{~nm}$ UV laser instead of the $\mathrm{Hg} / \mathrm{Xe}$ lamp, which is also suitable for $2 \mathrm{AP}$ excitation. However, although the fluorescence emission signals of our samples in the Tjump spectrometer with $280 \mathrm{~nm}$ laser excitation were much higher than the $314 \mathrm{~nm}$ excitation with the $\mathrm{Hg} / \mathrm{Xe}$ lamp, we inadvertently also excited Trp residues in the MutS $\alpha$ protein, thus complicating our interpretation of the observed relaxation kinetics, as discussed below.

T-jump relaxation kinetics on the MutS $\alpha-37$ TDNA complex were observed in the temperature range $28-39^{\circ} \mathrm{C}$ (final temperature). A representative relaxation trace for the complex and corresponding control measurements on free 2AP samples are shown in Fig. 5.13. In the complex, we observed biphasic kinetics after the initial rapid drop in fluorescence, with first an increase in the $2 \mathrm{AP} / \mathrm{Trp}$ fluorescence, with relaxation time constants in the range of 1-6 ms, followed by a decrease in the 2AP/Trp fluorescence, with relaxation time constants in the range of $22-36 \mathrm{~ms}$ signal (Fig. 5.13A). No relaxation kinetics were observed on the free $2 \mathrm{AP}$ samples over the entire temperature range. 


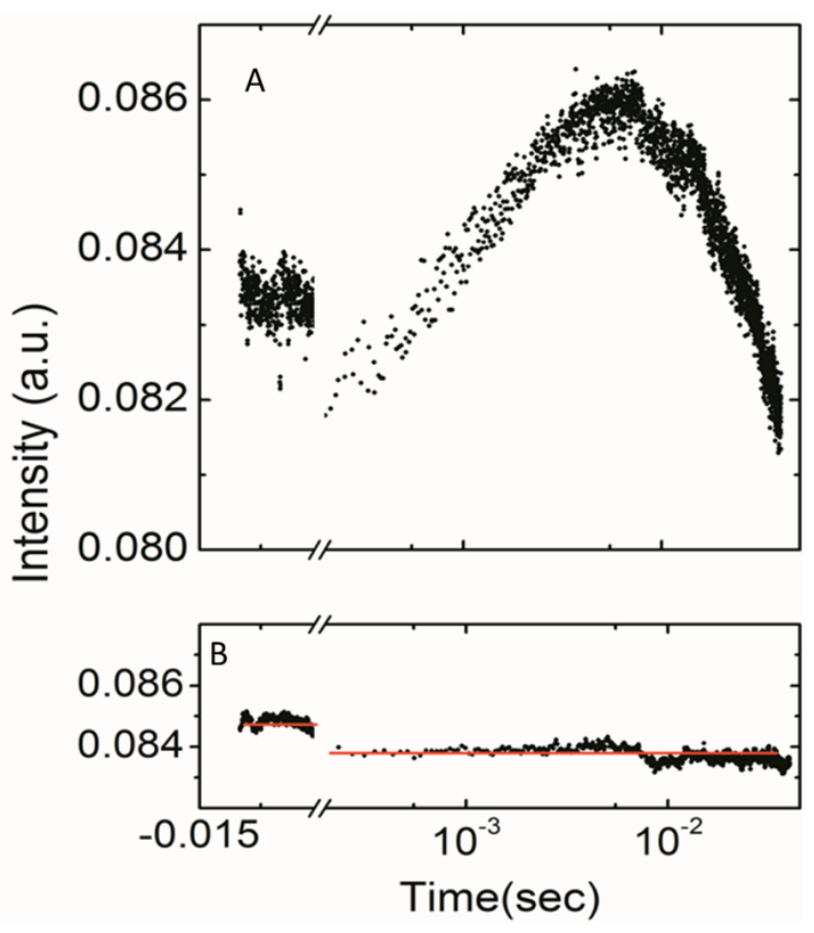

Fig.5.13. T-jump on yeast MutS $\alpha$-T-bulge DNA complex with 2AP probe using UV laser. (A) Relaxation kinetics in response to a $4^{\circ} \mathrm{C}$ T-jump, from $25^{\circ} \mathrm{C}$ to $29^{\circ} \mathrm{C}$, with relaxation times of $1.6 \mathrm{~ms}$ and $22 \mathrm{~ms}$. (B) Control experiment on free 2AP shows no kinetics other than the slow T-jump recovery. Note that the excitation wavelength for this experiment is $280 \mathrm{~nm}$ (UV laser).

In Fig. 5.14, we compare the relaxation rates obtained from the T-jump measurements on MutS $\alpha-37$ TDNA samples with those obtained on Taq MutS complexed with 2AP and FRET-labelled DNA and with previously reported bp opening rates. Interestingly, we find that the rapid phase observed in the MutS $\alpha-37$ TDNA complex has some overlap with intrinsic bp opening dynamics on normal (matched) B-DNA, suggesting that these dynamics perhaps reflect nonspecific rather than specific interactions of MutS with DNA. Since these measurements were carried out with a $280 \mathrm{~nm}$ probe source, where both $2 \mathrm{AP}$ and Trp are excited, we cannot separate the 2AP-reported DNA conformational dynamics from the Trpreported protein dynamics. Further studies, such as $280 \mathrm{~nm}$ excitation on MutS bound to unlabelled DNA, to monitor only Trp fluorescence changes, or $314 \mathrm{~nm}$ excitation on higher concentration samples, to monitor only $2 \mathrm{AP}$ fluorescence changes, as well as FRET measurements on end-labelled DNA with the MutS $\alpha$ protein, are needed to accurately piece 
together the various conformational changes and their relevant time-scales during mismatch recognition by MutS.

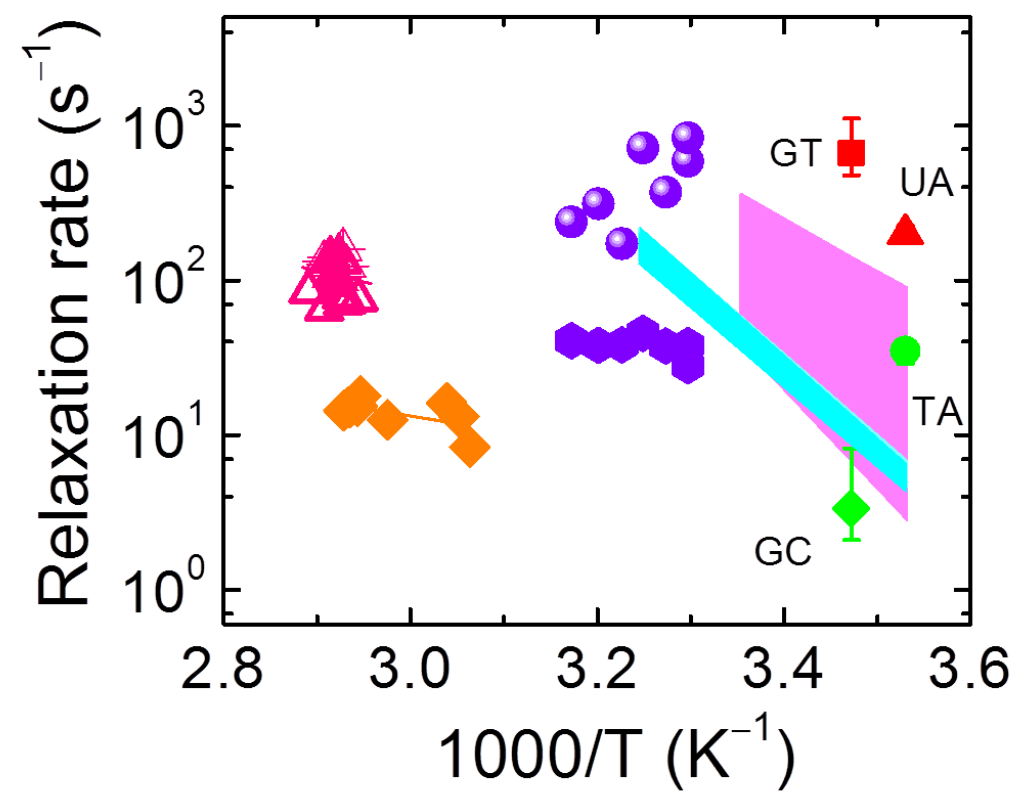

Fig.5.14. Comparison of relaxation rates for all three different samples: TDNA (2AP) -Taq MutS (pink); TFT (FRET) - Taq MutS (orange ) and 37TDNA (2AP) - MutS $\alpha$ (purple). The pink and cyan bars are described in Fig. 1.4, Chapter I.

\subsection{Discussion}

We have used laser T-jump perturbation to unveil previously unresolved dynamics of mismatch recognition by MutS with sub-microsecond time resolution, which is at least three orders of magnitude faster than previous studies on this complex, that were carried out using conventional stopped-flow or smFRET. While stopped-flow studies were unable to resolve unimolecular DNA bending/kinking dynamics of MutS-mismatch interactions, the smFRET studies on Taq MutS bound to DNA containing a G-T mismatch, carried out at room temperature, did report on very slow DNA bending/unbending dynamics, on time-scales of seconds or longer. The time-resolution of the smFRET measurements were limited to $>100$ ms. 
Here, we report relaxation kinetics in the $1-100 \mathrm{~ms}$ time scales during mismatch (Tbulge) recognition by $\mathrm{Taq}$ MutS, in the temperature range $56-72^{\circ} \mathrm{C}$, where the protein has previously been shown to have its highest activity [49]. Our results reveal 2AP-reported nucleotide dynamics at the mismatch site at $\sim 8 \mathrm{~ms}$ (at $\sim 70{ }^{\circ} \mathrm{C}$ ) and FRET-reported DNA bending dynamics on $\sim 70 \mathrm{~ms}$ (at similar temperatures). Both these kinetic steps are considerably slower than the intrinsic bp opening dynamics at mismatched sites, as measured by NMR, indicating that spontaneous kinking at the mismatch sites is not sufficient to overcome the free energy barrier to form the specific complex. The very slow (>seconds) DNA bending kinetics observed in the smFRET measurements are a puzzle, and may be a consequence of a much higher free energy barrier encountered by the Taq protein, designed by nature to function at $65^{\circ} \mathrm{C}-80^{\circ} \mathrm{C}$, in its attempt to form a recognition complex at room temperature.

We also measured mismatch (T-bulge) recognition dynamics by a eukaryotic MutS homolog S. cerevisae Msh2-Msh6 (MutS $\alpha$ ), designed to function close to room temperature. These studies were carried out on 2AP-labelled DNA adjacent to the T-bulge mismatch, similar to the 2AP studies with Taq MutS. However, the probe source in these studies, at 280 $\mathrm{nm}$, inadvertently excited both $2 \mathrm{AP}$ and the intrinsic Trp residues in the protein, and revealed complex biphasic kinetics with amplitudes of opposite signs. While accurate interpretation of these kinetics require further measurements, to separate the 2AP and Trp fluorescence signatures, we can draw some tentative conclusions based on comparison of the observed rates with the relaxation rates measured for the Taq MutS complexes, and with insights gained from our previous studies with IHF (Chapter 3) and Rad4 (Chapter 4). We find that the fast phase measured on the MutS $\alpha-37$ TDNA complex overlaps with intrinsic bp opening rates on normal DNA (Fig. 5.13). It is tempting to suggest that this fast phase is from conformational changes in the protein (detected by Trp fluorescence) during nonspecific 
bending of DNA by MutS, as seen in AFM studies, and rate-limited by intrinsic DNA dynamics. The slower phase observed on the MutS $\alpha-37$ TDNA complex could then be 2APreported DNA conformational dynamics in the vicinity of the T-bulge mismatch, similar to the 2AP-reported dynamics on the Taq MutS-TDNA complex. The very slow DNA bending dynamics captured by FRET measurements on the Taq MutS-FRTDNA complex would then be the formation of the ultimate recognition complex. A similar three-step mechanism was also observed in our T-jump studies on DNA damage recognition by Rad4 (discussed in Chapter 4).

\subsection{Conclusion}

This study presents the first observation of conformational dynamics in the MutSDNA complex in the time range from $10 \mu$ s to tens of ms, on time-scales that are relevant for mismatch recognition. Our study, carried out on two different MutS proteins, from $T$. aquaticus and S. cerevisiae, suggest at least three sequential events during mismatch (Tbulge) recognition: a rapid event $\left(\sim 1.2 \mathrm{~ms}\right.$ at $30^{\circ} \mathrm{C}$ in $\left.\mathrm{MutS} \alpha\right)$ occurring on time-scales similar to intrinsic bp opening dynamics of normal (matched) B-DNA, that we propose corresponds to a nonspecific DNA interrogation step; local dynamics at the vicinity of the mismatch site $\left(26.4 \mathrm{~ms}\right.$ at $30^{\circ} \mathrm{C}$ in MutS $\alpha$ and $\sim 8 \mathrm{~ms}$ at $70^{\circ} \mathrm{C}$ in $\mathrm{Taq}$ MutS), which we identify as a precursor to the recognition process; and a slower overall DNA bending step ( $\sim 70 \mathrm{~ms}$ at $68^{\circ} \mathrm{C}$ in $\mathrm{Taq}$ MutS), attributed to final rearrangements in the protein and DNA to form the ultimate recognition complex. It remains to be investigated how these relaxation dynamics will be affected when we alter the mismatch, and whether the rates at which the mismatches are recognised will correlate with previously reported mismatch repair efficiencies. 


\section{References}

1. Wang H, H.J., Signaling from DNA mispairs to mismatchrepair excision sites despite intervening blockades. EMBO J 2004. 23: p. 2126-2133.

2. McCulloch SD., K.T., The fidelity of DNA synthesis by eukaryotic replicative and translesion synthesis polymerases. Cell Res. 2008. 18: p. 148-161.

3. Buermeyer, A.B., Deschênes, Suzanne M., Baker, S.M., Lisaky, R.M., Mammalian DNA Mismatch Repair. Annual Review of Genetics, 1999. 33: p. 553-564.

4. Jiricny J1, M.G., DNA repair defects in colon cancer. Curr Opin Genet Dev., 2003. 13: p. 61-69.

5. Yang, W., Structure and mechanism for DNA lesion recognition. Cell Res, 2008. 18(1): p. 184-97.

6. Li, G.-M., Mechanisms and functions of DNA mismatch repair. Cell Research, 2008. 18: p. 85-98.

7. Juan Cristóbal Conde-Pérezprina, M.Á.L.-G., and Mina Konigsberg, DNA Mismatch Repair System: Repercussions in Cellular Homeostasis and Relationship with Aging. Oxid Med Cell Longev, 2012. 2012.

8. Isaacs, R.J. and H.P. Spielmann, A model for initial DNA lesion recognition by NER and MMR based on local conformational flexibility. DNA Repair (Amst), 2004. 3(5): p. 455-64.

9. Wang, H., et al., DNA bending and unbending by MutS govern mismatch recognition and specificity. Proc. Natl. Acad. Sci. U S A, 2003. 100(25): p. 14822-7.

10. Tessmer, I., et al., Mechanism of MutS searching for DNA mismatches and signaling repair. J Biol Chem, 2008. 283(52): p. 36646-54.

11. Nag, N., B.J. Rao, and G. Krishnamoorthy, Altered dynamics of DNA bases adjacent to a mismatch: a cue for mismatch recognition by MutS. J Mol Biol, 2007. 374(1): p. 39-53.

12. $\mathrm{Mu}, \mathrm{D}$., et al., Recognition and repair of compound DNA lesions (base damage and mismatch) by human mismatch repair and excision repair systems. Mol Cell Biol, 1997. 17(2): p. 760-9.

13. Lynch, H.T., et al., Genetics, natural history, tumor spectrum, and pathology of hereditary nonpolyposis colorectal cancer: an updated review. Gastroenterology, 1993. 104(5): p. 1535-49.

14. Han, H.J., et al., Germline mutations of hMLH1 and hMSH2 genes in Korean hereditary nonpolyposis colorectal cancer. J Natl Cancer Inst, 1996. 88(18): p. 13179.

15. Caskey, C.T., et al., Triplet repeat mutations in human disease. Science, 1992. 256(5058): p. 784-789.

16. Bowater, R.P. and R.D. Wells, The intrinsically unstable life of DNA triplet repeats associated with human hereditary disorders. Prog Nucleic Acid Res Mol Biol, 2001. 66: p. 159-202.

17. Mitas, M., Trinucleotide repeats associated with human disease. Nucleic Acids Res, 1997. 25(12): p. 2245-54.

18. Richards, R.I. and G.R. Sutherland, Repeat offenders: simple repeat sequences and complex genetic problems. Hum Mutat, 1996. 8(1): p. 1-7.

19. Schofield, M.J., et al., The Phe-X-Glu DNA binding motif of MutS. The role of hydrogen bonding in mismatch recognition. J Biol Chem, 2001. 276(49): p. 45505-8. 
20. Antony, E. and M.M. Hingorani, Asymmetric ATP binding and hydrolysis activity of the Thermus aquaticus MutS dimer is key to modulation of its interactions with mismatched DNA. Biochemistry, 2004. 43(41): p. 13115-28.

21. Junop, M.S., et al., Composite active site of an ABC ATPase: MutS uses ATP to verify mismatch recognition and authorize DNA repair. Mol. Cell, 2001. 7(1): p. 1-12.

22. Obmolova, G., et al., Crystal structures of mismatch repair protein MutS and its complex with a substrate DNA. Nature, 2000. 407(6805): p. 703-10.

23. Lamers, M.H., et al., The crystal structure of DNA mismatch repair protein MutS binding to a Gx T mismatch. Nature, 2000. 407(6805): p. 711-7.

24. Natrajan, G., et al., Structures of Escherichia coli DNA mismatch repair enzyme MutS in complex with different mismatches: a common recognition mode for diverse substrates. Nucleic Acids Res, 2003. 31(16): p. 4814-21.

25. Warren, J.J., et al., Structure of the human MutSalpha DNA lesion recognition complex. Mol Cell, 2007. 26(4): p. 579-92.

26. Kramer, B., W. Kramer, and H.J. Fritz, Different base/base mismatches are corrected with different efficiencies by the methyl-directed DNA mismatch-repair system of E. coli. Cell, 1984. 38(3): p. 879-87.

27. Edelbrock, M.A., S. Kaliyaperumal, and K.J. Williams, DNA mismatch repair efficiency and fidelity are elevated during DNA synthesis in human cells. Mutat Res, 2009. 662(1-2): p. 59-66.

28. Harfe, B.D. and S. Jinks-Robertson, DNA mismatch repair and genetic instability. Annu Rev Genet, 2000. 34: p. 359-399.

29. Sixma, T.K., DNA mismatch repair: MutS structures bound to mismatches. Curr. Opin. Struct. Biol., 2001. 11(1): p. 47-52.

30. Lopez de Saro, F.J., et al., The beta sliding clamp binds to multiple sites within MutL and MutS. J Biol Chem, 2006. 281(20): p. 14340-9.

31. Kunkel, T.A. and D.A. Erie, DNA mismatch repair. Annu Rev Biochem, 2005. 74: p. 681-710.

32. Sass, L.E., et al., Single-molecule FRET TACKLE reveals highly dynamic mismatched DNA-MutS complexes. Biochemistry, 2010. 49(14): p. 3174-90.

33. Crothers DM, Z.B., Theory of the melting transition of synthetic polynucleotides: evaluation of the stacking free energy. J Mol Biol, 1964. 116: p. 1-9.

34. Schofield MJ, B.F., Nayak S, Du C, Kool ET, Hsieh and P., The Phe-X-Glu DNA binding motif of MutS. The role of hydrogen bonding in mismatch recognition. J Biol Chem, 2001. 276: p. 45505-45508.

35. Yang, W., Poor base stacking at DNA lesions may initiate recognition by many repair proteins. DNA Repair (Amst), 2006. 5(6): p. 654-66.

36. Isaacs, R.J., W.S. Rayens, and H.P. Spielmann, Structural differences in the NOEderived structure of $G-T$ mismatched DNA relative to normal DNA are correlated with differences in (13)C relaxation-based internal dynamics. J Mol Biol, 2002. 319(1): p. 191-207.

37. Patel, D.J., et al., Dynamics of DNA duplexes containing internal G.T, G.A, A.C, and T.C pairs: hydrogen exchange at and adjacent to mismatch sites. Fed Proc, 1984. 43(11): p. 2663-70.

38. Pardi, A., et al., Kinetics for exchange of imino protons in the d(C-G-C-G-A-A-T-T-C$G-C-G)$ double helix and in two similar helices that contain a $G$. T base pair, $d(C-G-$

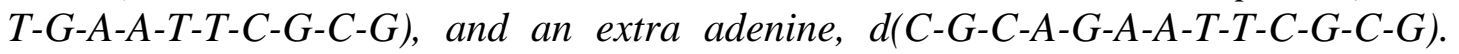
Biochemistry, 1982. 21(25): p. 6567-74. 
39. Patel, D.J., A. Pardi, and K. Itakura, DNA conformation, dynamics, and interactions in solution. Science, 1982. 216(4546): p. 581-90.

40. Moe, J.G. and I.M. Russu, Kinetics and energetics of base-pair opening in 5'd(CGCGAATTCGCG)-3' and a substituted dodecamer containing G.T mismatches. Biochemistry, 1992. 31(36): p. 8421-8.

41. Bhattacharya, P.K., J. Cha, and J.K. Barton, $1 H$ NMR determination of base-pair lifetimes in oligonucleotides containing single base mismatches. Nucleic Acids Res, 2002. 30(21): p. 4740-50.

42. Bishop, D.K., J. Andersen, and R.D. Kolodner, Specificity of mismatch repair following transformation of Saccharomyces cerevisiae with heteroduplex plasmid DNA. Proc Natl Acad Sci U S A, 1989. 86(10): p. 3713-7.

43. Parker, B.O. and M.G. Marinus, Repair of DNA heteroduplexes containing small heterologous sequences in Escherichia coli. Proc Natl Acad Sci U S A, 1992. 89(5): p. 1730-4.

44. Huang, S.N. and D.M. Crothers, The role of nucleotide cofactor binding in cooperativity and specificity of MutS recognition. J Mol Biol, 2008. 384(1): p. 31-47.

45. Su, S.S. and P. Modrich, Escherichia coli mutS-encoded protein binds to mismatched DNA base pairs. Proc Natl Acad Sci U S A, 1986. 83(14): p. 5057-61.

46. Jacobs-Palmer, E. and M.M. Hingorani, The effects of nucleotides on MutS-DNA binding kinetics clarify the role of MutS ATPase activity in mismatch repair. J. Mol. Biol., 2007. 366(4): p. 1087-98.

47. Biswas, I. and P. Hsieh, Identification and characterization of a thermostable MutS homolog from Thermus aquaticus. J Biol Chem, 1996. 271(9): p. 5040-8.

48. Antony, E., et al., Contribution of Msh2 and Msh6 subunits to the asymmetric ATPase and DNA mismatch binding activities of Saccharomyces cerevisiae Msh2-Msh6 mismatch repair protein. DNA Repair (Amst), 2006. 5(2): p. 153-62.

49. Hsieh, I.B.a.P., Identification and Characterization of a Thermostable MutS Homolog from Thermus aquaticus. THE JOURNAL OF BIOLOGICAL CHEMISTRY, 1996, 271(9) pp. 5040-5048

50. Antony, E. and M.M. Hingorani, Mismatch recognition-coupled stabilization of Msh2-Msh6 in an ATP-bound state at the initiation of DNA repair. Biochemistry, 2003. 42(25): p. 7682-93. 


\section{Appendix (A)}

\subsection{Energetic cost for bending DNA}

\section{Sequence dependent DNA elasticity}

The stiffness of DNA is often expressed in terms of its persistence length. Persistence length is the length where a DNA segment can be physically modelled as a straight rod. The persistence length of the DNA is $150 \mathrm{bp}(45-50 \mathrm{~nm})$ at $100 \mathrm{mM}$ salt. Below this length the DNA can be treated as a flexible elastic rod that is resistant to any significant bending and above this length the DNA properties can only be described statistically, like a three-dimensional random walk.

DNA molecule when it packed inside the cell and unbound to any protein is a long molecular thread, with a diameter of $\sim 2 \mathrm{~nm}$ and with a length in the order of few centimetres long. Inside the cell the negative charge in DNA back bone is screened by counter ions.

The persistence length $\mathrm{L}$ is related to the bending modulus $\mathrm{B}$ as

$$
L_{p}=\frac{B}{K_{B} T}
$$

Where we assume the DNA is a homogenous elastic rod.

$B=Y I$ and $\mathrm{Y}$ is the Young's modulus of the material, $\mathrm{I}$ is the moment of inertia of the crosssection.

For a rod with a circular cross- section of radius $\mathrm{r}, I=\frac{\Pi}{4} r^{4}$. If $\mathrm{r}=1 \mathrm{~nm}$ for a B-DNA, the Young modulus of DNA will be $300 \mathrm{MPa}$. 
The Bending energy $E_{b e n d}$ stored in an elastic rod of length $L$ that is bent by an angle $\theta$, with a radius of curvature $\mathrm{R}$ is given by

$$
\frac{E_{\text {bend }}}{L}=\frac{1}{2} B\left(\frac{1}{R}\right)^{2}=\frac{1}{2} B\left(\frac{\theta}{L}\right)^{2}
$$

From equipartition theory and equation A1

$$
<\theta^{2}>=\frac{K_{B} T L}{B}=\frac{L}{L_{p}}
$$

Thus the bending energy in terms of persistence length is given by

$$
E_{\text {bend }}=\frac{1}{2} K_{B} T\left(\frac{L_{P}}{L}\right) \theta^{2}
$$

From this equation we can calculate the free energy cost of bending a DNA. In the case of IHF the H'DNA is bend by $180^{\circ}$ and therefore the $\mathrm{E}_{\text {bend }} \approx 21 \mathrm{~K}_{\mathrm{B}} \mathrm{T}=13 \mathrm{kcal} / \mathrm{mol}$. Here we assumed the elastic rod description is valid for the short H' sequence.

\subsection{DNA Geometry}

To enable a complete description of DNA geometry a common reference frame with respect to idealized base-pair coordinates has been established. The parameters commonly used to describe DNA geometry based on such a reference frame are described below [1-3] (fig.1.1),

(1) The six bp parameters that define the positions and orientations of the two bases relative to ideal bp geometry - shear, buckle, stretch, propeller, stagger and opening.

(2) The six bp step parameters to define the relative positions and orientations of the two consecutive base pairs - shift, tilt, slide, roll, rise, and twist. 
(3) The six helical parameters that describe the geometry with respect to the helical axis- Xdisplacement, y-displacement, inclination, tip, helical rise and helical twist (not shown).

Base pair parameters
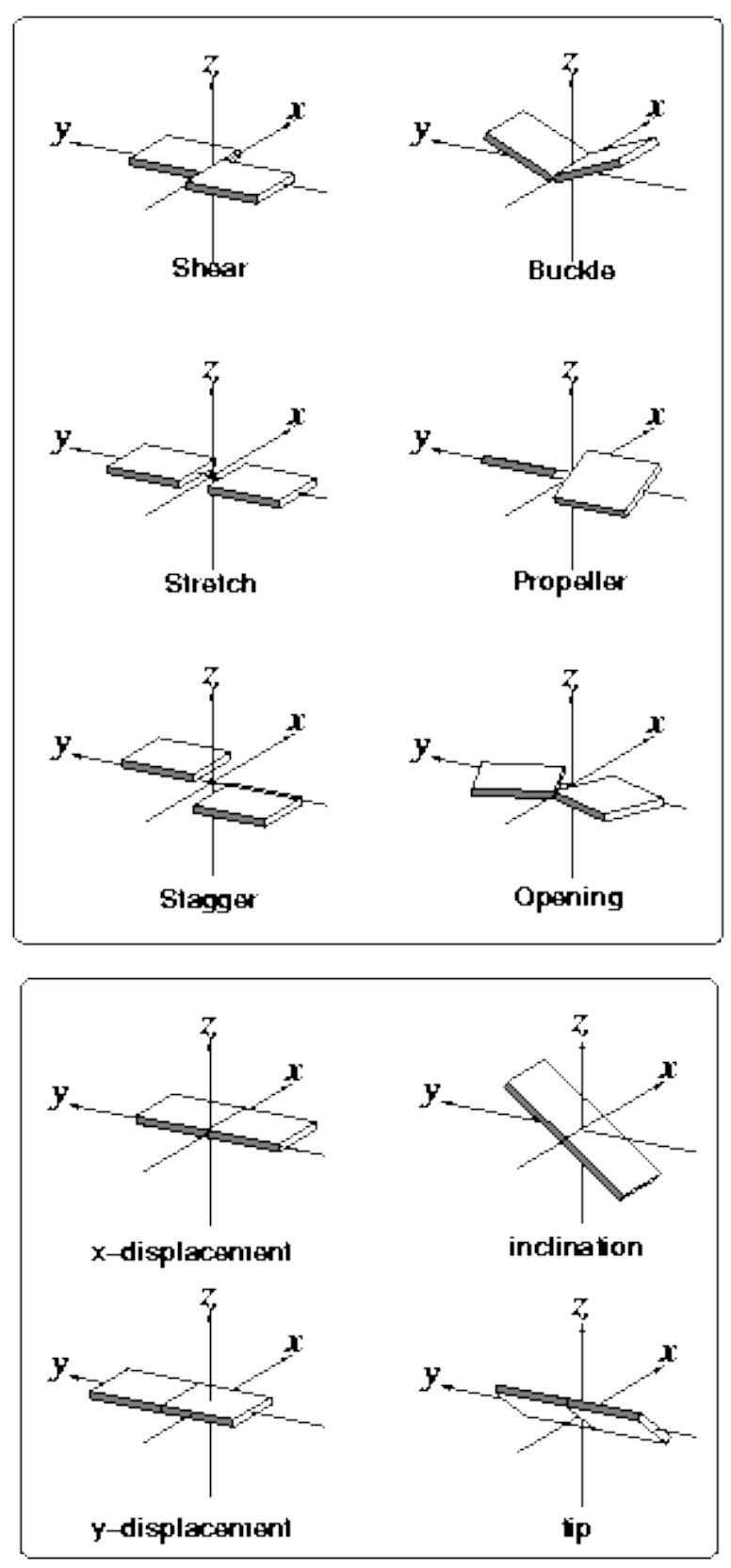

step parameters
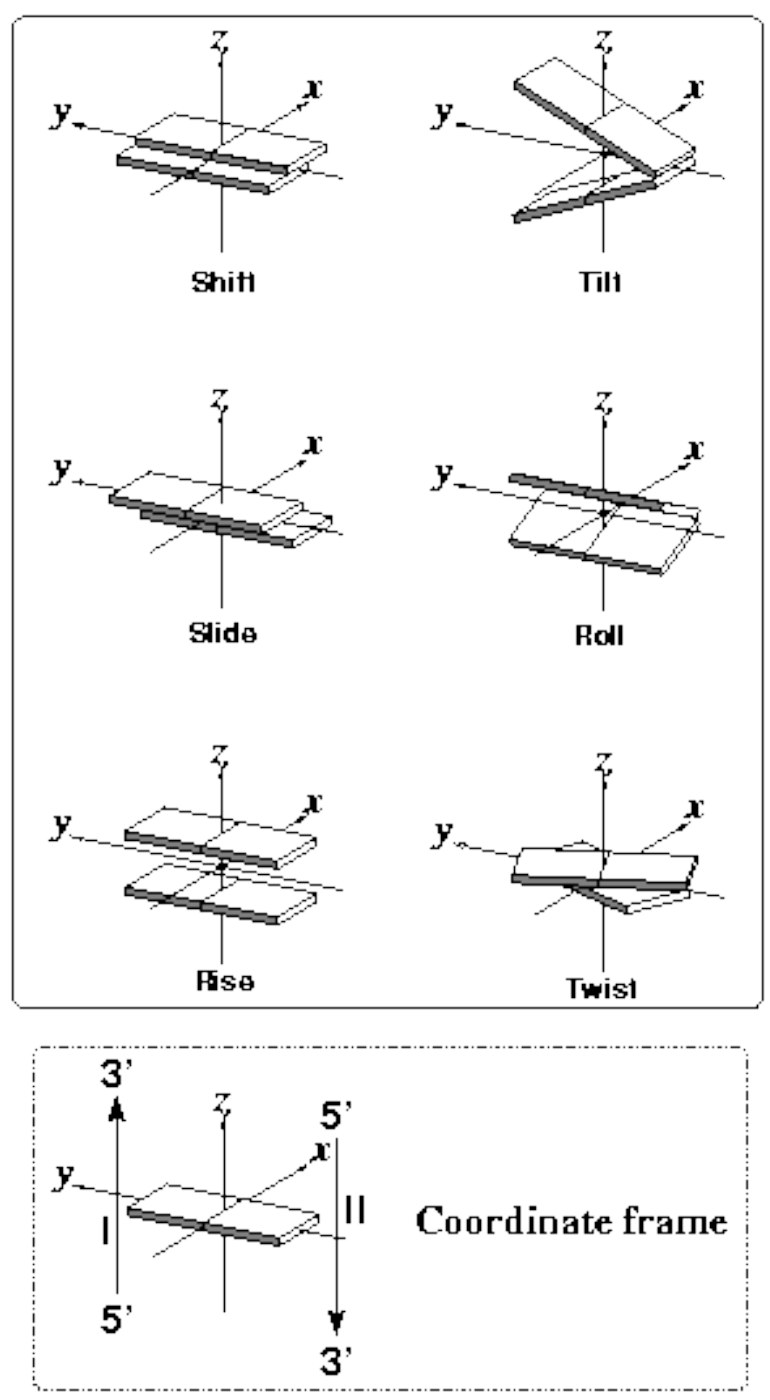

Images created with 3DNA illustrating positive values of designated parameters

Fig.1. 1. Illustration of parameters describing the geometry of DNA [4]. If a base or base pair is taken as a rigid block, six parameters are required to rigorously describe the position and orientation of one block relative to another. The six base-pair parameters describe the relative positions of the two bases in a pair. The six step parameters describe the local stacking geometry between neighbouring base pairs. Six helical parameters describe the position and orientation of a base pair with respect to the helical axis 
(helical rise and helical twist are not shown). The step and helical parameter sets are interrelated: from either set, the other can be deduced. The values of local vs helical rise and twist can be quite different in structures that deviate significantly from B-DNA. Figure reproduced from [5] with permission.

Table I. Diffusion constants (D) and residence times per base of various DNA-binding proteins on normal DNA duplexes.

\begin{tabular}{|c|c|c|c|}
\hline Protein & $\mathrm{D}\left(\mathrm{m}^{2} \mathrm{~s}^{-1}\right)$ & $\mathrm{D}\left(\mathrm{bp}^{2} \mathrm{~s}^{-1}\right)$ & Residence time $(\mu \mathrm{s})$ \\
\hline hUNG[6] & & $6 \times 10^{3}$ & 83.3 \\
\hline hOGG1[7] & $6 \times 10^{-13}$ & $5.2 \times 10^{6}$ & 0.1 \\
\hline MutM[7] & $5 \times 10^{-14}$ & $4.3 \times 10^{5}$ & 1.2 \\
\hline Msh2-Msh6[8] & $\begin{array}{l}9 \times 10^{-14} \\
-2 \times 10^{-16}\end{array}$ & $\begin{array}{l}7.8 \times 10^{5} \\
-1.7 \times 10^{3}\end{array}$ & $\begin{array}{l}0.6 \\
-289\end{array}$ \\
\hline LacI repressor[9] & $\begin{array}{l}1.3 \times 10^{-13} \\
-2.3 \times 10^{-16}\end{array}$ & $\begin{array}{l}1.1 \times 10^{6} \\
-2 \times 10^{3}\end{array}$ & $\begin{array}{l}0.4 \\
-251.3\end{array}$ \\
\hline $\begin{array}{l}\text { p53[10] } \\
\text { (range) }\end{array}$ & & $\begin{array}{l}2.6 \times 10^{6} \\
\left(1 \times 10^{7}-1 \times 10^{6}\right)\end{array}$ & $\begin{array}{l}0.2 \\
0.05-0.5\end{array}$ \\
\hline EcoRV[11] & $(1.2-0.9) \times 10^{-14}$ & $\begin{array}{l}1.0 \times 10^{5} \\
-7.8 \times 10^{4}\end{array}$ & $\begin{array}{l}4.8 \\
-6.4\end{array}$ \\
\hline Fpg[12] & $\begin{array}{l}1 \times 10^{-13} \\
-1 \times 10^{-15}\end{array}$ & $\begin{array}{l}8.7 \times 10^{5} \\
-8.7 \times 10^{3}\end{array}$ & $\begin{array}{l}0.6 \\
-57.8\end{array}$ \\
\hline
\end{tabular}




\section{References}

1. Rice, P.A. and C.C. Correll, Protein-Nucleic Acid Interactions. 2008, Cambridge: The Royal Society of Chemistry.

2. Chalikian, T.V., et al., The hydration of nucleic acid duplexes as assessed by a combination of volumetric and structural techniques. Biopolymers, 1999. 50(5): p. 45971.

3. Olson, W.K. and V.B. Zhurkin, Modeling DNA deformations. Curr. Opin. Struct. Biol., 2000. 10(3): p. 286-97.

4. Olson WK, B.M., Burley SK, Dickerson RE, Gerstein M, Harvey SC, Heinemann U, Lu XJ, Neidle S, Shakked Z, Sklenar H, Suzuki M, Tung CS, Westhof E, Wolberger C, Berman HM., A standard reference frame for the description of nucleic acid base-pair geometry. J Mol Biol, 2001. 313: p. 229-237.

5. Lu, X.J., Olson, W.K., 3DNA: a software package for the analysis, rebuilding and visualization of three-dimensional nucleic acid structures. Nucleic Acids Research, 2003. 31: p. 5108-5121.

6. Schonhoft, J.D. and J.T. Stivers, Timing facilitated site transfer of an enzyme on DNA. Nat Chem Biol, 2012. 8(2): p. 205-10.

7. Blainey, P.C., et al., A base-excision DNA-repair protein finds intrahelical lesion bases by fast sliding in contact with DNA. Proc Natl Acad Sci U S A, 2006. 103(15): p. 5752-7.

8. Gorman, J., et al., Dynamic basis for one-dimensional DNA scanning by the mismatch repair complex Msh2-Msh6. Mol Cell, 2007. 28(3): p. 359-70.

9. Wang, Y.M., R.H. Austin, and E.C. Cox, Single molecule measurements of repressor protein 1D diffusion on DNA. Phys. Rev. Lett., 2006. 97(4): p. 048302.

10. Tafvizi, A., et al., Tumor suppressor p53 slides on DNA with low friction and high stability. Biophys. J., 2008. 95(1): p. L01-3.

11. Bonnet, I., et al., Sliding and jumping of single EcoRV restriction enzymes on noncognate DNA. Nucleic Acids Res., 2008. 36(12): p. 4118-27.

12. Nelson, S.R., et al., Two glycosylase families diffusively scan DNA using a wedge residue to probe for and identify oxidatively damaged bases. Proc Natl Acad Sci U S A, 2014. 111(20): p. E2091-9. 


\section{Appendix (B)}

\subsection{Equilibrium perturbation using temperature jump (T-jump)}

Let us assume the simplest model of a two state equilibrium

$$
A \stackrel{K_{+}}{\stackrel{K_{-}}{\rightleftarrows} B}
$$

In the T-jump method the temperature dependence of the equilibrium constant is given according to the Van't Hoff equation

$$
\left(\frac{\partial \ln K}{\partial T}\right)_{p}=\frac{\Delta H}{R T^{2}}
$$

Where $\Delta H$ is the enthalpy different between two species in $\mathrm{kcal} / \mathrm{mole}, \mathrm{K}$ is the equilibrium constant and $\mathrm{R}$ is the gas constant.

Rearrange equation B1

$$
\partial \ln K=\frac{\partial K}{K}=\frac{\Delta H}{R T^{2}} \partial T
$$

For finite but small change in $\mathrm{K}(\Delta K \ll K)$

We can write

$$
\frac{\Delta K}{K}=\frac{\Delta H}{R T^{2}} \Delta T
$$

At $25^{\circ} \mathrm{C}$,

$$
\begin{aligned}
& \left(R T^{2}\right)^{-1}=5.67 * 10^{-6} \text { mole } \mathrm{cal}^{-1} \mathrm{deg}^{-2} \\
& \frac{\Delta \boldsymbol{K}}{\boldsymbol{K}}=\mathbf{5 . 6 7} * \mathbf{1 0}^{-\mathbf{6}} \Delta \boldsymbol{H} \Delta \boldsymbol{T}
\end{aligned}
$$


From this equation it is evident that the relative change in $\mathrm{K}$ for a given temperature jump $\Delta T$ is proportional to $\Delta H$. This explicitly tells that an enthalpy difference is required for there to be any measurable change in the chemical composition of equilibrium. It also suggests that sensitivity depends on entropy as well via the equilibrium constant.

If we assume $K \sim 1, \Delta T=15^{\circ} \mathrm{C}$ and there is only $4 \%$ change in $\mathrm{K}$, then we can observe any two states separated by as little as $0.5 \mathrm{kcal} . \mathrm{mole}^{-1}[1]$. It is worth noting here that the thermal energy at room temperature is $0.6 \mathrm{kcal} \cdot \mathrm{mole}^{-1}$.

\subsection{Temperature jump relaxation kinetics}

The relaxation kinetics measurement are based on rapid perturbation of the equilibrium concentrations of the reactants

For a two state reversible process,

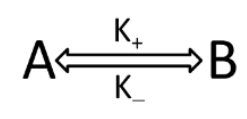

Since the deviations from equilibrium are typically relatively small, the relaxation process can be described by first - order, linear differential equations

$$
\Delta A(t)=\Delta A(0) e^{-K_{r e l} t}
$$

Where $\Delta A(t)=\left(A(t)-A_{e q}\right)$ is the deviation of the concentration of species A from equilibrium, Krel is the observed relaxation rate and is given by $K_{r e l}=K_{+}+K_{-}$and 
$\Delta A(t)=-\Delta B(t)$. The observed kinetic contains contributions from both the forward and backward reaction.

In order to obtain the forward and backwards rate constants it is necessary to determine the equilibrium constant for the reaction as well,

$$
K_{e q}=\frac{K_{+}}{K_{-}}
$$

We obtain the equilibrium constant by steady state measurements using fluorescence spectroscopy.

In general the reaction with $\mathrm{n}$ different states,

$$
A \stackrel{K 1_{+}}{\Longleftrightarrow} A 2 \stackrel{k 2_{+}}{\Longleftrightarrow} A 3 \stackrel{K 3_{+}}{\Longleftrightarrow} A 4 \stackrel{k 4_{+}}{\Longleftrightarrow} A 5 \stackrel{K 5_{+}}{\Longleftrightarrow} A 6 \stackrel{K 6_{+}}{\Longleftrightarrow} A 7
$$

will exhibit (n-1) relaxation rates, which are found as eigenvalues of the reaction rate matrix.

\section{Relaxation kinetics obtained from a master equation (referred from Ref [2])}

The transitions between the various microstates in the ensemble can be described in terms of a set of coupled differential equations,

$$
\frac{d p_{i}}{d t}=\sum_{j \neq i}\left[k_{j \rightarrow i} p_{j}-k_{i \rightarrow j} p_{i}\right]
$$

where $p_{i}\left(p_{j}\right)$ is the population of the $i_{\text {th }}\left(j_{t h}\right)$ microstate and $k_{j_{\rightarrow}}$ and $k_{i_{\rightarrow j}}$ are the rates for transitions from state $\mathrm{j}$ to state $\mathrm{i}$ and from state $\mathrm{i}$ to state $\mathrm{j}$, respectively.

We can rewrite the above equation in Matrix form, 


$$
\frac{d \mathbf{P}}{d t}=\mathbf{M} \cdot \mathbf{P}
$$

where $P$ is a column vector $\operatorname{col}\left(\mathrm{p}_{1}, \ldots, \mathrm{p}_{\Omega}\right)$, and $\mathrm{M}$ is a $\Omega \times \Omega$ rate matrix with $\mathrm{M}_{\mathrm{ij}}=\mathrm{k}_{\mathrm{j} \rightarrow \mathrm{l}}, \mathrm{i} \neq \mathrm{j}$ as the off-diagonal elements and $M_{i j}=\sum_{j \neq i} k_{i \rightarrow j}$ as the diagonal elements. The time - dependent solution of the rate equations yields the change in population as a function of time $\mathrm{P}(\mathrm{t})$, and is obtained by diagonalizing the matrix $\mathrm{M}$ to obtain its eigenvalues $\left(\lambda_{\mathrm{i}}\right)$ and eigenvectors $\left(\mathrm{U}_{\mathrm{i}}\right)$. The solution to Eq.8 can be written as:

$$
\mathbf{P}(t)=\exp (\mathbf{M} t) \mathbf{P}(0)=\mathbf{U} \exp (\lambda t) \mathbf{U}^{-1} \mathbf{P}(0)
$$

Where $\exp (\lambda t)$ is a $\Omega x \Omega$ is a diagonal matrix with $\exp \left(\lambda_{j} t\right)$ as the diagonal matrix elements, $\mathbf{U}$ is a matrix consisting of the eigenvectors, and $\mathbf{P}(0)$ is the column vector representing the populations of all microstates at $t=0$.

\subsection{Fluorescence resonance energy transfer (FRET)}

Fluorescence resonance energy transfer (FRET) is a photo physical process in which energy is transferred from a donor molecule (D) in an excited state to another molecule, the acceptor (A). The energy flows from donor to the acceptor by nonradiative long range ( 10-100 $\AA$ ) dipoledipole coupling process. FRET occurs between two appropriately chosen fluorophores attached

to a biomolecule only when the distance separating them is less than $\sim 100 \AA$. The FRET efficiency (E) of energy transfer between a donor-acceptor pair depends strongly on the relative distance and orientation of the donor/acceptor dipoles, making this technique extremely useful in probing time-dependent changes in the shape/conformation and separation of interacting biomolecules[3-6]. 

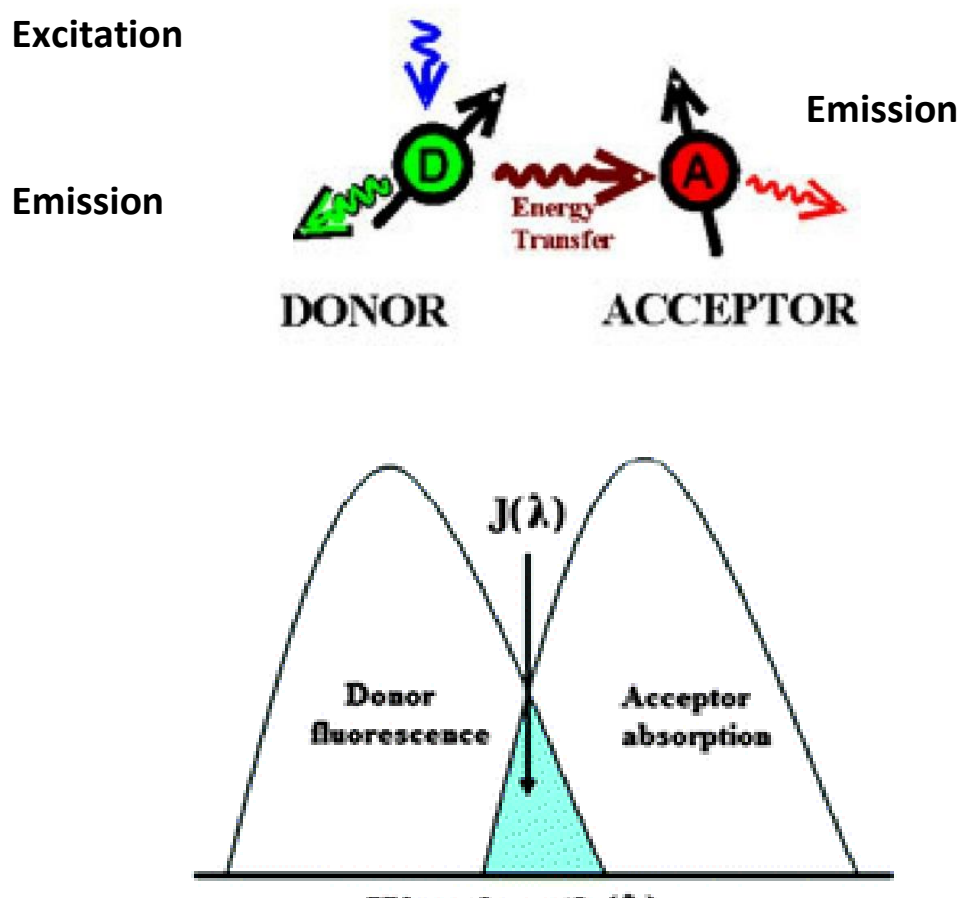

Wavelength $(\lambda)$

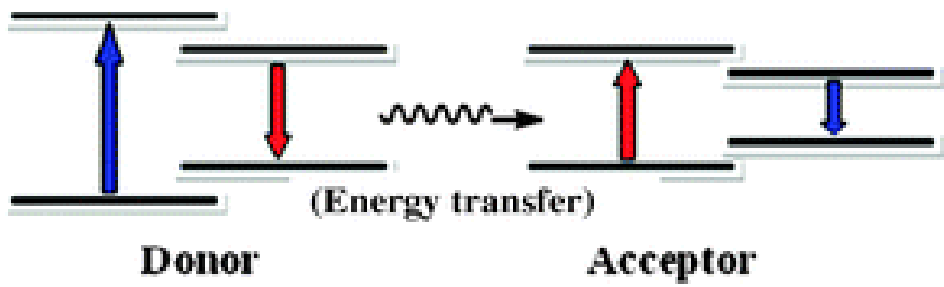

Fig.2. 1 Schematic representation of FRET process. FRET will occur only if the emission fluorescence spectrum of donor overlaps with excitation spectrum of the acceptor. The overlap integral is marked as $\mathrm{J}(\lambda)$ 


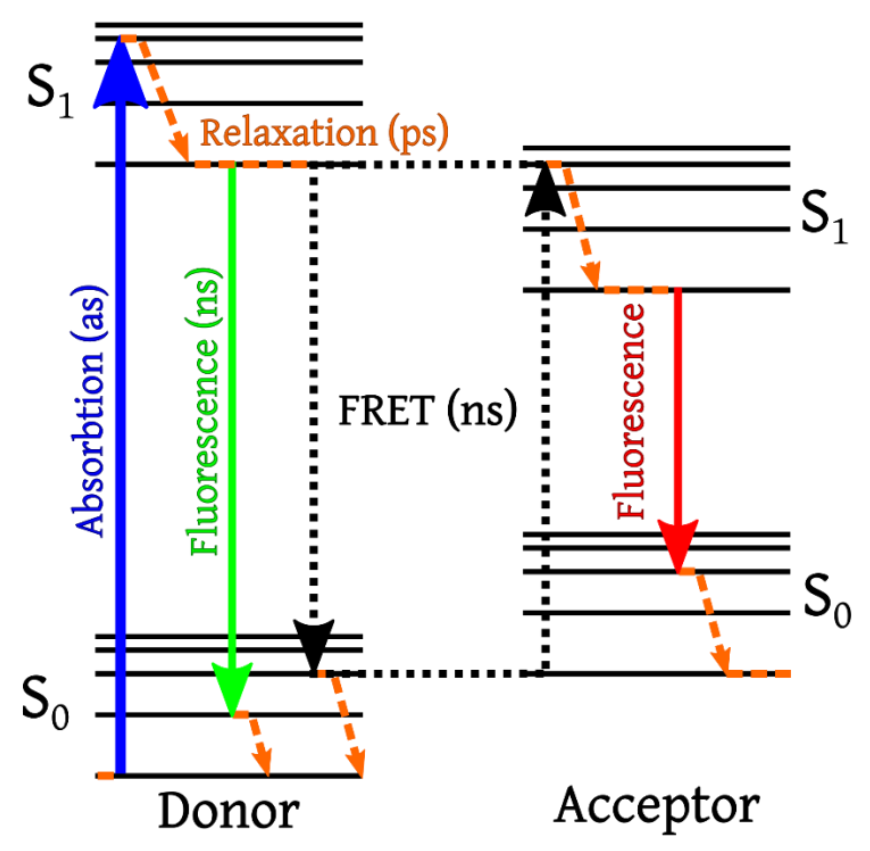

Fig.2. 2. Jablonski diagram for FRET energy transfer

The FRET efficiency (E) between an excited donor and a ground state acceptor strongly depends on the distance between fluorophores $\left(\mathrm{R}_{\mathrm{DA}}{ }^{-6}\right)$ and Foster critical distance $\left(\mathrm{R}_{\mathrm{o}}\right)$, which in turn depends on the quantum yield of the donor $\left(Q_{D}\right)$, the donor/acceptor spectral overlap integral $(J(\lambda))$, the refractive index of the medium $(n)$ and most importantly the geometric factor $(\kappa)$ which takes into consideration the orientation of donor and acceptor transition dipoles. This property of FRET makes it a very useful tool to monitor small changes in the distance between the donor-acceptor molecules.

$$
E=\frac{1}{\left(1+\left(\frac{R_{D A}}{R_{0}}\right)^{6}\right)}
$$

Where $\mathrm{R}_{\mathrm{o}}$ is the characteristic distance (Föster distance) where FRET efficiency is $50 \%, \mathrm{R}_{\mathrm{o}}$ can be calculated for any pair of fluorescence molecules. Figure 2.3 shows the FRET efficiency as a function of distance between fluorophores. 


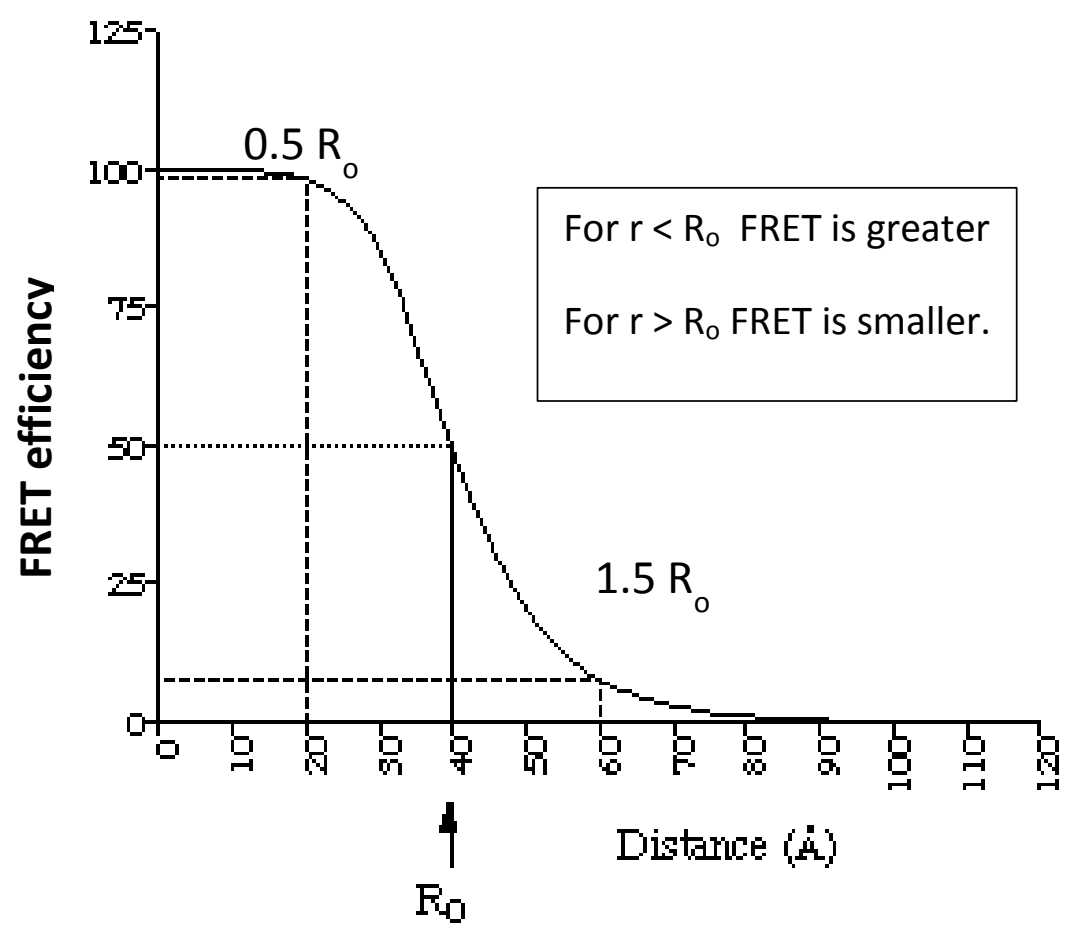

Fig.2.3. FRET efficiency versus distance. The FRET efficiency varies with sixth power of distance between donor and acceptor. As a results there are steep fall in FRET with increasing distance. The foster radius $\left(\mathrm{R}_{\mathrm{o}}\right)$ is the distance at which 50\% FRET occurs. Owing to the strong distance dependence, FRET is usually detected only when the two fluorophore are closer than $1.5 \mathrm{R}_{\mathrm{o}}$. This distance dependence curve is valid only when $\kappa$ is assumed to be a constant. i.e, the relative orientation of the dipoles is fixed or the two dipoles are freely rotating.

The value of $\mathrm{R}_{\mathrm{o}}$ for any pair of fluorophore in aqueous medium is determined by $[9,10]$.

$$
R_{o}=\left[2.8 * 10^{17} * \kappa^{2} * Q_{D} * \varepsilon_{A} * J(\lambda)\right]^{\frac{1}{6}} n m
$$

Where $k^{2}$, the Geometric factor, takes the direction of the donor and acceptor transition dipoles into consideration and is described by $\kappa=e_{1} \cdot e_{2}-3\left(e_{1} \cdot e_{12}\right)\left(e_{12} \cdot e_{2}\right)$ (where $e_{1}$ and $e_{2}$ are the unit vectors of the donor and acceptor transition dipoles and $e_{12}$ is the unit vectors between their canters). $\kappa^{2}$ can vary between 0 and 4 . If the donor and acceptor are parallel $k^{2}$ will be larger 
and if they are perpendicular $\kappa^{2}$ will be smaller. Usually $\kappa^{2}$ is assumed to be $2 / 3$, which is the average value integrated over all possible angles $[9,10], Q_{D}$ is the donor quantum yield, $\varepsilon_{A}$ is the maximal acceptor extinction coefficient in $\mathrm{Mol}^{-1} \mathrm{~cm}^{-1}, J(\lambda)$ is the spectral overlap integral between the normalized donor fluorescence $F_{D}(\lambda)$ and the acceptor excitation spectra $E_{A}(\lambda)$ and it is given by,

$$
J(\lambda)=\int F_{D}(\lambda) * E_{A}(\lambda) * \lambda^{4} d \lambda
$$

The range of possible $R_{o}$ value is limited to $4-6 \mathrm{~nm}[7,8]$. For most FRET pairs $R_{o}=5 \mathrm{~nm}$. $\kappa^{2}=2 / 3$ only if the donor and acceptor dipoles are freely rotating. Therefore when monitoring conformational changes or interaction processes in nucleic acid containing systems using FRET, the most common method is to covalently attach donor and acceptor molecules via flexible linkers to two different positions and to assume that $\kappa^{2}=2 / 3$ [9].

\section{FRET efficiency of $\mathrm{tC}^{\mathrm{O}}-\mathrm{tC}_{\text {nitro }}$}

The recently developed cysteine base analog $\mathrm{tC}^{\mathrm{O}}, \mathrm{tC}_{\text {nitro }}$ are rigidly stacked within the duplex DNA helix and consequently it gives excellent control of their exact position and orientations. When the FRET is measured as a function of varying base separation, this pair gives a very distinct distance dependent FRET changes compare to the traditional fluorophores, the FRET varies with the distance in a step wise fashion. This is because as the distance changes the orientation of the dyes are also changing due to DNA helicity and we no longer can assume that $\kappa$ is a constant. Fig. 2.4 shows FRET versus distance for $\mathrm{tC}^{\mathrm{O}}, \mathrm{tC}_{\text {nitro }}$ pair measured for 12 different bp separations. The data is fitted using eq 6 and 7 with phase angle $\alpha$, and overlap 
integral $J_{D A}$ as fit parameters. The phase angle is defined as the angle between the transition dipole moments of the donor on one strand and the acceptor on the other, looking along the DNA helix long-axis and when there are no bases separating the donor and the acceptor (i.e. the acceptor is the neighboring base of the guanine that base-pairs with the donor). Considering how the donor and acceptor are oriented in the helix, the phase angle can be translated to an angle describing the difference in the orientation of the transition dipole moments of the donor and acceptor within their three-ring systems [9].

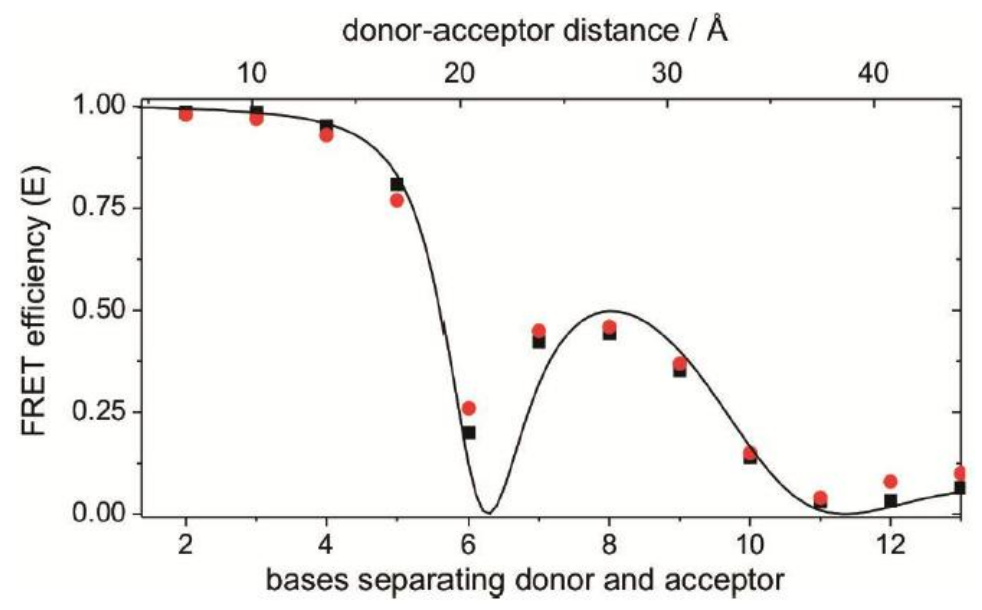

Fig.2.4. Efficiency of energy transfer for the base analog FRET-pair $\mathrm{tC}^{\mathrm{o}}-\mathrm{tC}_{\text {niro }}$ estimated using decreases in $\mathrm{tC}^{\circ}$, donor, emission (red circles) and $\mathrm{tC}^{\circ}$ average emission lifetimes (black squares) as the two analogs are separated by 2 to 13 bases in a DNA duplex. Curve fitting using equation 13 and 14 with $\alpha$ and $J_{D A}$ as fit parameters is shown as solid line. Excitation wavelength $370 \mathrm{~nm}$.

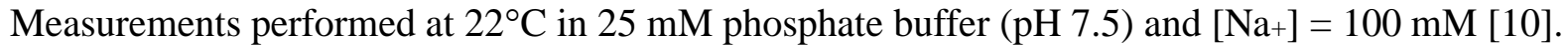


For these FRET pairs the orientation factor $\kappa$ is given by $[9,11]$,

$$
\kappa_{D A}=\cos \left(n_{D A} \beta+\alpha\right)-3\left(\frac{a \bullet \sin \left(n_{D A} \beta+\alpha\right)}{R_{D A}}\right)^{2}
$$

where $n_{D A}$ is the number of base pairs in between the donor and acceptor, $a$ is the distance between the center of the DNA helix to the centre of the chromophore (4 $\AA$ ), $R_{D A}$ is the donor acceptor distance and it is given by eq $14[9,11], \alpha$ is a fitted phase angle, and $\beta$ is the helical rise angle $\left(34.3^{\circ} / \mathrm{base}\right.$ pair).

$$
R_{D A}=\sqrt{2 a^{2}\left(1-\cos \left(n_{D A} \beta+\alpha\right)\right)+\left(b\left(n_{D A}+1\right)\right)^{2}}
$$

where $b$ is the helical rise (3.4 $\AA$ /base pair). 


\section{References}

1. Kubelka, J., Time-resolved methods in biophysics. 9. Laser temperature-jump methods for investigating biomolecular dynamics. Photochem. Photobiol. Sci., 2009. 8(4): p. 499-512.

2. Kuznetsov, S.V. and A. Ansari, A kinetic zipper model with intrachain interactions applied to nucleic acid hairpin folding kinetics. Biophys. J., 2012. 102: p. 101-111.

3. Parkhurst, L.J., et al., Time-resolved fluorescence resonance energy transfer studies of DNA bending in double-stranded oligonucleotides and in DNA-protein complexes. Biopolymers, 2001. 61(3): p. 180-200.

4. Hillisch, A., M. Lorenz, and S. Diekmann, Recent advances in FRET: distance determination in protein-DNA complexes. Curr. Opin. Struct. Biol., 2001. 11(2): p. 201-207.

5. Rasnik, I., S.A. McKinney, and T. Ha, Surfaces and orientations: much to FRET about? Acc Chem Res, 2005. 38(7): p. 542-8.

6. Preus, S. and L.M. Wilhelmsson, Advances in quantitative FRET-based methods for studying nucleic acids. Chembiochem, 2012. 13(14): p. 1990-2001.

7. Patterson, G.H.e.a., forster distances between green fluorescent protein pairs. Anal. Biochem. , 2000. 284: p. 438-440.

8. Wu, P., and Brand, L., Resonance energy transfer methods and applications. Anal.Biochem., 1994. 218: p. 1-13.

9. Borjesson, K., et al., Nucleic acid base analog FRET-pair facilitating detailed structural measurements in nucleic acid containing systems. J Am Chem Soc, 2009. 131(12): p. 4288-93.

10. Borjesson, K., et al., Nucleic acid base analog FRET-pair facilitating detailed structural measurements in nucleic acid containing systems. Chem Soc, 2009. 131 SRC - GoogleScholar: p. 4288-4293.

11. Carlsson, C., Larsson, A., Bjorkman, M., Jonsson, M., Albinsson, B.,, Biopolymers, 1997. 41: p. 481-494. 


\section{YOGAMBIGAI VELMURUGU (YOGA)}

SES 1612, Department of Physics, University of Illinois at Chicago $845 \mathrm{~W}$ Taylor Street, M/C273

Chicago, IL 60607
Phone: 312-996-5555(Lab)

312-413-8224(Off)

Email:VYOGAM2@UIC.EDU

\section{EDUCATION}

Ph.D. Physics, University of Illinois at Chicago, expected January, 2016

Thesis: Dynamics and mechanism of DNA- bending proteins in binding site recognition.

M.S. Physics, University of Illinois at Chicago, 2007

M.Phil (Physics) Department of Physics, University of Peradeniya, Sri Lanka / Department of Chemistry, Denmark Technical University, Lyngby, Denmark, 2005.

Thesis: Electrochemical and electromechanical behavior of Polypyrrole films

BSc (Physics Honors) University of Jaffna, Sri Lanka 1999

\section{RESEARCH EXPERIENCE}

- Research Assistant (2007-present) Department of Physics, University of Illinois, Chicago Adviser: Prof. Anjum Ansari

Protein-DNA interaction dynamics, RNA pseudoknot kinetics, DNA hairpin kinetics

- Research Assistant (2001-2005) Department of Physics, University of Peradeniya, SriLanka/ Department of Chemistry, DTU, Denmark

Advicers: Prof. M.A.Careem, Prof. Steen Skaarup

Electronically conduction polymers

- Research Assistant (part-time) (2001-2005), Post Graduate institute(PGIS), University of Peradeniya, Sri Lanka

Demonstrating advanced conducting polymer research techniques to graduate students

\section{TEACHING EXPERIENCE}

- Teaching Assistant (2000-2001) Department of Physics, University of Jaffna, Sri Lanka (conducting discussion classes for undergraduates to assist in problem solving, conducting undergraduate experiments, tutoring, and grading homework and exams).

- Teaching Assistant (2005-present) Department of Physics, University of Illinois, Chicago (Conducting laboratory experiments and discussion sections in physics for undergraduates)

\section{AWARDS/HONORS}

- Chancellor's Graduate Research Fellowship, UIC (2013)

- James Kouvel Fellowship, UIC (2013)

- Chancellor's Graduate Research Fellowship, UIC (2012)

- Provost Award, UIC (2012)

- Student Travel Grant from WISE, UIC (2010)

- International Science Program (ISP) Fellowship, UPPSALA University, Sweden to conduct polymer research at Denmark Technical University, Denmark (2002-2003). 
- NSF grant, Sri Lanka to carry out electronically conducting polymer research at university of Peradeniya, Sri Lanka, 2001-2005.

- The Best Research Presenter Award in the annual research sessions conducted by the University of Peradeniya, Sri Lanka (two-time recipient).

- Marhapola scholarship (1995-1999) for merit score in university entrance exam, Sri Lanka to study under graduate program.

\section{PUBLICATIONS}

*Co first author

1. X.Chen*, Y. Velmurugu* G. Zheng, B.S Park, Y. Shim, Y. Kim, L. Liu, B. V Houten, C. $\mathrm{He}$, A. Ansari, J.Min, "Kinetic gating mechanism of DNA damage recognition by Rad4/XPC", Nature Communications, 6, 5849, 2015.

Highlighted in several science news websites such as:

- Science 360, the NSF News site (http://news.uic.edu/damaged-dna-may-stallpatrolling-molecule-to-initiate-repair).

- SciGuru Science News (http://www.sciguru.org/newsitem/18318/damaged-dnamay-stall-patrolling-molecule-initiate-repair)

- Science Daily (http://www.sciencedaily.com/releases/2015/01/150128141423.htm)

- Phys Org (http://phys.org/news/2015-01-dna-stall-patrolling-molecule.html)

- Biology News Net (http://www.biologynews.net/link-entry.php)

- UIC News (http://news.uic.edu/taking-a-closer-look-at-damaged-dna)

2. $\underline{Y}$ Velmurugu* ${ }^{*}$ X. Chen*, A.Patel, P.Slogoff-Sevilla, J.Min, and A.Ansari "Twist-open mechanism of DNA damage recognition by Rad4/XPC nucleotide excision repair complex" (under PNAS review).

3. Y. Velmurugu, P. Vivas, S. V. Kuznetsov, P. A. Rice, A. Ansari, "Search mechanism of DNA binding site by an architectural DNA-bending protein IHF" (in preparation).

4. Paula Vivas, Y. Velmurugu, Serguei V. Kuznetsov, Phoebe A. Rice, Anjum Ansari, "Global analysis of ion dependence unveils hidden steps in DNA binding and bending by integration host factor", J. Phy. Chem.139, 1219-1227, 2013.

5. R. Narayanan, L. Zhu, Y. Velmurugu, J. Roca, S. V. Kuznetsov, G. Prehna, L. Lapidus, A. Ansari, "Exploring the energy landscape of nucleic acid hairpins using laser temperature-jump and microfluidic mixing", J. Am. Chem. Soc. 134, 18952-63, 2012.

6. P. Vivas*, Y. Velmuruqu${ }^{*}$, S. V. Kuznetsov, P. A. Rice, A. Ansari, "Mapping the Transition State in DNA Bending by IHF", J.Mol.Biol, 418, 300-15, 2012

7. R. Narayanan, Y. Velmurugu, S. V. Kuznetsov, A. Ansari, "Fast folding of RNA pseudoknots initiated by laser temperature-jump", J. Am. Chem. Soc. 133, 18767-74, 2011.

8. M. A. Careem, Y. Velmurugu, S. Skaarup, K. West, "A voltammetry study on the diffusion of counter ions in polypyrrole films", Journal of Power Sources, 159, 210-214, 2006.

9. Y. Velmurugu, S. Skaarup "Ion and solvent Transport in Polypyrrole: Experimental Test of Osmotic Model", Ionics 11, 370-374, 2005.

10. Y. Velmurugu, M. A. Careem, S. Skaarup, K. West, "Study of lon Movement in polypyrrole Films Using Electrochemical Quartz Microbalance Measurements", The Science and Technology of lons in Motion, 1073-1080, 2004. 
11. L. West, M. M. Bay, M. Nielsen, Y. Velmurugu, S. Skaarup, "Electronic Conductivity of Polypyrrole - Dodecyl Benzene Sulfonate Complexes", Phys. Chem. B, 108, 1500115008, 2004.

\section{CONFERENCES}

1. Y. Velmurugu, X. Chen, A. Patel, P. Slogoff-Sevilla, J. Min, and A. Ansari, Twist-open mechanism of DNA damage recognition by Rad4/XPC nucleotide excision repair complex, Poster presentation, BPS annual meeting, Feb 2015, Baltimore, Maryland, USA

2. J. Roca, Y. Velmurugu, R. Narayanan, P. Narayanan, S. Kouznetsov, A. Ansari, RNA Pseudoknot Folding Energy Landscape Elucidated with T-Jump Measurements and Kinetic Modeling, Poster presentation, BPS annual meeting, Feb 2015, Baltimore, Maryland, USA.

3. Y. Velmurugu, X. Chen, J. Min, A. Ansari, DNA damage recognition by nucleotide excision repair protein XPC, Poster presentation, The International Year of Crystallography 2014 (IYCr2014) Symposium, Oct. 2014, Chicago, Illinois, USA.

4. Y. Velmurugu, X. Chen, J. Min, A. Ansari, Dynamics of DNA damage recognition by nucleotide excision repair protein XPC, Poster presentation, BPS Annual session, Feb. 2014, San Francisco, California, USA.

5. Y. Velmurugu, R. Narayanan, J. Roca, J. Liu, S. V. Kuznetsov, A. Ansari, Laser T-jump studies of RNA pseudoknots reveal parallel folding/unfolding pathways, Poster presentation, Gordon research conference, Biopolymers, June 2012, Newport, Rhode Island, USA

6. Y. Velmurugu, X. Chen, C.Hall, J.Min, and A. Ansari, DNA-bending and base-flipping dynamics during lesion recognition by XPC, Poster presentation, Gordon research conference, Biopolymers, June 2012, Newport, Rhode Island, USA

7. R. Narayanan, Y. Velmurugu, S.V. Kuznetsov, A. Ansari, Tying RNA up in pseudoknots, Poster presentation, ZING conference on Nucleic Acids, November, 2010, Cancun, Mexico.

8. Y. Velmurugu, R. Narayanan, S.V. Kuznetsov, M. Hingorani, A. Ansari, Finding the right 'Mis'match : Millisecond conformational dynamics of MutS-DNA complex during DNA damage recognition, Poster presentation, BPS Annual session, Feb. 2010, San Francisco, California, USA.

9. P.Vivas, Y. Velmurugu , S. V. Kuznetsov, P. A. Rice, A. Ansari, Dynamics of DNAbending in binding site recognition by IHF, Poster presentation, BPS Annual session ,Feb. 2010, San Francisco, California, USA.

10. P. Vivas, Y.Velmurugu, S. V. Kuznetsov, A. Ansari, What is the role of DNA "bendability" in the indirect read-out mechanism of protein-DNA interactions? , Poster presentation, BPS Annual session, March. 2009, Boston, USA

11. M.A. Careem, Y. Velmurugu, S.Skaarup, K. West, A voltammetry study on the diffusion of counter ions in polypyrrole films, Oral presentation, International conference on materials for advanced technologies (ICMAT), July 2005, Singapore

12. Y. Velmurugu, S.Skaarup, Ion and Solvent Transport in Polypyrrole: Experimental Test of Osmotic Model, Oral presentation, International Conference on Functional Materials and Devices, June 2005, Kuala Lumpur, Malaysia.

13. Y. Velmurugu, S. Skaarup, M.A. Careem, "Reproducibility of the Electrochemical Doping Process of Polypyrrole / Dodecylbenzenesulfonate", Oral presentation, annual research sessions of SLAAS, December 2004, Sri Lanka. 
14. Y. Velmurugu, R.L.N. Chandrakanthi, M.A.Careem, S. Skaarup, "A Study of redox mechanism of Polypyrrole / Dodecylbenzenesulfonate polymer films using Optical bsorption and cyclic voltammetry technique", Oral presentation , annual research sessions, University of Peradeniya, October 2004, Sri Lanka.

15. Y. Velmurugu, S. Skaarup, M.A.Careem, "Effective Diffusion Coefficient of lons in Polypyrrole Films", Oral presentation, annual research sessions, University of Peradeniya, October 2003, Sri Lanka.

16. Y. Velmurugu, R.L.N.Chandrakanthi, M.A.Careem, "Electrochemically Synthesized Conducting Polyaninline for Electronic and Optical Applications", Oral presentation, annual research session, University of Peradeniya, October 2001, Sri Lanka. 


\section{Copyright}

Clearance Center

New Insights into the Transition Pathway from Nonspecific to Specific Complex of DNA with Escherichia Coli Integration Host Factor†

Author: $\quad$ Paula Vivas, Serguei V. Kuznetsov, Anjum Ansari

Publication: The Journal of Physical Chemistry B

Publisher: American Chemical Society

Date: $\quad$ May 1, 2008

Copyright @ 2008, American Chemical Society

\section{PERMISSION/LICENSE IS GRANTED FOR YOUR ORDER AT NO CHARGE}

This type of permission/license, instead of the standard Terms \& Conditions, is sent to you because no fee is being charged for your order. Please note the following:

- Permission is granted for your request in both print and electronic formats, and translations.

- If figures and/or tables were requested, they may be adapted or used in part.

- Please print this page for your records and send a copy of it to your publisher/graduate school.

- Appropriate credit for the requested material should be given as follows: "Reprinted (adapted) with permission from (COMPLETE REFERENCE CITATION). Copyright (YEAR) American Chemical Society." Insert appropriate information in place of the capitalized words.

- One-time permission is granted only for the use specified in your request. No additional uses are granted (such as derivative works or other editions). For any other uses, please submit a new request.

If credit is given to another source for the material you requested, permission must be obtained from that source.

\section{BACK}

CLOSE WINDOW

Copyright ( 92015 Copyright Clearance Center, Inc. All Rights Reserved. Privacy statement. Terms and Conditions.

Comments? We would like to hear from you. E-mail us at customercare@ copyright.com 



\section{Copyright}

Clearance Center

Title:

Author:

Nucleic Acid Base Analog

FRET-Pair Facilitating

Detailed Structural

Measurements in Nucleic Acid

Containing Systems

Karl Börjesson, Søren Preus, Afaf H. El-Sagheer, et al

Publication: Journal of the American Chemical Society

Publisher: American Chemical Society

Date: $\quad$ Apr 1, 2009

Copyright (C) 2009, American Chemical

Society
Logged in as:

Velmurugu

Yogambigai

Account \#:

3000985545

LOGOUT

\section{PERMISSION/LICENSE IS GRANTED FOR YOUR ORDER AT NO CHARGE}

This type of permission/license, instead of the standard Terms \& Conditions, is sent to you because no fee is being charged for your order. Please note the following:

- Permission is granted for your request in both print and electronic formats, and translations.

- If figures and/or tables were requested, they may be adapted or used in part.

- Please print this page for your records and send a copy of it to your publisher/graduate school.

- Appropriate credit for the requested material should be given as follows: "Reprinted (adapted) with permission from (COMPLETE REFERENCE CITATION). Copyright (YEAR) American Chemical Society." Insert appropriate information in place of the capitalized words.

- One-time permission is granted only for the use specified in your request. No additional uses are granted (such as derivative works or other editions). For any other uses, please submit a new request.

If credit is given to another source for the material you requested, permission must be obtained from that source.

\section{BACK}

CLOSE WINDOW

Copyright (C) 2015 Copyright Clearance Center, Inc. All Rights Reserved. Privacy statement. Terms and Conditions.

Comments? We would like to hear from you. E-mail us at customercare@ copyright.com 



$\begin{array}{lll}\text { Title: } & \begin{array}{l}\text { Base Flipping Free Energy } \\ \text { Profiles for Damaged and } \\ \text { Undamaged DNA }\end{array} & \begin{array}{l}\text { Logged in as: } \\ \text { Velmurugu Yogambigai } \\ \text { Account \#: }\end{array} \\ \begin{array}{ll}\text { Han Zheng, Yuqin Cai, Shuang } \\ \text { Ding, et al }\end{array} & 3000985545 \\ \text { Author: } & \\ \text { Publication: } & \text { Chemical Research in Toxicology } & \\ \begin{array}{l}\text { Publisher: } \quad \text { American Chemical Society } \\ \text { Date: }\end{array} & \text { Dec } 1,2010 \\ \text { Copyright } \odot \text { 2010, American Chemical Society } & \end{array}$

\section{PERMISSION/LICENSE IS GRANTED FOR YOUR ORDER AT NO CHARGE}

This type of permission/license, instead of the standard Terms \& Conditions, is sent to you because no fee is being charged for your order. Please note the following:

- Permission is granted for your request in both print and electronic formats, and translations.

- If figures and/or tables were requested, they may be adapted or used in part.

- Please print this page for your records and send a copy of it to your publisher/graduate school.

- Appropriate credit for the requested material should be given as follows: "Reprinted (adapted) with permission from (COMPLETE REFERENCE CITATION). Copyright (YEAR) American Chemical Society." Insert appropriate information in place of the capitalized words.

- One-time permission is granted only for the use specified in your request. No additional uses are granted (such as derivative works or other editions). For any other uses, please submit a new request.

If credit is given to another source for the material you requested, permission must be obtained from that source. 


\section{Copyright}

Clearance Center

New Insights into the Transition Pathway from Nonspecific to Specific Complex of DNA with Escherichia Coli Integration Host Factor†

Author: $\quad$ Paula Vivas, Serguei V. Kuznetsov, Anjum Ansari

Publication: The Journal of Physical Chemistry B

Publisher: American Chemical Society

Date: $\quad$ May 1, 2008

Copyright @ 2008, American Chemical Society

\section{PERMISSION/LICENSE IS GRANTED FOR YOUR ORDER AT NO CHARGE}

This type of permission/license, instead of the standard Terms \& Conditions, is sent to you because no fee is being charged for your order. Please note the following:

- Permission is granted for your request in both print and electronic formats, and translations.

- If figures and/or tables were requested, they may be adapted or used in part.

- Please print this page for your records and send a copy of it to your publisher/graduate school.

- Appropriate credit for the requested material should be given as follows: "Reprinted (adapted) with permission from (COMPLETE REFERENCE CITATION). Copyright (YEAR) American Chemical Society." Insert appropriate information in place of the capitalized words.

- One-time permission is granted only for the use specified in your request. No additional uses are granted (such as derivative works or other editions). For any other uses, please submit a new request.

If credit is given to another source for the material you requested, permission must be obtained from that source.

\section{BACK}

CLOSE WINDOW

Copyright ( 92015 Copyright Clearance Center, Inc. All Rights Reserved. Privacy statement. Terms and Conditions.

Comments? We would like to hear from you. E-mail us at customercare@ copyright.com 



\section{Copyright}

Clearance Center

Title:

Author:

Nucleic Acid Base Analog

FRET-Pair Facilitating

Detailed Structural

Measurements in Nucleic Acid

Containing Systems

Karl Börjesson, Søren Preus, Afaf H. El-Sagheer, et al

Publication: Journal of the American Chemical Society

Publisher: American Chemical Society

Date: $\quad$ Apr 1, 2009

Copyright (C) 2009, American Chemical

Society
Logged in as:

Velmurugu

Yogambigai

Account \#:

3000985545

LOGOUT

\section{PERMISSION/LICENSE IS GRANTED FOR YOUR ORDER AT NO CHARGE}

This type of permission/license, instead of the standard Terms \& Conditions, is sent to you because no fee is being charged for your order. Please note the following:

- Permission is granted for your request in both print and electronic formats, and translations.

- If figures and/or tables were requested, they may be adapted or used in part.

- Please print this page for your records and send a copy of it to your publisher/graduate school.

- Appropriate credit for the requested material should be given as follows: "Reprinted (adapted) with permission from (COMPLETE REFERENCE CITATION). Copyright (YEAR) American Chemical Society." Insert appropriate information in place of the capitalized words.

- One-time permission is granted only for the use specified in your request. No additional uses are granted (such as derivative works or other editions). For any other uses, please submit a new request.

If credit is given to another source for the material you requested, permission must be obtained from that source.

\section{BACK}

CLOSE WINDOW

Copyright (C) 2015 Copyright Clearance Center, Inc. All Rights Reserved. Privacy statement. Terms and Conditions.

Comments? We would like to hear from you. E-mail us at customercare@ copyright.com 



\section{Copyright Clearance Center \\ Logged in as: \\ Dynamics and Mechanism of DNA-Bending Proteins in Binding Site Recognition \\ the language of science \\ Title: \\ Author: Anjum Ansari
Publication: Springer eBook \\ Publisher: Springer \\ Date: Jan 1, 2010 \\ Copyright @ 2010, Springer Science+Business Media, LLC \\ Velmurugu Yogambigai \\ Account \#: 3000985545 \\ LOGOUT}

\section{Order Completed}

Thank you very much for your order.

This is a License Agreement between Velmurugu Yogambigai ("You") and Springer ("Springer"). The license consists of your order details, the terms and conditions provided by Springer, and thepayment terms and conditions.

\section{Get the printable license.}

\begin{tabular}{ll} 
License Number & 3825570506030 \\
\hline License date & Mar 10, 2016 \\
\hline Licensed content publisher & Springer \\
\hline Licensed content publication & Springer eBook \\
\hline Licensed content title & Dynamics and Mechanism of DNA-Bending Proteins in Binding Site Recognition \\
\hline Licensed content author & Anjum Ansari \\
\hline Licensed content date & Jan 1, 2010 \\
\hline Type of Use & Thesis/Dissertation \\
\hline Portion & Figures/tables/illustrations \\
\hline $\begin{array}{l}\text { Number of } \\
\text { figures/tables/illustrations }\end{array}$ & 1 \\
\hline Author of this Springer article & No \\
\hline Original figure numbers & Figure 6.1 \\
\hline $\begin{array}{l}\text { Title of your thesis / } \\
\text { dissertation }\end{array}$ & Dynamics and mechanism of DNA -bending proteins in binding site recognition \\
\hline Expected completion date & Jan 2016 \\
\hline Estimated size(pages) & 150 \\
\hline Total & 0.00 USD \\
\hline
\end{tabular}

\section{CLOSE WINDOW}

Copyright (c) 2016 Copyright Clearance Center, Inc. All Rights Reserved. Privacy statement. Terms and Conditions. Comments? We would like to hear from you. E-mail us at customercare@copyright.com 


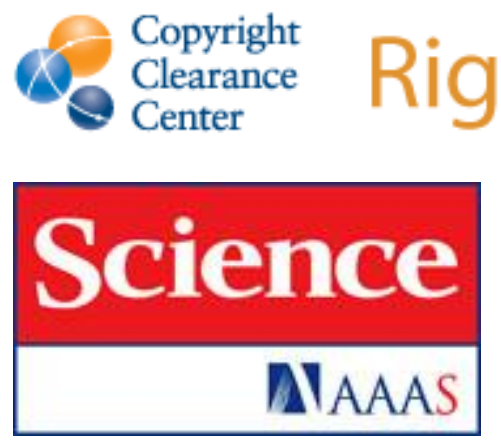

Title:

Structure and Flexibility

Logged in as:

Adaptation in Nonspecific and Specific Protein-DNA Complexes

Author: Charalampos G.

Kalodimos,Nikolaos

Biris,Alexandre M. J. J.

Bonvin,Marc M.

Levandoski,Marc

Guennuegues,Rolf

Boelens, Robert Kaptein

Publication: Science

Publisher: The American Association for the Advancement of Science

Date: Jul 16, 2004

Copyright @ 2004, The American

Association for the Advancement of

Science
Velmurugu

Yogambigai

Account \#:

3000985545

LOGOUT

Order Completed

Thank you very much for your order.

This is a License Agreement between Velmurugu Yogambigai ("You") and The American Association for the Advancement of Science ("The American Association for the Advancement of Science"). The license consists of your order details, the terms and conditions provided by The American Association for the Advancement of Science, and the payment terms and conditions.

Get the printable license.

License Number

License date

Licensed content publisher

Licensed content publication

Licensed content title

Licensed content author
3825990353852

Mar 11, 2016

The American Association for the Advancement of Science

Science

Structure and Flexibility Adaptation in Nonspecific and Specific Protein-DNA Complexes

Charalampos G. Kalodimos,Nikolaos Biris,Alexandre M. J. J. Bonvin,Marc M. Levandoski,Marc Guennuegues, Rolf Boelens, Robert Kaptein 


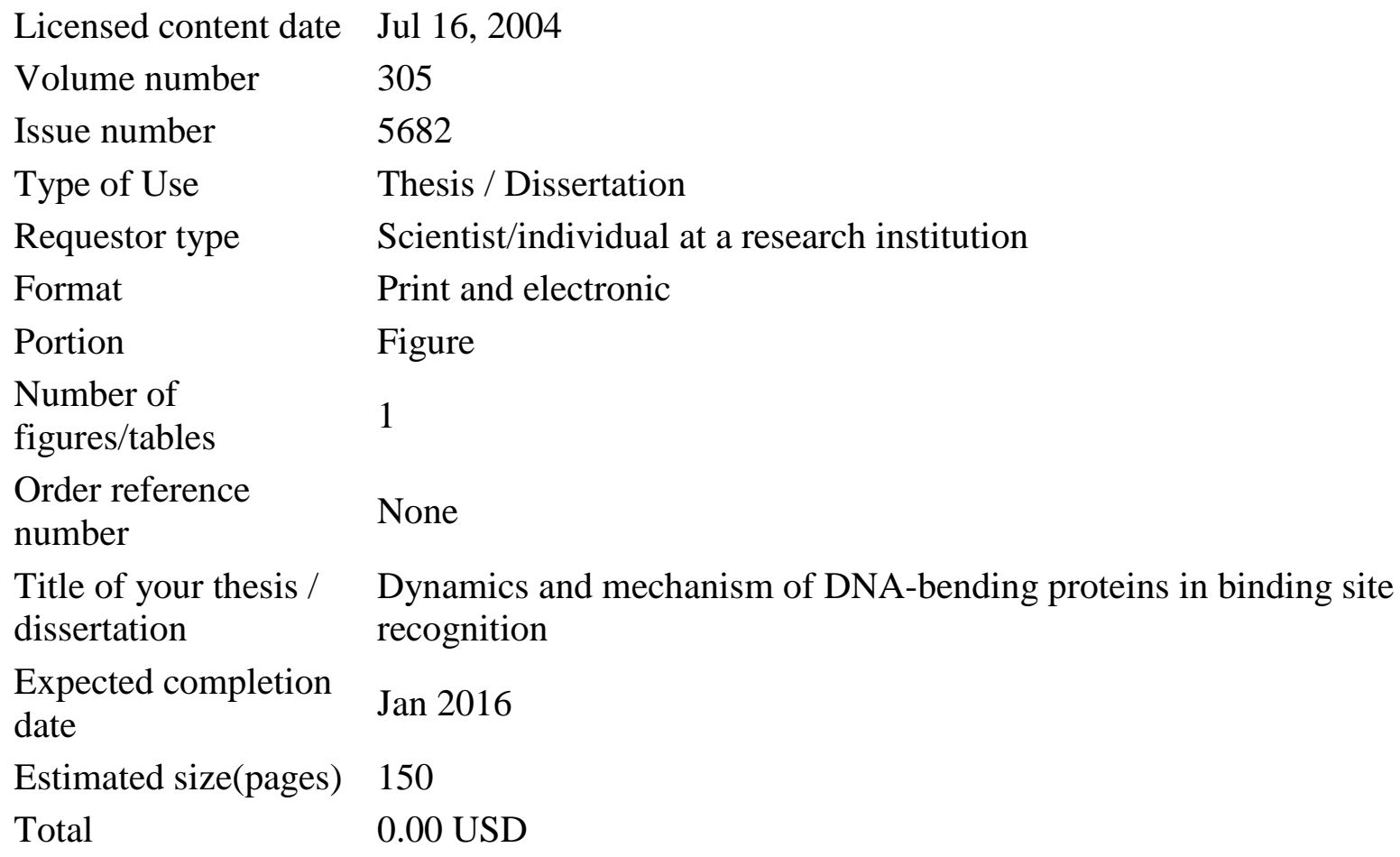

\section{ORDER MORE... CLOSE WINDOW}

Copyright (C) 2016 Copyright Clearance Center, Inc. All Rights Reserved. Privacy statement. Terms and Conditions.

Comments? We would like to hear from you. E-mail us at customercare@ copyright.com 


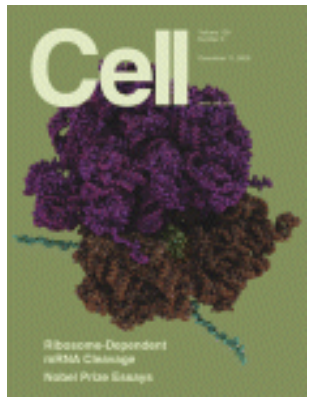

Title:

Crystal Structure of an IHF-

Logged in as:

DNA Complex: A ProteinInduced DNA U-Turn

Author:

Phoebe A Rice,Shu-wei Yang,Kiyoshi

Mizuuchi,Howard A Nash

Publication: Cell

Velmurugu

Yogambigai

Account \#: 3000985545

\section{LOGOUT}

Publisher: Elsevier

Date: 27 December 1996

Copyright (C) 1996 Cell Press. Published by

Elsevier Inc. All rights reserved.

Order Completed

Thank you very much for your order.

This is a License Agreement between Velmurugu Yogambigai ("You") and Elsevier ("Elsevier"). The license consists of your order details, the terms and conditions provided by Elsevier, and the payment terms and conditions.

Get the printable license.

License Number

License date

Licensed content publisher

Licensed content publication

Licensed content title

Licensed content author

Licensed content date

Licensed content volume number

Licensed content issue number

Number of pages

Type of Use

Portion

Number of figures/tables/illustrations

Format

Are you the author of this Elsevier article?
3826000007901

Mar 11, 2016

Elsevier

Cell

Crystal Structure of an IHF-DNA Complex: A ProteinInduced DNA U-Turn

Phoebe A Rice,Shu-wei Yang,Kiyoshi

Mizuuchi,Howard A Nash

27 December 1996

87

7

12

reuse in a thesis/dissertation

figures/tables/illustrations

both print and electronic

No 
Will you be translating?

Original figure numbers

Title of your thesis/dissertation

Expected completion date

Estimated size (number of pages)

Elsevier VAT number

Permissions price

VAT/Local Sales Tax

Total
No

Fig2a

Dynamics and mechanism of DNA-bending proteins in binding site recognition

Jan 2016

150

GB 494627212

0.00 USD

0.00 USD / 0.00 GBP

0.00 USD

\section{ORDER MORE... CLOSE WINDOW}

Copyright (C) 2016 Copyright Clearance Center, Inc. All Rights Reserved. Privacy statement. Terms and Conditions.

Comments? We would like to hear from you. E-mail us at customercare@ copyright.com 


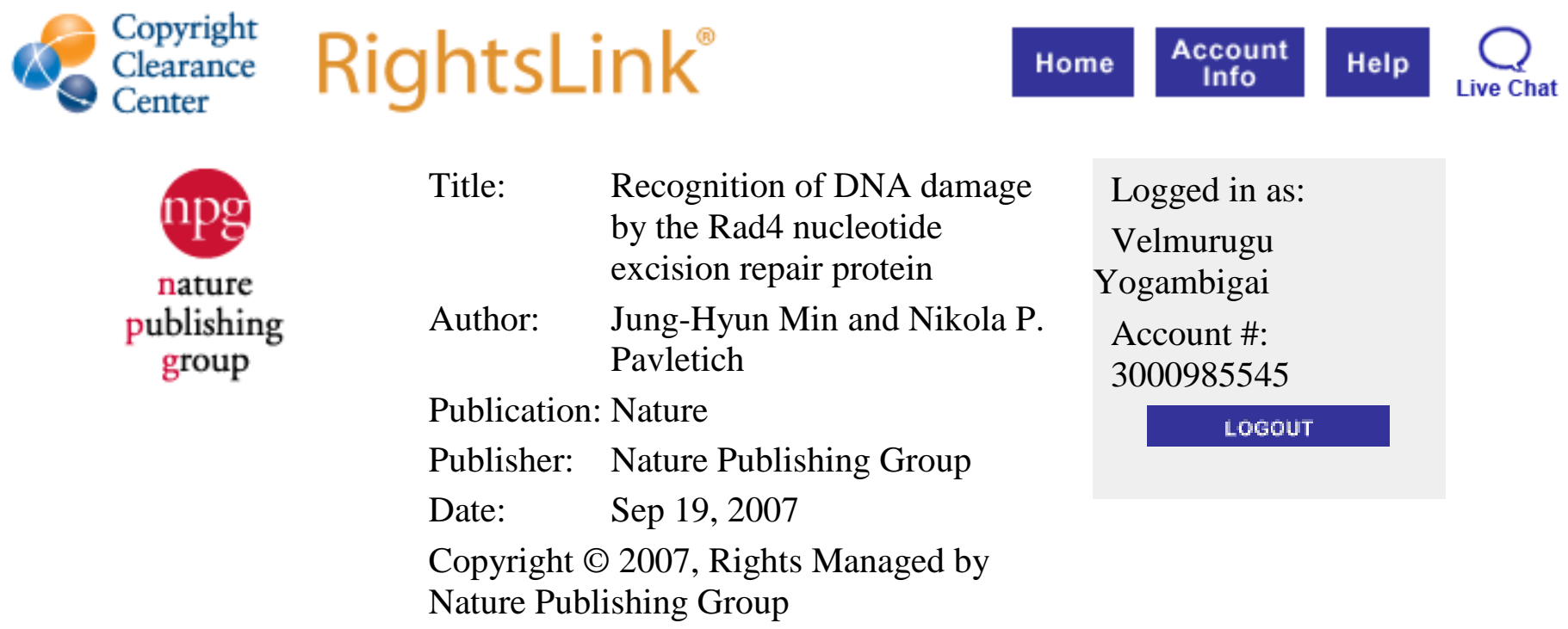

Order Completed

Thank you very much for your order.

This is a License Agreement between Velmurugu Yogambigai ("You") and Nature Publishing Group ("Nature Publishing Group"). The license consists of your order details, the terms and conditions provided by Nature Publishing Group, and the payment terms and conditions.

Get the printable license.

License Number

License date

Licensed content publisher

Licensed content publication

Licensed content title

Licensed content author

Licensed content date

Type of Use

Volume number

Issue number

Requestor type

Format

Portion
3826010285455

Mar 11, 2016

Nature Publishing Group

Nature

Recognition of DNA damage by the Rad4 nucleotide excision repair protein

Jung-Hyun Min and Nikola P. Pavletich

Sep 19, 2007

reuse in a dissertation / thesis

449

7162

academic/educational

print and electronic

figures/tables/illustrations

Number of figures/tables/illustrations 1

High-res required

no

Figures

Crystal structure of Rad4-DNA

Author of this NPG article

no 
Your reference number

Title of your thesis / dissertation

Expected completion date

Estimated size (number of pages)

Total

\section{ORDER MORE...}

Copyright (C) 2016 Copyright Clearance Center, Inc. All Rights Reserved. Privacy statement. Terms and Conditions.

Comments? We would like to hear from you. E-mail us at customercare@ copyright.com
None

Dynamics and mechanism of DNA-bending proteins in binding site recognition

150

0.00 USD
Jan 2016

\section{CLOSE WINDOW}

E-mail us at customercare@copyright.com 


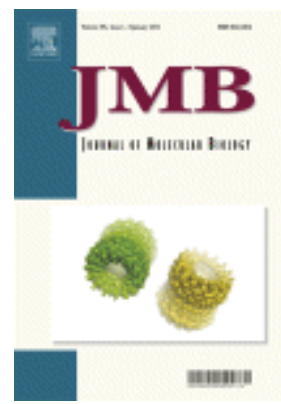

Title:

Mapping the Transition State

Logged in as:

for DNA Bending by IHF

Author:

Paula Vivas, Yogambigai

Velmurugu

Velmurugu,Serguei V.

Kuznetsov,Phoebe A.

Yogambigai

Rice,Anjum Ansari

Publication: Journal of Molecular Biology

Publisher: Elsevier

Date: $\quad 18$ May 2012

Copyright (C) 2012 Elsevier Ltd. All rights reserved.

\section{Order Completed}

Thank you very much for your order.

This is a License Agreement between Velmurugu Yogambigai ("You") and Elsevier ("Elsevier"). The license consists of your order details, the terms and conditions provided by Elsevier, and the payment terms and conditions.

Get the printable license.

License Number

License date

Licensed content publisher

Licensed content publication

Licensed content title

Licensed content author

Licensed content date

Licensed content volume number

Licensed content issue number

Number of pages

Type of Use

Portion

Number of figures/tables/illustrations 7

Format

Are you the author of this Elsevier article?

Will you be translating?
3776720364829

Dec 26, 2015

Elsevier

Journal of Molecular Biology

Mapping the Transition State for DNA Bending by IHF

Paula Vivas, Yogambigai Velmurugu,Serguei V.

Kuznetsov,Phoebe A. Rice,Anjum Ansari

18 May 2012

418

5

16

reuse in a thesis/dissertation

figures/tables/illustrations

both print and electronic

Yes

No 
Original figure numbers

Title of your thesis/dissertation

Expected completion date

Estimated size (number of pages)

Elsevier VAT number

Permissions price

VAT/Local Sales Tax

Total

\section{ORDER MORE...}

CLOSE WINDOW

Fig.1, Fig.2, Fig.4(a), Fig.5, Fig.7(a), fig.8(a),(b),Fig.10

Dynamics and mechanism of DNA-bending proteins in binding site recognition

Jan 2016

150

GB 494627212

0.00 USD

$0.00 \mathrm{USD} / 0.00 \mathrm{GBP}$

0.00 USD

Copyright (C) 2015 Copyright Clearance Center, Inc. All Rights Reserved. Privacy statement. Terms and Conditions.

Comments? We would like to hear from you. E-mail us at customercare@ copyright.com 


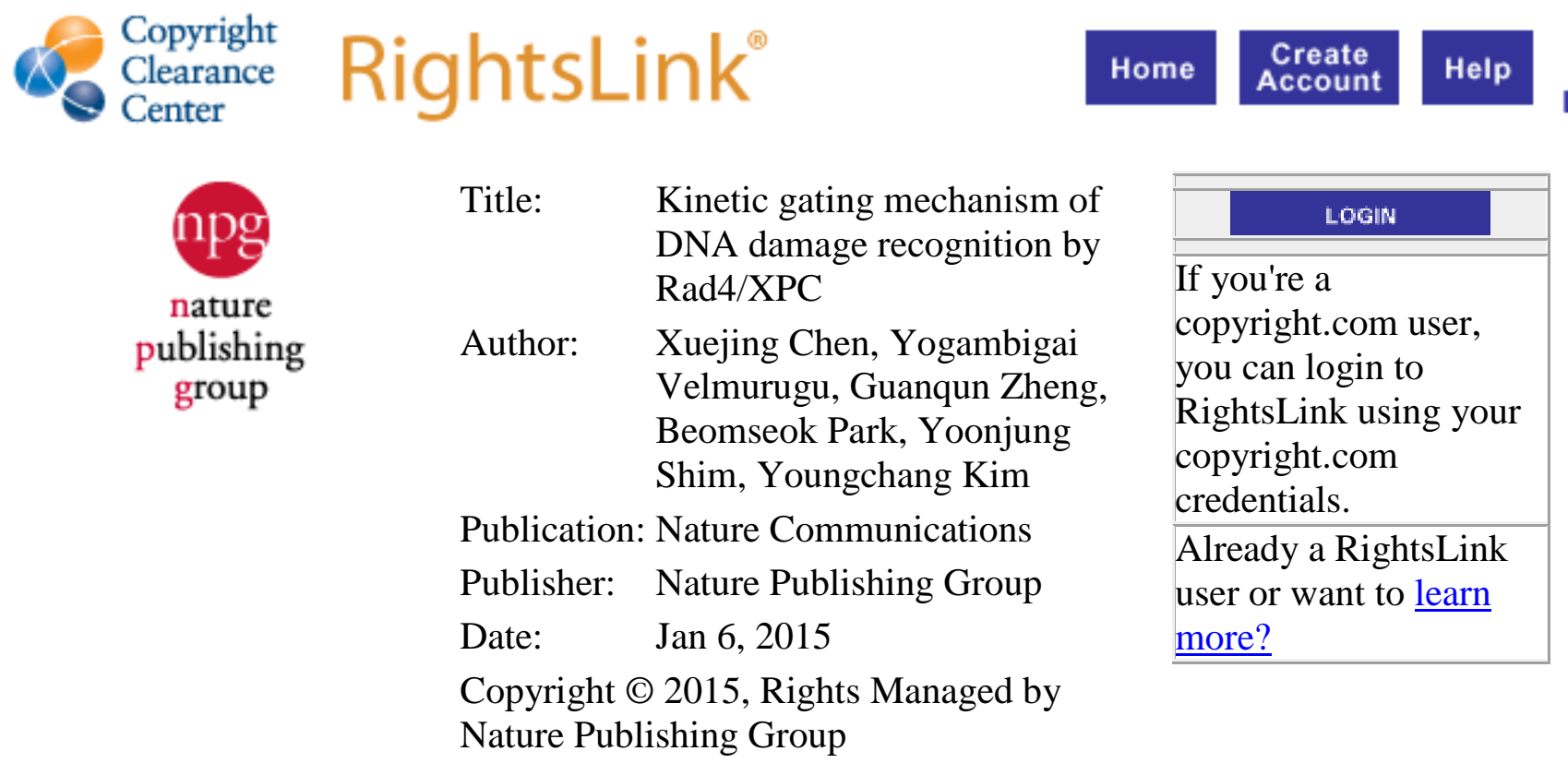

Author Use

Authors of NPG articles do not require permission to use content from their article in most cases as stated in the author's guidelines.

Authors wishing to use their article for commercial purposes must request permission in the normal way.

For further questions, please contact NPG's permissions department: permissions@ nature.com

\section{BACK}

\section{CLOSE WINDOW}

For commercial reprints of this content, please select the Order Commercial Reprints link located beside the Rights and Permissions link on the Nature Publishing Group Web site. 


\section{Copyright Clearance Center \\ Logged in as: \\ Dynamics and Mechanism of DNA-Bending Proteins in Binding Site Recognition \\ the language of science \\ Title: \\ Author: Anjum Ansari
Publication: Springer eBook \\ Publisher: Springer \\ Date: Jan 1, 2010 \\ Copyright @ 2010, Springer Science+Business Media, LLC \\ Velmurugu Yogambigai \\ Account \#: 3000985545 \\ LOGOUT}

\section{Order Completed}

Thank you very much for your order.

This is a License Agreement between Velmurugu Yogambigai ("You") and Springer ("Springer"). The license consists of your order details, the terms and conditions provided by Springer, and thepayment terms and conditions.

\section{Get the printable license.}

\begin{tabular}{ll} 
License Number & 3825570506030 \\
\hline License date & Mar 10, 2016 \\
\hline Licensed content publisher & Springer \\
\hline Licensed content publication & Springer eBook \\
\hline Licensed content title & Dynamics and Mechanism of DNA-Bending Proteins in Binding Site Recognition \\
\hline Licensed content author & Anjum Ansari \\
\hline Licensed content date & Jan 1, 2010 \\
\hline Type of Use & Thesis/Dissertation \\
\hline Portion & Figures/tables/illustrations \\
\hline $\begin{array}{l}\text { Number of } \\
\text { figures/tables/illustrations }\end{array}$ & 1 \\
\hline Author of this Springer article & No \\
\hline Original figure numbers & Figure 6.1 \\
\hline $\begin{array}{l}\text { Title of your thesis / } \\
\text { dissertation }\end{array}$ & Dynamics and mechanism of DNA -bending proteins in binding site recognition \\
\hline Expected completion date & Jan 2016 \\
\hline Estimated size(pages) & 150 \\
\hline Total & 0.00 USD \\
\hline
\end{tabular}

\section{CLOSE WINDOW}

Copyright (c) 2016 Copyright Clearance Center, Inc. All Rights Reserved. Privacy statement. Terms and Conditions. Comments? We would like to hear from you. E-mail us at customercare@copyright.com 


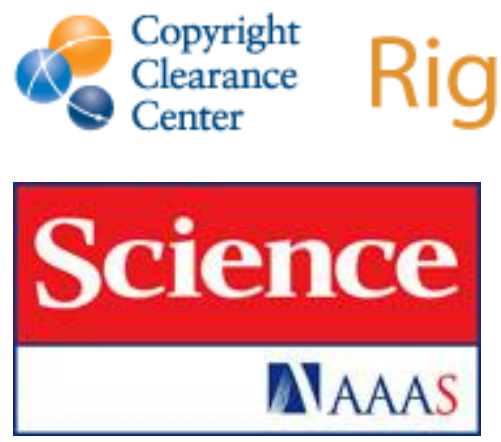

Title:

Structure and Flexibility

Logged in as:

Adaptation in Nonspecific and Specific Protein-DNA Complexes

Author: Charalampos G.

Kalodimos,Nikolaos

Biris,Alexandre M. J. J.

Bonvin,Marc M.

Levandoski,Marc

Guennuegues,Rolf

Boelens, Robert Kaptein

Publication: Science

Publisher: The American Association for the Advancement of Science

Date: Jul 16, 2004

Copyright @ 2004, The American

Association for the Advancement of

Science
Velmurugu

Yogambigai

Account \#:

3000985545

LOGOUT

Order Completed

Thank you very much for your order.

This is a License Agreement between Velmurugu Yogambigai ("You") and The American Association for the Advancement of Science ("The American Association for the Advancement of Science"). The license consists of your order details, the terms and conditions provided by The American Association for the Advancement of Science, and the payment terms and conditions.

Get the printable license.

License Number

License date

Licensed content publisher

Licensed content publication

Licensed content title

Licensed content author
3825990353852

Mar 11, 2016

The American Association for the Advancement of Science

Science

Structure and Flexibility Adaptation in Nonspecific and Specific Protein-DNA Complexes

Charalampos G. Kalodimos,Nikolaos Biris,Alexandre M. J. J. Bonvin,Marc M. Levandoski,Marc Guennuegues, Rolf Boelens, Robert Kaptein 


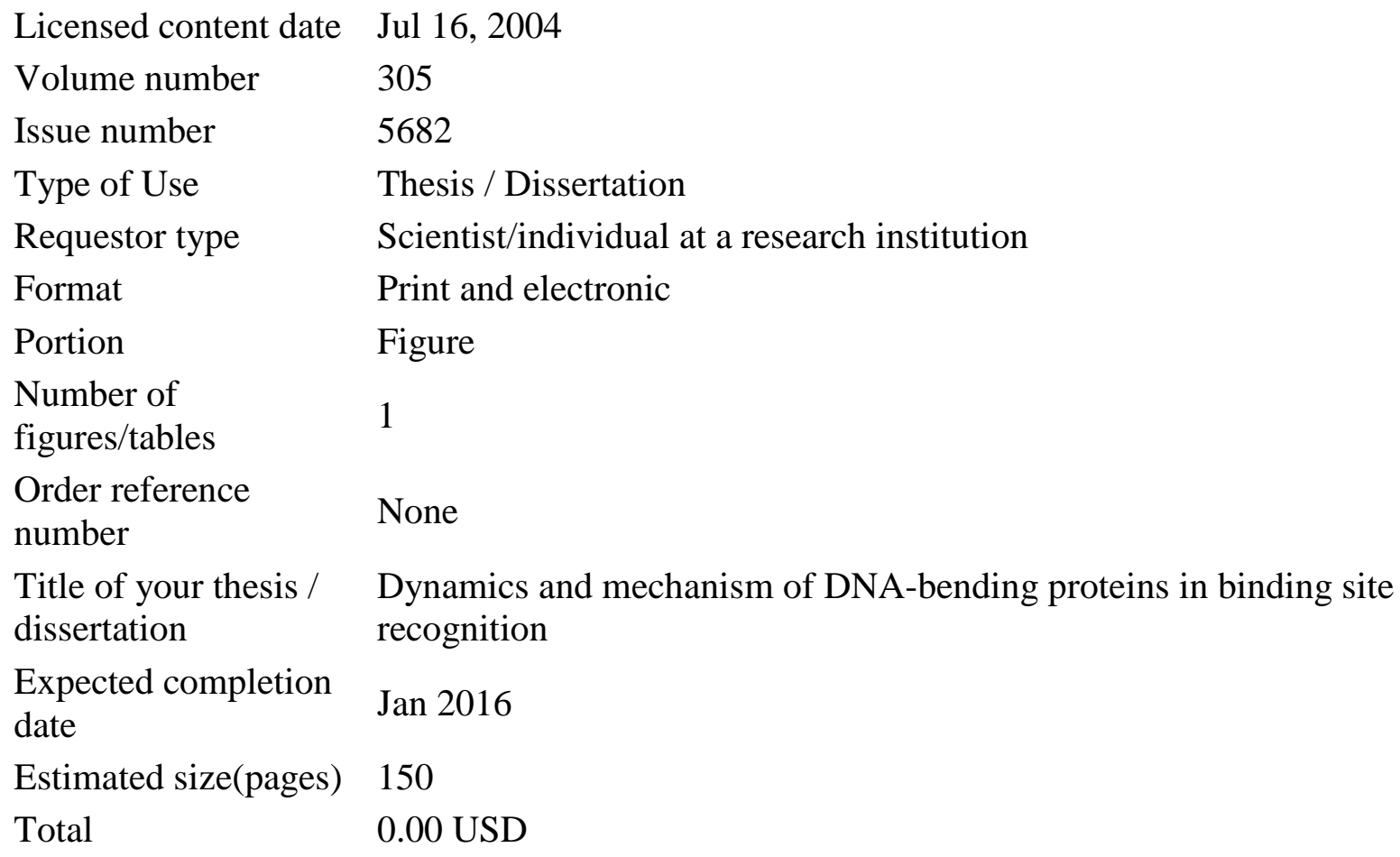

\section{ORDER MORE... CLOSE WINDOW}

Copyright (C) 2016 Copyright Clearance Center, Inc. All Rights Reserved. Privacy statement. Terms and Conditions.

Comments? We would like to hear from you. E-mail us at customercare@ copyright.com 


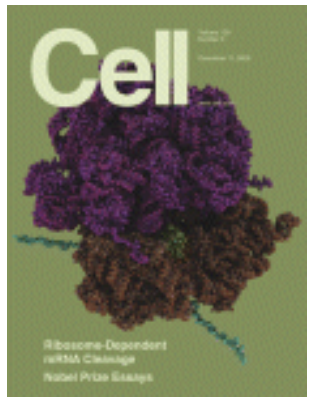

Title:

Crystal Structure of an IHF-

Logged in as:

DNA Complex: A ProteinInduced DNA U-Turn

Author:

Phoebe A Rice,Shu-wei Yang,Kiyoshi

Mizuuchi,Howard A Nash

Publication: Cell

Velmurugu

Yogambigai

Account \#: 3000985545

\section{LOGOUT}

Publisher: Elsevier

Date: 27 December 1996

Copyright (C) 1996 Cell Press. Published by

Elsevier Inc. All rights reserved.

Order Completed

Thank you very much for your order.

This is a License Agreement between Velmurugu Yogambigai ("You") and Elsevier ("Elsevier"). The license consists of your order details, the terms and conditions provided by Elsevier, and the payment terms and conditions.

Get the printable license.

License Number

License date

Licensed content publisher

Licensed content publication

Licensed content title

Licensed content author

Licensed content date

Licensed content volume number

Licensed content issue number

Number of pages

Type of Use

Portion

Number of figures/tables/illustrations

Format

Are you the author of this Elsevier article?
3826000007901

Mar 11, 2016

Elsevier

Cell

Crystal Structure of an IHF-DNA Complex: A ProteinInduced DNA U-Turn

Phoebe A Rice,Shu-wei Yang,Kiyoshi

Mizuuchi,Howard A Nash

27 December 1996

87

7

12

reuse in a thesis/dissertation

figures/tables/illustrations

both print and electronic

No 
Will you be translating?

Original figure numbers

Title of your thesis/dissertation

Expected completion date

Estimated size (number of pages)

Elsevier VAT number

Permissions price

VAT/Local Sales Tax

Total
No

Fig2a

Dynamics and mechanism of DNA-bending proteins in binding site recognition

Jan 2016

150

GB 494627212

0.00 USD

0.00 USD / 0.00 GBP

0.00 USD

\section{ORDER MORE... CLOSE WINDOW}

Copyright (C) 2016 Copyright Clearance Center, Inc. All Rights Reserved. Privacy statement. Terms and Conditions.

Comments? We would like to hear from you. E-mail us at customercare@ copyright.com 


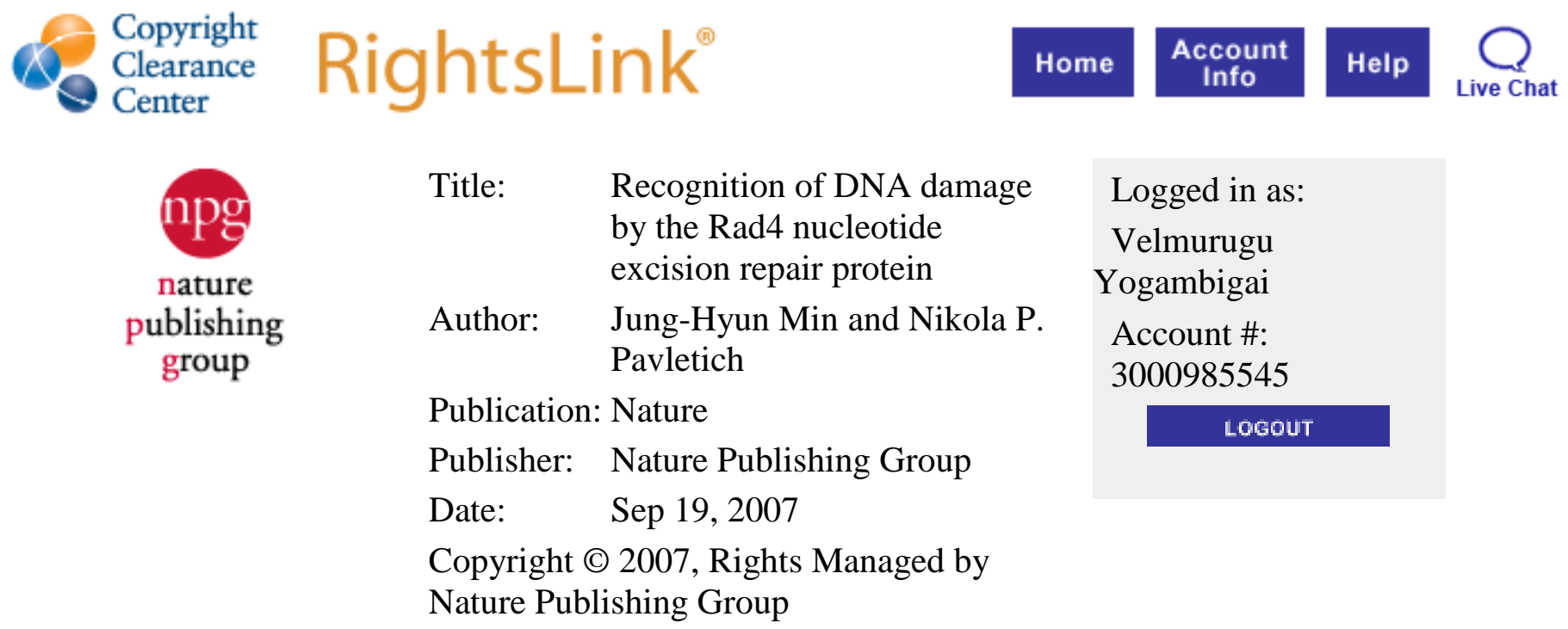

Order Completed

Thank you very much for your order.

This is a License Agreement between Velmurugu Yogambigai ("You") and Nature Publishing Group ("Nature Publishing Group"). The license consists of your order details, the terms and conditions provided by Nature Publishing Group, and the payment terms and conditions.

Get the printable license.

License Number

License date

Licensed content publisher

Licensed content publication

Licensed content title

Licensed content author

Licensed content date

Type of Use

Volume number

Issue number

Requestor type

Format

Portion
3826010285455

Mar 11, 2016

Nature Publishing Group

Nature

Recognition of DNA damage by the Rad4 nucleotide excision repair protein

Jung-Hyun Min and Nikola P. Pavletich

Sep 19, 2007

reuse in a dissertation / thesis

449

7162

academic/educational

print and electronic

figures/tables/illustrations

Number of figures/tables/illustrations 1

High-res required

no

Figures

Crystal structure of Rad4-DNA

Author of this NPG article

no 
Your reference number

Title of your thesis / dissertation

Expected completion date

Estimated size (number of pages)

Total

\section{ORDER MORE...}

Copyright (C) 2016 Copyright Clearance Center, Inc. All Rights Reserved. Privacy statement. Terms and Conditions.

Comments? We would like to hear from you. E-mail us at customercare@ copyright.com
None

Dynamics and mechanism of DNA-bending proteins in binding site recognition

150

0.00 USD
Jan 2016

\section{CLOSE WINDOW}

E-mail us at customercare@copyright.com 


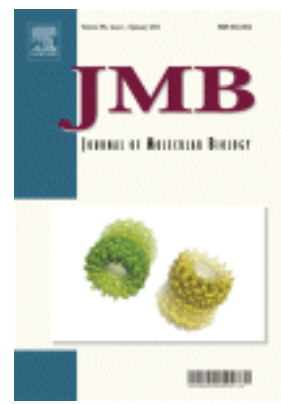

Title:

Mapping the Transition State

Logged in as:

for DNA Bending by IHF

Author:

Paula Vivas, Yogambigai

Velmurugu

Velmurugu,Serguei V.

Kuznetsov,Phoebe A.

Yogambigai

Rice,Anjum Ansari

Publication: Journal of Molecular Biology

Publisher: Elsevier

Date: $\quad 18$ May 2012

Copyright (C) 2012 Elsevier Ltd. All rights reserved.

\section{Order Completed}

Thank you very much for your order.

This is a License Agreement between Velmurugu Yogambigai ("You") and Elsevier ("Elsevier"). The license consists of your order details, the terms and conditions provided by Elsevier, and the payment terms and conditions.

Get the printable license.

License Number

License date

Licensed content publisher

Licensed content publication

Licensed content title

Licensed content author

Licensed content date

Licensed content volume number

Licensed content issue number

Number of pages

Type of Use

Portion

Number of figures/tables/illustrations 7

Format

Are you the author of this Elsevier article?

Will you be translating?
3776720364829

Dec 26, 2015

Elsevier

Journal of Molecular Biology

Mapping the Transition State for DNA Bending by IHF

Paula Vivas, Yogambigai Velmurugu,Serguei V.

Kuznetsov,Phoebe A. Rice,Anjum Ansari

18 May 2012

418

5

16

reuse in a thesis/dissertation

figures/tables/illustrations

both print and electronic

Yes

No 
Original figure numbers

Title of your thesis/dissertation

Expected completion date

Estimated size (number of pages)

Elsevier VAT number

Permissions price

VAT/Local Sales Tax

Total

\section{ORDER MORE...}

CLOSE WINDOW

Fig.1, Fig.2, Fig.4(a), Fig.5, Fig.7(a), fig.8(a),(b),Fig.10

Dynamics and mechanism of DNA-bending proteins in binding site recognition

Jan 2016

150

GB 494627212

0.00 USD

$0.00 \mathrm{USD} / 0.00 \mathrm{GBP}$

0.00 USD

Copyright (C) 2015 Copyright Clearance Center, Inc. All Rights Reserved. Privacy statement. Terms and Conditions.

Comments? We would like to hear from you. E-mail us at customercare@ copyright.com 


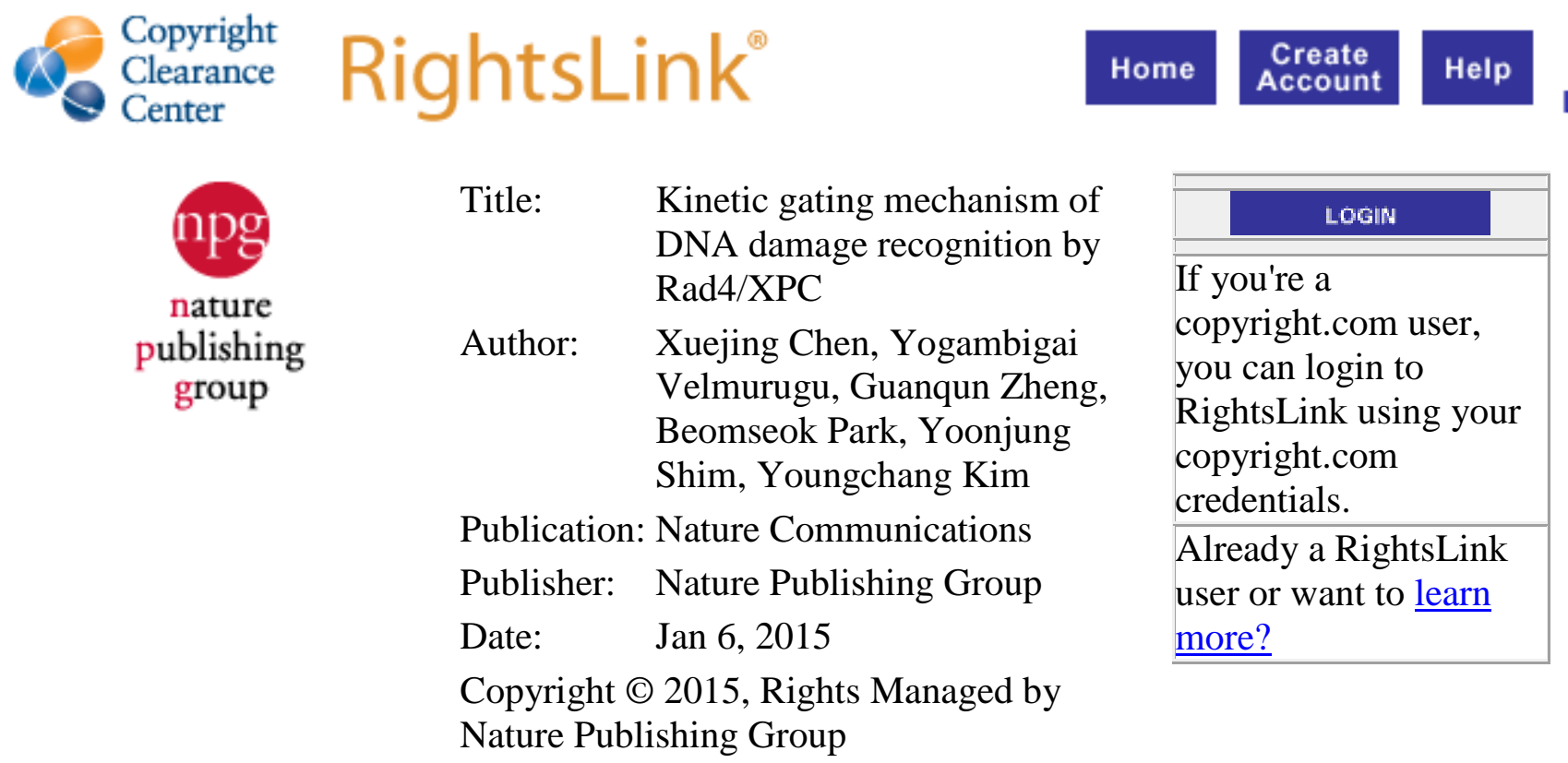

Author Use

Authors of NPG articles do not require permission to use content from their article in most cases as stated in the author's guidelines.

Authors wishing to use their article for commercial purposes must request permission in the normal way.

For further questions, please contact NPG's permissions department: permissions@ nature.com

\section{BACK}

\section{CLOSE WINDOW}

For commercial reprints of this content, please select the Order Commercial Reprints link located beside the Rights and Permissions link on the Nature Publishing Group Web site. 